\begin{tabular}{|c|c|c|c|c|c|c|c|}
\hline NOME & PARTIDO & UF & CARGO & $\begin{array}{l}\text { ÁREA DO } \\
\text { IMÓVEL }\end{array}$ & $\begin{array}{l}\text { № DE } \\
\text { IMÓVEIS }\end{array}$ & $\begin{array}{l}\text { TERRAS NO } \\
\text { INCRA (HA) } \\
\text { /No IMÓVEIS }\end{array}$ & EMPRESAS AGRONEGOCIO OU OUTRO BEM RURAL \\
\hline TEOTONIO BRANDÃO VILELA FILHO & PSDB & AL & Senador & 477,1 & 25 & $34(1)$ & $\begin{array}{l}\text { AÇÕES: USINAS REUNIDAS SERESTA S.A.; SOCŐCO S.A. INDUSTRIA } \\
\text { ALIMENTICIA; SOCÔCO AGROCINDUSTRIA DA AMAZONIA; QUOTAS } \\
\text { DE CAPITAL DE ADUBOPS BOA SORTE; CAPITAL DA } \\
\text { AGROPECUARIA INDIANA; QUOTAS CAPITAL AGUA MINERAL } \\
\text { FONTES DAS MADEIRAS LTDA }\end{array}$ \\
\hline $\begin{array}{l}\text { JOÃO THOMÉ VERÇOSA MESTRINHO } \\
\text { DE MEDEIROS RAPOSO }\end{array}$ & PMDB & AM & Deputado Federal & 1522 & 0 & 0 & $\begin{array}{l}\text { Terreno A Margem Esquerda Do Rio Manacapuru Adquirido Em 1998; } 4 \\
\text { Terrenos A Margem Direita Do Parana Do Sumauma Supia; Participacao } \\
\text { De } 98 \text { Do Capital Social Da Empresa Agropecuaria Thira Ltda Cnpj } 84478 \\
833000152\end{array}$ \\
\hline BENEDITO DIAS DE CARVALHO & PPB & AP & Deputado Federal & $\mathrm{NI}$ & 2 & & Participação em 50\% nas Fazendas São Luiz e Liberdade, Chaves/PA \\
\hline EDSON SAMPAIO PIMENTA & PC DO B & BA & Deputado Federal & 6 & & & $\begin{array}{l}\text { PROPRIEDADE AGRICOLA ENERGIA SOLAR - IBICOARA; FAZENDA } \\
\text { ENERGIA SOLAR - IBICOARA ; COTAS SICOOB; TERRENO } 6 \text { HÁ }\end{array}$ \\
\hline CORIOLANO SOUSA SALES & PMDB & BA & Deputado Federal & 520 & 1 & & $\begin{array}{l}\text { QUOTAS DE CAPITAL DA COOPERATIVA DE CRÉDITO RURAL } \\
\text { CONQUISTA LTDA. Em } 2010 \text { declarou Um Imovel Rural Denominado } \\
\text { Pedra Branca No Municipio De Presidente Janio Quadros Bahia R } \$ \\
400.000,00\end{array}$ \\
\hline COLBERT MARTINS DA SILVA FILHO & PPS & BA & Deputado Federal & $425,57+5 \mathrm{NI}$ & 10 & $785(6)$ & \\
\hline SEVERIANO ALVES DE SOUZA & PDT & BA & Deputado Federal & 488 & 3 & & $\begin{array}{l}1500 \text { Quotas de capital Social da Mariquita Agroindustria Comércio de } \\
\text { Combustível e Serviços LTDA CNPJ 16359/001-12 }\end{array}$ \\
\hline JOÃO ALMEIDA DOS SANTOS & PSDB & BA & Deputado Federal & $1298+\mathrm{NI}$ & 3 & & $\begin{array}{l}\text { QUOTAS - COOPERATIVAS DE CRÉDITO RURAL DE EUNAPÓLIS } \\
\text { LTDA }\end{array}$ \\
\hline CÉSAR AUGUSTO RABELLO BORGES & PFL & BA & Senador & 886,6 & 3 & & $\begin{array}{l}\text { Fazenda Provisao Com 645,36 Ha Incorporada Ao Conj Maravilha/Silencio; } \\
\text { Condominio Fazenda Corcovado/Cj Maravilha/Silencio E Condominio De } \\
\text { Imoveis Waldomiro Borges; Fazenda Corcovado, 73,10 Ha, Ipiau, Ba; } \\
\text { Fazenda Cachoeirinha, } 265 \text { Ha, Ibirataia, BA; Do Imovel Cj } \\
\text { Maravilha/Silencio Com 548,50 Ha, Jequie, BA }\end{array}$ \\
\hline TASSO RIBEIRO JEREISSATI & PSDB & CE & Senador & 91,44 & 1 & & $\begin{array}{l}\text { imóvel denominado "Lagoa" em Paracurú; Agropecuaria Jereissati Saldo } \\
\text { De Emprestimo no valor de } \mathrm{R} \$ 4.007 .561,70\end{array}$ \\
\hline IZALCI LUCAS FERREIRA & PR & DF & Deputado Federal & ? & ? & & $\begin{array}{l}\text { 50\% CHACARA } 13 \text { /RESERVA G GLEBA } 03 \text { PICAG; NO LOTE NA } \\
\text { CHACARA NA RESERVA G GLEBA } 3 \text { ARAEA /RURAL SOMA VALOR R } \$ \\
608.000,00\end{array}$ \\
\hline $\begin{array}{l}\$ \text { JOSÉ FUSCALDI CESÍLIO } \\
\end{array}$ & PSD & DF & Deputado Federal & 1248 & 2 & $5.239(4)$ & $\begin{array}{l}10.000 \text { cabeças de gado misto; participação de } 3 \% \text { da empresa Itatico } \\
\text { comércio de alimentos LTDA }\end{array}$ \\
\hline RODRIGO SOBRAL ROLLEMBERG & PSB & DF & Senador & 209 & 5 & & \\
\hline
\end{tabular}




\begin{tabular}{|c|c|c|c|c|c|c|c|}
\hline NOME & PARTIDO & UF & CARGO & $\begin{array}{l}\text { ÁREA DO } \\
\text { IMÓVEL }\end{array}$ & $\begin{array}{l}\text { № DE } \\
\text { IMÓVEIS }\end{array}$ & $\begin{array}{l}\text { TERRAS NO } \\
\text { INCRA (HA) } \\
\text { /NN IMÓVEIS }\end{array}$ & EMPRESAS AGRONEGOCIO OU OUTRO BEM RURAL \\
\hline PAULO ROBERTO FOLETTO & PSB & ES & Deputado Federal & ? & & & $\begin{array}{l}50 \% \text { DE UM TERRENO RURAL SITUADO EM SAO PEDRO FRIO } \\
\text { COLATINA ES; CENTO DE UM TERRENO RURAL SITUADO MONTE } \\
\text { BELO COLATINA ES; } 50 \text { POR CENTO DE UM TERRENO RURAL } \\
\text { SITUADO EM CORREGO TAQUARAPOCA COLATINA; UM TERCO POR } \\
\text { CENTO DE UM TERRENO RURAL SITUADO EM SAO PEDRO FRIO } \\
\text { PANCAS R } \$ 114262,00 ; \text { SICOOB ES }\end{array}$ \\
\hline MARCELINO AYUB FRAGA & PMDB & ES & Deputado Federal & $974,41+2 \mathrm{NI}$ & 6 & & $\begin{array}{l}\text { 463.566,86 AÇÕES DA EMPRESA FAZENDA REUNIDAS CAIADO } \\
\text { FRAGA }\end{array}$ \\
\hline $\begin{array}{l}\text { NILTON GOMES DE OLIVEIRA (NILTON } \\
\text { BAIANO) }\end{array}$ & PPB & ES & Deputado Federal & 1799 & 2 & $107(!)$ & Terra em Conceição do Araguaia/PA \\
\hline $\begin{array}{l}\text { RAQUEL FIGUEIREDO ALESSANDRI } \\
\text { TEIXEIRA }\end{array}$ & PSDB & GO & Deputado Federal & 2250,06 & 2 & & AÇÃO DO COUNTRY CLUBE DE GOIÁS \\
\hline JOSÉ RIBAMAR COSTA ALVES & PSB & MA & Deputado Federal & 3033,72 & 2 & & \\
\hline $\begin{array}{l}\text { JOSE ALBERTO OLIVEIRA VELOSO } \\
\text { FILHO }\end{array}$ & PMDB & MA & Deputado Federal & 0 & 0 & & 24 VACAS NELORE PO COM REGISTRO VALOR R $\$ 72.000,00$ \\
\hline NICE LOBÁO & DEM & $\mathrm{MA}$ & Deputado Federal & & & & ESPOSA DE EDSON LOBÃO \\
\hline CARLOS ORLEANS BRANDAO JUNIOR & PSDB & MA & Deputado Federal & & & $250(1)$ & $\begin{array}{l}\text { ALTERAÇÃO CONTRATUAL NAS QUOTAS DE CAPITAL DA FIRMA } \\
\text { COAGRI LTDA R\$39.000,00; FOI Diretor, COAGRI - Colinas } \\
\text { Agropecuária Indústria e Comércio Ltda; SECRETÁRIO DE } \\
\text { AGRICULTURA DO ESTADO DO MARANHÃO }\end{array}$ \\
\hline JOAO CASTELO RIBEIRO GONCALVES & PSDB & MA & Deputado Federal & 0 & & $6.018(2)$ & $\begin{array}{l}\text { AÇÖES DA FAZENDA MODELO S.A. CODÓ/MA EM NOME DA ESPOSA; } \\
271 \text { QUOTAS DE CAPITAL DA GRANOLEO S.A. }\end{array}$ \\
\hline ANTONIO JOAQUIM ARAUJO FILHO & PPB & $\mathrm{MA}$ & Deputado Federal & 8727,6 & 4 & $289(1)$ & 5650 CABEÇAS DE GADO VACUM \\
\hline PEDRO FERNANDES RIBEIRO & PFL & MA & Deputado Federal & 550 & 2 & & 51 CABEÇAS DE GADO BOVINO \\
\hline REMI ABREU TRINTA & $\mathrm{PL}$ & MA & Deputado Federal & 0 & & $12(1)$ & $\begin{array}{l}\text { 67 CABEÇAS DE BUFALOS; } 56 \text { CABEÇAS DE GADO COMUM, } \\
\text { DECLAROU UM MINIFÚNDIO NO INCRA }\end{array}$ \\
\hline ROSEANA SARNEY MURAD & PFL & MA & Senador & $9,12+\mathrm{NI}$ & 2 & & \\
\hline MARIO DE OLIVEIRA & PSC & MG & Deputado Federal & & & & $\begin{array}{l}\text { 500 CABEÇAS DE NOVILHAS/BEZERROS, EM TERRENO DE } \\
\text { TERCEIRO NO ESTADO DE TOCANTIS - R\$500,00 CADA. }\end{array}$ \\
\hline OSMANIO PEREIRA DE OLIVEIRA & PSDB & MG & Deputado Federal & 830,35 & 1 & & $\begin{array}{l}622 \text { cabeças de gado incorporadas a Fazenda Primorosa. Fazenda } \\
\text { Primorosa recebida da dissolução da empresa Primorosa Agropecuaria } \\
\text { LTDA em Itapetininga BA }\end{array}$ \\
\hline MURILO ZAUITH & PFL & MS & Deputado Federal & 958 & 2 & & cotas capital da cooperativa de crédito rural Dourados LTDA \\
\hline RAMEZ TEBET & PMDB & MS & Senador & 8068,64 & 10 & & \\
\hline DILSO SPERAFICO & PSDB & MS & Deputado Federal & ? & ? & ? & $\begin{array}{l}\text { Irmão do Deputado Federal Dilseu João Sperafico, ambos são donos da } \\
\text { empresa agropecuária Sperafico terras no INCRA }\end{array}$ \\
\hline ROGERIO LUCIO SOARES DA SILVA & PMDB & MT & Deputado Federal & 16960,44 & 10 & $17.811(11)$ & \\
\hline
\end{tabular}




\begin{tabular}{|c|c|c|c|c|c|c|c|}
\hline NOME & PARTIDO & UF & CARGO & $\begin{array}{l}\text { ÁREA DO } \\
\text { IMÓVEL }\end{array}$ & $\begin{array}{c}\text { № DE } \\
\text { IMÓVEIS }\end{array}$ & $\begin{array}{l}\text { TERRAS NO } \\
\text { INCRA (HA) } \\
\text { /NNo IMÓVEIS } \\
\end{array}$ & EMPRESAS AGRONEGOCIO OU OUTRO BEM RURAL \\
\hline OLÁVIO SILVA ROCHA & PMDB & PA & Deputado Federal & $\mathrm{NI}$ & 2 & $6.548(2)$ & $\begin{array}{l}\text { Faz. Três Poderes Rodovia Transamazônica, Km 215, Vicinal Belmonte } \\
\text { Km 80, Novo Repartimento }\end{array}$ \\
\hline WANDENKOLK PASTEUR GONCALVES & PSDB & PA & Deputado Federal & 0 & 1 & $117(1)$ & Associação Rural de Altamira, 1976-2006; Diretor, Instituto Teotônio Vilela \\
\hline BENJAMIM GOMES MARANHAO NETO & PMDB & PB & Deputado Federal & 69 & 2 & & $\begin{array}{l}\text { PROPRIETARIO DE } 132 \text { CABEÇAS DE GADO; IMÓVEL RURAL } \\
\text { EXPLORADO SÃO MATEUS E FAZENDA JARDIM - ARARUNA } \\
\text { ARARUNA PB }\end{array}$ \\
\hline $\begin{array}{l}\text { AGUINALDO VELLOSO BORGES } \\
\text { RIBEIRO }\end{array}$ & PP & PB & Deputado Federal & & & & $\begin{array}{l}\text { FILHO DE RURALISTA } 10 \text { CABEÇAS DE GADO DE CRIA E RECRIA R } \$ \\
20.000,00 \text { FOI SECRETÁRIO DE AGRICULTURA DO ESTADO DO } \\
\text { MARANHÃO }\end{array}$ \\
\hline $\begin{array}{l}\text { FERNANDO BEZERRA DE SOUZA } \\
\text { COELHO FILHO }\end{array}$ & PSB & PE & Deputado Federal & & & & SOBRINHO NETO DE OSWALDO SOUZA COELHO RURALISTA \\
\hline JOSÉ SEVERIANO CHAVES & PMDB & PE & Deputado Federal & 757,21 & 2 & & \\
\hline EDUARDO FRANCISCO SCIARRA & PFL & PR & Deputado Federal & & & & $\begin{array}{l}\text { AÇÕES DA PARANAENSE COLONIZAÇÃO AGROPECUARIA } \\
\text { INDUSTRIAL DO ACRE; Participacao De } 10 \text { Ponto } 15 \text { Porcento Da } \\
\text { Empresa Reflorestamento Cascavel Ltda; Pariticipacao De } 10 \text { Ponto } 15 \\
\text { Porcento Na Sociedade Reflorestamento Parana Ltda }\end{array}$ \\
\hline GUSTAVO BONATO FRUET & PMDB & PR & Deputado Federal & 1310,2 & 3 & & \\
\hline AFFONSO ALVES DE CAMARGO NETO & PSDB & PR & Deputado Federal & 0 & 0 & & $\begin{array}{l}\text { 4875 COTAS DA EMPRESA PARANA PECUARIA COM SEDE EM } \\
\text { CURITIBA; Sua família paterna, formada por pecuaristas e donos de } \\
\text { frigoríficos, fornecera quadros políticos ao antigo Partido Republicano } \\
\text { Paranaense }\end{array}$ \\
\hline & & & & & & & $\begin{array}{l}\text { Saldo Das Contas Patrimoniais Na Cocamar- Cooperativa Dos } \\
\text { Cafeicultores De Maringa Ltda, Com Sede E Foro Em Maringa - Pr; Quotas } \\
\text { Capital Na Empresa Agrodiamente Pecuária E Agroflorestal Ltda, Com } \\
\text { Sede Em Curitiba; Credito Pela Venda A Prazo De } 621.970 \text { Quotas De } \\
\text { Capital No Valor Nominal De R } \$ 1,00 \text { Cada Uma Na Empresa } \\
\text { Agrodiamante Pecuária E Agroflorestal Ltda }(\mathrm{R} \$ 181.191,79)\end{array}$ \\
\hline OSMAR FERNANDES DIAS & PDT & PR & Senador & 936,98 & 5 & & \\
\hline MAURO NAZIF RASUL & PSB & $\mathrm{RO}$ & Deputado Federal & $3 \mathrm{NI}$ & 3 & & 531 Cabeças De Gado \\
\hline ERNANDES SANTOS AMORIM & PTB & RO & Deputado Federal & & & & $\begin{array}{l}300 \text { Caprinos E Ovinos, } 29 \text { Suínos, } 1936 \text { Cabeças De Gado Bovinos Ou } \\
\text { Bufalinos, } 2 \text { Lotes Rurais Na Br } 421 \text { Linha C-35 - Montenegro }\end{array}$ \\
\hline GIOVANI CHERINI & PDT & RS & Deputado Federal & & & & $\begin{array}{l}\text { CAPITAL COOP. COAGRISOL; CAPITAL INTEGRALIZADO COTRIJAL; } \\
\text { IMOVEL RURAL - SAO LOURENÇO DO SUL R } \$ 190.570,00 ; 75,4 \mathrm{HA} \\
\text { TERRAS, CAMPOS, MATOS E LAVOURA - SAO LOURENÇO DO SUL; } \\
\text { PROPRIEDADE RURAL SAO LOURENÇO DO SUL SOMAM } 300.472,00\end{array}$ \\
\hline ELISEU LEMOS PADILHA & PMDB & RS & Deputado Federal & 1853,2 & 3 & & $\begin{array}{l}\text { SESMARIA DA LAVRINHA OU ROSARIO, MUNICÍPIO DE PONTES E } \\
\text { LACERDA/MT }\end{array}$ \\
\hline
\end{tabular}




\begin{tabular}{|c|c|c|c|c|c|c|c|}
\hline NOME & PARTIDO & UF & CARGO & $\begin{array}{l}\text { ÁREA DO } \\
\text { IMÓVEL }\end{array}$ & $\begin{array}{l}\text { № DE } \\
\text { IMÓVEIS }\end{array}$ & $\begin{array}{l}\text { TERRAS NO } \\
\text { INCRA (HA) } \\
\text { /NN IMÓVEIS }\end{array}$ & EMPRESAS AGRONEGOCIO OU OUTRO BEM RURAL \\
\hline & & & & & & & $\begin{array}{l}\text { UM CAVALO MECÂNICO COM CARRETA SCANIA; } 100 \text { Tarrefas Moita } \\
\text { Bonita; Sitio Agricola Campo Grande Moita Bonita Se } 50 \text { Tarrefas; } 60 \\
\text { Tarrefas Pov Jacoca Ribeiropolis; } 524 \text { Tarreas Monte Santo Monte Alegre; } \\
600 \text { Tarrefas Pedro Alexandre Bahia; Rural } 22824 \text { Hectares Jacoca Moita } \\
\text { Bonita; Rural } 400 \text { Tarrefas Pedro Alexandre Bahia; } 6 \text { Tarrefas Nossa } \\
\text { Senhora Da Gloria Se; Rural } 380 \text { Tarrefas Velani Riberopolis Se; Rural } 7 \\
\text { Tarefas Moita Bonita; Sitio } 2 \text { Tarrefas Moita Bonita }\end{array}$ \\
\hline JOÃO BOSCO DA COSTA & PSDB & SE & Deputado Federal & 21,71 & 10 & $380(2)$ & \\
\hline JOSÉ CARLOS MACHADO & PFL & SE & Deputado Federal & $?$ & 6 & $524(2)$ & $\begin{array}{l}\text { Semoventes i Bovinos, Bufalinos, Asininos, Eqüinos E Muares - } 1.332 \\
\text { Cabeças X R } \$ 600,00(R \$ 799.200,00)\end{array}$ \\
\hline PAULO ROBERTO GOMES MANSUR & PP & SP & Deputado Federal & & & & $\begin{array}{l}9683 \text { Cabeças de gado; } 4000 \text { Sacos de Sorgo; } 15000 \text { Sacas de Soja; } 300 \\
\text { Sacas de feijão; } 1080 \text { Sacos de sorgo Estoque Prod.Agricolas; } 01 \text { Gado / } \\
\text { Cercas e Pastagens Financiado; } 02 \text { Silos p/ Armazenagem, Marca Pagé } \\
\text { Modelo AS 9114; equipamentos agrpicolas, Pivos, tratores, reboques } \\
\text { agrícolas caminhões }\end{array}$ \\
\hline $\begin{array}{l}\text { JOÃO EDUARDO DADO LEITE DE } \\
\text { CARVALHO }\end{array}$ & PDT & SP & Deputado Federal & $683+\mathrm{NI}$ & 2 & & $\begin{array}{l}\text { fração de } 1 / 15 \text { fazenda Santa maria do Barreiro - Auriflama SP; Areas de } \\
\text { terras 583,0 HA - Porto Velho RO; Veiculo caminhão cavalo mecanico } \\
\text { ARD-0340 ano } 2001 \text {; Veiculo caminhão cavalo mecanico ano } 98 \text { ARD- } \\
\text { 0332; participação societaria 16,66 porcento quotas sociais hmr madeiras } \\
\text { Itda; }\end{array}$ \\
\hline RICARDO IZAR JUNIOR & PV & SP & Deputado Federal & & & & FILHO DE RICARDO NAZIZ IZAR DEPUTADO FEDERALRURALISTA \\
\hline RICARDO NAGIB IZAR & PTB & SP & Deputado Federal & $132,69+?$ & 3 & & \\
\hline ALOYSIO NUNES FERREIRA FILHO & PSDB & SP & Deputado Federal & 875,13 & 3 & $1825(3)$ & $\begin{array}{l}\text { Em } 2010 \text { declarou Imovel Rural Com Bemfeitorias No Municipio De } \\
\text { Guzolandia-SP R } \$ 1.124 .235,00\end{array}$ \\
\hline FRANCISCO MARCELO ORTIZ FILHO & PV & SP & Deputado Federal & 796,7 & 4 & $64(2)$ & $\begin{array}{l}500 \text { AÇÕES DA BUNGE ALIMENTOS AS E SEBDO OE } 13924 \text { E PE } \\
252.000 ;\end{array}$ \\
\hline JOSE ARISTODEMO PINOTTI & PMDB & SP & Deputado Federal & 9094 & 3 & & $\begin{array}{l}\text { FAZENDA EM COMUTAMA/ AM E JARAGUARI/MS; COTAS DE } \\
\text { CAPITAL HARAS GRAMADO S/C LTDA; } 160.000 \text { COTAS DE CAPITAL } \\
\text { DA FIRMA AGROPECUARIA SÃO MIGUEL LTDA REGISTRADO NA } \\
\text { JUNTA COMERCIAL DO DISTRITO FEDERAL }\end{array}$ \\
\hline MICHEL MIGUEL ELIAS TEMER LULIA & PMDB & SP & Deputado Federal & $500+2 ?$ & 3 & & DIREITOS POSSESSÓRIOS DE TERRENOS EM ALTO PARAÍSO/GO \\
\hline JOSÉ MAURÍCIO RABELO & PSD & TO & Deputado Federal & 73 & 2 & & $\begin{array}{l}60 \text { CABEÇAS DE GADO NA FAZENDA PROGRESSO EM ALIANÇA DO } \\
\text { TOCANTINS/TO; } 50 \text { CABECCAS DE GADO NA FAZENDA CHIQUITITITAS } \\
\text { EM FORMOSO DO ARAGUAIA/TO }\end{array}$ \\
\hline AMARILDO MARTINS DA SILVA & PPB & TO & Deputado Federal & 917,6 & 2 & & FAZENDA EM PORTO NACIONAL/TO \\
\hline VICENTE ALVES DE OLIVEIRA & PR & TO & SENADOR & $2864+?$ & 2 & & \\
\hline
\end{tabular}




\begin{tabular}{|c|c|c|c|c|c|c|c|c|c|c|c|c|c|c|}
\hline \multicolumn{15}{|c|}{ ANEXO C - NOME, PARTIDO, GESTÃO, PROFISSÃO, ÁREA DAS TERRAS, VALOR DAS TERRAS E VALOR DO PATRIMÔNIO } \\
\hline моме & PARTIDO & UF & 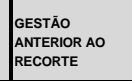 & GESTĀol & GESTĀoll & GEST̄̄ôIII & GESTĀolv & 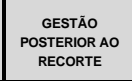 & 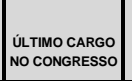 & $\begin{array}{c}\text { PRBFFSAOA } \\
\text { DECLAAADA }\end{array}$ & 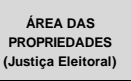 & VALOR DAS TERRAS & \begin{tabular}{|c|} 
VALOR PATRMIMÓNIO \\
TOTAL
\end{tabular} & $\begin{array}{l}\text { \%VALOR DAS TERRAS } \\
\text { PATRIMONONO }\end{array}$ \\
\hline $\begin{array}{l}\text { SERGIO OE OLVERAA CUNHA } \\
\text { (PETECAOO) }\end{array}$ & PMN, 1993 & $\mathrm{AC}$ & 0 & 10 & \% & o & $2007-2011$ & o & SENADOR & 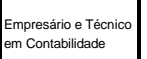 & 0,00 & Rs 0,00 & Rs 282.719,00 & $0.00 \%$ \\
\hline MARCIO MGGUEL BITTAR & $\begin{array}{l}\text { PMDB, 1993-1999; } \\
\text { PPS 1999-2009; } \\
\text { PSDD, 2009 , }\end{array}$ & $\mathrm{AC}$ & 0 & 1999-2003 & 。 & o & o & $2011-2015$ & $\begin{array}{l}\text { DEEUTADO } \\
\text { FEDERAL }\end{array}$ & Agropecuarista & $3.842,00$ & RS 108.653.97 & Rs $390.511,31$ & $27,82 \%$ \\
\hline SERGIO SEBASTIÄO DE BARROS & $\begin{array}{l}\text { Pot, 1966-199; } \\
\text { PSSOBB, 1999 }\end{array}$ & $\mathrm{AC}$ & 0 & 0 & | & & 0 & o & 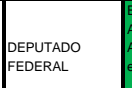 & 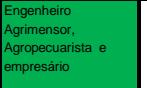 & $8.236,37$ & RS 1.228.083,68 & Rq 2.555.831,15 & $48,05 \%$ \\
\hline $\begin{array}{l}\text { JOĀO TOTA SOARES DE } \\
\text { FIGUERREDO }\end{array}$ & 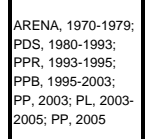 & $\mathrm{AC}$ & |1991-1995 & |1997-1999 & |1999-2003 & $2003-2007$ & & $0^{\circ}$ & $\begin{array}{l}\text { DEEUTADO } \\
\text { FEDERAL }\end{array}$ & $\begin{array}{l}\text { Memblo o o o oder } \\
\text { Execuluo }\end{array}$ & $2.900,00$ & Rs 2.144.957,09 & R\$3 3.591.692,18 & $59,72 \%$ \\
\hline OLAVO CaLEEROS FLHO & 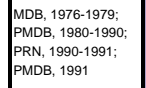 & AL & |1991-1995 & |1995-1999 & |1999-2003 & 2003-2007 & 2007-2011 & 0 & 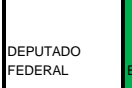 & Engenheiro Agrônomo & 0,00 & $\mathrm{R} \$ 0,00$ & R\$ 4.143.165.51 & $0.00 \%$ \\
\hline $\begin{array}{l}\text { JOSÉ RENAN VASCONCELOS } \\
\text { CALHERIRS }\end{array}$ & 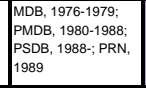 & $\mathrm{AL}$ & $\mid \begin{array}{l}1983-1987 ; 1987 . \\
1991\end{array}$ & |1995-1999 & |1999-2003 & $2003-2007$ & $2011-2015$ & $\begin{array}{l}2 \\
20101-2015 ; ; 2015-\end{array}$ & SENADOR & Aatrogado & 0,00 & R\& 0,00 & Rq 2.182.877,98 & $0,00 \%$ \\
\hline JoĀo CALDAS DA SILVA & 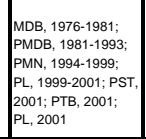 & AL & 0 & & |1999-2003 & 2003-2007 & & & $\begin{array}{l}\text { DEEUTADO } \\
\text { FEDERAL }\end{array}$ & Aavogado & 0,00 & R\& 0,00 & R\$ $213.600,00$ & $0,00 \%$ \\
\hline LUI DANAAS LIMA & \begin{tabular}{|l|} 
PMDB, 1986-1989; \\
PRN, 1989-1990; \\
PSS, 1990-1993; \\
PSD, 199-1999; \\
PST, 1999-2001; \\
PRRB, 2000; PTB, \\
2001 \\
\end{tabular} & $\mathrm{AL}$ & \begin{tabular}{|l}
$1991-1995$ \\
\end{tabular} & |1995-1999 & |1999-2003 & $20003-2007$ & & 0 & $\begin{array}{l}\text { DeFUTAOD } \\
\text { EEDERAL }\end{array}$ & Aagrónome & 0,00 & RS $186.655,34$ & R\& $653.855,12$ & $28.55 \%$ \\
\hline BENEDITO DE LIRA & 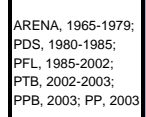 & AL & 0 & |1995-1999 & 0 & 2003-2007 & 2007-2011 & $2011-2015$ & SENADOR & 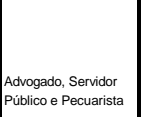 & $2 \mathrm{NI}$ & R\$ $360.018,00$ & R\$ $551.937,16$ & $65,23 \%$ \\
\hline 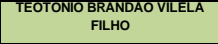 & PSOB & AL & 1986-1994 & 1995-1999 & $1999-2003$ & 2003-2006 & 0 & $T$ & SENADOR & Economista & 47,10 & Rs 592.562.83 & R\$ 14.623.903,.60 & $4.05 \%$ \\
\hline $\begin{array}{l}\text { CARLOS ALEERTO MORERAR DE } \\
\text { MENDONCA CANUTO }\end{array}$ & PMDB AL & AL & 0 & 0 & 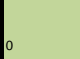 & 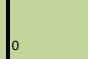 & 2007-2011 & 10 & \begin{tabular}{|l} 
DEPUTADO \\
FEDERAL
\end{tabular} & Agropecuarista & 13,00 & RS 1.091.469,00 & Rq 2.342.068,39 & $46,60 \%$ \\
\hline ARTHUR CÉSAR PEREERA DE & 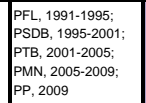 & AL & & & & & & $2011-2015$ & $\begin{array}{l}\text { DEEUTADO } \\
\text { FEDERAAL }\end{array}$ & 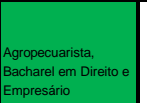 & $4 \mathrm{NI}$ & RS 1 $13522.000,00$ & R\$2 20866.666,56 & $64,79 \%$ \\
\hline
\end{tabular}




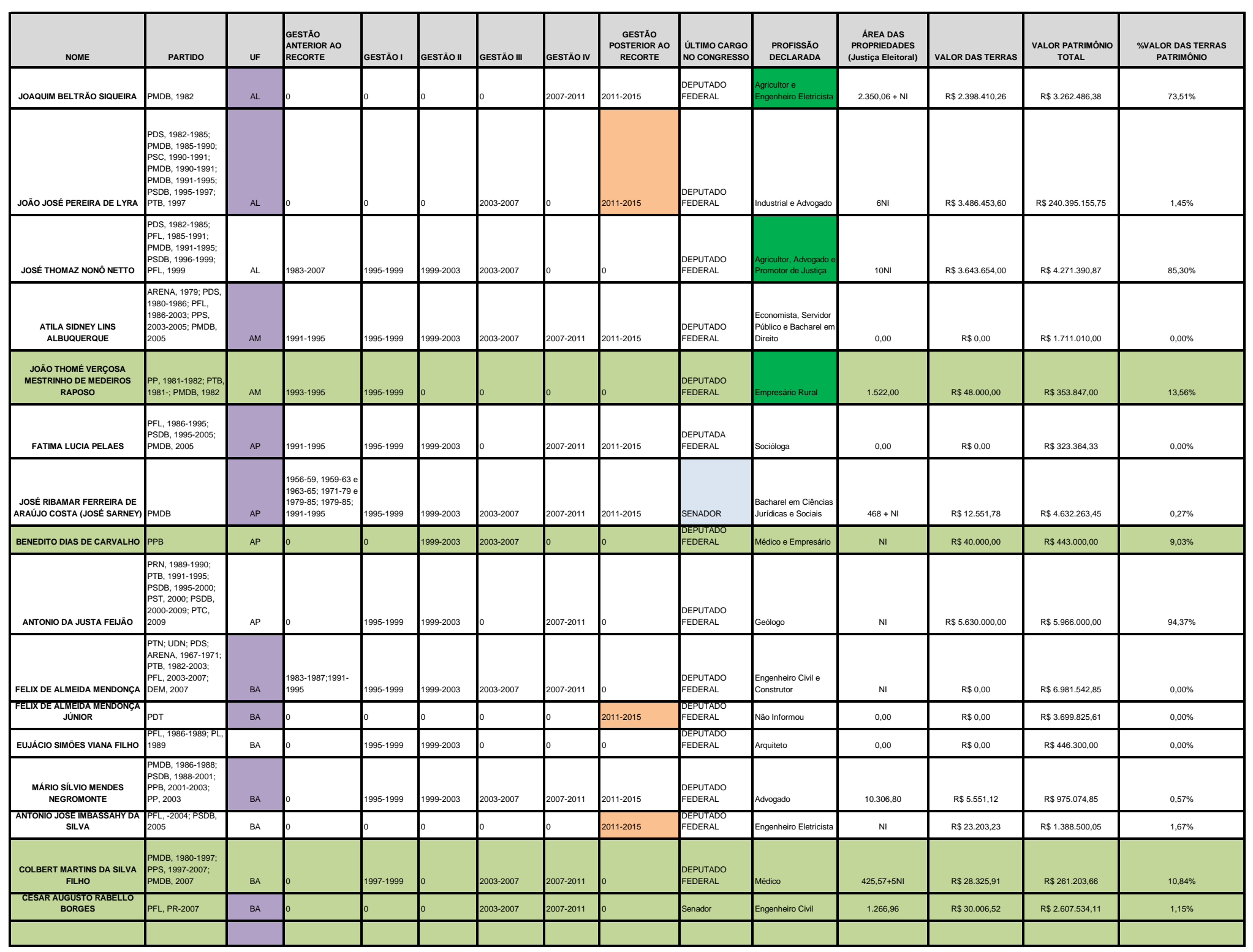




\begin{tabular}{|c|c|c|c|c|c|c|c|c|c|c|c|c|c|c|}
\hline NOME & PARTIDO & UF & $\begin{array}{l}\text { ANTERIOR } \\
\text { ANTER }\end{array}$ & GESTÃol & GESTÃo II & GESTĀo III & GESTÃo IV & \begin{tabular}{|l|l|} 
POSTERIIOR \\
POSTE
\end{tabular} & UútTimo cargo & DECLARADA & $(H A)$ & RS & $\begin{array}{l}\text { PATRIMOONONIO } \\
\text { PAL }\end{array}$ & TERARSPAPTRIMOONIONIO \\
\hline $\begin{array}{l}\text { JOĀO CARLLOS PAOLLLO } \\
\text { BACELAR }\end{array}$ & \begin{tabular}{|l|} 
PDS, 1980-1984; \\
PFL, 1985-1986; \\
PMDB, 1992; PSC, \\
1992-1997; PFL, \\
1997-2005; PL, 2005
\end{tabular} & BA & $\left.\right|_{1995} ^{1987-1991 ; 1991-}$ & | 1997-1999 & $2000-2003$ & 2003-2007 & 0 & o & $\begin{array}{l}\text { DEPUTADO } \\
\text { FEDERAL }\end{array}$ & 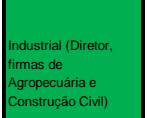 & $2 \mathrm{NI}$ & R\$ $37.900,00$ & $\mathrm{R} \$ 1.171 .491,00$ & $3,24 \%$ \\
\hline EDSON SAMPAII PIMENTA & PC DOB & $\mathrm{BA}$ & 0 & 0 & 0 & 0 & 0 & $2011-2015$ & FEDERAL & Agricultor & $6+3 \mathrm{NI}$ & R\$ 57.000,00 & $\mathrm{R} \$ 1.022 .924,45$ & $5,57 \%$ \\
\hline $\begin{array}{l}\text { JUSMARITEREZINHA DE SOUZA } \\
\text { OLVEIRA }\end{array}$ & 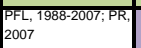 & BA & 0 & 0 & 0 & 0 & 2007-2011 & 0 & $\begin{array}{l}\text { DEPUTADA } \\
\text { FEDERAL }\end{array}$ & $\begin{array}{l}\text { Eoucadoraa } \\
\text { agricultora }\end{array}$ & $\mathrm{NI}$ & Rs $60.000,00$ & $\mathrm{R} \$ 660.371,00$ & $9,09 \%$ \\
\hline JOĀo FELIPE DE SOUZA LEĀo & 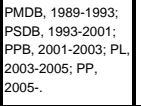 & BA & 0 & |1995-1999 & |1999-2003 & 2003-2007 & $2007-2011$ & $2011-2015$ & \begin{tabular}{|l} 
DEFUTADO \\
FEDERAL
\end{tabular} & Empresário & 0,00 & $\mathrm{R} \$ 61.506,36$ & $\mathrm{R} \$ 1.040 .968,02$ & $5,91 \%$ \\
\hline $\begin{array}{l}\text { JOĀO CARLOS PAOLILO } \\
\text { BACELAR FLLHO }\end{array}$ & $\begin{array}{l}\text { PL, 2002-2007; PR, } \\
\text { 2007 }\end{array}$ & $\mathrm{BA}$ & 0 & 0 & 0 & 0 & $2007-2011$ & 2011-2015 & $\begin{array}{l}\text { DEPUTADO } \\
\text { EEDERAL }\end{array}$ & $\begin{array}{l}\text { Engegherio } \\
\text { (Empresario do } \\
\text { Agronogegcioio) }\end{array}$ & NI & Rs $70.900,00$ & $\mathrm{R} \$ 1.171 .491,00$ & $6,05 \%$ \\
\hline $\begin{array}{l}\text { ARTHUR DE OLIVEIRA MAIA DA } \\
\text { SLIVA }\end{array}$ & $\begin{array}{l}\text { PSC, 1988-1989; } \\
\text { PMDB, 1989-1997; } \\
\text { PSDB, 1997-2007; } \\
\text { PMDB, 2007 }\end{array}$ & BA & 0 & 0 & 0 & 0 & 0 & $2011-2015$ & $\begin{array}{l}\text { DEPUTADO } \\
\text { FEDERAL }\end{array}$ & Advogado & $\mathrm{NI}$ & $\mathrm{R} \$ 76.581,37$ & $\mathrm{R} \$ 852.160,46$ & $8,99 \%$ \\
\hline ROBERTO PEREIRA DE BRITTO & ${ }_{2005}^{\mathrm{PLT}, 995-20005 ; \mathrm{PP}}$ & BA & 0 & 0 & 0 & 0 & $2007-2011$ & $2011-2015$ & 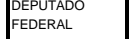 & 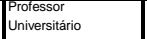 & $1.105+5 \mathrm{NI}$ & R\$ $86.635,84$ & R\$ 532.581,47 & $16,27 \%$ \\
\hline $\begin{array}{l}\text { JOSÉ CARLOS LEĀo de } \\
\text { ARAUJO }\end{array}$ & 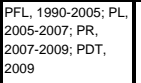 & BA & 0 & 0 & 0 & 2003-2007 & 2007-2011 & $2011-2015$ & $\begin{array}{l}\text { DEPUTADO } \\
\text { FEDERAL }\end{array}$ & \begin{tabular}{|l} 
Radialista e \\
Administrador
\end{tabular} & $\mathrm{NI}$ & $\mathrm{R} \$ 94.736,52$ & $\mathrm{R} \$ 641.514,34$ & $14,77 \%$ \\
\hline JOSÉ NUNES SOARES & DEM, 1996 & $\mathrm{BA}$ & 0 & 0 & 0 & 0 & 0 & $2011-2015$ & 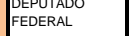 & Empresário & $2 \mathrm{NI}$ & $\operatorname{RS} 107.607,81$ & $\mathrm{R} \$ 4.893 .779,12$ & $2,20 \%$ \\
\hline SAULO PEDROSA DE ALMEIDA & $\begin{array}{l}\text { PPDDB, 1987-1990; } \\
\text { PSDB, } 1900\end{array}$ & $\mathrm{BA}$ & 0 & 0 & $1999-2003$ & 0 & 0 & o & $\begin{array}{l}\text { DEEUTAADO } \\
\text { FEDERAL }\end{array}$ & 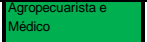 & $\mathrm{NI}$ & R\$ $110.000,00$ & $\mathrm{R} \$ 1.110 .000,00$ & $9,91 \%$ \\
\hline SEVERIANO ALVES DE SOUZA & $\begin{array}{l}\text { PFL, 1988-1990; } \\
\text { PLDBB 1990-1993; } \\
\text { PDT, 1993-2009; } \\
\text { PMDB, 2009. }\end{array}$ & BA & 0 & |1995-1999 & 0 & 2003-2007 & $2007-2011$ & o & $\begin{array}{l}\text { DEPUTADO } \\
\text { FEDERAL }\end{array}$ & \begin{tabular}{|l} 
Advogadoe \\
Procurador Federal
\end{tabular} & 488,00 & RS $120.000,00$ & $\mathrm{R} \$ 380.000,00$ & $31,58 \%$ \\
\hline $\begin{array}{l}\text { JoĀo LUIZ CoORREIA ARGốLO } \\
\text { DOS SANTOS }\end{array}$ & \begin{tabular}{|l|} 
PFL, 1999-2001; PP, \\
2001
\end{tabular} & BA & 0 & 0 & 0 & 0 & 0 & $2011-2015$ & $\begin{array}{l}\text { DEPUTADO } \\
\text { FEDERAL }\end{array}$ & $\begin{array}{l}\text { Pecuarista } \\
\text { Empresario e } \\
\text { Administrador }\end{array}$ & $13+2 \mathrm{NI}$ & $\operatorname{R} \$ 160.000,00$ & $\mathrm{R} \$ 1.103 .561,05$ & $14,50 \%$ \\
\hline MARCOS ANTONIO MEDRADO & \begin{tabular}{|l|} 
MDB, 1974-1979; \\
PMDBB, 1980-1989; \\
PRN, 1990-1991; \\
PDC, 1991-1993; \\
PP, 1993-2005; \\
PDT, 2005
\end{tabular} & BA & 1991-1995 & |1995-1996 & 0 & 0 & 2007-2011 & 2011-2015 & $\begin{array}{l}\text { DEPUTADO } \\
\text { FEDERAL }\end{array}$ & $\begin{array}{l}\begin{array}{l}\text { Administrador de } \\
\text { Empresas e Radialistat }\end{array} \\
\text { Enas }\end{array}$ & $\mathrm{NI}$ & R\$ $172.000,00$ & $\mathrm{R} \$ 850.000,00$ & $20,24 \%$ \\
\hline PAULO ROBERTO LUZ BRAGA & $\begin{array}{l}\text { PDS, 1981-1986; } \\
\text { PFL, 1986 }\end{array}$ & $B A$ & 0 & 0 & |1999-2003 & 0 & 0 & o & $\begin{array}{l}\text { DEPUTADO } \\
\text { FEDERAL }\end{array}$ & $\begin{array}{l}\text { Adgropecuarista } \mathrm{e} \\
\text { Adminstrador de } \\
\text { Empresas }\end{array}$ & $5.062,70$ & $\mathrm{R} \$ 226.037,58$ & $\mathrm{R} \$ 714.223,33$ & $31,65 \%$ \\
\hline $\begin{array}{l}\text { LEUR ANTONIO DE BRTTO } \\
\text { LOMANTO }\end{array}$ & $\begin{array}{l}\text { ARENA, 1975-1979; } \\
\text { PDS, 1980-1985; } \\
\text { PFL, 1986-2000; } \\
\text { PMDB, 2000 }\end{array}$ & BA & 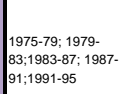 & |1995-1999 & 1999-2003 & 0 & 0 & 10 & $\begin{array}{l}\text { DEPUTADO } \\
\text { FEDERAL }\end{array}$ & Advogado & 53,00 & $\mathrm{R} \$ 340.016,61$ & $\mathrm{R} \$ 479.223,60$ & $70,95 \%$ \\
\hline $\begin{array}{l}\text { FRANCISTOONIO ALVES PINTO } \\
(07 / 101 / 1943 \text { a 16:102/2010) }\end{array}$ & $\begin{array}{l}\text { PMDB, 1985-2000; } \\
\text { PFL, 2000 }\end{array}$ & BA & 0 & 0 & |1999-2003 & 0 & 0 & 10 & $\begin{array}{l}\text { DEPUTADO } \\
\text { FEDERAL }\end{array}$ & Pecuarista & $1.004,00$ & $\mathrm{R} \$ 363.000,00$ & $\mathrm{R} \$ 457.500,00$ & $79,34 \%$ \\
\hline JOĀO ALMEIDA DOS SANTOS & $\begin{array}{l}\text { MDBB, 1970-1979; } \\
\text { PMDB, 1980-1997; } \\
\text { PSDB, 1997 }\end{array}$ & BA & |1991-1995 & |1995-1999 & |1999-2003 & 2003-2007 & 2007-2011 & o & $\begin{array}{l}\text { DEFUTADO } \\
\text { FEDERAL }\end{array}$ & Geólogo & $1.298+\mathrm{NI}$ & R\$ $396.986,39$ & R\$ 899.548,89 & $44,13 \%$ \\
\hline CORIOLANO SOUSA SALES & 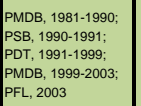 & BA & 0 & | 1995-1999 & |1999-2003 & 2003-2007 & 0 & 0 & $\begin{array}{l}\text { DEPUTADO } \\
\text { FEDERAL }\end{array}$ & Advogado & 520,00 & R\$ $400.000,00$ & $R \$ 789.910,44$ & $50,64 \%$ \\
\hline
\end{tabular}




\begin{tabular}{|c|c|c|c|c|c|c|c|c|c|c|c|c|c|c|}
\hline NOME & PARTIDO & UF & $\begin{array}{l}\text { GESTÃo } \\
\text { ANTERIOR }\end{array}$ & GESTÃol & GESTĀOII & GESTÃóOII & GESTÄO IV & \begin{tabular}{|l} 
GESTÃo \\
POSTERRIOR
\end{tabular} & Uútimo cargo & $\begin{array}{l}\text { PROFISSĀo } \\
\text { DEELARADA }\end{array}$ & $\begin{array}{l}\text { AREA DAS TERRAS } \\
\text { (HA) }\end{array}$ & $\begin{array}{c}\text { VALOR DAS TERRAS } \\
\text { RS }\end{array}$ & $\begin{array}{l}\text { VALOR DO } \\
\text { PATRIMÓNIO }\end{array}$ & $\begin{array}{c}\text { VALOR DAS } \\
\text { TERAASPATRIMOONIO }\end{array}$ \\
\hline OZIEL ALVES DE OLIVEIRA & 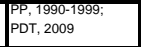 & $\mathrm{BA}$ & 0 & 0 & 0 & 0 & 0 & $2011-2015$ & $\begin{array}{l}\text { DEFPTAAD } \\
\text { FEDERAL }\end{array}$ & 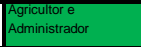 & $2 \mathrm{NI}$ & R\$ 550.625,00 & RS 57.4432,83 & $95,86 \%$ \\
\hline AROLDO CEDRAZ DE OLIVEIRA & $\begin{array}{l}\text { MDB, 1974-1979; } \\
\text { PMBD, } 1988-1999 ; \\
\text { PRN, 1989-1993; } \\
\text { PFL, 1993 }\end{array}$ & BA & 1991-1995 & |1995-1999 & |1999-2003 & 2003-2007 & 2007-2011 & 0 & $\begin{array}{l}\text { DEPUTADO } \\
\text { FEDERAL }\end{array}$ & \begin{tabular}{|l} 
Profóssor \\
Juiversitário, \\
Empresário e Médico \\
Veterinário
\end{tabular} & $820+4 \mathrm{NI}$ & R\$ 623.473,53 & $\mathrm{R} \$ 1.076 .205,57$ & $57,93 \%$ \\
\hline LUCIO QUADROS VIEIRA LIMA & PMDB, 1993 & $\mathrm{BA}$ & 0 & 0 & 0 & 0 & 0 & $2011-2015$ & $\begin{array}{l}\text { DEPUTADO } \\
\text { FEDERAL }\end{array}$ & $\begin{array}{l}\text { Engenheir A A Arónor } \\
\text { ecacauicultor }\end{array}$ & $\mathrm{NI}$ & RS 624.142,62 & $R \$ 4.981 .631,77$ & $12,53 \%$ \\
\hline FERNANDO DANTAS TORRES & \begin{tabular}{|l} 
PTdoB, 2000-2005; \\
PRTM, 2005-2009; \\
DEM, 2009
\end{tabular} & BA & 0 & 0 & 0 & 0 & 0 & $2011-2015$ & $\begin{array}{l}\text { DEPUTADO } \\
\text { FEDERAL }\end{array}$ & Empresário & $\mathrm{NI}$ & RS 722.080,00 & $\mathrm{R} \$ 2.685 .800,69$ & $26,89 \%$ \\
\hline FÁBIO LOUREIRO SOUTO & \begin{tabular}{|l} 
PFLL, 1997-2007; \\
DEM, 2007
\end{tabular} & BA & 0 & 0 & 0 & 2003-2007 & $2007-2011$ & $2011-2015$ & $\begin{array}{l}\text { DEEUTADO } \\
\text { FEDERAL }\end{array}$ & Economista & $5 \mathrm{NI}$ & R\$ $735.000,00$ & $\mathrm{R} \$ 4.552 .601,21$ & $16,14 \%$ \\
\hline GEDDEL QUADROS VIEIRA LIMA & PMDB, 1990 & $\mathrm{BA}$ & 1991-1995 & | 1995-1999 & |1999-2003 & 2003-2007 & $2007-2011$ & 0 & $\begin{array}{l}\text { DEPUTADO } \\
\text { EEDERAL }\end{array}$ & \begin{tabular}{|l} 
Emplisistrador \\
Pecuarista
\end{tabular} & $8.900,70$ & RS 779.990,24 & \begin{tabular}{|l|l|} 
Rs & $3.798 .442,64$ \\
\end{tabular} & $20,53 \%$ \\
\hline JAIME FERNANDES FLLHO & PFL & BA & ? & 0 & |1999-2003 & $?$ & ? & ? & $\begin{array}{l}\text { DEPUTADO } \\
\text { FEDERAL }\end{array}$ & $?$ & $187+4 \mathrm{NI}$ & R\$ $880.206,52$ & \begin{tabular}{ll|} 
RS & $1.780 .790,00$ \\
\end{tabular} & $49,43 \%$ \\
\hline JOĀo DURVAL CARNEIRO & \begin{tabular}{|l|} 
PSD (1954-1965); \\
ARENA (1966- \\
1980); PDS (1980- \\
1991); PMN(1991- \\
1999) PDT (1998- \\
presente)
\end{tabular} & BA & $\mid \begin{array}{l}1975-1979 ; 1979- \\
1983\end{array}$ & 0 & o & 0 & $2007-2011$ & $2011-2015$ & SENADOR & $\begin{array}{l}\text { iciurgáa-0en } \\
\text { Pecuarista }\end{array}$ & $\mathrm{NI}$ & R\$ 1.252.945,63 & $\mathrm{R} \$ 2.136 .137,29$ & $58,65 \%$ \\
\hline JOSE ALVES ROCHA & $\begin{array}{l}\text { AAREAA, 1970-1980; } \\
\text { POS, 198-1985; } \\
\text { PFL, 1985-2007; } \\
\text { 2007. }\end{array}$ & BA & 0 & |1995-1999 & |1999-2003 & 2003-2007 & $2007-2011$ & 0 & 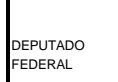 & Médico & $92+26 \mathrm{NI}$ & R\$ 1.294.666,35 & $\mathrm{R} \$ 2.323 .691,87$ & $55,72 \%$ \\
\hline $\begin{array}{l}\text { SÉRGIO LUIS LACERDA DE } \\
\text { BRITO }\end{array}$ & 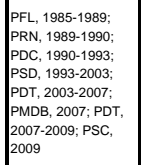 & BA & 0 & 0 & o & 0 & 2007-2011 & $2011-2015$ & $\begin{array}{l}\text { DEPUTADO } \\
\text { FEDERAL }\end{array}$ & \begin{tabular}{|l} 
Servidor Público, \\
Administrador de \\
Empresas e \\
Empresário
\end{tabular} & $8.000,00$ & $\mathrm{R} \$ 1.400 .200,00$ & $\mathrm{R} \$ 1.547 .264,95$ & $90,50 \%$ \\
\hline $\begin{array}{l}\text { JOSE LOURENCOO MORAIS DA } \\
\text { SILVA }\end{array}$ & 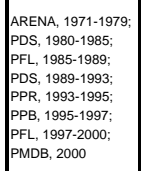 & $\mathrm{BA}$ & $\mid \begin{array}{l}1983-1987 ; 1987- \\
1999 ; 1991-1995\end{array}$ & |1996-1999 & |1999-2003 & 0 & 0 & 0 & \begin{tabular}{|l} 
DEEUTADO \\
FEDERAL
\end{tabular} & $\begin{array}{l}\text { Economistae } \\
\text { Empresario }\end{array}$ & $19.387,00$ & $\mathrm{R} \$ 2.455 .969,10$ & \begin{tabular}{|l|l|} 
R\$ & $6.383 .998,20$ \\
\end{tabular} & $38,47 \%$ \\
\hline $\begin{array}{l}\text { PAULO SÉRGIO PARANHHOS DE } \\
\text { MAGALHÄES }\end{array}$ & $\begin{array}{l}\text { PFL,-2007; DEM, } \\
2007\end{array}$ & BA & 0 & 0 & |1999-2003 & 2003-2007 & $2007-2011$ & $2011-2015$ & $\begin{array}{l}\text { DEPUTADO } \\
\text { FEDERAL }\end{array}$ & $\begin{array}{l}\text { Administrador de } \\
\text { Empresas }\end{array}$ & ${ }_{12 \mathrm{NI}}$ & R\$ $11.061 .443,00$ & R\$ 14.046.149,19 & $78,75 \%$ \\
\hline TASSO RIBEERO JEREISSATI & PSDB & $\mathrm{CE}$ & 0 & 0 & & 2003-2007 & $2007-2011$ & 0 & SENADOR & Empresário & 0,00 & R\& 0.00 & 63.537.081,27. & $0.00 \%$ \\
\hline $\begin{array}{l}\text { JOSÉ ARNON CRUZ BEZZRRA } \\
\text { DE MENEZES }\end{array}$ & \begin{tabular}{|l|} 
PFL, 1988-1993; \\
PSBB, $1994-2003 ;$ \\
PTB, 2003
\end{tabular} & $\mathrm{CE}$ & 10 & |1995-1999 & |1999-2003 & 2003-2007 & 2007-2011 & $2011-2015$ & $\begin{array}{l}\text { DEPUTADO } \\
\text { FEDERAL }\end{array}$ & Médico & 0,00 & Rs 0,00 & $R \$ 2.006 .879,63$ & $0,00 \%$ \\
\hline $\begin{array}{l}\text { CARLOS MAURO CABBAL } \\
\text { BENEVIDES }\end{array}$ & $\begin{array}{l}\text { PSD, 1994-1966; } \\
\text { MDB, } 1966-1979 ; \\
\text { PMDB, 1980; }\end{array}$ & $C E$ & 0 & 0 & |1999-2003 & 2003-2007 & 2007-2011 & $2011-2015$ & $\begin{array}{l}\text { DEPUTADO } \\
\text { FEDERAL }\end{array}$ & \begin{tabular}{|l} 
Advogado, Jornalista \\
e Professor
\end{tabular} & $\mathrm{NI}$ & Rs 0,00 & R\$ 854.559.42 & $0,00 \%$ \\
\hline RAIMUNDO GOMES DE MATOS & PDS; PFL; PSDB & $\mathrm{CE}$ & 0 & |1997-1998 & 1999-2003 & 2003-2007 & $2007-2011$ & $2011-2015$ & $\mid \begin{array}{l}\text { FEDERAAL } \\
\text { FEDER }\end{array}$ & Médico & $\mathrm{NI}$ & $\mathrm{R} \$ 4.200,00$ & R\$ 556.210,57 & $0,76 \%$ \\
\hline JOSÉ LINHARES PONTE & \begin{tabular}{|l|} 
PDC, 1988-1989; \\
PSBB, 1990-1992; \\
PST, 1992-1993; PP, \\
1993-1995; PB, \\
1995-2003; PP, \\
2003 \\
\end{tabular} & $\mathrm{CE}$ & |1991-1995 & |1995-1999 & |1999-2003 & 2003-2007 & $2007-2011$ & $2011-2015$ & $\begin{array}{l}\text { DEFUTADO } \\
\text { FEDERAL }\end{array}$ & Padre & 38,00 & $R \$ 36.738,00$ & R\$ $990.314,00$ & $3,71 \%$ \\
\hline
\end{tabular}




\begin{tabular}{|c|c|c|c|c|c|c|c|c|c|c|c|c|c|c|}
\hline NOME & PARTIDO & UF & \begin{tabular}{|l|l} 
GESTÃo \\
ANTERIOR
\end{tabular} & GESTÃol & GESTĀol & GESTĀO III & GESTÃo IV & $\begin{array}{l}\begin{array}{l}\text { GESTÃo } \\
\text { POSTERIOR }\end{array} \\
\end{array}$ & ÚlTimo cargo & $\begin{array}{l}\text { PROFISSĀ̃o } \\
\text { DECLARADA }\end{array}$ & $\begin{array}{c}\text { AREA DAS TERRAS } \\
(\mathrm{HA})\end{array}$ & $\begin{array}{c}\text { VALOR DAS TERRAS } \\
\text { RS }\end{array}$ & $\begin{array}{l}\text { VALOR DO } \\
\text { PATRMMONIO }\end{array}$ & $\begin{array}{c}\text { VALOR DAS } \\
\text { TERRASPATRIMOONIO }\end{array}$ \\
\hline $\begin{array}{l}\text { FRANCISCO ROMMEL FENÓ DE } \\
\text { SA }\end{array}$ & $\begin{array}{l}\text { PMDB, 1986-1989; } \\
\text { PSSB, } 1999-2003 ; \\
\text { PTB, 2003 }\end{array}$ & $\mathrm{CE}$ & 0 & |1995-1999 & |1999-2003 & 20003-2007 & 2007-2011 & 0 & \begin{tabular}{|l} 
DEEUTADO \\
FEDERAL
\end{tabular} & \begin{tabular}{|l|} 
Médico o \\
Administrador \\
Hospitalar
\end{tabular} & ${ }_{6 N 1}$ & $\mathrm{R} \$ 41.458,38$ & R\$ 386.713 .27 & $10,72 \%$ \\
\hline $\begin{array}{l}\text { VICENTE FERREIRA DE ARRUDA } \\
\text { COELHO }\end{array}$ & $\begin{array}{l}\text { PSDB, 1993-2007; } \\
\text { PR, 2007 }\end{array}$ & $\mathrm{CE}$ & 0 & |1995-1999 & |1999-2003 & 2003-2007 & 2007-2011 & $2011-2015$ & $\begin{array}{l}\text { DEPUTADO } \\
\text { FEDERAL }\end{array}$ & $\begin{array}{l}\text { Advogado, Jornalista } \\
\text { e Cientista Politico }\end{array}$ & $4 \mathrm{NI}$ & $\mathrm{R} \$ 55.517,16$ & R\$ $761.867,82$ & $7,29 \%$ \\
\hline $\begin{array}{l}\text { RAAMUNDO ANTONONO DE } \\
\text { MACEDO (RAIMUNDÄO) }\end{array}$ & PMDB & $\mathrm{CE}$ & 0 & 0 & o & 0 & 0 & $2011-2015$ & $\begin{array}{l}\text { DEPUTADO } \\
\text { FEDERAL }\end{array}$ & Médico & 9NI & R\$ $136.014,53$ & $787.029,98$ & $17,28 \%$ \\
\hline ANIBAL FERREIRA GOMES & $\begin{array}{l}\text { PMDB, 1982-1997; } \\
\text { PSDB, } 1997-1999 ; \\
\text { PMDB, 1999; }\end{array}$ & $\mathrm{CE}$ & 0 & |1995-1999 & 1999-2003 & 20003-2007 & 2007-2011 & 2011-2015 & $\begin{array}{l}\text { DEPUTADO } \\
\text { FEDERAL }\end{array}$ & $\mid \begin{array}{l}\text { Agropecuarista e } \\
\text { Cirurgiào Dentista }\end{array}$ & $24+7 \mathrm{NI}$ & Rs 242.365,92 & $\mathrm{R} \$ 6.805 .631,36$ & $3,56 \%$ \\
\hline ROBERTO SOARES PESSOA & $\begin{array}{l}\text { PFL, 1990-2003; PL, } \\
2003\end{array}$ & $\mathrm{CE}$ & 0 & |1995-1999 & |1999-2003 & 2003-2007 & 10 & 0 & $\begin{array}{l}\text { DEPUTADO } \\
\text { FEDERAL }\end{array}$ & $\begin{array}{l}\text { A Aropenecuarista, } \\
\text { Economista e } \\
\text { Contador }\end{array}$ & $8 \mathrm{NI}$ & R\$ $301.021,72$ & $\mathrm{R} \$ 11.559 .714,10$ & $2,60 \%$ \\
\hline FRANCISCO PINHEIRO LANDIM & $\mid \begin{array}{l}\text { PMOO3, -2003, PSL, } \\
2003\end{array}$ & $\mathrm{CE}$ & $1991-1995$ & 1995-1999 & 1999-2003 & $2003-2007$ & D & 0 & $\mid \begin{array}{l}\text { FEDERALL } \\
\text { pePALO }\end{array}$ & Empresário & $4.032,00$ & RS $365.001,20$ & $\begin{array}{|ll|}\text { R\$ } & 8.568 .810,76 \\
\end{array}$ & $4,26 \%$ \\
\hline GENECIAS MATEUS NORONHA & PMDB, 1995 & $\mathrm{CE}$ & 0 & 0 & 0 & 0 & 0 & $2011-2015$ & $\begin{array}{l}\text { DEEQTADO } \\
\text { FEDERAL }\end{array}$ & Empresário & 3,00 & RS $391.171,00$ & $\begin{array}{|ll|}\text { R\$ } & 4.664 .525,89 \\
\end{array}$ & $8,39 \%$ \\
\hline EUNICIO LOPES OLVEERA & $\begin{array}{l}\text { MMDB, } 1981 \\
\text { PMD19 }\end{array}$ & $\mathrm{CE}$ & 0 & 0 & 1999-2003 & 2003-2007 & $2007-2011$ & $2011-2019$ & SENADOR & \begin{tabular}{|l|l} 
Agropecuarars \\
Empresári
\end{tabular} & $73 \mathrm{NI}$ & RS 1.069.569,89 & $\begin{array}{|ll|}\text { Rs } & 25.639 .317,78 \\
\end{array}$ & $4,17 \%$ \\
\hline JAQUELINE MARIA RORIZ & $\begin{array}{l}\text { PSDB, 2005-2009; } \\
\text { PMN, 2009-2010 }\end{array}$ & DF & 0 & 0 & 0 & 0 & 0 & $2011-2015$ & $\begin{array}{l}\text { DEPUTADA } \\
\text { FEDERAL }\end{array}$ & Pedagoga & 0,00 & R\$ 0,00 & $\begin{array}{ll}\text { R\$ } & 2.846 .951,47 \\
\end{array}$ & $0,00 \%$ \\
\hline IZALCI LUCAS FERREIRA & 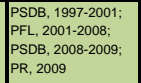 & DF & 0 & 0 & 0 & 10 & 2007-2011 & 2011-2015 & $\begin{array}{l}\text { DEPUTADO } \\
\text { FEDERAL }\end{array}$ & Professor e Contador & $\mathrm{NI}$ & R\$ $120.000,00$ & $\mathrm{R} \$ 6.362 .764,83$ & $1,89 \%$ \\
\hline $\begin{array}{l}\text { RODRIGO SOBBAL } \\
\text { ROLLEMBERG }\end{array}$ & PSB, 1985 & DF & 0 & 0 & 0 & 0 & 0 & $\begin{array}{l}2011-2015 ; 2015- \\
2019\end{array}$ & SENADOR & Funcionário Público & 209,00 & Rs 263.043,00 & Rs $671.484,00$ & $39,17 \%$ \\
\hline JOSÉ FUSCALDI CESILIO & $\begin{array}{l}\text { PSC, 1998-2001; } \\
\text { PSD, 2001-2003; } \\
\text { PTR, 2003-2055; PL, } \\
\text { 2005; PTB, 2005 }\end{array}$ & DF & 0 & 0 & o & 2003-2007 & 2007-2011 & 0 & $\begin{array}{l}\text { DEPUTADO } \\
\text { FEDERAL }\end{array}$ & \begin{tabular}{|l|} 
Fazenderioo \\
Comerciante
\end{tabular} & $1.248,00$ & R\$ $1.000 .000,00$ & $\mathrm{NI}$ & ? \\
\hline $\begin{array}{l}\text { JOAQUUM DOMNGOS RORIZ } \\
\text { (RENUNCIOU EM 2007) }\end{array}$ & $\mid \begin{array}{l}\text { MDB, 1974; РМDB. } \\
\text { 1987 }\end{array}$ & DF & 1987 & 0 & 0 & 0 & 2007-2011 & 2011-2015 & SENADOR & Empresário & NI & $R \$ 100.000,00$ & $\mathrm{R} \$ 5.241 .152,60$ & $1,91 \%$ \\
\hline CAMLLO COLA & $\begin{array}{l}\text { ARENA, 1964; PDS, } \\
\text { 1980;; PMDB, } 1986\end{array}$ & ES & 0 & 0 & 0 & 0 & $2007-2011$ & $2011-2015$ & $\begin{array}{l}\text { DEEUTAAD } \\
\text { FEDERAL }\end{array}$ & \begin{tabular}{|l} 
Administrador Ri \\
Empresario de
\end{tabular} & 0,00 & Rs 0,00 & \begin{tabular}{|l|l|} 
RS & $1.562 .032,32$ \\
\end{tabular} & $0,00 \%$ \\
\hline MARCUS ANTONIO VICENTE & $\begin{array}{l}\text { ARENA; PFLL; PSDB } \\
\text { 2001; PPB, 2001- } \\
\text { 2003; PTB, } 2003\end{array}$ & ES & 0 & | 1997-1999 & |1999-2003 & 2003-2007 & o & 0 & $\begin{array}{l}\text { DEEUTADO } \\
\text { FEDERAL }\end{array}$ & Advogado & 0,00 & Rs 0,00 & $\begin{array}{ll}\text { R\$ } & 633.158,32 \\
\end{array}$ & $0,00 \%$ \\
\hline MARCELINO AYUB FRAGA & PMDB, 1979 & ES & 0 & 0 & 0 & 2003-2007 & 0 & 0 & $\mid \begin{array}{l}\text { FEPDERAL } \\
\text { FEDEL }\end{array}$ & Agropecuarista & $974,41+2 \mathrm{NI}$ & $\mathrm{R} \$ 5.808 .97$ & R\$ 711.454,72 & $0,82 \%$ \\
\hline JOĀO MIGUEL FEU ROSA & \begin{tabular}{|l} 
ARENA, 1974-1980; \\
PSS, 1980-1985; \\
PFL, , 1985-1988; \\
PPS, 1993; PSDB, \\
1999-2003; PP, \\
2003
\end{tabular} & ES & 0 & |1995-1999 & |1999-2003 & 2003-2007 & o & 0 & 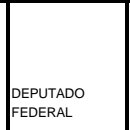 & $\begin{array}{l}\begin{array}{l}\text { Profosssor } \\
\text { Univestitháio } \\
\text { Engenheiro }\end{array} \\
\end{array}$ & $\mathrm{NI}$ & R\$ $32.870,93$ & R\$ $478.445,78$ & $6,87 \%$ \\
\hline PAULO ROBERTO FOLETTO & PSB, 1990 & ES & 0 & 0 & 0 & 10 & 0 & $2011-2015$ & $\begin{array}{l}\text { EDPETADC } \\
\text { FEDERAL }\end{array}$ & Médico & $4 \mathrm{NI}$ & R\$ 81.289,00 & R\$ 1.337.695.00 & $6,08 \%$ \\
\hline JOSÉ CARLOS ELLAS & MDB; РMDB; РTB & ES & 0 & 1995-1999 & 1999-2003 & $2003-2004$ & 0 & 0 & \begin{tabular}{|l} 
DEEDERALO \\
FEDER
\end{tabular} & Agricultor e Bancário & 103,21 & Rs 104.285,69 & 594.287,54 & $17,55 \%$ \\
\hline $\begin{array}{l}\text { NLITON GOMES OLVEERA } \\
\text { (NLTTON BAAANO) }\end{array}$ & \begin{tabular}{|l|} 
PMDB, 1984-1995; \\
PSC, 1991-1992; \\
PPB, 1995-2003; \\
PP, 2003-.
\end{tabular} & ES & |1991-1995 & |1995-1999 & |1999-2003 & 2003-2007 & 0 & 0 & \begin{tabular}{|l} 
DEPUTADO \\
FEDERAL
\end{tabular} & $\begin{array}{l}\text { Medico e Proféssor } \\
\text { Universitário }\end{array}$ & $1.799,00$ & $?$ & $?$ & \#VALOR! \\
\hline LEONARDO MOURA VILELA & $\begin{array}{l}\text { PPB, 1996-2003; } \\
\text { PP, 2003-2005; } \\
\text { PDOB 2005 }\end{array}$ & Go & 0 & 0 & o & 2003-2007 & 2007-2011 & $2011-2015$ & $\begin{array}{l}\text { DEPUTADO } \\
\text { FEDERAL }\end{array}$ & $\begin{array}{l}\text { Médico, Pecuarista e } \\
\text { Profossor }\end{array}$ & 0,00 & R\$ 0,00 & R\$ $576.587,08$ & $0,00 \%$ \\
\hline LUIZ JOSE BITTENCOURT & $\begin{array}{l}\text { PDC, 1990-1993; } \\
\text { PMDB, 1993 }\end{array}$ & Go & 10 & 0 & 1999-2003 & 2003-2007 & 2007-2011 & 10 & $\begin{array}{l}\text { DEPUTADO } \\
\text { FEDERAL }\end{array}$ & \begin{tabular}{|l} 
Engenenheiro Civil e \\
Professor \\
Universitário
\end{tabular} & 0,00 & R\$ 0,00 & $490.836,31$ & $0,00 \%$ \\
\hline
\end{tabular}




\begin{tabular}{|c|c|c|c|c|c|c|c|c|c|c|c|c|c|c|}
\hline NOME & PARTIDO & UF & $\begin{array}{l}\text { GESTÃo } \\
\text { ANTERIOR }\end{array}$ & GESTÃol & GESTĀol & GESTÃo III & GESTÃo IV & \begin{tabular}{|l} 
GESTÃo \\
POSTERIOR
\end{tabular} & ÚlTIMO CARGo & $\begin{array}{l}\text { PROFISSÃo } \\
\text { DEELARADA }\end{array}$ & $\begin{array}{c}\text { AREA DAS TERRAS } \\
\text { (HA) }\end{array}$ & $\begin{array}{c}\text { VALOR DAS TERRAS } \\
\text { RS }\end{array}$ & $\begin{array}{l}\text { VALOR DO } \\
\text { PATRIMÓNIO }\end{array}$ & $\begin{array}{c}\text { VALOR DAS } \\
\text { TERAASPATRMIMONIO }\end{array}$ \\
\hline $\begin{array}{l}\text { DEMOSTENES LÁAZARO XAVIER } \\
\text { TORBES }\end{array}$ & DEM & Go & 0 & 0 & 0 & 2003-2007 & $2007-2011$ & $\begin{array}{l}\text { 2011-2015;2015- } \\
2019\end{array}$ & SENADOR & Procurador de Justiça & 0,00 & Rs 0,00 & \begin{tabular}{|l|l|} 
R\$ & $374.964,60$ \\
\end{tabular} & $0,00 \%$ \\
\hline JOÃo CAMPOS DE ARAúso & PSDB, 2002 & GO & 0 & 0 & 0 & 2003-2007 & 2007-2011 & $2011-2015$ & $\begin{array}{l}\text { DEPUTADO } \\
\text { FEDERAL }\end{array}$ & $\begin{array}{l}\text { Delegado de Policia } \\
\text { de Classe Especial }\end{array}$ & 18,70 & $\mathrm{R} \$ 25.000,00$ & R\$ $775.412,20$ & $3,22 \%$ \\
\hline $\begin{array}{l}\text { MARCELO DE ARAU广J MELO. } \\
\text { INESC }\end{array}$ & РмDВ, 1980 & Go & 0 & 0 & 0 & 0 & 2007-2011 & 0 & $\begin{array}{l}\text { DEPUTADO } \\
\text { FEDERAL }\end{array}$ & Nấo Intormou & $\mathrm{NI}$ & R\$ $10.121,00$ & R\$ 59.183,00 & $17,10 \%$ \\
\hline 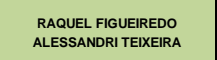 & PSDB & Go & 0 & 0 & 0 & 2003-2007 & $2007-2011$ & 0 & $\begin{array}{l}\text { DEPUTADO } \\
\text { FEDERAL }\end{array}$ & $\begin{array}{l}\text { Protessora } \\
\text { Univssitaria }\end{array}$ & $2.250,06$ & R\$ 56.331,60 & \begin{tabular}{ll|} 
Rs & $622.627,41$ \\
\end{tabular} & $9,05 \%$ \\
\hline LEANDRO VILELA VELLOSO & РмDB & Go & 0 & 0 & 0 & $2003-2007$ & $2007-2011$ & $2011-2015$ & \begin{tabular}{|l} 
DEEUAADO \\
FEDERAL
\end{tabular} & Agricultor & $\mathrm{NI}$ & $\mathrm{R} \$ 92.592,48$ & $\mathrm{R} \$ 460.650,96$ & $20,10 \%$ \\
\hline HEULER ABREU CRUVINEL & DEM, 2009 & Go & 0 & 0 & 0 & 0 & 0 & $2011-2015$ & $\begin{array}{l}\text { DEPUTADO } \\
\text { FEDERAL }\end{array}$ & $\mid \begin{array}{l}\text { Verde, FESURV, Rio } \\
\text { verde, GO, Brasil) }\end{array}$ & 528,00 & R\$ 100.000,00 & $\mathrm{R} \$ 208.750,59$ & $47,90 \%$ \\
\hline JOSÉ GOMES DA ROCHA & \begin{tabular}{|l|} 
MDB, 1976-1979; \\
PMD, 1980-1986; \\
PDC, 1986-1989; \\
PRN, 1989-1995; \\
PSD, , $995-1999 ;$ \\
PMDB, 1999
\end{tabular} & Go & |1989-1991 & |1995-1999 & |1999-2003 & 0 & 0 & 0 & $\begin{array}{l}\text { DEPUTADO } \\
\text { FEDERAL }\end{array}$ & $\begin{array}{l}\text { agropecuarista e } \\
\text { Fazendelio }\end{array}$ & $1.297,00$ & $\mathrm{R} \$ 495.277,68$ & R\$ 728.183,62 & $68,02 \%$ \\
\hline $\begin{array}{l}\text { CARLOS ALEERTOOLEREADA } \\
\text { SILVA }\end{array}$ & PSDB, 1989 & Go & 0 & 0 & 0 & $2003-2007$ & $2007-2011$ & $2011-2015$ & $\begin{array}{l}\text { JEPDUAADC } \\
\text { FEDERAL }\end{array}$ & Radialista & $393,2+3 \mathrm{NI}$ & RS 613.200,00 & $\mathrm{R} \$ 2.043 .457,91$ & $30,01 \%$ \\
\hline SANDRO ANTÓNIO SCODRO & $\begin{array}{l}\text { PMOB, 1988-1999; } \\
\text { PFL, 2000-2003; L, } \\
\text { 20203-2007; PR; } \\
\text { 2007 }\end{array}$ & GO & 0 & |1995-1999 & 10 & 2003-2007 & 2007-2011 & 2011-2015 & $\begin{array}{l}\text { DEPUTADO } \\
\text { FEDERAL }\end{array}$ & $\begin{array}{l}\begin{array}{l}\text { Administrador de } \\
\text { Empresas }\end{array} \\
\text { Ens }\end{array}$ & $10.918,20$ & R\$ 733.793,53 & $\mathrm{R} \$ 70.992 .163,06$ & $1,03 \%$ \\
\hline LÚCIA VÂNIA ABRĀo COSTA & 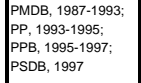 & GO & $\mid \begin{array}{l}1987-1991 ; \text {; } 1991- \\
1995\end{array}$ & 0 & |1999-2003 & 0 & $2007-2011$ & $\begin{array}{l}2011-2015 ; 2015- \\
2019\end{array}$ & SENADORA & Jornalista & $34,7+8 \mathrm{NI}$ & R\$ $880.436,35$ & 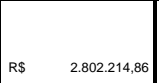 & $31,42 \%$ \\
\hline ROBERTO EGIIIO BALLESTRA & \begin{tabular}{|l|} 
ARENA, 1966-1979; \\
PSS, 1980-1985; \\
PDC, 1986-1993; \\
PPR, 1993-1995; \\
PPB, 1995-2003; \\
PP, 2003
\end{tabular} & Go & $\begin{array}{l}1987-1991 ; \text {; } 1991 . \\
1995\end{array}$ & |1995-1999 & |1999-2003 & 2003-2007 & $2007-2011$ & $2011-2015$ & \begin{tabular}{|} 
DEFUTADO \\
FEDERAL
\end{tabular} & 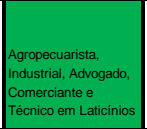 & $1.320,80$ & R\$ $1.021 .744,50$ & \begin{tabular}{|l|l|} 
R\$ & $3.884 .883,85$ \\
\end{tabular} & $26,30 \%$ \\
\hline JOVAIR DE OLVEIRA ARANTES & $\begin{array}{l}\text { PMDB, 1983-1989; } \\
\text { PSSB, , } 990-2003 ; \\
\text { PTB, 2003 }\end{array}$ & GO & 0 & |1995-1999 & 1999-2003 & 2003-2007 & 2007-2011 & 2011-2015 & $\begin{array}{l}\text { DEEPUTADO } \\
\text { FEDERAL }\end{array}$ & Cirurgiäo Dentista & 794,50 & R\$ 1.404.717,00 & $\mathrm{R} \$ 2.723 .019,00$ & $51,59 \%$ \\
\hline RONALDO RAMOS CAIADO & $\begin{array}{l}\text { PFLL, 1986-1988; } \\
\text { PCC, , } 9898 \text { PSS, } \\
\text { 1989-1991 PFL, } \\
\text { 1991-2007; PEM, } \\
\text { 2007 }\end{array}$ & Go & |1991-1995 & 0 & |1999-2003 & 2003-2007 & 2007-2011 & $2011-2015$ & $\begin{array}{l}\text { DEEUTADO } \\
\text { FEDERAL }\end{array}$ & \begin{tabular}{|l} 
Médico, Protessore e \\
Produtor Rural
\end{tabular} & $5.172,20$ & R\$ $1.722 .865,50$ & $\begin{array}{ll}\text { R\$ } & 5.950 .666,62\end{array}$ & $28,95 \%$ \\
\hline $\begin{array}{l}\text { IRIS DE ARAUUO REZENDE } \\
\text { MACHADO (DONA IRIIS) }\end{array}$ & PMDB, 1980 & Go & 0 & 0 & |1999-2003 & 2006-2007 & $2007-2011$ & 2011-2015 & $\begin{array}{l}\text { DEPUTADA } \\
\text { FEDERAL }\end{array}$ & Administrador Público & 25.308,90 & R\$ 2.818.696,47 & $\begin{array}{ll}\text { R\$ } & 14.173 .468,69\end{array}$ & $19,89 \%$ \\
\hline $\begin{array}{l}\text { TRIS DE ARACUO REZENDE } \\
\text { MACHADO }\end{array}$ & PMDB & Go & 0 & 1995-1999 & $1999-2003$ & 0 & $2007-2011$ & 0 & SENADOR & 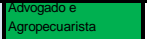 & 25.308 .90 & RS 2.882.696,47 & $\begin{array}{|ll|}\text { RS } & 14.638 .948,31 \\
\end{array}$ & 19,69\% \\
\hline REMI ABREU TRRNTA & 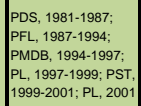 & MA & 0 & |1995-1999 & |1999-2003 & 2003-2007 & 0 & 10 & $\begin{array}{l}\text { DEPUTADO } \\
\text { FEDERAL }\end{array}$ & $\begin{array}{l}\text { Médico e Cirurgiäo- } \\
\text { Dentista }\end{array}$ & 0,00 & R\& 0.00 & $\mathrm{R} \$ 5.664 .907,60$ & $0,00 \%$ \\
\hline $\begin{array}{l}\text { JOAO CASTELO RIBEEIRO } \\
\text { GONCALVES }\end{array}$ & PSDB, 1997 & MA & $\begin{array}{l}\mid \begin{array}{l}1971-1975 ; 1975- \\
1979 ;\end{array} \\
\end{array}$ & & $1999-2003$ & $2003-2007$ & 0 & 0 & $\begin{array}{l}\text { DEFPTIAOO } \\
\text { FEDEALL }\end{array}$ & $\begin{array}{l}\text { Acarogadoe } \\
\text { Empresário }\end{array}$ & 0,00 & Rs 0.00 & R\$ 3.964.295.45 & $0,00 \%$ \\
\hline NICE LOBĀO & $\begin{array}{l}\text { PST, 1996-1997; } \\
\text { PFL 1997-2077; } \\
\text { DEN, 2077-2011; } \\
\text { PSD, 2011 }\end{array}$ & MA & 0 & 10 & |1999-2003 & 2003-2007 & 2007-2011 & 2011-2015 & $\begin{array}{l}\text { DEPUTADO } \\
\text { FEDERAL }\end{array}$ & Servidora Pública & 0,00 & $\mathrm{R} \$ 0,00$ & $\mathrm{R} \$ 2.209 .719,78$ & $0,00 \%$ \\
\hline $\begin{array}{l}\text { CARLOS ORLEANS BRANDAO } \\
\text { JUNIOR }\end{array}$ & $\begin{array}{l}\text { PFL, 2002-2004; } \\
\text { PSDB, 2006 }\end{array}$ & MA & 10 & 10 & 0 & 10 & 2007-2011 & 2011-2015 & $\begin{array}{l}\text { DEPUTADO } \\
\text { FEDERAL }\end{array}$ & $\begin{array}{l}\text { Empresárío, } \\
\text { Funcionárí Publico e } \\
\text { Médico Vetetrinário }\end{array}$ & 0,00 & $\mathrm{R} \$ 0,00$ & R\$ 291.004,85 & $0,00 \%$ \\
\hline
\end{tabular}




\begin{tabular}{|c|c|c|c|c|c|c|c|c|c|c|c|c|c|c|}
\hline NoME & PARTIDO & uF & \begin{tabular}{|l}
$\begin{array}{l}\text { GESTÃo } \\
\text { ANTERIOR }\end{array}$ \\
\end{tabular} & GESTÃoI & GESTÃol II & GESTÃo III & GESTÃo IV & \begin{tabular}{|l|l}
$\begin{array}{l}\text { GESTÃO } \\
\text { POSTERIOR }\end{array}$ \\
\end{tabular} & Último cargo & $\begin{array}{l}\text { PROFISSÁO } \\
\text { DECLARADA }\end{array}$ & $\begin{array}{c}\text { AREA DAS TERRAS } \\
\text { (HA) }\end{array}$ & \begin{tabular}{|c|} 
VALOR DAS TERRAS \\
RS
\end{tabular} & $\begin{array}{l}\text { VALOR DO } \\
\text { PATRIMÓNIO }\end{array}$ & $\begin{array}{c}\text { VALOR DAS } \\
\text { TERRASPATRIMOONOOO }\end{array}$ \\
\hline $\begin{array}{l}\text { JOSE ALBERTO OLIVERA } \\
\text { VELOSO FILHO }\end{array}$ & $\begin{array}{l}\text { PDT, 2007-2009; } \\
\text { PMDB, 2009; }\end{array}$ & MA & 0 & 0 & 0 & 0 & 0 & $2011-2015$ & $\begin{array}{l}\text { DEPUTADO } \\
\text { FEDERAL }\end{array}$ & Bacharel em Diretio & 0,00 & R\$ 0,00 & R\$ 177.753 .53 & $0,00 \%$ \\
\hline GASTĀO DIAS VIERRA & $\begin{array}{l}\text { PMDB, 1985-1990; } \\
\text { PSC, 1990-1994; } \\
\text { PMDB, 1994 }\end{array}$ & MA & 0 & |1995-1999 & |1999-2003 & 2003-2007 & $2007-2011$ & 0 & $\begin{array}{l}\text { DEPUTADO } \\
\text { fEDERAL }\end{array}$ & Advogado & 0,00 & R\$ 0,00 & $421.180,19$ & $0,00 \%$ \\
\hline JOSÉ VIEIRA LINS & $\begin{array}{l}\text { PP, 2003-2005; } \\
\text { PsDB, 2005-2007; } \\
\text { PP, 2007-2008; } \\
\text { S.PART., 2009; PR, } \\
\text { 2009 }\end{array}$ & MA & 0 & 0 & 0 & 0 & $2009-2011$ & $2011-2015$ & $\begin{array}{l}\text { DEPUTADO } \\
\text { FEDERAL }\end{array}$ & \begin{tabular}{|l} 
Pecuarista e \\
Empresário
\end{tabular} & $10.147,56$ & $\mathrm{R} \$ 4.373,45$ & $\mathrm{R} \$ 8.176 .954,00$ & $0,05 \%$ \\
\hline $\begin{array}{l}\text { LOURRVAL MENDES DA } \\
\text { FONSECA FLLHO }\end{array}$ & 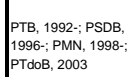 & MA & 0 & 0 & 0 & 0 & 2007-2011 & $2011-2015$ & $\begin{array}{l}\text { DEPUTADO } \\
\text { FEDERAL }\end{array}$ & \begin{tabular}{|l} 
Pollitico e Delegadodo de \\
Polici ac liasse \\
Especial
\end{tabular} & $2 \mathrm{NI}$ & $\mathrm{R} \$ 11.600,00$ & $\mathrm{R} \$ 234.005,84$ & $4,96 \%$ \\
\hline EDISON LOBĀO & $\begin{array}{l}\text { ARENA; PDS, 1980- } \\
\text { 1981; PFL (1987); } \\
\text { PDS }\end{array}$ & MA & $\begin{array}{l}\text { 1979-1983; } 1983- \\
\text { 1987 }\end{array}$ & |1995-1999 & $\mid 1999-2003$ & 10 & 2007-2011 & 2011-2019 & SENADOR & Advogado e Jornalista & $2 \mathrm{NI}$ & R\$ $12.067,60$ & $\mathrm{R} \$ 5.093 .842,25$ & $0,24 \%$ \\
\hline $\begin{array}{l}\text { PEDRO NOVAIS LIMA } \\
\text { (LICENCIADO) }\end{array}$ & $\begin{array}{l} \\
\text { ARENA, 1977-1979; } \\
\text { PMDB, 1980-1989; } \\
\text { PDC, 1989-1993; } \\
\text { PPR, 1993-1994; } \\
\text { PMDB, 1994 }\end{array}$ & MA & $\left.\right|_{1995} ^{1983-1984 ; \text {; } 1991-}$ & |1995-1999 & 1999-2003 & 2003-2007 & $2007-2011$ & $2011-2015$ & $\begin{array}{l}\text { DEPUTADO } \\
\text { FEDERAL }\end{array}$ & Advogado & 182,50 & $\operatorname{RS} 12.500,00$ & $\mathrm{R} \$ 6.382 .972,59$ & $0,20 \%$ \\
\hline HÉLIO BATISTA DOS SANTOS & PSDB, 2001 & MA & 0 & 0 & 0 & o & 0 & $2011-2015$ & $\begin{array}{l}\text { DEPPTAAD } \\
\text { FEDERAL }\end{array}$ & Agropecuarista & ${ }_{2 \mathrm{NI}}$ & R\$ $\$ 4.000,00$ & RS 87.809.78 & $72.88 \%$ \\
\hline $\begin{array}{l}\text { PAULO CELSO FONSECA } \\
\text { MARINHO }\end{array}$ & $\begin{array}{l}\text { PMDB, 1980-1999; } \\
\text { PSC, 1990-1999; } \\
\text { FLL 1999-2003; PL, } \\
\text { 2003 }\end{array}$ & MA & |1991-1992 & |1995-1999 & |1999-2003 & 2003-2007 & 10 & 0 & $\begin{array}{l}\text { DEPUTADO } \\
\text { FEDERAL }\end{array}$ & \begin{tabular}{|l|} 
Advogado e Servidor \\
Pubilico en
\end{tabular} & $80,26+3 \mathrm{NI}$ & $\mathrm{R} \$ 66.854,11$ & $\mathrm{R} \$ 271.454,48$ & $24,63 \%$ \\
\hline PEDRO FERNANDESS RIBEIRO & $\begin{array}{l}\text { PSDB, 1988-1995; } \\
\text { PSDD 1995-1999; } \\
\text { FFL, 199-2003; } \\
\text { PTB, 20002 }\end{array}$ & MA & 0 & 0 & |1999-2003 & 2003-2007 & $2007-2011$ & $2011-2015$ & $\begin{array}{l}\text { DEPUTADO } \\
\text { FEDERAL }\end{array}$ & \begin{tabular}{|l} 
Engenheiro Civile \\
Bancário
\end{tabular} & ${ }_{5 \mathrm{NI}}$ & $344.000,00$ & R\$ $994.000,00$ & $34,61 \%$ \\
\hline $\begin{array}{l}\text { ANTONOO JoAQUIM ARAUSO } \\
\text { FLLHO }\end{array}$ & \begin{tabular}{|l} 
PDS, 1981-1986; \\
PFL, 1986-1997; PL, \\
1997-1998; PPB, \\
1998-2003; PP, \\
2003-2005; PTB, \\
2005; PSBB, 2005
\end{tabular} & MA & 0 & |1995-1999 & 1999-2002 & 2003-2007 & 10 & 0 & $\begin{array}{l}\text { DEPUTADO } \\
\text { FEDERAL }\end{array}$ & Médico & $8.727,60$ & $349.000,00$ & R\$ 488.000,00 & $72,71 \%$ \\
\hline $\begin{array}{l}\text { FRANCISCO DE ASSIS } \\
\text { MLLHOMEM }\end{array}$ & 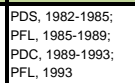 & MA & $\begin{array}{l}\text { 1987-1991; } 1991- \\
1995\end{array}$ & & 1999-2003 & 10 & 10 & 0 & $\begin{array}{l}\text { DEPUTADO } \\
\text { FEDERAL }\end{array}$ & $\begin{array}{l}\text { Agropecuarista e } \\
\text { Engenheiro Agrónomo }\end{array} \mid$ & $8.165,00$ & RS $396.717,00$ & $\mathrm{R} \$ 1.421 .007,00$ & $27,92 \%$ \\
\hline ROSEANA SARNEY MURAD & PFL & MA & 1991-1995 & 0 & 0 & 2003-2007 & $2007-2011$ & 0 & SENADOR & Sociobloga & $9,12+\mathrm{NI}$ & $844.707,00$ & R\$ 4.810.438,40 & $17,56 \%$ \\
\hline $\begin{array}{l}\text { ELISEU BARROSO DE } \\
\text { CARVALO MOURA }\end{array}$ & $\begin{array}{l}\text { PFLL, 1987-1997; PL, PL } \\
\text { 1979-1999; PB, } \\
\text { 1999-2003; PP, } \\
\text { 20203 }\end{array}$ & MA & 0 & |1995-1999 & |1999-2003 & 0 & $l_{0}$ & 0 & $\begin{array}{l}\text { DEPUTAAD } \\
\text { FEDERAL }\end{array}$ & $\mid \begin{array}{l}\text { Engegnheritr Civile } \\
\text { Pecouarista }\end{array}$ & $2540+\mathrm{NI}$ & $\mathrm{R} \$ 3.750 .000,00$ & $\mathrm{R} \$ 9.203 .500,00$ & $40,75 \%$ \\
\hline $\begin{array}{l}\text { JOSÉ DE RIIAAMAR DA COSTA } \\
\text { ALVES }\end{array}$ & $\begin{array}{l}\text { PMDB, 1983-1997; } \\
\text { PDT, 1997-2001; } \\
\text { PSB, 2001 }\end{array}$ & MA & 0 & lo & 0 & 2003-2007 & 2007-2011 & $2011-2015$ & 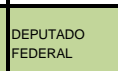 & Médico & $3.033,72$ & & & \#DIVIo! \\
\hline VITTORIO MEDIOLI & $\begin{array}{l}\text { PSDB, 1989-2005; } \\
\text { PV, } 2005\end{array}$ & MG & $1991-1995$ & |1995-1999 & |1999-2003 & $2003-2007$ & 0 & 0 & $\begin{array}{l}\text { DEPUTADO } \\
\text { FEDERAL }\end{array}$ & Empresário & 0,00 & R\$ 0,00 & $\mathrm{R} \$ 36.756 .876,28$ & $0,00 \%$ \\
\hline $\begin{array}{l}\text { ELISEU RESENDE (07/02/1929 a } \\
01102 / 07)\end{array}$ & \begin{tabular}{|l} 
PDS, 1980-1993; \\
PFL, 1993
\end{tabular} & MG & 0 & |1995-1999 & |1999-2003 & 2003-2007 & 2007-2011 & 0 & SENADOR & Engengeiro Civil & 0,00 & Rs 0,00 & $\mathrm{R} \$ 2.918 .546,50$ & $0,00 \%$ \\
\hline $\begin{array}{l}\text { LEONARDO LEMOS BARROS } \\
\text { QUiNTĀo }\end{array}$ & $\begin{array}{l}\text { PMDB, 1999-2001; } \\
\text { PSB 2001-2003; } \\
\text { PFL, 2003; PMDB, } \\
\text { 20203 }\end{array}$ & MG & 10 & ]$_{0}$ & 10 & & 2007-2011 & $2011-2015$ & $\begin{array}{l}\text { DEPUTADO } \\
\text { FEDERAL }\end{array}$ & Empresário & 0,00 & R\$ 0,00 & $\mathrm{R} \$ 2.637 .840,47$ & $0,00 \%$ \\
\hline
\end{tabular}




\begin{tabular}{|c|c|c|c|c|c|c|c|c|c|c|c|c|c|c|}
\hline Nоме & PARtioo & uF & 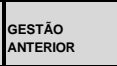 & GESTÃol & GESTÃoul & GESTĀo III & GESTÃon & 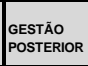 & Uultimo cafGo & \begin{tabular}{|l}
$\begin{array}{c}\text { Profissắ } \\
\text { DECLAAADA }\end{array}$ \\
\end{tabular} & $\begin{array}{c}\text { AREA DAS TERAAS } \\
(\text { HA })\end{array}$ & $\begin{array}{c}\text { VALOR DAS TERAAS } \\
\text { RS }\end{array}$ & $\begin{array}{l}\text { VALOR Do } \\
\text { PATrmOMONO }\end{array}$ & $\begin{array}{c}\text { VaLLo DAS } \\
\text { TERAAPPATRMOMOMO }\end{array}$ \\
\hline Еемав ватIтTа Мовева | & 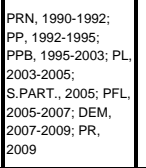 & мG & |991-1995 & o & 1999-2003 & 2003-2007 & $2007-2011$ & o & $\begin{array}{l}\text { DEFUADOO } \\
\text { FEDERAL }\end{array}$ & 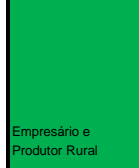 & 0,00 & R\& 0.00 & R\$ 2.089.758,30 & $0.00 \%$ \\
\hline \begin{tabular}{|l|} 
EENAARD D DE VASCONCELLOS \\
SANTANA
\end{tabular} & 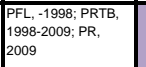 & ма & 0 & $l^{\circ}$ & 0 & & 0 & $2011-2015$ & $\begin{array}{l}\text { DEEUTADO } \\
\text { FEDERAL }\end{array}$ & Adovgagato & 0,00 & R\$ 0,00 & Rs 1.021.804,15 & $0,00 \%$ \\
\hline $\begin{array}{l}\text { STlot ANATHIO } \\
\text { MATTOS }\end{array}$ & 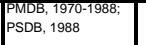 & ма & $0_{0}$ & $l_{0}$ & $1999-2003$ & $2003-2007$ & $2007-2011$ & $0^{0}$ & 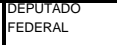 & Senvidor Pubilico & 0.00 & Rs 0,00 & Rs 933.468 .40 & $0.00 \%$ \\
\hline $\begin{array}{l}\text { JOSE RAFAEL GUERRA PINTO } \\
\text { COELHO }\end{array}$ & PSDB, 1988 & мa & 0 & o & 1999-2003 & $2003-2007$ & $2007-2011$ & 0 & 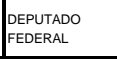 & 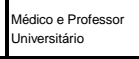 & 0,00 & R\$ 0,00 & $\mathrm{R} \$ 737.113,04$ & $0,00 \%$ \\
\hline & \begin{tabular}{|c|c|} 
PFL, 1998 \\
\end{tabular} & ма & 0 & 0 & $1999-2003$ & 0 & 0 & 0 & $\begin{array}{l}\text { DEFUADO } \\
\text { EEDERAL }\end{array}$ & Empresario & 0,00 & Rs 0,00 & Rs 490.077,56 & $0,00 \%$ \\
\hline 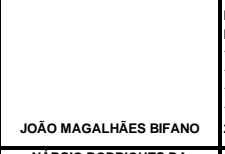 & 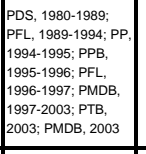 & ма & 0 & | 1996-1999 & 1999-2003 & $2003-2007$ & $2007-2011$ & $2001-2015$ & $\begin{array}{l}\text { DEUUATOO } \\
\text { EEEERAL }\end{array}$ & 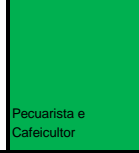 & 2NI & R\$5 $5.648,95$ & RS $46.071,18$ & $12.26 \%$ \\
\hline 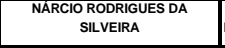 & PSDB, 1993 & ма & 0 & $1995-1998$ & 1999-2003 & $2003-2007$ & $2007-2011$ & $2011-2015$ & 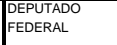 & \begin{tabular}{|l} 
Jonalista \\
\end{tabular} & N & F\$ $8.099,70$ & R8 1.822.729,18 & $0.44 \%$ \\
\hline \begin{tabular}{|l|l|} 
LUI FERNANDO RAMOS FARIA \\
\end{tabular} & 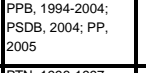 & мG & 10 & 0 & 0 & 0 & $2007-2011$ & $2011-2015$ & $\begin{array}{l}\substack{\text { DEEUTADO } \\
\text { FEDERAL }} \\
\end{array}$ & 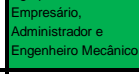 & $\mathrm{N}$ & RS 21.583.33 & Rs 1.142.311,89 & $1,53 \%$ \\
\hline WALTER DA ROCHA TOSTA & $\begin{array}{l}\text { PTR, I996-1997; } \\
\text { PMN, 1997 }\end{array}$ & мG & $0_{0}$ & o & 0 & $0^{0}$ & $0^{0}$ & $2011-2015$ & $\begin{array}{l}\text { DeFUTAOO } \\
\text { EEDERAL }\end{array}$ & Autronomo & ${ }^{3 \mathrm{NI}}$ & RS 21.900.00 & Rs 880.519,68 & $2,49 \%$ \\
\hline NEWTON CARDOSO & MOB; РP; РМОв & мa & |1979-1983; & |995-1996 & 0 & 0 & 0 & $2011-2015$ & $\begin{array}{l}\text { DEEUTADO } \\
\text { FEDERAL }\end{array}$ & 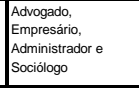 & N & Rs 3 $30.000,00$ & R\$ 7 7.:956.890,08 & $0,04 \%$ \\
\hline RENZO DO AMARAL BRAZ & PP, 1999 & мG & 10 & o & 0 & 0 & o & $2011-2015$ & 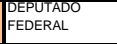 & 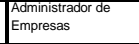 & 3N1 & R5 $34,450,000$ & RS 1.509.06, 40 & $2,28 \%$ \\
\hline JAIME MARTINS FLLHO & 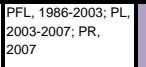 & мG & 0 & |995-1999 & $1999 \cdot-2003$ & $2003-2007$ & 2007-2011 & $2001-2015$ & 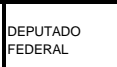 & 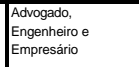 & 0,00 & Rs $39.024,91$ & Rq 2.690.247,33 & $1,45 \%$ \\
\hline OLAVO BLLAC PINTO NETO & 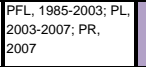 & мa & 0 & {[} & 0 & & $2007-2011$ & $2011-2015$ & 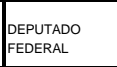 & Adovogado & 487,76 & Rs 69994,23 & R8 8.877.604,04 & $0,79 \%$ \\
\hline $\begin{array}{l}\text { JOSE SANTANA DE } \\
\text { VASCONCELOS }\end{array}$ & 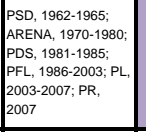 & ма & 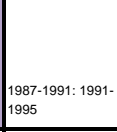 & |995-1999 & 0 & $2003-2007$ & $2007-2011$ & 0 & $\begin{array}{l}\text { DEEUTADO } \\
\text { EEDERALL }\end{array}$ & 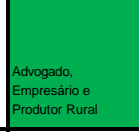 & $1.242,82$ & R5 $76.328,18$ & Rs 1.195.077,97 & $6,39 \%$ \\
\hline $\begin{array}{l}\text { DIMAS FABAAN TOLEDO } \\
\text { JUNOOR }\end{array}$ & PP & ма & $1^{\circ}$ & o & $0^{\circ}$ & 10 & o & $2011-2015$ & $\begin{array}{l}\text { DEFUUADO } \\
\text { EEDERAL }\end{array}$ & Naio intormou & $2002 \mathrm{NI}$ & RS 101.125.00 & R8 1.139.599.,34 & $8.87 \%$ \\
\hline OSMANIO PEREIRA DE OLVEIRA & 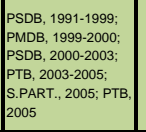 & ма & |991-1995 & |995-1999 & 1999-2003 & $2003-2007$ & & & $\begin{array}{l}\text { DEEUAADO } \\
\text { EEDERAL }\end{array}$ & 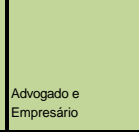 & 830,35 & R\$ 113.591,69 & $122.905,00$ & \\
\hline OSE SILVA SAARES (ZZZ SILVA) & PPT, 2008 & MG & & & & & & $2011-2015$ & $\begin{array}{l}\text { DEEUTADO } \\
\text { FEDERAL }\end{array}$ & 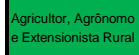 & 323,40 & $\mathrm{R} \$ 148.637,88$ & RS 730.477 .88 & $20,35 \%$ \\
\hline
\end{tabular}




\begin{tabular}{|c|c|c|c|c|c|c|c|c|c|c|c|c|c|c|}
\hline NOME & PARtido & UF & $\begin{array}{l}\text { GESTÃo } \\
\text { ANTERIOR }\end{array}$ & GESTÃoI & GESTĀol & GESTÃo III & GESTÃo IV & \begin{tabular}{|l} 
GESTäo \\
POSTERIOR
\end{tabular} & ÚLTIMO CARGO & $\begin{array}{l}\text { PROFISSÃo } \\
\text { DEELARADA }\end{array}$ & $\begin{array}{c}\text { AREA DAS TERRAS } \\
\text { (HA) }\end{array}$ & $\begin{array}{c}\text { VALOR DAS TERRAS } \\
\text { RS }\end{array}$ & $\begin{array}{l}\text { VALOR DO } \\
\text { PATRIMOONIO }\end{array}$ & $\begin{array}{c}\text { VALOR DAS } \\
\text { TERAASPATRMIMONIO }\end{array}$ \\
\hline $\begin{array}{l}\text { MARCIO REINALDO DIAS } \\
\text { MORERA }\end{array}$ & 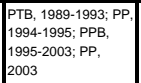 & MG & 0 & |1995-1999 & |1999-2003 & 2003-2005 & 2007-2011 & $2011-2015$ & $\begin{array}{l}\text { DEPUTADO } \\
\text { FEDERAL }\end{array}$ & $\begin{array}{l}\text { Economista e Analistal } \\
\text { de Orçamentios }\end{array}$ & $204,14+\mathrm{NI}$ & R\$ $148.849,71$ & $\mathrm{R} \$ 1.795 .774,69$ & $8,29 \%$ \\
\hline RODRIGO BATISTA DE CASTRO & PSDB & MG & 0 & 0 & 0 & 0 & 0 & $2011-2015$ & $\begin{array}{l}\text { DEPUTADO } \\
\text { FEDERAL }\end{array}$ & $\mid \begin{array}{l}\text { Advogado } \\
\text { Administrador de } \\
\text { Empresas }\end{array}$ & $57+\mathrm{NI}$ & R\$ $159.614,85$ & $\mathrm{R} \$ 1.195 .798,41$ & $13,35 \%$ \\
\hline LAEL VIEIRA VARELLA & 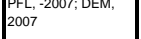 & MG & $\left.\right|_{1995} ^{1987-19}$ & 1995-1999 & $1999-2003$ & $2003-2007$ & $2007-2011$ & $2011-2015$ & $\begin{array}{l}\text { DEPPUADO } \\
\text { FEDERAL }\end{array}$ & Empresário & $\mathrm{NI}$ & R\$ 165.737,36 & $\mathrm{R} \$ 10.399 .182,94$ & $1,59 \%$ \\
\hline PAULO PIAU NOGUERA & \begin{tabular}{|l|} 
PFL, 1992-2003; PP, \\
2003-2005; PPS, \\
2005-2007; PMDB, \\
2007
\end{tabular} & MG & 0 & 0 & 0 & 0 & $2007-2011$ & $2011-2015$ & $\begin{array}{l}\text { DEPUTADO } \\
\text { FEDERAL } \\
\end{array}$ & e Produtor Rural & $2 \mathrm{NI}$ & Rs 221.000,00 & $\mathrm{R} \$ 430.181,15$ & $51,37 \%$ \\
\hline CLEUBER BRANDĀO CARNERO & \begin{tabular}{|l|} 
PDS; ARENA; PFL, \\
1985-2005; PTB, \\
2005
\end{tabular} & MG & 0 & 0 & |1999-2003 & 2003-2007 & 0 & 0 & $\begin{array}{l}\text { DEPUTADO } \\
\text { FEDERAL }\end{array}$ & $\begin{array}{l}\text { A Avvogado, Protessor } \\
\text { Prododtor Purate le } \\
\text { Comerciante }\end{array}$ & $542,28+2 \mathrm{NI}$ & R\$ 231.442,34 & R\$ 701.771,01 & $32,98 \%$ \\
\hline $\begin{array}{l}\text { GERALDO THADEU PEREERA } \\
\text { DOS SANTOS }\end{array}$ & \begin{tabular}{|l|} 
PSDB, 1989-2001; \\
PPS, 2001
\end{tabular} & MG & 0 & 0 & 0 & 2003-2007 & $2007-2011$ & $2011-2015$ & $\begin{array}{l}\text { DEPUTADO } \\
\text { EEDERAL }\end{array}$ & Denista & $\mathrm{NI}$ & Rs 236.915,10 & R\$ 858.366,99 & $27,60 \%$ \\
\hline $\begin{array}{l}\text { ALEXANDRE SILVEIRA DE } \\
\text { OLIVEIRA }\end{array}$ & \begin{tabular}{|l|} 
PL, 1999-2005; PPS, \\
2005
\end{tabular} & MG & 0 & 0 & 0 & 0 & $2007-2011$ & $2011-2015$ & $\begin{array}{l}\text { DEPUTADO } \\
\text { FEDERAL }\end{array}$ & $\begin{array}{l}\text { Comerciante e } \\
\text { Funcionaríi Püblico }\end{array}$ & $\mathrm{NI}$ & R\$ 240.000,00 & $\mathrm{R} \$ 1.382 .666,91$ & $17,36 \%$ \\
\hline $\begin{array}{l}\text { ODELMO LEÄO CARNEIRO } \\
\text { SOBRINHO }\end{array}$ & $\begin{array}{l}\text { PRN, 1990-1993; } \\
\text { PP, 1993-1995; } \\
\text { PPB, 1995-2003; } \\
\text { PP, 2003 }\end{array}$ & MG & |1991-1995 & | 1995-1999 & |1999-2003 & 2003-2005 & 0 & 0 & $\begin{array}{l}\text { DEEUTADO } \\
\text { FEDERAL }\end{array}$ & $\begin{array}{l}\text { Bancáric } \\
\text { Rural }\end{array}$ & $3.399,97+2 \mathrm{NI}$ & Rs $308.117,61$ & R\$ $835.460,55$ & $36,88 \%$ \\
\hline $\begin{array}{l}\text { ADEMIR CAMLOO PRATES } \\
\text { RODRIGUES }\end{array}$ & \begin{tabular}{|l|} 
PSDB, 1991-1997; \\
PPS, 1997-2005; PL, \\
2005; PDT, 2005
\end{tabular} & MG & 0 & 0 & 0 & 2003-2007 & 2007-2011 & $2011-2015$ & $\begin{array}{l}\text { DEPUTADO } \\
\text { FEDERAL }\end{array}$ & \begin{tabular}{|l|} 
Advogado, \\
Administrador \\
Holspitalar, Médico \\
Ginecologista, \\
Obbstetra do Trabalho \\
e Legista
\end{tabular} & $1.852,90$ & R\$ $315.591,43$ & R\$ 507.215,23 & $62,22 \%$ \\
\hline CARLALLE JESUS PEDROSA & PSDB, 2001 & MG & 0 & 0 & 0 & 0 & 0 & $2011-2015$ & $\begin{array}{l}\text { DEEUTAADO } \\
\text { FEDERAL }\end{array}$ & Comerciante & $3 \mathrm{NI}$ & R\$ $320.899,17$ & $\mathrm{R} \$ 4.301 .660,18$ & $7,46 \%$ \\
\hline MAURO RIBEIRO LOPES & $\begin{array}{l}\text { PFL, 1986-1997; } \\
\text { PMDB, 1997 }\end{array}$ & MG & 0 & | 1995-1999 & |1999-2003 & 2003-2005 & $2007-2011$ & $2011-2015$ & $\begin{array}{l}\text { DEPUTADO } \\
\text { EEDERAL }\end{array}$ & \begin{tabular}{|l} 
Empresário e \\
Bacharel em Direito
\end{tabular} & 204,14 & R\$ $341.039,20$ & $\mathrm{R} \$ 4.383 .573,27$ & $7,78 \%$ \\
\hline AELTON JOSÉ DE FREITAS & \begin{tabular}{|l|} 
PSD, 1992-1996; \\
PMDB, 1996-2003; \\
LL, 2003-2007; PR, \\
2007 \\
\end{tabular} & MG & 0 & 0 & |1999-2003 & 2003-2007 & $2007-2011$ & $2011-2015$ & $\begin{array}{l}\text { DEFUTADO } \\
\text { FEDERAL }\end{array}$ & 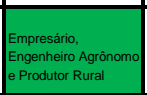 & ${ }_{5 \mathrm{NI}}$ & $\mathrm{R} \$ 349.602,82$ & $\mathrm{R} \$ 5.468 .418,98$ & $6,39 \%$ \\
\hline $\begin{array}{l}\text { CARLOS CARMO ANDRADES } \\
\text { MELLES }\end{array}$ & \begin{tabular}{|l|} 
PFL, \\
DEM, 2007
\end{tabular} & MG & 0 & |1995-1999 & |1999-2003 & 2003-2007 & 2007-2011 & $2011-2015$ & $\begin{array}{l}\text { DEPUTADO } \\
\text { FEDERAL }\end{array}$ & \begin{tabular}{|l|} 
Empresárío e \\
Engenheiro Agrónomomo
\end{tabular} & $5 \mathrm{NI}$ & $\mathrm{R} \$ 378.870,86$ & $\mathrm{R} \$ 2.5455 .232,43$ & $14,89 \%$ \\
\hline DANLLO DE CASTRO & PSDB, 1994 & MG & 0 & | 1995-1999 & 1999-2003 & 2003-2007 & 0 & 0 & FEDERAL & Bancário & $258,34+\mathrm{NI}$ & R\$ 417.864,27 & R\$ $\quad 1.586 .858,22$ & $26,33 \%$ \\
\hline JOSÉ MLLTĀO COSTA & $\begin{array}{l}\text { PMDB, 1985-1986; } \\
\text { PFL, 1986-1988; }\end{array}$ & MG & 0 & 0 & |1999-2003 & 2003-2007 & 0 & 0 & $\begin{array}{l}\text { DEPUTADO } \\
\text { FEDERAL }\end{array}$ & Servidor Público & 556,51 & R\$ $419.231,43$ & R\$ 895.231,43 & $46,83 \%$ \\
\hline MARCOS MONTES CORDEIRO & $\begin{array}{l}\text { PFLL, 1995-2007; } \\
\text { DEM, 2007 }\end{array}$ & MG & 0 & 0 & 10 & 10 & 2007-2011 & $2011-2015$ & $\begin{array}{l}\begin{array}{l}\text { DEPUTADO } \\
\text { FEDERAL }\end{array} \\
\end{array}$ & \begin{tabular}{|l|} 
Medicina e Médico do \\
Trabalho
\end{tabular} & $3 \mathrm{NI}$ & $\mathrm{R} \$ 460.802,51$ & $\mathrm{R} \$ 2.307 .547,27$ & $19,97 \%$ \\
\hline HERCULANO ANGHINETTII & $\begin{array}{l}\text { PSDB, 1995; PPB, } \\
\text { 1995-2003; PP, }\end{array}$ & MG & 0 & | 1995-1999 & |1999-2003 & 2003-2007 & 0 & 0 & \begin{tabular}{|l}
$\begin{array}{l}\text { DEPUTADO } \\
\text { FEDERAL }\end{array}$ \\
\end{tabular} & $\begin{array}{l}\text { Corretor de Segur } \\
\text { Procoutor Rural }\end{array}$ & $3 \mathrm{NI}$ & R\$ $470.000,00$ & $\mathrm{R} \$ 2.785 .627,27$ & $16,87 \%$ \\
\hline ARACELY DE PAULA & \begin{tabular}{|l|} 
PFL, - 2003; ARENA \\
PDS: PL, 2003- \\
2007; PR, 2007
\end{tabular} & MG & |1991-1995 & |1995-1999 & 1999-2003 & 2003-2007 & 2007-2011 & $2011-2015$ & $\begin{array}{l}\text { DEPUTADO } \\
\text { FEDERAL }\end{array}$ & $\mid \begin{array}{l}\text { Avogogadoe } \\
\text { Pecuarista }\end{array}$ & $368,26+3 \mathrm{NI}$ & R\$ 529.383,13 & $R \$ 3.179 .527,37$ & $16,65 \%$ \\
\hline JOSE SARAIVA FELPEE & $\begin{array}{l}\text { MDB, 1979-1981; } \\
\text { PMDB, 1981 }\end{array}$ & MG & 0 & | 1995-1999 & |1999-2003 & 2003-2007 & $2007-2011$ & $2011-2015$ & $\begin{array}{l}\text { DEPUTADO } \\
\text { FEDERAL }\end{array}$ & $\begin{array}{l}\text { Médico e Professsor } \\
\text { Universitatrío }\end{array}$ & $4 \mathrm{NI}$ & R\$ $616.869,80$ & $\mathrm{R} \$ 2.385 .644,69$ & $25,86 \%$ \\
\hline $\begin{array}{l}\text { DIEGO LEONARDDO DE ANDRADE } \\
\text { CARVALHO }\end{array}$ & PR, 2006 & MG & 0 & 0 & 0 & 0 & 0 & $2011-2015$ & \begin{tabular}{|l} 
DEPUTADO \\
FEDERAL
\end{tabular} & $\begin{array}{l}\begin{array}{l}\text { Administradar de } \\
\text { Empresas }\end{array} \\
\end{array}$ & $20+3 \mathrm{NI}$ & R\$ $680.0000,00$ & $\mathrm{R} \$ 1.583 .653,75$ & $42,94 \%$ \\
\hline 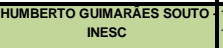 & \begin{tabular}{|l} 
1966-1979; PDS, \\
1980-1984; PFL,
\end{tabular} & MG & $\begin{array}{l}\text { 1983; 1983-1987; } \\
\text { 1987-1991; 1991- }\end{array}$ & 1995 & 0 & 0 & 2007-2011 & 0 & FEDERAL & Advogado e Contador & $\mathrm{NI}$ & R\$ $645.414,26$ & R\$ $\quad 2.663 .913,89$ & $24,23 \%$ \\
\hline MARIO LUCIO HERINGER - INESC & PDT, 2001 & MG & 0 & 0 & 0 & $2003-2007$ & $2007-2011$ & 0 & $\begin{array}{l}\text { DEEUTAAO } \\
\text { FEDERAL }\end{array}$ & \begin{tabular}{|l} 
Amimisitrador \\
Hospitalar
\end{tabular} & $\mathrm{NI}$ & R\$ $358.570,51$ & $\begin{array}{|ll|}\text { Rs } & 4.745 .076,47 \\
\end{array}$ & $7,56 \%$ \\
\hline MARIO DE OLIVEIRA & $\begin{array}{l}\text { PTR, 1991; PP, } \\
\text { 1993-1995; PPB, }\end{array}$ & MG & $\begin{array}{l}1983-1987 ; 1987- \\
1991 ; 1991-1995\end{array}$ & | 1995-1999 & |1999-2003 & 0 & $2007-2011$ & 0 & $\begin{array}{l}\begin{array}{l}\text { DEPUTADO } \\
\text { FEDERAL }\end{array} \\
\end{array}$ & Ministro Evangêlico & $\mathrm{NI}$ & RS 687.445,91 & RS & \\
\hline $\begin{array}{l}\text { BONIFACIO JoSÉ TAMMM DE } \\
\text { ANDRADA }\end{array}$ & \begin{tabular}{|l|} 
UDN, 1954-1965; \\
AREAA 1966-1979; \\
PES, 1980-1993; \\
PST, , $9944-19966 ;$ \\
PSDB, 1997
\end{tabular} & MG & 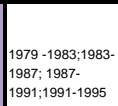 & |1995-1999 & |1999-2003 & 2003-2007 & 2007-2011 & & $\begin{array}{l}\text { DEPUTADO } \\
\text { FEDERAL }\end{array}$ & $\begin{array}{l}\text { Advogado e Professor } \\
\text { Universtário }\end{array}$ & $924,44+3 \mathrm{NI}$ & $\mathrm{R} \$ 932.152,33$ & $\mathrm{R} \$ 9.691 .069,66$ & $9,62 \%$ \\
\hline
\end{tabular}




\begin{tabular}{|c|c|c|c|c|c|c|c|c|c|c|c|c|c|c|}
\hline NOME & PARTIDO & UF & $\begin{array}{l}\text { GESTÄO } \\
\text { ANTERIOR }\end{array}$ & GESTĀOI & GESTĀOII & GESTĀO III & GESTÃo IV & \begin{tabular}{|l} 
GESTÃo \\
POSTERIOR
\end{tabular} & ÚLTIMO CARGO & $\begin{array}{l}\text { PROFISSÃO } \\
\text { DECLARADA }\end{array}$ & $\begin{array}{c}\text { AREA DAS TERRAS } \\
\text { (HA) }\end{array}$ & $\begin{array}{c}\text { VALOR DAS TERRAS } \\
\text { RS }\end{array}$ & $\begin{array}{l}\text { VALLR DO } \\
\text { PATRIMOONIO }\end{array}$ & $\begin{array}{l}\text { VALOR DAS } \\
\text { TERRASPATRRIMONIO }\end{array}$ \\
\hline $\begin{array}{l}\text { DOMINGOS SAVIO CAMPOS } \\
\text { RESENDE }\end{array}$ & PSDB, 1988 & MG & 0 & 0 & 0 & 0 & 0 & 2011-2015 & $\begin{array}{l}\text { DEPUTADO } \\
\text { FEDERAL }\end{array}$ & Médico Veterinário & $2 \mathrm{NI}$ & $\mathrm{R} \$ 1.800 .000,00$ & $\mathrm{R} \$ 3.168 .605,62$ & $56,81 \%$ \\
\hline $\begin{array}{l}\text { ANTONIO EUSTAQUUIO ANDRADE } \\
\text { FERRERA }\end{array}$ & PMDB, 1987 & MG & 0 & 0 & 0 & 0 & 2007-2011 & 2011-2015 & \begin{tabular}{|l}
$\begin{array}{l}\text { DEPUTADO } \\
\text { FEDERAL }\end{array}$ \\
\end{tabular} & \begin{tabular}{|l} 
Engenheir Civile \\
Produtor Rural
\end{tabular} & $8 \mathrm{NI}$ & $\mathrm{R} \$ 1.823 .756,06$ & $\mathrm{R} \$ 3.122 .713,65$ & $58,40 \%$ \\
\hline ROMEL ANIZZO JORGE & $\begin{array}{l}\text { PDS, 1980-1990; } \\
\text { PRN, 1990-1994; }\end{array}$ & MG & |1991-1995 & | 1995-1999 & |1999-2003 & 2003-2007 & 0 & 0 & \begin{tabular}{|l} 
DEPUTAAO \\
FEDERAL
\end{tabular} & $\begin{array}{l}\text { Agropecuarista e } \\
\text { Empresasrio Rural }\end{array}$ & $3 \mathrm{NI}$ & R\$ $1.854 .978,10$ & $\mathrm{R} \$ 5.694 .412,49$ & $32,58 \%$ \\
\hline ROMEU FERREIRA DE QUEEROZ & $\begin{array}{l}\text { PPS, 1990-1993; } \\
\text { PTB, 1993-1995; }\end{array}$ & MG & 1991-1995 & | 1995-1999 & |1999-2003 & 2003-2007 & 0 & 0 & $\begin{array}{l}\text { DEEUTAAO } \\
\text { FEDERAL }\end{array}$ & $\begin{array}{l}\text { Advogadoe e } \\
\text { Empresário }\end{array}$ & $3725,37+\mathrm{NI}$ & R\$ $2.127 .501,22$ & $\mathrm{R} \$ 6.545 .661,11$ & $32,50 \%$ \\
\hline SILAS BRASILEIRO & PMDB, 1982 & MG & 0 & | 1995-1999 & |1999-2003 & 2003-2007 & 2009-20011 & 0 & $\begin{array}{l}\text { DEPUTAAO } \\
\text { FEDERAL }\end{array}$ & 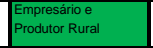 & $836,13+2 \mathrm{NI}$ & Rs $3.929 .667,44$ & $\mathrm{R} \$ 6.564 .394,73$ & $59,86 \%$ \\
\hline $\begin{array}{l}\text { WALDEMIR MOKA MIRANDA DE } \\
\text { BRITO }\end{array}$ & MDB; PMDB, 1981 & Ms & 0 & 0 & |1999-2003 & 2003-2007 & 2007-2011 & 0 & $\begin{array}{l}\begin{array}{l}\text { DEPUTADO } \\
\text { FEDERAL }\end{array} \\
\end{array}$ & Médico e Professor & 0,00 & Rs 0,00 & R\$ $340.997,41$ & $0,00 \%$ \\
\hline $\begin{array}{l}\text { MARÇAL GONCALVES LETTE } \\
\text { FLLHO }\end{array}$ & $\begin{array}{l}\text { PSDB, 1997-1999; } \\
\text { PMDB, 1999 }\end{array}$ & Ms & 0 & | 1996-1999 & |1999-2003 & 0 & 2009-2011 & 2011-2015 & \begin{tabular}{|l} 
DEPUTADO \\
FEDERAL
\end{tabular} & Advogado e Radialista & 86,00 & R\$ $\$ 0.0000,00$ & $\mathrm{R} \$ 2.160 .564,03$ & $2,78 \%$ \\
\hline LUIZ HENRIQUE MANDETTA & $\begin{array}{l}\text { PMBB, 2003-2009; } \\
\text { DEM, 2009 }\end{array}$ & Ms & 0 & 0 & 0 & 0 & 0 & $2011-2015$ & $\begin{array}{l}\text { DEEUTADOO } \\
\text { FEDERAL }\end{array}$ & Médico & 805,00 & R\$ 406.390,47 & $\begin{array}{|ll|}\mathrm{R} \$ & 539.617,57 \\
\end{array}$ & $75,31 \%$ \\
\hline EDSON GIROTO & $\begin{array}{l}\text { PMBD, 1997-2009; } \\
\text { PR, 2010 }\end{array}$ & ms & 0 & 0 & 0 & 0 & 0 & 2011-2015 & $\begin{array}{l}\text { DEEUTAAD } \\
\text { FEDERAL }\end{array}$ & Engenheiro Civil & 206,56 & R\$ $820.283,00$ & $\mathrm{R} \$ 1.767 .386,40$ & $46,41 \%$ \\
\hline MURLLO ZAUTTH & PFL, 2001 & Ms & 0 & 0 & 0 & 2003-2007 & 0 & 0 & $\mid \begin{array}{l}\text { DEEDERAL } \\
\text { FEUEA }\end{array}$ & Engenherio Civil & 958,00 & R\$ $942.414,19$ & R\$ $7.351 .791,04$ & $12,82 \%$ \\
\hline $\begin{array}{l}\text { FLLVIII AUGGSTT COEEHO } \\
\text { DERZI (30/06/1951 a 13/08/2001) }\end{array}$ & \begin{tabular}{|l|} 
PFL, 1991-1993; \\
PST, 1993; PP, 1993
\end{tabular} & Ms & |1991-1995 & |1995-1999 & 1999-2001 & 0 & 0 & 0 & \begin{tabular}{|l} 
DEEUTAAO \\
FEDERAL
\end{tabular} & $\begin{array}{l}\text { Administrador de } \\
\text { Empresas e }\end{array}$ & $45.572,00$ & R\$ $1.733 .734,00$ & \begin{tabular}{|l|l|} 
RS & $3.082 .056,08$ \\
\end{tabular} & $56,25 \%$ \\
\hline $\begin{array}{l}\text { RAMEZ TEBET (07711/1/1936- } \\
\text { 1771/1/2006) }\end{array}$ & PMDB & Ms & 0 & 0 & 0 & 2003-2006 & 0 & 0 & Senador & Advogado & $8.068,64$ & 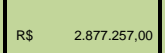 & $\mathrm{R} \$ 4.304 .235,43$ & $66,85 \%$ \\
\hline REINALDO AZAMBUJA SILVA & PSDB, 1995 & Ms & 0 & 0 & 0 & 0 & 0 & $2011-2015$ & $\begin{array}{l}\text { DEEUTTADO } \\
\text { FEDERAL } \\
\end{array}$ & Agropecuarista & $2.504,00$ & $R \$ 22.600 .000,00$ & 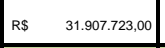 & $70,83 \%$ \\
\hline $\begin{array}{l}\text { DAGOBERTO NOGUEERA FLHHO - } \\
\text { INESC }\end{array}$ & PDT, 1993 & Ms & 0 & 10 & o & jo & 2007-2011 & 0 & $\begin{array}{l}\text { DEPUTADO } \\
\text { FEDEAAL }\end{array}$ & Nấo Intormou & $1.715,00$ & R\$ $918.510,28$ & $\begin{array}{|ll|}\text { RS } & 1.381 .414 .97 \\
\end{array}$ & $66,49 \%$ \\
\hline DILSO SPERAFICO & \begin{tabular}{|l|}
$\begin{array}{l}\text { PFL, 1988-1993; } \\
\text { PMDD, 1993-1997; } \\
\text { PSDB, 1997 }\end{array}$ \\
PST
\end{tabular} & Ms & 0 & | 1995-1999 & to & o & 0 & 0 & $\begin{array}{l}\text { DEEUTADO } \\
\text { FEDERAL }\end{array}$ & \begin{tabular}{|l|} 
Engenhhirio Agrónnomo \\
e Prodoutor Rural
\end{tabular} & ? & $?$ & ? & ? \\
\hline $\begin{array}{l}\text { JONAS PNHEERP DA SLILA } \\
\text { (22201/1941 a 19:02:2008) }\end{array}$ & $\begin{array}{l}\text { PDS, 1982-1985; } \\
\text { PFL, 1985 }\end{array}$ & мт & $\mid \begin{array}{l}1983-1987,1,1987- \\
19991,1991-1995\end{array}$ & | 1995-1999 & |999-2003 & 2003-2007 & 2007-2008 & 0 & SENADOR & \begin{tabular}{|l|} 
Extensionista Rurale \\
Medico Veternanario
\end{tabular} & 9,77 & $\mathrm{R} \$ 3.314,74$ & R\$7 717.700,38 & $0,46 \%$ \\
\hline RICARTE DE FREITAS JUNIOR & \begin{tabular}{|l} 
PLL, 1987-1994; \\
PSBB, 1994-2003; \\
PTB, 2003
\end{tabular} & мт & 0 & 0 & |1999-2003 & 2003-2007 & 2010-2011 & 0 & $\begin{array}{l}\text { DEEUTADO } \\
\text { FEDERAL }\end{array}$ & Advogado & 69,65 & RS $119.879,45$ & R\$ $370.413,62$ & $32,36 \%$ \\
\hline $\begin{array}{l}\text { WELLINGTON ANTONIO } \\
\text { FAGUNDES }\end{array}$ & \begin{tabular}{|l|} 
PDS, 1980-1985; \\
PDT, 1985-1987; PL, \\
1987-1999; PSDB, \\
1999-2001; PL, 2001 \\
2007; PR, 2007 \\
\end{tabular} & мт & |1991-1995 & | 1995-1999 & |1999-2003 & 2003-2007 & 2007-2011 & $2011-2015$ & \begin{tabular}{|l} 
DEFUTADO \\
FEDERAL
\end{tabular} & 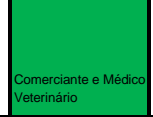 & $2.133,70$ & R\$ $187.371,92$ & $\mathrm{R} \$ 7.255 .285,56$ & $2,58 \%$ \\
\hline $\begin{array}{l}\text { ELIENE JOSÉ DE LIMA (ELIENE } \\
\text { LIMA) }\end{array}$ & $\begin{array}{l}\text { PPT, , } 99191-1993 ; \\
\text { PSB, 1993-2005; } \\
\text { PP, 2005-. }\end{array}$ & мт & 0 & 0 & 0 & 0 & 2007-2011 & 2011-2015 & $\begin{array}{l}\text { DEPUTADO } \\
\text { FEDERAL }\end{array}$ & \begin{tabular}{|l} 
Engenheiro Civil e \\
Profoessor
\end{tabular} & $4+2 \mathrm{NI}$ & R\$ $300.000,00$ & R\$ 565.000,00 & $53,10 \%$ \\
\hline $\begin{array}{l}\text { ROGÉRIO LUCIO SOARES DA } \\
\text { SILVA }\end{array}$ & $\begin{array}{l}\text { PRN, 1989-1993; } \\
\text { PPR, 1993-1995; } \\
\text { PPB, 995; PFL, } \\
\text { 1995-2001; PMDB, } \\
\text { 2001-2003; PPS, } \\
\text { 2003-2007; PP, } \\
\text { 2007 }\end{array}$ & мт & 0 & | 1995-1999 & 2002 & 2003-2007 & 2008 & 0 & $\begin{array}{l}\text { DEFUTADO } \\
\text { FEDERAL }\end{array}$ & \begin{tabular}{|l} 
Agricultor e \\
Empresario
\end{tabular} & $16.960,44$ & $463.248,05$ & $\mathrm{R} \$ 1.309 .499,50$ & $35,38 \%$ \\
\hline CARLOS GOMES BEZERRA & $\begin{array}{l}\text { PPBB, 1960-1964; } \\
\text { MDB, 1966-1980; } \\
\text { PMDB, 1980 }\end{array}$ & мт & 1979-1983 & |995-1999 & |999-2003 & 2003-2011 & 2007-2011 & $2011-2015$ & $\begin{array}{l}\text { DEFUTADO } \\
\text { FEDERAL }\end{array}$ & \begin{tabular}{|l|l}
$\mid \begin{array}{l}\text { Industrial, Advogado o } \\
\text { Profoessor }\end{array}$ \\
\end{tabular} & $4.665,00$ & RS 557.856,94 & $\mathrm{R} \$ 18.431 .261,49$ & $3,03 \%$ \\
\hline HOMERO ALVES PEREIRA & $\begin{array}{l}\text { P2B, 1994; PPS, } \\
\text { 2001-2007; PR, }\end{array}$ & мт & 0 & 0 & 0 & 0 & 2007-2011 & 2011-2015 & FEDERAL & $\begin{array}{l}\text { agropecuária } \\
\text { Agra }\end{array}$ & $8.329,90$ & R\$ $1.271 .498,00$ & $\mathrm{R} \$ 2.342 .845,21$ & $54,27 \%$ \\
\hline JÚLIO JOSÉ DE CAMPOS & $\begin{array}{l}\text { ARENA, 1972-1979; } \\
\text { PDS, 1980-1985; } \\
\text { PFL, 1986 }\end{array}$ & мт & $\mid \begin{array}{l}1979-1983 ; \text {; } 1987- \\
1991 ;\end{array}$ & | 1995-1999 & 0 & 10 & 0 & 2011-2015 & $\begin{array}{l}\text { DEPUTADO } \\
\text { FEDERAL }\end{array}$ & \begin{tabular}{|l|} 
Empresáário \\
Engenherio Arônomomo
\end{tabular} & $8443+\mathrm{NI}$ & $\mathrm{R} \$ 1.458 .180,97$ & $\mathrm{R} \$ 12.894 .052,71$ & $11,31 \%$ \\
\hline BLAIRO BORGES MAGGI & PR & мт & 0 & 0 & o & 0 & 0 & $\begin{array}{l}2011-2015 ; 2015- \\
2019\end{array}$ & SENADOR & Nấo Intormou & $2.433,45$ & R\$ 2.197.323,45 & 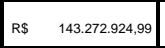 & $1,53 \%$ \\
\hline JAYME VERISSIMO CAMPOS & DЕM & мт & 10 & 10 & 10 & 10 & $2007-2011$ & $2011-2015$ & SENADOR & Nâo Informou & $24796+4 N I$ & RS 6.794.161,52 & R\$ 14.142.116,37 & $48,04 \%$ \\
\hline
\end{tabular}




\begin{tabular}{|c|c|c|c|c|c|c|c|c|c|c|c|c|c|c|}
\hline NOME & PARTIDO & UF & $\begin{array}{l}\text { GESTAO } \\
\text { ANTERIOR }\end{array}$ & GESTÃol & GESTĀoll & GESTÃO III & GESTÃo IV & $\begin{array}{l}\text { GESTAO } \\
\text { POSTERIOR }\end{array}$ & ÚLTiMO CaRGo & $\begin{array}{l}\text { PROFISSAO } \\
\text { DECLARADA }\end{array}$ & $\begin{array}{l}\text { AREA DAS TERRAS } \\
\text { (HA) }\end{array}$ & $\begin{array}{c}\text { VALOR DAS TERRAS } \\
\text { RS }\end{array}$ & $\begin{array}{l}\text { VALLRDO } \\
\text { PATRIMOONIO }\end{array}$ & $\begin{array}{c}\text { VALOR DAS } \\
\text { TERRASPATRRMONONIO }\end{array}$ \\
\hline $\begin{array}{l}\text { WANDENKOLK PASTEUR } \\
\text { GONĢALVES }\end{array}$ & $\begin{array}{l}\text { PP, 1995; PSDB, } \\
\text { 1996-2006; PSDB, }\end{array}$ & $\mathrm{PA}$ & 0 & 0 & 0 & 0 & 2007-2011 & 2011-2015 & $\begin{array}{l}\text { DEPUTADO } \\
\text { FEDERAL }\end{array}$ & |Engenheiro Agrönomo & 0,00 & R\$ 0,00 & R\$ $514.661,46$ & $0,00 \%$ \\
\hline $\begin{array}{l}\text { ELCIONN THEREZINHA ZAHLUTH } \\
\text { BARBALHO }\end{array}$ & PMDB, 1981 & $\mathrm{PA}$ & 0 & | 1995-1999 & |1999-2003 & 0 & 2007-2011 & $2011-2015$ & $\begin{array}{l}\text { DEPUTADA } \\
\text { FEDERAL }\end{array}$ & Pedagoga & 0,00 & $R \$ 0,00$ & $\mathrm{R} \$ 4.5199 .084,88$ & $0,00 \%$ \\
\hline JOSÉ BENTTO PRIANTE JÚNIOR & PMDB, 1984 & $\mathrm{PA}$ & 0 & |1995-1999 & 1999-2003 & 2003-2007 & 0 & $2011-2015$ & FEDERAL & Advogado & 0,00 & $\mathrm{R} \$ 0,00$ & Rs $610.651,11$ & $0,00 \%$ \\
\hline ASDRUBAL MENDES BENTES & $\begin{array}{l}\text { ARENA, 1976-1979; } \\
\text { PMDB, 1980 }\end{array}$ & PA & |1987-1991 & |1997-1999 & $2001-2003$ & 2003-2007 & $2007-2011$ & $20011-2015$ & \begin{tabular}{|l} 
DEPUTADO \\
FEDERAL
\end{tabular} & \begin{tabular}{|l|} 
Advogado e Servidor \\
Puiblico
\end{tabular} & 0,00 & $\mathrm{R} \$ 0,00$ & R\$ $443.617,40$ & $0,00 \%$ \\
\hline $\begin{array}{l}\text { ZENALDO REDREGGUES } \\
\text { COUTINHO JUUNIOR }\end{array}$ & PDS; PTB; PSDB & $\mathrm{PA}$ & 0 & 0 & 1999-2003 & 2003-2007 & $2007-2011$ & $2011-2015$ & $\begin{array}{l}\text { DEPUTADO } \\
\text { FEDERAL }\end{array}$ & Advogado & $\mathrm{NI}$ & RS $112.930,00$ & Rs 678.926,.21 & $16,63 \%$ \\
\hline JOAQUUM DE LIRA MAIA & $\begin{array}{l}\text { PFL, 1999-2003; } \\
\text { PMDB, 2003-2005; } \\
\text { PFL, 2005-2007; } \\
\text { DEM, 2007-. }\end{array}$ & $\mathrm{PA}$ & 0 & 0 & o & 10 & 2007-2011 & 0 & $\begin{array}{l}\text { DEPUTADO } \\
\text { FEDERAL }\end{array}$ & Engenheiro Agrônomo & $313,91+5 \mathrm{NI}$ & R\$ $120.529,32$ & $\mathrm{R} \$ 1.233 .716,93$ & $9,77 \%$ \\
\hline JOSUÉ BENGTSON & PTB, 1997 & $\mathrm{PA}$ & 0 & 0 & 1999-2003 & 2003-2007 & o & $2011-2015$ & FEDERAL & Pastor Evangelico & $3850+4 \mathrm{NI}$ & R\$ $394.928,08$ & $\mathrm{R} \$ 1.170 .155,78$ & $33,75 \%$ \\
\hline MÁRIO COUTO FLLHO & PSDB & $\mathrm{PA}$ & 0 & 0 & 10 & 0 & 2007-2011 & $2011-2015$ & SENADOR & Nāo Informou & 528,00 & R\$ $400.000,00$ & R\$ 598.852,49 & $66,79 \%$ \\
\hline ANIVALDO JUVENLL VALE & $\begin{array}{l}\text { PPP, 1999-1995; } \\
\text { PPB, 1995-1999; } \\
\text { PSDB, 1997 }\end{array}$ & $\mathrm{PA}$ & 0 & | 1995-1999 & |1999-2003 & 2003-2007 & o & 0 & $\begin{array}{l}\text { DEEUTADO } \\
\text { FEDERAL }\end{array}$ & \begin{tabular}{|l} 
Agropecuaristae \\
Servidor Publico
\end{tabular} & $4.956,36+2 \mathrm{NI}$ & R\$ 571.745,46 & R\$ 877.284,19 & $65,62 \%$ \\
\hline OLAVIO SILVA ROCHA & 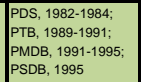 & $\mathrm{PA}$ & 0 & | 1995-1999 & 10 & 10 & o & 0 & $\begin{array}{l}\text { DEPUTADO } \\
\text { FEDERAL }\end{array}$ & Pecuarista & $\mathrm{NI}$ & R\$ $1.805 .577,20$ & $\mathrm{R} \$ 2.215 .577,22$ & $77,09 \%$ \\
\hline JADER FONTENELLE BARBALHO & $\begin{array}{l}\text { MODB, 1966-1981; } \\
\text { PMDB, } 1981\end{array}$ & $\mathrm{PA}$ & $\begin{array}{l}\mid 1975-1979 ; 1979- \\
183 ;\end{array}$ & 0 & 0 & $2003-2007$ & $2007-2011$ & 0 & $\begin{array}{l}\text { DEPUTADO } \\
\text { FEDERAL }\end{array}$ & $\begin{array}{l}\text { Advogadoo e } \\
\text { Empresário }\end{array}$ & $3{ }_{3 N}$ & R\$ $1.828 .653,10$ & $\mathrm{R} \$ 4.583 .434,21$ & $39,90 \%$ \\
\hline GIOVANNI CORREA QUEEROZ & $\begin{array}{l}\text { ARENA, 1975-1983; } \\
\text { PDT, 1986 }\end{array}$ & $\mathrm{PA}$ & 1991-1995 & 1995-1999 & $1999-2003$ & 10 & $2007-2011$ & $2011-2015$ & $\begin{array}{l}\text { DEPUTADO } \\
\text { FEDERAL }\end{array}$ & $\mid \begin{array}{l}\text { Aqropeci } \\
\text { Médico }\end{array}$ & $6.216,94+2 \mathrm{NI}$ & $\mathrm{R} \$ 8.020 .000,00$ & $\mathrm{R} \$ 10.421 .200,00$ & $76,96 \%$ \\
\hline RONALDO JOSÉ DA CUNHA LIMA & $\begin{array}{l}\text { MDB, -1980; PTB, } \\
\text { 1959-1969; PMDB, } \\
1980-2001 ; \text { PSDB, } \\
2001\end{array}$ & PB & 0 & 0 & o & 2003-2007 & 2007-2011 & 0 & $\begin{array}{l}\text { DEPUTADO } \\
\text { FEDERAL }\end{array}$ & $\begin{array}{l}\text { Advogado, Promotor } \\
\text { de Justica, Profosssor } \\
\text { de Diretio e Professor } \\
\text { de Português }\end{array}$ & 0,00 & $\mathrm{R} \$ 0,00$ & RS & $0,00 \%$ \\
\hline $\begin{array}{l}\text { MARCONDES IRAN BENEVIDES } \\
\text { GADELHA }\end{array}$ & $\begin{array}{l}\text { MDB, 1968-1982; } \\
\text { PDS, 1982-1984; } \\
\text { PFL, 1983-2003; } \\
\text { PTB, 2003-2005; } \\
\text { PSB, 2005-2009; } \\
\text { PSC, 2009 }\end{array}$ & PB & $\left|\begin{array}{l}1971-1975 ; \text {; } 975- \\
1979 ; \text { 1979-1983; }\end{array}\right|$ & 0 & |1999-2003 & 2003-2007 & 2007-2011 & 0 & $\begin{array}{l}\text { DEPUTADO } \\
\text { FEDERAL }\end{array}$ & Médico & 2,00 & R\$ $15.000,00$ & R\$ $836.719,89$ & $1,79 \%$ \\
\hline ENIVALDO RIBEIRO & $\begin{array}{l}\text { PDS, 1987-1991; } \\
\text { PDT, 1991-1993; }\end{array}$ & PB & 0 & |1995-1999 & |1999-2003 & 2003-2007 & $2007-2011$ & 0 & $\begin{array}{l}\text { DEPUTADO } \\
\text { FEDERAL }\end{array}$ & 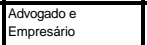 & $24,2+2 \mathrm{NI}$ & $\mathrm{R} \$ 34.800,03$ & $\mathrm{R} \$ 710.555,75$ & $4,90 \%$ \\
\hline JOSÉ WELLINGTON ROBERTO & \begin{tabular}{|l|} 
PL, 2003-2007; PR, \\
2007
\end{tabular} & PB & 0 & 1998 & |999-2002 & 2003-2007 & 2007-2011 & $2011-2015$ & $\begin{array}{l}\text { DEPUTADO } \\
\text { FEDERAL }\end{array}$ & Empresário & 934,00 & $\mathrm{R} \$ 41.638,37$ & R\$ 1.981.809.26 & $2,10 \%$ \\
\hline $\begin{array}{l}\text { VITAL DO REGGO FLLHO } \\
\text { (VITALZINHO) }\end{array}$ & 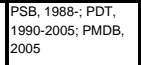 & PB & 0 & 0 & 0 & 10 & 2007-2011 & $\mid \begin{array}{l}2011-2015 ; 2011- \\
2019\end{array}$ & SENADOR & Advogado e Médico & $\mathrm{NI}$ & $\mathrm{R} \$ 150.000,00$ & R\$ 888.842,05 & $16,88 \%$ \\
\hline MANDO ABILIO VIEIRA & $\begin{array}{l}\text { ARENA, 1982-1988; } \\
\text { PMDB, 1989-1990; } \\
\text { PFL, 1991-1992; } \\
\text { PMDB, 1993-2001; } \\
\text { PSDBB, 2001-2006; } \\
\text { PTB, 2006 }\end{array}$ & PB & 0 & | 1995-1999 & |1999-2003 & 20003-2007 & $2007-2011$ & 0 & \begin{tabular}{|l} 
DEFUTADO \\
FEDERAL
\end{tabular} & Médico & ${ }_{12 \mathrm{NI}}$ & RS 186.222,83 & R\$ 992.474,76 & $18,76 \%$ \\
\hline $\begin{array}{l}\text { BENJAMIN GOONES MARANHAO } \\
\text { NETO }\end{array}$ & PMDB, 1989 & $\mathrm{~PB}$ & 0 & 0 & o & $2003-2007$ & o & $2011-2015$ & $\begin{array}{l}\text { DEPUTADO } \\
\text { FEDERAL }\end{array}$ & Cirurgiáa-Dentista & 69,00 & RS 194.666,69 & ? & ? \\
\hline EFRAMM DE ARAUJO DE MORAIS & PDSPFL; PFL & PB & 1991-1995 & |1995-1999 & 1999-2003 & 2003-2007 & $2007-2011$ & 0 & SENADOR & Engenheiro & $1.059,00$ & R\$ $251.200,00$ & $\mathrm{R} \$ 2.550 .576,00$ & $9,85 \%$ \\
\hline CARLOS MARQUES DUNGA & $\begin{array}{l}\text { PPS, } 19828-1986 ; \\
\text { PFL, 1986-1990; }\end{array}$ & PB & 0 & 0 & 1999-2003 & 2003-2007 & 0 & 0 & \begin{tabular}{|l} 
FEDEERALO \\
FEDAL
\end{tabular} & $\mid \begin{array}{l}\text { Avogoado, Pe } \\
\text { e Professor }\end{array}$ & $2.044,20$ & R\$ $304.000,00$ & $\mathrm{R} \$ 1.542 .862,03$ & $19,70 \%$ \\
\hline ADAUTO PEREIRA DE LIMA & \begin{tabular}{|l|} 
ARENA; UDN; PDS, \\
1979-1990; PFL, \\
1990
\end{tabular} & PB & \begin{tabular}{|l|} 
1983-1987; $1987-$ \\
$1991 ; 1991-1995$
\end{tabular} & | 1995-1999 & | 1999-2003 & |2003-2007 & 10 & 10 & $\begin{array}{l}\text { DEPUTADO } \\
\text { FEDERAL }\end{array}$ & $\begin{array}{l}\text { Agropecuarista e } \\
\text { Industrial }\end{array}$ & $7,5+9 \mathrm{9N1}$ & $\operatorname{Rs} 485.746,74$ & R\$ $\quad 3.924 .676,40$ & $12,38 \%$ \\
\hline
\end{tabular}




\begin{tabular}{|c|c|c|c|c|c|c|c|c|c|c|c|c|c|c|}
\hline NOME & PARTIDO & UF & $\begin{array}{l}\text { GESTÄO } \\
\text { ANTERIOR }\end{array}$ & GESTÃol & GESTĀol & GESTÃo III & GESTÃo IV & \begin{tabular}{|l} 
GESTäo \\
POSTERIOR
\end{tabular} & ÚlTIMO CARGo & $\begin{array}{l}\text { PROFISSÃo } \\
\text { DEELARADA }\end{array}$ & $\begin{array}{c}\text { AREA DAS TERRAS } \\
\text { (HA) }\end{array}$ & $\begin{array}{c}\text { VALOR DAS TERRAS } \\
\text { RS }\end{array}$ & $\begin{array}{l}\text { VALOR DO } \\
\text { PATRIMOONIO }\end{array}$ & $\begin{array}{c}\text { VALOR DAS } \\
\text { TERAASPATRMIMONIO }\end{array}$ \\
\hline $\begin{array}{c}\text { JOSÉ TARGINO MARANHĀO (ZÉ } \\
\text { MARANHÂO) }\end{array}$ & 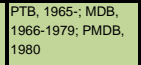 & PB & $\mid \begin{array}{l}1983-1987: 1987-7 \\
1991 ; 1999-1995\end{array}$ & 0 & 0 & 2003-2007 & 0 & 0 & SENADOR & $\begin{array}{l}\text { Advogado, Pecuarista } \\
\text { e Empresariro }\end{array}$ & $11 \mathrm{NI}$ & R\$ 1.741.392,24 & $R \$ 7.429 .880,68$ & $23,44 \%$ \\
\hline $\begin{array}{c}\text { AGUUNALDOVELLOSO BORGES } \\
\text { RiBERO }\end{array}$ & pp & $\mathrm{PB}$ & 0 & 0 & 0 & 0 & 0 & $2011-2015$ & $\begin{array}{l}\text { DEPUTADO } \\
\text { FEDERAL }\end{array}$ & Administrador & $24,2+2 \mathrm{NI}$ & $\mathrm{R} \$ 3.575 .094,34$ & $?$ & $?$ \\
\hline $\begin{array}{l}\text { FERNANDO BEZERRA DE SOUZA } \\
\text { COELH FLLHO }\end{array}$ & PSB & $\mathrm{PE}$ & 0 & 0 & 0 & 0 & $2007-2011$ & $2011-2015$ & $\begin{array}{l}\text { DEPUTADO } \\
\text { FEDERAL }\end{array}$ & $\begin{array}{l}\text { Administrador de } \\
\text { Empresas }\end{array}$ & 0,00 & Rs 0,00 & $\mathrm{R} \$ 482.361,00$ & $0,00 \%$ \\
\hline RICARDO FERREIRA FUUZA & \begin{tabular}{|l} 
ARENA, 1970-1979; \\
PDSS, 1980-1985; \\
PFL, 1986-2001; \\
PPB, 2001-2003; \\
PP, 2003
\end{tabular} & PE & 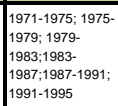 & 10 & |1999-2003 & 2003-2007 & o & 0 & $\begin{array}{l}\text { DEPUTADO } \\
\text { FEDERAL }\end{array}$ & Advogado & 0,00 & R\$ 0,00 & $\begin{array}{|ll|}R \$ & 13.953 .258,67\end{array}$ & $0,00 \%$ \\
\hline $\begin{array}{l}\text { JOSE MENDONCA BEZERRA } \\
\text { (18:01/1936 a 24:04:2011) }\end{array}$ & $\begin{array}{l}\text { ARENA, 1966-1979; } \\
\text { PDS, , 980-1985; } \\
\text { PFL, 1986-2007; } \\
\text { DLM, 2007 }\end{array}$ & $\mathrm{PE}$ & $\begin{array}{l}\text { 1979-1983; 1983- } \\
\text { |987; } 1987-1991 ;\end{array}$ & |1995-1999 & |1999-2003 & 2003-2007 & 2007-2011 & 0 & $\begin{array}{l}\text { DEPUTADO } \\
\text { FEDERAL }\end{array}$ & 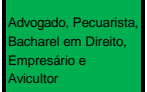 & 0,00 & R\$ 0,00 & $\mathrm{R} \$ 1.393 .238,55$ & $0,00 \%$ \\
\hline $\begin{array}{c}\text { SEVERINO JosÉ CaVALCANTI } \\
\text { FERREIRA }\end{array}$ & \begin{tabular}{|l} 
UDN, 1962-1966; \\
ARENA, 1966-1979; \\
PDS, 1980-1987; \\
PDC, 1987-1990; \\
PL, 1990-1992; \\
PRR, 1992-1993; \\
PFL, 1994-1995; \\
PBB, 1995-2003; \\
PP, 2003-.
\end{tabular} & $\mathrm{PE}$ & 0 & |1995-1999 & |1999-2003 & 2003-2007 & 0 & 0 & 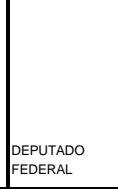 & \begin{tabular}{|l} 
Membro do Poder \\
Legislativo
\end{tabular} & 0,00 & Rs 0,00 & $\mathrm{R} \$ 173.174,99$ & $0,00 \%$ \\
\hline $\begin{array}{l}\text { LUZZ PIAUHYLINO DE MELLO } \\
\text { MONTERIRO }\end{array}$ & $\begin{array}{l}\text { PMDB, 1986-1990; } \\
\text { PSB, 1990-1995; } \\
\text { PSD, 195-2003; } \\
\text { PTB, 2093-2004; } \\
\text { PDT, 2004 }\end{array}$ & PE & |1991-1995 & | 1995-1999 & |1999-2003 & 2003-2007 & o & 0 & 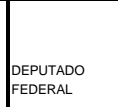 & Advogado & ${ }^{2 \mathrm{NI}}$ & $\mathrm{R} \$ 6.608,24$ & $146.739,00$ & $4,50 \%$ \\
\hline JOĀO BASTOS COLAÇO DIAS & 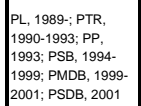 & PE & \begin{tabular}{|l}
$1991-1992,1995-$ \\
1999
\end{tabular} & |1995-1999 & |1999-2003 & 10 & ]$_{0}$ & 0 & $\begin{array}{l}\text { DEPUTADO } \\
\text { FEDERAL }\end{array}$ & $\begin{array}{l}\text { Proppitetario de } \\
\text { Establecimento } \\
\text { Comercial }\end{array}$ & $\mathrm{NI}$ & $R \$ 11.333,87$ & \begin{tabular}{|l|l|}
$R \$$ & $217.133,73$ \\
\end{tabular} & $5,22 \%$ \\
\hline OSWALDO DE SOUZA COELHO & $\begin{array}{l}\text { ARENA, 1966-1979; } \\
\text { PDS, 1980-1955; }\end{array}$ & $\mathrm{PE}$ & $\begin{array}{l}\text { 1983; 1983-1987; } \\
1987-1991 ; 1991-\end{array}$ & 1995-1999 & $1999-2003$ & $2003-2007$ & 0 & 0 & $\begin{array}{l}\text { DEEUTAAD } \\
\text { FEDERAL }\end{array}$ & \begin{tabular}{|l} 
Adrogado, II \\
Pecuarista
\end{tabular} & $1900+N 1$ & RS 101.886,58 & $\mathrm{R} \$ 3.806 .490,92$ & $2,68 \%$ \\
\hline JOSÉ MUCIO MONTERO & $\begin{array}{l}\text { PDSS 1980; PFL, } \\
\text { 1991-2000; ; PSB, } \\
\text { 20201-2003; PTB, } \\
\text { 2003 }\end{array}$ & $\mathrm{PE}$ & |1991-1995 & |1995-1999 & |1999-2003 & 2003-2007 & 2007-2011 & 0 & $\begin{array}{l}\text { DEPUTADO } \\
\text { FEDERAL }\end{array}$ & Engenheiro & $\mathrm{NI}$ & R\$ $105.108,00$ & $\mathrm{R} \$ 878.715,10$ & $11,96 \%$ \\
\hline LUIZ GONZAGA PATRIOTA & $\begin{array}{l}\text { MDB, 1968-1980; } \\
\text { PMDB, 1980-1989; } \\
\text { PDT, 1989-1991; } \\
\text { PSB, 1991 }\end{array}$ & $\mathrm{PE}$ & \begin{tabular}{|l} 
1981-1987, 1995- \\
1999
\end{tabular} & |1995-1999 & |999-2003 & 2003-2007 & 2007-2011 & 0 & \begin{tabular}{|l} 
DEEUTADO \\
FEDERAL
\end{tabular} & $\left|\begin{array}{l}\text { Acuogado, Jornalista, } \\
\text { Amministarood de } \\
\text { Empresas e Contador }\end{array}\right|$ & ${ }_{12 \mathrm{NI}}$ & R\$ 105.500,00 & $\mathrm{R} \$ 3.215 .532,52$ & $3,28 \%$ \\
\hline $\begin{array}{l}\text { ANTONONO CARLOS VIERA DOS } \\
\text { SANTOS (CARLOS BATATA. } \\
\text { INESC) }\end{array}$ & \begin{tabular}{|l|} 
PSB, 1986-1999; \\
PSBB, 1999-2005; \\
PFL, 2005
\end{tabular} & PE & 0 & o & 1999-2003 & 2003-2007 & 0 & 0 & \begin{tabular}{|l} 
DEEUTADO \\
FEDERAL
\end{tabular} & $\begin{array}{l}\text { Agropecuarista e } \\
\text { Médico Veterinário }\end{array}$ & 0,00 & R\$ 147.907,55 & $R \$ 4.299 .593,75$ & $3,44 \%$ \\
\hline JOSÉ SEVERIANO CHAVES & \begin{tabular}{|l|} 
PSDB, 1989-1994; \\
PSB, 1994-1995; \\
PSDB 1966; PMDB, \\
1996-2003; PTB, \\
2003
\end{tabular} & $\mathrm{PE}$ & 0 & |1995-1999 & |999-2003 & 2003-2007 & 2007-2011 & 2011-2015 & $\begin{array}{l}\text { DEPUTADO } \\
\text { FEDERAL }\end{array}$ & Engenheiro Civil & 757,21 & RS 214.154,97 & $R \$ 7.403 .209,73$ & $2,89 \%$ \\
\hline $\begin{array}{l}\text { AUGUSTO RODRIIUESS } \\
\text { COUTTINHO DE MELO }\end{array}$ & $\begin{array}{l}\text { PFL, 1990; DEM, } \\
2007\end{array}$ & PE & jo & 10 & 0 & 0 & to & $2011-2015$ & $\begin{array}{l}\text { DEPUTADO } \\
\text { FEDERAL }\end{array}$ & Engenheiro Civil & $3 \mathrm{NI}$ & R\$ $305.870,00$ & $\mathrm{R} \$ 1.103 .837,60$ & $27,71 \%$ \\
\hline $\begin{array}{l}\text { SEVERRINO SERGIO ESTELITA } \\
\text { GUERRA (SERGIO GUERRA) }\end{array}$ & \begin{tabular}{|l} 
PDT, 1985-1988; \\
PSB, 1989-1999;
\end{tabular} & PE & |1991-1995 & |1995-1999 & 0 & 10 & 10 & $2011-2015$ & $\begin{array}{l}\text { DEPUTADO } \\
\text { FEDERAL }\end{array}$ & \begin{tabular}{|l} 
Pecuarista $\mathrm{e}$ \\
Professor
\end{tabular} & $206+\mathrm{NI}$ & R\$ 525.000,00 & $\mathrm{R} \$ 3.014 .044,83$ & $17,42 \%$ \\
\hline
\end{tabular}




\begin{tabular}{|c|c|c|c|c|c|c|c|c|c|c|c|c|c|c|}
\hline NOME & PARTIDO & UF & $\begin{array}{l}\text { GESTÄO } \\
\text { ANTERIOR }\end{array}$ & GESTÃol & GESTĀol & GESTÃo III & GESTÃo IV & \begin{tabular}{|l} 
GESTäo \\
POSTERIOR
\end{tabular} & ÚLTIMO CARGO & $\begin{array}{l}\text { PROFISSÃo } \\
\text { DEELARADA }\end{array}$ & $\begin{array}{c}\text { AREA DAS TERRAS } \\
\text { (HA) }\end{array}$ & $\begin{array}{c}\text { VALOR DAS TERRAS } \\
\text { RS }\end{array}$ & $\begin{array}{l}\text { VALOR DO } \\
\text { PATRIMOONIO }\end{array}$ & $\begin{array}{c}\text { VALOR DAS } \\
\text { TERAASPATRMIMONIO }\end{array}$ \\
\hline INOCÉNCIO GOMES DE OLVEIRA & \begin{tabular}{|l} 
ARENA, 1975-1979; \\
PSD, 1980-1985; \\
PFLL, ,985-2005; \\
PMDB, 2005; PL, \\
2005-2007; PR, \\
2007
\end{tabular} & PE & $\begin{array}{l}\text { 1979-1993: 1983- } \\
\text { 1988; } \\
\text { 1991-1997-1995-1991; }\end{array}$ & |1995-1999 & |999-2003 & 2003-2007 & 2007-2011 & $2011-2015$ & $\begin{array}{l}\text { DEPUTADO } \\
\text { FEDERAL }\end{array}$ & Médico & $2 \mathrm{~N} 1+0,25$ & R\$ 1.296.562,54 & $\mathrm{R} \$ 6.901 .519,44$ & $18,79 \%$ \\
\hline $\begin{array}{l}\text { MARCOS ANTONIO DE BARROS } \\
\text { (DE JESUS) }\end{array}$ & $\begin{array}{l}\text { PTB, 1997-1999; } \\
\text { PST, 1999-2000; } \\
\text { PSDB, 2000; PL, } \\
\text { 2000-2005; PFL, } \\
\text { 2005-2006; } \\
\text { S.PART., 2006 }\end{array}$ & PE & 0 & 0 & |999-2003 & 2003-2007 & 0 & 0 & \begin{tabular}{|l} 
DEFUTADO \\
FEDERAL
\end{tabular} & $\begin{array}{l}\text { Radialista, Pastor } \\
\text { Evangeilico e Auxiliar } \\
\text { de Enfermagem }\end{array}$ & 0,00 & $\mathrm{No}$ & $\mathrm{NI}$ & \#VALOR! \\
\hline $\begin{array}{l}\text { JOAOVICENERE DE MACEDO } \\
\text { CLAUINO }\end{array}$ & PSDB, 1997-2000; & $\mathrm{Pl}$ & 0 & 0 & 0 & 0 & $2007-2011$ & $2011-2015$ & SENADOR & Economista & 0,00 & R\& 0,00 & R\$ 656.399,77 & $0,00 \%$ \\
\hline JOSÉ FRANCIISCO PAES LANDIM & $\begin{array}{l}\text { PDS, 1982-1985; } \\
\text { PFL, 1987-2004; }\end{array}$ & $\mathrm{Pl}$ & $\begin{array}{l}\text { 1987-1991, 1991- } \\
\text { 1995 1995-1999 }\end{array}$ & |1995-1999 & |1999-2003 & 2003-2007 & $2007-2011$ & 0 & $\begin{array}{l}\begin{array}{l}\text { DEPUTADO } \\
\text { FEDERAL }\end{array} \\
\end{array}$ & Advogado e Professor & 0,00 & R\& 0,00 & $\mathrm{R} \$ 7.411,59$ & $0,00 \%$ \\
\hline HERACLTO DE SOUZA FORTES & \begin{tabular}{|l|}
$1979-1982 ;$ PMDB, \\
1982; PFL, 1994
\end{tabular} & $\mathrm{Pl}$ & $\begin{array}{l}1982-1983 ; ; 983- \\
1987 ; 1987-1989 ;\end{array}$ & | 1995-1999 & |1999-2003 & 2003-2007 & 0 & 0 & SENADOR & Servidor Público & 53,79 & $\mathrm{R} \$ 1.341,85$ & $\mathrm{R} \$ 7.924 .463,70$ & $0,02 \%$ \\
\hline ATLA FRETAS LIRA & \begin{tabular}{|l} 
ARENA, 1976-1979; \\
PDS, 1980-1984;
\end{tabular} & $\mathrm{PI}$ & $\begin{array}{l}1987-1991 ; \text {; } 1991- \\
1995\end{array}$ & & $\begin{array}{l}1999-2003 ; \\
2003-2007\end{array}$ & 2003-2007 & 2007-2001 & $2011-2015$ & \begin{tabular}{|l} 
DEFUTADO \\
FEDERAL
\end{tabular} & \begin{tabular}{|l|}
$\begin{array}{l}\text { Administrador de } \\
\text { Empresas }\end{array}$ \\
\end{tabular} & $4 \mathrm{NI}$ & $\mathrm{R} \$ 94.720,83$ & R\$ 562.988,06 & $16,82 \%$ \\
\hline $\begin{array}{l}\text { IRACEMA MARIA POORTELLA } \\
\text { NUNES NOGUEIRA LLMAA }\end{array}$ & pp & $\mathrm{Pl}$ & 0 & 0 & 0 & 0 & 0 & $2011-2015$ & $\begin{array}{l}\text { DEPUTADO } \\
\text { FEDERAL }\end{array}$ & $\begin{array}{l}\begin{array}{l}\text { Professora e } \\
\text { Empresária }\end{array} \\
\end{array}$ & $\mathrm{NI}$ & $\mathrm{R} \$ 150.000,00$ & $R \$ 2.115 .997,82$ & $7,09 \%$ \\
\hline MARCELO COSTA E CASTRO & \begin{tabular}{|l|} 
PDSB, 1991; PMR, \\
1993-1997; PMDB,
\end{tabular} & $\mathrm{PI}$ & 0 & 0 & 1999-2003 & $2003-2007$ & 2007-2011 & $2011-2015$ & $\begin{array}{l}\text { DEEUTAAD } \\
\text { FEDERAL }\end{array}$ & Médico & $1151,2+25 \mathrm{NI}$ & R\$ 177. 419,17 & Rs 629.274,77 & $28,19 \%$ \\
\hline BENEDITO DE CARVALHO SA & $\begin{array}{l}\text { PDS, 1990-1992; } \\
\text { PP, 1992-1995; }\end{array}$ & $\mathrm{Pl}$ & |1991-1995 & |1995-1999 & |1999-2003 & 2003-2007 & 2007-2011 & 0 & $\begin{array}{l}\text { DEPUTADO } \\
\text { FEDERAL }\end{array}$ & Médico & 60,00 & $R \$ 185.000,05$ & $\mathrm{R} \$ 496.500,05$ & $37,26 \%$ \\
\hline CIRO NOGUEIRA LIMA FILHO & \begin{tabular}{|l|} 
P2L, $1994-2004 ; ; P P$, \\
204
\end{tabular} & $\mathrm{Pl}$ & 0 & 1995-1999 & $1999-2003$ & $2003-2007$ & $2007-2011$ & $2011-2019$ & SENADOR & Advogado & 130,00 & R\$ 187.608,00 & $\mathrm{R} \$ 1.972 .460,32$ & $9,51 \%$ \\
\hline $\begin{array}{c}\text { THEMISTOCLES DE SAMPAIO } \\
\text { PEREIRA }\end{array}$ & $\begin{array}{l}\text { MDB, 1966-1979; } \\
\text { PMDB, } 1980\end{array}$ & $\mathrm{Pl}$ & 0 & 0 & |1999-2003 & 0 & $2009-2011$ & 0 & $\begin{array}{l}\text { DEPUTADO } \\
\text { FEDERAL }\end{array}$ & $\begin{array}{l}\begin{array}{l}\text { Agricultor, Advogado e } \\
\text { Pecuarista }\end{array} \\
\text { a }\end{array}$ & $7.765,61+4 \mathrm{NI}$ & R\$ 943.000,00 & $\mathrm{R} \$ 2.248 .606,18$ & $41,94 \%$ \\
\hline \begin{tabular}{|l|} 
JULIO CESAR DE CARVALHO \\
LIMA
\end{tabular} & $\begin{array}{l}1985-2007 ; \text { DEM, } \\
2007\end{array}$ & $\mathrm{Pl}$ & 0 & |1995-1999 & 0 & 2003-2007 & 2007-2011 & $2011-2015$ & $\begin{array}{l}\begin{array}{l}\text { DEPUTADO } \\
\text { FEDERAL }\end{array} \\
\end{array}$ & $\begin{array}{l}\begin{array}{l}\text { Advogado, Profess } \\
\text { e Produtor Rural }\end{array} \\
\end{array}$ & $185,5+5 \mathrm{NI}$ & R\$ 1.090.472,56 & $R \$ 3.091 .931,01$ & $35,27 \%$ \\
\hline 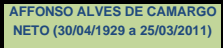 & \begin{tabular}{|l|} 
1967-1978; PP, \\
1979-1981; ; PMB,
\end{tabular} & PR & 0 & | 1995-1999 & |1999-2003 & 2003-2007 & 2007-2011 & 0 & $\begin{array}{l}\text { DEPUTADO } \\
\text { FEDERAL }\end{array}$ & Engenheiro Civil & 0,00 & R\& 0.00 & $\mathrm{R} \$ 7.761 .261,43$ & $0,00 \%$ \\
\hline EDUARDO FRANCISCO SCIARRA & DEM & PR & 0 & 0 & 0 & 2003-2007 & 2007-2011 & $2011-2015$ & $\begin{array}{l}\text { DEPUTADO } \\
\text { FEDERAL }\end{array}$ & \begin{tabular}{|l} 
Engenheiro Civil e \\
Empresário
\end{tabular} & 0,00 & Rs 0,00 & $\mathrm{R} \$ 2.677 .733,28$ & $0,00 \%$ \\
\hline \begin{tabular}{|c|} 
JACOB ALFREDO STOFFELS \\
KAEFER
\end{tabular} & PSDB & $\mathrm{PR}$ & 0 & 10 & 0 & 0 & $2007-2011$ & $2011-2015$ & \begin{tabular}{|l} 
DEPUTAAO \\
FEDERAL
\end{tabular} & Nầo Intormou & 0,00 & Rs 0,00 & R\$ $95.728 .260,00$ & $0,00 \%$ \\
\hline NELSON MEURER & $\begin{array}{l}\text { 1981-1993; PP, } \\
\text { 1993-1995; PPB, }\end{array}$ & PR & 0 & |1995-1999 & & 2003-2007 & $2007-2011$ & 0 & \begin{tabular}{|l} 
DEPUTADO \\
FEDERAL
\end{tabular} & Agropecuarista & 0,00 & Rs 0,00 & $\begin{array}{|ll|}\text { R\$ } & 1.516 .749,51 \\
\end{array}$ & $0,00 \%$ \\
\hline HOMERO BARBOSA NETO & 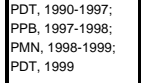 & PR & 0 & 0 & 0 & 0 & $2007-2011$ & 0 & $\begin{array}{l}\text { DEPUTADO } \\
\text { FEDERAL }\end{array}$ & Jornalista e Radialista & 0,00 & Rs 0,00 & R\$ $544.489,73$ & $0,00 \%$ \\
\hline HERMES PARCIANELLO & $\begin{array}{l}\text { MDB, 1976; PMDB, } \\
\text { 1982 }\end{array}$ & PR & 0 & |1995-1999 & |1999-2003 & 2003-2007 & 2007-2011 & $2011-2015$ & $\begin{array}{l}\text { DEPUTADO } \\
\text { FEDERAL }\end{array}$ & \begin{tabular}{|l} 
Bacharel em Ciências \\
Contábeis
\end{tabular} & 0,00 & $\mathrm{R} \$ 0,00$ & $348.983,00$ & $0,00 \%$ \\
\hline JOSÉ RODRIGUES BORBA & $\begin{array}{l}\text { ARENA; PMDB; } \\
\text { PTB, 1993-1999; } \\
\text { PMDB, 1999 }\end{array}$ & PR & 0 & |1995-1999 & |1999-2003 & 2003-2007 & 0 & 0 & \begin{tabular}{|} 
DEEPUTADO \\
fEDERAL
\end{tabular} & $\begin{array}{l}\text { Pecuarista e } \\
\text { Comerciante }\end{array}$ & 0,00 & Rs 0,00 & R\$ $287.861,99$ & $0,00 \%$ \\
\hline OSMAR JOSE SERRAGLIO & $\begin{array}{l}\text { MDB, 1978-1981; } \\
\text { PMDB, } 1981 \text {; }\end{array}$ & PR & 0 & 0 & |1999-2003 & 2003-2007 & 2007-2011 & $2011-2015$ & $\begin{array}{l}\text { DEPUTADO } \\
\text { FEDERAL }\end{array}$ & $\begin{array}{l}\begin{array}{l}\text { Advogadoe Professor } \\
\text { Universitário }\end{array} \\
\end{array}$ & $1 \mathrm{NI}$ & $\mathrm{R} \$ 2.526,10$ & \begin{tabular}{ll|} 
R\$ & $2.101 .177,00$ \\
\end{tabular} & $0,12 \%$ \\
\hline $\begin{array}{l}\text { RICARDO JOSÉ MAGALHĀES DE } \\
\text { BARROS - INESC }\end{array}$ & $=\left[\begin{array}{l}\text { PPLL, 1988-1997; } \\
\text { PPB, 1907-2003; }\end{array}\right.$ & PR & 0 & |1995-1999 & |1999-2003 & 2003-2007 & 2007-2011 & 0 & $\begin{array}{l}\text { DEPUTADO } \\
\text { FEDERAL }\end{array}$ & $\begin{array}{l}\text { Engenheiro Civil e } \\
\text { Empresário }\end{array}$ & $5.159,00$ & $90.000,00$ & $\mathrm{R} \$ 1.921 .118,59$ & $4,68 \%$ \\
\hline GUSTAVO BONATO FRUET & \begin{tabular}{|l|} 
PMDB, 1991-2004; \\
S.PART, 2005; \\
PSDB, 2005
\end{tabular} & PR & 0 & 0 & |1999-2003 & 2003-2007 & $2007-2011$ & 10 & $\begin{array}{l}\text { DEPUTADO } \\
\text { FEDERAL }\end{array}$ & Advogado & $1.310,20$ & 12.548.87 & $\mathrm{R} \$ 1.882 .938,69$ & $0,67 \%$ \\
\hline LEOPOLDO COSTA MEYER & \begin{tabular}{|l|}
$\begin{array}{l}\text { PL, 1988-1997; } \\
\text { PSDB, } \\
\text { PSB, 20097-2009; }\end{array}$ \\
PS,
\end{tabular} & PR & 0 & 0 & 0 & 0 & 10 & $2011-2015$ & $\begin{array}{l}\text { DEEUTADO } \\
\text { FEDERAL }\end{array}$ & Engenheiro Civil & 4,03 & $\mathrm{R} \$ 29.320,00$ & R\$2 2.161.007,74 & $1,36 \%$ \\
\hline WERNER WANDERER (nativo) & $\begin{array}{l}\text { PTB, 1965; ARENA, } \\
\text { 1966-1977; PSS, } \\
\text { 1980-1985; PFL, } \\
1985\end{array}$ & PR & 1991-1995 & | 1995-1999 & |1999-2003 & 10 & & 10 & $\begin{array}{l}\text { DEPUTADO } \\
\text { FEDERAL }\end{array}$ & \begin{tabular}{|l} 
Agricultor e \\
Empresário
\end{tabular} & 206,50 & $\mathrm{R} \$ 46.752,20$ & $811.089,23$ & $5,76 \%$ \\
\hline
\end{tabular}




\begin{tabular}{|c|c|c|c|c|c|c|c|c|c|c|c|c|c|c|}
\hline NOME & PARTIDO & uF & \begin{tabular}{|l} 
GESTÃo \\
ANTERIOR
\end{tabular} & GESTĀoI & GESTĀoll & GESTÃO III & GESTÃo IV & $\begin{array}{l}\begin{array}{l}\text { GESTÃó } \\
\text { POSTERIOR }\end{array} \\
\end{array}$ & ÚLTIMO CARGO & $\begin{array}{l}\text { PROFISSĀO } \\
\text { DECLARADA }\end{array}$ & $\begin{array}{c}\text { AREA DAS TERRAS } \\
(H A)\end{array}$ & $\begin{array}{c}\text { VALOR DAS TERRAS } \\
\text { RS }\end{array}$ & $\begin{array}{l}\text { VALOR DO } \\
\text { PATRIMÓNIO }\end{array}$ & $\begin{array}{c}\text { VALOR DAS } \\
\text { TERRASPATRRIMOONIO }\end{array}$ \\
\hline $\begin{array}{l}\text { MAX ROSENMANN (29/1/1/1944 a a } \\
\text { 25/10/2008) }\end{array}$ & 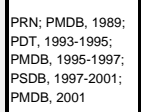 & PR & $\mid \begin{array}{l}1987-1991 ; 1991- \\
1995\end{array}$ & |1995-1999 & 1999-2003 & 2003-2007 & 2007-2011 & 10 & $\begin{array}{l}\text { DEPUTADO } \\
\text { FEDERAL }\end{array}$ & $\begin{array}{l}\text { Advogado e } \\
\text { Empresário }\end{array}$ & ${ }_{8 \mathrm{NI}}$ & $\mathrm{R} \$ 55.017,60$ & $\mathrm{R} \$ 3.669 .659,24$ & $1,50 \%$ \\
\hline IRIS XAVIER SIMŌES & \begin{tabular}{|l|}
$1995-1996 ;$ PSDB, \\
1996-1997; PTB,
\end{tabular} & $\mathrm{PR}$ & 0 & 10 & |1999-2003 & $2003-2011$ & 2007-2011 & ]$_{0}$ & $\begin{array}{l}\text { DEPUTADO } \\
\text { FEDERAL }\end{array}$ & Radialista & $120,94+2 \mathrm{NI}$ & $\mathrm{R} \$ 56.770,00$ & $\begin{array}{|ll|}\text { RS } & 659.958,20 \\
\end{array}$ & $8,60 \%$ \\
\hline LUCIANO PIZZATTO & $\begin{array}{l}\text { PRN, 1990-1991; } \\
\text { PFL, 1991-; DEM, }\end{array}$ & $\mathrm{PR}$ & 1991-1995 & |1995-1999 & 1999-2003 & 2007 & 0 & 0 & $\begin{array}{l}\text { DEPUTADO } \\
\text { FEDERAL }\end{array}$ & \begin{tabular}{|l|}
$\begin{array}{l}\text { Empresárío e } \\
\text { Engenheiro Florestal }\end{array}$ \\
\end{tabular} & 3 & R\$ 124.303,02 & $\begin{array}{|ll|}\text { R\$ } & 6.415 .038,80 \\
\end{array}$ & $1,94 \%$ \\
\hline $\begin{array}{l}\text { MOACIR MICHELETTO } \\
\text { (25/11/1942 a 30:01/2012) }\end{array}$ & $\begin{array}{l}\text { ARENA, 1973-1980; } \\
\text { PP, 1981-1982; } \\
\text { PMDBB, 1982 }\end{array}$ & $\mathrm{PR}$ & 1993-1995 & |1997-1999 & |1999-2003 & $2003-2007$ & $2007-2011$ & $2011-2015$ & $\begin{array}{l}\text { DEPUTADO } \\
\text { FEDERAL }\end{array}$ & 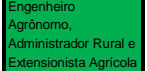 & 49,10 & RS 169.051,78 & 1.238.423,84 & $13,65 \%$ \\
\hline LUIS CARLOS JORGE HAULY & \begin{tabular}{|l|} 
PMDB, 1980-1991; \\
PST, 1991-1992; PP,
\end{tabular} & $\mathrm{PR}$ & 1991-1995 - & |1995-1999 & |1999-2003 & 2003-2007 & 2007-2011 & $2011-2015$ & $\begin{array}{l}\text { DEPUTADO } \\
\text { FEDERAL }\end{array}$ & \begin{tabular}{|l|}
$\begin{array}{l}\text { Economista e } \\
\text { Profeessor }\end{array}$ \\
\end{tabular} & $166,25+\mathrm{NI}$ & RS 176.386,93 & 777.902,85 & $22,67 \%$ \\
\hline ALEX CANZIANI SILVEIRA & 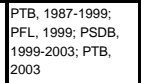 & $\mathrm{PR}$ & 0 & 0 & 1999-2003 & $2003-2007$ & $2007-2011$ & 2011-2015 & $\begin{array}{l}\text { DEPUTADO } \\
\text { FEDERAL }\end{array}$ & $\begin{array}{l}\begin{array}{l}\text { Registrador de } \\
\text { Imovevis }\end{array} \\
\end{array}$ & $962,55+\mathrm{N1}$ & R\$ $353.405,62$ & $\mathrm{R} \$ 1.580 .972 .56$ & $22.35 \%$ \\
\hline $\begin{array}{l}\text { FRANCISCO OTAVIO BECERTT } \\
\text { (CHICODA DPINCEAA) }\end{array}$ & $\begin{array}{l}\text { 1992-1995; PTB, } \\
\text { 19995-1999; }\end{array}$ & $\mathrm{PR}$ & $l_{0}$ & \begin{tabular}{|c|}
$1995-19999$ \\
\end{tabular} & \begin{tabular}{|c|}
$1999-2003$ \\
\end{tabular} & 2003-2007 & $2007-2011$ & $0_{0}$ & $\begin{array}{l}\text { DEFUTADO } \\
\text { FEDERAL }\end{array}$ & Contador & $174+\mathrm{N1}$ & RS 416.655 .00 & $\begin{array}{|ll|}\text { RS } & 1.161 .712 .24 \\
\end{array}$ & $35.87 \%$ \\
\hline ABELARDO LUZZ LUPION MELLOO & $\begin{array}{l}\text { PRN, 1990-1992; } \\
\text { PFL, 1992-2007; } \\
\text { DEM, 2007 }\end{array}$ & PR & 1992 e 1994-1995 & |1995-1999 & 1999-2003 & 2003-2007 & $2007-2011$ & $2011-2015$ & $\begin{array}{l}\text { DEPUTADO } \\
\text { FEDERAL }\end{array}$ & $\begin{array}{l}\text { Agropeceuarista e } \\
\text { Empresário }\end{array}$ & ${ }_{2 \mathrm{NI}}$ & RS 444.142,81 & $\begin{array}{|ll|}\text { RS } & 5.898 .464,13 \\
\end{array}$ & $7,53 \%$ \\
\hline ALLARO FERNANDES DIAS & \begin{tabular}{|l|} 
PMDB, 1982; 1991, \\
PST; 1994, PSDB
\end{tabular} & $\mathrm{PR}$ & $\mid$ & 0 & 1999-2003 & $2003-2007$ & $2007-2011$ & $2011-2015$ & SENADOR & Näo Intormou & 288,07 & $\mathrm{RS} 456.540,88$ & $\mathrm{R} \$ 1.904 .924,82$ & $23,97 \%$ \\
\hline EDMAR DE SOUZA ARRUDA & PSC, 2009 & PR & 0 & 0 & 0 & 0 & 0 & $2011-2015$ & \begin{tabular}{|l} 
DEEUTAAO \\
FEDEALL
\end{tabular} & Economista & $1.230,35+4 N 1$ & $\operatorname{RS} 636.070,77$ & R\$ 12.396.842.85 & $5,13 \%$ \\
\hline $\begin{array}{l}\text { AlRTON BERBARDO ROVEDA- } \\
\text { INESC }\end{array}$ & $\begin{array}{l}\text { PDT, 1995-1999; } \\
\text { PFL, 1999-2000; }\end{array}$ & $\overline{P R}$ & 0 & 0 & 1999-2003 & 2003-2007 & 2007-2011 & 0 & $\begin{array}{l}\text { DEPUTADO } \\
\text { FEDERAL }\end{array}$ & $\begin{array}{l}\text { Comerciante e } \\
\text { Minerador }\end{array}$ & $? ?$ & $291.145,12$ & $\mathrm{R} \$ 3.634 .755,79$ & $8,01 \%$ \\
\hline OSMAR FERNANDES DIAS & PDT & PR & 0 & 1995-1999 & 1999-2003 & $2003-2007$ & 2007-2011 & 0 & SENADOR & Engenheiro & 936,98 & $\begin{array}{|ll|}\mathrm{R} & 661.812 .85 \\
\end{array}$ & R\$5.191.343,92 & $12,75 \%$ \\
\hline $\begin{array}{l}\text { CEZAR AUGUSTOCAROLLO } \\
\text { SILVESTRI }\end{array}$ & $\begin{array}{l}\text { PDI;PSDB;PTB; } \\
\text { PPS }\end{array}$ & PR & 0 & 10 & 10 & 2003-2007 & $2007-2011$ & 2011-2015 & $\begin{array}{l}\text { DEPUTADO } \\
\text { FEDERAL }\end{array}$ & $\begin{array}{l}\text { Agropecuaristae } \\
\text { Engenheiro Civil }\end{array}$ & $1.570,57$ & $\mathrm{R} \$ 1.294 .164,73$ & $\mathrm{R} \$ 2.026 .710,25$ & $63,86 \%$ \\
\hline DILCEU JOĀO SPERAFICO & $\begin{array}{l}\text { PDS, 1980-1993; } \\
\text { PP, 1993-19955; } \\
\text { PBP, } 1995-2003 ; \\
\text { PP, 2003 }\end{array}$ & $\mathrm{PR}$ & 0 & |1995-1999 & |1999-2003 & 2003-2007 & 0 & 2011-2015 & $\begin{array}{l}\text { DEPUTADO } \\
\text { FEDERAL }\end{array}$ & 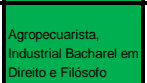 & ${ }_{6 \mathrm{NI}}$ & R\$ $1.870 .850,50$ & $\begin{array}{|ll|}\text { RS } & 7.680 .293,03 \\
\end{array}$ & $24,36 \%$ \\
\hline LUIZ CARLOS SETIM & $\begin{array}{l}\text { PFLL, 1996-2007; } \\
\text { DEM, 2007 }\end{array}$ & $\overline{P R}$ & 0 & 0 & 0 & 0 & $2007-2011$ & $2011-2015$ & $\begin{array}{l}\begin{array}{l}\text { DEPUTADO } \\
\text { FEDERAL }\end{array} \\
\end{array}$ & $\begin{array}{l}\begin{array}{l}\text { Advogadoe } \\
\text { Administrador de }\end{array} \\
\end{array}$ & $2.132,48+\mathrm{NI}$ & R\$ 2.191.574,50 & $\begin{array}{|ll|}\text { RS } & 6.008 .493,60 \\
\end{array}$ & $36,47 \%$ \\
\hline ODILLIO BALBINOTTI & $\begin{array}{l}\text { ARENA, 1973-1988; } \\
\text { PELL, 1988-1992; } \\
\text { PDC, 1992-1993; } \\
\text { PDT, 1993-1995; } \\
\text { PTR, 1996-1997; } \\
\text { PSDB, 1997-2003; } \\
\text { PMDB, 2003 }\end{array}$ & PR & 0 & |1995-1999 & 1999-2003 & 2003-2007 & $2007-2011$ & 0 & $\begin{array}{l}\text { DEPUTADO } \\
\text { fEDERAL }\end{array}$ & \begin{tabular}{|l} 
Agropecuarista e \\
Empresário
\end{tabular} & $98,36+2 \mathrm{NI}$ & R\$ 2.649.072,00 & $\begin{array}{|ll|}\text { RS } & 16.066 .180,57 \\
\end{array}$ & $16,49 \%$ \\
\hline NELSON PADOVANI & PSC & PR & 0 & 0 & 0 & 0 & 0 & $2011-2015$ & $\begin{array}{l}\text { EEPTUADO } \\
\text { FEDERAL }\end{array}$ & Empresário & $45,33+3 \mathrm{NI}$ & $\mathrm{R} \$ 3.645 .344,17$ & R\$ 7.970.860.81 & $45,73 \%$ \\
\hline LEONARDO CARNEIRO PICCIAN| & PMDB, 2000 & RJ & 0 & 0 & 10 & 2003-2007 & $2007-2011$ & $2011-2015$ & $\begin{array}{l}\text { DEPUTADO } \\
\text { FEDERAL }\end{array}$ & \begin{tabular}{|l} 
Empresáron \\
Pecuarista
\end{tabular} & 0,00 & R\$ 0,00 & $\mathrm{R} \$ 1.356 .101,20$ & $0,00 \%$ \\
\hline ALEXANDRE JOSÉ DOS SANTOS & $\begin{array}{l}\text { PP, 2003-2005; } \\
\text { PMDB, 2005 }\end{array}$ & RJ & 0 & |1995-1999 & 1999-2003 & 2003-2007 & $2007-2011$ & $2011-2015$ & \begin{tabular}{|l} 
DEPUTADO \\
FEDERAL
\end{tabular} & Empresário & $\mathrm{NI}$ & $\mathrm{R} \$ 6.939,72$ & R\$ 743.411,93 & $0,93 \%$ \\
\hline $\begin{array}{l}\text { LUIZ EDUARDO ALMEEA DE } \\
\text { OLIVERA (LUISINHO) }\end{array}$ & PPB & RJ & 2 & ? & ? & 2003-2007 & ${ }_{0}$ & & $\begin{array}{l}\text { DEPUTADO } \\
\text { FEDEAAL }\end{array}$ & & $\mathrm{NI}$ & RS $36.000,00$ & RS $292.458,00$ & $12,31 \%$ \\
\hline $\begin{array}{l}\text { PAULO FERNANDO FEIJO } \\
\text { TORRES }\end{array}$ & $\begin{array}{l}\text { PTB, 1990-1993; } \\
\text { PSOB, 1993-2009; }\end{array}$ & RJ & 0 & |1995-1999 & 1999-2003 & 2003-2007 & 0 & 0 & $\begin{array}{l}\text { DEPUTADO } \\
\text { FEDERAL }\end{array}$ & Engenheiro Mecânico & ${ }_{54+\mathrm{NI}}$ & Rs $309.198,00$ & R\$ 853.448,19 & $36,23 \%$ \\
\hline $\begin{array}{l}\text { CARLOS ALEERTO DE SOUZA } \\
\text { ROSADO SOS }\end{array}$ & $\begin{array}{l}\text { PFL, 1986-2007; } \\
\text { DEM, 2007 }\end{array}$ & $\mathrm{RN}$ & 0 & |1995-1999 & 1999-2003 & $2003-2007$ & 2007-2011 & 2011-2015 & $\begin{array}{l}\text { DEPUTADO } \\
\text { FEDERAL }\end{array}$ & $\begin{array}{l}\text { Profofssor } \\
\text { Universtiánio e }\end{array}$ & 784,80 & $\mathrm{R} \$ 11.658,46$ & Rs 499.714,52 & $2,33 \%$ \\
\hline JOSE AGRIPINO MAIA & PFL, DEM,RN & $\mathrm{RN}$ & 1987-1995 & 1995-1999 & 1999-2003 & $2003-2007$ & $2007-2011$ & $\begin{array}{l}2011-2015 ; 20 \\
2019\end{array}$ & SENADOR & Engenheiro Civil & $\mathrm{NI}$ & R\$ $12.500,00$ & $\mathrm{R} \$ 4.225 .168,10$ & $0,30 \%$ \\
\hline
\end{tabular}




\begin{tabular}{|c|c|c|c|c|c|c|c|c|c|c|c|c|c|c|}
\hline NOME & PARTIDO & UF & $\begin{array}{l}\text { GESTÄO } \\
\text { ANTERIOR }\end{array}$ & GESTĀOI & GESTĀOII & GESTĀO III & GESTÃo IV & \begin{tabular}{|l} 
GESTÃo \\
POSTERIOR
\end{tabular} & ÚLTIMO CARGO & $\begin{array}{l}\text { PROFISSÃo } \\
\text { DEELARADA }\end{array}$ & $\begin{array}{c}\text { AREA DAS TERRAS } \\
\text { (HA) }\end{array}$ & $\begin{array}{c}\text { VALOR DAS TERRAS } \\
\text { RS }\end{array}$ & $\begin{array}{l}\text { VALOR DO } \\
\text { PATRIMOONIO }\end{array}$ & $\begin{array}{l}\text { VALLR DAS } \\
\text { TERAASPATRIMOONIO }\end{array}$ \\
\hline $\begin{array}{l}\text { HENRRIOUE EDUARDO LYYAA } \\
\text { ALVES }\end{array}$ & $\begin{array}{l}\text { MDB, 1971-1979; } \\
\text { PP, 1980-1982; } \\
\text { PMDDB, 1982 }\end{array}$ & RN & $\begin{array}{l}1971-1975 ; 1975- \\
1979 ; 1979-1983 ; \\
1983-1987 ; ; 987- \\
1991 ; 19991-1995 \\
199\end{array}$ & |1995-1999 & |999-2003 & 2003-2007 & 2007-2011 & $2011-2015$ & \begin{tabular}{|l} 
DEFUTADO \\
FEDERAL
\end{tabular} & Bacharel em Direito & 32,00 & $R \$ 75.500,00$ & $\mathrm{R} \$ 5.584 .304,96$ & $1,35 \%$ \\
\hline João DA SILVA MAIA & $\begin{array}{l}\mid \mathrm{P}, 2000-2007 ; \mathrm{PR}, \\
2007\end{array}$ & $\mathrm{RN}$ & 0 & 0 & 0 & 0 & 2007-2011 & $2011-2015$ & $\begin{array}{l}\text { DEEUTADOO } \\
\text { FEDERAL }\end{array}$ & Economista & $35,54+\mathrm{NI}$ & $R \$ 151.048,00$ & $\mathrm{R} \$ 14.116 .205,00$ & $1,07 \%$ \\
\hline $\begin{array}{l}\text { NELLO SILVEIRA DIAS } \\
\text { (22:01/1945 a 20/107/2007) }\end{array}$ & $\begin{array}{l}\text { PPB, 2001-2003; } \\
\text { PP, 2003 }\end{array}$ & $\mathrm{RN}$ & 0 & 0 & 0 & $2003-2007$ & $2007-2011$ & 0 & $\begin{array}{l}\text { DEPUTADO } \\
\text { FEDERAL }\end{array}$ & Agropecuarista & $4 \mathrm{NI}$ & $\mathrm{R} \$ 200.000,00$ & $\mathrm{R} \$ 420.000,00$ & $47,62 \%$ \\
\hline REDITÁRIO CASSOL & $\begin{array}{l}\text { ARENA, 1966-1979; } \\
\text { PMDB, 1986-1988; } \\
\text { PMB, 1990-1991; } \\
\text { PTR, 1991-1993; } \\
\text { PRL, 1992-; PSD, } \\
\text { 1993-1994; PP, } \\
\text { 1994 }\end{array}$ & RO & 1991-1995 & 0 & o & 0 & 0 & $\mid \begin{array}{l}2011-2015 ; 2015- \\
2019\end{array}$ & SENADOR & $\begin{array}{l}\mid \text { Industrial } \\
\text { Inter }\end{array}$ & 0,00 & Rs 0.00 & $\mathrm{R} \$ 8.972 .914,00$ & $0,00 \%$ \\
\hline CONFÚCIO AIRES MOURA & PMDB, 1983 & RO & 0 & |1995-1999 & |999-2003 & 2003-2007 & 0 & 0 & $\begin{array}{l}\text { DEPUTADO } \\
\text { FEDERAL }\end{array}$ & \begin{tabular}{|l} 
Médico, Pecuarista e \\
Professor
\end{tabular} & 0,00 & R\$ 0,00 & $\mathrm{R} \$ 8.554 .881,14$ & $0,00 \%$ \\
\hline $\begin{array}{l}\text { NILTON BALBIIO(NILTON } \\
\text { CAPIXABA) }\end{array}$ & $\begin{array}{l}\text { PPLL, 1995--1997; } \\
\text { PTB, } 1997\end{array}$ & Ro & 0 & 10 & |1999-2003 & 2003-2007 & 0 & $2011-2015$ & $\begin{array}{l}\text { DEPPTAAO } \\
\text { FEDERAL }\end{array}$ & Empresário & $3 \mathrm{NI}$ & RS 104.243,44 & $R \$ 1.505 .778,39$ & $6,92 \%$ \\
\hline $\begin{array}{l}\text { RUBENS MORERAR MENDES } \\
\text { FLLHO }\end{array}$ & $\begin{array}{l}\text { ARENA, 1976;; ;DT, } \\
\text { 1995; PFL, 1999-; } \\
\text { PPS, 2005 }\end{array}$ & RO & 0 & 10 & |999-2003 & 0 & 2007-2011 & 2011-2015 & $\begin{array}{l}\text { DEPUTADO } \\
\text { FEDERAL }\end{array}$ & $\begin{array}{l}\text { A Aropeceuarista e } \\
\text { Advogado }\end{array}$ & $3 \mathrm{NI}$ & $\mathrm{R} \$ 104.243,44$ & $\mathrm{R} \$ 1.505 .778,39$ & $6,92 \%$ \\
\hline IVO NARCISO CASSOL & PP & Ro & 0 & 0 & 0 & 0 & 0 & 2019 & SENADOR & Empresário & $6 \mathrm{NI}$ & R\$ $118.300,00$ & 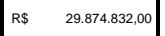 & $0,40 \%$ \\
\hline $\begin{array}{c}\text { SERGIO SIQUEIRA DE } \\
\text { CARVALHO (23/03/1995 a } \\
? 2 / 2 / 2003)\end{array}$ & $\begin{array}{l}\text { PMDB, 1986-1997; } \\
\text { PSDB, 1997-2003 }\end{array}$ & RO & 0 & 0 & |999-2003 & 2003 & 0 & 0 & $\begin{array}{l}\text { DEPUTADO } \\
\text { FEDERAL }\end{array}$ & Médico & 624,40 & $\mathrm{R} \$ 127.500,00$ & 487.200,83 & $26,17 \%$ \\
\hline VALDIR RAUPP DE MATOS & РмDв Rо & Ro & 0 & 0 & 0 & 2003-2007 & $2007-2011$ & $2011-2019$ & SENADOR & Administrador & $\mathrm{NI}$ & R\$ $32.819,67$ & $728.607,29$ & $4,50 \%$ \\
\hline $\begin{array}{l}\text { MARINHA CELAA ROCHA RAUPP } \\
\text { DE MATOS -NESCC }\end{array}$ & $\begin{array}{l}\text { PMDB, 1986-1994; } \\
\text { PSDB, 1994-2001; } \\
\text { PMDB, 2001 }\end{array}$ & RO & 0 & | 1995-1999 & |999-2003 & 2003-2007 & 2007-2011 & 2011-2015 & $\begin{array}{l}\text { DEPUTADA } \\
\text { FEDERAL }\end{array}$ & $\begin{array}{l}\text { em Assuntos } \\
\text { Efocucaionais e } \\
\text { Psicologa e }\end{array}$ & 0,00 & $\mathrm{R} \$ 0,00$ & $\begin{array}{|ll|}\text { RS } & 1.979 .751 .88 \\
\end{array}$ & $0,00 \%$ \\
\hline ERNANDES SANTOS AMORIM & РTB, 2005 & Ro & 0 & 0 & 0 & 0 & 2007-2011 & 0 & $\mid \begin{array}{l}\text { FEDERAL } \\
\text { DEVOAOA }\end{array}$ & Pecuarista & $\mathrm{NI}$ & R\$ $150.000,00$ & 185.000,00 & $81,08 \%$ \\
\hline MAURO NAZZF RASUL & PSB, 2000 & Ro & 0 & 0 & 0 & 0 & $2007-2011$ & $2011-2015$ & 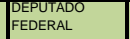 & Médico & 3N1 & $\begin{array}{ll}\text { R\$ } & 157,750,00 \\
\end{array}$ & RS $697.417,00$ & $22,62 \%$ \\
\hline $\begin{array}{l}\text { EXPEDITOGONCCALVES } \\
\text { FERREIRA JUNIOR }\end{array}$ & $\begin{array}{l}\text { PL, 1989-1995; PPB, } \\
\text { 1995-1997; PL, }\end{array}$ & Ro & 1987 & | 1995-1999 & |1999-2003 & 2003-2007 & 2007-2011 & 2011-2015 & SENADOR & Professor & 121,00 & R\$ 210.000,00 & \begin{tabular}{|l|l|} 
RS & $1.165 .750,62$ \\
\end{tabular} & $18,01 \%$ \\
\hline AGNALDO MUNIZ & $\mid \begin{array}{l}\text { PDT, } 1997-2000 ; \\
\text { PSS, 200-2005; } \\
\text { P, 2005-2010; } \\
\text { PSC, 2010; }\end{array}$ & RO & 0 & 0 & |1999-2003 & 2003-2007 & $2010-2011$ & 0 & \begin{tabular}{|l} 
DEFUTADO \\
FEDERAL
\end{tabular} & Advogado & $2.798,00$ & Rs 213.000,00 & RS 253.000,00 & $84,19 \%$ \\
\hline $\begin{array}{l}\text { PAULLO CESAR JUSTO } \\
\text { QUARTIERO }\end{array}$ & $\begin{array}{l}\text { PDT,-2007; DEM, } \\
\text { 2007 }\end{array}$ & $\mathrm{RR}$ & 0 & 10 & 0 & 0 & 0 & $2011-2015$ & $\begin{array}{l}\begin{array}{l}\text { DEPUTADO } \\
\text { FEDERAL }\end{array} \\
\end{array}$ & Engenheiro Agrônomo & 0,00 & R\$ 0,00 & $\mathrm{R} \$ 8.010 .010,00$ & $0,00 \%$ \\
\hline LUCIANO DE SOUZA CASTRO & $\begin{array}{l}1990-1991 ; \mathrm{P} F \mathrm{~L}, \\
1991-1993 ; \mathrm{PPR},\end{array}$ & $\mathrm{RR}$ & |1993-1995 & | 1995-1999 & |1999-2003 & 2003-2007 & 2007-2011 & 2011-2015 & $\begin{array}{l}\text { DEPUTADO } \\
\text { FEDERAL }\end{array}$ & $\begin{array}{l}\text { Econonistae } \\
\text { Administrador }\end{array}$ & 0,00 & R\$ 0,00 & $\mathrm{R} \$ 1.300 .621,37$ & $0,00 \%$ \\
\hline ALCESTE MADEIRA DE ALMEIDA & $\begin{array}{l}\text { PDT, 1987-1990; } \\
\text { PTB, 1990-1995; }\end{array}$ & $\mathrm{RR}$ & 1991-1995 & |1995-1999 & |999-2003 & 2003-2007 & 0 & 0 & $\begin{array}{l}\text { DEPUTADO } \\
\text { FEDERAL }\end{array}$ & Médico & 0,00 & R\$ 0,00 & R\$ 859.893,44 & $0,00 \%$ \\
\hline LUIZ BARBOSA ALVES & $\begin{array}{l}\text { PRT, } 9939-1-1995 ; \\
\text { PPBB, } \\
\text { PFL, 19959-1999; }\end{array}$ & $\mathrm{RR}$ & 0 & | 1995-1999 & |999-2003 & 0 & 0 & 0 & \begin{tabular}{|l} 
DEFUTADO \\
FEDERAL
\end{tabular} & Empresário & $958,96+\mathrm{NI}$ & $R \$ 10.430,61$ & $R \$ 1.209 .413,33$ & $0,86 \%$ \\
\hline $\begin{array}{l}\text { FRANCISCD DE ASIS } \\
\text { RODRIGUES }\end{array}$ & $\begin{array}{l}\text { PTB, 1989-1995; } \\
\text { PSD, 1995; PPB, }\end{array}$ & $\mathrm{RR}$ & |1991-1995 & |1995-1999 & |1999-2003 & 2003-2007 & 2007-2011 & 0 & $\begin{array}{l}\begin{array}{l}\text { DEPUTADO } \\
\text { FEDERAL }\end{array} \\
\end{array}$ & Engenheiro Agrônomo & $2 \mathrm{NI}$ & R\$20.000,00 & $R \$ 1.939 .730,56$ & $1,03 \%$ \\
\hline $\begin{array}{l}\text { SALOMÄO AFONSO DE SOUZA } \\
\text { CRUZ }\end{array}$ & \begin{tabular}{|l|} 
PFL, 1985-1990; \\
PSDBB, 1990-1991; \\
PFL, 1991-1996; \\
PDBB, 1996-1999; \\
PPB, 1999-2001; \\
PFL, 2001
\end{tabular} & $\mathrm{RR}$ & 0 & |1995-1999 & |999-2002 & 0 & o & 0 & $\begin{array}{l}\text { DEPUTADO } \\
\text { FEDERAL }\end{array}$ & Engenheiro & $2600+\mathrm{NI}$ & $\mathrm{R} \$ 41.964,26$ & $241.756,30$ & $17,36 \%$ \\
\hline JOHNATHAN PERERA DE JESUS & PRB, 2009 & $\mathrm{RR}$ & 0 & 0 & 0 & 0 & 0 & $2011-2015$ & $\begin{array}{l}\text { DEPUTADO } \\
\text { FEDERAL }\end{array}$ & Empresário & $958,2+\mathrm{NI}$ & RS 126.000,00 & R\$ 556.149,98 & $22,66 \%$ \\
\hline $\begin{array}{c}\text { FRANCISCO MOZARLLDO DE } \\
\text { MELO CAVALCANTI }\end{array}$ & \begin{tabular}{|c|}
$1970-1979 ;$ \\
$1980-1984 ;$ PLS, 1990
\end{tabular} & $\mathrm{RR}$ & $\begin{array}{l}1983-1987 ; \text {; } 987- \\
1991\end{array}$ & 0 & |1999-2003 & 2003-2007 & 2007-2011 & $2011-2015$ & SENADOR & Médico e Professsor & $\mathrm{NI}$ & RS 355.134,00 & $\mathrm{R} \$ 1.344 .5266,54$ & $26,41 \%$ \\
\hline RAUL DA SILVA LIMA SOBRINHO & \begin{tabular}{|l|}
$2005 ;$ PMDB, 2005- \\
2009
\end{tabular} & $\mathrm{RR}$ & 0 & 0 & 0 & 0 & 0 & 2011-2015 & $\begin{array}{l}\text { DEPUTADO } \\
\text { FEDERAL }\end{array}$ & Administrador & $\mathrm{NI}$ & R\$ 658.000,00 & $\mathrm{R} \$ 1.096 .369,28$ & $60,02 \%$ \\
\hline
\end{tabular}




\begin{tabular}{|c|c|c|c|c|c|c|c|c|c|c|c|c|c|c|}
\hline NOME & PARtido & UF & $\begin{array}{l}\text { GESTAO } \\
\text { ANTERIOR }\end{array}$ & GESTÃol & GESTĀoll & GESTÃO III & GESTÃo IV & $\begin{array}{l}\text { GESTAO } \\
\text { POSTERIOR }\end{array}$ & Último cafago & $\begin{array}{l}\text { PROFISAO } \\
\text { DECLARADA }\end{array}$ & $\begin{array}{c}\cos _{\text {AREA DAS TERRAS }} \\
(\text { HÁ) }\end{array}$ & $\begin{array}{l}\text { VALOR DAS TERRAS } \\
\text { RS }\end{array}$ & $\begin{array}{l}\text { VALLRDO } \\
\text { PATRIMOONIO }\end{array}$ & $\begin{array}{c}\text { VALOR DAS } \\
\text { TERRASPATRRMONONIO }\end{array}$ \\
\hline DARCI POMPEO DE MATTOS & $\begin{array}{l}\text { MDDB, 19/6-19/9; } \\
\text { PDT, } 1979\end{array}$ & RS & 0 & 0 & |1999-2003 & 2003-2007 & 2007-2011 & & $\begin{array}{l}\text { DEPUTADO } \\
\text { FEDERAL }\end{array}$ & Advogado e Bancário & 0,00 & $R \$ 0,00$ & $\mathrm{R} \$ 1.430 .812,21$ & $0,00 \%$ \\
\hline ONYX DORNELLES LORENZONI & $\left.\right|_{2007} ^{1997-2007 ; \text { DEM, }}$ & RS & 0 & 0 & 0 & $2003-2007$ & $2007-2011$ & $2011-2015$ & \begin{tabular}{|l} 
DEPPTAAD \\
FEDERAL
\end{tabular} & $\mid \begin{array}{l}\mid \begin{array}{l}\text { Empresásio } \\
\text { veterinario }\end{array} \\
\end{array}$ & 0,00 & $\mathrm{R} \$ 0,00$ & R\$ 800.439,06 & $0,00 \%$ \\
\hline $\begin{array}{l}\text { JULIO CESAR REDECKER } \\
\text { (12/07/1956 a 17/07/2007) }\end{array}$ & $\begin{array}{l}\text { PDS, 1980-1993; } \\
\text { PPR, 1993-1995; }\end{array}$ & RS & 0 & |1995-1999 & |1999-2003 & 2003-2007 & 2007 & 0 & \begin{tabular}{|l} 
DEPUTADO \\
FEDERAL
\end{tabular} & $\begin{array}{l}\text { Universitário e } \\
\text { Empresário }\end{array}$ & 0,00 & $\mathrm{R} \$ 0,00$ & RS $675.630,00$ & $0,00 \%$ \\
\hline DARCIIIO PAULO PERONDI & PMDB, 1993 & RS & 0 & 1995-1999 & 1999-2003 & 2003-2007 & $2007-2011$ & $2011-2015$ & $\mid \begin{array}{l}\text { DEPPTAAO } \\
\text { FEDERAL }\end{array}$ & Médico & 0,00 & $R \$ 0,00$ & R\$ 215.962,52 & $0,00 \%$ \\
\hline RENATO DELMAR MOLLING & PP, 1993. & RS & 0 & 0 & 0 & 0 & $2007-2011$ & $2011-2015$ & $\mid \begin{array}{l}\text { FEDEERAL } \\
\text { FEDEL }\end{array}$ & 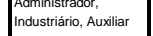 & 49,00 & RS $18.081,82$ & R\$ 586.627,24 & $3.08 \%$ \\
\hline SÉRGio IVAN MORAES & PTB, 1988 & RS & 0 & 0 & 0 & 0 & $2007-2011$ & $2011-2015$ & FEDERAL & Nâo Intorrmou & $1+\mathrm{NI}$ & $\mathrm{R} \$ 21.000,00$ & R\$ 203.043,69 & $10,34 \%$ \\
\hline VILSON LUIZ COVATTI & PP, 1982 & RS & 0 & 0 & 0 & 0 & $2007-2011$ & $2011-2015$ & $\begin{array}{l}\text { DEPUTAAO } \\
\text { FEDERAL }\end{array}$ & Advogado & ${ }_{2 \mathrm{NI}}$ & $\mathrm{R} \$ 42.862,77$ & R\$ $947.996,33$ & $4,52 \%$ \\
\hline ELISEU LEMOS PADLLHA & \begin{tabular}{|l|}
$\begin{array}{l}\text { MDB, 1966; PMDB, } \\
\text { 1986 }\end{array}$ \\
\end{tabular} & RS & 0 & |1995-1999 & o & 2003-2007 & $2007-2011$ & $2011-2015$ & $\begin{array}{l}\text { DEPUTADO } \\
\text { FEDERAL }\end{array}$ & \begin{tabular}{|l} 
Advogadaó e \\
Empresario
\end{tabular} & $1.853,20$ & \begin{tabular}{|ll} 
RS & $55.517,82$ \\
\end{tabular} & $\mathrm{R} \$ 2.668 .415,73$ & $2,08 \%$ \\
\hline CEZAR AUGUSTO SCHIRMER & $\begin{array}{l}\text { MDB, 1972-1981; } \\
\text { PMDB, } 1981\end{array}$ & RS & 0 & 10 & |1999-2003 & 2003-2007 & 2007-2011 & 0 & $\begin{array}{l}\text { DEPUTADO } \\
\text { FEDERAL }\end{array}$ & $\begin{array}{l}\text { Advogado e Professor } \\
\text { Universitário }\end{array}$ & 187,86 & $\mathrm{R} \$ 62.457,05$ & R\& 777.457,05 & $8,03 \%$ \\
\hline TELMO JOSÉ KIRST & 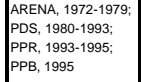 & RS & $\begin{array}{l}\text { 1979-1983; 1983- } \\
\text { 1987; } 1987-1991 ; \\
\text { 1991-1995 }\end{array}$ & |1995-1999 & |1999-2003 & 0 & 0 & 0 & $\begin{array}{l}\text { DEFUTADO } \\
\text { FEDERAL }\end{array}$ & Industriale Advogado & 25,10 & $\mathrm{R} \$ 99.442,30$ & R\$ $\quad 917.585,04$ & $10,84 \%$ \\
\hline PAULO JOSE GOUVEA & $\begin{array}{l}\text { PTB, 1997-1999; } \\
\text { PST, 1999; PL, } 1999\end{array}$ & RS & 0 & 0 & |1999-2003 & 2003-2007 & o & 0 & $\begin{array}{l}\text { DEPUTADO } \\
\text { FEDEAAL }\end{array}$ & Radialista & 1,00 & RS $110.000,00$ & R\$ $412.964,00$ & $26,64 \%$ \\
\hline NELSON MARCHEZAN JÚNIOR & PSDB, 2001 & RS & 0 & 0 & 0 & 2003-2007 & 0 & $2011-2015$ & $\begin{array}{l}\text { DEPPTAAC } \\
\text { FEDERAL }\end{array}$ & Advogado & 106,00 & RS 124.240,45 & RS 195.533.59 & $63,54 \%$ \\
\hline $\begin{array}{l}\text { JOĀo AUGUSTO RIBEIRO } \\
\text { NARDES }\end{array}$ & 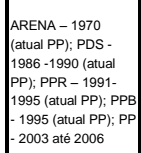 & RS & 0 & |1995-1999 & |1999-2003 & 2003-2005 & o & 0 & $\begin{array}{l}\text { DEPUTADO } \\
\text { FEDERAL }\end{array}$ & $\begin{array}{l}\text { Administrador de } \\
\text { Empresas }\end{array}$ & 220,00 & $\mathrm{R} \$ 142.696,84$ & $704.725,88$ & $20,25 \%$ \\
\hline JOSÉ AFONSO EBERT HAMM & $\begin{array}{l}\mathrm{PPB}, 1995-; \mathrm{PP}, \\
1995\end{array}$ & RS & 0 & 0 & 0 & 2003-2007 & 2007-2011 & 2011-2015 & $\begin{array}{l}\text { DEPUTADO } \\
\text { FEDERAL }\end{array}$ & Engenheiro Agrônomo | & $119+N \mid$ & R\$ $203.369,83$ & R\$ $495.447,52$ & $41,05 \%$ \\
\hline $\begin{array}{l}\text { ADOLFO ANTONOOFETTER } \\
\text { JUNIOR }\end{array}$ & $\begin{array}{l}\text { PDS, 1982-1993; } \\
\text { PPR, 1993-1995; }\end{array}$ & RS & |1991-1995 & |1995-1999 & |1999-2003 & 0 & 0 & 0 & \begin{tabular}{|l} 
DEPUTADO \\
FEDERAL
\end{tabular} & \begin{tabular}{|l|} 
Empressas e \\
Engenheiro Agrónomomo
\end{tabular} & 598,60 & Rs 220.656,88 & R\$ 1.287.402,93 & $17,14 \%$ \\
\hline GIOVANI CHERINI & PDT, 1988 & $\mathrm{RS}$ & 0 & 0 & 0 & 0 & 0 & $2011-2015$ & | & $\begin{array}{l}\text { Iecnólogo em } \\
\text { Cooperativismo }\end{array}$ & $75,4+\mathrm{NI}$ & $\begin{array}{ll}\text { R\$ } & 491.042,00\end{array}$ & $\mathrm{R} \$ 1.203 .646,00$ & $40,80 \%$ \\
\hline LUIZ CARLOS HEINZE & $\begin{array}{l}\text { PPS, 1992-1995; } \\
\text { PPB, 1995-2003; } \\
\text { PP, 2003 }\end{array}$ & RS & 0 & 0 & |1999-2003 & 2003-2007 & 2007-2011 & $2011-2015$ & $\begin{array}{l}\text { DEPUTADO } \\
\text { FEDERAL }\end{array}$ & $\begin{array}{l}\text { Enggenherio Agríno } \\
\text { e produtor Rural }\end{array}$ & $1.543,00$ & $\mathrm{R} \$ 1.285 .083,23$ & $\mathrm{R} \$ 1.841 .261,75$ & $69,79 \%$ \\
\hline LEONEL ARCANGELO PAVAN & \begin{tabular}{|l|} 
PMDB, 1982-1985; \\
PDT, 1986
\end{tabular} & sc & 0 & |1995-1997 & o & 2003-2007 & 2007-2011 & 0 & SENADOR & Comerciante & 0,00 & R\$ 0,00 & $\mathrm{R} \$ 2.468 .266,48$ & $0,00 \%$ \\
\hline HUGO MATHAS BIEHL & $\begin{array}{l}\text { PDS, 1980-1993; } \\
\text { PPR, 1993-1995; }\end{array}$ & $\mathrm{sc}$ & |1991-1995 & |1995-1999 & |1999-2003 & 0 & 0 & 0 & $\begin{array}{l}\text { DEPUTADO } \\
\text { FEDERAL }\end{array}$ & 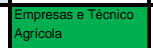 & 0,00 & $R \$ 0,00$ & $R \$ 2.213 .112,68$ & $0,00 \%$ \\
\hline GERVASIO JOSE DA SILVA & $\begin{array}{l}\text { PDS, 1980-1987; } \\
\text { PL, 1987-1990; PFL, } \\
\text { 1990-2007; DEM, } \\
\text { 2007; PSDB, 2007 }\end{array}$ & $\mathrm{sc}$ & 0 & 0 & |1999-2003 & 2003-2007 & 2007-2011 & 0 & \begin{tabular}{|} 
DEPUTADO \\
FEDERAL
\end{tabular} & \begin{tabular}{|l|} 
Empreseário, Contador \\
e Corretor de Inóveis
\end{tabular} & 0,00 & Rs 0,00 & R\$ $938.889,00$ & $0,00 \%$ \\
\hline ODACIR ZONTA & \begin{tabular}{|l|} 
ARENA, 1967-1981; \\
PPS, 1981-1993; \\
PPR, 1993-1995; \\
PPB, 1995-2003; \\
PP, 2003
\end{tabular} & sc & 10 & o & o & 2003-2007 & $2007-2011$ & 0 & $\begin{array}{l}\text { DEEUTADO } \\
\text { FEDERAL }\end{array}$ & 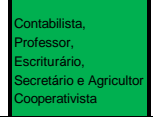 & 0,00 & Rs 0,00 & R\$ $564.476,74$ & $0,00 \%$ \\
\hline ESPERIIIĀO AMIN HELOU FLLHO & $\mathrm{PP}$ & sc & 1983; 1991-1995 & |1995-1998 & 10 & 0 & 0 & $2011-2015$ & $\begin{array}{l}\text { DEPUTADO } \\
\text { FEDERAL }\end{array}$ & \begin{tabular}{|l|}
$\begin{array}{l}\text { Advogado, Professor } \\
\text { Universitáaio e } \\
\text { Administrador }\end{array}$ \\
\end{tabular} & 8,30 & R\$ 16.655,34 & $\mathrm{R} \$ 1.646 .754,53$ & $1,01 \%$ \\
\hline CASILDO JOĀO MALDANER & РMDB & $\mathrm{sc}$ & 1987 & 10 & 0 & 0 & $2007-2011$ & $2011-2015$ & SENADOR & Comerciante & ${ }_{2 \mathrm{NI}}$ & $\mathrm{R} \$ 73.234,66$ & $\mathrm{R} \$ 2.863 .731,92$ & $2,56 \%$ \\
\hline
\end{tabular}




\begin{tabular}{|c|c|c|c|c|c|c|c|c|c|c|c|c|c|c|}
\hline NOME & PARTIDO & UF & $\begin{array}{l}\left.\right|_{\text {AESTAO }} ^{\text {GESTERIOR }} \\
\text { ANT }\end{array}$ & GESTÃOI & GEstāol & GESTÃO III & GESTÄOIV & $\begin{array}{l}\text { GESTAO } \\
\text { POSTERIOR }\end{array}$ & Último cargo & $\begin{array}{l}\text { PROFISAO } \\
\text { DECLARADA }\end{array}$ & $\begin{array}{c}\text { AREA DAS TERRAS } \\
\text { (HA) }\end{array}$ & \begin{tabular}{|c|}
$\begin{array}{c}\text { VALOR DAS TERRAS } \\
\text { RS }\end{array}$ \\
\end{tabular} & $\begin{array}{l}\text { VALOR DO } \\
\text { PATRIMOONIO }\end{array}$ & $\begin{array}{c}\text { VALOR DAS } \\
\text { TERAASPATRMIMONIO }\end{array}$ \\
\hline JOĀO BATISTA MATOS & PP; PMDB & sc & 0 & | 1995-1999 & |1999-2003 & 2003-2007 & $2007-2011$ & 0 & $\begin{array}{l}\text { DEPUTADO } \\
\text { FEDEAAL }\end{array}$ & \begin{tabular}{|l|} 
Administrtador de \\
Empresas P Professsor
\end{tabular} & 14,64 & RS $116.980,78$ & $\mathrm{R} \$ 1.322 .709,01$ & $8,84 \%$ \\
\hline JOĀO ALBERTO PIZZOLATTI & $\begin{array}{l}\text { PPR, 1993-1995; } \\
\text { PPB, 1995-2003; }\end{array}$ & sc & 0 & | 1995-1999 & |1999-2003 & 2003-2007 & 2007-2011 & 2011-2015 & \begin{tabular}{|l} 
DEPUTADO \\
FEDERAL
\end{tabular} & \begin{tabular}{|l} 
Engenheiro Civile \\
Servidor Puiblico
\end{tabular} & 248,72 & RS $132.000,00$ & $\mathrm{R} \$ 1.077 .163,32$ & $12,25 \%$ \\
\hline VALDIR COLATTO & $\begin{array}{l}\text { MDB, } 1980 ; \text {; PMDB, } \\
1981\end{array}$ & sc & $\begin{array}{l}1989-1990 ; 1993- \\
1995\end{array}$ & | 1995-1999 & 10 & 2005-2007 & 2007-2011 & 10 & $\begin{array}{l}\text { DEPUTADO } \\
\text { FEDERAL }\end{array}$ & | Engenheiro Agrơnomol & $2.265,13+1 \mathrm{NI}$ & $\operatorname{R\$ } 236.365,85$ & $\mathrm{R} \$ 1.399 .105,16$ & $16,89 \%$ \\
\hline RONALDO JOSÉ BENEDET & РМDв & sc & 0 & 0 & 0 & 0 & 0 & $2011-2015$ & FEDERAL & Advogado & $125+\mathrm{NI}$ & R\$ 246.003,21 & $\mathrm{R} \$ 1.597 .961,97$ & $15,39 \%$ \\
\hline EDSON BEZ DE OLIVEIRA & PMDB, 1985 & sc & 0 & 1995-1999 & 1999-2003 & $2003-2007$ & $2007-2011$ & $2011-2015$ & $\begin{array}{l}\text { DEVUTADO } \\
\text { FEDERAL }\end{array}$ & $\left|\begin{array}{l}\text { A Aricicutor, Profosssor, } \\
\text { Bancário e Contador }\end{array}\right|$ & $11 \mathrm{NI}$ & R\$ 887.222,31 & $\mathrm{R} \$ 3.337 .918,69$ & $26,58 \%$ \\
\hline JOSÉ TELES DE MENDONÇA A & $\begin{array}{l}\text { PDS, 1980-1993; } \\
\text { PPR, 193-1955; }\end{array}$ & SE & $1991-1995$ & 1995-1999 & $1999-2003$ & 0 & o & 0 & $\begin{array}{l}\text { DEPUTADO } \\
\text { FEDERAL }\end{array}$ & $\begin{array}{l}\text { Agropecuarista e } \\
\text { Empresário }\end{array}$ & 5N1 & R\$51.065,13 & RS 194.199,49 & $26,30 \%$ \\
\hline $\begin{array}{l}\text { JOSÉ CLEONANACII DA } \\
\text { FONSECA }\end{array}$ & 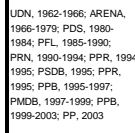 & SE & $\mid \begin{array}{l}1987-1991 ; 1991- \\
\text { 1995; } 1995-\end{array}$ & | 1995-1999 & |1999-2003 & 2003-20007 & o & 0 & $\begin{array}{l}\text { DEPUTADO } \\
\text { FEDERAL }\end{array}$ & 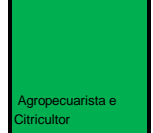 & ${ }_{6 \mathrm{NI}}$ & $\mathrm{R} \$ 52.475,23$ & R\$ $106.193,49$ & $49,41 \%$ \\
\hline JOSÉ ALMEIDA LIMA & $\begin{array}{l}\text { MOB, 1970-1986; } \\
\text { PSB 1986-1989; } \\
\text { POT, 1989-2004; } \\
\text { PPSB, 2004-2045; } \\
\text { PMDB, 2005 }\end{array}$ & SE & 0 & 0 & 0 & 0 & o & 2011-2015 & \begin{tabular}{|l} 
DEFUTADO \\
FEDERAL
\end{tabular} & $\begin{array}{l}\text { Advogado e Professor } \\
\text { de Direito }\end{array}$ & 378,98 & R\$ $58.348,00$ & $2.089 .580,00$ & $2,79 \%$ \\
\hline JOSÉ CARLOS MACHADO & DEM, 2007 & $\mathrm{SE}$ & 0 & 0 & 0 & 2003-2007 & $2007-2011$ & 0 & FEDERAL & $\begin{array}{l}\text { Engennerec } \\
\text { Empresario li e }\end{array}$ & $6 \mathrm{NI}$ & $\begin{array}{|ll|}\text { R } & 111.943,83 \\
\end{array}$ & R\$ $6.273 .665,40$ & $1,78 \%$ \\
\hline JOĀO BOSCO DA COSTA & $\begin{array}{l}\text { PPR, 1999-1995; } \\
\text { PPB, 1995-2001; }\end{array}$ & SE & 0 & 0 & 0 & 2003-2007 & 0 & 0 & $\begin{array}{l}\text { DEEUTADO } \\
\text { FEDERAL }\end{array}$ & \begin{tabular}{|l} 
Adminisistrador de \\
Empresas
\end{tabular} & 22.869 .87 & $\begin{array}{|ll|}\text { R\$ } & 223.255,17 \\
\end{array}$ & $\mathrm{R} \$ 1.912 .687,18$ & $11,67 \%$ \\
\hline JORGE ALBERTO TELES PRADO & $\begin{array}{l}\text { PRN, 1990-1991; } \\
\text { PMDB, } 1993\end{array}$ & SE & 0 & 0 & |1999-2003 & 2003-2007 & 2007-2011 & & $\begin{array}{l}\text { DEPUTAAO } \\
\text { FEDERAL }\end{array}$ & Médico & 580,00 & R\$ $332.496,23$ & $\mathrm{R} \$ 1.158 .500,93$ & $28,70 \%$ \\
\hline RICARDO IZAR JUNIOR & $\mid \begin{array}{l}\text { Pv, 2009-2011; } \\
\text { PSD, 2011 }\end{array}$ & $\mathrm{SP}$ & 0 & 0 & 0 & 0 & 0 & 2011-2015 & $\begin{array}{l}\text { DEPUTADO } \\
\text { FEDERAL }\end{array}$ & \begin{tabular}{|l} 
Economistae \\
Empresarioio
\end{tabular} & 0,00 & Rs 0,00 & $\begin{array}{|ll|}\text { RS } & 2.683 .168,05 \\
\end{array}$ & $0,00 \%$ \\
\hline $\begin{array}{l}\text { JOSE ALDO REBELO } \\
\text { FIGUERREDO }\end{array}$ & $\begin{array}{l}\text { PMDB, 1980-1985; } \\
\text { PCDoB, 1985 }\end{array}$ & $\mathrm{SP}$ & |1991-1995 & |1995-1999 & |1999-2003 & 2003-2007 & 2007-2011 & $2011-2015$ & \begin{tabular}{|l} 
DEPUTADO \\
FEDERAL
\end{tabular} & Jornalista & 0,00 & Rs 0,00 & R\$ 376.372 .54 & $0,00 \%$ \\
\hline RODRIGO GARCIA & DEM, 1994 & SP & 0 & 0 & 0 & 0 & 0 & $2011-2015$ & 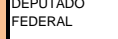 & Não Intormou & 0,00 & Rs 0,00 & $R \$ 4.391 .456,34$ & $0,00 \%$ \\
\hline $\begin{array}{l}\text { ALINE LEMOS CORREA DE } \\
\text { OLIVEIRA ANDRADE }\end{array}$ & PP, 2003 & $\mathrm{SP}$ & 0 & 0 & 0 & 0 & 2007-2011 & $2011-2015$ & $\begin{array}{l}\text { DEPUTADA } \\
\text { FEDERAL }\end{array}$ & Empresária & 0,00 & R\$ 0,00 & $\mathrm{R} \$ 2.241 .726,72$ & $0,00 \%$ \\
\hline $\begin{array}{l}\text { RUBEENEUTONOOLLEIRA LIMA } \\
\text { (NEUTON LLMA) }\end{array}$ & $\begin{array}{l}\text { PDT, 1999-1999; } \\
\text { PFL, 1999-2003; }\end{array}$ & $\mathrm{sP}$ & 0 & 1995-1999 & |999-2003 & 2003-2007 & 0 & 0 & $\begin{array}{l}\text { DEEUTADO } \\
\text { FEDERAL }\end{array}$ & Advogado & 0,00 & R\& 0,00 & $\begin{array}{|ll|}\text { RS } & 1.527 .587,66 \\
\end{array}$ & $0,00 \%$ \\
\hline LUIZ ANTONIO FLEURY FILHO & \begin{tabular}{|l} 
PTB, 19955 -1995; \\
PTB
\end{tabular} & $\mathrm{sP}$ & 0 & 0 & 1999-2003 & 2003-2007 & 0 & 0 & \begin{tabular}{|l} 
FEPDIADO \\
FEDERAL
\end{tabular} & Advogado & 0,00 & R\$ 0,00 & $\mathrm{R} \$ 1.032 .132,60$ & $0,00 \%$ \\
\hline VALDEMAR CORAUCI SOBRINHO & $\begin{array}{l}\text { ARENA, 1976-1982; } \\
\text { PDSS, 1982-1985; } \\
\text { PFF, 1985-1994; PL, } \\
\text { 19944-1995; PFL, } \\
\text { 19995 }\end{array}$ & SP & 0 & | 1995-1999 & |1999-2003 & 2003-2007 & o & 0 & $\begin{array}{l}\text { DEPUTADO } \\
\text { FEDERAL }\end{array}$ & 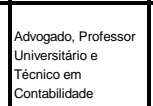 & 0,00 & R\$ 0,00 & $609.885,73$ & $0,00 \%$ \\
\hline $\begin{array}{l}\text { LUIZ ANTONOO DE MEDEROS } \\
\text { NETO }\end{array}$ & $\begin{array}{l}\text { PFL, 1997-2000; PL. } \\
\text { 2001 }\end{array}$ & SP & 0 & 0 & |999-2003 & 2003-2007 & o & 0 & $\begin{array}{l}\text { DEPUTADO } \\
\text { FEDERAL }\end{array}$ & \begin{tabular}{|l|} 
Metalúricico e Torneiro \\
Mecanico
\end{tabular} & 0,00 & R\$ 0,00 & \begin{tabular}{|l|l|} 
R\$ & $131.169,11$ \\
\end{tabular} & $0,00 \%$ \\
\hline $\begin{array}{l}\text { FRANCISCO MARCELO ORTIZ } \\
\text { FLLHO }\end{array}$ & PV, 1996 & $\mathrm{SP}$ & 0 & 0 & 0 & 2003-2007 & 2007-2011 & 0 & \begin{tabular}{|l} 
DEPUTADO \\
FEDERAL
\end{tabular} & \begin{tabular}{|l|}
$\begin{array}{l}\text { Advogado e Professor } \\
\text { Universitário }\end{array}$ \\
\end{tabular} & 796,70 & $\begin{array}{|ll|}\mathrm{R} \$ & 6.939,73 \\
\end{array}$ & $\mathrm{R} \$ 2.057 .071,57$ & $0,34 \%$ \\
\hline $\begin{array}{l}\text { ALOYSIO NUNES FERREIRA } \\
\text { FLLHO }\end{array}$ & $\begin{array}{l}\text { PMDB, 1980-1997; } \\
\text { PSDB, 1997 }\end{array}$ & SP & 0 & | 1995-1999 & |999-2003 & 2003-2007 & 2007-2011 & $\begin{array}{l}2011-2015 ; 2011- \\
2019\end{array}$ & SENADOR & \begin{tabular}{|l|}
$\begin{array}{l}\text { Procurador do Estado } \\
\text { de São Paulo }\end{array}$ \\
\end{tabular} & 875,13 & $\mathrm{R} \$ 6.939,73$ & $\mathrm{R} \$ 2.057 .071,57$ & $0,34 \%$ \\
\hline $\begin{array}{l}\text { MLLTON ANTONO CASQUEL } \\
\text { MONTI }\end{array}$ & $\begin{array}{l}\text { PMDB, 1980-2003; } \\
\text { PLL, 2003-2077; PR, } \\
\text { 2007 }\end{array}$ & $\mathrm{SP}$ & 10 & 0 & |1999-2003 & 2003-2007 & 2007-2011 & 2011-2015 & $\begin{array}{l}\text { DEPUTADO } \\
\text { FEDERAL }\end{array}$ & $\begin{array}{l}\text { Economistae } \\
\text { Empresário }\end{array}$ & $\mathrm{NI}$ & R\$ $10.000,00$ & 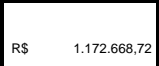 & $0,85 \%$ \\
\hline $\begin{array}{l}\text { JOAO EDUARDO DADO LETTE DE } \\
\text { CARVALHO }\end{array}$ & $\begin{array}{l}\text { PSDC, 1999-2000; } \\
\text { PMDB, 2000-2001; }\end{array}$ & SP & 10 & 10 & $2001-2003$ & 2003-2007 & 2007-2011 & $2011-2015$ & $\begin{array}{l}\text { DEPUTADO } \\
\text { FEDERAL }\end{array}$ & $\begin{array}{l}\text { Agente Fiscal de } \\
\text { Rendas }\end{array}$ & $683+\mathrm{NI}$ & $115.000,00$ & $\mathrm{R} \$ 1.000 .719,49$ & $11,49 \%$ \\
\hline
\end{tabular}




\begin{tabular}{|c|c|c|c|c|c|c|c|c|c|c|c|c|c|c|}
\hline NOME & Partido & UF & \begin{tabular}{|l} 
Gestrío \\
ANTERHOR
\end{tabular} & GESTÃol & GESTĀoll & GESTT̄ôIII & GESTÃóolv & 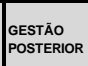 & Último cafGo & $\begin{array}{l}\text { Profissã̃o } \\
\text { DECLAAADA }\end{array}$ & \begin{tabular}{|c|}
$\substack{\text { AREA DAS TERRAS } \\
(\text { HA } A}$ \\
\end{tabular} & 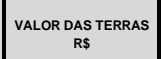 & $\begin{array}{l}\text { VALOR DO } \\
\text { PATRMOMONIO }\end{array}$ & $\begin{array}{c}\text { VALOR DAS } \\
\text { TERAASPATRMOOMOMO }\end{array}$ \\
\hline $\begin{array}{l}\text { ANTONOO DUARTE NOGUERA } \\
\text { JuNIOR }\end{array}$ & 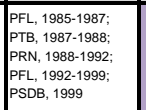 & SP & 0 & $0^{\circ}$ & o & $\sigma_{0}$ & 2007-2011 & $2011-2015$ & 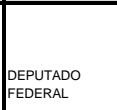 & 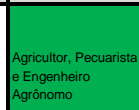 & $\mathrm{NI}$ & R\$ $178.087,29$ & $\mathrm{R} \$ 1.1188 .806,68$ & 15.92\% \\
\hline $\begin{array}{l}\text { ARNALDO CALIL PEEREA } \\
\text { JAROMIM }\end{array}$ & 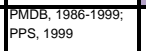 & $\overline{\mathrm{SP}}$ & 0 & jo & 0 & 0 & $2007-2011$ & $2011-2015$ & 隹 & \begin{tabular}{|l} 
Engoenthero \\
profossor
\end{tabular} & $3 \mathrm{NM}$ & Rs 185.640.27 & R5 1.542.555, .62 & $12,03 \%$ \\
\hline JUNI ABE & 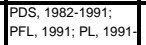 & $\mathrm{sp}$ & $j_{0}$ & 0 & 10 & 0 & 10 & $2011-2015$ & $\begin{array}{l}\text { DEEUTADO } \\
\text { EEEERAL }\end{array}$ & 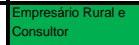 & $4 \mathrm{NI}$ & RS 2677.967,30 & R5 2.208.390,12 & $11,13 \%$ \\
\hline $\begin{array}{l}\text { EDSON EDNHO COELHO } \\
\text { ARALUO }\end{array}$ & 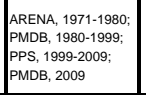 & SP & 0 & 1995-1999 & \begin{tabular}{|l|l|}
$199-2001$ \\
\end{tabular} & & 0 & $2011-2015$ & $\begin{array}{l}\substack{\text { EEPUTADO } \\
\text { FEDERAL }} \\
\text { E }\end{array}$ & Advogasto P Protessor & 11N1 & R\$ $320.196,03$ & R\$ 1.1.24.256,37 & $28,48 \%$ \\
\hline 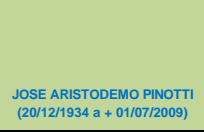 & 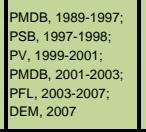 & SP & 0 & 1995-1999 & & 2003-2007 & 2007-2011 & 10 & 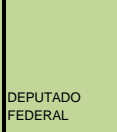 & Mádico & $9.094,00$ & 388.624,38 & R8 7.861.153.23 & $4,94 \%$ \\
\hline FrAnCISCOO GRAZZANO NETTO & $\begin{array}{l}\text { PMOBB, 1980-1988; } \\
\text { PSOB, 1988 }\end{array}$ & $\mathrm{sp}$ & 0 & 0 & \begin{tabular}{|l}
$1999-2003$ \\
\end{tabular} & $2005-2007$ & 0 & 0 & $\begin{array}{l}\text { DeFUDADO } \\
\text { EDEERAL }\end{array}$ & 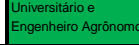 & 420,07 & RS 4 415.965.00 & $\begin{array}{|ll|}\text { RS } & 696.663,00 \\
\end{array}$ & $59,71 \%$ \\
\hline JOSÉ CARLLOS VAZ D DE LMA & PSDB, 1990 & $\mathrm{sP}$ & 0 & 0 & 0 & 0 & 0 & $2011-2015$ & 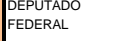 & 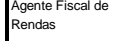 & $2 \mathrm{NI}$ & RS 544.085,00 & R\& 1.081.541,16 & $50.31 \%$ \\
\hline 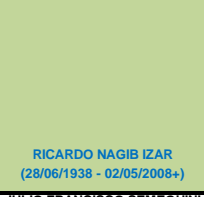 & 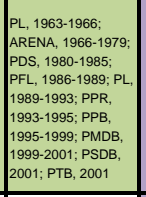 & $\mathrm{sP}$ & $\begin{array}{l}1987-199 ; ; 1991- \\
1995\end{array}$ & |1995-1999 & 1999-2003 & $2003-2007$ & $2007-2011$ & 10 & $\begin{array}{l}\text { DeFuUADO } \\
\text { EEEEARL }\end{array}$ & \begin{tabular}{|l} 
Adrogaado \\
\end{tabular} & $132.69+\mathrm{NI}$ & 695.858.69 & R\$ 2.624.294.53 & $26.52 \%$ \\
\hline 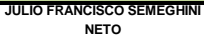 & PSDB, 1990 & $\mathrm{sp}$ & & 0 & $1999-2003$ & $2003-2007$ & $2007-2011$ & $2011-2015$ & 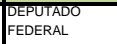 & 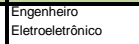 & $3 \mathrm{NN}$ & RS 1.388.948.00 & R\$ 2.748.985,52 & 50,53\% \\
\hline 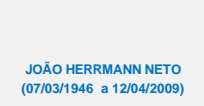 & 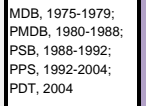 & $\mathrm{SP}$ & 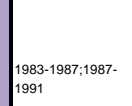 & 0 & |1999-2003 & $2003-2007$ & 2009 & 0 & $\begin{array}{l}\text { DEEUATAD } \\
\text { FEDERAL }\end{array}$ & Engenher & $6.399,77+\mathrm{N1}$ & RS 1.921.672.88 & R\$ 27.752.887,.52 & $6.90 \%$ \\
\hline $\begin{array}{l}\text { PAULO RoBEERTO GOMES } \\
\text { MANSUR }\end{array}$ & 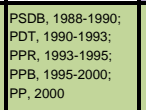 & SP & |1991-1995 & |1995-1997 & o & $\sigma_{0}$ & 2007-2011 & $2011-2015$ & 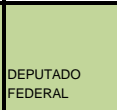 & 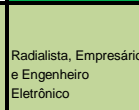 & $420+\mathrm{NI}$ & $\begin{array}{ll}\mathrm{R} \$ & 2.935 .510,70 \\
\end{array}$ & R\$ 16.229.185.27 & $18.09 \%$ \\
\hline NELSON MARQUEZZZELU & 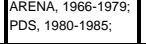 & $\mathrm{sP}$ & |1991-1995 & |1995-1999 & \begin{tabular}{|l}
$1999-2003$ \\
\end{tabular} & $2003-2007$ & $2007-2011$ & $2011-2015$ & $\begin{array}{l}\text { DeFUTAOO } \\
\text { EEDERAL }\end{array}$ & 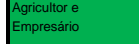 & $2.288,02$ & RS $3.046 .649,71$ & \begin{tabular}{|ll} 
RS & $6.990 .000,92$ \\
\end{tabular} & $43.5 \% \%$ \\
\hline $\begin{array}{l}\text { PAULO o eEAR DE OLNERA } \\
\text { LIMA }\end{array}$ & $\begin{array}{l}\text { PFL, 1986-1999; } \\
\text { PMDB, 1999; }\end{array}$ & $\mathrm{SP}$ & |1992-1994 & |1995-1999 & |1999-2003 & 2003-2007 & 2008 & 0 & $\begin{array}{l}\text { DEEUTADO } \\
\text { FEDERAL }\end{array}$ & 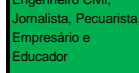 & $1.77 .20+2 \mathrm{NI}$ & Rs 5. $195.749,23$ & $\begin{array}{ll}\text { R\$ } & 26.141 .278 ., 84\end{array}$ & $19.88 \%$ \\
\hline ETVALDO VADD̄o GOMES & 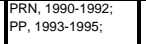 & $\mathrm{sp}$ & \begin{tabular}{|l}
$1991-1995$ \\
\end{tabular} & 1995-1999 & 1999-2003 & 2003-2007 & 2007-2011 & o & 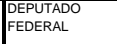 & 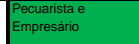 & $9 \mathrm{ONI}$ & RS $116.400 .000,00$ & \begin{tabular}{|ll} 
RS & $192.511 .136,64$
\end{tabular} & $60.46 \%$ \\
\hline $\begin{array}{l}\text { CARLOS EDUADD TORRES } \\
\text { GOMES (EDUARDO GOMES) }\end{array}$ & 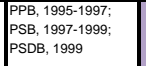 & то & 0 & 0 & 0 & $2003-2007$ & 2007-2011 & $2011-2015$ & 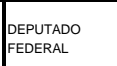 & 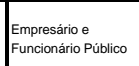 & 0.00 & Rs 0,00 & R\$ $481.100,00$ & $0,00 \%$ \\
\hline IGOR PUGLESI AVELINO & Рм0B, 1997 & то & 0 & 0 & $\begin{array}{l}1999-2003 \\
\end{array}$ & $\Gamma$ & 0 & 0 & 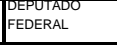 & 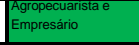 & 0.00 & Rs 0,00 & $363.000,00$ & $0.00 \%$ \\
\hline DARCI MAATTNS COELHO & $\mid$ & то & 1993-1995 & $1995-1999$ & 1999-2003 & $2003-2007$ & $\sigma_{0}$ & $\sigma_{0}$ & $\begin{array}{l}\text { DEEUAAD } \\
\text { FEDERAAL }\end{array}$ & 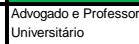 & 0.00 & Rs 0,00 & $\begin{array}{|ll|}\text { RS } & 205,423,15 \\
\end{array}$ & $0.00 \%$ \\
\hline CESAR HANNA HALUM & 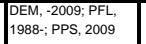 & то & $T_{0}$ & 10 & ${ }_{0}$ & 0 & $\sigma_{0}$ & $2011-2015$ & 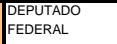 & Mestic Veterinatio & 177,36 & RS 160.000,00 & R8 $1.682 .501,29$ & $9,51 \%$ \\
\hline
\end{tabular}




\begin{tabular}{|c|c|c|c|c|c|c|c|c|c|c|c|c|c|c|}
\hline NOME & PARTIDO & UF & $\begin{array}{l}\text { GESTÃo } \\
\text { ANTERIOR }\end{array}$ & GESTÃol & GESTĀol & GESTÃô II & GESTÃon & $\begin{array}{l}\text { GESTÃo } \\
\text { POSTERIOR }\end{array}$ & ÚLTIMO CARGO & $\begin{array}{l}\begin{array}{l}\text { PROFISSÃ̃o } \\
\text { DECLARADA }\end{array} \\
\end{array}$ & $\begin{array}{c}\text { AREA DAS TERRAS } \\
(\mathrm{HA})\end{array}$ & $\begin{array}{c}\text { VALOR DAS TERRAS } \\
\text { RS }\end{array}$ & $\begin{array}{l}\text { VALOR DO } \\
\text { PATRIMOONOIO }\end{array}$ & $\begin{array}{c}\text { VALOR DAS } \\
\text { TERRASPPATRIMONIOIO }\end{array}$ \\
\hline JOSÉ MAURICIO RABELO & \begin{tabular}{|l} 
PDC, 1992-2001; \\
PSD, 2001-2003: \\
PTB, 2003; PL, 2003
\end{tabular} & то & 0 & o & o & 2003-2007 & o & 0 & \begin{tabular}{|} 
DEPUTADO \\
FEDERAL
\end{tabular} & $\begin{array}{l}\text { Radialista, Professor, } \\
\text { Empresário e } \\
\text { Lavrador }\end{array}$ & 73,00 & $180.000,00$ & R\$ $\$ 48.000,00$ & $40,18 \%$ \\
\hline $\begin{array}{l}\text { JOĀO BATISTA DE JESUS } \\
\text { RIBEIRO J }\end{array}$ & 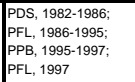 & то & 0 & |1995-1999 & |1999-2003 & 2003-2007 & 2007-2011 & & SENADOR & Empresário & $1.056,09$ & R\$ $201.203,00$ & $\mathrm{R} \$ 1.936 .579,75$ & $10,39 \%$ \\
\hline $\begin{array}{l}\text { MiRANDA (Cassado, nāo } \\
\text { assumiu) }\end{array}$ & PMDB & то & 0 & 0 & 0 & 0 & 0 & $2011-2015$ & SENADOR & Empresário & $\mathrm{NI}$ & R\$ $215.000,00$ & $\mathrm{R} \$ 2.170 .911,03$ & $9,90 \%$ \\
\hline LAZARO BOTELHO MARTINS & PP, 2003 & то & 0 & 0 & 0 & 0 & $2007-2011$ & $2011-2015$ & \begin{tabular}{|c|c|}
$\mid$ FEVUTADO \\
FEDERLL
\end{tabular} & \begin{tabular}{|l|l} 
Peceuarista e \\
Empresário
\end{tabular} & $2.129,90$ & $\operatorname{RS} 219.600,00$ & R\$ 672.819,38 & $32,64 \%$ \\
\hline $\begin{array}{l}\text { LEOMAR DE MELO } \\
\text { QUUNTANILHA }\end{array}$ & $\begin{array}{l}\text { PDC, 1993; PPR, } \\
1993\end{array}$ & то & $\begin{array}{l}\text { 1989-1991, 1991- } \\
\text { 1995 }\end{array}$ & |1995-1999 & |1999-2003 & 2003-2007 & $2007-2011$ & 0 & SENADOR & Bancário & $3.622,73+\mathrm{NI}$ & $\operatorname{Rs} 263.844,64$ & $\mathrm{R} \$ 1.936 .579,75$ & $13,62 \%$ \\
\hline KATIA REGINA DE ABREU & \begin{tabular}{|l} 
PPB, 1995-1998; \\
PLL, 1998; PSD \\
2011
\end{tabular} & то & 0 & o & $2000-2002$ & 2003-2007 & 2007-2011 & $2011-2015$ & SENADORA & \begin{tabular}{|l|} 
Empresária Rural e \\
Psicóloga
\end{tabular} & $2.485,11+2 \mathrm{NI}$ & $\mathrm{R} \$ 274.854,19$ & $\mathrm{R} \$ 437.182,19$ & $62,87 \%$ \\
\hline LAUREZ DA ROCHA MOREIRA & PSB, 2007 & то & 0 & o & o & 0 & 2007-2011 & $2011-2015$ & $\begin{array}{l}\text { DEPUTADO } \\
\text { FEDERAL }\end{array}$ & Advogado & 786,75 & RS 707.760,00 & $\mathrm{R} \$ 1.060 .834,32$ & $66,72 \%$ \\
\hline $\begin{array}{l}\text { JOSE DOS SANTTS FREIRE } \\
\text { JUNIOR }\end{array}$ & $\mid$ & то & $\left.\right|_{1995} ^{1989-1991 ; 1991-}$ & |1995-1999 & |1999-2003 & ]$_{0}$ & 2007-2011 & 0 & $\left.\right|_{\text {FEDERALL }} ^{\text {DEPUAD }}$ & $\mid$\begin{tabular}{|l} 
Agropecuararit \\
Economista
\end{tabular} & $1.145,40+\mathrm{NI}$ & R\$ $849.004,56$ & $\mathrm{R} \$ 3.460 .627,14$ & $24,53 \%$ \\
\hline $\begin{array}{l}\text { IRAJA SILVESTRE FLLO (RAJA } \\
\text { ABREU) }\end{array}$ & DEM, 2001 & то & 0 & 0 & 0 & 0 & 0 & $2011-2015$ & \begin{tabular}{|c|c|} 
DEPQTAAO \\
FEDERAL
\end{tabular} & $\begin{array}{l}\text { Empresátioe } \\
\text { Produtor Rural }\end{array}$ & 2NI & R\$ $1.007 .833,33$ & $\mathrm{R} \$ 1.386 .738,95$ & $72,68 \%$ \\
\hline RAAMUNDO COIMBRA JÚNIOR & $\begin{array}{l}\text { PMDB, } 2005 \text { - } \\
\text { PND, }\end{array}$ & то & 0 & 0 & 0 & 0 & o & $2011-2015$ & 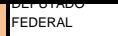 & \begin{tabular}{|l|l} 
Jornalista \\
\end{tabular} & $1.360,00$ & R\$ 2.000.000,00 & $\mathrm{R} \$ 3.076 .960,00$ & $65,00 \%$ \\
\hline VICENTE ALVES DE OLIVERA & $\mid \begin{array}{l}\text { PFL, 1979-2005; } \\
\text { PSDB, 2005-2007; }\end{array}$ & то & 0 & 0 & 0 & 0 & $2007-2011$ & 0 & SENADOR & $\begin{array}{l}\text { Empresastio } \\
\text { Comercial }\end{array}$ & $2.864+\mathrm{NI}$ & $\mathrm{R} \$ \quad 7.791 .120,00$ & $\mathrm{R} \$ 9.600 .717,68$ & $81,15 \%$ \\
\hline OSVALDO DE SOUZA REIS & $\begin{array}{l}\text { PTR, 1991-1993; } \\
\text { PP, 1993-1995; }\end{array}$ & то & |1991-1995 & |1995-1999 & |1999-2003 & 2003-2007 & $2007-2011$ & 0 & $\begin{array}{l}\text { DEPUTADO } \\
\text { FEDERAL }\end{array}$ & Empresário & $13.854,14$ & $\mathrm{R} \$ 10.320 .000,00$ & $\mathrm{R} \$ 22.737 .636,03$ & $45,39 \%$ \\
\hline AMARLLDO MARTINS DA SILVA & $\begin{array}{l}\text { PSB, 2003; PSC, } \\
\text { 2003-2005; PDB, }\end{array}$ & то & 0 & 10 & |1999-2003 & 2003-2007 & 0 & 0 & $\begin{array}{l}\text { DEPUTADO } \\
\text { FEDERAL }\end{array}$ & \begin{tabular}{|l} 
Empresário e éécn \\
em Contabilidade
\end{tabular} & 917,60 & $?$ & $?$ & \#VALOR! \\
\hline & & & & & & & & & & & & & & \\
\hline
\end{tabular}

\section{LEGENDA DA TABELA}

RURALISTAS QUE EXERCEM PROFISSÕES LIGADAS A ATIVIDADE AGROPECUÁRIA, OU AGRONEGÓCIO

RURALISTAS QUE PASSARAM A PERTENCER A BANCADA A PARTIR DE 2010, FORA DO PERÍODO DA ANÁLISE

PARLAMENTARES RURALISTAS FALECIDOS

PARLAMENTARES QUE TEM PARENTES NA POLITICA

BIOGRAFIAS INDISPONÍVEIS (2 DEPUTADOS)

RURALISTAS IDENTIFICADOS FORA DA LISTA DO DIAP 


\begin{tabular}{|c|c|c|c|c|c|c|c|c|c|c|c|c|}
\hline GEocodiaO & parlamentar & PARTIDO & FONTE & IMÓVEL & MUNICiPIO & uF & ÁREA & CATEGORIA FUNDIÁRIAA & ÁREA REGISTRADA & $\begin{array}{l}\text { AREA DECLARADA } \\
\text { COMO POSSE }\end{array}$ & $\begin{array}{l}\text { REGISTRO EM NOME DE } \\
\text { OUTRAS PESSOAS }\end{array}$ & $\begin{array}{l}\text { VincuLo como } \\
\text { PARLAMENTAR }\end{array}$ \\
\hline 120020 & $\begin{array}{l}\text { JOAO TOTA SOARES DE } \\
\text { FIGUEIRREDO }\end{array}$ & PPAC & INCRA 2003 & FAZENDA MORADA NOVA & CRUZEIRO DO SUL & $\overline{A C}$ & 3206 & $\begin{array}{l}\text { GRANDE PROPRIEDADE } \\
\text { PRODUTIVA }\end{array}$ & 3206 & 0 & & \\
\hline 120050 & MARCIO MIGUEL BITTAR & PSDBAC & INCRA 2003 & OLHO D AGUA & SENA MADUREIRA & $\mathrm{AC}$ & 1000 & $\begin{array}{l}\text { MÉDAA PROPRIEDADE } \\
\text { IMPRODUTIVA }\end{array}$ & 1000 & 0 & & \\
\hline 120080 & SERGIO SEBASTIAO DE BARROS & PMNAC & INCRA 2003 & FAZENDA SANTA TEREZINHA & PORTO ACRE & $\mathrm{AC}$ & 2012 & $\begin{array}{l}\text { GRANDE PROPRIIEDADE } \\
\text { PRODUTIVA }\end{array}$ & 2012 & 0 & & \\
\hline 120040 & SERGIO SEBASTIAO DE BARROS & PMNAC & INCRA 2003 & SITIO JARI & RIO BRANCO & $A C$ & 67 & MiNIFÚNDIO & 67 & 0 & & \\
\hline 270420 & $\begin{array}{l}\text { CARLOS ALBERTO MOREERA DE } \\
\text { MENDONĢA CANUTO }\end{array}$ & PMDB AL & INCRA 2003 & SITIO TIPI & LMOEERO DE ANADIA & AL & 19 & MINIFÚNDIO & 0 & 19 & $\begin{array}{l}\text { CARLOS ALBERTO MOREIRA } \\
\text { DE MENDONCA CANUTO }\end{array}$ & \\
\hline 270040 & $\begin{array}{l}\text { CARLOS ALBERTO MOREIRA DE } \\
\text { MENDONĢA CANUTO }\end{array}$ & PMDB AL & INCRA 2003 & FAZENDA SAO PAULO & ATALAIA & AL & 116 & $\begin{array}{l}\text { MÉDIA PROPRIEDADE } \\
\text { PRODUTIVA }\end{array}$ & 116 & 0 & $\begin{array}{l}\text { CAALOS ALBERTO MOREERA } \\
\text { DE MENDONCA CANUTO }\end{array}$ & \\
\hline 270040 & $\begin{array}{l}\text { CARLOS ALBERTO MOREERA DE } \\
\text { MENDONĢA CANUTO }\end{array}$ & PMDB AL & INCRA 2003 & FAZENDA SAO ROQUEI & ATALAIA & AL & 126 & $\begin{array}{l}\text { MÉDDAA PROPRIEDADE } \\
\text { PRODUTIVA }\end{array}$ & 126 & 0 & $\begin{array}{l}\text { CAALOS ALBERTO MOREIRA } \\
\text { DE MENDONCA CANUTO }\end{array}$ & \\
\hline 270690 & $\begin{array}{l}\text { CARLOS ALEERTO MOREIRA DE } \\
\text { MENDONÇA CANUTO }\end{array}$ & PMDB AL & INCRA 2003 & FAZENDA SANTO ANTONIO & PLLAR & AL & 150 & $\begin{array}{l}\text { MÉDA PROPRIEDADE } \\
\text { PRODUTIVA }\end{array}$ & 150 & 0 & $\begin{array}{l}\text { CARLOS ALEERTO MOREIRA } \\
\text { DE MENDONCA CANUTO }\end{array}$ & \\
\hline 270040 & $\begin{array}{l}\text { CARLOS ALBERTT MOREERA DE } \\
\text { MENDONĢA CANUTO }\end{array}$ & PMDB AL & INCRA 2003 & FAZENDA FLOR DO PARABBA & ATALAIA & AL & 168 & $\begin{array}{c}\text { MÉDIA PROOPRIEDADE } \\
\text { PRODUTIVA }\end{array}$ & 168 & 0 & $\begin{array}{l}\text { CARLOS ALBERTO MOREIRA } \\
\text { DE MENDONCA CANUTO }\end{array}$ & \\
\hline 270690 & $\begin{array}{l}\text { CARLOS ALBERTO MOREIRA DE } \\
\text { MENDONĢA CANUTO }\end{array}$ & PMDB AL & INCRA 2003 & FAZENDA SANTA TEREZINHA II & PLLAR & AL & 217 & $\begin{array}{l}\text { GRANDE PROPRIEDADE } \\
\text { PRODUTIVA }\end{array}$ & 217 & 0 & $\begin{array}{l}\text { CARLOS ALBERTO MOREIRA } \\
\text { DE MENDONCA CANUTO }\end{array}$ & \\
\hline 270420 & $\begin{array}{l}\text { CARLOS ALBERTO MOREERA DE } \\
\text { MENDONĢA CANUTO }\end{array}$ & PMDB AL & INCRA 2003 & FAZ NOSSA SENHORA APARECIDA & LIMOEIRO DE ANADIA & AL & 218 & $\begin{array}{l}\text { MÉDIA PROPRIEDADE } \\
\text { PRODUTIVA }\end{array}$ & 218 & 0 & $\begin{array}{l}\text { CARLOS ALBERTO MOREIRA } \\
\text { DE MENDONCA CANUTO }\end{array}$ & \\
\hline 270430 & JOAO JOSE PERERAR DE LYRA & PтB AL & INCRA 2003 & SITIO SAO GONCALO & MACEIÓ & AL & 13 & PEQUENA PROPRIEDADE & 13 & 0 & & \\
\hline 270230 & JOAO JOSE PERERAR DE LYRA & PTB AL & INCRA 2003 & FAZENDA GUAXUMA & CORURIPE & AL & 30 & PEQUENA PROPRIEDADE & 30 & 0 & & \\
\hline 270430 & JOAO JOSE PEREIRA DE LYRA & PTBAL & INCRA 2003 & SITIO SAO GONCALO & MACEIÓ & AL & 39 & $\begin{array}{l}\text { MÉDIA PROPRIEDADE } \\
\text { IMPRODUTIVA }\end{array}$ & 39 & 0 & & \\
\hline 270930 & JOAO JOSE PEREIRA DE LYRA & PTB AL & INCRA 2003 & FAZENDEA SANTO INACIO & UNIĪOO DOS PALMARES & $\mathrm{AL}$ & 90 & $\begin{array}{l}\text { MÉDIA PROPRIEDADE } \\
\text { PRODUTIVA }\end{array}$ & 90 & 0 & & \\
\hline 270680 & $\begin{array}{l}\text { JoSE THOMAZ DA SLLVA NONO } \\
\text { NETO }\end{array}$ & DEM AL & INCRA 2003 & SITIO MARACUJA & PIAÇABUÇU & $\overline{\mathrm{AL}}$ & 5 & MINIFÚNDIO & 5 & 0 & & \\
\hline 270230 & $\begin{array}{l}\text { JOSE THOMAZ DA SLLVA NONO } \\
\text { NETO }\end{array}$ & DEM AL & INCRA 2003 & BALANCA & CORURIPE & AL & 8 & MINIFÚNDIO & 8 & 0 & & \\
\hline 270230 & $\begin{array}{l}\text { JOSE THOMAZ DA SLLVA NONO } \\
\text { NETO }\end{array}$ & DEM AL & INCRA 2003 & BALANCA & CORURIPE & AL & 21 & MINIFÚNDIO & 19 & 0 & & \\
\hline 270230 & $\begin{array}{l}\text { JoSE THOMAZ DA SILVA NONO } \\
\text { NETO }\end{array}$ & DEM AL & INCRA 2003 & BANANNEIRAS & CORURIPE & $\mathrm{AL}$ & 30 & PEQUENA PROPRIEDADE & 30 & 0 & & \\
\hline 270230 & $\begin{array}{l}\text { JoSE THOMAZ DA SILVA NONO } \\
\text { NETO }\end{array}$ & DEM AL & INCRA 2003 & FAZENDA VER O MAR & CORURIPE & AL & 39 & PEQUENA PROPRIEDADE & 39 & 0 & & \\
\hline 270230 & $\begin{array}{l}\text { JOSE THOMAZ DA SLLVA NONO } \\
\text { NETO }\end{array}$ & DEM AL & INCRA 2003 & BANANERAS & CORURIPE & AL & 44 & PEQUENA PROPRIEDADE & 44 & 0 & & \\
\hline 270230 & $\begin{array}{l}\text { JOSE THOMAZ DA SLLVA NONO } \\
\text { NETO }\end{array}$ & DEMAL & INCRA 2003 & FAZENDA BALANCA & CORURIPE & AL & 119 & PEQUENA PROPRIEDADE & 119 & 0 & & \\
\hline 270090 & $\begin{array}{l}\text { JoSE THOMAZ DA SILVA NONO } \\
\text { NETO }\end{array}$ & DEMAL & INCRA 2003 & SERRA DAS PORTEIRAS & BELO MONTE & AL & 441 & $\begin{array}{l}\text { MÉDIA PROPRIEDADE } \\
\text { IMPRODUTIVA }\end{array}$ & ${ }_{441}$ & 0 & & \\
\hline 270230 & $\begin{array}{l}\text { JoSE THOMAZ DA SILVA NONO } \\
\text { NeTO }\end{array}$ & DEMAL & INCRA 2003 & FAZENDA BALANCA IX & CORURIPE & AL & 16 & MINIFÚNDIO & 16 & 0 & & \\
\hline 270550 & OLAVO CALHEIROS FILHO & PMDB AL & INCRA 2003 & FAZ CAPOEERAO & MURICI & $\overline{\mathrm{AL}}$ & 350 & $\begin{array}{c}\text { GRANDE PROPRIEDADE } \\
\text { PRODUTIVA }\end{array}$ & 350 & 0 & & \\
\hline 270550 & OLAVO CALHEIROS FLLHO & PMDB AL & INCRA 2003 & BOA VISTA & MURIII & AL & 869 & $\begin{array}{l}\text { GRANDE PROPRIEDADE } \\
\text { PRODUTIVA }\end{array}$ & 500 & 0 & & \\
\hline
\end{tabular}




\begin{tabular}{|c|c|c|c|c|c|c|c|c|c|c|c|c|}
\hline GEOCODIGO & PARLAMENTAR & PARTIDO & FONTE & IMÓvEL & MUNICIPPO & $\mathrm{uF}$ & AREA & CATEGORIA FUNDIÁRIA & AREA REGISTRADA & $\begin{array}{l}\text { AREA DECLARADA } \\
\text { COMO POSSE }\end{array}$ & $\begin{array}{l}\text { REGISTRO EM NOME DE } \\
\text { OUTRAS PESSOAS }\end{array}$ & $\begin{array}{l}\text { vinCULO COMO } \\
\text { PARLAMENTAR }\end{array}$ \\
\hline 270870 & $\begin{array}{l}\text { TEOTONIO BRANDÃO VILELA } \\
\text { FLHO }\end{array}$ & PSDB AL & INCRA 2003 & SITIO PRAIA DE SAO MIGUEL & SÃo MIGUEL DOS MLLAGRES & $\mathrm{AL}$ & 34 & PEQUENA PROPRIEDADE & 0 & 0 & & \\
\hline 292580 & AROLDO CEDRAZ DE OLVEIRA & PFLBA & INCRA 2003 & FAZ VARGEM DA PEDDA & QUEIMADAS & $\mathrm{BA}$ & 4 & PEQUENA PROPRIEDADE & 0 & 4 & 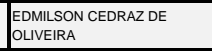 & \begin{tabular}{|l} 
PARENTESCO NÄO \\
IDENTIFICADO
\end{tabular} \\
\hline 290840 & AROLDO CEDRAZ DE OLVEIRA & PFLBA & INCRA 2003 & FAZENDA LARANJEIRA & CONGEIÇÃO DO COITÉ & BA & 7 & MINIFÚNDIO & 7 & 0 & 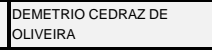 & \\
\hline 290840 & AROLDO CEDRAZ DE OLVEIRA & PFLBA & INCRA 2003 & FAZENDA RIACHO DO MARTINS & CONCEIC̣ĀO DO COITÉ & $\mathrm{BA}$ & 7 & MINIFÚNDIIO & 7 & 7 & $\begin{array}{l}\text { DEMETRIO CEDRAZ DE } \\
\text { oulvEIRA }\end{array}$ & $\begin{array}{l}\text { PARENTECO NäO } \\
\text { IDENTIFICADO }\end{array}$ \\
\hline 270350 & AROLDO CEDRAZ DE OLVEIRA & PFL BA & INCRA 2003 & FAZENDA MACAMBBRA & JACUPE & BA & 10 & MINIFÚNDIIO & 0 & 10 & MATILDES CEDRAZ OLIVEIRA & $\begin{array}{l}\text { PARENTESCO NÃO } \\
\text { IDENTIFICADO }\end{array}$ \\
\hline 292580 & AROLDO CEDRAZ DE OLVEIRA & PFLBA & INCRA 2003 & ROCA PAU D ARCO & QUEIMADAS & BA & 11 & $\begin{array}{l}\text { PEQQUENA PROPRIEDADE } \\
\end{array}$ & 0 & 11 & 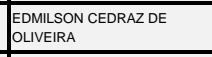 & $\begin{array}{l}\text { PARENTESCON NäO } \\
\text { IDENTIFICADO }\end{array}$ \\
\hline 290840 & AROLDO CEDRAZ DE OLVEIRA & PFLBA & INCRA 2003 & PINDOBEIRA & CONGEIÇĀO DO COITÉ & $\mathrm{BA}$ & 20 & MINIFÚNDIIO & 20 & 0 & RAIMUNDO CEDRAZ OLIVEIRA & \begin{tabular}{|l} 
PAEENTESCO NÄO \\
IDENTIFICADO
\end{tabular} \\
\hline 292120 & AROLDO CEDRAZ DE OLIVIRA & PFLBA & INCRA 2003 & ENGENHO VELHO & MIGUEL CALMON & BA & 25 & PEQUENA PROPRIIEDADE & 8 & 16 & $\begin{array}{l}\text { JOSELITO CEDRAZ DE } \\
\text { OLIVEIRA }\end{array}$ & $\begin{array}{l}\text { PARENTESCO NÃO } \\
\text { IDENTIIICADO }\end{array}$ \\
\hline 291080 & AROLDO CEDRAZ DE OLIVERA & PFLBA & INCRA 2003 & FAZENDA SACO DO CAPITAO & FEIRA DE SANTANA & $\overline{B A}$ & 26 & MINIFÚNDIO & 0 & 26 & JASMO CEDRAZ DE OLIVEIRA & $\begin{array}{l}\text { PARENTESCO NÃOO } \\
\text { IDENTIIIICADO }\end{array}$ \\
\hline 292580 & AROLDO CEDRAZ DE OLIVERA & PFLBA & INCRA 2003 & FAZ CEDRAZ & QUEIMADAS & $\mathrm{BA}$ & 27 & PEQUENA PROPRIIEDADE & 0 & 27 & $\begin{array}{l}\begin{array}{l}\text { EDMLSON CEDRAZ DE } \\
\text { OOLVIIRA }\end{array} \\
\end{array}$ & $\begin{array}{l}\text { PARENTESCO NÃO } \\
\text { IDENTIFICADO }\end{array}$ \\
\hline 292580 & AROLDO CEDRAZ DE OLIVEIRA & PFL BA & INCRA 2003 & FAZENDA DE CIMA & QUEIMADAS & BA & 27 & MINIFÚNDIO & 27 & 0 & $\begin{array}{l}\text { MARIA MADALENA CEDRAZ } \\
\text { DE OLVEIRA }\end{array}$ & $\begin{array}{l}\text { PARENTESCO NÃO } \\
\text { IDENTIFICADO }\end{array}$ \\
\hline 290840 & AROLDO CEDRAZ DE OLIVEIRA & PFLBA & INCRA 2003 & FAZENDA BELA VISTA & CONCEIÇĀO DO COITÉ & BA & 38 & MINIFÚNDIO & 38 & 0 & ALBINO CEDRAZ DE OLIVEIRA & $\begin{array}{l}\text { PARENTESCO NÃO } \\
\text { IDENTIFICADO }\end{array}$ \\
\hline 290840 & AROLDO CEDRAZ DE OLIVEIRA & PFLBA & INCRA 2003 & FAZENDA DOIS IRMAOS & CONG̣EICYÃO DO COITÉ & $\mathrm{BA}$ & 52 & PEQUENA PROPRIEDADE & 52 & 0 & $\begin{array}{l}\text { DEMETRO CEDRAZ DE } \\
\text { ouIVEIRA: }\end{array}$ & $\begin{array}{l}\text { PARENTECO NÄO } \\
\text { IDENTIFICADO }\end{array}$ \\
\hline 292010 & AROLDO CEDRAZ DE OLVEIRA & PFLBA & INCRA 2003 & FAZENDA FLORESTA & MAIRI & BA & 66 & PEQUENA PROPRIEDADE & 66 & 0 & $\begin{array}{l}\text { UBALDINO CEDRAZ DE } \\
\text { oLIVIVIRA: }\end{array}$ & $\begin{array}{l}\text { PARENTESCO NÃOO } \\
\text { IDENTIFICADO }\end{array}$ \\
\hline 292120 & AROLDO CEDAAZ DE OLIVEIRA & PFL BA & INCRA 2003 & PEIXE & MIGUEL CALMON & BA & 73 & PEQUENA PROPRIIEDADE & 23 & 50 & $\begin{array}{l}\text { JoSELITO CEDRAZ DE } \\
\text { joLvERAA }\end{array}$ & $\begin{array}{l}\text { PARENTESCO NÃOO } \\
\text { IDENTIFICADO }\end{array}$ \\
\hline 292630 & AROLDO CEDRAZ DE OLIVEIRA & PFLBA & INCRA 2003 & FAZ PANORAMA DA QUIXABEIRA GRANDE & RIACHĀO DO JACUIPE & BA & 86 & $\begin{array}{l}\text { PEQUENA PROPRIEDADE } \\
\end{array}$ & 0 & 86 & $\begin{array}{l}\text { ANTONIO CEDRAZ DE } \\
\text { OLIVEEAR }\end{array}$ & $\begin{array}{l}\text { PARENTESCON NäO } \\
\text { IDENTIFICADO }\end{array}$ \\
\hline 293300 & AROLDO CEDRAZ DE OLVEIRA & PFLBA & INCRA 2003 & FAZENDA ALTO BONITO & VALENTE & $\mathrm{BA}$ & 87 & PEQUENA PROPRIEDADE & 87 & 0 & & \\
\hline 292580 & AROLDO CEDRAZ DE OLVEIRA & PFLBA & INCRA 2003 & FAZENDA MARIA & QUEIMADAS & BA & 132 & PEQUENA PROPRIEDADE & 132 & 0 & $\begin{array}{l}\text { ZENOBIO CEDRAZ DE } \\
\text { OLIVEIRA }\end{array}$ & 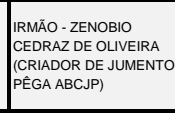 \\
\hline 291650 & AROLDO CEDRAZ DE OLVEIRA & PFLBA & INCRA 2003 & FAZENDA Vo EMLIA & ITAPICURU & BA & 178 & $\begin{array}{l}\text { MÉDDA PROPRIEDADE } \\
\text { IMPROOUTIVA }\end{array}$ & 178 & 0 & ZENÓBIO CEDRAZ OLVEIRA & 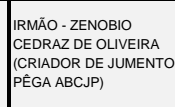 \\
\hline 292580 & AROLDO CEDRAZ DE OLVEIRA & PFLBA & INCRA 2003 & FAZENDA LAGOA DO BOI & QUEIMADAS & BA & 220 & $\begin{array}{c}\text { MÉEDAA PROPRIEDADE } \\
\text { IMPROOUTIVA } \\
\end{array}$ & 220 & 0 & $\begin{array}{l}\begin{array}{l}\text { EDUARDO CDRRZ DE } \\
\text { oLIVERA }\end{array} \\
\end{array}$ & \begin{tabular}{|l} 
RMMäO- EDUARDO \\
CEDRAZ DE OLIVERA
\end{tabular} \\
\hline 291700 & AROLDO CEDRAZ DE OLVEIRA & PFLBA & INCRA 2003 & FAZENDA ZE JOAO & IтÚBA & BA & 394 & $\begin{array}{l}\text { MÉDIA PROPRIEDADE } \\
\text { IMPROOUTIVA }\end{array}$ & 394 & 0 & ZENOBIO CEDRAZ OLVEIRA & 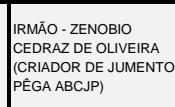 \\
\hline 292580 & AROLDO CEDRAZ DE OLIVEIRA & PFLBA & INCRA 2003 & FAZENDA FORTUNA & QUEIMADAS & BA & 435 & $\begin{array}{l}\text { MÉEDA PROPRIEDADE } \\
\text { IMPROOUTIVA } \\
\end{array}$ & 440 & 0 & & \\
\hline 292800 & AROLDO CEDRAZ DE OLVEIRA & PFLBA & INCRA 2003 & FAZENDA VARGEM GRANDE & SANTALUZ & $\mathrm{BA}$ & 582 & $\begin{array}{c}\text { MÉDIA PROPRIEDADE } \\
\text { IMPRODUTIVA } \\
\end{array}$ & 582 & 0 & GIILO CEDRAZ DE OLIVEIRA & $\begin{array}{l}\text { PARENTECO NÄO } \\
\text { IDENTIFICADO }\end{array}$ \\
\hline 292580 & AROLDO CEDRAZ DE OLIVEIRA & PFLBA & INCRA 2003 & FAZENDA VARZEA DA PEDRA & QUEIMADAS & BA & 611 & $\begin{array}{c}\text { MÉDIA PROPRIEDADE } \\
\text { IMPROOUTIVA }\end{array}$ & 611 & 0 & GILDO CEDRAZ DE OLIVEIRA & $\begin{array}{l}\text { PARENTECO NÄO } \\
\text { IDENTIFICADO }\end{array}$ \\
\hline 292800 & AROLDO CEDRAZ DE OLIVEIRA & PFL BA & INCRA 2003 & FAZENDA CAMPO FORMOSO & SANTALUZ & BA & 805 & $\begin{array}{c}\text { GRANDE PROPRIIDADE } \\
\text { IMPRODUTIVA }\end{array}$ & 805 & 0 & $\begin{array}{l}\text { EDUARDO CEDRAZ DE } \\
\text { oLIVIVIRA }\end{array}$ & 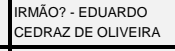 \\
\hline 292800 & AROLDO CEDRAZ DE OLVEIFA & PFL BA & INCRA 2003 & FAZENDA CAMPO FORMOSO & SANTALUZ & $\mathrm{BA}$ & 805 & $\begin{array}{c}\text { GRANDE PROPRIIDADAE } \\
\text { IMPRODUTIVA }\end{array}$ & 805 & 0 & \begin{tabular}{|l} 
EDUARDO CEDRAZ DE \\
oLIVIRA
\end{tabular} & $\begin{array}{l}\text { IRMAOOO? EDUARDO } \\
\text { CEDRAZ DE OLVEIIRA }\end{array}$ \\
\hline
\end{tabular}




\begin{tabular}{|c|c|c|c|c|c|c|c|c|c|c|c|c|}
\hline GEOCODIGO & PARLAMENTAR & PARTIDO & FONTE & IMÓVEL & MUNICIPIO & uF & AREA & CATEGORIA FUNDIÁRIA & ÁREA REGISTRADA & $\begin{array}{l}\text { AंREA DECLARADA } \\
\text { COMO POSSE }\end{array}$ & $\begin{array}{l}\text { REGISTRO EM NOME DE } \\
\text { OUTRAS PESSOAS }\end{array}$ & $\begin{array}{l}\text { vincuLo com } 0 \\
\text { PARLAMENTAR }\end{array}$ \\
\hline 292800 & AROLDO CEDRAZ DE OLVEEIRA & PFL BA & INCRA 2003 & FAZENDA QUIXABA & SANTALUZ & BA & 816 & $\begin{array}{l}\text { GRANDE PROPRIEDADE } \\
\text { IMPRODUTIVA }\end{array}$ & 816 & 0 & \begin{tabular}{|l} 
ZENOBIO CEDRAZ DE \\
OLIVERA
\end{tabular} & 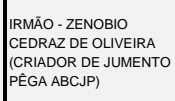 \\
\hline 292580 & AROLDO CEDRAZ DE OLVEIIRA & PFL BA & INCRA 2003 & FAZENDA NOCA & QUEIMADAS & BA & 843 & $\begin{array}{l}\text { GRANDE PROPRIEDADE } \\
\text { IMPRODUTIVA }\end{array}$ & 843 & 0 & $\begin{array}{l}\text { ZENOBIO CEDAAZ DE } \\
\text { ouvVIRA }\end{array}$ & 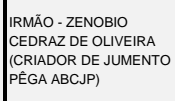 \\
\hline 290840 & AROLDO CEDRAZ DE OLVVEIRA & PFLBA & INCRA 2003 & FAZENDA PEDRA VERMELHA & CONCEIÇÃO DO COITÉ & BA & 924 & $\begin{array}{l}\text { GRANDE PROPRIEDADE } \\
\text { IMPRODUTIVA }\end{array}$ & 924 & 0 & $\begin{array}{l}\text { ZENOBIO CEDRAZ DE } \\
\text { OLIVEIRA }\end{array}$ & 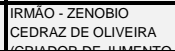 \\
\hline 290680 & AROLDO CEDRAZ DE OLVEERA & PFLBA & INCRA 2003 & FAZENDA CAMPO DO GATO & CANSANĢĀo & BA & 1089 & $\begin{array}{l}\text { GRANDE PROPRIEDADE } \\
\text { IMPRODUTIVA }\end{array}$ & 551 & 537 & $\begin{array}{l}\text { EDUARDO CEDRAZ DE } \\
\text { OLIVERA' }\end{array}$ & $\begin{array}{l}\text { IRMAOOP- EDUARDO } \\
\text { CEDRAZ DE OLVEERA }\end{array}$ \\
\hline 291080 & $\begin{array}{l}\text { COLBERT MARTINS DA SLIVA } \\
\text { FLLHO }\end{array}$ & PPS BA & INCRA 2003 & FAZENDA AMPARO & FEIRA DE SANTANA & BA & 21 & MMINIÚNDIO & 21 & 0 & COLBERT MARTINS DA SLIVA & $\begin{array}{l}\text { PAI. COLBERT MARTINS } \\
\text { DA SLIVA FLHO }\end{array}$ \\
\hline 291080 & $\begin{array}{l}\text { COLBERT MARTINS DA SILVA } \\
\text { FLLHO }\end{array}$ & PPS BA & INCRA 2003 & FAZENDA LAGOA DO MEIO & FEIRA DE SANTANA & BA & 44 & PEQUENA PROPRIEDADE & 44 & 0 & COLBERT MARTINS DA SILVA & $\begin{array}{l}\text { PAI- COLBERT MARTINS } \\
\text { DA SILVA FLHO }\end{array}$ \\
\hline 291080 & $\begin{array}{l}\text { COLBERT MARTINS DA SILVA } \\
\text { FILHO }\end{array}$ & PPS BA & INCRA 2003 & FAZENDA CEDRO II & FEIRA DE SANTANA & BA & 47 & PEQUENA PROPRIIEDADE & 47 & 0 & COLBERT MARTINS DA SLVVA & $\begin{array}{l}\text { PAI. COLBERT MARTINS } \\
\text { DA SLIVA FLHO }\end{array}$ \\
\hline 291080 & $\begin{array}{l}\text { COLBERTT MARTINS DA SILVA } \\
\text { FLLHO }\end{array}$ & PPS BA & INCRA 2003 & FAZENDA CEDROI & FEIRA DE SANTANA & BA & 120 & PEQUENA PROPRIEDADE & 120 & 0 & COLBERT MARTINS DA SLIVA & $\begin{array}{l}\text { PAI-COLBERT MARTINS } \\
\text { DA SLIVA FLHO }\end{array}$ \\
\hline 291080 & $\begin{array}{l}\text { COLBERT MARTINS DA SILVA } \\
\text { FLLHO }\end{array}$ & PPS BA & INCRA 2003 & FAZENDA BARRA & FEIRA DE SANTANA & BA & 143 & $\begin{array}{l}\text { MÉDIA PROPRIEDADE } \\
\text { IMPROOUTIVA }\end{array}$ & 143 & 0 & COLBERT MARTINS DA SIVVA & $\begin{array}{l}\text { PAI. COLBERT MARTTNS } \\
\text { DA SLIVA FLLOO }\end{array}$ \\
\hline 29280 & $\begin{array}{l}\text { COLBERT MARTINS DA SILVA } \\
\text { FLLHO }\end{array}$ & PPS BA & INCRA 2003 & FAZENDA SAO JOSE & NOVA ITARANA & BA & 410 & $\begin{array}{l}\text { MÉDIA PROPRIEDADE } \\
\text { IMPRODUTIVA }\end{array}$ & 410 & 0 & COLBERT MARTINS DA SLIVA & $\begin{array}{l}\text { PAI. COLBERT MARTTNS } \\
\text { DA SLIVA FLHO }\end{array}$ \\
\hline 290630 & FABIO LOUREIRO SOUTO & DEMBA & INCRA 2003 & FAZENDA LAGOS & Canavieiras & BA & 0 & PEQUENA PROPRIEDADE & 0 & 0 & paulo ganem Souto & $\begin{array}{l}\begin{array}{l}\text { PAI DE FABIO LOUREIRO } \\
\text { souto }\end{array} \\
\end{array}$ \\
\hline 290630 & FABIO LOUREIRO SOUTO & DEMBA & INCRA 2003 & FAZENDA MONGOOOS ॥ & canavieiras & $\mathrm{BA}$ & 3 & MIIIFÚNDIO & 3 & 0 & Paulo genem Souto & $\begin{array}{l}\begin{array}{l}\text { PAI DE FABIO LOUREIRO } \\
\text { souTo }\end{array} \\
\end{array}$ \\
\hline 290630 & FABIO LOUREIRO SOUTO & DEM BA & INCRA 2003 & FAZENDA MONGOOOS I & CANAVIEIRAS & BA & 13 & MINIFÚNDIO & 13 & 0 & Paulo ganem souto & $\begin{array}{l}\begin{array}{l}\text { PAI DE FABIO LOUREIRO } \\
\text { SOUTO }\end{array} \\
\end{array}$ \\
\hline 290630 & FABIO LOUREIRO SOUTO & DEM BA & INCRA 2003 & FAZ ESPLANADA & Canavieiras & BA & 60 & PEQUENA PROPRIEDADE & 60 & 0 & RODRIGO LOUREIRO SOUTO & \begin{tabular}{|l|} 
IRMÄO- FÁB̈IO \\
LOUREIRO SOUTO
\end{tabular} \\
\hline 292805 & FABIO LoUREIRO SOUTO & DEM BA & INCRA 2003 & FAZENDA REDENCAO & SANTA LUZIA & BA & 114 & $\begin{array}{l}\text { MÉDIA PROPRIEDADE } \\
\text { IMPROOUTIVA }\end{array}$ & 0 & 114 & $\begin{array}{l}\text { ZZABEL CAROLINA LOUREIRO } \\
\text { SOUTO }\end{array}$ & $\begin{array}{l}\text { FILAA-FABIO } \\
\text { LOUREIRO SOUTO }\end{array}$ \\
\hline 290630 & FABIO LOUREIRO SOUTO & DEM BA & INCRA 2003 & FAZ VISTA ALEGRE & CANAVIEIRAS & $\mathrm{BA}$ & 204 & $\begin{array}{l}\text { MÉDIA PROPPRIEDADE } \\
\text { PRRODUTIVA } \\
\end{array}$ & 204 & 0 & VITOR LOUREIRO SOUTO & \begin{tabular}{|l} 
IRMĀO- FÁB̈IO \\
LOUREIRO SOUTO \\
\end{tabular} \\
\hline 292805 & FABIO LOUREIRO SOUTO & DEM BA & INCRA 2003 & FAZENDA AGUAS CLARAS & SANTALUZIA & BA & 268 & $\begin{array}{l}\text { MÉDIA PROPRIEDADE } \\
\text { IMPRODUTIVA }\end{array}$ & 0 & 268 & $\begin{array}{l}\text { |ZABEL CAROLINA LOUREIRO } \\
\text { SOUTO }\end{array}$ & \begin{tabular}{|l}
$\begin{array}{l}\text { FLLHA-FABIO } \\
\text { LOUREIRO SOUTO }\end{array}$ \\
\end{tabular} \\
\hline 292805 & FABIO LOUREIRO SOUTO & DEM BA & INCRA 2003 & CONJUNTO SANTO ANTONIO & SANTA LUZIA & BA & 346 & $\begin{array}{l}\text { GRANDE PROPRIEDADE } \\
\text { PRODUTIVA }\end{array}$ & 346 & 0 & Paulo ganem Souto & 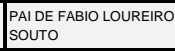 \\
\hline 291280 & FRANCISTOONIO ALVES PINTO & PMDB BA & INCRA 2003 & FAZ SENHOR DO BOMFIM & IBRAPUÁ & $\mathrm{BA}$ & 512 & $\begin{array}{l}\text { MÉDIA PROPPIEDADE } \\
\text { PROOUTIVA }\end{array}$ & 512 & 0 & & \\
\hline 291550 & GEDDEL QUADROS VIEIRA LIMA & РMDB ВA & INCRA 2003 & FAZENDA FELIZ VITORIA & ITAJuipe & BA & 25 & PEQUENA PROPRIEDADE & 0 & 25 & $\begin{array}{l}\text { AFRISIO DE SOUSA VIEIRA } \\
\text { LIMA }\end{array}$ & $\begin{array}{l}\text { PAI DE GEDDEL } \\
\text { OUADROS VEIRA LIMA }\end{array}$ \\
\hline 291550 & GEDDEL QUADROS VIEIRA LIMA & PMDB BA & INCRA 2003 & FAZENDA AMARALINA & itAuUUipe & BA & 41 & PEQUENA PROPRIEDADE & 41 & 0 & $\begin{array}{l}\text { AFRISIO DE SOUSA VIEIRA } \\
\text { LIMA }\end{array}$ & $\begin{array}{l}\text { PAIDE GEDDEL } \\
\text { QUADROS VEIRA LIMA }\end{array}$ \\
\hline 291360 & GEDDEL QUADROS VEIRA LIMA & РMDB ВA & INCRA 2003 & FAZENDA YPIRANGA BOA VISTA & LHEÉus & $\overline{B A}$ & 59 & PEQUENA PROPRIIEDADE & 59 & 0 & $\begin{array}{l}\text { AFRISIO DE SOUSA VIEIRA } \\
\text { LIMA }\end{array}$ & $\begin{array}{l}\text { PAIDE GEDDEL } \\
\text { QUADROS VEIRA LIMA }\end{array}$ \\
\hline 291230 & GEDDEL QUADROS VIEIRA LIMA & PMDB BA & INCRA 2003 & FAZENDA PANCADINHA & Biıcui & BA & 113 & PEQUENA PROPRIEDADE & 113 & 0 & $\begin{array}{l}\text { AFRISIO DE SOUSA VIEIRA } \\
\text { LIMA }\end{array}$ & $\begin{array}{l}\text { PAI DE GEDDEL } \\
\text { QUADROS VIERA LIMA }\end{array}$ \\
\hline 291230 & GEDDEL QUADROS VIEIRA LMA & PMDB BA & INCRA 2003 & FAZENDA RECREIO & IBICUI & BA & 172 & 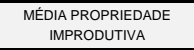 & 172 & 0 & & \\
\hline 291230 & GEDDEL QUADROS VIEIRA LMA & РМDB ВA & INCRA 2003 & FAZENDA BELA VISTA & isicui & BA & 220 & $\begin{array}{c}\text { MÉDIA PROPRIEDADE } \\
\text { IMPRODUTIVA }\end{array}$ & 220 & 0 & $\begin{array}{l}\text { AFRISIO DE SOUSA VIEIRA } \\
\text { LIMA }\end{array}$ & 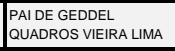 \\
\hline 291230 & GEDDEL QUADROS VIEIRA LIMA & РМDB ВA & INCRA 2003 & FAZENDA RIO BONITO & isicui & $\mathrm{BA}$ & 262 & $\begin{array}{c}\text { MÉDIA PROPRIEDADE } \\
\text { IMPROOUTIVA } \\
\text { The }\end{array}$ & 0 & 262 & & \\
\hline 291230 & GEDDEL QUADROS VIEIRA LMA & РMDB BA & INCRA 2003 & FAZENDA PANCADINHA & Biıcui & BA & 328 & $\begin{array}{l}\text { MÉDIA PROPRIEDADE } \\
\text { IMPRODUTIVA }\end{array}$ & 328 & 0 & & \\
\hline 291970 & GEDDEL QUADROS VIEIRA LIMA & РМDB BA & INCRA 2003 & FAZENDA VALE DO CARAIM & MACARANI & BA & 352 & 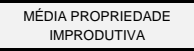 & 352 & 0 & $\begin{array}{l}\text { AFRISIO DE SOUSA VIEIRA } \\
\text { LIMA }\end{array}$ & $\begin{array}{l}\text { PAI DE GEDDEL } \\
\text { OUADROS VEIRA LIMA }\end{array}$ \\
\hline 291970 & GEDDEL QUADROS VIEIRA LIMA & PMDB BA & INCRA 2003 & FAZENDA TABAJARA & MACARANI & BA & 891 & $\begin{array}{l}\text { MÉDIA PROPPIEDADE } \\
\text { PROOUTIVA }\end{array}$ & 891 & 0 & $\begin{array}{l}\text { AFRISIO DE SOUZA VIEIRA } \\
\text { LIMA }\end{array}$ & $\begin{array}{l}\text { PAIDE GEDDEL } \\
\text { QuADROS VIERA LIMA }\end{array}$ \\
\hline
\end{tabular}




\begin{tabular}{|c|c|c|c|c|c|c|c|c|c|c|c|c|}
\hline GEOCODIGO & PARLAMENTAR & PARTIDO & FONTE & IMóveL & MUNICIPIO & UF & ÁREA & CATEGORIA FUNDIÁRIAA & AREA REGISTRADA & $\begin{array}{l}\text { AREA DECLARADA } \\
\text { COMO POSSE }\end{array}$ & $\begin{array}{l}\text { REGISTRO EM NOME DE } \\
\text { OUTRAS PESSOAS }\end{array}$ & $\begin{array}{l}\text { viNcuLO COMO O } \\
\text { PARLAMENTAR }\end{array}$ \\
\hline 290910 & GEDDEL QUADROS VIEIRA LIMA & PMDB BA & INCRA 2003 & FAZENDA VARGEM GRANDE DIAMANTE & CORIBE & $\mathrm{BA}$ & 1989 & $\begin{array}{c}\text { GRANDE PROPRIEDADE } \\
\text { IMPRODUTIVA }\end{array}$ & 1989 & 0 & $\begin{array}{l}\text { AFRIIIIO DE SOUSA VIEIRA } \\
\text { LIMA }\end{array}$ & $\begin{array}{l}\text { PAI DE GEDDEL } \\
\text { QUADROS VIEIRA LIMA }\end{array}$ \\
\hline 291560 & JaIME FERNANDES FLLHO & PFLBA & INCRA 2003 & FAZENDA SANTO ONOFRE & itamaraju & $\mathrm{BA}$ & 187 & $\begin{array}{c}\text { MÉDIA PROPRIEDADE } \\
\text { IMPROOUTIVA }\end{array}$ & 0 & 187 & & \\
\hline 291560 & JAIME FERNANDES FLLHO & PFL BA & INCRA 2003 & FAZENDA GOIANA SAO PEDRO & itamarauU & BA & 918 & $\begin{array}{c}\text { GRANDE PROPRIIDADE } \\
\text { PRODUTIVA }\end{array}$ & 974 & 0 & & \\
\hline 291530 & JAMME FERNANDES FLLHO & PFLBA & INCRA 2003 & FAZENDA ALANCA & ITAGIMIRIM & BA & 1917 & $\begin{array}{l}\text { GRANDE PROPRIEDADE } \\
\text { PRODUTIVA }\end{array}$ & 1917 & 0 & JAIME MACIEL FERNANDES & \begin{tabular}{|l|} 
JaAME MACIEL \\
FIRNANDES - JAIME \\
FERNANDES FILHO \\
DELAROOU AZENDAA \\
ALIANÇA EM ITAGIMIRIM
\end{tabular} \\
\hline 291530 & JAIME FERNANDES FLLHO & PFLBA & INCRA 2003 & FAZENDA COLUMBIA & ITAGIMIRIM & BA & 2304 & $\begin{array}{l}\text { GRANDE PROPRIEDADE } \\
\text { PRODUTVA }\end{array}$ & 2304 & 0 & $\begin{array}{l}\begin{array}{l}\text { BABBARA FERNANDES } \\
\text { SUAREZ }\end{array} \\
\text { SER }\end{array}$ & \begin{tabular}{|l|} 
BARBARA FERNANDES \\
SUAREZ - - SAME \\
FERNANDES DECLAROU \\
FAAENDACOLUMBA EM \\
ITAGIMIRIM
\end{tabular} \\
\hline 292420 & JOÃO BOSCO DA COSTA & PSDB SE & INCRA 2003 & FAZENDA BELA VISTA & PEDRO ALEXANDRE & $\mathrm{BA}$ & 169 & PEQUENA PROPRIEDADE & 121 & 48 & JOAO BOSCO DA COSTA & \\
\hline 292420 & JOĀO BOSCO DA COSTA & PSDB SE & INCRA 2003 & FAZENDA BELA VISTA & PEDRO ALEXANDRE & BA & 211 & PEQUENA PROPRIEDADE & 121 & 0 & JOAO BOSCO DA COSTA & \begin{tabular}{|l|} 
JOAO BOSCO DA COSTA \\
DECLAROU TERRAS \\
NESTE MUNIIPIO
\end{tabular} \\
\hline 291050 & JOAO CARLOS PAOLLLO BACELAR & PLBA & INCRA 2003 & LOTE 77 & ENTRE RIOS & $\mathrm{BA}$ & 24 & MINIF́UNDIO & 24 & 0 & $\begin{array}{l}\text { JoAQUUM RUYY PAULLLO } \\
\text { BACELAR }\end{array}$ & \\
\hline 240510 & JOAO CARLOS PAOLLLO BACELAR & PLBA & INCRA 2003 & FAZ LAGOA ESCURA & JANDAira & $\mathrm{BA}$ & 50 & PEQUENA PROPRIEDADE & 0 & 50 & \begin{tabular}{|l} 
JOAQUUM RUI PAOLLOO \\
BACELAR
\end{tabular} & \\
\hline 291050 & JOAO CARLOS PAOLLLO BACELAR & PLBA & INCRA 2003 & RETIRO & ENTRE RIOS & $\mathrm{BA}$ & 70 & PEQUENA PROPRIEDADE & 70 & 0 & $\begin{array}{l}\text { JOAOCARLOS PAOLLO } \\
\text { BACELAR S }\end{array}$ & \\
\hline 240510 & JOAO CARLOS PAOLLO BACELAR & PLBA & INCRA 2003 & BIRIBEIRA & JANDAirA & BA & 176 & $\begin{array}{c}\text { MÉDIA PROPRIEDADE } \\
\text { IMPROOUTIVA } \\
\end{array}$ & 0 & 176 & $\begin{array}{l}\text { JJAQUUIM RUI PAOLLOO } \\
\text { BACELAR }\end{array}$ & \\
\hline 291050 & JOAO CARLOS PAOLLO BACELAR & PLBA & INCRA 2003 & FAZENDA BAIXAO & ENTRE RIOS & $\mathrm{BA}$ & 200 & 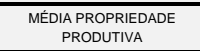 & 0 & 0 & $\begin{array}{l}\text { TOMAZ ALAARA BACELAR } \\
\text { ALMEIIAA }\end{array}$ & \\
\hline 291050 & JOAO CARLOS PAOLLO BACELAR & PLBA & INCRA 2003 & SAO JOAO & ENTRE RIOS & BA & 200 & $\begin{array}{l}\text { MÉDIA PROPRIEDADE } \\
\text { IMPROOUTIVA }\end{array}$ & 200 & 0 & $\begin{array}{l}\text { JOAOCAARLOS PAOLLOO } \\
\text { BACELAR }\end{array}$ & \\
\hline 240510 & JOAO CARLOS PAOLLOO BACELAR & PLBA & INCRA 2003 & FAZENDA ANGRILA & Jandaira & $\mathrm{BA}$ & 363 & $\begin{array}{l}\text { MÉDIA PROPRIEDADE } \\
\text { IMPROOUTIVA }\end{array}$ & 363 & 0 & $\begin{array}{l}\text { JoAQUIM RUY PAULLLO } \\
\text { BACELAR }\end{array}$ & \\
\hline 291050 & JoAO CARLOS PAOLLO BACELAR & PLBA & INCRA 2003 & FDAZENDA SOCORRO & ENTRE RIOS & $\mathrm{BA}$ & 700 & $\begin{array}{c}\text { GRANDE PROPRIEDADE } \\
\text { IMPRODUTIVA }\end{array}$ & 646 & 53 & $\begin{array}{l}\text { JOAQUIM RUY PAUULLO } \\
\text { BACELAR }\end{array}$ & \\
\hline 291470 & JOAO DURVAL CARNEIRO & РDT BA & INCRA 2003 & FAZENDA SAO JOSE & ITABERABA & $\mathrm{BA}$ & 143 & PEQUENA PROPRIEDADE & 143 & 0 & & \\
\hline 291080 & JOAO DURVAL CARNEIRO & РDT BA & INCRA 2003 & FAZENDA NOVA ESPERANCA & FEIRA DE SANTANA & BA & 412 & 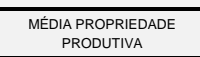 & 412 & 0 & & \\
\hline 290700 & \begin{tabular}{|l|} 
JoĀO LUIZ CORREIA ARGÓLO \\
DOS SANTOS
\end{tabular} & PPBA & INCRA 2003 & FAZENDA MARINGA & CARDEAL DA SILVA & $\mathrm{BA}$ & 242 & $\begin{array}{l}\text { MÉDIA PROPRIEDADE } \\
\text { PRODUTIVA }\end{array}$ & 242 & 0 & & \\
\hline 290910 & JOSE ALVES ROCHA & PFLBA & INCRA 2003 & IITIO USINA & CORIBE & $\mathrm{BA}$ & 3 & MINIFÚNDIO & 3 & 0 & & \\
\hline 291720 & JOSE ALVES ROCHA & PFL BA & INCRA 2003 & LAGEDAO & IтuAçu & BA & 35 & MINIIÉNDIO & 35 & 0 & & \\
\hline 290660 & JOSE ALVES ROCHA & $\begin{array}{ll}\text { PFLBA } \\
\end{array}$ & INCRA 2003 & FAZ LAGOA DO BEZERRO & CANDiBA & $\mathrm{BA}$ & 70 & PEQUENA PROPRIEDADE & 70 & 0 & & \\
\hline 290910 & JOSE ALVES ROCHA & PFL BA & INCRA 2003 & VOLTA DO REGO & CORIBE & $\mathrm{BA}$ & 111 & PEQUENA PROPRIEDADE & 111 & 0 & & \\
\hline 290910 & JOSE ALVES ROCHA & PFLBA & INCRA 2003 & FAZENDA LAGED I ITAMBURIL & CORIBE & $\mathrm{BA}$ & 197 & PEQUENA PROPRIEDADE & 197 & 0 & & \\
\hline 290910 & JOSE ALVES ROCHA & PFL BA & INCRA 2003 & FAZENDA FORMOSO & CORIBE & BA & 233 & PEQUENA PROPRIEDADE & 233 & 0 & & \\
\hline 290910 & JOSE ALVES ROCHA & PFL BA & INCRA 2003 & FAZENDA FORMOSO & CORIBE & $\mathrm{BA}$ & 233 & PEQUENA PROPRIEDADE & 233 & 0 & & \\
\hline 290910 & JOSE ALVES ROCHA & PFLBA & INCRA 2003 & FAZENDA BONITO & CORIBE & $\mathrm{BA}$ & 500 & $\begin{array}{l}\text { MÉDIA PROPRIIEADE } \\
\text { IMPROOUTIVA }\end{array}$ & 500 & 0 & & \\
\hline 290910 & JOSE ALVES ROCHA & PFLBA & INCRA 2003 & FAZENDA JACU & CORIBE & $\mathrm{BA}$ & 557 & $\begin{array}{l}\text { MÉDIA PROPRIEDADE } \\
\text { IMPROOUTIVA }\end{array}$ & 557 & 0 & & \\
\hline
\end{tabular}




\begin{tabular}{|c|c|c|c|c|c|c|c|c|c|c|c|c|}
\hline GEOCODIGO & PARLAMENTAR & PARTIDO & FONTE & IMÓVEL & MUNICIPPO & UF & AREA & CATEGORIA FUNDIÁRIIA & ÁREA REGISTRADA & $\begin{array}{l}\text { ÁREA DECLARADA } \\
\text { COMO POSSE }\end{array}$ & $\begin{array}{l}\text { REGISTRO EM NOME DE } \\
\text { OUTRAS PESSOAS }\end{array}$ & $\begin{array}{l}\text { viNCULO COM O } \\
\text { PARLAMENTAR }\end{array}$ \\
\hline 290910 & JOSE ALVES ROCHA & PFLBA & INCRA 2003 & FAZENDA JACU & CORIBE & $\mathrm{BA}$ & 557 & $\begin{array}{c}\text { MÉDIA PROPRIEDADE } \\
\text { IMPROUUTIVA }\end{array}$ & 557 & 0 & & \\
\hline 292170 & JOSE CARLOS LEAO DE ARAUJO & PDT BA & INCRA 2003 & FAZ LAGOA DA PEDRA BRANCA & MORRO DO CHAPÉU & BA & 174 & PEQUENA PROPRIEDADE & 174 & 0 & & \\
\hline 292170 & JOSE CARLOS LEAO DE ARAUJO & PDT BA & INCRA 2003 & FAZ LAGOA DA PEDRA BRANCA & MORRO DO CHAPÉU & BA & 174 & PEQUENA PROPRIEDADE & 174 & 0 & & \\
\hline 292100 & JoSE CARLOS LEAO DE ARAUJO & PDT BA & INCRA 2003 & FAZENDA SAO JOSE & MATA DE SÃO JOÃO & BA & 338 & $\begin{array}{c}\text { GRANDE PROPRIEDADE } \\
\text { PRODUTIVA }\end{array}$ & 272 & 338 & & \\
\hline 292100 & JOSE CARLOS LEAO DE ARAUJO & PDT BA & INCRA 2003 & FAZENDA SAO JOSE & MATA DE SÃo JOẤ & BA & 338 & $\begin{array}{c}\text { GRANDE PROPRIEDADE } \\
\text { PRODUTIVA }\end{array}$ & 272 & 338 & & \\
\hline 290950 & $\begin{array}{l}\text { JoSE LOURENCSO MORAIS DA } \\
\text { SLIVA }\end{array}$ & РмDв вА & INCRA 2003 & FAZENDA BPMAIM & CRAVOLÁNDIA & BA & 100 & $\begin{array}{l}\text { PEQUENA PROPRIEDADE } \\
\end{array}$ & 100 & 0 & & \\
\hline 292660 & $\begin{array}{l}\text { JOSE LOURENÇO MORAIS DA } \\
\text { SILVA }\end{array}$ & PMDB BA & INCRA 2003 & FAZENDA COVA & RIBEIRA DO POMBAL & BA & 578 & $\begin{array}{l}\text { GRANDE PROPRIIEDADE } \\
\text { IMPRODUTIVA }\end{array}$ & 607 & 0 & & \\
\hline 290440 & $\begin{array}{l}\text { JOSE LOURENÇO MORAIS DA } \\
\text { SILVA }\end{array}$ & РмDв вA & INCRA 2003 & FAZENDA SAO LOURENCO & BREJOLÂNDIA & BA & 798 & 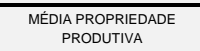 & 798 & 0 & & \\
\hline 290440 & $\begin{array}{l}\text { JOSE LOURENÇO MORAIS DA } \\
\text { SILVA }\end{array}$ & РМDв ВA & INCRA 2003 & FAZENDA SAO PAULOI & BREJOLÂNDIA & $\overline{B A}$ & 858 & $\begin{array}{l}\text { MÉDIA PROPRIEDADE } \\
\text { PRODUTIVA }\end{array}$ & 858 & 0 & & \\
\hline 293030 & $\begin{array}{l}\text { JOSE LOURENÇO MORAIS DA } \\
\text { SILVA }\end{array}$ & РмDB вA & INCRA 2003 & FAZENDA SAO PAULO ॥ & SERRA DOURADA & $\mathrm{BA}$ & 871 & $\begin{array}{l}\text { MÉDIA PROPRIEDADE } \\
\text { IMPROOUTIVA }\end{array}$ & 871 & 0 & & \\
\hline 290440 & $\begin{array}{l}\text { JOSE LOURENÇO MORAIS DA } \\
\text { SILVA }\end{array}$ & РMDB BA & INCRA 2003 & FAZ SAO MARTINHO II & BREJOLÁNDIA & BA & 2224 & $\begin{array}{l}\text { GRANDE PROPRIEDADE } \\
\text { IMPROOUTIVA }\end{array}$ & 160 & 2224 & & \\
\hline 290440 & $\begin{array}{l}\text { JOSE LOURENÇO MORAIS DA } \\
\text { SILVA }\end{array}$ & PмDв ва & INCRA 2003 & FAZENDA SAO MARTINHO & BREJOLÂNDIA & BA & 2267 & $\begin{array}{c}\text { GRANDE PROPRIEDADE } \\
\text { IMPRODUTIVA }\end{array}$ & 2267 & 0 & & \\
\hline 293090 & $\begin{array}{l}\text { JOSE LOURENÇO MORAIS DA } \\
\text { sLLVA }\end{array}$ & РMDв вА & INCRA 2003 & FAZENDA SAO JOSE & TABOCAS DO BREJO VELHO & BA & 2870 & $\begin{array}{c}\text { GRANDE PROPRIIEDADE } \\
\text { IMPRODUTIVA }\end{array}$ & 2870 & 0 & & \\
\hline 291070 & $\begin{array}{l}\text { JOSE LOURENÇO MORAIS DA } \\
\text { SILVA }\end{array}$ & РмDв BA & INCRA 2003 & FAZENDA SIPTTUBA & EUCLIDES DA CUNHA & $\mathrm{BA}$ & 8883 & $\begin{array}{c}\text { GRANDE PROPRIIDADE } \\
\text { IMPROOUTIVA }\end{array}$ & 8883 & 0 & & \\
\hline 293030 & JOSE NUNES SOARES & DEM BA & INCRA 2003 & FAZENDA PONTA DAGUA & SERRA DOURADA & BA & 3 & MINIFÚNDIO & 0 & 3 & & \\
\hline 293030 & JOSE NUNES SOARES & DEM BA & INCRA 2003 & FAZENDA CARABAAS DE CIMA & SERRA DOURADA & BA & 37 & MINIF́ñolo & 37 & 0 & & \\
\hline 293030 & JOSE NUNES SOARES & DEMBA & INCRA 2003 & FAZENDA BAIXO & SERRA DOURADA & $\mathrm{BA}$ & 50 & MINIF́́NDIO & 0 & 50 & & \\
\hline 292300 & JOSE NUNES SOARES & DEM BA & INCRA 2003 & FAZ SANTO ANTONIO DA FAVELA & Nova Vị̧osa & BA & 67 & PEQUENA PROPRIEDADE & 67 & 0 & & \\
\hline 291070 & JOSE NUNES SOARES & DЕм вА & INCRA 2003 & FAZ SAO JOSE & EUCLIDES DA CUNHA & BA & 300 & $\begin{array}{l}\text { MÉDIA PROPRIEDADE } \\
\text { IMPROOUTIVA } \\
\end{array}$ & 300 & 0 & & \\
\hline 291070 & JOSE NUNES SOARES & DEMBA & INCRA 2003 & FAZ SAO GABRIEU & EUCLIDES DA CUNHA & $\mathrm{BA}$ & 450 & 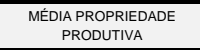 & 450 & 0 & & \\
\hline 291070 & JOSE NUNES SOARES & DEM BA & INCRA 2003 & FAZ BOM JARDIM & EUCLIDES DA CUNHA & BA & 3300 & $\begin{array}{c}\text { GRANDE PROPRIEDADE } \\
\text { IMPROOUTIVA }\end{array}$ & 3300 & 0 & & \\
\hline 291230 & $\begin{array}{l}\text { LEUR ANTONIO DE BRITO } \\
\text { LOMANTO }\end{array}$ & РMDв BA & INCRA 2003 & FAZ RANCHO ALEGRE & isicui & BA & 208 & $\begin{array}{l}\text { MÉEDAA PROPRIEDADE } \\
\text { IMPROOUTIVA }\end{array}$ & 208 & 0 & & \\
\hline 292540 & LUCIO QUADROS VIEIRA LIMA & РмDB BA & INCRA 2003 & FAZENDA TABAJARA & POTIRAGUÁ & $\mathrm{BA}$ & 750 & 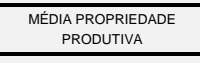 & 750 & 0 & RAFAEL AMOEDO AMOEDO & 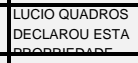 \\
\hline 291840 & LUIZ GONZAGA PATRIOTA & PSB PE & INCRA 2003 & ROCA CAMPO DOS CAVALOS & JuAZEIRO & BA & 12 & MINIF́ÚNDIO & 12 & 0 & & \\
\hline 293325 & OSVALDO DE SOUZA COELHO & PFLPE & INCRA 2003 & FAZENDA CACHOERIINHA & VEREDA & $\mathrm{BA}$ & 152 & $\begin{array}{l}\text { MÉDIA PROPRIEDADE } \\
\text { IMPROOUTIVA }\end{array}$ & 0 & 152 & osvaldo de SOUZA CoElHO & \\
\hline 293270 & $\begin{array}{l}\text { PAULOO SERGIO PARANHOS DE } \\
\text { MAGALAAES }\end{array}$ & DEMBA & INCRA 2003 & FAZENDA BOM SOSSEGO & URUÇUCA & $\mathrm{BA}$ & 34 & PEQUENA PROPRIEDADE & 34 & 0 & $\begin{array}{l}\text { PAULO SERGIO PARANHOS } \\
\text { DE MAGALHAES }\end{array}$ & \\
\hline 291490 & $\begin{array}{l}\text { PAULO SERGIO PARANHOS DE } \\
\text { MAGALAAES }\end{array}$ & DEM BA & INCRA 2003 & CONJ PEDRO R MARIA & ITACARÉ & BA & 56 & PEQUENA PROPRIEDADE & 56 & 0 & $\begin{array}{l}\text { PAULO SERGIO PARANHOS } \\
\text { DE MAGALHAES }\end{array}$ & \\
\hline 291490 & $\begin{array}{l}\text { PAULO SERGIO PARANHOS DE } \\
\text { MAGGLLAES }\end{array}$ & DEM BA & INCRA 2003 & FAZENDA BOM JESUS & ITACARE & $\mathrm{BA}$ & 56 & PEQUENA PROPRIEDADE & 56 & 0 & $\begin{array}{l}\text { PAULO SERGIO PARANHOS } \\
\text { DE MAGALHAES }\end{array}$ & \\
\hline 293270 & $\begin{array}{l}\text { PAULO SERGIO PARANHOS DE } \\
\text { MAGGLAAES }\end{array}$ & DEMBA & INCRA 2003 & FAZENDA DOIS IRMAOS & URUÇUCA & $\mathrm{BA}$ & 262 & $\begin{array}{c}\text { MÉDIA PROPRIEDADE } \\
\text { IMPROOUTIVA }\end{array}$ & 262 & 0 & $\begin{array}{l}\text { PAULO SERGIO PARANHOS } \\
\text { DE MAGALHAES }\end{array}$ & \\
\hline 291490 & $\begin{array}{l}\text { PAULO SERGIO PARANHOS DE } \\
\text { MAGGLAAES }\end{array}$ & DEMBA & INCRA 2003 & FAZENDA NOVA FLORENTINA & ITAETÉ & BA & 600 & $\begin{array}{c}\text { MÉDIA PROPRIEDADE } \\
\text { IMPROUUTIVA }\end{array}$ & 600 & 0 & $\begin{array}{l}\text { PAULO SERGIO PARANHOS } \\
\text { DE MAGALHAES }\end{array}$ & \\
\hline
\end{tabular}




\begin{tabular}{|c|c|c|c|c|c|c|c|c|c|c|c|c|}
\hline GEOCODIGO & PARLAMENTAR & PARTIDO & FONTE & IMÓvel & MuNICiPIO & UF & AREA & CATEGORIA FUNDIÁRIAA & AREA REGISTRADA & $\begin{array}{l}\text { AREA DECLARADA } \\
\text { COMO POSAEE }\end{array}$ & $\begin{array}{l}\text { REGIITRR EM NOME DE } \\
\text { OUTRAS PESSOAS }\end{array}$ & $\begin{array}{l}\text { viNCULO com o } \\
\text { PARLAMENTAR }\end{array}$ \\
\hline 290380 & ROBERTO PEREIRA DE BRITO & PP BA & INCRA 2003 & UMBuzeiro & BOA VISTA DO TUPIM & BA & 53 & MINIÉ́NDIO & 53 & 0 & ROBERTO PEREIRA DE BRITO & \\
\hline 230750 & EUNICIO LOPES DE OLVERAA & PMDB CE & INCRA 2003 & SITIO VARZEA GRANDE & LAVRAS DA MANGGBERA & $\mathrm{CE}$ & 0 & PEQUENA PROPRIEDADE & 0 & 50 & & \\
\hline 230750 & EUNICIO LOPES DE OLVEIRA & PMDB CE & INCRA 2003 & SITIO VARZEA GRANDE & LAVRAS DA MANGABEIRA & $\mathrm{CE}$ & 5 & MINIF́́NDIO & 5 & 0 & & \\
\hline 231270 & FRANCISCO PINHEIRO LANDIM & РМDВ СE & INCRA 2003 & SITIO CAMPOS & SENADOR POMPEU & $\mathrm{CE}$ & 84 & PEQUENA PROPRIEDADE & 84 & 0 & & \\
\hline 230835 & FRANCISCO PINHEIRO LANDIM & PMDB CE & INCRA 2003 & SIITO SAO PEDRO & MLHÄ & $\mathrm{CE}$ & 279 & $\begin{array}{c}\text { MÉDIA PROPRIEDADE } \\
\text { IMPROOUTIVA }\end{array}$ & 284 & 0 & & \\
\hline 230835 & FRANCISCO PINHEIRO LANDIM & РМDВ СE & INCRA 2003 & SITIO SERRA VERDE & MLLÄ & $\mathrm{CE}$ & 280 & $\begin{array}{c}\text { MÉDIA PROPRIEDADE } \\
\text { IMPROOUTIVA } \\
\end{array}$ & 284 & 0 & & \\
\hline 231030 & GENECIAS MATEUS NORONHA & PMDBCE & INCRA 2003 & SITIO PARAMBU & PARAMBU & $\mathrm{CE}$ & 41 & MINIFÚNDIO & 41 & 0 & & \\
\hline 231030 & GENECIAS MATEUS NORONHA & PMDBCE & INCRA 2003 & FAZENDA UMBUZERTO & PARAMBU & $\mathrm{CE}$ & 1067 & $\begin{array}{l}\text { MÉDIA PROPRIEDADE } \\
\text { IMPRODUTIVA }\end{array}$ & 1067 & 0 & $\begin{array}{l}\text { LOURENCO DA COSTA } \\
\text { LETAO FEITOSA }\end{array}$ & 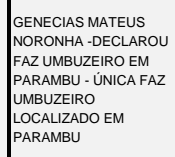 \\
\hline 230820 & JOSE LNHARES PONTE & PPCE & INCRA 2003 & SITIO BOM JARDIM & MERUOCA & $\mathrm{CE}$ & 8 & MINIFÚNDIO & 8 & 0 & JOSE LINHARES PONTE & \\
\hline 230800 & JOSE LNHARES PONTE & PPCE & INCRA 2003 & MUMBABA DE CIMA & MASSAPEE & $\mathrm{CE}$ & 15 & MINIF́́NDIO & 0 & 0 & VILMAR LINHARES PONTE & \\
\hline 230800 & JOSE LINHARES PONTE & PPCE & INCRA 2003 & MUMBABA DE CIMA & MASSAPÉ & $\mathrm{CE}$ & 16 & MIIIFÚ́NDIO & 0 & 0 & VILMAR LINHARES PONTE & \\
\hline 231290 & JOSE LNHARES PONTE & PPCE & INCRA 2003 & ТЕLHA & SOBRAL & CE & 163 & PEQUENA PROPRIEDADE & 0 & 163 & VILMAR LNHARES PONTE & \\
\hline 230800 & JOSE LINHARES PONTE & PPCE & INCRA 2003 & FAZENDA SAO NARCISIO & MASSAPÉ & $\mathrm{CE}$ & 176 & PEQUENA PROPRIEDADE & 176 & 0 & $\begin{array}{l}\text { FRANCISCO LINHARES } \\
\text { PONTES }\end{array}$ & \\
\hline 231020 & LUIS BARBOSA ALVES & PSDB RR & INCRA 2003 & amuados & PARACURU & $\mathrm{CE}$ & 75 & PEQUENA PROPRIEDADE & 0 & 75 & LUIZ BARBOSA ALVES & \\
\hline 230170 & $\begin{array}{l}\text { RAMUNNDO ANTONIO DE MACEDO } \\
\text { (RAMUNDAOO) }\end{array}$ & РМDB CE & INCRA 2003 & SITIO SERROTE DO CACHIMBO & AURORA & $\mathrm{CE}$ & 42 & MINIFÚNDIO & 42 & 0 & VICENTE NETO LEMOS & 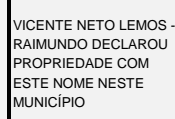 \\
\hline 230170 & \begin{tabular}{|l|} 
RAMUUNDO ANTONIO DE MACEDO \\
(RAMUNDAOO)
\end{tabular} & PMDB CE & INCRA 2003 & SITIO PACOTE & AURORA & $\mathrm{CE}$ & 538 & $\begin{array}{l}\text { MÉDIA PROPRIEDADE } \\
\text { PRODUTIVA }\end{array}$ & 538 & 0 & VICENTE NETO LEMOS & 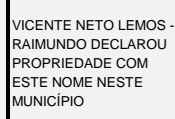 \\
\hline 230765 & ROBERTO SOARES PESSOA & PLCE & INCRA 2003 & MUCUNA & MARAcanaú & $\mathrm{CE}$ & 17 & PEQUENA PROPRIEDADE & 17 & 0 & ROBERTO SOARES PESSOA & \\
\hline 230900 & ROBERTO SOARES PESSOA & PLCE & INCRA 2003 & SITIO SIREMA & мисамво & CE & 49 & MINIÉ́NDIO & 49 & 0 & ROBERTO SOARES PESSOA & \\
\hline 230900 & ROBERTO SOARES PESSOA & PLCE & INCRA 2003 & SITIO SIRIEMA & мисамво & CE & 49 & MINIFÚNDIO & 49 & 0 & & \\
\hline 230765 & ROBERTO SOARES PESSOA & PLCE & INCRA 2003 & GRANJA SAO FRANCISCO V VI VII & MARACANAU & $\mathrm{CE}$ & 85 & $\begin{array}{l}\text { MÉDIA PROPRIEEADE } \\
\text { PRODUTIVA }\end{array}$ & 85 & 0 & ROBERTO SOARES PESSOA & \\
\hline 230765 & ROBERTO SOARES PESSOA & PLCE & INCRA 2003 & GRANJA SAO FRANCIISCO V VI VIII & MARACANAÚ & $\mathrm{CE}$ & 85 & $\begin{array}{l}\text { MÉDIA PROPRIEDADE } \\
\text { PRODUTIVA }\end{array}$ & 85 & 0 & & \\
\hline 230765 & ROBERTO SOARES PESSOA & PLCE & INCRA 2003 & MUCUNA & MARACANAÚ & $\mathrm{CE}$ & 17 & PEQUENA PROPRIEDADE & 17 & 0 & & \\
\hline 230220 & $\begin{array}{l}\text { VICENTE FERREIRA DE ARRUDA } \\
\text { COELHO }\end{array}$ & PRCE & INCRA 2003 & FAZENDA EZEQUIEL & $\begin{array}{l}\text { BEBERIBEE } \\
\end{array}$ & $\overline{C E}$ & 56 & PEQUENA PROPRIEDADE & 56 & 0 & $\begin{array}{l}\text { ELIEZER OLIVEIRA DE } \\
\text { ARRUDA COELHO }\end{array}$ & $\begin{array}{l}\text { IRMĀO-VICENTE } \\
\text { ARRUDA COELHO }\end{array}$ \\
\hline 230470 & $\begin{array}{l}\text { VICENTE FERREIRA DE ARRUDA } \\
\text { COELHO }\end{array}$ & PRCE & INCRA 2003 & FAZ BAIXA ESCURA & GRANJA & $\mathrm{CE}$ & 313 & $\begin{array}{c}\text { MÉDIA PROPRIEDADE } \\
\text { IMPROOUTIVA }\end{array}$ & 202 & 114 & $\begin{array}{l}\text { ELIEZER OLIVEIRA DE } \\
\text { ARRUDA COELHO }\end{array}$ & $\begin{array}{l}\text { IRMAOO-VICENTE } \\
\text { ARRUDA COELHO }\end{array}$ \\
\hline 230470 & $\begin{array}{l}\begin{array}{l}\text { VICENTE FERREIRA DE ARRUDA } \\
\text { COELHO }\end{array} \\
\end{array}$ & PRCE & INCRA 2003 & FAZ SALGADINHO & GRANJA & $\mathrm{CE}$ & 40 & MINIIÉNDIO & 0 & 40 & $\begin{array}{l}\text { ELIEZER OLVEIRA DE } \\
\text { ARRUDA COELHO }\end{array}$ & \begin{tabular}{|l|} 
IRMAOO- VICENTE \\
ARRUDA COELLHO
\end{tabular} \\
\hline
\end{tabular}




\begin{tabular}{|c|c|c|c|c|c|c|c|c|c|c|c|c|}
\hline GEOCODIGO & PARLAMENTAR & PARTIDO & FONTE & IMÓvel & MUNICIPIO & UF & AREA & CATEGORIA FUNDIÁRIAA & AREA REGISTRADA & $\begin{array}{l}\text { AREA DECLARADA } \\
\text { COMO POSAEE }\end{array}$ & $\begin{array}{l}\text { REGIITRR EM NOME DE } \\
\text { OUTRAS PESSOAS }\end{array}$ & $\begin{array}{l}\text { viNCULO com o } \\
\text { PARLAMENTAR }\end{array}$ \\
\hline 230470 & $\begin{array}{l}\text { VICENTE FERREIRA DE ARRUDA } \\
\text { COELHO }\end{array}$ & PRCE & INCRA 2003 & FAZENDA KM OITO & GRANuA & $\mathrm{CE}$ & 178 & PEQUENA PROPRIEDADE & 178 & 0 & $\begin{array}{l}\text { ELIEZER OLVEIRA DE } \\
\text { ARRUDA COELHO }\end{array}$ & $\begin{array}{l}\text { IRMĀO-VICENTE } \\
\text { ARRUDA COELHO }\end{array}$ \\
\hline 231290 & $\begin{array}{l}\text { VICENTE FERREIRA DE ARRUDA } \\
\text { COELHO }\end{array}$ & PRCE & INCRA 2003 & FAZENDA ACUDE & SOBRAL & $\mathrm{CE}$ & 1010 & 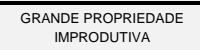 & 1010 & 0 & $\begin{array}{l}\text { LUCIANO DE ARRUDA } \\
\text { COELHO }\end{array}$ & $\begin{array}{l}\text { GRAU DE PARENTESCO } \\
\text { NAO IDENTIFICADO }\end{array}$ \\
\hline 521308 & $\begin{array}{l}\text { CARLOS ALBERTO LERÉlA DA } \\
\text { SILVA }\end{array}$ & PSDB GO & INCRA 2003 & FAZ QUEIXADA DO CURRIOLA & minaçu & Go & 15 & MINIIEÚNDIO & 15 & 0 & $\begin{array}{l}\text { MARIA DALVA VALERIO DA } \\
\text { COSTA): }\end{array}$ & 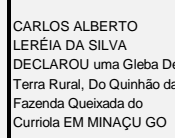 \\
\hline 521308 & $\begin{array}{l}\text { CARLOS ALBERTO LERÉ|A DA } \\
\text { SILVA }\end{array}$ & PSDB GO & INCRA 2003 & FAZ QUEIXADA DO CURRIIOLA & MINAC̣U & Go & 61 & PEQUENA PROPRIEDADE & 25 & 36 & JOAOBATISTA FERREIRA); & 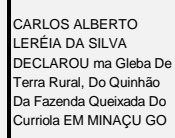 \\
\hline 521308 & $\begin{array}{l}\text { CARLOS ALEERTO LERÉ|A DA } \\
\text { SILVA }\end{array}$ & PSDB GO & INCRA 2003 & FAZ QUEIXADA DO CORRIOLA & MINAÇU & Go & 527 & $\begin{array}{l}\text { MÉDIA PROPRIEDADE } \\
\text { IMPRODUTIVA }\end{array}$ & 0 & 527 & RUBENS TOPAL); & 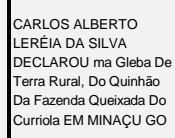 \\
\hline 521308 & $\begin{array}{l}\text { CARLOS ALBERTO LERÉIA DA } \\
\text { SILVAA }\end{array}$ & PSDB GO & INCRA 2003 & FAZENDA SANTA RITA & Minaçu & Go & 871 & $\begin{array}{l}\text { GRANDE PROPRIEDADE } \\
\text { IMPRODUTIVA }\end{array}$ & 871 & 0 & $\begin{array}{l}\text { RUY RODRIGUES DE } \\
\text { CASTRO); }\end{array}$ & 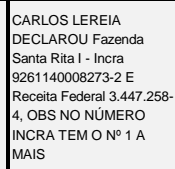 \\
\hline 521720 & \begin{tabular}{|l|} 
EEMOSTENES LAZARO XAVIER \\
TORRES
\end{tabular} & PFL GO & INCRA 2003 & FAZ MACAUBA & PIRANHAS & Go & 242 & $\begin{array}{c}\text { MÉDIA PROPRIEDADE } \\
\text { IMPROOUTIVA }\end{array}$ & 242 & 0 & & \\
\hline 520580 & EUNICIO LOPES DE OLIVEIRA & PMDB CE & INCRA 2003 & FAZENDA CATINGUEIRO GRANDE & CORUMBÁ DE GOIÁS & Go & 2 & MiNIFÚnDIO & 2 & 0 & $\begin{array}{l}\text { PRACIII) SARDINHA DA } \\
\text { cOSTAA; }\end{array}$ & \\
\hline 520580 & EUNICIO LOPES DE OLIVEIRA & PMDBCE & INCRA 2003 & FAZENDA BEIJA MAO & CORUMBÁ DE GOIÁS & Go & 3 & MINIÉ́NDIO & 3 & & & \\
\hline 520580 & EUNICIO LOPES DE OLIVEIRA & РМDB CE & INCRA 2003 & FAZENDA CATINGUEIRO GRANDE & CORUMBÁ DE GOIÁS & Go & 4 & MINIFÚNDIO & 4 & 0 & \begin{tabular}{|l} 
ANTONOO SARDINHA DA \\
COSTA:
\end{tabular} & \\
\hline 520580 & EUNICIO LOPES DE OLIVEIRA & РМDB СE & INCRA 2003 & FAZENDA CATINGUEIro GRANDE & CORUMBÁ DE GOIÁS & Go & 5 & MINIFÚNDIO & 5 & 0 & $\begin{array}{l}\text { CARLOS MANOEL ALVES DA } \\
\text { COSTA): }\end{array}$ & \\
\hline 520580 & EUNICIO LOPES DE OLIVEIRA & PMDBCE & INCRA 2003 & FAZENDA CATINGUEIRO GRANDE & CORUMBÁ DE GOIÁS & Go & 5 & MINIFÚNDIO & 5 & 0 & GERGINO ALVES DA COSTA); & \\
\hline 520580 & EUNICIO LOPES DE OLIVEIRA & РМDВ СE & INCRA 2003 & FAZENDA CATINGUEIRO GRANDE & CORUMBÁ DE GOIÁS & Go & 5 & MINIFÚNDIO & 5 & 0 & MARIA JOSE DA COSTA); & \\
\hline 520580 & EUNICIO LOPES DE OLIVEIRA & PMDB CE & INCRA 2003 & FAZENDA CATINGUEIRO GRANDE & CORUMBÁ DE GOIÁS & Go & 6 & MINIFÚNDIO & 6 & 0 & $\begin{array}{l}\text { LAUDEMIRO SARDINHA DA } \\
\text { COSTA): }\end{array}$ & \\
\hline 520580 & EUNICIO LOPES DE OLIVEIRA & РМDВ СE & INCRA 2003 & FAZENDA BEIAA MAO & CORUMBÁ DE GOIÁS & Go & 7 & MINIF́ĆNDIO & 7 & & & \\
\hline 520580 & EUNICIO LOPES DE OLIVERA & РMDB СE & INCRA 2003 & FAZENDA CATINGUEIRO GRANDE & CORUMBÁ DE GOIÁS & Go & 12 & MINIFÚNDIO & 12 & 0 & ALAOR PEREIRA DE MORAIS); & 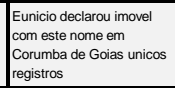 \\
\hline 520580 & EUNICIO LOPES DE OLIVEIRA & РМDВ СE & INCRA 2003 & FAZENDA CATINGUEIRO GRANDE & CORUMBÁ DE GOIÁS & Go & 15 & MINIIÉnDIO & 15 & 0 & $\begin{array}{l}\text { LAUDERICO ALVES DA } \\
\text { COSTA): }\end{array}$ & \\
\hline 520580 & EUNICIO LOPES DE OLVEIRA & РМDB СE & INCRA 2003 & FAZENDA LAGES E CONGONHA & CORUMBÁ DE GOIÁS & Go & 19 & MINIFÚNDIO & 0 & 19 & & \\
\hline 520580 & EUNICIO LOPES DE OLIVEIRA & PMDBCE & INCRA 2003 & FAZENDA BEIAA MAO & CORUMBÁ DE GOÁÁS & Go & 22 & MINIFÚNDIO & 22 & 22 & & \\
\hline 521390 & EUNICIO LOPES DE OLIVERA & PMDB CE & INCRA 2003 & FAZENDA CATINGUEIRO GRANDE & MOSSAMEDES & Go & 24 & PEQUENA PROPRIEDADE & 24 & $\overline{0}$ & $\begin{array}{l}\begin{array}{l}\text { GERALDO MARQUES } \\
\text { FERREIRA); }\end{array} \\
\text { FRE }\end{array}$ & \\
\hline 520580 & EUNICIO LOPES DE OLVEREA & РМDB СE & INCRA 2003 & FAZENDA CATINGUEIRO GRANDE & CORUMBÁ DE GOIÁS & Go & 27 & MINIF́ÚNDIO & 27 & 0 & MANOEL ALVES MAGALLAEES: & \\
\hline
\end{tabular}




\begin{tabular}{|c|c|c|c|c|c|c|c|c|c|c|c|c|}
\hline GEOCODIGO & PARLAMENTAR & PARTIDO & FONTE & IḾ́VEL & MUNICIPIO & UF & ÁREA & CATEGORIA FUNDIÁRIIA & ÁREA REGISTRADA & $\begin{array}{l}\text { ÁREA DECLARADA } \\
\text { COMO POSSE }\end{array}$ & $\begin{array}{l}\text { REGISTRO EM NOME DE } \\
\text { OUTRAS PESSOAS }\end{array}$ & $\begin{array}{l}\text { viNcULO COMO O } \\
\text { PARLAMENTAR }\end{array}$ \\
\hline 520580 & EUNICIO LOPES DE OLIVEIRA & РМDв СE & INCRA 2003 & FAZENDA CATINGUEIRO GRANDE & CORUMBÁ DE GOIÁS & Go & 28 & MINIFÚNDIO & 28 & 0 & $\begin{array}{l}\text { LOURIVAL SARDINHA DA } \\
\text { COSTA): }\end{array}$ & \\
\hline 520580 & EUNICIO LOPES DE OLIVEIRA & PMDB CE & INCRA 2003 & CONG DOS BUENOS OU BUENOS E OLARIA & CORUMBÁ DE GOÁs & Go & 30 & MINIF́ÚNDIO & 0 & 30 & & \\
\hline 520580 & EUNICIO LOPES DE OLIVEIRA & PMDB CE & INCRA 2003 & FAZENDA BEINA MAO & CORUMBÁ DE GOÁÁS & Go & 38 & PEQUENA PROPRIEDADE & 38 & & & \\
\hline 520580 & EUNICIO LOPES DE OLIVEIRA & РMDв СE & INCRA 2003 & FAZENDA CATINGUEIRO GRANDE & CORUMBÁ DE GOIÁS & Go & 48 & PEQUENA PROPRIEDADE & 48 & 0 & $\begin{array}{l}\text { PEDRO ALVES DA COSTA } \\
\text { FLLHO); }\end{array}$ & \\
\hline 520580 & EUNICIO LOPES DE OLIVEIRA & PMDB CE & INCRA 2003 & FAZENDA GONGONHAS DOS ALVES & CORUMBÁ DE GOÍ́S & Go & 61 & PEQUENA PROPRIEDADE & 0 & 61 & & \\
\hline 520580 & EUNICIO LOPES DE OLIVEIRA & PMDB CE & INCRA 2003 & FAZENDA BEIJA MAO & CORUMBÁ DE GOIÁS & Go & 62 & PEQUENA PROPRIEDADE & 62 & & & \\
\hline 520580 & EUNICIO LOPES DE OLIVEIRA & РМDв СE & INCRA 2003 & FAZENDA CONGONHA DOS ALVES & CORUMBÁ DE GOIÁS & Go & 74 & PEQUENA PROPRIEDADE & 74 & 0 & & \\
\hline 520580 & EUNICIO LOPES DE OLIVEIRA & РMDB CE & INCRA 2003 & FAZENDA PASTO GRANDE & CORUMBÁ DE GOIÁS & Go & 77 & PEQUENA PROPRIEDADE & 77 & 0 & & \\
\hline 520580 & EUNICIO LOPES DE OLVEIRA & PMDB CE & INCRA 2003 & MATA DO CORUMBA & CORUMBÁ DE GOÁS & Go & 83 & PEQUENA PROPRIEDADE & 83 & 0 & (MOACIR BELCHIOR); & 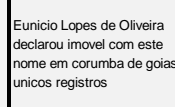 \\
\hline 520580 & EUNICIO LOPES DE OLIVEIRA & PMDB CE & INCRA 2003 & FAZ MATA DO CORUMBA & CORUMBÁ DE GOIÁS & Go & 211 & $\begin{array}{l}\text { MÉDIA PROPRIEDADE } \\
\text { PRODUTIVA }\end{array}$ & 211 & 0 & MOACIR BELCHIOR); & 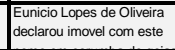 \\
\hline 520030 & EUNICIO LOPES DE OLIVEIRA & PMDB CE & INCRA 2003 & FAZENDA SAO LUIZ & $\overline{\text { ALEXÂNIA }}$ & Go & 250 & $\begin{array}{l}\text { MÉDIA PROPRIEDADE } \\
\text { PRODUTIVA }\end{array}$ & 250 & 0 & & \\
\hline 520580 & EUNICIO LOPES DE OLIVEIRA & РMDв СE & INCRA 2003 & FAZENDA CATINGUEIRO GRANDE & CORUMBÁ DE GOIÁS & Go & 385 & $\begin{array}{c}\text { MÉDIA PROPRIEDADE } \\
\text { IMPROOUTIVA } \\
\end{array}$ & 385 & 0 & ALONSO ANTONIO DE FARIA); & 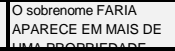 \\
\hline 520580 & EUNICIO LOPES DE OLVEERA & PMDB CE & INCRA 2003 & FAZENDA MATA DO CORUMBA & CORUMBÁ DE GOÁS & Go & 551 & $\begin{array}{l}\text { GRANDE PROPRIEDADE } \\
\text { IMPRODUTIVA }\end{array}$ & 551 & 0 & (MOACIR BELCHIOR); & 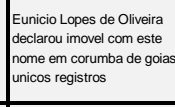 \\
\hline 520580 & EUNICIO LOPES DE OLIVEIRA & PMDB CE & INCRA 2003 & FAZENDA SANTA MONICA & CORUMBÁ DE GOÍÁS & Gо & 1075 & $\begin{array}{c}\text { GRANDE PROPRIIDADE } \\
\text { PRODUTIVA }\end{array}$ & 1075 & 0 & & \\
\hline 522020 & IGOR PUGLIESI AVELINO & РмDв то & INCRA 2003 & FAZENDA SAO FRANCISCO & SÃo MIGUEL DO ARAGUAIA & G० & 678 & $\begin{array}{l}\text { MÉDAA PROPRIEDADE } \\
\text { PRODUTIVA }\end{array}$ & 678 & 0 & $\begin{array}{l}\text { FRANCISCO ANTONIO } \\
\text { PUGLESI); }\end{array}$ & $\begin{array}{l}\text { PARENTESCONÃOO } \\
\text { IDENTFIIIADO }\end{array}$ \\
\hline 521483 & GOR PUGLLESI AVELINO & РМОв то & INCRA 2003 & FAZENDA BORDA DO CAMPO & NOVA CRIXÁS & Go & 1015 & $\begin{array}{c}\text { GRANDE PROPRIIEADE } \\
\text { PRODUTIVA }\end{array}$ & 1015 & 0 & PAULO FERNANDO PUGLESI): & $\begin{array}{l}\text { PARENTESCO NÃOO } \\
\text { IDENTIFICADO }\end{array}$ \\
\hline 520380 & $\begin{array}{l}\text { FRIS REZENDE E DONA IRIS } \\
\text { REZENDE }\end{array}$ & РмDв G० & INCRA 2003 & FAZ ELDORADO OU RETIRO & BRITÃNIA & Gо & 324 & $\begin{array}{l}\text { MÉDIA PROPRIEDADE } \\
\text { PRODUTIVA }\end{array}$ & 312 & 0 & & \\
\hline 522140 & $\begin{array}{l}\begin{array}{l}\text { PRIS REZENDE E DONA IRIS } \\
\text { REZENDE }\end{array} \\
\text { ReN }\end{array}$ & РМОв GO & INCRA 2003 & FAZ BOA VISTA DO RIBEERAO & TRINDADE & Go & 774 & $\begin{array}{c}\text { GRANDE PROPRIEDADE } \\
\text { PRODUTIVA }\end{array}$ & 774 & 0 & & \\
\hline 520380 & 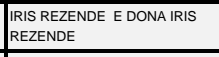 & РмDв G० & INCRA 2003 & FAZ MUtiRaMA & BRITÂANA & Go & 2894 & $\begin{array}{c}\text { GRANDE PROPRIEDADE } \\
\text { PROOUTIVA }\end{array}$ & 2894 & 0 & & \\
\hline 520830 & $\begin{array}{l}\text { JOSE DOS SANTOS FREIRE } \\
\text { JUNIOR }\end{array}$ & РмDв то & INCRA 2003 & FAZENDA PEQUIZEIRO & DIVINÓPOLIS DE GOIÁS & Gо & 895 & $\begin{array}{l}\text { MÉDIA PROPRIEDADE } \\
\text { PRODUTIVA } \\
\end{array}$ & 895 & 0 & \begin{tabular}{|l} 
JOSE DOS SANTOS FREIRE \\
JUNIOR);
\end{tabular} & \\
\hline 521350 & $\begin{array}{l}\text { JOSE DOS SANTOS FREIRE } \\
\text { JUNIOR }\end{array}$ & РмDв То & INCRA 2003 & FAZENDA MOCAMBO & MONTE ALEGRE DE GOIÁS & G० & 2023 & $\begin{array}{c}\text { GRANDE PROPRIEDADE } \\
\text { PRODUTIVA }\end{array}$ & 2023 & 0 & JOSE DOS SANTOS FREIRE): & \\
\hline 521350 & $\begin{array}{l}\text { JOSE DOS SANTOS FREIRE } \\
\text { JUNIOR }\end{array}$ & Рмов то & INCRA 2003 & FAZENDA RETIRO & MONTE ALEGRE DE GOÁÁS & Go & 2178 & $\begin{array}{l}\text { GRANDE PROPRIEDADE } \\
\text { PROOUTIVA }\end{array}$ & 2178 & ${ }^{0}$ & $\begin{array}{l}\text { JoSE DOS SANTOS FREIRE } \\
\text { JuNIOR): }\end{array}$ & \\
\hline 522230 & JOSÉ FUSCALDI CESIILIO & PSD DF & INCRA 2003 & FAZENDA BAIXAO & VILA PROPICIO & Go & 574 & $\begin{array}{c}\text { GRANDE PROPRIIEADDE } \\
\text { PRODUTIVA }\end{array}$ & 245 & 574 & $\begin{array}{l}\text { FILHO-ALESSANDRO JOSE } \\
\text { CESILO); }\end{array}$ & \\
\hline 521560 & JOSÉ FUSCALDI CESIILO & PSD DF & INCRA 2003 & FAZENDA MESTRE DARMAS & PADRE BERNARDO & Go & 837 & $\begin{array}{l}\text { GRANDE PROPRIIEADDE } \\
\text { PRODUTIVA }\end{array}$ & 837 & 0 & $\begin{array}{l}\text { FLLHO-ALESSANDRO JOSE } \\
\text { CESILO); }\end{array}$ & \\
\hline 522230 & JOSÉ FUSCALDI CESILIO & PSD DF & INCRA 2003 & FAZ CASA BLANCA & VILA PROPICIO & Go & 1640 & $\begin{array}{c}\text { GRANDE PROPRIEDADE } \\
\text { PRODUTIVA }\end{array}$ & 1645 & 0 & $\begin{array}{l}\text { FILHO- ALESSANDRO JOSE } \\
\text { CESLIIO); }\end{array}$ & \\
\hline 521560 & JOSÉ FUSCALDI CESILLIO & PSD DF & INCRA 2003 & FAZENDA BAIXAO & PADRE BERNARDO & Go & 2188 & $\begin{array}{c}\text { GRANOE PROPRIEDADE } \\
\text { PROOUTIVA }\end{array}$ & 2188 & 0 & $\begin{array}{l}\text { FILHO-ALESSANDRO JOSE } \\
\text { (EESLIOO); }\end{array}$ & \\
\hline 520425 & JOSE GOMES DA ROCHA & PSD GO & INCRA 2003 & ILHA DO ZE GOMES & CACHOEIRA DOURADA & Go & 9 & MINIFÚNDIO & 0 & 9 & & \\
\hline
\end{tabular}




\begin{tabular}{|c|c|c|c|c|c|c|c|c|c|c|c|c|}
\hline GEOCODIGO & PARLAMENTAR & PARTIDO & FONTE & IMÓVEL & MUNICIPIO & UF & ÁREA & CATEGORIA FUNDIÁRIAA & ÁREA REGISTRADA & $\begin{array}{l}\text { AREA DELCARAUA } \\
\text { COMO POSSE }\end{array}$ & $\begin{array}{l}\text { REGISTRO EM NOME DE } \\
\text { OUTRAS PESSOAS }\end{array}$ & $\begin{array}{l}\text { VincULO COM O } \\
\text { PARLAMENTAR }\end{array}$ \\
\hline 521250 & $\begin{array}{l}\text { JOSÉ RIBAMAR FERREIRA DE } \\
\text { ARAÚJO COSTA }\end{array}$ & PMDB AP & INCRA 2003 & FAZENDA AGUA QUENTE & LUZIÁNIA & go & 8 & MIIIFÚNDDIO & 8 & 0 & $\begin{array}{l}\text { JOSE AMERICANO DO } \\
\text { BRASLIL: }\end{array}$ & 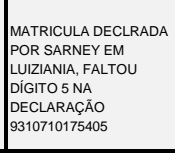 \\
\hline 520725 & LEANDRO VILELA VELLOSO & РмDB GO & INCRA 2003 & FAZ LEBRE & DOVERLÂNDIA & Go & 2659 & $\begin{array}{l}\text { GRANDE PROPRIEDDAE } \\
\text { IMPRODUUTIVA }\end{array}$ & 2659 & 0 & $\begin{array}{l}\text { ERVANDA MARIA DE } \\
\text { RESENDE LUCIANO); }\end{array}$ & 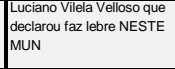 \\
\hline 520870 & LUCIA VANIA ABRÃOO COSTA & PSD GO & INCRA 2003 & FAZ CAVEIRAS & GOIÂNIA & Go & 3 & MIIIFÚNDIO & 3 & 0 & PEDRO ABRAO FLLHO); & \\
\hline 520870 & LUCIA VANIA ABRÃO COSTA & PSDGO & INCRA 2003 & Faz caverira & GOIÂNIA & Go & 7 & PEQUENA PROPRIEDADE & 7 & 0 & 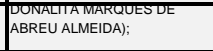 & \\
\hline 520870 & LUCIA VANIA ABRÃOO COSTA & PSD GO & INCRA 2003 & FAZENDA SALINO & GOIÂNIA & Go & 11 & PEQUENA PROPRIEDADE & 11 & 0 & ABDALA ABRAO); & 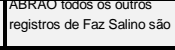 \\
\hline 520870 & LUCIA VANIA ABRĀOO COSTA & PSDGO & INCRA 2003 & $\begin{array}{l}\text { FAZENDA SANTA RITA } \\
\end{array}$ & GOIÂNIA & go & 14 & PEQUENA PROPRIEDADE & 0 & 14 & PEDRO ABRAO FLLHO): & \\
\hline 520870 & LUCIA VANIA ABRĀOO COSTA & PSD GO & INCRA 2003 & \begin{tabular}{|l} 
FAZENDA SANTA RITA \\
\end{tabular} & GOIÂNIA & go & 14 & PEQUENA PROPRIEDADE & 0 & 14 & PEDRO ABRAO FLLHO); & \\
\hline 520870 & LUCIA VANIA ABRÃO COSTA & PSD GO & INCRA 2003 & FAZENDA SANTA RITA & GOIÂNIA & Go & 17 & PEQUENA PROPRIEDADE & 17 & 0 & PEDRO ABRAO FLLHO); & \\
\hline 520995 & LUCIA VANIA ABRÃO COSTA & PSD GO & INCRA 2003 & FAZENDA BOA VISTA & INDIARA & go & 65 & PEQUENA PROPRIEDADE & 65 & 0 & PEDRO ABRAO FILHO); & \\
\hline 520660 & LUCIA VANIA ABRÄO COSTA & PSD GO & INCRA 2003 & FAZENDA SAO PEDRO & CUMARI & go & 103 & PEQUENA PROPRIEDADE & 103 & 0 & PEDRO ABRAO); & \\
\hline 520870 & LUCIA VANIA ABBĀO COSTA & PSD GO & INCRA 2003 & FAZENDA SALINO & GOIÂNIA & Go & 261 & IMPRODUTIVA & 261 & 0 & ABDALA ABRAO); & ABAÁO \\
\hline 521483 & RONALDO RAMOS CAIADO & DEM GO & INCRA 2003 & FAZ SEGREDO & Nova CRIXÁs & gо & 754 & $\begin{array}{c}\text { MÉDIA PROPOAIIDADE } \\
\text { PROOUTIVA }\end{array}$ & 754 & 0 & & \\
\hline 521040 & RONALDO RAMOS CAIADO & DEM GO & INCRA 2003 & FAZ CONQUISTA & ITABERAi & Go & 795 & $\begin{array}{c}\text { GRANDE PROPRIIIDADE } \\
\text { IMPRODUTIVA }\end{array}$ & 0 & 795 & & \\
\hline 521280 & RONALDO RAMOS CAAADO & DEMGO & INCRA 2003 & FAZ ESCALADA & MARA ROSA & Go & 968 & $\begin{array}{c}\text { GRANDE PROPRIEDADE } \\
\text { IMPRODUTIVA }\end{array}$ & 968 & ${ }^{\circ}$ & & \\
\hline 521483 & RONALDO RAMOS CAADO & DEM GO & INCRA 2003 & FAZ SANTA MARIA & NOVA CRIXÁs & 6o & 1120 & $\begin{array}{c}\text { GRANDE PROOPRIEDADE } \\
\text { PRODUTIVA }\end{array}$ & 1120 & 0 & & \\
\hline 522020 & $\begin{array}{l}\text { SANDRO ANTÓNIO SCODRO } \\
\text { (SANDRO MABEL) }\end{array}$ & PR GO & INCRA 2003 & FAZENDA SANTA CAMLA & SÃo MIGUEL DO ARAGUAIA & Go & 1860 & 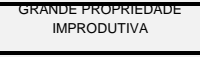 & 1860 & 0 & $\begin{array}{l}\text { SSANOVOAONT/ } \\
\text { ESCOORO): }\end{array}$ & 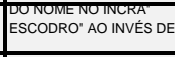 \\
\hline 520580 & EUNICIO LOPES DE OLIVEIRA & РMDB CE & INCRA 2003 & FAZENDA BEIA MAO & CORUMBÁ DE GOIÁS & Go & 12 & MIIIIÉNDIO & 12 & 0 & 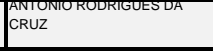 & \\
\hline 520580 & EUNICIO LOPES DE OLIVEIRA & PMDB CE & INCRA 2003 & FAZENDA S JOAO OU S LUIZ & CORUMBÁ DE GOÁS & Go & 50 & PEQUENA PROPRIIEDADE & 50 & 0 & (RON PINTO DE FARAA); & 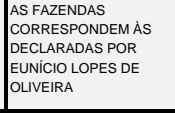 \\
\hline 520580 & EUNICIO LOPES DE OLIVEIRA & PMDB CE & INCRA 2003 & FAZENDA MATO DA CRUZ & CORUMBÁ DE GOIÁS & Go & 66 & PEQUENA PROPRIEDADE & 66 & 0 & $\begin{array}{l}\text { INOCENCIA DA COSTA } \\
\text { RAMOS }\end{array}$ & 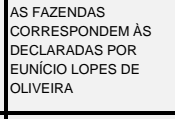 \\
\hline 520580 & EUNICIO LOPES DE OLIVEIRA & РMDB CE & INCRA 2003 & 'FAZENDA MANGABEIRA' & 'CORUMBÁ DE GOIÁS' & Go & 70 & PEQUENA PROPRIEDADE & 70 & 0 & & \\
\hline 520580 & EUNICIO LOPES DE OLIVEIRA & PMDB CE & INCRA 2003 & FAZ SAO LUIZ OU SAO JOAO & CORUMBÁ DE GOIÁS & Go & 96 & PEQUENA PROPRIEDADE & 96 & 0 & IRON PINTO DE FARIA); & 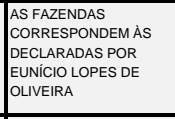 \\
\hline 520580 & EUNICIO LOPES DE OLIVEIRA & PMDB CE & INCRA 2003 & FAZENDA SAO GERALDO & CORUMBÁ DE GOÁS & Go & 98 & PEQUENA PROPRIEDADE & 98 & 0 & $\begin{array}{l}\text { DIMAS GOMES DO } \\
\text { NASCIMENTO } \\
\end{array}$ & 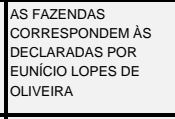 \\
\hline 520580 & EUNICIO LOPES DE OLIVEIRA & РMDB CE & INCRA 2003 & FAZENDA CONGONHAS DOS ALVES & CORUMBÁ DE GOIÁS & Go & 121 & PEQUENA PROPRIEDADE & 121 & 0 & & \\
\hline 520580 & EUNICIO LOPES DE OLIVEIRA & РMDB CE & INCRA 2003 & Faz BAU SEPULLUURA E SAo LUIZ & CORUMBÁ DE GOIÁS & go & 182 & 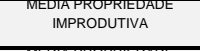 & 164 & 18 & NICANOR MOREIRA FARIAS); & 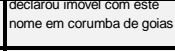 \\
\hline 520580 & EUNICIO LOPES DE OLIVEIRA & PMDB CE & INCRA 2003 & FAZENDA MANUELA & CORUMBÁ DE GOIÁS & Go & 193 & IMPRODUTIVA & 193 & 0 & & \\
\hline
\end{tabular}




\begin{tabular}{|c|c|c|c|c|c|c|c|c|c|c|c|c|}
\hline GEocoDiGo & PARLAMENTAR & PARTIDO & FONTE & IMÓveL & MUNICIPIO & uF & AREA & CATEGORIA FUNDIÁRIA & ÁREA REGISTRADA & $\begin{array}{l}\text { AREADEECLARAVA } \\
\text { COMO POSSE }\end{array}$ & $\begin{array}{l}\text { REGISTRO EM NOME DE } \\
\text { OUTRAS PESSOAS }\end{array}$ & $\begin{array}{l}\text { vinCULO COMO } \\
\text { PARLAMENTAR }\end{array}$ \\
\hline 520580 & EUNICIO LOPES DE OLIVEIRA & PMDBCE & INCRA 2003 & FAZENDA CONGONHA & CORUMBÁ DE GOIÁS & go & 200 & $\begin{array}{l}\text { IMPRODUTIVA } \\
\text { IMPA }\end{array}$ & 200 & 0 & & \\
\hline 520580 & EUNICIO LOPES DE OLIVEIRA & РМDB СE & INCRA 2003 & FAZENDA RETTRO E CERCADO & CORUMBÁ DE GOÁS & go & 257 & $\begin{array}{l}\text { IMPRODUTIVA } \\
\text { IPRA }\end{array}$ & 257 & 0 & & \\
\hline 520580 & EUNICIO LOPES DE OLIVEIRA & PMDBCE & INCRA 2003 & FAZENDA 3 B CONGONHA DOS ALVES & CORUMBÁ DE GOÁÁS & Go & 259 & $\begin{array}{l}\text { EUAAROPRIEDAD } \\
\text { IMPRODUTIVA }\end{array}$ & 259 & 0 & & \\
\hline 520580 & EUNICIO LOPES DE OLIVERA & РМDB CE & INCRA 2003 & Fazenda bau & CORUMBÁ DE GOÁS & Go & 338 & $\begin{array}{l}\text { EUAAPGOPIEUAD } \\
\text { IMPRODUTIVA }\end{array}$ & 0 & 338 & & \\
\hline 520580 & EUNICIO LOPES DE OLIVEIRA & PMDB CE & INCRA 2003 & FAZENDA OLARIA E MATO DO MEIO & CORUMBÁ DE GOIÁS & Go & 342 & $\begin{array}{l}\text { EDAAFOPRIEAL } \\
\text { IMPRODUTIVA }\end{array}$ & 0 & 342 & & \\
\hline 520580 & EUNICIO LOPES DE OLIVEIRA & РMDВ СE & INCRA 2003 & FAZENDA BUENOS & CORUMBÁ DE GOÁS & Go & 396 & IMPRODUTIVA & 0 & 396 & & \\
\hline 520580 & EUNICIO LOPES DE OLIVEIRA & PMDB CE & INCRA 2003 & FAZENDA SAO GERALDO & CORUMBÁ DE GOÁAS & Go & 474 & $\begin{array}{l}\text { EDIAPROPRIEDAL } \\
\text { IMPRODUTIVA }\end{array}$ & 474 & 0 & DARLA N MACEDO SOUZA & $\begin{array}{l}\text { COOREESTONTUDMAS } \\
\text { DECLARADAS POR }\end{array}$ \\
\hline 520580 & EUNICIO LOPES DE OLIVEIRA & PMDB CE & INCRA 2003 & FAZENDA SAO JOAO & CORUMBÁ DE GOIÁS & Go & 595 & IMPRODUTIVA & 226 & 369 & & \\
\hline 210330 & $\begin{array}{l}\text { SNITUNOTL } \\
\text { FLLHO }\end{array}$ & PPB MA & INCRA 2003 & BOI NAO BERRA & cODó & MA & 289 & $\begin{array}{l}\text { IEUAPFOPRAEOHC } \\
\text { IMPRODUTVA }\end{array}$ & 289 & 0 & $\begin{array}{l}\text { AlvUUN } \\
\text { FLLHO }\end{array}$ & \\
\hline 210350 & $\begin{array}{l}\text { CARLOS SRLEA } \\
\text { JUNIOR }\end{array}$ & PSDB MA & INCRA 2003 & FAZENDA TAMBURILL & COLINAS & MA & 250 & PEQUENA PROPRIEDADE & 250 & 0 & & \\
\hline 210140 & 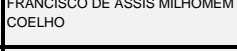 & PFLMA & INCRA 2003 & FAZENDA BREJAO & BALSAS & MA & 560 & 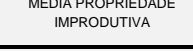 & 560 & 0 & 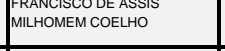 & \\
\hline 210005 & HÉLIO BATISTA DOS SANTOS & PSDB MA & INCRA 2003 & FAZENDA BOM JESUS & AÇALLÂANDIA & MA & 98 & PEQUENA PROPRIEDADE & 98 & 0 & & \\
\hline 211210 & $\begin{array}{l}\text { JOOACAATIELO IIBERO } \\
\text { GONCALVES }\end{array}$ & PSDB MA & INCRA 2003 & FAZENDAS SAO DOMINGOS & TIMBIRAS & MA & 2232 & 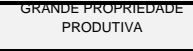 & 2168 & 64 & & \\
\hline 210845 & $\begin{array}{l}\text { JOAOCASTELC } \\
\text { GONCALVES }\end{array}$ & PSDB MA & INCRA 2003 & FAZENDA BOA ESPERANCA & PERITORÓ & MA & 3786 & $\begin{array}{l}\text { GRANUE PROPRIEA } \\
\text { PRODUIVA }\end{array}$ & 3781 & 0 & & \\
\hline 210290 & JOSE GOMES DA ROCHA & PSD GO & INCRA 2003 & FAZENDA BELA VISTA & CARUTAPERA & MA & 562 & $\begin{array}{l}\text { WEUEAPRHOPFIIIVAD } \\
\text { IMPRODUTIVA }\end{array}$ & 562 & 0 & JOSE GOMES ROCHA & \\
\hline 210140 & JOSE GOMES DA ROCHA & PSD GO & INCRA 2003 & FAZ SAN RODRIGO & BALLAS & MA & 634 & $\begin{array}{l}\text { MIEUAPROPREIEUAD } \\
\text { IMPRODTIVA }\end{array}$ & 634 & 0 & JJSE GOMES DA ROCHA & \\
\hline 211200 & JOSE GOMES DA ROCHA & PSD GO & INCRA 2003 & FAZENDA NOVA OLINDA & TASSO FRAGOSO & MA & 1237 & $\begin{array}{l}\text { GRANDE PROPRIEDADE } \\
\text { IMPRODUTIVA }\end{array}$ & 1237 & 0 & JOSE GOMES DA ROCHA & 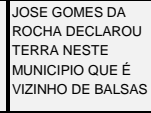 \\
\hline 210140 & JOSE GOMES DA ROCHA & PSD GO & INCRA 2003 & FAZENDA SANTA LUZIA DO RIO BALSAS & BALSAS & MA & 4595 & $\begin{array}{l}\text { PRODUTIVA } \\
\text { PRA }\end{array}$ & 4595 & 0 & JOSE GOMES DA ROCHA & \\
\hline 210750 & 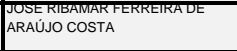 & PMDBAP & INCRA 2003 & FAZ CURUPU & PAÇO DO LUMIAR & MA & 1600 & $\begin{array}{l}\text { GFAIUEPROPRTIEADE } \\
\text { IMPRODUTIVA }\end{array}$ & 1600 & 0 & & $\begin{array}{l}\text { FARINAIVOJ JOSE } \\
\text { MACIERA SARNEY }\end{array}$ \\
\hline 210300 & 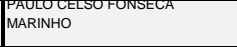 & PLMA & INCRA 2003 & FAZENDA RETIRO & CAXAAS & MA & 1075 & 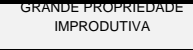 & 1075 & 0 & & \\
\hline 211120 & REMI ABREU TRINTA & PLMA & INCRA 2003 & SITIO PALOMA PAOLA & SÄO JOSÉ DE RIBAMAR & MA & 12 & MINIFÚNDIO & 12 & 0 & REMI ABREU TRINTA & \\
\hline 313440 & AELTON JOSE DE FREITAS & PPMG & INCRA 2003 & FAZ MONTE BELO & $\frac{1 \text { TURAMA }}{2}$ & MG & 24 & MIIIFUNDIO & 0 & 0 & & \\
\hline 313440 & AELTON JOSE DE FREITAS & $\frac{P P M G}{P}$ & INCRA 2003 & FAZ MONTE ALTO & ITURAMA & MG & 31 & PEQUENA PROPRIEDADE & 0 & 0 & & \\
\hline 313440 & AELTON JOSE DE FREITAS & $\begin{array}{l}\text { PPMG } \\
\text { PP }\end{array}$ & INCRA 2003 & FAZ MONTE ALTO & ITURAMA & MG & 64 & PEQUENA PROPRIEDADE & 0 & 0 & & \\
\hline 313862 & AELTON JOSE DE FREITAS & PPMG & INCRA 2003 & FAZ AGUA BOA & LIMEIRA DO OESTE & MG & 214 & $\begin{array}{l}\text { MEDIAPROPRIEDADE } \\
\text { IMPROUUTVA }\end{array}$ & 0 & 0 & & \\
\hline 313862 & AELTON JOSE DE FREITAS & $\begin{array}{l}\text { PPMG } \\
\text { PP }\end{array}$ & INCRA 2003 & FAZ RECANTO ALEGRE & LIMEIRA DO OESTE & MG & 240 & $\begin{array}{l}\text { MEDIA PROPRIEDADE } \\
\text { PRODUTIVA }\end{array}$ & 0 & 0 & & \\
\hline 311340 & ANIVALDO JUVENLL VALE & PSDB PA & INCRA 2003 & CORREGo do SUICO & CARATINGA & MG & 5 & $\begin{array}{l}\text { MEUIAPROPRIIIURAC } \\
\text { IMPRODUTIVA }\end{array}$ & 150 & 0 & ANIVALDO JUVENLL VALE & $\begin{array}{l}\text { REEEEIA } \\
\text { GERAIS }\end{array}$ \\
\hline 317100 & 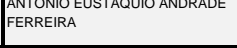 & Рмов ма & INCRA 2003 & FAZENDA SALOBO & VAZANTE & MG & 0 & MINIFÚNDIO & 0 & 22 & LOURIIVL AMERIICO DE MELO & \\
\hline 317100 & 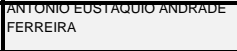 & РмDв МG & INCRA 2003 & FAZENDA SALOBO-ROCHEDO E PALMITO & VAzANTE & MG & 0 & PEQUENA PROPRIIEDADE & 119 & 0 & 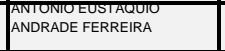 & \\
\hline 312950 & ARACELY DE PAULA & PRMG & INCRA 2003 & FAZ CACHOEIRA GRANDE & IBIA & MG & & $\begin{array}{l}\text { PEQUENA PROPRIEDADE } \\
\end{array}$ & & & & \\
\hline
\end{tabular}




\begin{tabular}{|c|c|c|c|c|c|c|c|c|c|c|c|c|}
\hline GEocodiaO & PaRLamentar & PARTIDO & FONTE & IMÓVEL & MUNICIPIO & UF & $\overline{\text { AREA }}$ & CATEGORIA FUNDIÁRIA & AREA REGISTRADA & $\begin{array}{l}\text { AREADEC PARAUI } \\
\text { COMO POSSE }\end{array}$ & $\begin{array}{l}\text { REGIISTRO EM NOME DE } \\
\text { OUTRAS PESSOAS }\end{array}$ & $\begin{array}{l}\text { viNCULO COM O } \\
\text { PARLAMENTAR }\end{array}$ \\
\hline 313290 & $\begin{array}{l}\text { CARLOS CARMO ANDRADE } \\
\text { MELLES }\end{array}$ & PMDBMG & INCRA 2003 & SIITIO SERRA NEGRA & ITAMOGI & MG & 27 & MINIFÚNDIO & 0 & $\overline{0}$ & & \\
\hline 316470 & $\begin{array}{l}\text { CARLOS CARMO ANDRADE } \\
\text { MELLES }\end{array}$ & PFLMG & INCRA 2003 & FAZ STO ANTONIO DO PAU DALLO & SAO SEBASTIAO DO PARAISO & MG & 76 & PEQUENA PROPRIEDADE & 0 & 0 & & \\
\hline 316470 & $\begin{array}{l}\text { CARLOS CARMO ANDRADE } \\
\text { MELLES }\end{array}$ & PMDB MG & INCRA 2003 & FAZ DIAMANTINA DE STO ANTONIO & SÁO SEBASTIÁO DO PARAISO & MG & 381 & $\begin{array}{l}\text { MEEUAPROPFIIIEAAUE } \\
\text { PRODUTIVA }\end{array}$ & 0 & $\overline{0}$ & & \\
\hline 310730 & CLEUBER BRANDAO CARNERO & PTBMG & INCRA 2003 & FAZENDA TIRIIIICA & BOCAIÚVA & MG & 0 & PEQUENA PROPRIEDADE & 41 & 0 & \begin{tabular}{|l|}
$\begin{array}{l}\text { CARLOS ELIISIO BRANDAO } \\
\text { CARNEIRO }\end{array}$ \\
\end{tabular} & $\begin{array}{l}\text { GRAUDE } \\
\text { PARENTENSCO NÄO } \\
\text { IDENTIFICADO }\end{array}$ \\
\hline \begin{tabular}{|l|}
310730 \\
\end{tabular} & CLLEUBER BAANDAO CARNEIRO & PTBMG & INCRA 2003 & FAZZNDA PEDREGULHO & BOCAIÚVA & MG & 0 & PEQUENA PROPRIEDADE & 87 & 0 & $\begin{array}{l}\text { LUCIIIAITY } \\
\text { CARNEIRO }\end{array}$ & $\begin{array}{l}\text { GRAUDE } \\
\text { PARENTENSCO NÃO }\end{array}$ \\
\hline 310730 & CLLEUBER BAANDAO CARNERO & PTBMG & INCRA 2003 & FAZENDA TIRIIIICA & BOCAIÚVA & MG & 0 & PEQUENA PROPRIEDADE & 104 & 0 & \begin{tabular}{|l|} 
MARINA DE FATIMA BRANDAO \\
CARNEIRO
\end{tabular} & $\begin{array}{l}\text { GAAUDED } \\
\text { PARENTENCO NÄO } \\
\text { IDENTIFICADO }\end{array}$ \\
\hline 310730 & CLEUBER BRANDAO CARNEIRO & PTBMG & INCRA 2003 & FAZ CERCADO DE BAIXO TIRIIIICA & BOCAIÚVA & MG & 0 & PEQUENA PROPRIEDADE & 137 & 0 & \begin{tabular}{|l|}
$\begin{array}{l}\text { CARLOS ELIISIO BRANDAO } \\
\text { CARNEIRO }\end{array}$ \\
\end{tabular} & $\begin{array}{l}\text { GRAUDE } \\
\text { PARENENSCO NÄO } \\
\text { IDENTIFICADO }\end{array}$ \\
\hline 310730 & CLEUGER BAANDAO CARNEIRO & PTBMG & INCRA 2003 & FAZENDA LAGOA & BOCAIÚVA & MG & 0 & PEQUENA PROPRIEDADE & 140 & 0 & \begin{tabular}{|l|}
$\begin{array}{l}\text { ANTONIO CARLOS BRANDAO } \\
\text { CARNEIRO }\end{array}$ \\
\end{tabular} & $\begin{array}{l}\text { GRAUDE } \\
\text { PAAENENSCO NÄO } \\
\text { IDENTIFICADO NÁ }\end{array}$ \\
\hline 312400 & DANLOO DE CASTRO & DEMMG & INCRA 2003 & SIITO BOM JARDIM & ERVALIA & MG & 18 & PEQUENA PROPRIEDADE & 0 & 0 & & \\
\hline 312400 & DANILO DE CASTRO & DEMMG & INCRA 2003 & FAZ CORREGO FRIO & ERVALLA & MG & 127 & PEQUENA PROPRIEDADE & 0 & 3 & & \\
\hline 310370 & DANILO DE CASTRO & DEMMG & INCRA 2003 & FAZ SAO DOMINGOS & ARAPONGA & MG & 184 & PEQUENA PROPRIEDADE & $\overline{0}$ & $\overline{0}$ & & \\
\hline 310720 & DIMAS FABIANO TOLEDO JUNIOR & PPMG & INCRA 2003 & SERTAO & BOCAINA DE MINAS & MG & 0 & PEQUENA PROPRIEDADE & 96 & 0 & DIMAS FABIANO TOLEDO & $\begin{array}{l}\text { PAIUE UIIIASTARB } \\
\text { TOLEDO JUNIOR }\end{array}$ \\
\hline 312230 & $\begin{array}{l}\text { DOMINGOS SAVIO CAMPOS } \\
\text { RESENDE }\end{array}$ & $\begin{array}{l}\text { PSDB MG } \\
\text { M }\end{array}$ & INCRA 2003 & FAZENDA FLORADA & DIIINÓPOLIS & MG & 14 & $\begin{array}{l}\text { TMEUIAPROPRIIEUAD } \\
\text { IMPRODUTIVA }\end{array}$ & 0 & 238 & Domingos savio & \\
\hline 312230 & $\begin{array}{l}\text { DOMINGOS SAVIO CAMPOS } \\
\text { RESENDE }\end{array}$ & PSDBMG & INCRA 2003 & FAZENDA FLORADA & DIIINÓPOLIS & MG & 14 & $\begin{array}{l}\text { MEEUAR PROPRIEDADE } \\
\text { PRODUTIVA }\end{array}$ & 0 & 235 & DOMINGOS SAVIO & \\
\hline 312940 & HERCULANO ANGHINETTI & PMDB MG & INCRA 2003 & FAZ ESTRELA DA MANHA & IBERTIOGA & MG & $\frac{120}{120}-x-3-1$ & PEQUENA PROPRIEDADE & 0 & 0 & & \\
\hline 313350 & JAIME MARTINS FILHO & PMDBMG & INCRA 2003 & GLEBA DO OLEO & TIAPECERICA & MG & 14 & $\begin{array}{l}\text { PEQUENA PROPRIEDADE } \\
\end{array}$ & 0 & 0 & & \\
\hline 313350 & JAIME MARTINS FLLHO & PMOB MG & INCRA 2003 & FAZ PEDRACA & $\begin{array}{l}\text { ITAPECERICA } \\
\text { I }\end{array}$ & MG & 19 & PEQUENA PROPRIEDADE & 0 & 0 & & \\
\hline 313350 & JAIME MARTINS FLLHO & PMDB MG & INCRA 2003 & FAZ PEDBACA & ITAPECERICA & MG & 19 & PEQUENA PROPRIEDADE & 0 & 0 & & \\
\hline 313350 & JAIME MARTINS FILHO & PMDBMG & INCRA 2003 & FAZ CAPOEIRA GRANDE & ITAPECERICA & MG & 25 & PEQUENA PROPRIEDADE & 0 & 0 & & \\
\hline 313350 & JAIME MARTINS FLLHO & PMDB MG & INCRA 2003 & FAZPEDRACA & ITAPECERICA & MG & 56 & PEQUENA PROPRIEDADE & 0 & 0 & & \\
\hline 314520 & JAIME MARTINS FLLHO & PMDBMG & INCRA 2003 & FAZ AMORIM & NOVA SERRANA & MG & 56 & PEQUENA PROPRIEDADE & 0 & 0 & & \\
\hline 420120 & $\begin{array}{l}\text { JOSE BONIFACIO TAMM DE } \\
\text { ANDRADA }\end{array}$ & PSDBMG & INCRA 2003 & CHACARA DOS ALMEIDAS & ANTÔNIO CARLOS & MG & 0 & MINIF́́NDIO & 20 & 0 & 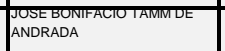 & \\
\hline 313910 & $\begin{array}{l}\text { JOSE BONIFACIO TAMM DE } \\
\text { ANDRADA }\end{array}$ & $\begin{array}{l}\text { PSDB MG } \\
\end{array}$ & INCRA 2003 & EStancia j b & MADRE DE DEUS DE MINAS & MG & 0 & PEQUENA PROPRIEDADE & 30 & 0 & $\begin{array}{l}\text { JOSEBONIIT } \\
\text { ANDRADA }\end{array}$ & \\
\hline 310560 & $\begin{array}{l}\text { JOSE BONIFACIO TAMM DE } \\
\text { ANDRADA }\end{array}$ & PSDBMG & INCRA 2003 & FAZENDA BORDA DO CAMPO & BARBACENA & MG & 0 & PEQUENA PROPRIEDADE & 45 & 0 & ANDRADA & \\
\hline 420120 & $\begin{array}{l}\text { JOSE BONIFACIO TAMM DE } \\
\text { ANDRADA }\end{array}$ & PSDBMG & INCRA & FAZENDA DO BELEM & ANTÔNIO CARLOS & MG & 11 & $\begin{array}{l}\text { TMEUAAPROPRIEVAL } \\
\text { IMPRODUTIVA }\end{array}$ & 125 & 0 & \begin{tabular}{|l} 
EOUIIFACIO \\
ANDRADA
\end{tabular} & \\
\hline 420120 & $\begin{array}{l}\text { JOSE BONIFACIO TAMM DE } \\
\text { ANDRADA }\end{array}$ & PSDBMG & INCRA 2003 & CHACARA DOS ALMEIDAS & ANTÓNIO CARLOS & MG & 20 & MIINFUUNDIO & 0 & 0 & & \\
\hline 313910 & $\begin{array}{l}\text { JOSE BONIFACIO TAMM DE } \\
\text { ANDRADA }\end{array}$ & PSDB MG & INCRA 2003 & ESTANCIA JB & MADRE DE DEUS DE MINAS & MG & 30 & PEQUENA PROPRIEDADE & 0 & 0 & & \\
\hline 420120 & $\begin{array}{l}\text { JOSE BONIFACIO TAMM DE } \\
\text { ANDRADA }\end{array}$ & $\begin{array}{l}\text { PSDBMG } \\
\end{array}$ & INCPA & FAZENDA DO CAMPO VERDE & ANTÔNIO CARLOS & MG & 39 & $\begin{array}{l}\text { MNIDEPROPRIIIEAF } \\
\text { IMPRODUTIVA }\end{array}$ & 432 & 0 & $\begin{array}{l}\text { JOSE BOUNI } \\
\text { ANDRADA }\end{array}$ & \\
\hline 420120 & $\begin{array}{l}\text { JOSE BONIFACIO TAMM DE } \\
\text { ANDRADA }\end{array}$ & PSDBMG & INCRA 2003 & FAZ DO CAMPO VERDE & ANTÓNIO CARLOS & MG & 432 & $\begin{array}{l}\text { GRANDE PROPRIEDADE } \\
\text { IMPRODUTIVA }\end{array}$ & & & & \\
\hline
\end{tabular}




\begin{tabular}{|c|c|c|c|c|c|c|c|c|c|c|c|c|}
\hline GEocodiao & PARLAMENTAR & PAATIDO & FONTE & IMÓVEL & MUNICIPIO & uF & AREA & CATEGORIA FUNDIÁRIAA & ÁREA REGISTRADA & $\begin{array}{l}\text { AREADEDCLAAAUA } \\
\text { COMO POSSE }\end{array}$ & $\begin{array}{l}\begin{array}{l}\text { REGISTRO EM NOME DE } \\
\text { OUTRAS PESSOAS }\end{array} \\
\text { OUTS }\end{array}$ & $\begin{array}{l}\text { viNCULO COM O } \\
\text { PARLAMENTAR }\end{array}$ \\
\hline 310560 & $\begin{array}{l}\text { JOSE BODIFACIO TAMM DE } \\
\text { ANDRADA }\end{array}$ & PSDBMG & INCRA 2003 & SITIO ESTIVA MAE MARIA & BARBACENA & MG & 0 & PEQUENA PROPRIEDADE & 67 & 0 & $\begin{array}{l}\text { JOSE RODRIGUES DE } \\
\text { OLVEIRA }\end{array}$ & 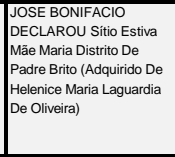 \\
\hline 314110 & JOSE MLITAO COSTA & PTBMG & INCRA 2003 & POUSADA DOS TUCANOS & MATOZINHOS & MG & 12 & PEQUENA PROPRIIEDADE & $\sigma^{\circ}$ & 0 & & \\
\hline 314110 & JOSE MLITAO COSTA & PTBMG & INCRA 2003 & POUSADA DOS TUCANOS & MATOZINHOS & MG & 0 & PEQUENA PROPRIEDADE & 12 & 0 & & \\
\hline 313170 & $\begin{array}{l}\text { JOSE SANTANA DE } \\
\text { VASCONCELOOS MOREIRA }\end{array}$ & DEMMG & $\begin{array}{ll}\text { INCRA } 2003 \\
\end{array}$ & FAZ DA FORTUNA & ITABIRA & MG & 641 & $\begin{array}{c}\text { GRANDE PROPPIEDADE } \\
\text { PRODUTVA }\end{array}$ & 0 & 0 & & \\
\hline 311455 & JOSÉ SILVA SOARES & PDTMG & INCRA 2003 & ESTANCIA PEQUI DA AGUA AMARELA & CARNERINHO & MG & 0 & PEQUENA PROPRIEDADE & 48 & 0 & JOSE SILVA SOARES & 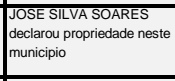 \\
\hline 313440 & $\begin{array}{l}\text { NETO } \\
\text { NETH }\end{array}$ & DEMAL & INCRA 2003 & FAZENDA MONTE ALTO III & ITURAMA & MG & 0 & MINIFÚNDIO & 22 & 0 & & \\
\hline 313440 & 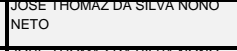 & DEM AL & INCRA 2003 & FAZENDA MONTE ALTO III & ITURAMA & MG & 0 & MINIFÚNDIO & 22 & 0 & & \\
\hline 313440 & 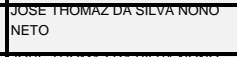 & DEM AL & INCRA 2003 & FAZENDA MONTE ALTO PERDIZES & ITURAMA & MG & 0 & PEQUENA PROPRIEDADE & 76 & 0 & & \\
\hline 313440 & 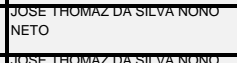 & DEM AL & INCRA 2003 & $\begin{array}{l}\text { FAZENDA MONTE ALTO PEROIZES } \\
\end{array}$ & ITURAMA & мG & 0 & PEQUENA PROPRIEDADE & 76 & 0 & & \\
\hline 313440 & 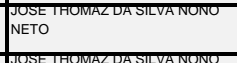 & DEM AL & INCRA 2003 & FAZENDA MONTE ALTO & ITURAMA & MG & 15 & 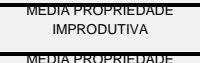 & 236 & 0 & & \\
\hline 313440 & $\begin{array}{l}\text { WOEST IHOWAZUA SLVA NONO } \\
\text { NETO }\end{array}$ & DEM AL & INCRA 2003 & FAZENDA MONTE ALTO & ITURAMA & MG & 15 & 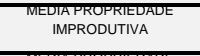 & 236 & 0 & & \\
\hline 313440 & 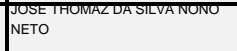 & DEM AL & INCRA 2003 & FAZENDA MONTE ALTO & ITURAMA & MG & 28 & $\begin{array}{l}\text { MEDIAPROPRIIEADV } \\
\text { IMPRODUIVA }\end{array}$ & 424 & 0 & & \\
\hline 313440 & $\begin{array}{l}\text { WOEST EROUAZUA SLVA NONO } \\
\text { NETO }\end{array}$ & DEM AL & INCRA 2003 & FAZENDA MONTE ALTO & ITURAMA & MG & 28 & $\begin{array}{l}\text { MEOEAPFOPFIIIUAUE } \\
\text { IMPROOUTVA }\end{array}$ & 424 & 0 & & \\
\hline 314390 & LAEL VEERA VARELLA & PMDB MG & INCRA 2003 & CHACARA LAGLORIA & MURAAE & MG & 25 & PEQUENA PROPRIEDADE & 0 & 0 & & \\
\hline 314390 & tAEL VEERA VARELLA & PMDB MG & INCRA 2003 & ESTANCIA BRASLLIA & MURIAE & MG & $\frac{44}{44}$ & PEQUENA PROPRIEDADE & 0 & 0 & & \\
\hline 312385 & $\begin{array}{l}\text { tEUNAREO } \\
\text { QUUNAOAO }\end{array}$ & РмОВ М̈ & INCRA 2003 & FAZENDA ATALAIA & ENTRE FOLHAS & MG & 0 & MIIIFÚNDIO & 14 & 0 & $\begin{array}{l}\text { EEBASTIAC } \\
\text { QUUNAO }\end{array}$ & 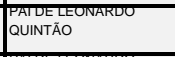 \\
\hline 317057 & 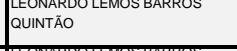 & РмОВ М̈ & INCRA 2003 & FAZENDA BETEL & VARGEM ALEGRE & MG & 9 & $\begin{array}{l}\text { PRAOUTIVA } \\
\text { PROPHOU }\end{array}$ & 329 & 0 & $\begin{array}{l}\text { SEBASTAOU } \\
\text { QuUNAOO }\end{array}$ & QUUNTAO \\
\hline 311340 & $\begin{array}{l}\text { QEUNATAOO } \\
\text { aü }\end{array}$ & РМОв МG & INCRA 2003 & FAZENDA OURO VERDE & CARATINGA & MG & 21 & $\begin{array}{l}\text { MEEDAPPORPRIIDVAV } \\
\text { IMPROOUTVA }\end{array}$ & 260 & 0 & $\begin{array}{l}\text { JEBASTAO } \\
\text { aUNTAO }\end{array}$ & 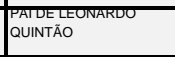 \\
\hline 316070 & LUZ FERNANDO RAMOS FARIA & PPMG & INCRA 2003 & FAZENDA COQUERROS & SANTOS DUMONT & ма & 16 & $\begin{array}{c}\text { MÉDIA PROPRIEDADE } \\
\text { IMPROOUTIVA }\end{array}$ & 219 & 0 & $\begin{array}{l}\text { PAULO ROBERTO RAMOS DE } \\
\text { FARIA }\end{array}$ & $\begin{array}{l}\text { LUIZ FERNANDO RAMMS } \\
\text { FARAA declarou tazenda } \\
\text { coguerios santos dumontt } \\
\text { me }\end{array}$ \\
\hline 311120 & MAURO RIBEIRO LOPES & DEMMG & INCRA 2003 & FAZZESTRETIO & CAMPO BELO & MG & 13 & MIIIFUNDIO & 0 & 0 & & \\
\hline 3111120 & $\begin{array}{l}\text { MAURO RIBEIRO LOPES } \\
\end{array}$ & DEMMG & INCRA 2003 & FAZ TECELAO & CAMPO BELO & MG & $\frac{102}{102}$ & PEQUENA PROPRIEDADE & 0 & 0 & & \\
\hline 311120 & MAURO RIBEIRO LOPES & DEMMG & INCRA 2003 & FAZENDA ESTREITO & САМРО ВЕLО & MG & 0 & MINIFÚNDIO & 13 & 0 & & \\
\hline 311120 & MAURO RIBEIRO LOPES & DEMMG & INCRA 2003 & FAZENDA TECELAO & САМРО вЕLО & MG & 0 & PEQUENA PROPRIEDADE & 42 & 0 & & \\
\hline 311120 & MAURO RIBEIRO LOPES & DEM MG & INCRA 2003 & FAZENDA TECELAO & САМРО ВеLо & MG & 0 & PEQUENA PROPRIEDADE & 102 & 0 & & \\
\hline 310665 & NEWTON CARDOSO & РМОВ МG & INCRA 2003 & FAZENDA MURIBECA & BERIZAL & MG & 29 & $\begin{array}{c}\text { MÉDIA PROPRILDADE } \\
\text { IMPRODUTINA } \\
\end{array}$ & 265 & 190 & JOSE AUGUSTO PESSOA & $\begin{array}{l}\text { NEWTON CARDOSO } \\
\text { UENCA AROPRIESADE } \\
\text { COM ETSENMMEN } \\
\text { ESTADO DE MG }\end{array}$ \\
\hline 315340 & $\begin{array}{l}\text { ODELMO LEAO CARNEIRO } \\
\text { SOBRINHO }\end{array}$ & PPMG & INCRA 2003 & FAZZENDA GAUCHA III & PRESIIENTE OLEGÁRIO & MG & зз & $\begin{array}{l}\text { MÉDIA PROPRIEDADE } \\
\text { PROOUTIVA }\end{array}$ & 644 & 0 & ROGERRIO LUIZ SEIBT & 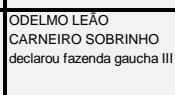 \\
\hline 317040 & OLAVO BILAC PINTO NETO & PRMG & INCRA 2003 & FAZENDA UNAI & unai & MG & 0 & PEQUENA PROPRIEDADE & 0 & 149 & $\begin{array}{l}\text { OLAVOBLALC PIVTONETOE } \\
\text { OUTROS }\end{array}$ & \\
\hline
\end{tabular}




\begin{tabular}{|c|c|c|c|c|c|c|c|c|c|c|c|c|}
\hline GEOCODIGO & PARLAMENTAR & PARTIDO & FONTE & IMóveL & MUNICIPPO & UF & AREA & CATEGORIA FUNDIÁRIAA & AREA REGISTRADA & $\begin{array}{l}\text { AREAUECLAARUUA } \\
\text { COMO POSSE }\end{array}$ & $\begin{array}{l}\text { REGISTRO EM NOME DE } \\
\text { OUTRAS PESSOAS }\end{array}$ & $\begin{array}{l}\text { vinculo com O } \\
\text { PARLAMENTAR }\end{array}$ \\
\hline 314800 & PAULO PAaU NoGUEIrA & РMDB MG & INCRA 2003 & FAZENDA RIBEIRAO DA MATA & PATOS DE MINAS & MG & 0 & MINIIÉNDIO & 9 & 0 & JAIRO GERALDO NOGUEIRA & PaI DE PAULo PIAU \\
\hline 314800 & PaUlo piau Nogueira & PMDB MG & INCRA 2003 & FAZENDA RIBEIIRAO DA MATA & PATOS DE MINAS & mG & 0 & PEQUENA PROPRIEDADE & 100 & 0 & JAIRO GERALDO NOGUEIRA & PaI DE PAULo piau \\
\hline 314390 & RENZO DO AMARAL BRAZ & PPMG & INCRA 2003 & GRANJA HASTENRETTER & MURAÉ & MG & 0 & MiNiÉñNolo & 17 & 0 & $\begin{array}{l}\text { MARIA REGINA BICALHO } \\
\text { HASTENREITER }\end{array}$ & 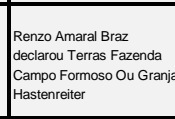 \\
\hline 314390 & RENZO DO AMARAL BRAZ & PPMG & INCRA 2003 & GRANJA HASTENREITER & MURIAÉ & MG & 0 & PEQUENA PROPRIEDADE & 28 & 0 & JOSE GERALDO DE FREITAS & 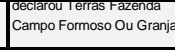 \\
\hline 313420 & ROMEL ANIZIO JORGE & PPMG & INCRA 2003 & FAZ MATAO & ITUUIUABA & MG & 180 & $\begin{array}{l}\text { GRANDE PROPRIEDADE } \\
\text { PRODUTIVA }\end{array}$ & $\overline{0}$ & 0 & & \\
\hline 313420 & ROMEL ANIZIO JORGE & PPMG & INCRA 2003 & FAZ OLHOS DAGUA & ITUIUTABA & MG & 343 & $\begin{array}{l}\text { MEDIA PROPRIEDADE } \\
\text { PRODUTIVA }\end{array}$ & 0 & 0 & & \\
\hline 313862 & ROMEL ANIZIO JORGE & PPMG & INCRA 2003 & FAZ THALITA & LIMEIRA DO OESTE & MG & 432 & $\begin{array}{l}\text { MEEDA PROPRIEDADE } \\
\text { PRODUTIVA }\end{array}$ & 0 & 0 & & \\
\hline 313862 & FOMEL ANIZIO JORGE & PPMG & INCRA 2003 & FAZ THALITAIII & LIMEIRA DO OESTE & MG & 976 & $\begin{array}{l}\text { GRANDE PROPRIEDADE } \\
\text { PRODUTIVA }\end{array}$ & 0 & 0 & & \\
\hline 311930 & ROMEU FERRERAA DE QUEROZZ & PTBMG & INCRA 2003 & FAZ SUCURI EARAUJOS & COROMANDEL & MG & 100 & PEQUENA PROPRIEDADE & 0 & 0 & & \\
\hline 311930 & ROMEU FERREIRA DE QUEROZZ & PTBMG & INCRA 2003 & FAZ ARCOS BURACAO & COROMANDEL & MG & 103 & PEQUENA PROPRIEDADE & 0 & 0 & & \\
\hline 314810 & ROMEU FERREIRA DE QUERROZ & PTBMG & INCRA 2003 & FAZ ESMERIL & PATROCINIO & MG & 223 & $\begin{array}{l}\text { MEEDAA PROPRIEDADE } \\
\text { PRODUTIVA }\end{array}$ & 0 & 0 & & \\
\hline 31930 & ROMEU FERREIRA DE QUEROZZ & PTBMG & INCRA 2003 & FAZ ARAUSO E SUCURI & COROMANDEL & MG & 233 & $\begin{array}{l}\text { MEDIA PROPRIEDADE } \\
\text { PRODUTIVA }\end{array}$ & 0 & 0 & & \\
\hline 311930 & ROMEU FERREIRA DE QUERORZ & PTBMG & INCRA 2003 & FAZ SERRA VERDE SUCURI E ARAUJ & COROMANDEL & MG & 440 & $\begin{array}{l}\text { MEDIA PROPRIEDADE } \\
\text { PRODUTIVA }\end{array}$ & 0 & 0 & & \\
\hline 311930 & ROMEU FERREIRA DE QUEROZZ & PTBMG & INCRA 2003 & FAZ CUCURI E ARAUJOS & $\begin{array}{l}\text { COROMANDEL } \\
\end{array}$ & MG & 903 & $\begin{array}{c}\text { GRANDE PROPRIIEADE } \\
\text { IMPRODUTIVA }\end{array}$ & 0 & 0 & & \\
\hline 344810 & SIILAS BRASILEIRO & PMDB MG & INCRA 2003 & FAZ SANTO ANTONIO & PATROCINIO & MG & 14 & MINIFUNDIO & 0 & 0 & & \\
\hline 344810 & SIILAS BRASILEIRO & PSDBMG & INCRA 2003 & FAZ OURO VERDE & PATROCINIO & $M G$ & 15 & MINIFUUNDIO & 0 & 0 & & \\
\hline 314810 & SILAS BRASILEIRO & PMDBMG & INCRA 2003 & FAZ ESMERIL TAMANDUA & $\begin{array}{l}\text { PATROCINIO } \\
\end{array}$ & MG & 23 & MINIFUUDDIO & 0 & 0 & & \\
\hline 314810 & SIILAS BRASILEIRO & PMDBMG & INCRA 2003 & FAZ ESMERIL & PATROCINIO & MG & 24 & MINIFUNDIO & $\overline{0}$ & 0 & & \\
\hline 310920 & SILAS BRASILEIRO & PMDBMG & INCRA 2003 & FAZ ROMARIA & BUENÓPOLIS & MG & 25 & MINIFUUNDIO & 0 & 473 & & \\
\hline 314810 & SIILAS BRASILEIRO & PMDBMG & INCRA 2003 & $\begin{array}{l}\text { FAZ FOLHADOS ESPIGAO } \\
\end{array}$ & PATROCINIO & MG & 29 & MINIFUNDIO & 0 & 0 & & \\
\hline 314810 & SILAS BRASILEIRO & PMDBMG & INCAA 2003 & $\begin{array}{l}\text { FAZ MUMBECA } \\
\end{array}$ & PATROCINIO & MG & 42 & MINIFUUNDIO & 0 & 0 & & \\
\hline 314810 & SILAS BRASILERO & PMDB MG & INCRA 2003 & FAZ CAMPO LIMPO & PATROCINIO & MG & 60 & $\overline{P E Q U E N A ~ P R O P R I E D A D E ~}$ & 0 & 0 & & \\
\hline 31930 & SILAS BRASILEIRO & PMDB MG & INCRA 2003 & FAZ TABUOES & COROMANDEL & $M G$ & 100 & PEQUENA PROPRIEDADE & 0 & 0 & & \\
\hline 314810 & SILAS BRASILEIRO & PMDBMG & INCRA 2003 & FAZ DAS POSSES & $\begin{array}{l}\text { PATROCINIO } \\
\end{array}$ & MG & 179 & $\begin{array}{c}\text { MEDIA PROPRIEDADE } \\
\text { IMPRODUTVA }\end{array}$ & 0 & 0 & & \\
\hline 314810 & SILAS BRASILEIRO & PMDB MG & INCRA 2003 & FAZ FOLHADOS & PATROCINIO & MG & 188 & $\begin{array}{l}\text { MEDIA PROPRIEDADE } \\
\text { IMPROOUTIVA }\end{array}$ & 0 & 0 & & \\
\hline 314810 & SILAS BRASILEIRO & PMDB MG & INCRA 2003 & FAZ CHAPADA & PATROCINIO & MG & 744 & $\begin{array}{c}\text { GRANDE PROPRIEDADE } \\
\text { PRODUTIVA }\end{array}$ & 0 & 0 & & \\
\hline 314810 & SILAS BRASILEIRO & PMDBMG & INCRA 2003 & FAZ BOA VISTA & $\begin{array}{l}\text { PATROCINIOO } \\
\end{array}$ & MG & 832 & $\begin{array}{c}\text { GRANDE PROPRIEDADE } \\
\text { PRODUTIVA }\end{array}$ & 0 & 0 & & \\
\hline 311930 & SILAS BRASILEIRO & PMDBMG & INCRA 2003 & FAZ CHAPADAO DOS BORGES & $\begin{array}{l}\text { COROMANDEL } \\
\end{array}$ & MG & 884 & $\begin{array}{c}\text { GRANDE PROPRIEDADE } \\
\text { PRODUTIVA }\end{array}$ & 0 & 0 & & \\
\hline 313370 & WALTER DA ROCHA TOSTA & PMNMG & INCRA 2003 & SITIO NOGUEIRA TOSTA & itatiançu & MG & 0 & MINIFÚNDIO & 3 & 0 & WALTER DA ROCHA TOSTA & \begin{tabular}{|l} 
WOALITEA \\
TOSTA
\end{tabular} \\
\hline 510787 & BLARO BORGES MAGGI & PRMT & INCRA 2003 & FAZENDA SAPEZZAL & SAPEZAL & мT & 9 & MINIF́́nDIO & 0 & 9 & ERAI MAGGI ISHEFFER & $\begin{array}{l}\text { BORGES MAGGI } \\
\end{array}$ \\
\hline
\end{tabular}




\begin{tabular}{|c|c|c|c|c|c|c|c|c|c|c|c|c|}
\hline GEocodiao & PARLAMENTAR & PAATIDO & FONTE & IMÓVEL & MUNICIPIO & $\mathrm{UF}$ & AREA & CATEGORIA FUNDIÁRIA & AREA REGISTRADA & $\begin{array}{l}\text { AREAEDCLAARADI } \\
\text { COMO POSSE }\end{array}$ & $\begin{array}{l}\text { REGISTRO EM NOME DE } \\
\text { OUTRAS PESSOAS }\end{array}$ & $\begin{array}{l}\text { viNCULO COM O } \\
\text { PARLAMENTAR }\end{array}$ \\
\hline 510263 & BLARO BORGES MAGGI & PRMT & INCRA 2003 & PARTE DA FAZENDA SAO FRANCISCO & CAMPO NOVO DO PARECIS & мт & 10 & MINIF́́NDIo & 10 & 0 & ERAI MAGGI SCHEFFER & $\begin{array}{l}\text { BORGES MAGGI } \\
\text { BOAG }\end{array}$ \\
\hline 510460 & BLARO BORGES MAGGI & PR MT & INCRA 2003 & FAZENDA SM 38 & ITIQUIRA & мт & 14 & MINIFÚNDIO & 14 & 0 & ANDRE ANTONIO MAGGI & $\begin{array}{l}\text { APIDEG } \\
\text { MAGGI }\end{array}$ \\
\hline 510460 & BLAIRO BORGES MAGGI & PR MT & INCRA 2003 & FAZENDA SM 7 & ITIQUIRA & мт & 242 & $\begin{array}{l}\text { EUTAPROPRIEDAL } \\
\text { IMPRODUTIVA }\end{array}$ & 242 & 0 & ANDRE ANTONIO MAGGI & $\begin{array}{l}\text { PAIDEBLAAITOBO } \\
\text { MAGGI }\end{array}$ \\
\hline 510787 & BLAARO BORGES MAGGI & PRMT & INCRA 2003 & FAZ SANTO ANTONIO & SAPEZAL & мт & 468 & $\begin{array}{l}\text { MÉDIA PROPRIEDADE } \\
\text { IMPROOUTIVA }\end{array}$ & & & & \\
\hline 510787 & BLARO BORGES MAGGI & PRMT & INCRA 2003 & FAZENDA GLEBA JUREMA II & SAPEZAL & мт & 500 & 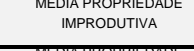 & 500 & 0 & ANDRE ANTONIO MAGGI & MAGGI \\
\hline 510787 & BLARO BORGES MAGGI & PR MT & INCRA 2003 & FAZENDA COMELI & SAPEZAL & мт & 502 & IMPROOUTIVA & 0 & 502 & ANDRE ANTONIO MAGGI & $\begin{array}{l}\text { AAIGGI } \\
\text { MAGG }\end{array}$ \\
\hline 510787 & BLARO BORGES MAGGI & PRMT & INCRA 2003 & FAZENDA AGUA DOCE & SAPEZAL & мт & 700 & $\begin{array}{l}\text { EDAPFRPRIERA } \\
\text { PRODUTIVA }\end{array}$ & 700 & 0 & 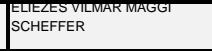 & $\begin{array}{l}\text { GRRADERARERNES } \\
\text { NAO IDENTIFICADO }\end{array}$ \\
\hline 510460 & BLARO BORGES MAGGI & PR MT & INCRA 2003 & FAZENDA SM 5B & ITIQUIRA & мт & 810 & PRODUTIVA & 810 & 0 & ANDRE ANTONIO MAGGI & MAGGI \\
\hline 510787 & BLAIRO BORGES MAGGI & PR MT & INCRA 2003 & FAZENDA DO CEZAR & SAPEZAL & мт & 1004 & 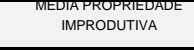 & 1004 & 0 & ANDRE ANTONIO MAGGI & MAGGI \\
\hline 510787 & BLAIRO BORGES MAGGI & PRMT & INCRA 2003 & FAZENDA SAPEZZAL & SAPEZAL & мт & 1012 & 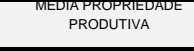 & 1012 & 0 & ELUSMAR MAGGI SCHEFFER & $\begin{array}{l}\text { JFIIIADEDEDARO } \\
\text { BORGES MAGGI }\end{array}$ \\
\hline 510460 & BLARO BORGES MAGGI & PR MT & INCRA 2003 & FAZENDA SM5B & ITIQUIRA & мт & 1210 & PRODUTIVA & 1210 & 0 & ANDRE ANTONIO MAGGI & MAGGI \\
\hline 510787 & BLARO BORGES MAGGI & PR MT & INCRA 2003 & SANTA BARBARA II & SAPEZAL & мт & 1210 & IMPRODUTIVA & 1210 & 0 & ANDRE ANTONIO MAGGI & MAGGI \\
\hline 510787 & BLAARO BORGES MAGGI & PR MT & INCRA 2003 & FAZENDA MATOS & SAPEZAL & мт & 1311 & $\begin{array}{l}\text { PRODUTIVA } \\
\text { PRTOE }\end{array}$ & 1311 & 0 & ANDRE ANTONIO MAGGI & 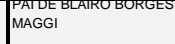 \\
\hline 510460 & BLAIRO BORGES MAGGI & PR MT & INCRA 2003 & FAZENDA SM 6 & ITIQUIRA & мт & 1333 & 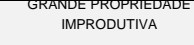 & 1333 & 0 & ANDRE ANTONIO MAGGI & \begin{tabular}{|l} 
PAIDEE \\
MAGGI
\end{tabular} \\
\hline 510460 & BLAIRO BORGES MAGGI & PRMT & INCRA 2003 & FAZENDA SM 6 A & ITIQUIRA & мт & 1435 & $\begin{array}{l}\text { ANUEFROOPREDUAUE } \\
\text { IMPROOUTIVA }\end{array}$ & 1435 & 0 & ANDRE ANTONIO MAGGI & 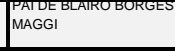 \\
\hline 510267 & BLARO BORGES MAGGI & PRMT & INCRA 2003 & FAZENDA SANTA FITA & CAMPO VERDE & мт & 1458 & 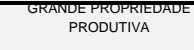 & 1458 & 0 & ERAI MAGGI SCHEFFER & 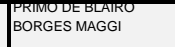 \\
\hline 510787 & BLAIRO BORGES MAGGI & PR MT & INCRA 2003 & FAZENDA ANA HELIO & SAPEZAL & мт & 1567 & 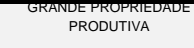 & 1567 & 1567 & JONAS SCHAEFFER MAGGI & $\begin{array}{l}\text { GGRAUDE PARENIES } \\
\text { NAOO IDENTIFICADO }\end{array}$ \\
\hline 510787 & BLARO BORGES MAGGI & PR MT & INCRA 2003 & FAZENDA PROGRESSO & SAPEZAL & мт & 1672 & $\begin{array}{r}\text { N629/93 } \\
\text { NAOCLSTA }\end{array}$ & 1672 & 0 & ERAI MAGGI SCHEFFER & BORGES MAGGI \\
\hline 510787 & BLARO BORGES MAGGI & PR MT & INCRA 2003 & FAZENDA DESCANSO & SAPEZAL & мт & 1969 & IMPRODUTIVA & 0 & 1969 & ANDRE ANTONIO MAGGI & $\begin{array}{l}\text { ADEGI } \\
\text { MAGCARO }\end{array}$ \\
\hline 510787 & BLARO BORGES MAGGI & PR MT & INCRA 2003 & FAZENDA PROGRESSOI & SAPEZAL & мт & 1999 & $\begin{array}{l}\text { ANINUEFROPRIIIUA } \\
\text { IMPRODUTIVA }\end{array}$ & 0 & 1999 & JONAS SCHAEFFER MAGGI & $\begin{array}{l}\text { GRAQDER PRERNIES } \\
\text { NAO IDENTIFICADO }\end{array}$ \\
\hline 510785 & BLARO BORGES MAGGI & PRMT & INCRA 2003 & Magnus & SĀo FÉLIX DO ARAGUAIA & мт & 2019 & IMPROOUTIVA & 2019 & 0 & ANTONIO MAGNUS MAGGI & $\begin{array}{l}\text { AAGGE } \\
\end{array}$ \\
\hline 510787 & BLARO BORGES MAGGI & PR MT & INCRA 2003 & AGROPECUARIA SAO PERDO IE II & SAPEZAL & мт & 2028 & PROOUTIVA & 2028 & 0 & ERAI MAGGI SCHEFFER & BORGES MAGGI \\
\hline 510267 & BLARO BORGES MAGGI & PRMT & INCRA 2003 & FAZENDA FARTURA & CAMPO VERDE & мт & 2112 & $\begin{array}{l}\text { AINUER ROOPRIEDAVE } \\
\text { PRODUTVA }\end{array}$ & 2112 & 0 & ELUSMAR MAGGI SCHEFFER & $\begin{array}{l}\text { FPFIIIAUEEBEARIRO } \\
\text { BORGES MAGGI }\end{array}$ \\
\hline 510267 & BLARO BORGES MAGGI & PR MT & INCRA 2003 & FAZENDA AGUA AZUL & CAMPO VERDE & мт & 2323 & $\begin{array}{l}\text { ANUER FOPFIIIE } \\
\text { PRODUVA }\end{array}$ & 2323 & 0 & ERAI MAGGI SCHEFFER & BORGES MAGGI \\
\hline 510787 & BLAARO BORGES MAGGI & PR MT & INCRA 2003 & FAZENDA ESPERANCA & SAPEZAL & мт & 2849 & $\begin{array}{l}\text { P PODUTIVA } \\
\text { PRA }\end{array}$ & 2849 & 0 & ERAI MAGGI SCHEFFER & $\begin{array}{l}\text { BORGES MAGGI } \\
\text { BaOG }\end{array}$ \\
\hline 510787 & BLARO BORGES MAGGI & РR MT & INCRA 2003 & FaZZNDA SANTA LUZIA & SAPEZAL & мт & 3060 & $\begin{array}{l}\text { ANUEFROPFIIID } \\
\text { PRODUTIVA }\end{array}$ & 3060 & 0 & ERAQUE MAGGI SCHEFFER & $\begin{array}{l}\text { PFIIIIODE BLAIRO } \\
\text { BORGES MAGGI }\end{array}$ \\
\hline 510787 & BLARO BORGES MAGGI & PR MT & INCRA 2003 & FAZZNDA SANTA LUZIA & SAPEZAL & мт & 3060 & $\begin{array}{l}\text { ANUERPOPPIIEU } \\
\text { PRODUTIVA }\end{array}$ & 3060 & 0 & ERAI MAGGI SCHEFFER & $\begin{array}{l}\text { |PRIIIOUE BLAIRO } \\
\text { BORGES MAGGI }\end{array}$ \\
\hline 510760 & BLARO BORGES MAGGI & PR MT & INCRA 2003 & FAZENDA PONTE DE PEDRA & RONDONÓPOLIS & мт & 7229 & $\begin{array}{l}\text { ANUERPOPPIIED } \\
\text { PRODUTVA }\end{array}$ & 7229 & 0 & ANDRE ANTONIO MAGGI & $\begin{array}{l}\text { PAIDEELAIISOBC } \\
\text { MAGGI }\end{array}$ \\
\hline 510770 & CARLOS GOMES BEZERRA & Рмов Мт & INCRA 2003 & FAZENDA PARAISO V & ROSÁRIIO OESTE & мт & 17 & MINIF́́NDIO & 0,00 & 17 & DIOGO GOMES BEZERRA & $\begin{array}{l}\text { GARUU UE PARENES } \\
\text { NAOO IDENTIFICADO }\end{array}$ \\
\hline 510770 & CARLOS GOMES BEZERRA & Рмов Мт & INCRA 2003 & FAZENDA PARAISO Iv & ROSÁRIIO OESTE & мт & 30 & MINIF́́nDIO & 0,00 & 30 & DIOGO GOMES BEZERRA & $\begin{array}{l}\text { GRAQ DERARENTES } \\
\text { NAO IDENTIFICADO }\end{array}$ \\
\hline 510770 & CARLOS GOMES BEZERRA & РмDв мт & INCRA 2003 & FAZENDA PARAISO III & ROSÁRIO OESTE & мт & 62 & MINIFÚNDIO & 0,00 & 62 & DIOGO GOMES BEZERRA & NÂO IDENTIFICADO \\
\hline 510630 & CARLOS GOMES BEZERRA & РМов Мт & INCRA 2003 & СHACARA ROMALU & paranatinga & мт & 78 & MINIFÚNDIO & 78,00 & 0 & 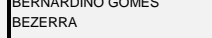 & 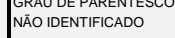 \\
\hline
\end{tabular}




\begin{tabular}{|c|c|c|c|c|c|c|c|c|c|c|c|c|}
\hline GEOCODIGO & PARLAMENTAR & PARTIDO & FONTE & IMÓVEL & MUNICIPPO & $\mathrm{UF}$ & AREA & CATEGORIA FUNDIÁRIIA & ÁREA REGISTRADA & $\begin{array}{l}\text { AREAUELCAAAUA } \\
\text { COMO POSSE }\end{array}$ & $\begin{array}{l}\text { REGISTRO EM NOME DE } \\
\text { OUTRAS PESSOAS }\end{array}$ & $\begin{array}{l}\text { viNCULO COM O } \\
\text { PARLAMENTAR }\end{array}$ \\
\hline 510770 & CARLOS GOMES BEZERRA & РМDB MT & INCRA 2003 & FAZENDA PARAISO II & ROSÁRIO OESTE & мт & 103 & PEQUENA PROPRIEDADE & 0,00 & 103 & DIOGO GOMES BEZERRA & $\begin{array}{l}\text { GRAAUEPARENES } \\
\text { NAO IDNTIFICADO }\end{array}$ \\
\hline 510760 & CARLOS GOMES BEZERRA & РМDВ Мт & INCRA 2003 & ESTANCIA BELA VISTA & RONDONÓPOLIS & мт & 122 & PEQUENA PROPRIEDADE & 122,00 & 0 & EMANOEL GOMES BEZERRA & NÄO IDENTIFICADO \\
\hline 510770 & CARLOS GOMES BEZERRA & РМDв Мт & INCRA 2003 & FAZENDA PARAISOI & ROSÁRIO OESTE & мт & 304 & PEQUENA PROPRIIEDADE & 304,00 & 0 & DIOGO GOMES BEZERRA & NAAO IDENTIFICADO \\
\hline 510645 & CARLOS GOMES BEZERRA & РМDВ Мт & INCRA 2003 & PARTE DA FAZENDA RANCHARIA & PLANALTO DA SERRA & мт & 358 & PEQUENA PROPRIEDADE & 0,00 & 358 & MAXIMO GoMES BEZERRA & 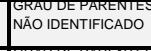 \\
\hline 510620 & CARLOS GOMES BEZERRA & РMDB MT & INCRA 2003 & PARTE RAZENDA RANCHARIA & NOVA BRASILÂNDIA & мт & 358 & PEQUENA PROPRIEDADE & 358,00 & 0 & OSVALDO GOMES BEZERRA & $\begin{array}{l}\text { GRAOUE PREENES } \\
\text { NÂO IDENTIFIIADO }\end{array}$ \\
\hline 510700 & CARLOS GOMES BEZERRA & РMDB MT & INCRA 2003 & FAZENDA BURITI ALEGRE & POXORÉO & мт & 400 & PRODUTIVA & 400,00 & 0 & 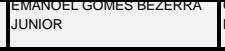 & 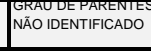 \\
\hline 510770 & CARLOS GOMES BEZERRA & РМDВ Мт & INCRA 2003 & FAZENDA AVANTE SAO JORGE & ROSÁRIO OESTE & мт & 614 & 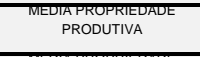 & 614,00 & 0 & ERASMO GOMES BEZERRA & $\begin{array}{l}\text { FGARUERPAREDNES } \\
\text { NAO IDENTIFICADO }\end{array}$ \\
\hline 510630 & CARLOS GOMES BEZERRA & РMDB MT & INCRA 2003 & FAZENDA CACHOEIRA DO NORTE & PARANATINGA & мт & 912 & 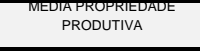 & 912,00 & 0 & ERASMO GOMES BEZERRA & $\begin{array}{l}\text { GRAOUE PREENES } \\
\text { NAOO IDENTIFIIADO }\end{array}$ \\
\hline 510645 & CARLOS GOMES BEZERRA & РMDB MT & INCRA 2003 & FAZENDA CAMPO BELO & PLANALTO DA SERRA & мт & 1490 & 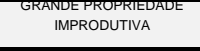 & 1490,00 & 0 & \begin{tabular}{|l} 
BEERERSBAC \\
\end{tabular} & 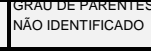 \\
\hline 510620 & CARLOS GOMES BEZERRA & РМDВ Мт & INCRA 2003 & FAZENDA SAO BENEDITO OU ACORIZAL & NOVA BRASILÂNDIA & мт & 1927 & 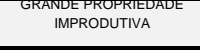 & 1927,00 & 0 & OSVALDO GOMES BEZERRA & $\begin{array}{l}\text { GRAOUDEPARENES } \\
\text { NAO IDNTIFICADO }\end{array}$ \\
\hline 510630 & CARLOS GOMES BEZERRA & РMDB MT & INCRA 2003 & LOTE CLAUdio & PARANATINGA & мт & 2537 & $\begin{array}{l}\text { IANOERROPRIIVOA } \\
\text { IMPROUTVA }\end{array}$ & 2537,00 & 0 & & \\
\hline 510630 & CARLOS GOMES BEZERRA & РMDB MT & INCRA 2003 & TANIAMAR & PARANATINGA & мт & 2581 & $\begin{array}{l}\text { RANUERROPRIIIUFA } \\
\text { IMPROOUTVA }\end{array}$ & 2581,00 & 0 & & \\
\hline 510630 & CARLOS GOMES BEZERRA & РMDB MT & INCRA 2003 & FAZ CACHOERAO & PARANATINGA & мт & 2899 & 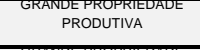 & 2999,00 & 0 & $\begin{array}{l}\text { EERRARTUI } \\
\text { BEZERAA }\end{array}$ & $\begin{array}{l}\text { GRAOUDEPARENES } \\
\text { NAO IDENTIFICADO }\end{array}$ \\
\hline 510630 & CARLOS GOMES BEZERRA & РмDв Мт & INCRA 2003 & GLEBA CAIAPO & PARANATINGA & мт & 4198 & IMPRODUTIVA & 4198,00 & 0 & ANTONIO GOMES BEZERRA & NÁO IDENTIFICADO \\
\hline 510630 & CARLOS GOMES BEZERRA & РмDв Мт & INCRA 2003 & FAZ TRES MORROS & PARANATINGA & мт & 4600 & IMPRODUTIVA & 0,00 & 4600 & & \\
\hline 510267 & $\begin{array}{l}\text { JOÄO SPERAFICO } \\
\text { JuLEU }\end{array}$ & PSDB MS E PP PR & INCRA 2003 & FAZENDA DIIINO PAI ETERNO & CAMPO VERDE & мт & ${ }_{134}$ & PEQUENA PROPRIEDADE & 134 & & LEVINO JOSE SPERAFICO & \\
\hline 510267 & $\begin{array}{l}\text { JOALO SPERAATIOOTE } \\
\text { JOÁ SPAFO }\end{array}$ & PSDB MS e PP PR & INCRA 2003 & FAZENDA RECANTO DA FELCIDADE & CAMPO VERDE & мт & ${ }_{150}$ & PEQUENA PROPRIEDADE & 150 & & DILCEU JOAO SPERAFICO & \\
\hline 510267 & 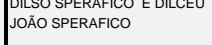 & PSDB MS e PP PR & INCRA 2003 & FAZENDA TUTRANA & CAMPO VERDE & мт & 239 & PEQUENA PROPRIEDADE & 0 & 23 & DILCEU JOAO SPERAFICO & \\
\hline 510590 & 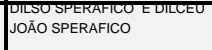 & PSDB MS e PP PR & INCRA 2003 & PARTE LOTE 10 GLEBA TRIVELATTO & NOBRES & мт & 363 & $\begin{array}{l}\text { MEUIAPROPRIIEDADE } \\
\text { IMPRODUTIVA }\end{array}$ & 363 & & ELOI SPERAFICO & \\
\hline 510267 & 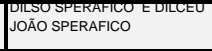 & PSDB MS e PP PR & INCRA 2003 & FAZENDA AGUA BRANCA & CAMPO VERDE & мт & 434 & 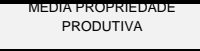 & 434 & & OLVINO JOSE SPERAFICO & \\
\hline 510267 & 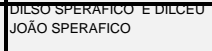 & PSDB MS e PP PR & INCRA 2003 & FAZENDA SANTA JULIANA & CAMPO VERDE & мт & 496 & $\begin{array}{l}\text { EUAPFROPRIEAA } \\
\text { PRODUTVA }\end{array}$ & 496 & & ELOI SPERAFICO & \\
\hline 510787 & $\begin{array}{l}\text { DJLLOSPREARTIOE } \\
\text { JOÄO SPERAFICO }\end{array}$ & PSDB MS E PP PR & INCRA 2003 & FAZENDA SPERAFICO ॥ & $\begin{array}{l}\text { SAPEZAL } \\
\end{array}$ & мт & 501 & $\begin{array}{l}\text { EUARPGOPRIEDAT } \\
\text { IMPRODUTIVA }\end{array}$ & 0 & 49 & 9 LEVINO JOSE SPERAFICO & \\
\hline 510787 & 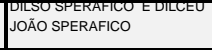 & PSDB MS e PP PR & INCRA 2003 & ALTO SAPEZAL & SAPEZAL & мт & 726 & $\begin{array}{l}\text { EUDAPFOPRIEDAL } \\
\text { IMPRODUTIVA }\end{array}$ & 0 & 72 & LEVINO JOSE SPERAFICO & \\
\hline 510455 & $\begin{array}{l}\text { DOLLO SPRERATCOE } \\
\text { JOÄO SPERAFICO }\end{array}$ & PSDB MS e PP PR & INCRA 2003 & CRUZERo do SUL & ITAÚBA & мт & ${ }_{1452}$ & 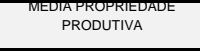 & 1452 & & DILCEU JOAO SPERAFICO & \\
\hline 510350 & $\begin{array}{l}\text { DOALO SPPERAFICO } \\
\text { JoÁO }\end{array}$ & PSDB MS $\mathrm{e}$ PP PR & INCRA 2003 & FAZENDA BIAZUS & Diamantino & мт & 2197 & $\begin{array}{l}\text { ATUERROPREVU } \\
\text { PRODUTVA }\end{array}$ & 1111 & 108 & LEVINO JOSE SPERAFICO & \\
\hline 510787 & JOÄO SPERAFICO & PSDB MS e PP PR & INCRA 2003 & LOTE 102 GLEBA CACORE & SAPEZAL & мт & 2362 & 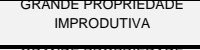 & 2361 & & DILSO SPERAFICO & \\
\hline 510787 & $\begin{array}{l}\text { JOÂO SPERAFICO } \\
\text { JoULCEU }\end{array}$ & PSDB MS e PP PR & INCRA 2003 & LOTE 101 GLEBA CACORE & SAPEZAL & мт & 2618 & IMPRODUTIVA & 2618 & & DIACIR ANTONOO SPERAFICO & \\
\hline 510480 & $\begin{array}{l}\text { JOLAO SPERAFICO EUILCEO } \\
\text { JOAO SER }\end{array}$ & PSDB MS e PP PR & INCRA 2003 & ESTANCIA MEZZOMO & JACIARA & мт & 3000 & 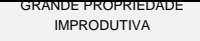 & 3000 & & DEVINO JOSE SPERAFICO & \\
\hline 510787 & 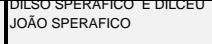 & PSDB MS e PP PR & INCRA 2003 & GLEBA FORTUNA & SAPEZAL & мт & 3000 & 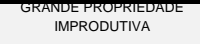 & 3000 & & DILCEU JOAO SPERAFICO & \\
\hline 510263 & $\begin{array}{l}\text { DODLOSOSPEAAFCOUE } \\
\text { JOÂO SPERAFICO }\end{array}$ & PSDB MS e PP PR & INCRA 2003 & FAZENDA SUPERAFICO & CAMPO NOVO DO PARECIS & мт & 7000 & $\begin{array}{l}\text { RAIIDERROPRIIEOA } \\
\text { IMPRODUTIVA }\end{array}$ & 7000 & & DENIS SPERAFICO & \\
\hline 510060 & HOMERO ALVES PEREIRA & PRMT & INCRA 2003 & FAZ PLANALTO & ALTO TAQUARI & мт & 242 & IMPRODUTIVA & 242,00 & 0 & & \\
\hline 510628 & HOMERO ALVES PEREIRA & РR MT & INCRA 2003 & FAZ J JUNIOR & NOVO SÃO JOAQUIM & мт & 840 & $\begin{array}{l}\text { TMEDIAPROPUIIEOAI } \\
\text { PRODUTIVA }\end{array}$ & 840,00 & 0 & & \\
\hline
\end{tabular}




\begin{tabular}{|c|c|c|c|c|c|c|c|c|c|c|c|c|}
\hline GEocodiao & PARLAMENTAR & PARTIDO & FONTE & IMÓvEL & MunICIPIO & UF & AREA & CATEGORIA FUNDIÁRIA & AREA REGISTRADA & $\begin{array}{l}\text { AREADEECLARAVA } \\
\text { COMO POSSE }\end{array}$ & $\begin{array}{l}\text { REGISTRO EM NOME DE } \\
\text { OUTRAS PESSOAS }\end{array}$ & $\begin{array}{l}\text { vinculo COM O } \\
\text { PARLAMENTAR }\end{array}$ \\
\hline 510628 & HOMERO ALVES PEREIRA & PRMT & INCRA 2003 & FAZ J JOYCE & NOVO SĀO JOAQUIM & мт & 900 & $\begin{array}{l}\text { REDARPOPORIEUAD } \\
\text { IMPRODUTIVA }\end{array}$ & 900,00 & 0 & & \\
\hline 510706 & $\begin{array}{l}\text { TRIS REZENDE E DONA IRIS } \\
\text { REZENDE }\end{array}$ & РMDB GO & INCRA 2003 & FAZENDA VALE DO XINGU & QUEREENCIA & мт & 394 & 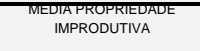 & 394 & 0 & ORLANDo ALVES CARNERO & PEZENDE \\
\hline 510706 & $\begin{array}{l}\text { IRIS REZENDE E DONA IRIS } \\
\text { REZENDE }\end{array}$ & РмDВ GO & INCRA 2003 & FAZENDA VALE DO XINGU II & QUEREENCIA & мт & 1000 & $\begin{array}{l}\text { EUAPFRPFIIEATI } \\
\text { PRODUTIVA }\end{array}$ & 1000 & 0 & ORLANDo ALVES CARNEIRO & 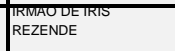 \\
\hline 290620 & $\begin{array}{l}\text { |RIS REZENDE E DONA IRIS } \\
\text { REZENDE }\end{array}$ & PMDB GO & INCRA 2003 & FAZENDA TRES RIOS & CANARANA & мт & 1037 & 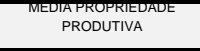 & 1037 & 0 & IRIS REZENDE MACHADO & \\
\hline 290620 & \begin{tabular}{|l} 
RISS REZENDE E DONA IRIS \\
REZENDE
\end{tabular} & РМОВ GO & INCRA 2003 & FAZENDA ESTRELA & Canarana & мт & 2906 & $\begin{array}{l}\text { GRANDE PROPRIEDADE } \\
\text { IMPRODUTIVA }\end{array}$ & 2906 & 0 & ARI GOSS DE OLVEIRA & 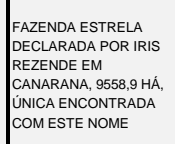 \\
\hline 290620 & $\begin{array}{l}\text { PRIS REZENDE E DONA IRIS } \\
\text { REZENDE }\end{array}$ & PMDB GO & INCRA 2003 & FAZENDA VALE DO XINGU & CANARANA & мт & 7386 & 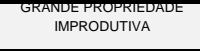 & 7416 & 0 & 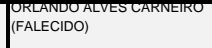 & 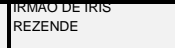 \\
\hline 510610 & JAYME VERISSIMO CAMPOS & DЕммт & INCRA 2003 & FAZENDA PEDRA 90 & NOSSA SENHORA DO LIVRAMENTO & мт & 291 & PEQUENA PROPRIEDADE & 291 & 0 & $\begin{array}{l}\text { AAYMEVEF } \\
\text { CAMPOS }\end{array}$ & \\
\hline 510770 & JAYME VERISSSIMO CAMPOS & DEMMT & INCRA 2003 & FAZENDA SANTA INES & ROSÁRIO OESTE & мт & 938 & $\begin{array}{l}\text { TEEUAPROPFFIIIVAUE } \\
\text { PRODUTIVA }\end{array}$ & 0 & 938 & $\begin{array}{l}\text { JAYME VERISSIMUUE } \\
\text { CAMPOS }\end{array}$ & \\
\hline 510770 & JAYME VERISSIMO CAMPOS & DЕмMT & INCRA 2003 & FAZENDA BONANZA & ROSÁRIO OESTE & мт & 2656 & $\begin{array}{l}\text { RAINUE PROPPIIIEVAD } \\
\text { IMPRODUTIVA }\end{array}$ & 2656 & 0 & $\begin{array}{l}\text { ARYME VERIISSIMODE } \\
\text { CAMPOS }\end{array}$ & \\
\hline 510025 & JAYME VERISSIMO CAMPOS & DEMMT & INCRA 2003 & FAZENDA ELDORADO & ALTA FLORESTA & мт & 2864 & $\begin{array}{l}\text { ANUE PROPRIIEU } \\
\text { PRODUTIVA }\end{array}$ & 2864 & 2864 & $\begin{array}{l}\text { JAYME VEFISSIIIOT } \\
\text { CAMPOS JUNIOR }\end{array}$ & $\begin{array}{l}\text { FLRODEAAYME } \\
\text { VERRSSIMO CAMPOS }\end{array}$ \\
\hline 510780 & JOĀO AUGUSTO RIBEIRO NARDES & PPRS & INCRA 2003 & SEM DENOMINACAO & SANTO ANTÔNIO DO LEVERGER & мт & 1 & MINIFÚNDIO & 0 & 1 & $\begin{array}{l}\text { JTANIIIINARITESDE } \\
\text { CARVALHO }\end{array}$ & $\begin{array}{l}\text { GRAU DE PARENTESCO } \\
\text { NAOO IDENTIFICADO }\end{array}$ \\
\hline 510780 & JOĀO AUGUSTO RIBEIRO NARDES & PPAS & INCRA 2003 & ITAPEVA & SANTO ANTÔNIO DO LEVERGER & мт & 8 & MINIIÚNDIO & 8 & 0 & 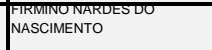 & $\begin{array}{l}\text { GRAU DE PARENTESCO } \\
\text { NAOO IDENTIFICADO }\end{array}$ \\
\hline 510704 & JoÃo AUGUSTO RIBEIRO NARDES & PPAS & INCRA 2003 & FAZENDA GETULIO VARGES & PRIMAVERA DO LESTE & мт & 200 & PEQUENA PROPRIEDADE & 200 & 0 & 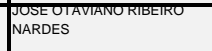 & $\begin{array}{l}\text { GRAU DE PARENTESCO } \\
\text { NAOO IDENTIFICADO }\end{array}$ \\
\hline 510780 & JOÃO AUGUSTO RIBEIRO NARDES & PPAS & INCRA 2003 & FAZENDA N S APARECIDA & SANTO ANTOONIO DO LEVERGGR & мт & 254 & PEQUENA PROPRIEDADE & 0 & 254 & \begin{tabular}{|l} 
DIILADAC \\
NARDES
\end{tabular} & $\begin{array}{l}\text { GRAU DE PARENTESCO } \\
\text { NAO IDENTIFICADO }\end{array}$ \\
\hline 510704 & JOĀO AUGUSTO RIBEIRO NARDES & PPAS & INCRA 2003 & FAZENDA LAGO AZUL & PRIMAVERA Do LESTE & мт & 538 & $\begin{array}{l}\text { EUAPROPFIEVY } \\
\text { PRODUTIVA }\end{array}$ & 538 & 0 & $\begin{array}{l}\text { JOSEOUA } \\
\text { NARDES }\end{array}$ & $\begin{array}{l}\text { GRAU DE PARENTESCO } \\
\text { NAOO IDENTIFICADO }\end{array}$ \\
\hline 510704 & JoÃo AUGUSTO RIBEIRO NARDES & PPAS & INCRA 2003 & FAZENDA MICHELAN & PRIMAVERA DO LESTE & мт & 621 & $\begin{array}{l}\text { EUAPPROPRIEVY } \\
\text { PRODUTIVA }\end{array}$ & 621 & 0 & $\begin{array}{l}\text { JPSEOAP } \\
\text { NARDES }\end{array}$ & $\begin{array}{l}\text { GRAU DE PARENTESCO } \\
\text { NAO IDENTIFICADO }\end{array}$ \\
\hline 510704 & Joäo AUGUSTO RIBEIRO NARDES & PPRS & INCRA 2003 & FAZENDA LAGO AZUL & PRIMAVERA DO LESTE & мт & 755 & 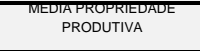 & 755 & 0 & $\begin{array}{l}\text { JOSEOUA } \\
\text { NARDES }\end{array}$ & $\begin{array}{l}\text { GRAU DE PARENTES } \\
\text { NÄO IDENTIFICADO }\end{array}$ \\
\hline 510267 & JOĀO AUGUSTO RIBEIRO NARDES & PSBRS & INCRA 2003 & FAZENDA SANTA LUZIA & CAMPO VERDE & мт & 763 & $\begin{array}{l}\text { DIAPRPPIIEA } \\
\text { PRODUTIVA }\end{array}$ & 763 & 0 & $\begin{array}{l}\text { JOSEOUA } \\
\text { NARDES }\end{array}$ & $\begin{array}{l}\text { GRAU DE PARENTESS } \\
\text { NÄO IDENTIFICADO }\end{array}$ \\
\hline 510704 & JOÄO AUGUSTO RIBEIRO NARDES & PPRS & INCRA 2003 & FAZZNDA RIO DA MATA & PRIMAVERA DO LESTE & мт & 1566 & 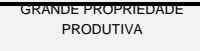 & 1566 & 0 & LAURA BATTISTI NARDES & $\begin{array}{l}\text { GRAU DE PARENTESC } \\
\text { NÄO IDENTIFICADO }\end{array}$ \\
\hline 510704 & JOÃO AUGUSTO RIBEIRO NARDESS & PPAS & INCRA 2003 & FAZENDA SAO LUIZ & PRIMAVERA DO LESTE & мт & 1997 & $\begin{array}{l}\text { GRAIUVE PROOFIIIEDADE } \\
\text { PRODUTIVA }\end{array}$ & 0 & 1997 & $\begin{array}{l}\text { JOEROTAVIANO RIIEETO } \\
\text { NARDES }\end{array}$ & $\begin{array}{l}\text { GRAU DE PARENTESCO } \\
\text { NAO IDENTIFICADO }\end{array}$ \\
\hline 510622 & 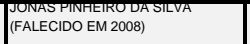 & DЕMмт & INCRA 2003 & LOTE 13 SANT ANA & Nova MUTUM & мт & 328 & PEQUENA PROPRIEDADE & 328,00 & 0 & & \\
\hline 510622 & 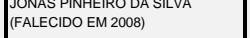 & DEM MT & INCRA 2003 & LOTE N 12 SANT ANA & Nova MUTUM & мт & 346 & PEQUENA PROPRIEDADE & 346,00 & 0 & & \\
\hline 510490 & JúLIO JOSÉ DE CAMPOS & DEMMT & INCRA 2003 & QULLOMBO & JANGADA & мт & 163 & PEQUENA PROPRIEDADE & 0 & 163 & $\begin{array}{l}\text { Jutrovor } \\
\text { caMPOS }\end{array}$ & $\begin{array}{l}\text { PAIUEJULIOTO } \\
\text { CAMPOS }\end{array}$ \\
\hline 510340 & JUULLO JOSÉ DE CAMPOS & DEMMT & INCRA 2003 & VARZEA DO MANGAVA & CUIABÁ & мт & 200 & $\begin{array}{l}\text { MEEUAPFOOPAIIIUAUE } \\
\text { IMPRODUTVA }\end{array}$ & 200 & 0 & JULIO JOSE DE CAMPOS & \\
\hline 510490 & JúLIO JOSÉ DE CAMPOS & DЕMмт & INCRA 2003 & SESMARIA QUILOMBO & JANGADA & мт & 764 & $\begin{array}{l}\text { MEUIAPROPFIIIUAD } \\
\text { IMPRODUTVA }\end{array}$ & 764 & 32 & $\begin{array}{l}\text { JuLIODOMMIIGGUSUE } \\
\text { CAMPOS }\end{array}$ & $\begin{array}{l}\text { PAIUEJULIOJOSEUE } \\
\text { CAMPOS }\end{array}$ \\
\hline 510490 & JúLIO JOSÉ DE CAMPOS & DEMMT & INCRA 2003 & FAZENDA SALOBA & JANGADA & мT & 2082 & $\begin{array}{l}\text { RAINDEF FOPRFIIIVDA } \\
\text { IMPRODUTIVA }\end{array}$ & 2082 & 0 & 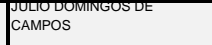 & $\begin{array}{l}\text { PAADE JULIOJOSE UE } \\
\text { CAMPOS }\end{array}$ \\
\hline 510160 & JúLOO JOSÉ DE CAMPOS & DЕмMT & INCRA 2003 & FAZENDA SAO JOSE DO PIQUIIII & BARĀo DE MELGAÇO & мт & 8443 & $\begin{array}{l}\text { PRODUTVA } \\
\text { PRA }\end{array}$ & 8443 & 0 & JULLO JOSE DE CAMPOS & \\
\hline 510170 & MARCOS MONTES CORDEIRO & DEMMG & INCRA 2003 & FAZENDA MINEIRA II & BARRA DO BUGRES & мт & 649 & $\begin{array}{l}\text { MÉDIA PROPRIEDADE } \\
\text { IMPROOUTIVA }\end{array}$ & 649 & 0 & MARCOS MONTES CORDEIRO & 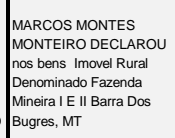 \\
\hline
\end{tabular}




\begin{tabular}{|c|c|c|c|c|c|c|c|c|c|c|c|c|}
\hline GEOCODIGO & PARLAMENTAR & PARTIDO & FONTE & IMÓvel & MUNICiPIO & uF & AREA & CATEGORIA FUNDIÁRIA & AREA REGISTRADA & $\begin{array}{l}\text { AREA DECLARADA } \\
\text { COMO POSSE }\end{array}$ & $\begin{array}{l}\text { REGISTRO EM NOME DE } \\
\text { OUTRAS PESSOAS }\end{array}$ & \begin{tabular}{|l} 
vinculo como \\
PARLAMENTAR
\end{tabular} \\
\hline 510170 & MARCOS MONTES CORDEIRO & DемMG & INCRA 2003 & FAZENDA MINEIRAI & BARRA DO BUGRES & мт & 726 & $\begin{array}{l}\text { MÉDIA PROPRIEDADE } \\
\text { PRODUTIVA }\end{array}$ & 726 & 0 & MARCOS MONTES CORDEIRO & 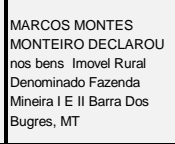 \\
\hline 510790 & RICARTE DE FREITAS JUNIOR & Ртв мт & INCRA 2003 & CHACARA DONA MARIA LOTES 308 E 309 & SINOP & мт & 24 & PEQUENA PROPRIEDADE & 24,00 & 0 & & \\
\hline 510790 & RICARTE DE FREITAS JUNIOR & Ртв мт & INCRA 2003 & SITIO SEU MENINO LOTE 162 & SINOP & мт & 60 & PEQUENA PROPRIEDADE & 60,00 & 0 & & \\
\hline 510794 & SILVA & РмDв Мт & INCRA 2003 & Lote SAO GABRIEL & TABAPORÃ & мт & 314 & PEQUENA PROPRIEDADE & 314 & 0 & SILVA & \\
\hline 510794 & $\begin{array}{l}\text { SILVA } \\
\text { SIVA }\end{array}$ & РмDв мт & INCRA 2003 & LOTE PRUDENTE & TABAPORÃ & мт & 484 & $\begin{array}{l}\text { IMPRODUTIVA } \\
\text { IMAPE }\end{array}$ & 484 & 0 & $\begin{array}{l}\text { FroGER } \\
\text { SLIVA }\end{array}$ & \\
\hline 510794 & SILVA & Рмов мт & INCRA 2003 & LOTE PRUDENTE & TABAPORÃ & мт & 605 & IMPROOUTINA & 605 & 0 & SILVA & \\
\hline 510794 & silva & РмDв Мт & INCRA 2003 & LOTE PRUDENTE & TABAPORÄ & мт & 605 & IMPRODUTIVA & 605 & 0 & SILVA & \\
\hline 510025 & $\begin{array}{l}\text { RILVEAIO LUCIOSOAF } \\
\text { SIVA }\end{array}$ & РмDВ Мт & INCRA 2003 & LOTE N 315-D & ALTA FLORESTA & мт & 870 & 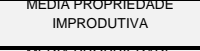 & 0 & 870 & $\begin{array}{l}\text { SOEGEEAII } \\
\text { SILVA }\end{array}$ & \\
\hline 510140 & 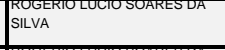 & РмDВ Мт & INCRA 2003 & FAZENDA CAFEZAL II & ARIPUANÃ & мт & 1370 & 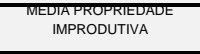 & 0 & 1370 & \begin{tabular}{|l} 
FuGERAII \\
SLLVA
\end{tabular} & \\
\hline 510794 & SILVA & РмDв Мт & INCRA 2003 & LOTE CRUZEiro do SUL & TABAPORÃ & мт & 1650 & IMPRODUTIVA & 1650 & 0 & SILVA & \\
\hline 510794 & SILVA & РмDв мт & INCRA 2003 & LOTE CRUZEIRO DO SUL & TABAPORÃ & мт & 2255 & IMPRODUTIVA & 2255 & 0 & SILVA & \\
\hline 510794 & 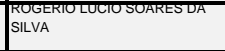 & РмDВ Мт & INCRA 2003 & LOTE CRUZEIRO DO SUL & TABAPORÃ & мт & 2288 & $\begin{array}{l}\text { GRAVUEFPOPPIIEAUE } \\
\text { IMPRODUTIVA }\end{array}$ & 2288 & 0 & 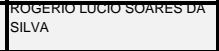 & \\
\hline 510626 & $\begin{array}{l}\text { RIIVEARA } \\
\text { SIIVA }\end{array}$ & РмDв Мт & INCRA 2003 & FAZENDA AMOR & Novo MUNDo & мт & 2371 & $\begin{array}{l}\text { IMPRODUTIVA } \\
\text { IMPADE }\end{array}$ & 0 & 2371 & $\begin{array}{l}\text { F RIVEARA } \\
\text { SILVA }\end{array}$ & \\
\hline 510025 & SILVAEA & РмDв Мт & INCRA 2003 & FAZENDA TIETE I & ALTA FLORESTA & мт & 4999 & $\begin{array}{l}\text { ANUE PROURIIIUAT } \\
\text { IMPRODUTVA }\end{array}$ & 0 & 4999 & $\begin{array}{l}\text { Filveri| } \\
\text { siLVA }\end{array}$ & \\
\hline 510310 & \begin{tabular}{|l|}
$\begin{array}{l}\text { SANDRO ANTŌNIO SCODRO } \\
\text { (SANDRO MABEL) }\end{array}$ \\
\end{tabular} & PR GO & INCRA 2003 & FAZENDA SANTA LUIZA & COCALINHO & мт & 9000 & 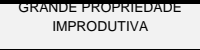 & 0 & 9000 & SANDRO ANTONIO SCODRO & $\begin{array}{l}\text { DEDCLAROUTOSSS } \\
9000 \text { HECTARES }\end{array}$ \\
\hline 510760 & FAGUNDES & PRMT & INCRA 2003 & FAZ LAGO AZUL & RONDONÓPOLIS & MT & 102 & PEQUENA PROPRIIEDADE & 102,00 & 0 & & \\
\hline 150830 & ANIVALDO JUVENIL VALE & PSDB PA & INCRA 2003 & COLONIA PIRIA FAZ TIPITINGA & VISEU & $\mathrm{PA}$ & 50 & MINIFÚNDIO & & 50 & & \\
\hline 150830 & ANIVALDO JUVENIL VALE & PSDB PA & INCRA 2003 & COLONIA PIRIA FAZ TIPINTINGA & VISEU & $\mathrm{PA}$ & 74 & MINIFÚNDIO & & 74 & & \\
\hline 150030 & ANIVALDO JUVENLI VALE & PSDB PA & INCRA 2003 & COLONIA PIRIA N 09 & VISEU & $\mathrm{PA}$ & 125 & PEQUENA PROPRIEDADE & & 125 & & \\
\hline 150830 & ANIVALDO JUVENIL VALE & PSDB PA & INCRA 2003 & FAZENDA TIPTININGA COLONIA PIRIA & VISEU & $\mathrm{PA}$ & 429 & $\begin{array}{l}\text { MÉDIA PROPRIEDADE } \\
\text { IMPROOUTIVA }\end{array}$ & 429 & 0 & ANIALDO JUVENLIL VALE & $\begin{array}{l}\text { NOME DIGITADO } \\
\text { ERRADO N INARA } \\
\text { "AANALDO" AO INVÉS DE } \\
\text { ANIVALDO } \\
\end{array}$ \\
\hline 150830 & ANIVALDO JUVENLL VALE & PSDB PA & INCRA 2003 & COLONIA PIRIA FAZ TIPTTINGA & VISEU & $\mathrm{PA}$ & 498 & $\begin{array}{l}\text { EEAP PROURREVUVE } \\
\text { IMPRODUTIVA }\end{array}$ & & 498 & & \\
\hline 150830 & ANIVALDO JUVENLL VALE & PSDB PA & INCRA 2003 & COLONIA PIRIA & VISEU & $\mathrm{PA}$ & 551 & 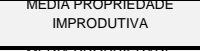 & & 551 & & \\
\hline 150830 & ANIVALDO JUVENIL VALE & PSDB PA & INCRA 2003 & COLONIA PIRIA & VISEU & $\mathrm{PA}$ & 575 & $\begin{array}{l}\text { IMPRODUTIVA } \\
\text { INATE }\end{array}$ & & 575 & & \\
\hline 150730 & CASILDO JOĀO MALDANER & PMDB SC & INCRA 2003 & GLEBA ALTAMIRA VI PROJETO TRAIRAO & SĀo FéLIX DO XINGU & $\mathrm{PA}$ & 1385 & $\begin{array}{l}\text { GRANDE PROPRIEDADE } \\
\text { PRODUTIVA }\end{array}$ & 1385 & 0 & $\begin{array}{l}\text { MARIA LUIZA CAMA PROENCA } \\
\text { FERNANDES }\end{array}$ & 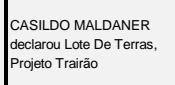 \\
\hline 150730 & CASILDO JOĀO MALDANER & PMDBsC & INCRA 2003 & GLEBA ALTAMIRA VI PROJETO TRAIRAO & SẪo FéLLXXo XINGU & $\mathrm{PA}$ & 1393 & $\begin{array}{c}\text { GRANDE PROPRIEDADE } \\
\text { IMPRODUTIVA }\end{array}$ & 1393 & 0 & $\begin{array}{l}\text { NELSON LUIZ PROENCA } \\
\text { FERNANDES }\end{array}$ & 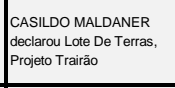 \\
\hline 150730 & CASLLDO JOĀo MALDANER & РMDB sC & INCRA 2003 & GLEBA ALTAMIRA VI PROJETO TRAIRAO & SĀO FÉLIX DO XINGU & PA & 1432 & $\begin{array}{l}\text { GRANDE PROPRIEDADE } \\
\text { PRODUTIVA }\end{array}$ & 1432 & 0 & PROENCA EROENCA LTDA & 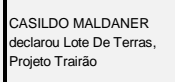 \\
\hline
\end{tabular}




\begin{tabular}{|c|c|c|c|c|c|c|c|c|c|c|c|c|}
\hline GEOCODIGO & PARLAMENTAR & PARTIDO & FONTE & IMÓvel & MuniciPIO & uF & ÁREA & CATEGORIA FUNDIÁRIAA & AREA REGISTRADA & $\begin{array}{l}\text { AREA DECLARADA } \\
\text { COMO POSSE }\end{array}$ & $\begin{array}{l}\text { REGISTRO EM NOME DE } \\
\text { OUTRAS PESSOAS }\end{array}$ & $\begin{array}{l}\text { viNCULO COM O } \\
\text { PARLAMENTAR }\end{array}$ \\
\hline 150563 & JOAO BATISTA DE JESUS RIIEERO & PR TO & INCRA 2003 & FAZENDA OURO VERDE & PIÇARRA & PA & 184 & PEQUENA PROPRIEDADE & 184 & 184 & & \\
\hline 150034 & JOÃO CAMPOS DE ARAÚJO & PSDB GO & INCRA 2003 & FAZENDA PROGRESSO & AGUA AZUL DO NORTE & PA & 261 & PEQUENA PROPRIEDADE & 261 & 0 & BENONE CAMPOS DE ARAUJC & $\begin{array}{l}\text { WAEDE JOAC } \\
\text { DE ARAUJO }\end{array}$ \\
\hline 150370 & \begin{tabular}{|l}
$\begin{array}{l}\text { NLITON GOMES DE OLVEIRA } \\
\text { (NLITON BAAANO) }\end{array}$ \\
\end{tabular} & PPB ES & INCRA 2003 & FAZENDA RECREIO & ITUPBAAnga & PA & 107 & PEQUENA PROPRIEDADE & 107 & 0 & NLLTON GOMES DE OLIVEIRA & 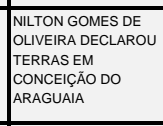 \\
\hline 150618 & OLÁVIO SILVA ROCHA & PMDB PA & INCRA 2003 & FAZENDA BOI BOM LOTE 96 & RONDON DO PARÁ & $\mathrm{PA}$ & 2435 & $\begin{array}{l}\text { GRANDERFOPFIIIUAOE } \\
\text { PRODUTIVA }\end{array}$ & 2435 & & & \\
\hline 150618 & OLÁVIO SILVA ROCHA & PMDB PA & INCRA 2003 & FAZENDA MARIA BONITA & RONDON DO PARÁ & $\mathrm{PA}$ & 4113 & $\begin{array}{l}\text { GRANOEPROPFIEOH } \\
\text { PROUTIVA }\end{array}$ & 44113 & & & \\
\hline 150370 & 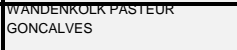 & PSDB PA & INCRA 2003 & FAZENDA SANTA RITA & ITUPRANGA & PA & 117 & PEQUENA PROPRIEDADE & 117 & 0 & & \\
\hline 251270 & ARMANDO ABILIO VIEIRA & Ртв Рв & INCRA 2003 & SITIO CAPIM DO CHEIRO & REMíio & PB & 25 & PEQUENA PROPRIEDADE & 25 & 0 & & \\
\hline 251270 & ARMANDO ABILIO VIEIRA & Ртв Рв & INCRA 2003 & & REMigio & PB & 25 & PEQUENA PROPRIEDADE & 25 & 0 & & \\
\hline 251270 & ARMANDO ABILIO VIEIRA & Ртв Рв & INCRA 2003 & SITIO GENIPAPO & REMiGio & PB & 30 & PEQUENA PROPRIEDADE & 30 & 0 & & \\
\hline 251270 & ARMANDO ABILIO VIEIRA & Ртв Рв & INCRA 2003 & & REMiGio & PB & 30 & PEQUENA PROPRIEDADE & 30 & 0 & & \\
\hline 250560 & ARMANDO ABILIO VIEIRA & Ртв Рв & INCRA 2003 & SITIO SABONETE & DIAMANTE & PB & 60 & PEQUENA PROPRIEDADE & 60 & 0 & & \\
\hline 250560 & ARMANDO ABILIO VIEIRA & Ртв Рв & INCRA 2003 & & DIAMANTE & Рв & 60 & PEQUENA PROPRIEDADE & 60 & 0 & & \\
\hline 250600 & ARMANDO ABILIO VIEIRA & Ртв Рв & INCRA 2003 & SITIO LAGOA DE PEDRA & ESPERANÇA & PB & 63 & $\begin{array}{l}\text { MEEDAPPROPIIEDAVE } \\
\text { IMPROOUTIVA }\end{array}$ & 63 & 0 & & \\
\hline 250250 & CARLOS MARQUES DUNGA & Ртв Рв & INCRA 2003 & & BOQUEIRÃo & PB & 517 & $\begin{array}{l}\text { PRODUTVA } \\
\text { PRA }\end{array}$ & 517 & 0 & & \\
\hline 260770 & $\begin{array}{l}\text { FRAACLISCUDE ASSIS } \\
\text { RODRIGUES }\end{array}$ & DEM RR & INCRA 2003 & IITIO CATINGUEIRA & ITAPETMM & PE & 6 & MINIF́UNDIO & 0 & 6 & 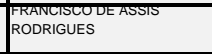 & \\
\hline 260515 & $\begin{array}{l}\text { F-FANCISCOUDE ASSIS } \\
\text { RODRIGUES }\end{array}$ & DEM RR & INCRA 2003 & SITIO AMARGOSA & DORMENTES & PE & 6 & MINIÉ́NDIO & 6 & 0 & $\begin{array}{l}\text { FrARACIICODVE ASSIS } \\
\text { RODRIGUES }\end{array}$ & \\
\hline 260990 & 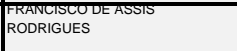 & DEM RR & INCRA 2003 & SITIO PAU DARCO FAZ AGUA PRETA & OURICURI & PE & 28 & MINIIÚNDIO & 28 & 0 & $\begin{array}{l}\text { FrANCISCOU } \\
\text { RODRIGUES }\end{array}$ & \\
\hline 261110 & $\begin{array}{l}\text { TFADVISCOUET } \\
\text { RORIGUES }\end{array}$ & DEM RR & INCRA 2003 & LOTE AGRICOLA N A5 22 & PETROLINA & $\overline{P E}$ & 34 & MINIF́́́NDIO & 34 & 0 & $\begin{array}{l}\text { FAREISCOE } \\
\text { RODRIGUES }\end{array}$ & \\
\hline 260875 & $\begin{array}{l}\text { FAAVIISCOUE ASSIS } \\
\text { RODRIGUES }\end{array}$ & DEM RR & INCRA 2003 & SITIO CACIMBINHA & LAGOA GRANDE & PE & 70 & PEQUENA PROPRIEDADE & 0 & 70 & $\begin{array}{l}\text { FrAIVISCOUVE ASSIS } \\
\text { RODRIGUES }\end{array}$ & \\
\hline 260300 & $\begin{array}{l}\text { THADCIISCDUE ASSIS } \\
\text { RODRIGUES }\end{array}$ & DEM RR & INCRA 2003 & BELA VISTA LOTE 895 & САВRово́ & PE & 101 & PEQUENA PROPRIEDADE & 117 & 0 & $\begin{array}{l}\text { FRAIRCLCOUDE ASSIS } \\
\text { RODRIGUES }\end{array}$ & \\
\hline 260300 & $\begin{array}{l}\text { FFARCLISCDUE ASSIS } \\
\text { RODRIGUES }\end{array}$ & DEM RR & INCRA 2003 & CACHOEIRINHA LOTE 1469 & САВвово́ & PE & 254 & 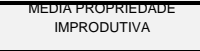 & 520 & 0 & 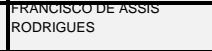 & \\
\hline 260070 & INOCÉNCIO GOMES DE OLVEIRA & PR PE & INCRA 2003 & FAZENDA CAETES & ALLANÇA & PE & 260 & 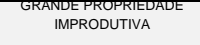 & 260 & 0 & & \\
\hline 261130 & JOÃO BASTOS COLAÇO DIAS & 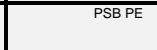 & INCRA 2003 & LOTE 1 Q A ENG CACHOEIRA S J NOVOO & Pомвоs & PE & 60 & PEQUENA PROPRIEDADE & 0 & 60 & GUSTAVO COLACO DIAS & 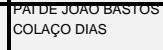 \\
\hline 260170 & JOSE MENDONÇA BEZERRA & PFLPE & INCRA 2003 & LUGAR ALTO LIMPO & BELO JARDIM & PE & 2 & MINIF́ÚNDIO & 2 & 0 & 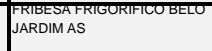 & \\
\hline 261300 & JOSE MENDONÇA BEZERRA & PFLPE & INCRA 2003 & SITIO INGA & SĀO BENTO DO UNA & PE & 13 & MINIF́́NDIO & 13 & 0 & JOSE MENDONCA BEZERRA & \\
\hline 260170 & JOSE MENDONÇA BEZERRA & PFLPE & INCRA 2003 & SITIO INGA & BELO JARDIM & PE & 60 & PEQUENA PROPRIEDADE & 60 & 0 & SEA & 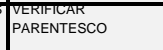 \\
\hline 261110 & JOSE MENDONĢA BEZERRA & PFLPE & INCRA 2003 & SITIO GRANJA SAO JOSE & PETROLINA & $\overline{P E}$ & 118 & PEQUENA PROPRIEDADE & 118 & 0 & ROBERTO JOSE BEZERRA & $\begin{array}{l}\text { PARENTESCOO } \\
\text { PARTR }\end{array}$ \\
\hline
\end{tabular}




\begin{tabular}{|c|c|c|c|c|c|c|c|c|c|c|c|c|}
\hline GEOCODIGO & PARLAMENTAR & PARTIDO & FONTE & IMóveL & MUNICIPIO & UF & AREA & CATEGORIA FUNDIÁRIAA & ÁREA REGISTRADA & $\begin{array}{l}\text { AREAUECLAARUUA } \\
\text { COMO POSSE }\end{array}$ & $\begin{array}{l}\text { REGISTRO EM NOME DE } \\
\text { OUTRAS PESSOAS }\end{array}$ & $\begin{array}{l}\text { VinCULO COM O } \\
\text { PARLAMENTAR }\end{array}$ \\
\hline 261110 & JOSE MENDONÇA BEZERRA & PFLPE & INCRA 2003 & SITIO TEMMOSO & PETROLINA & $\mathrm{PE}$ & 420 & $\begin{array}{l}\text { EUARPIOPRAERAC } \\
\text { IMPRODUTVA }\end{array}$ & 641 & 0 & ROBERTO JOSE BEZERRA & $\begin{array}{l}\text { VARERIILAR } \\
\text { PARESESO } \\
\end{array}$ \\
\hline 261280 & JOSÉ MUCIO MONTERRO FLLHO & PFLPE & INCRA 2003 & FAZENDA CHICA BOA & SANTA TEREZINHA & $\mathrm{PE}$ & 773 & $\begin{array}{l}\text { PRODUTIVA } \\
\text { PRA }\end{array}$ & 773 & 0 & & \\
\hline 261110 & LUIZ GONZAGA PATRIOTA & PSB PE & INCRA 2003 & FAZENDA PONTA DA SERRA DO MEIO & PETROLINA & $\mathrm{PE}$ & 154 & PEQUENA PROPRIEDADE & 154 & 0 & & \\
\hline 261110 & LUIZ GONZAGA PATRIOTA & PSB PE & INCRA 2003 & FAZENDA CACHOEIRA & PETROLINA & PE & 574 & $\begin{array}{l}\text { MEDIPAROPRELUVOE } \\
\text { IMPRODUTVA }\end{array}$ & 574 & 0 & & \\
\hline 261110 & OSVALDO DE SOUZA COELHO & $\begin{array}{l}P F L P E \\
\text { PLE }\end{array}$ & INCRA 2003 & SITIO ganaAu & PETROLINA & $\overline{P E}$ & 18 & MINIF́́nDIO & 0 & 0 & Geraldo de SOUZA CoELHO & \\
\hline 260890 & 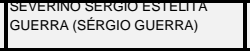 & PSDBPE & INCRA 2003 & FAZENDA PEDRA VERDE & LIMOEIRO & PE & 96 & 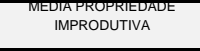 & 96 & 0 & & \\
\hline 260530 & INOCÉNCIO GOMES DE OLIVERA & PRPE & INCRA 2003 & FAZENDA MUNDURI & EXU & PE & 1170 & 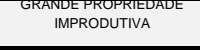 & 1170 & 0 & & \\
\hline 260530 & INOCÊNCIO GOMES DE OLIVEIRA & PR PE & INCRA 2003 & FAZENDA PADRE CICERO & EXu & $\mathrm{PE}$ & 2659 & 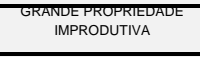 & 2659 & 0 & & \\
\hline 260530 & INOCÉNCIO GOMES DE OLIVEIRA & PR PE & INCRA 2003 & SITIO LAGOA SECA & EXu & PE & 9 & MINIFÚNDIO & 9 & 0 & & \\
\hline 260890 & INOCÉNCIO GOMES DE OLIVERA & PR PE & INCRA 2003 & FAZENDA N S DA CONCEICAO & LIMOËво & $\mathrm{PE}$ & 220 & $\begin{array}{l}\text { PRODUTIVA } \\
\text { PRA }\end{array}$ & 220 & 0 & & \\
\hline 261390 & INOCÉNCIO GOMES DE OLIVEIRA & PR PE & INCRA 2003 & FAZENDA ESTRELINHA & SERRA TALHADA & $\mathrm{PE}$ & 178 & $\begin{array}{l}\text { EUAAPVOPLEVAL } \\
\text { IMPRODUTVA }\end{array}$ & 178 & 0 & & \\
\hline 261390 & INOCÉNCIO GOMES DE OLIVEIRA & PR PE & INCRA 2003 & FAZENDA PAPAGAIO & SERRA TALHADA & PE & 482 & IMPRODUTIVA & 482 & 0 & & \\
\hline 261390 & INOCÉNCIO GOMES DE OLIVEIRA & PRPE & INCRA 2003 & FAZENDA SAO JOAO II & SERRA TALHADA & PE & 581 & $\begin{array}{l}\text { EUAA POPORHEDAL } \\
\text { IMPRODUTIVA }\end{array}$ & 581 & 0 & & \\
\hline 221130 & CIRO NOGUERRA LIMA FLHO & PP PI & INCRA 2003 & FAZENDA SAO MARCELO & VALENÇA DO PIAUÍ & $\mathrm{PI}$ & 590 & $\begin{array}{l}\text { EUARPIOPRHEVAC } \\
\text { IMPRODUTVA }\end{array}$ & 590 & 0 & $\begin{array}{l}\text { DOSENA } \\
\text { NUNES }\end{array}$ & 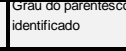 \\
\hline 221100 & CIRO NOGUEIRA LIMA FLHO & PP PI & INCRA 2003 & JUNCO SERAFIM & TERESINA & $\mathrm{Pl}$ & 822 & IMPRODUTIVA & 822 & 0 & & \\
\hline 221100 & GIRO NOGUEIRA LIMA FLHO & PP PI & INCRA 2003 & FAZENDA JUNCO & TERESINA & $\mathrm{PI}$ & 1741 & IMPRODUTIVA & 1741 & 0 & & \\
\hline 220350 & CIRO NOGUERRA LIMA FLHO & PP PI & INCRA 2003 & FAZENDA Nova AFRICA & ELESBĀO VELOSO & $\mathrm{PI}$ & 1842 & $\begin{array}{l}\text { RANUEFROOPREDAUE } \\
\text { IMPRODUTIVA }\end{array}$ & 1842 & 0 & $\begin{array}{l}\text { DOESNANA } \\
\text { NUNES }\end{array}$ & $\begin{array}{l}\text { Graud o p pare } \\
\text { identificado }\end{array}$ \\
\hline 221000 & JoSE Francisco PAES LANDIM & Ртв РI & INCRA 2003 & BOQUEIRAO E MALHADA & SĀO JOÃO DO PIAUÍ & $\mathrm{PI}$ & 72 & PEQUENA PROPRIEDADE & 72 & 0 & 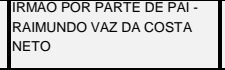 & \\
\hline 220535 & JOSE FRANCISCO PAES LANDIM & Ртв Р1 & INCRA 2003 & FRADES & JOĀO COSTA & $\mathrm{PI}$ & 169 & PEQUENA PROPRIEDADE & 169 & 0 & $\begin{array}{l}\text { PAES LANDIM } \\
\text { PFATU }\end{array}$ & \\
\hline 220192 & JOSE FRANCISCO PAES LANDIM & Pтв Р1 & INCRA 2003 & LAGOA DAS POMBAS & BONFIM DO PIAUI & $\mathrm{PI}$ & 173 & PEQUENA PROPRIEDADE & 173 & 0 & LANDIM & \\
\hline 220535 & JOSE FrANCISCO PAES LANDIM & РТв Р1 & INCRA 2003 & SAO PEDRO & JOĀO COSTA & $\mathrm{PI}$ & 183 & PEQUENA PROPRIEDADE & 183 & 0 & 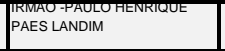 & \\
\hline 220535 & JOSE FRANCISCO PAES LANDIM & РТВ РI & INCRA 2003 & BAIXA DO CAXE IDALINO E PINTADO & JOÄO COSTA & $\mathrm{PI}$ & 1590 & $\begin{array}{l}\text { GRANDE PROPRIEDADE } \\
\text { IMPRODUTIIVA }\end{array}$ & 1641 & 0 & $\begin{array}{l}\text { RAMAO PRO PATTE ED PAI- } \\
\text { RAMUND VAZ DA COSTA } \\
\text { NETO }\end{array}$ & \\
\hline 220450 & JUULIO CESAR DE CARVALHO LIMA & DEMPI & INCRA 2003 & SITIO ARACAS & GUADALUPE & $\mathrm{PI}$ & 26 & MINIFÚNDIO & 26 & 0 & & \\
\hline 221100 & JúLIO CESAR DE CARVALHO LIMA & DEMPI & INCRA 2003 & EURECA & TERESINA & $\mathrm{PI}$ & 50 & PEQUENA PROPRIEDADE & 50 & 0 & & \\
\hline 220080 & JúLIO CESAR DE CARVALHO LIMA & DEMPI & INCRA 2003 & PINTADA & ANTÔNIO ALMEIDA & $\mathrm{Pl}$ & 65 & MINIFÚNDIO & 65 & 0 & & \\
\hline 221100 & JúLO CESAR DE CARVALHO LIMA & DEMPI & INCRA 2003 & CANAA & TERESINA & $\mathrm{PI}$ & 135 & 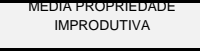 & 135 & 0 & & \\
\hline 220450 & JúLIO CESAR DE CARVALHO LIMA & DEMPI & INCRA 2003 & BREJO DOS CACADOS & GUADALUPE & $\mathrm{PI}$ & 148 & PEQUENA PROPRIEDADE & 148 & 0 & & \\
\hline 221110 & JúLIO CESAR DE CARVALHO LIMA & DEMPI & INCRA 2003 & BOM JESUS & UNĀo & $\mathrm{PI}$ & 198 & IMPRODUTIVA & 198 & 0 & & \\
\hline 220080 & JúLIO CESAR DE CARVALHO LIMA & DEMPI & INCRA 2003 & PINTADA & ANTÔNIO ALMEIDA & $\mathrm{PI}$ & 354 & $\begin{array}{l}\text { IMPRODUTIVA } \\
\text { IMAPAOAT }\end{array}$ & 354 & 0 & & \\
\hline 221100 & JúLIO CESAR DE CARVALHO LIMA & DEMPI & INCRA 2003 & AROEIRAS & TERESINA & $\mathrm{PI}$ & 487 & 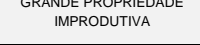 & 487 & 0 & & \\
\hline 220080 & JúLOO CESAR DE CARVALHO LIMA & DEMPI & INCRA 2003 & SANTA ROSA & ANTÔNIO ALMEIDA & PI & 622 & $\begin{array}{l}\text { IMPRODUTIVA } \\
\text { IMPOA }\end{array}$ & 622 & 0 & & \\
\hline
\end{tabular}




\begin{tabular}{|c|c|c|c|c|c|c|c|c|c|c|c|c|}
\hline GEOCODIGO & PARLAMENTAR & PARTIDO & FONTE & IMÓVEL & MUNICIPPO & UF & AREA & CATEGORIA FUNDIÁRIIA & AREA REGISTRADA & $\begin{array}{l}\text { AREAUELCAAAUA } \\
\text { COMO POSSE }\end{array}$ & $\begin{array}{l}\text { REGISTRO EM NOME DE } \\
\text { OUTRAS PESSOAS }\end{array}$ & $\begin{array}{l}\text { ViNCULO COMO } \\
\text { PARLAMENTAR }\end{array}$ \\
\hline 220080 & JÚLIO CESAR DE CARVALHO LIMA & DEMPI & INCRA 2003 & QUEBRA BUNDA & ANTÔNIO ALMEIDA & $\mathrm{PI}$ & 665 & 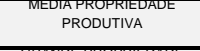 & 665 & 0 & & \\
\hline 220450 & JúLLO CESAR DE CARVALHO LIMA & DEMPI & INCRA 2003 & FLORES & GUADALUPE & $\mathrm{PI}$ & 1141 & IMPROOUTIVA & 1141 & 0 & & \\
\hline 220920 & MARCELO COSTA E CASTRO & РMDB РI & INCRA 2003 & FAZENDA COMETA & SANTA FILOMENA & $\mathrm{PI}$ & 1000 & $\begin{array}{l}\text { EUAAROPPIEVAD } \\
\text { IMPRODUTIVA }\end{array}$ & 1000 & 0 & & \\
\hline 221100 & 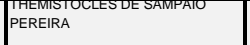 & PMDB PI & INCRA 2003 & Junco & TERESINA & $\mathrm{PI}$ & 3 & MINIFÚNDIO & 3 & 0 & & \\
\hline 220120 & $\begin{array}{l}\text { PEEMISAO } \\
\text { PEREA }\end{array}$ & PMDB PI & INCRA 2003 & LAGOA DE LAGES & BARAAS & $\mathrm{Pl}$ & 5 & MINIFÚNDIO & 5 & 0 & & \\
\hline 220067 & PEREIRA & РМОВ РІ & INCRA 2003 & SANTA ROSA & MORRO DO CHAPEU DO PIAUI & $\mathrm{PI}$ & 6 & MINIFÚNDIO & 6 & 0 & & \\
\hline 220370 & $\begin{array}{l}\text { PIELISTOU } \\
\text { PERERA }\end{array}$ & PMDB PI & INCRA 2003 & ALto Bonito & ESPERANTINA & $\mathrm{PI}$ & 12 & MINIFÚNDIO & 12 & 0 & & \\
\hline 220370 & $\begin{array}{l}\text { PEREIRA } \\
\text { PERA }\end{array}$ & PMDB PI & INCRA 2003 & CANTO DA VELHA & ESPERANTINA & $\mathrm{PI}$ & 14 & MINIFÚNDIO & 14 & 0 & & \\
\hline 220370 & $\begin{array}{l}\text { PEREIRA } \\
\text { PERASO }\end{array}$ & PMDB PI & INCRA 2003 & CURRALINHO 2 & ESPERANTINA & $\mathrm{PI}$ & 23 & MINIIF́́ndIO & 23 & 0 & & \\
\hline 220370 & PEREIRA & РMDB PI & INCRA 2003 & MuLungus & ESPERANTINA & $\mathrm{Pl}$ & 25 & MINIIÉNDIO & 25 & 0 & & \\
\hline 220370 & $\begin{array}{l}\text { PEEMISTO } \\
\text { PERARA }\end{array}$ & PMDB PI & INCRA 2003 & СURRALINHO & ESPERANTINA & $\mathrm{Pl}$ & 34 & MINIFÚNDIO & 34 & 0 & & \\
\hline 220370 & 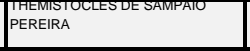 & PMDB PI & INCRA 2003 & BARROCAO & ESPERANTINA & $\mathrm{PI}$ & 37 & MINIIÉNDIO & 37 & 0 & & \\
\hline 220370 & 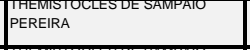 & PMDB PI & INCRA 2003 & CURRALINHO 3 & ESPERANTINA & $\mathrm{PI}$ & 38 & MINIIÉNDIO & 38 & 0 & & \\
\hline 220150 & PEREIRA & PMDB PI & INCRA 2003 & VARJota & BATALHA & $\mathrm{Pl}$ & 47 & MINIF́ÚNDIO & 47 & 0 & & \\
\hline 220370 & $\begin{array}{l}\text { PEREIRA } \\
\end{array}$ & РмDв РІ & INCRA 2003 & BURITI DOS NEGROS & ESPERANTINA & $\mathrm{Pl}$ & 52 & MINIFÚNDIO & 52 & 0 & & \\
\hline 220370 & $\begin{array}{l}\text { PEREIRA } \\
\text { PERES SAMPAIO }\end{array}$ & РMDB PI & INCRA 2003 & IpAnema & ESPERANTINA & $\mathrm{PI}$ & 53 & MINIIÉNDIO & 53 & 0 & & \\
\hline 220150 & 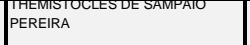 & PMDB PI & INCRA 2003 & CATINGUEIRAS & BATALHA & $\mathrm{PI}$ & 61 & PEQUENA PROPRIEDADE & 61 & 0 & & \\
\hline 220150 & $\begin{array}{l}\text { PEREIRA } \\
\end{array}$ & PMDB PI & INCRA 2003 & CARAIBAS & BATALHA & $\mathrm{Pl}$ & 95 & PEQUENA PROPRIEDADE & 95 & 0 & & \\
\hline 220150 & \begin{tabular}{|l} 
PEREIRA \\
\end{tabular} & PMDB PI & INCRA 2003 & PAu diarco & BATALHA & $\mathrm{PI}$ & 108 & PEQUENA PROPRIEDADE & 108 & 0 & & \\
\hline 220370 & $\begin{array}{l}\text { PEREIRA } \\
\text { PERES SAMPAIO }\end{array}$ & PMDB PI & INCRA 2003 & LADEIRA GRANDE & ESPERANTINA & $\mathrm{PI}$ & 145 & PEQUENA PROPRIEDADE & 145 & 0 & & \\
\hline 220370 & 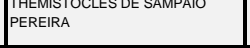 & PMDB PI & INCRA 2003 & ACUDINHO & ESPERANTINA & $\mathrm{Pl}$ & 145 & PEQUENA PROPRIEDADE & 145 & 0 & & \\
\hline 220370 & $\begin{array}{l}\text { PEREIRA } \\
\text { PERAS }\end{array}$ & PMDB PI & INCRA 2003 & ALEGRE & ESPERANTINA & $\mathrm{Pl}$ & 148 & PEQUENA PROPRIEDADE & 148 & 0 & & \\
\hline 220667 & 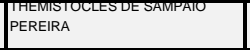 & PMDB PI & INCRA 2003 & GINGA DE FORA & MORRO DO CHAPEU DO PIAUI & $\mathrm{PI}$ & 150 & PEQUENA PROPRIEDADE & 0 & 150 & & \\
\hline 220370 & PEREIRA & РMDB РI & INCRA 2003 & CAPOTE & ESPERANTINA & $\mathrm{Pl}$ & 150 & PEQUENA PROPRIEDADE & 150 & 0 & & \\
\hline 220667 & 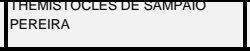 & PMDB РI & INCRA 2003 & CANTO DA SERRAGEM E ARACAS & MORRO DO CHAPEU DO PIAUí & $\mathrm{PI}$ & 150 & PEQUENA PROPRIEDADE & 150 & 150 & & \\
\hline 220370 & $\begin{array}{l}\text { PEREIRA } \\
\text { PERA }\end{array}$ & РMDB PI & INCRA 2003 & ALTO VERMELHO & ESPERANTINA & $\mathrm{Pl}$ & 183 & PEQUENA PROPRIEDADE & 183 & 0 & & \\
\hline 220370 & $\begin{array}{l}\text { PEREIRA } \\
\text { PERA }\end{array}$ & РMDB РI & INCRA 2003 & CURRALINHO 1 & ESPERANTINA & $\mathrm{PI}$ & 203 & PEQUENA PROPRIEDADE & 203 & 0 & & \\
\hline 220150 & ES EAMIPAIO & PMDB PI & INCRA 2003 & SANTA ADELAIDE & BATALHA & $\mathrm{PI}$ & 204 & PEQUENA PROPRIEDADE & 204 & 0 & & \\
\hline 220150 & $\begin{array}{l}\text { PEREIRA } \\
\text { PERECLE SAMPAIO }\end{array}$ & PMDB PI & INCRA 2003 & SANTO ANTONIO & BATALHA & $\mathrm{PI}$ & 311 & $\begin{array}{l}\text { MEUIAPROPFHIIIDATC } \\
\text { IMPRODTVA }\end{array}$ & 311 & 0 & & \\
\hline 220580 & $\begin{array}{l}\text { IHEMISTOCLESUE SAINPAIO } \\
\text { PEREIRA }\end{array}$ & PMDB PI & INCRA 2003 & SAO FRANCISCO E BOA VISTA & LUZLÂANDIA & $\mathrm{PI}$ & 330 & 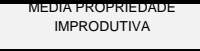 & 330 & 0 & & \\
\hline 220067 & PEREIRA & РМОВ РІ & INCRA 2003 & BOA VISTA & MORRO DO CHAPEU DO PIAUI & $\mathrm{PI}$ & 700 & IMPRODUTIVA & 700 & 0 & & \\
\hline 220540 & $\begin{array}{l}\text { PAEMISTO } \\
\text { PERERARA }\end{array}$ & PMDB PI & INCRA 2003 & NOVO AMANHECER & JOAQUIM PIRES & $\mathrm{Pl}$ & 1205 & $\begin{array}{l}\text { GRAIIUDPROORTIIVUA } \\
\text { IMPRODUTVA }\end{array}$ & 0 & 1200 & & \\
\hline
\end{tabular}




\begin{tabular}{|c|c|c|c|c|c|c|c|c|c|c|c|c|}
\hline GEOCODIGO & PARLAMENTAR & PARTIDO & FONTE & IMóveL & MUNICIPPO & $\mathrm{UF}$ & AREA & CATEGORIA FUNDIÁRIAA & AREA REGISTRADA & $\begin{array}{l}\text { AREA UECLAARUA } \\
\text { COMO POSSE }\end{array}$ & $\begin{array}{l}\text { REGISTRO EM NOME DE } \\
\text { OUTRAS PESSOAS }\end{array}$ & $\begin{array}{l}\text { vinculo com O } \\
\text { PARLAMENTAR }\end{array}$ \\
\hline 220667 & 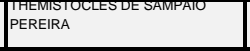 & РмОв РІ & INCRA 2003 & BOA VISTA E SALOBRO & MORRO DO CHAPEU DO PIAUÍ & $\mathrm{PI}$ & 1717 & 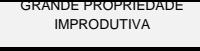 & 1754 & 0 & & \\
\hline 220115 & $\begin{array}{l}\text { BIAAREOS } \\
\text { BARBOS }\end{array}$ & PSDB PR & INCRA 2003 & FAZENDA ECONOMIZA IV & BAIXA GRANDE DO RIBEIRO & $\mathrm{PI}$ & 5136 & 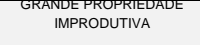 & 5136 & 0 & R.J. SOLETTI & $\begin{array}{l}\text { WARROS declarou esta } \\
\text { BARALAE }\end{array}$ \\
\hline 330450 & $\begin{array}{l}\text { EEONARDO CARNERORO } \\
\text { MONTERO PIICIAANII }\end{array}$ & PMDB RJ & INCRA 2003 & FAZENDA SAUDADE & RIO DAS FLORES & RJ & 318 & $\begin{array}{l}\text { EVIAFFOPFIIEAD } \\
\text { IMPRODUTIVA }\end{array}$ & 318 & 0 & JORGE SAYED PICCIANI & $\begin{array}{l}\text { PAIDELEEONARED } \\
\text { CARNEIRO PICCIANI }\end{array}$ \\
\hline 330450 & $\begin{array}{l}\text { EOONARDO CARNERO } \\
\text { MONTERO PICCIANI }\end{array}$ & PMDB RJ & INCRA 2003 & FAZZNDA SANTA MARIA & RIO DAS FLORES & RJ & 396 & $\begin{array}{l}\text { GRAVUE PFOPFIIEDAUE } \\
\text { PRODUTIVA }\end{array}$ & 396 & 0 & JORGE SAYED PICCIANI & $\begin{array}{l}\text { PAIUELEOUNARUIO } \\
\text { CARNEIRO PICCIANI }\end{array}$ \\
\hline 330450 & $\begin{array}{l}\text { EEONARDO CARNEROO } \\
\text { MONTERO PIICGIANII }\end{array}$ & PMDB RJ & INCRA 2003 & FAZENDA PRIMAVERA & RIO DAS FLORES & RJ & 677 & 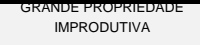 & 677 & 0 & JORGE SAYED PICCIANI & $\begin{array}{l}\text { PAAULLEEONARED } \\
\text { CARNEIRO PICCIANI }\end{array}$ \\
\hline 240800 & $\begin{array}{l}\text { CARLOS ALBERTO DE SOUZA } \\
\text { ROSADO }\end{array}$ & PFLRN & INCRA 2003 & POCO DO MEIO & MOSSORÓ & $\mathrm{RN}$ & 554 & 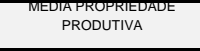 & 554 & 0 & & \\
\hline 110002 & CONEÚCIO ARES MOURA & РмDв во & INCRA 2003 & FAZ SAO FRANCISCO & ARIQUEMES & Rо & 150 & PEQUENA PROPRIEDADE & 150 & 0 & & \\
\hline 110002 & CONFÚCIO AIRES MOURA & РмDв во & INCRA 2003 & FAZ SAO FRANCISCO & ARIQUEMES & RO & 236 & PEQUENA PROPRIEDADE & 236 & 0 & & \\
\hline 110002 & CONFÚCIO AIRES MOURA & РмDB во & INCRA 2003 & FAZ SAO FRANCISCO & ARIQUEMES & RO & 237 & PEQUENA PROPRIEDADE & ${ }_{237}$ & 0 & & \\
\hline 110002 & CONFÚCIO AIRES MOURA & РMDB RO & INCRA 2003 & FAZENDA SANTOS MARIAA & ARIQUEMES & RO & 980 & $\begin{array}{l}\text { RANDEFROPFIIIEA } \\
\text { IMPROOUTIVA }\end{array}$ & 980 & 0 & & \\
\hline 110002 & ERNANDES SANTOS AMORIM & Ртв во & INCRA 2003 & FAZENDA POUSO FELIZ & ARIQUEMES & RO & 247 & IMPRODUTIVA & 247 & 0 & & \\
\hline 110029 & IVO NARCISO CASSOL & PP Rо & INCRA 2003 & SITIO NARCISO & SANTA LUZIA D'OESTE & Rо & 106 & PEQUENA PROPRIEDADE & 106 & 0 & & \\
\hline 110029 & |VO NARCISO CASSOL & PP RO & INCRA 2003 & SITIO SAO GERALDO & SANTA LUZIA D'OESTE & Ro & 188 & PEQUENA PROPRIEDADE & 188 & 0 & & \\
\hline 110029 & REDITÁRIO CASSOL & PP во & INCRA 2003 & FAZENDA ARIPUANA & SANTA LUZIA DOESTTE & Rо & 36 & MINIIÉNDIO & 36 & 0 & $\begin{array}{l}\text { AASSOL } \\
\text { CASELA }\end{array}$ & NAOO IDENTFIFACADO \\
\hline 110008 & REDITÁRIO CASSOL & PPRO & INCRA 2003 & SITIO PE DE SERRA & COSTA MARQUES & Rо & 48 & MINIFÚNDIO & 0 & 48 & ORIDES ANTONIO CASSOL & NÁO IDENTFICACADO \\
\hline 110029 & REDITÁRIO CASSOL & PP RO & INCRA 2003 & SITIO SAO JOAO & SANTA LUZIA D'OESTE & Rо & 48 & MINIFÚNDIO & 48 & 0 & $\begin{array}{l}\text { DANIISE ROSAIICASSOC } \\
\text { MEIRA }\end{array}$ & $\begin{array}{l}\text { FILLADE REUTIARIIO } \\
\text { CASSOL }\end{array}$ \\
\hline 110012 & REDITÁRIO CASSOL & PP Rо & INCRA 2003 & SITIO BOA ESPERANCA & Jl-PARANÁ & RO & 50 & MINIFÚNDIO & 0 & 50 & VALDIR JOAO CASSOL & $\begin{array}{l}\text { GRAQDDOPARENESC } \\
\text { NAOO IDENTFIIICADO }\end{array}$ \\
\hline 110045 & REDITÁRIO CASSOL & PР во & INCRA 2003 & & BURITIS & Rо & 51 & MINIFÚNDIO & 0 & 51 & OLDAR JOSE CASSOL & $\begin{array}{l}\text { GRAQDODAFENTESC } \\
\text { NAOO IDENTFICACADO }\end{array}$ \\
\hline 110029 & REDITÁRIO CASSOL & PP RO & INCRA 2003 & FAZENDA ARIPUANA & SANTA LUZIA DOEESTE & RO & 105 & PEQUENA PROPRIEDADE & 105 & 0 & DARGLLA TEREZINHA CASSOL & $\begin{array}{l}\text { FILADED REDIAFIT } \\
\text { CASSOL }\end{array}$ \\
\hline 110029 & REDITÁRIO CASSOL & PP RO & INCRA 2003 & FAZENDA ARIPUANA & SANTA LUZIA D'OESTE & RO & 114 & PEQUENA PROPRIEDADE & 114 & 219 & DARCLLA TEREZINHA CASSOL & $\begin{array}{l}\text { FILAADEREEUTARIO } \\
\text { CASSOL }\end{array}$ \\
\hline 110029 & REDITÁRIO CASSOL & PP Rо & INCRA 2003 & SITIO MIRANDA & SANTA LUZIA D'OESTE & Rо & ${ }_{187}$ & PEQUENA PROPRIEDADE & 187 & 0 & & \\
\hline 110029 & REDITÁRIO CASSOL & PP RO & INCRA 2003 & SITIO SAO JOSE & SANTA LUZIA D'OESTE & Rо & 199 & PEQUENA PROPRIEDADE & 199 & 0 & CEZAR CASSOL & $\begin{array}{l}\text { FASSOL } \\
\text { CALUIIAT }\end{array}$ \\
\hline 110029 & REDITÁRIO CASSOL & PP RO & INCRA 2003 & FAZENDA FENIX & SANTA LUZIA D'OESTE & RO & 408 & $\begin{array}{l}\text { MÉDIA PROPRIEDADE } \\
\text { IMPROOUTIVA }\end{array}$ & 0 & 408 & $\begin{array}{l}\text { DARCLLKA TEREZINHA } \\
\text { CASSOL }\end{array}$ & 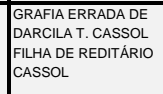 \\
\hline 110029 & REDITÁRIO CASSOL & PP RO & INCRA 2003 & FAZENDA RIO FORMOSO & SANTA LUZIA DOEESTE & RO & 408 & $\begin{array}{l}\text { MEDIAPROPRIIIDDAD } \\
\text { IMPRODUTIVA }\end{array}$ & 0 & 408 & \begin{tabular}{|l|} 
DARCLLA TEREZINHA CASSOL \\
\end{tabular} & $\begin{array}{l}\text { FILARDEE REDITARIO } \\
\text { CASSOL }\end{array}$ \\
\hline 110080 & RUBENS MOREIRA MENDES FILHO & PPS RO & INCRA 2003 & FAZENDA TRES CAPELAS & CANDEEAS DO JAMARII & RO & 876 & $\begin{array}{l}\text { MMEUAAPROPFIEVAUE } \\
\text { IMPROUTIVA }\end{array}$ & 876 & 0 & 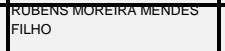 & \\
\hline 110030 & VALDIR COLATTO & РMDB SC & INCRA 2003 & FAZENDA ELDA & VILHENA & Rо & 99 & PEQUENA PROPRIEDADE & 99 & 0 & & \\
\hline 110030 & |VALIR COLATTO & РMDв SC & INCRA 2003 & FAZENDA BANDEIRANTE & VILHENA & Ro & 100 & PEQUENA PROPRIEDADE & 100 & 0 & & \\
\hline 110030 & VALDIR COLATTO & PMDB SC & INCRA 2003 & AGROPECUARIA XANXERE & VILHENA & RO & 1063 & $\begin{array}{l}\text { AWUEF HOPFIEU } \\
\text { PRODUTVA }\end{array}$ & 1063 & 0 & & \\
\hline 110009 & $\begin{array}{l}\text { VAADUIFRAAPPPMARII } \\
\text { RAUPP DE MATOS }\end{array}$ & РмDB во & INCRA 2003 & FAZ BARRETOS & ESPIGÃO DIOESTE & Ro & 1634 & $\begin{array}{l}\text { RANDEFROPFIIIUA } \\
\text { IMPRODUTIVA }\end{array}$ & 1634 & 0 & JOAO RAUPP DE MATTOS & \\
\hline 110018 & 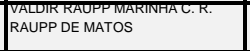 & РмDв во & INCRA 2003 & FAZENDA JR & PIMENTA BUENO & Rо & 419 & 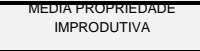 & 0 & 419 & JOAO RAUPP DE MATTOS & \\
\hline 110014 & $\begin{array}{l}\text { RAUPP DE MATOS } \\
\text { RALA }\end{array}$ & Рмов во & INCRA 2003 & IITIO CATARINENSE & NOVA BRASLLÂNDIA DIOESTE & Rо & 48 & MINIIÉNDIO & 0 & 48 & VILMAR RAUPP DE MATOS & \\
\hline 110014 & $\begin{array}{l}\text { RAUPP DE MATOS } \\
\text { RATS }\end{array}$ & РмDв во & INCRA 2003 & IITIO SAO CRISTOVAO & NOVA BRASLLANDDIA DIOESTE & во & 49 & Minifündo & 0 & 49 & VALDIR RAUPP DE MATOS & \\
\hline
\end{tabular}




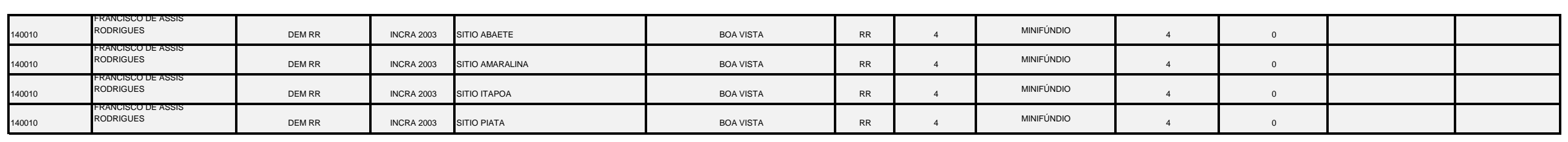




\begin{tabular}{|c|c|c|c|c|c|c|c|c|c|c|c|c|}
\hline GEOCODIGO & PARLAMENTAR & PARTIDO & FONTE & IMÓVEL & MUNICIPIO & uF & AREA & CATEGORIA FUNDIÁRIAA & ÁREA REGISTRADA & $\begin{array}{l}\text { AEEA DEELAFAUA } \\
\text { COMO POSSE }\end{array}$ & $\begin{array}{l}\text { REGISTRO EM NOME DE } \\
\text { OUTRAS PESSOAS }\end{array}$ & $\begin{array}{l}\text { ViNCULO COM O } \\
\text { PARLAMENTAR }\end{array}$ \\
\hline 140060 & $\begin{array}{l}\text { FRANCISCO DE ASSIS } \\
\text { RODRIGUES }\end{array}$ & DEMrR & INCRA 2003 & SITIO LIMA & SÃo LuIz & $\mathrm{RR}$ & 103 & PEQUENA PROPRIEDADE & 0 & 103 & $\begin{array}{l}\text { ROBERT SALLINGER } \\
\text { NOBREGA LIMA }\end{array}$ & 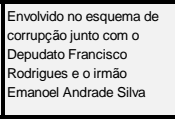 \\
\hline 140005 & $\begin{array}{l}\text { ROARVIISCOUE } \\
\text { RODRGUES }\end{array}$ & DEMrR & INCRA 2003 & POtiREtAMA & ALTO ALEGRE & $\mathrm{RR}$ & 107 & PEQUENA PROPRIEDADE & 107 & 0 & ANTONIO NONO RODRIGUES & 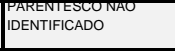 \\
\hline 140005 & $\begin{array}{l}\text { ROADVISCOUE } \\
\text { RODIGUES }\end{array}$ & DEMrR & INCRA 2003 & FAZ SAO LUIz & ALTO ALEGRE & $\mathrm{RR}$ & 281 & PEQUENA PROPRIEDADE & 281 & 0 & ANTONIO NONO RODRIGUES & $\begin{array}{l}\text { IDENTIFICADO } \\
\text { IARE }\end{array}$ \\
\hline 140060 & 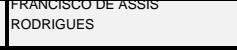 & DEMrR & INCRA 2003 & FAZENDA CACIMBA NOVAI & SẪo LUIz & $\mathrm{RR}$ & 300 & PEQUENA PROPRIEDADE & 0 & 300 & & \\
\hline 140060 & 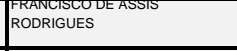 & DEM RR & INCRA 2003 & FAZENDA CACINBA NOVA II & SẪo LUIZ & $\mathrm{RR}$ & 400 & PEQUENA PROPRIEDADE & 0 & 400 & & \\
\hline 140060 & $\begin{array}{l}\text { FRANCISCO DE ASSIS } \\
\text { RODRIGUES }\end{array}$ & DEM RR & INCRA 2003 & FAZENDA SAO SEBASTIAO & SÃo LUIZ & $\mathrm{RR}$ & 472 & $\begin{array}{l}\text { MÉDIA PROPRIEDADE } \\
\text { IMPROOUTVA }\end{array}$ & 0 & 469 & EMANOEL ANDRADE SLIVA & $\begin{array}{l}\text { FRANCISCO } \\
\text { RODRILUES TEM IRMÃO } \\
\text { COME STE NOME }\end{array}$ \\
\hline 140030 & $\begin{array}{l}\text { RODRIGUES } \\
\text { RADE ASTIS }\end{array}$ & DEMrR & INCRA 2003 & FAZ SAO RAMUUNDOI & mucauai & $\mathrm{RR}$ & 491 & 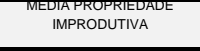 & 491 & 0 & ANTONIO NONO RODRIGUES & $\begin{array}{l}\text { PAERNTESCONAO } \\
\text { DEENTICADO }\end{array}$ \\
\hline 140005 & $\begin{array}{l}\text { FRANCISCO DE ASIS } \\
\text { RoDRIGUES }\end{array}$ & DEM RR & INCRA 2003 & SITIO SERRINHA II & ALTO ALEGRE & $\mathrm{RR}$ & 1027 & $\begin{array}{l}\text { MÉDIA PROPRIIEDADE } \\
\text { PRODUTIVA }\end{array}$ & 1027 & 0 & ANTONIO NONO RODRIGUES & 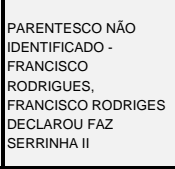 \\
\hline 140005 & $\begin{array}{l}\text { FODANIIICEODDE ASSIS } \\
\text { RODIGUS }\end{array}$ & DEMrR & INCRA 2003 & FAZ SANTA LUZIA & ALTO ALEGRE & $\mathrm{RR}$ & 1916 & 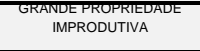 & 1876 & 0 & ANTONIO NONO RODRIGUES & $\begin{array}{l}\text { PARENIESCEONAC } \\
\text { DENTIFICADO }\end{array}$ \\
\hline 140015 & $\begin{array}{l}\text { RODRIGUES } \\
\text { ROASES }\end{array}$ & DEMrR & INCRA 2003 & FAZ SAO FRANCISCO DE ASSIS & BONFIM & $\mathrm{RR}$ & 2604 & $\begin{array}{l}\text { INUEFHOPFIED } \\
\text { PRODUTIVA }\end{array}$ & 2608 & 0 & ANTONIO NONO RODRIGUES & 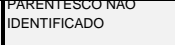 \\
\hline 140010 & RAUL DA SILVA LMA SOBRINHO & PMDB RR & INCRA 2003 & FAZENDA SEGREDO ॥ & BOA VISTA & $\mathrm{RR}$ & 1697 & IMPROOUTIVA & 0 & 1702 & SOBRINHO & \\
\hline 140010 & $\begin{array}{l}\text { TALCWIAO AFOUSOUE SOUSA } \\
\text { CRUZ }\end{array}$ & Ртв RR & INCRA 2003 & FAZENDA SAO SEBASTIAO & BOA VISTA & $\mathrm{RR}$ & 1183 & $\begin{array}{l}\text { EDVARFOPFIIEADVE } \\
\text { IMPRODUIVA }\end{array}$ & 1183 & 0 & & \\
\hline 431800 & LUIZ CARLOS HENZE & PPRS & INCRA 2003 & RN DAS TUNAS ESESMARIA DA BOA VISTA & SÃO BORJA & RS & 433 & $\begin{array}{l}\text { GRANUE PROPTIIEDAD } \\
\text { PROOUTIAA }\end{array}$ & 433 & 0 & & \\
\hline 420620 & EDSON BEZ DE OLIVEIRA & PMDB SC & INCRA 2003 & $?$ & gravatal & sc & 6 & MIIIFÚNDIO & 6 & 0 & & \\
\hline 420620 & EDSON BEZ DE OLVEIRA & PMDB SC & INCRA 2003 & \begin{tabular}{|l} 
TERMAS Do GRAVATAL \\
\end{tabular} & GrAvatal & $\mathrm{sc}$ & 10 & MINIFÚNDIO & 10 & 0 & MACHADO & 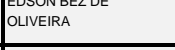 \\
\hline 420620 & EDSON BEZ DE OLIVEIRA & PMDB SC & INCRA 2003 & $?$ & GRAVATAL & sc & 24 & PEQUENA PROPRIEDADE & 24 & 0 & & \\
\hline 420620 & EDSON BEZ DE OLIVEIRA & PMDB SC & INCRA 2003 & $?$ & GRAVATAL & sc & 70 & IMPRODUTIVA & 70 & 0 & & \\
\hline 421430 & ESPERIDIIOA AMIN HELOU FLLHO & PP SC & INCRA 2003 & SITIO DE RANCHO QUEIMADO & RANCHO QUEIMADO & sc & 6 & MIIIFÚNDIO & 6 & 0 & & \\
\hline 421660 & GERVASIO JOSE DA SILVA & PFLSC & INCRA 2003 & $?$ & SÃO JOSÉ & $\mathrm{sc}$ & 25 & PEQUENA PROPRIEDADE & 25 & 0 & & \\
\hline 420420 & HUGO MATHAS BІЕНL & PPB SC & INCRA 2003 & $?$ & CHAPECÓ & sc & 12 & MIIIFÚNDIO & 12 & 0 & & \\
\hline 421320 & 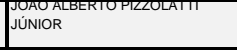 & pPsc & INCRA 2003 & JOÃo ALBERTO PIZZOLATTI JÚNIIOR & POMERODE & sc & 8 & MINIFÚNDIO & 8 & 0 & & \\
\hline 421650 & Jö́o BATISTA MATOS & PMDB SC & INCRA 2003 & FAZENDA BAIOS & SĀO JOAQUIM & $\mathrm{sc}$ & 18 & MIIIFÚNDIO & 18 & 0 & & \\
\hline 420690 & JoÄ́ BATISTA MATOS & PMDB SC & INCRA 2003 & LTS 4354364374388499440441 & iBriama & sc & 153 & $\begin{array}{l}\text { PRODUTIVA } \\
\text { PRA }\end{array}$ & 153 & 0 & & \\
\hline 420300 & Jö̈o Batista MATOS & PMDBSC & INCRA 2003 & FAZENDA CACHOEIRA ANTIGA & CAÇADOR & $\mathrm{sc}$ & 215 & IMPRODUTIVA & 215 & 0 & & \\
\hline 421760 & RONALDO JOSÉ BENEDET & PMDBSC & INCRA 2003 & SITIO FUNDO DOS GROTOES & SIDERÓPOLIS & sc & 11 & MIIIFǗNDIO & 11 & 0 & & \\
\hline 421760 & RONALDO JOSÉ BENEDET & PMDBSC & INCRA 2003 & SIITO DAS LARANJEIRAS & SIDERÓPOLIS & sc & 11 & MIIIFÚNDIO & 11 & 0 & & \\
\hline 421760 & RONALDO JOSÉ BENEDET & PMDBSC & INCRA 2003 & SITIO DO RIO PERCIO & SIDERÓPOLIS & $\mathrm{sc}$ & 11 & MINIFÚNDIO & 11 & 0 & & \\
\hline
\end{tabular}




\begin{tabular}{|c|c|c|c|c|c|c|c|c|c|c|c|c|}
\hline GEOCODIGO & PARLAMENTAR & PARTIDO & FONTE & IMóveL & MUNICIPPO & UF & ÁREA & CATEGORIA FUNDIÁRIA & AREA REGISTRADA & $\begin{array}{l}\text { AREAUECLAARUUA } \\
\text { COMO POSSE }\end{array}$ & $\begin{array}{l}\text { REGISTRO EM NOME DE } \\
\text { OUTRAS PESSOAS }\end{array}$ & $\begin{array}{l}\text { viNcuLO COMO O } \\
\text { PARLAMENTAR }\end{array}$ \\
\hline 421760 & RONALDO JOSÉ BENEDET & PMDB sc & INCRA 2003 & RIO SITIO DO MANIN & SIDERÓPOLIS & sc & 14 & PEQUENA PROPRIEDADE & 14 & 0 & & \\
\hline 421760 & RONALDO JOSÉ BENEDET & PMDB SC & INCRA 2003 & QUERENCIA DO BOLERO & SIDERÓPOLIS & sc & 23 & PEQUENA PROPRIEDADE & 23 & 0 & & \\
\hline 420010 & VALDIR COLATTO & PMDB SC & INCRA 2003 & FAZENDA COLATTO & ABELARDO LUZ & sc & 16 & MINIFÚNDIO & 16 & 0 & & \\
\hline 260450 & JOÃO BASTOS COLAÇO DIAS & PSBPE & INCRA 2003 & ENGENHO MACACOS & CHā GRANDE & SE & 9 & MINIÉ́NDIO & 9 & 0 & GUSTAVO COLACO DIAS & $\begin{array}{l}\text { PAIUE JOAUBAS } \\
\text { COLAÇQ DIAS }\end{array}$ \\
\hline 260450 & JOÃO BASTOS COLAÇO DIAS & $\begin{array}{l}\text { PSBPE } \\
\end{array}$ & INCRA 2003 & MACACOS & CHĀ GRANDE & SE & 53 & PEQUENA PROPRIIEDADE & 53 & 0 & GUSTAVO COLACO DIAS & 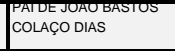 \\
\hline 260450 & JOĀO BASTOS COLAÇO DIAS & $\begin{array}{l}\text { PSB PE } \\
\end{array}$ & INCRA 2003 & FAZENDA AROEIRAS & CHĀ GRANDE & SE & 54 & PEQUENA PROPRIEDADE & 54 & 0 & GUSTAVO COLACO DIAS & COLACGO DIAS \\
\hline 260450 & JOĀO BASTOS COLAÇO DIAS & $\begin{array}{l}\text { PSB PE } \\
\text { PSE }\end{array}$ & INCRA 2003 & FAZENDA AROEIRAS & CHĀ GRANDE & SE & 119 & $\begin{array}{l}\text { MEDARPROPFIIIDAL } \\
\text { IMPRODUTVA }\end{array}$ & 119 & 0 & $\begin{array}{l}\text { GESTAT } \\
\text { NETO }\end{array}$ & $\begin{array}{l}\text { PAIDE JOADBAS } \\
\text { COLAÇO DIAS }\end{array}$ \\
\hline 261130 & JOẤO BASTOS COLAÇO DIAS & PSBPE & INCRA 2003 & SIITIO CAJOCA & РомвоS & SE & 218 & $\begin{array}{l}\text { MEDARPROPFIIIVALC } \\
\text { IMPROUTIVA }\end{array}$ & 218 & 0 & $\begin{array}{l}\text { GULILFERIIE BASTOSCOLAT } \\
\text { DAAS }\end{array}$ & 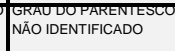 \\
\hline 280290 & JOSÉ CARLOS MACHADO & DEMSE & INCRA 2003 & SITIOO BatULA & ItABaAanA & SE & 6 & MINIF́́ndIO & 6 & 0 & JOSE CARLOS MACHADO & $\begin{array}{l}\text { SAO AS MESMAS } \\
\text { PROPRIEDADES } \\
\text { DECLARADAS A } \\
\text { RECEITA }\end{array}$ \\
\hline 280290 & JOSÉ CARLOS MACHADO & DEMSE & INCRA 2003 & FAZENDA TELHA & ITABAIANA & SE & 518 & $\begin{array}{l}\text { GRANDE PROPRIEDADE } \\
\text { IMPRODUTIVA }\end{array}$ & 518 & 0 & JOSE CARLOS MACHADO & $\begin{array}{l}\text { SÃO AS MESMAS } \\
\text { PROPRIEDADES } \\
\text { DECLARAADS A } \\
\text { RECEITA }\end{array}$ \\
\hline 280067 & JOSÉ CLEONÂNCIO DA FONSECA & PMDBSE & INCRA 2003 & SITIO JABOTICABAL & BoQUIM & SE & 12 & MINIIÚNDIO & 12 & 0 & & \\
\hline 280067 & JOSÉ CLEONÂNCIO DA FONSECA & PMDBSE & INCRA 2003 & FAZENDA PALMEIRA & BoQuim & SE & 34 & PEQUENA PROPRIIEDADE & 34 & 0 & & \\
\hline 280040 & JOSÉ CLEONÂNCIO DA FONSECA & PMDB SE & INCRA 2003 & SITIO SAO JOSE & araUúa & SE & 55 & PEQUENA PROPRIIEDADE & 55 & 0 & & \\
\hline 280067 & JOSÉ CLEONÂNCIO DA FONSECA & PMDB SE & INCRA 2003 & FAZENDA PILOES & Boquim & SE & 160 & $\begin{array}{l}\text { DEAPFOPRIEEA } \\
\text { PRODUTVA }\end{array}$ & 0 & 160 & & \\
\hline 280067 & JOSÉ CLEONÂNCIO DA FONSECA & PMDB SE & INCRA 2003 & FAZENDA PILOES & Boquim & SE & 160 & PRODUTIVA & 0 & 160 & & \\
\hline 280320 & JOSÉ CLEONÂNCIO DA FONSECA & PMDB SE & INCRA 2003 & FAZ ATALAIA & ITAPORANGA DAJUUDA & SE & 454 & IMPRODUTIVA & 454 & 0 & & \\
\hline 280320 & JOSÉ CLEONÂNCIO DA FONSECA & PMDB SE & INCRA 2003 & FAZ ATALAIA & ITAPORANGA DAAJUDA & SE & 454 & 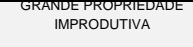 & 454 & 0 & & \\
\hline 280067 & JOSÉ CLEONÂNCIO DA FONSECA & PMDB SE & INCRA 2003 & SITIO JABOTICABAL & Boquim & SE & 12 & MINIÉNDIO & 12 & 0 & & \\
\hline 280067 & JOSÉ CLEONÂNCIO DA FONSECA & PMDB SE & INCRA 2003 & FAZENDA PALMEIRA & BoQuim & $\overline{\mathrm{SE}}$ & 34 & PEQUENA PROPRIEDADE & 34 & 0 & & \\
\hline 280040 & JOSÉ CLEONÂNCIO DA FONSECA & PMDB SE & INCRA 2003 & SITIO SAO JOSE & araúá & SE & 55 & PEQUENA PROPRIEDADE & 55 & 0 & & \\
\hline 351750 & ALOYSIO NUNES FERREIRA FILHO & PSDB SP & INCRA 2003 & FAZENDA SANTANA & guAPIAÇU & $\mathrm{SP}$ & 66 & $\begin{array}{l}\text { MEDVARFOFPIIIDAVE } \\
\text { IMPRODUTIVA }\end{array}$ & 66 & 0 & ALOYSIO NUNES FERREIRA & $\begin{array}{l}\text { PAIDEALCY } \\
\text { FERREIRA }\end{array}$ \\
\hline 351890 & ALOYSIO NUNES FERREIRA FILHO & PSDB SP & INCRA 2003 & FAZENDA MARIMBONDO & GUZOLÂNDIA & $\mathrm{sP}$ & 135 & PEQUENA PROPRIEDADE & 135 & 0 & $\begin{array}{l}\text { ALLYSIO NUNESTERTEIRA } \\
\text { FLLO }\end{array}$ & $\begin{array}{l}\text { PADUEADOYSTO } \\
\text { FERREIRA }\end{array}$ \\
\hline 354025 & ALOYSIO NUNES FERREIRA FILHO & PSDB SP & INCRA 2003 & FAZENDA BOA ESPERANCA & PONTALINDA & $\mathrm{sP}$ & 1624 & 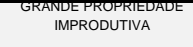 & 1624 & 0 & ALOYSIO NUNES FERREIRA & $\begin{array}{l}\text { PADUEALO } \\
\text { FERREIRA }\end{array}$ \\
\hline 354660 & EDSON EDINHO COELHO ARAúJO & РмDB SP & INCRA 2003 & ESTANCIA ARACAJU III & SANTA FÉ DO SUL & $\mathrm{SP}$ & 36 & PEQUENA PROPRIEDADE & 36 & 0 & EMIDIO ANTONIO ARAUJO & $\begin{array}{l}\text { PAR-FUSOUVEDUVIVI } \\
\text { COELHO ARAUUJO }\end{array}$ \\
\hline 354660 & EDSON EDINHO COELHO ARAÚJO & РмDB SP & INCRA 2003 & ESTANCIA ARACAJUI & SANTA FÉ DO SUL & $\mathrm{SP}$ & 106 & PEQUENA PROPRIEDADE & 106 & 0 & EMIDIO ANTONIO ARAUJO & $\begin{array}{l}\text { COELHO ARAÚJO } \\
\text { CoEs }\end{array}$ \\
\hline 351520 & ETIVALDO VADÃo GOMES & PP SP & INCRA 2003 & CHACARA APARECIDA & ESTRELA D'OESTE & $\mathrm{SP}$ & 2 & PEQUENA PROPRIEDADE & & & & \\
\hline 351520 & ETVALDO VADẪo GOMES & PP SP & INCRA 2003 & CHACARA MOLINA & ESTRELA D'OESTE & SP & 6 & PEQUENA PROPRIEDADE & & & & \\
\hline 351520 & ETIVALDO VADÃO GOMES & PP SP & INCRA 2003 & CHACARA RECREIO & ESTRELA D'OESTE & $\mathrm{SP}$ & 12 & PEQUENA PROPRIEDADE & & & & \\
\hline 351520 & ETIVALDO VADĀO GOMES & PP SP & INCRA 2003 & CHACARA SAO JOAO & ESTRELA D'OESTE & $\mathrm{SP}$ & 16 & PEQUENA PROPRIEDADE & & & & \\
\hline 351520 & ETIVALDO VADĀO GOMES & PP SP & INCRA 2003 & CHACARA RECREIO & ESTRELA D'OESTE & SP & 18 & PEQUENA PROPRIEDADE & & & & \\
\hline
\end{tabular}




\begin{tabular}{|c|c|c|c|c|c|c|c|c|c|c|c|c|}
\hline GEOCODIGO & PARLAMENTAR & PARTIDO & FONTE & IMÓVEL & MUNICIPPO & UF & AREA & CATEGORIA FUNDIÁRIA & AREA REGISTRADA & $\begin{array}{l}\text { AREAUELCAAAUA } \\
\text { COMO POSSE }\end{array}$ & $\begin{array}{l}\text { REGISTRO EM NOME DE } \\
\text { OUTRAS PESSOAS }\end{array}$ & $\begin{array}{l}\text { viNCULO COM O } \\
\text { PARLAMENTAR }\end{array}$ \\
\hline 351520 & ETIVALDO VADÃO GOMES & PP SP & INCRA 2003 & FAZ TURBLLHÂO & ESTRELA D'OESTE & $\mathrm{SP}$ & 27 & PEQUENA PROPRIEDADE & & & & \\
\hline 352480 & ETIVALDO VADÃO GOMES & PP SP & INCRA 2003 & FAZ TURBILHÃo & JALES & $\mathrm{sP}$ & 27 & PEQUENA PROPRIEDADE & & & & \\
\hline 351520 & ETIVALDO VADÃo GOMES & PP SP & INCRA 2003 & SITIO ALVORADA & ESTRELA D'OESTE & $\mathrm{sP}$ & 100 & PEQUENA PROPRIEDADE & & & & \\
\hline 350330 & FRANCISCO GRAZIANO NETO & PSDBSP & INCRA 2003 & FAZ SANTA CLEMENTINA & ARARAS & $\mathrm{SP}$ & 8 & PEQUENA PROPRIIEDADE & & & & \\
\hline 351840 & $\begin{array}{l}\mid \text { FAAUSIS } \\
\text { FLLHO }\end{array}$ & PVSP & INCRA 2003 & SITIO XIKOTE & GUARATINGUETÁ & $\mathrm{SP}$ & 19 & MINIF́́nDIO & 19 & 0 & $\begin{array}{l}\text { FIFANC } \\
\text { FLHO }\end{array}$ & \\
\hline 351360 & $\begin{array}{l}\text { FLAHO } \\
\end{array}$ & PVSP & INCRA 2003 & FAZENDA MORADA DO SOL & CUNHA & $\mathrm{SP}$ & 45 & PEQUENA PROPRIIEDADE & 17 & 28 & $\begin{array}{l}\text { FLLHO } \\
\end{array}$ & \\
\hline 353870 & JOĀO HERRMANN NETO & PDT SP & INCRA 2003 & CHACARA POMPEIA & PIRACICABA & $\mathrm{SP}$ & 1 & MINIF́́ndIO & 1 & 0 & JOAO HERRMANN NETO & \\
\hline 355870 & JOĀO HERRMANN NETO & PDT SP & INCRA 2003 & CHACARA POMPEIA & PIRACICABA & $\mathrm{SP}$ & 10 & PEQUENA PROPRIEDADE & 10 & 0 & JOAO HERRMANN NETO & \\
\hline 350430 & JOĀO HERRMANN NETO & PDT SP & INCRA 2003 & FAZENDA SAO SEBASTIAO & Avai & $\mathrm{sP}$ & 14 & PEQUENA PROPRIEDADE & 14 & 0 & JOAO HERRMANN NETO & \\
\hline 350430 & JOÄO HERRMANN NETO & PDT SP & INCRA 2003 & FAZENDA SAO SEBASTIAO & Avai & $\mathrm{sP}$ & 14 & PEQUENA PROPRIEDADE & 14 & 0 & JOAO HERRMANN NETO & \\
\hline 354110 & JOĀO HERRMANN NETO & PDT SP & INCRA 2003 & SITIO BELA VISTA & PRESIDENTE ALVES & $\mathrm{sP}$ & 15 & MINIFÚNDIO & 15 & 0 & JOAO HERRMANN NETO & \\
\hline 350430 & JOĀO HERRMANN NETO & PDT SP & INCRA 2003 & FAZENDA SAO SEBASTIAO & AVAi & $\mathrm{sP}$ & 18 & PEQUENA PROPRIIEDADE & 18 & 0 & JOAO HERRMANN NETO & \\
\hline 350430 & JOĀO HERRMANN NETO & PDT SP & INCRA 2003 & FAZENDA SAO SEBASTIAO & AVai & $\mathrm{SP}$ & 282 & $\begin{array}{l}\text { PRODUTIVA } \\
\text { PRADE }\end{array}$ & 282 & 0 & JOAO HERRMANN NETO & \\
\hline 353060 & JUNJ ABE & DEMSP & INCRA 2003 & SITIO ABE & MOJ DAS CRUZES & $\mathrm{SP}$ & 3 & MINIF́́NDIO & 3 & 0 & & \\
\hline 353060 & JUNJ ABE & DEM SP & INCRA 2003 & JUNJ ABE & MOJ DAS CRUZES & $\mathrm{sP}$ & 9 & PEQUENA PROPRIIEDADE & 9 & 0 & & \\
\hline 353060 & JUNJI ABE & DEM SP & INCRA 2003 & FAZENDA ABE & MOJ DAS CRUZES & $\mathrm{SP}$ & 116 & 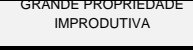 & 116 & 0 & & \\
\hline 353930 & NELSON MARQUEZELLI & Ртв SP & INCRA 2003 & FAZ CACHOEIRA & PIRASSUNUNGA & $\mathrm{SP}$ & 52 & PEQUENA PROPRIEDADE & & & & \\
\hline 351370 & NELSON MARQUEZZELI & Pтв SP & INCRA 2003 & FAZ SERRINHA & DESCALVADO & $\mathrm{sP}$ & 74 & 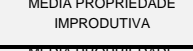 & & & & \\
\hline 353930 & NELSON MARQUEZELLI & PтB SP & INCRA 2003 & ISNTANCIA NS APARECIDA & PIRASSUNUNGA & $\mathrm{SP}$ & 92 & 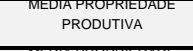 & 92 & 0 & & \\
\hline 351270 & NELSON MARQUEZELLI & Ртв SP & INCRA 2003 & FAZ SAO FRANCISCO & CORUMBataí & $\mathrm{sP}$ & 185 & 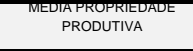 & 185 & 0 & & \\
\hline 353930 & NELSON MARQUEZZELI & Pтв SP & INCRA 2003 & FAZ CAMPO ALEGRE & PIRASSUNUNGA & $\mathrm{sP}$ & 209 & $\begin{array}{l}\text { EUAAPUPRIVEAL } \\
\text { IMPRODUTVA }\end{array}$ & & & & \\
\hline 351370 & NELSON MARQUEZZELI & Ртв SP & INCRA 2003 & FAZ SANTA ROSA ALTA & DESCALVADO & $\mathrm{sP}$ & 605 & PRODUTIVA & & & & \\
\hline 354140 & PAULO CESAR DE OLVEIRA LIMA & PMDB SP & INCRA 2003 & CHACARA AMANDA LIMA & PRESIIENTE PRUDENTE & $\mathrm{sP}$ & 2 & MINIF́́NDIO & 2 & 0 & $\begin{array}{l}\text { FLLHOIII } \\
\end{array}$ & DE OLIVERA LIMA \\
\hline 354140 & PAULO CESAR DE OLVEIRA LIMA & PMDB SP & INCRA 2003 & RANCHO ALEGRE & PRESIIDNTE PRUDENTE & $\mathrm{SP}$ & 3 & MINIF́UNDIO & 3 & 0 & $\begin{array}{l}\text { FLLHOIII } \\
\end{array}$ & DE OLIVERA LIMA \\
\hline 354140 & PAULO CESAR DE OLIVERA LIMA & PMDB SP & INCRA 2003 & CHACARA SAO CrISTOVAO & PRESIDENTE PRUDENTE & $\mathrm{sP}$ & 5 & MINIFÚNDIO & & & & \\
\hline 350130 & PAULO CESAR DE OLIVERA LIMA & PMDB SP & INCRA 2003 & HARAS UNIAO & ALVARES MACHADO & $\mathrm{SP}$ & 9 & MINIIÉNDIO & 9 & 0 & CARAPEBA & \\
\hline 354140 & PAULO CESAR DE OLIVEIRA LIMA & PMDB SP & INCRA 2003 & CHACARA OLIVEIRA LIMA & PRESIIDNTE PRUDENTE & $\mathrm{SP}$ & 13 & MINIF́ÚNDIO & 13 & 0 & $\begin{array}{l}\text { ARtrIIII } \\
\text { FLLHO }\end{array}$ & $\begin{array}{l}\text { PAIDERPAUOOCES } \\
\text { DE OLIVERA LIMA }\end{array}$ \\
\hline 354140 & PAULO CESAR DE OLIVEIRA LIMA & PMDB SP & INCRA 2003 & RANCHO SÃO FRANCISCO DE ASSIS & PRESIIENTE PRUDENTE & $\mathrm{sP}$ & 14 & MINIFÚNDIO & & & & \\
\hline 353920 & PAULO CESAR DE OLIVEIRA LIMA & PMDB SP & INCRA 2003 & EStANCIA Gr & PIRAPOZINHO & $\mathrm{sP}$ & 54 & PEQUENA PROPRIEDADE & & & & \\
\hline 350910 & PAULO CESAR DE OLIVERA LIMA & PMDB SP & INCRA 2003 & FAZ INDIANA & caluá & $\mathrm{SP}$ & 1476 & $\begin{array}{l}\text { GRAIUEF FOPPIIEDAVE } \\
\text { IMPRODUTVA }\end{array}$ & & & & \\
\hline 171110 & ANIBAL FERREIRA GOMES & PMDBCE & INCRA 2003 & CHACARA VISTA RICA & ITAPORÃ DO TOCANTINS & то & 13 & MINIF́́̄NDIO & 13 & 0 & ANTONIO FERREIRA GOMES & IDENTIFICADO \\
\hline 170220 & ANIBAL FERREIRA GOMES & PMDB CE & INCRA 2003 & FAZENDA BACURI & ARAGUATINS & то & 80 & PEQUENA PROPRIEDADE & 80 & 0 & ANTONIO FERREIRA GOMES & $\begin{array}{l}\text { |PARENIESCONAC } \\
\text { IDENTIFICADO }\end{array}$ \\
\hline
\end{tabular}




\begin{tabular}{|c|c|c|c|c|c|c|c|c|c|c|c|c|}
\hline GEOCODIGO & PARLAMENTAR & PARTIDO & FONTE & IMÓVEL & MUNICIPIO & UF & AREA & CATEGORIA FUNDIÁRIAA & ÁREA REGISTRADA & $\begin{array}{l}\text { AREA UECLAARUA } \\
\text { COMO POSSE }\end{array}$ & $\begin{array}{l}\text { REGISTRO EM NOME DE } \\
\text { OUTRAS PESSOAS }\end{array}$ & $\begin{array}{l}\text { vinculo com O } \\
\text { PARLAMENTAR }\end{array}$ \\
\hline 170025 & ANIBAL FERREIRA GOMES & РМDВ СE & INCRA 2003 & FAZENDA SAO PEDRO & ABREULÁNDIA & то & 108 & PEQUENA PROPRIEDADE & 108 & 0 & JOSE FERREIRA GOMES & 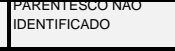 \\
\hline 171050 & ANIBAL FERREIRA GOMES & РМDB CE & INCRA 2003 & FAZENDA XANADU & ITACAJÁ & то & 186 & PEQUENA PROPRIEDADE & 186 & 0 & LUCAS FERREIRA GOMES & 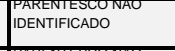 \\
\hline 170700 & ANIBAL FERREIRA GOMES & PMDB CE & INCRA 2003 & FAZENDA BURITI CUMPRIIDO LOTE 01' & DIANÓPOLIS & то & 206 & PEQUENA PROPRIEDADE & 206 & 0 & ISRAEL FERREIRA GOMES & IDENTIFICADO \\
\hline 170950 & ANIBAL FERREIRA GOMES & PMDB CE & INCRA 2003 & FAZENDA SANTA LUIZA & GURUPI & то & 228 & PEQUENA PROPRIEDADE & 228 & 0 & GESSIMAR FERREIRA GOMES & IDENTIFICADO \\
\hline 170710 & ANIBAL FERREIRA GOMES & PMDB CE & INCRA 2003 & FAZENDA SANTA TEREZA & DIIINÓPOLIS DO TOCANTINS & то & 264 & PEQUENA PROPRIEDADE & 264 & 0 & $\begin{array}{l}\text { a GOMANU } \\
\text { GOMES }\end{array}$ & IDENTIFICADO \\
\hline 171660 & ANIBAL FERREIRA GOMES & РМDB CE & INCRA 2003 & FAZ SANTA EDWIRGES & PEIXE & то & 484 & $\begin{array}{l}\text { MÉDIA PROPRIEDADE } \\
\text { IMPROOUTIVA } \\
\end{array}$ & 484 & 0 & $\begin{array}{l}\text { ANA BRAGA - ANIBAL } \\
\text { FERRERAA GOMES DECLAROU } \\
\text { FAZAZANA EDEWRGENS NO } \\
\text { TOCANTINS }\end{array}$ & \\
\hline 171050 & ANIBAL FERREIRA GOMES & PMDBCE & INCRA 2003 & FAZ MALIBU & ITACAJA & то & 678 & IMPRODUTIVA & 678 & 0 & JOSE FERREIRA GOMES & IDENTIFICADO \\
\hline 170240 & ANIBAL FERREIRA GOMES & PMDBCE & INCRA 2003 & FAZENDA CANTINHO & ARRAIAS & Tо & 1425 & IMPRODUTIVA & 1425 & 0 & LEONIDO FERREIRA GOMES') & IDENTIFICADO \\
\hline 171050 & ANIBAL FERREIRA GOMES & РМDB CE & INCRA 2003 & FAZ MALIBU & ITACAJA & то & 1819 & IMPRODUTIVA & 1838 & 0 & JOSE FERREIRA GOMES & $\begin{array}{l}\text { PDENTFICADO } \\
\text { IDETI }\end{array}$ \\
\hline 171050 & ANIBAL FERREIRA GOMES & РМDB СE & INCRA 2003 & FAZ MALIBU & ITACAJÁ & то & 1967 & IMPROOUTTYA & 1967 & 0 & JOSE FERREIRA GOMES & IDENTIFICADO \\
\hline 170210 & CESAR HANNA HaLUM & PPS Tо & INCRA 2003 & FAZENDA BOA ESPERANCA & ARAguaina & то & 47 & MINIFÚNDIO & 0 & 47 & CESAR HANNA HaLUM & \\
\hline 171820 & IGOR PUGLIESI AVELINO & Рмов то & INCRA 2003 & FAZENDA FARTURA & PORTO NACIONAL & то & 73 & MINIF́́NDIO & 73 & 0 & $\begin{array}{l}\text { AEVARES } \\
\text { TAVANG }\end{array}$ & IDENTIFICADO \\
\hline 171610 & IGOR PUGLLESI AVELINO & Рмов то & INCRA 2003 & FAZENDA PANORAMA & PARÁSO DO TOCANTINS & то & 77 & MINIIÚNDIO & 0 & 77 & JURI PUGLESI AVELINO & IDENTIFICADO \\
\hline 171820 & IGOR PUGLIESI AVELINO & РмОв то & INCRA 2003 & FAZENDA BREJINHO & PORTO NACIONAL & то & 105 & PEQUENA PROPRIEDADE & 105 & 0 & 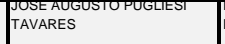 & 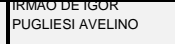 \\
\hline 171888 & IGOR PUGLESI AVELINO & Рмов то & INCRA 2003 & FAZENDA VISTA BONITA & SANTA MARIA DO TOCANTINS & то & 607 & 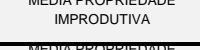 & 607 & 0 & ORION PUGLESI TAVARES & IDENTIFICADO \\
\hline 171050 & IGOR PUGLIESI AVELINO & РмОв то & INCRA 2003 & FAZENDA BACABA & ITACAJÁ & то & 675 & $\begin{array}{l}\text { EUAAROPRIEAL } \\
\text { IMPRODUTVA }\end{array}$ & 675 & 0 & MARIO GIOVANNI PUGLIESI & $\begin{array}{l}\text { DDENTIFICADO } \\
\text { IDEST }\end{array}$ \\
\hline 171050 & IGOR PUGLIESI AVELINO & РмОв то & INCRA 2003 & FAZENDA VEREDA & ITACAJÁ & то & 675 & IMPRODUTIVA & 675 & 0 & MARIO GIOVANNI PUGLIESI & $\begin{array}{l}\text { IENTIFICADO } \\
\text { IDEN }\end{array}$ \\
\hline 171320 & IGOR PUGLIESI AVELINO & РмОВ то & INCRA 2003 & FAZENDA GORGULHO & MIRACEMA DO TOCANTINS & то & 990 & IMPRODUTIVA & 990 & 0 & TAVARES & IENTIFICADO \\
\hline 171888 & GOR PUGLIESI AVELINO & РмОв то & INCRA 2003 & FAZENDA MATO VERDE & SANTA MARIA DO TOCANTINS & то & 1303 & 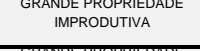 & 1302 & 0 & ORION PUGLESI TAVARES & $\begin{array}{l}\text { PAENTIFICADO } \\
\end{array}$ \\
\hline 171790 & JOAO BATISTA DE JESUS RIBEERO & PRTO & INCRA 2003 & FAZENDA MAR AZUL & PONTE ALTA DO TOCANTINS & то & 1506 & IMPRODUTIVA & $1.506,00$ & 0 & & \\
\hline 171790 & JOAO BATISTA DE JESUS RIBEIRO & PR Tо & INCRA 2003 & FAZENDA IBIPORAN & PONTE ALTA DO TOCANTINS & то & 1506 & IMPRODUTIVA & $1.506,00$ & 0 & & \\
\hline 170240 & $\begin{array}{l}\text { JunIOR } \\
\text { Justis }\end{array}$ & РмОВ то & INCRA 2003 & FAZ SANTO INACIO DO OLHO GRANDE & ARRAAIS & то & 450 & 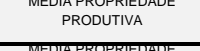 & 450,00 & 0 & FEIRE & \\
\hline 170240 & $\begin{array}{l}\text { JUNIOR } \\
\text { JaNAS FRERE }\end{array}$ & РМОв то & INCRA 2003 & FAZENDA ILHA DO CANTO & ARRAIAS & то & 450 & 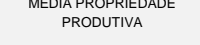 & 450,00 & 0 & $\begin{array}{l}\text { PAI IJS } \\
\text { FRERE }\end{array}$ & \\
\hline 170240 & $\begin{array}{l}\text { JUSEIOR SAIUSTFIIIE } \\
\text { JUN }\end{array}$ & Рмов то & INCRA 2003 & FAZENDA TIRIRIICA & ARRAIAS & то & 885 & $\begin{array}{l}\text { EDAPFRPPIIEA } \\
\text { PRODUTIVA }\end{array}$ & 885,00 & 0 & 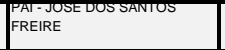 & \\
\hline 170240 & JUENOR & Рмов то & INCRA 2003 & FAZENDA BOA VISTA & ARRAIAS & то & 1098 & $\begin{array}{l}\text { PRODUTIVA } \\
\text { PRA }\end{array}$ & $1.098,00$ & 0 & 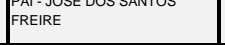 & \\
\hline 170240 & JUNIOR & Рмов то & INCRA 2003 & FAZENDA SOSSEGO & ARRAIAS & то & 1923 & $\begin{array}{l}\text { ANUEFPOPFIIIU } \\
\text { PRODUTIVA }\end{array}$ & $1.923,00$ & 0 & 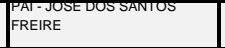 & \\
\hline 170240 & 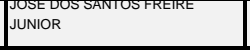 & РмОв то & INCRA 2003 & FAZENDA SANTO ANTONIO & ARRAIAS & то & 8226 & 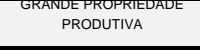 & $8.226,00$ & 0 & $\begin{array}{l}\substack{\text { APII JUSE } \\
\text { FRERE }} \\
\end{array}$ & \\
\hline 170310 & JOSE GOMES DA ROCHA & PSD GO & INCRA 2003 & FAZ SOBRAL & BARROLÁNDIA & то & 83 & PEQUENA PROPRIEDADE & 83 & 0 & & \\
\hline 170625 & KATIA REGINA ABREU & DЕल TO & INCRA 2003 & FAZENDA SAO LUIZ & CRIXÁs DO TOCANTINS & то & 46 & MINIFÚNDIO & 46 & 0 & $\begin{array}{l}\text { HENRIOUE RODRIGUES } \\
\text { ARAUJO }\end{array}$ & 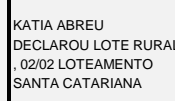 \\
\hline
\end{tabular}




\begin{tabular}{|c|c|c|c|c|c|c|c|c|c|c|c|c|}
\hline GEOCODIGO & PARLAMENTAR & PARTIDO & FONTE & IMóvel & Municipio & uF & ÁREA & CATEGORIA FUNDIÁAIIA & AREA REGISTRADA & $\begin{array}{l}\text { AREA DECLARADA } \\
\text { COMO POSSE }\end{array}$ & $\begin{array}{l}\text { REGISTRO EM NOME DE } \\
\text { OUTRAS PESSOAS }\end{array}$ & $\begin{array}{l}\text { viNCULO CoM O } \\
\text { PARLAMENTAR }\end{array}$ \\
\hline 171800 & KATIA REGINA ABREU & DЕMTO & INCRA 2003 & FAZENDA SAO LUIZ LOTEAMENTO CRIXAS & PORTO ALEGRE DO TOCANTINS & то & 46 & MINIFÚNDIO & 46 & 0 & $\begin{array}{l}\text { HENRIOUE RODRIGUES } \\
\text { ARAUJO }\end{array}$ & 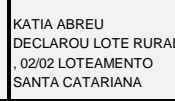 \\
\hline 170625 & KATIA REGINA ABREU & DEMTO & INCRA 2003 & LOTE 23-E GLEBA 2 LOTEAMENTO CRIXA & CRIXÁS DO TOCANTINS & то & 73 & MINIFÚNDIO & 73 & 0 & CRISTINA DIAS DE SOUZA & 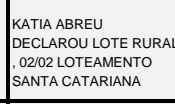 \\
\hline 170035 & KATAA REGINA ABREU & DEMTO & INCRA 2003 & FAZENDA ITAPEMA & ALIANÇA DO TOCANTINS & то & 129 & PEQUENA PROPRIEDADE & 129,00 & & & \\
\hline 170610 & KATIA REGINA ABREU & DЕМ TO & INCRA 2003 & FAZENDA SOMBRA DA TARDE & CRISTALÂNDIA & то & 174 & $\begin{array}{l}\text { PEQUENA PROPRIEDADE } \\
\end{array}$ & 174 & 0 & CRISTINA DAAS DE SOUZA & 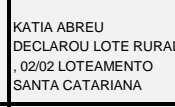 \\
\hline 170625 & KATIA REGINA ABREU & DEMTO & INCRA 2003 & FAZENDA SAO PAULO LOTEAMENTO CRIXA & CRIXÁs DO TOCANTINS & то & 400 & $\begin{array}{c}\text { MÉDIA PROPRIEDADE } \\
\text { IMPROOUTIVA } \\
\end{array}$ & 400 & 0 & EMIDIO TEXERA NETO & 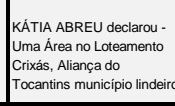 \\
\hline 170900 & KATIA REGINA ABREU & DEMTO & INCRA 2003 & $\begin{array}{l}\text { LOTEAMENTO SANTA CATARINA } \\
\end{array}$ & GOATINS & Tо & 2301 & $\begin{array}{l}\text { GRANDE PROPRIIEDADE } \\
\text { IMPRODUTIVA }\end{array}$ & 2297 & 0 & $\begin{array}{l}\text { JJSE APARECIDO DO } \\
\text { NASCIMENTO }\end{array}$ & 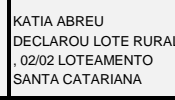 \\
\hline 170900 & KATIA REGINA ABREU & DEMTO & INCRA 2003 & L22 LOTEAMENTO FAZ STA CATARINA & GOAATNS & то & 2990 & $\begin{array}{l}\text { GRANDE PROPRIIEDADE } \\
\text { IMPRODUTIVA }\end{array}$ & 2990 & 0 & $\begin{array}{l}\text { SOLORRIICO S A INDUSTRIAE } \\
\text { COMERCIO }\end{array}$ & 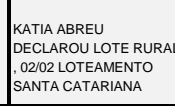 \\
\hline 170730 & LAUREZ DA ROCHA MOREIRA & РSв TO & INCRA 2003 & FAZENDA VALE DO TUCUM & DUERÉ & то & 546 & $\begin{array}{l}\text { MEDAA PROPRIEDADE } \\
\text { PRODUTIVA }\end{array}$ & 546 & 0 & & \\
\hline 170730 & LAUREZ DA ROCHA MOREIRA & РSB TO & INCRA 2003 & FAZENDA BOM JARDIM & DUERÉ & то & 966 & $\begin{array}{l}\text { MEDIA PROPRIEDADE } \\
\text { PRODUTIVA }\end{array}$ & 966 & 966 & & \\
\hline 170230 & LEOMAR DE MELO QUINTANILHA & PRTO & INCRA 2003 & FAZENDA REFUGIOS DAS CUNHAS & ARAPOEMA & то & 1213 & $\begin{array}{l}\text { GRANDE PROPRIIEADE } \\
\text { IMPRODUTIVA }\end{array}$ & $1.213,00$ & & & \\
\hline 170730 & LEOMAR DE MELO QUINTANILHA & PRTO & INCRA 2003 & FAZZNDA PONDEROSA & DUERÉ & то & 1998 & $\begin{array}{l}\text { GRANDE PROPRIEDADE } \\
\text { PROOUTIVA }\end{array}$ & $1.998,00$ & 0 & & \\
\hline 171320 & $\begin{array}{l}\text { ODELMO LEAO CARNEIRO } \\
\text { SOBRRINHO }\end{array}$ & PPMG & INCRA 2003 & FAZENDA AGUA AZUL. & MIRACEMA DO TOCANTINS & то & 902 & 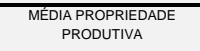 & 902 & 0 & $\begin{array}{l}\text { ODELMO LEAO CARNEIRO } \\
\text { SOBRINHO }\end{array}$ & $\begin{array}{l}\text { OOELLO LEAO } \\
\text { CARNEIRO SOBRINHO }\end{array}$ \\
\hline 171320 & $\begin{array}{l}\text { ODELMO LEAO CARNEIRO } \\
\text { SOBRRINHO }\end{array}$ & $\frac{P P M G}{P P G}$ & INCRA 2003 & FAZENDA AGUA AZUL & MIRACEMA DO TOCANTINS & то & 902 & $\begin{array}{l}\text { MEDDA PROPRIEDADE } \\
\text { PRODUTIVA }\end{array}$ & 902 & 0 & $\begin{array}{l}\text { ODELMO LEAO CARNEIRO } \\
\text { SOBRINHO }\end{array}$ & \\
\hline 170190 & RAMUUNDO COIMBRA JÚNIOR & PPS TO & INCRA 2003 & FAZENDA ITAPORA & ARAGUACEMA & то & 335 & $\begin{array}{c}\text { MEDIA PROPRIEDADE } \\
\text { IMPROOUTIVA }\end{array}$ & 335 & 0 & \begin{tabular}{|l|} 
RAMUNDO COIMBRA JUNIOR \\
\end{tabular} & \\
\hline 171840 & RAIMUNDO COIMBRA JÚNIOR & Рмов то & INCRA 2003 & FAZENDA SANTA MARIA & PRESIDENTE KENNEDY & то & 82 & PEQUENA PROPRIEDADE & 82 & 0 & MARIA ALENCAR COIMBRA & 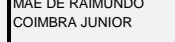 \\
\hline 170820 & RONALDO RAMOS CAIADO & DEM GO & INCRA 2003 & AGROVILA 3 PROJ R F DO ARAGUAIA & FORMOSO DO ARAGUAIA & то & 562 & $\begin{array}{c}\text { MÉDIA PROPRIEDADE } \\
\text { IMPROOUTIVA }\end{array}$ & 562 & 0 & & \\
\hline 170820 & VALDIR COLATTO & PMDBSC & INCRA 2003 & PROJETO RIO FORMOSO 5 E 6 & FORMOSO DO ARAGUAIA & то & 64 & MINIFÚNDIO & 64 & 0 & $\begin{array}{l}\text { PAtRiCIA NASCIMENTO } \\
\text { VaALADO }\end{array}$ & 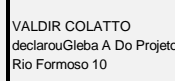 \\
\hline 170820 & VALDIR COLATTO & PMDBSC & INCRA 2003 & PROJETO RIO FORMOSO MODULO G4 & FORMOSO DO ARAGUAIA & то & 135 & PEQUENA PROPRIEDADE & 135 & 0 & FLAVIA CAMOES LABOISSIIERE & 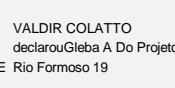 \\
\hline 170820 & VALDIR COLATTO & PMDBSC & INCRA 2003 & PROJETO RIO FORMOSO MODULO 84 & FORMOSO DO ARAGUAIA & то & 139 & PEQUENA PROPRIEDADE & 139 & 0 & FLAVIA CAMOES LABOISSIERE & 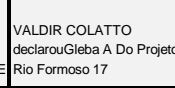 \\
\hline 170820 & VALDIR COLATTO & PMDB SC & INCRA 2003 & PROJETO RIO FORMOSO MODULO F2 & FORMOSO DO ARAGUAIA & то & 144 & PEQUENA PROPRIEDADE & 144 & 0 & BYRON PENHA PAES LEME & 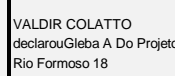 \\
\hline
\end{tabular}




\begin{tabular}{|c|c|c|c|c|c|c|c|c|c|c|c|c|}
\hline GEoconico & PARLLMENTAR & PARTIDO & FoNTE & Imóvel & Muncipiro & uF & AREA & CATEGORIA FUNDDÁRIA & AREA REGISTRADA & $\begin{array}{l}\text { AREA DEELARADA } \\
\text { COMO POSSEE }\end{array}$ & $\begin{array}{l}\text { REGistro EM NOME DE } \\
\text { OUTRAAS PESSOAS }\end{array}$ & $\begin{array}{l}\text { Vinculo como } \\
\text { PARLAMENTAR }\end{array}$ \\
\hline 170820 & $\begin{array}{l}\mid \text { VALDIR COLATTO } \\
\end{array}$ & PMDBSC & INCAA 2003 & PROJETO RIO FORMOSO 3 ETAPA & FORMOSO DO ARAGUAAA & то & 181 & PEQUENA PROPPRIEADE & 181 & 0 & PEDRO BRAZ BERTONI & 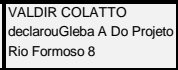 \\
\hline 170820 & $\begin{array}{l}\text { VALLIR CoLATtO } \\
\end{array}$ & PMDBSC & INCAA 2003 & PROJETO RIO FORMOSO 3 ETAPA & FORMOSO DO ARAGUAAA & то & 209 & PEQUENA PROPRIEDADE & 209 & $\circ$ & 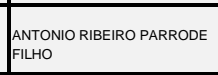 & 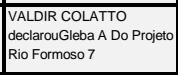 \\
\hline 170820 & $\begin{array}{l}\text { VALDir CoLATTO } \\
\end{array}$ & PMDB SC & INCAR 2003 & PROJETO RIO FORMOSO 3 ETAPA & FORMOSO DO ARAGUAAA & то & 210 & PEQUENA PROPRIEAADE & 210 & 0 & \begin{tabular}{|l} 
ANTOMO EOION FELIXE \\
SOUSA
\end{tabular} & 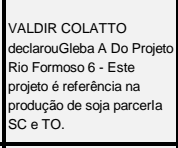 \\
\hline 170820 & $\begin{array}{l}\text { Valdir CoLATTO } \\
\text { A }\end{array}$ & РMOBS SC & INCAA 2003 & $\begin{array}{l}\text { PROJETT RIO FORMOSO } 3 \text { З EEAAPA } \\
\end{array}$ & FORMOSO DO ARAGUAAA & то & 215 & PEQUENA PROOPREEADAE & 215 & 0 & 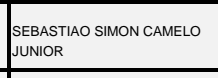 & 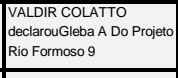 \\
\hline 170820 & $\begin{array}{l}\text { VALLIR COLATTO } \\
\end{array}$ & PMDB SC & INCAR 2003 & PROJETO RIO FORMOSO GLEBAS E11EF11 & FORMOSO DO ARAGUAAA & то & 271 & PEQUENA PROPRREDADE & 271 & 0 & \begin{tabular}{|l} 
ULZZEENANDO LOBO \\
TOURANO
\end{tabular} & 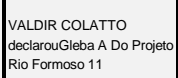 \\
\hline
\end{tabular}


ANEXO E - TERRAS DOS PARLAMENTARES RURALISTAS, DECLARADAS À JUSTIÇA ELEITORAL (1998, 2002, 2006 OU 2010)

\begin{tabular}{|c|c|c|c|c|c|c|c|c|}
\hline GEOCODIGO & MUNICIPIO & UF & PARLAMENTAR & PARTIDO & FONTE & JIMOVEL & AREA & TCATEGORIA FUNDIÁRIA \\
\hline 120013 & BUJARI & $\mathrm{AC}$ & $\begin{array}{l}\text { SERGIO SEBASTIAO DE } \\
\text { BARROS }\end{array}$ & PMN AC & REC FEDERAL 2006 & AREA RURAL FAZENDA CAROLINA & $6.224,37$ & GRANDE PROPRIEDADE \\
\hline 120013 & BUJARI & $\mathrm{AC}$ & $\begin{array}{l}\text { SERGIO SEBASTIAO DE } \\
\text { BARROS }\end{array}$ & PMN AC & REC FEDERAL 2006 & $5 \%$ AREA RURAL FAZ JARAGUÁ & 50,00 & MINIFUNDIO \\
\hline 120013 & BUJARI & AC & $\begin{array}{l}\text { SERGIO SEBASTIAO DE } \\
\text { BARROS }\end{array}$ & PMN AC & REC FEDERAL 2006 & LOTE RURAL & 3,49 & MINIFUNDIO \\
\hline 120020 & CRUZEIRO DO SUL & $A C$ & $\begin{array}{l}\text { JOAO TOTA SOARES DE } \\
\text { FIGUEIREDO }\end{array}$ & PP AC & REC FEDERAL 2006 & \begin{tabular}{|l|}
$\begin{array}{l}\text { UMA TERRA RURAL DENOMINADA SERINGAL } \\
\text { INVEJA }\end{array}$ \\
\end{tabular} & $\mathrm{NI}$ & NÃO INFORMOU (NI) \\
\hline 120020 & CRUZEIRO DO SUL & $A C$ & $\begin{array}{l}\text { JOAO TOTA SOARRES DE } \\
\text { FIGUEIREDO }\end{array}$ & PPAC & REC FEDERAL 2006 & $\begin{array}{l}\text { FAZENDA MORADA NOVA AS MARGENS DO } \\
\text { RIO MÔA }\end{array}$ & $2.900,00$ & GRANDE PROPRIEDADE \\
\hline 120020 & CRUZEIRO DO SUL & $A C$ & $\begin{array}{l}\text { JOAO TOTA SOARRES DE } \\
\text { FIGUEIREDO }\end{array}$ & PPAC & REC FEDERAL 2006 & UMA ÁREA RURAL AS MARGENS DO RIO MÔA & 100,00 & PEQUENA PROPRIEDADE \\
\hline 120020 & CRUZEIRO DO SUL & $\mathrm{AC}$ & $\begin{array}{l}\text { JOAO TOTA SOARRES DE } \\
\text { FIGUEIREDO }\end{array}$ & PP AC & REC FEDERAL 2006 & $\begin{array}{l}\text { AREA RURAL LOC NA ESTRADA D } \\
\text { AEROPORTO FAZENDA MORADA NOVA }\end{array}$ & 37,50 & MINIFUNDIO \\
\hline 120080 & PORTO ACRE & $A C$ & $\begin{array}{l}\text { SERGIO SEBASTIAO DE } \\
\text { BARROS }\end{array}$ & PMN AC & REC FEDERAL 2006 & FAZ SANTA TEREZINHAIRACEMA & $2.012,00$ & GRANDE PROPRIEDADE \\
\hline 120080 & PORTO ACRE & $\mathrm{AC}$ & $\begin{array}{l}\text { SERGIO SEBASTIAO DE } \\
\text { BARROS }\end{array}$ & PMN AC & REC FEDERAL 2006 & UMA ÁREA RURAL & 5,80 & MINIFUNDIO \\
\hline 120050 & SENA MADUREIRA & $\mathrm{AC}$ & $\begin{array}{l}\text { EDSON EDINHO COELHO } \\
\text { ARAÚJO }\end{array}$ & PMDB SP & REC FEDERAL 2010 & Uma Gleba De Terra, Sena Madueira, Acre & $\mathrm{NI}$ & NÃO INFORMOU (NI) \\
\hline 120050 & SENA MADUREIRA & $\mathrm{AC}$ & $\begin{array}{l}\text { EDSON EDINHO COELHO } \\
\text { ARAÚJJO }\end{array}$ & PMDB SP & REC FEDERAL 2010 & $\begin{array}{l}50 \text { Por Cento De Uma Gleba De Terra, Sena } \\
\text { Madureira, Acre }\end{array}$ & $\mathrm{NI}$ & NÄO INFORMOU (NI) \\
\hline 120050 & SENA MADUREIRA & $\mathrm{AC}$ & MARCIO MIGUEL BITTAR & PSDB AC & REC FEDERAL 1998 & FAZ NA BR-364 & $\mathrm{NI}$ & NÃO INFORMOU (NI) \\
\hline 120050 & SENA MADUREIRA & $\mathrm{AC}$ & MARCIO MIGUEL BITTAR & PSDB AC & REC FEDERAL 1998 & ÁREA RURAL CÓRREGO DO OURO I & $3.842,00$ & GRANDE PROPRIEDADE \\
\hline 120050 & SENA MADUREIRA & $\mathrm{AC}$ & MARCIO MIGUEL BITTAR & PSDB AC & REC FEDERAL 1998 & FAZ OLHO D'ÁGUA & $1.000,00$ & MÉDIA PROPRIEDADE \\
\hline 120050 & SENA MADUREIRA & $\mathrm{AC}$ & MARCIO MIGUEL BITTAR & PSDB AC & REC FEDERAL 1998 & 50\% COLONIA SĀOJOSÉ GLEBA LOBĀO & 22,95 & MINIFUNDIO \\
\hline 270040 & Atalaia & AL & JOÃO JOSÉ PEREIRA DE LYRA & PTB AL & REC FEDERAL 2010 & $\begin{array}{l}\text { Fazenda Santa Thereza E Moita Redonda R } \$ \\
2.349 .000,00\end{array}$ & $\mathrm{NI}$ & NÄO INFORMOU (NI) \\
\hline 270040 & ATALAIA & $\mathrm{AL}$ & $\begin{array}{l}\text { CARLOS ALBERTO MOREIRA } \\
\text { DE MENDONÇA CANUTO }\end{array}$ & PMDB AL & REC FEDERAL 2010 & Fazenda Flor Do Paraiba - Atalaia-Al & $\mathrm{NI}$ & NÃO INFORMOU (NI) \\
\hline 270040 & Atalaia & AL & $\begin{array}{l}\text { CARLOS ALBERTO MOREIRA } \\
\text { DE MENDONÇA CANUTO }\end{array}$ & PMDB AL & REC FEDERAL 2010 & Fazenda São Roque, $125 \mathrm{Ha}$ - Atalaia - Al & 125,00 & MÉDIA PROPRIEDADE \\
\hline 270040 & Atalaia & $\mathrm{AL}$ & $\begin{array}{l}\text { CARLOS ALBERTO MOREIRA } \\
\text { DE MENDONÇA CANUTO }\end{array}$ & PMDB AL & REC FEDERAL 2010 & Fazenda São Paulo - Atalaia - Al & $\mathrm{NI}$ & NÃO INFORMOU (NI) \\
\hline 270070 & BATALHA & $\mathrm{AL}$ & LUIZ DANTAS LIMA & PMDB AL & REC FEDERAL 2010 & Propriedade Bebedouro & $\mathrm{NI}$ & NÃO INFORMOU (NI) \\
\hline 270230 & CORURIPE & AL & JOÃO JOSÉ PEREIRA DE LYRA & PTB AL & REC FEDERAL 2010 & \begin{tabular}{|l|}
$\begin{array}{l}\text { Fazenda Guaxuma li, Zona Rural, Coruripe, } \\
\text { Alagoas }\end{array}$ \\
\end{tabular} & $\mathrm{NI}$ & NÃO INFORMOU (NI) \\
\hline 270230 & CORURIPE & AL & \begin{tabular}{|l|} 
JOSÉ THOMAZ DA SILVA NONÔ \\
NETTO
\end{tabular} & DEMAL & REC FEDERAL 2010 & Faz. Ver O Mar & $\mathrm{NI}$ & NÃO INFORMOU (NI) \\
\hline 270230 & CORURIPE & $\mathrm{AL}$ & \begin{tabular}{|l|} 
JOSÉ THOMAZ DA SILVA NONÔ \\
NETTO
\end{tabular} & DEM AL & REC FEDERAL 2010 & Faz. Bananeiras & $\mathrm{NI}$ & NÃO INFORMOU (NI) \\
\hline 270230 & CORURIPE & AL & \begin{tabular}{|l|} 
JOSÉ THOMAZ DA SILVA NONÔ \\
NETTO
\end{tabular} & DEMAL & REC FEDERAL 2010 & Faz. Balança & $\mathrm{NI}$ & NÄO INFORMOU (NI) \\
\hline 270230 & CORURIPE & AL & $\begin{array}{l}\text { CARLOS ALBERTO MOREIRA } \\
\text { DE MENDONÇA CANUTO } \\
\end{array}$ & PMDB AL & REC FEDERAL 2010 & Fazenda Santa Terezinha, $25,5 \mathrm{Ha}$ - Coruripe-Al & 25,50 & MINIFUNDIO \\
\hline 270380 & JOAQUIM GOMES & AL & $\begin{array}{l}\text { CARLOS ALBERTO MOREIRA } \\
\text { DE MENDONÇA CANUTO }\end{array}$ & PMDB AL & REC FEDERAL 2010 & Fazenda Padre Cicero - Joaquim Gomes & $\mathrm{NI}$ & NÃO INFORMOU (NI) \\
\hline 270380 & JOAQUIM GOMES & AL & $\begin{array}{l}\text { CARLOS ALBERTO MOREIRA } \\
\text { DE MENDONÇA CANUTO }\end{array}$ & PMDB AL & REC FEDERAL 2010 & $\begin{array}{l}\text { Imóvel Ruraldenominado Sitio Vermelho - Joaquim } \\
\text { Gomes - Al }\end{array}$ & $\mathrm{NI}$ & NÃO INFORMOU (NI) \\
\hline 270380 & JOAQUIM GOMES & AL & $\begin{array}{l}\text { CARLOS ALBERTO MOREIRA } \\
\text { DE MENDONÇA CANUTO }\end{array}$ & PMDB AL & REC FEDERAL 2010 & $\begin{array}{l}\text { Imóvel Rural Denominado São Sebastião - Joaquim } \\
\text { Gomesm - Al }\end{array}$ & $\mathrm{NI}$ & NÃO INFORMOU (NI) \\
\hline 270400 & JUNQUEIRO & AL & $\begin{array}{l}\text { TEOTONIO BRANDÃO VILELA } \\
\text { FLLHO }\end{array}$ & PSDB AL & REC FEDERAL 2010 & PROPRIEDADE LAGOA SECA, COM $438 \mathrm{HA}$. & 438,00 & MÉDIA PROPRIEDADE \\
\hline 270470 & MARECHAL DEODORO & AL & $\begin{array}{l}\text { TEOTONIO BRANDÃO VILELA } \\
\text { FLLHO }\end{array}$ & PSDB AL & REC FEDERAL 2010 & $\begin{array}{l}\text { FAIXA DE TERRA 06/07 DO SITIO BEIRA MAR } \\
\text { 2, NA MASSAGUEIRA }\end{array}$ & $\mathrm{NI}$ & NÄO INFORMOU (NI) \\
\hline 270470 & MARECHAL DEODORO & AL & $\begin{array}{l}\text { TEOTONIO BRANDÃO VILELA } \\
\text { FLLHO }\end{array}$ & PSDB AL & REC FEDERAL 2010 & $\begin{array}{l}\text { FAIXA DE TERRA } 12 \text { A } 19 \text { DO SITIO BARRA } \\
\text { MAR 2, MASSAGUEIRA }\end{array}$ & $\mathrm{NI}$ & NÃO INFORMOU (NI) \\
\hline 270470 & MARECHAL DEODORO & $\mathrm{AL}$ & $\begin{array}{l}\text { TEOTONIO BRANDÃO VILELA } \\
\text { FILHO }\end{array}$ & PSDB AL & REC FEDERAL 2010 & $\begin{array}{l}\text { FAIXA DE TERRA } 10 \text { E } 20 \text { DO SITIO BARRA } \\
\text { MAR 2, MASSAGUEIRA }\end{array}$ & $\mathrm{NI}$ & NÃO INFORMOU (NI) \\
\hline 270470 & MARECHAL DEODORO & $\mathrm{AL}$ & $\begin{array}{l}\text { TEOTONIO BRANDÃO VILELA } \\
\text { FILHO }\end{array}$ & PSDB AL & REC FEDERAL 2010 & $\begin{array}{l}\text { FAIXA DE TERRA PARTE MAIOR DO SITIO } \\
\text { BARRA MAR 2, MASSAGUEIRA }\end{array}$ & $\mathrm{NI}$ & NÃO INFORMOU (NI) \\
\hline 270470 & MARECHAL DEODORO & $\mathrm{AL}$ & $\begin{array}{l}\text { TEOTONIO BRANDÃO VILELA } \\
\text { FLLHO }\end{array}$ & PSDB AL & REC FEDERAL 2010 & $1 / 7$ DO IMOVEL RURAL CUMBE & $\mathrm{NI}$ & NÄO INFORMOU (NI) \\
\hline 270550 & MURICI & $\mathrm{AL}$ & $\begin{array}{l}\text { CARLOS ALBERTO MOREIRA } \\
\text { DE MENDONÇA CANUTO } \\
\end{array}$ & PMDB AL & REC FEDERAL 2010 & Fazenda Pindoba, Murici - Al & $\mathrm{NI}$ & NÃO INFORMOU (NI) \\
\hline 270680 & PIAÇABUÇU & AL & \begin{tabular}{|l|} 
JJOSÉ THOMAZ DA SILVA NONÔ \\
NETTO
\end{tabular} & DEM AL & REC FEDERAL 2010 & Sitio. Maracuja & $\mathrm{NI}$ & NÄO INFORMOU (NI) \\
\hline 270680 & PIAÇABUÇU & AL & \begin{tabular}{|l|} 
JOSÉ THOMAZ DA SILVA NONÔ \\
NETTO
\end{tabular} & DEM AL & REC FEDERAL 2010 & Faz. Santa Tereza & $\mathrm{NI}$ & NÃO INFORMOU (NI) \\
\hline 270680 & PIAÇABUÇU & AL & \begin{tabular}{|l|} 
JOSÉ THOMAZ DA SILVA NONÔ \\
NETTO
\end{tabular} & DEMAL & REC FEDERAL 2010 & Sitio Barra Das Laranjeiras & $\mathrm{NI}$ & NÄO INFORMOU (NI) \\
\hline 270690 & PILAR & AL & $\begin{array}{l}\text { ARTHUR CÉSAR PEREIRA DE } \\
\text { LIRA }\end{array}$ & PMDB AL & REC FEDERAL 2010 & Sitio Nicodemos & $\mathrm{NI}$ & NÃO INFORMOU (NI) \\
\hline 270690 & PILAR & AL & JOÄO JOSÉ PEREIRA DE LYRA & PTB AL & REC FEDERAL 2010 & Parte Da Fazenda Imburi, Pilar/AI R $\$ 10.000,00$ & $\mathrm{NI}$ & NÃO INFORMOU (NI) \\
\hline 270690 & PILAR & AL & JOÃO JOSÉ PEREIRA DE LYRA & PTB AL & REC FEDERAL 2010 & Fazenda Imburi, $R \$ 60.000,00$ & 40,00 & MÉDIA PROPRIEDADE \\
\hline 270690 & PILAR & $\mathrm{AL}$ & $\begin{array}{l}\text { CARLOS ALBERTO MOREIRA } \\
\text { DE MENDONÇA CANUTO } \\
\end{array}$ & PMDB AL & REC FEDERAL 2010 & Fazenda Santa Terezinha, $192,85 \mathrm{Ha}$ - Pilar -Al & 192,85 & GRANDE PROPRIEDADE \\
\hline 270690 & PILAR & AL & $\begin{array}{l}\text { CARLOS ALBERTO MOREIRA } \\
\text { DE MENDONÇA CANUTO }\end{array}$ & PMDB AL & REC FEDERAL 2010 & Fazenda Graussu - Pilar - Al & $\mathrm{NI}$ & NÃO INFORMOU (NI) \\
\hline 270690 & PILAR & AL & $\begin{array}{l}\text { CARLOS ALBERTO MOREIRA } \\
\text { DE MENDONÇA CANUTO }\end{array}$ & PMDB AL & REC FEDERAL 2010 & Fazenda Santo Antonio - Pilar - Al & $\mathrm{NI}$ & NÃO INFORMOU (NI) \\
\hline
\end{tabular}




\begin{tabular}{|c|c|c|c|c|c|c|c|c|}
\hline 270870 & $\begin{array}{l}\text { SĀ̃O MIGUEL DOS } \\
\text { MLLAGRES }\end{array}$ & $\mathrm{AL}$ & $\begin{array}{l}\text { TEOTONIO BRANDÃO VILELA } \\
\text { FLLHO } \\
\end{array}$ & PSDB AL & REC FEDERAL 2010 & SITIO DE COQUEIRO CURTUME & $\mathrm{NI}$ & NÃO INFORMOU (NI) \\
\hline 270870 & $\begin{array}{l}\text { SĀ̃O MIGUEL DOS } \\
\text { MLAGRES }\end{array}$ & $\mathrm{AL}$ & $\begin{array}{l}\text { TEOTONIO BRANDĀO VILELA } \\
\text { FLLHO }\end{array}$ & PSDB AL & REC FEDERAL 2010 & SITIO SÃO JOSE & $\mathrm{NI}$ & NÄO INFORMOU (NI) \\
\hline 270870 & $\begin{array}{l}\text { SĀO MIGUEL DOS } \\
\text { MLAGRES }\end{array}$ & AL & $\begin{array}{l}\text { TEOTONIO BRANDĀO VILELA } \\
\text { FLLHO }\end{array}$ & PSDB AL & REC FEDERAL 2010 & 1/3 DO SITIO CURTUME & $\mathrm{NI}$ & NĀO INFORMOU (NI) \\
\hline 270870 & $\begin{array}{l}\text { SAAO MIGUEL DOS } \\
\text { MLAGRES }\end{array}$ & AL & $\begin{array}{l}\text { TEOTONIO BRANDĀO VILELA } \\
\text { FLLHO }\end{array}$ & PSDB AL & REC FEDERAL 2010 & SITIO DE COQUEIROS SAO MIGUEL & $\mathrm{NI}$ & NÃO INFORMOU (NI) \\
\hline 270880 & SÃ̃O SEBASTIĀo & AL & $\begin{array}{l}\begin{array}{l}\text { ARTHUR CÉSAR PEREIRA DE } \\
\text { LIRA }\end{array} \\
\end{array}$ & PMDB AL & REC FEDERAL 2010 & $50 \%$ Faz Sta Maria & $\mathrm{NI}$ & NÄO INFORMOU (NI) \\
\hline 270880 & SÃ̃o SEBASTIẪo & AL & $\begin{array}{l}\text { ARTHUR CÉSAR PEREIRA DE } \\
\text { LIRA }\end{array}$ & PMDB AL & REC FEDERAL 2010 & Faz Pedras & $\mathrm{NI}$ & NÃO INFORMOU (NI) \\
\hline 270930 & UNIÄO DOS PALMARES & AL & JOÃO JOSÉ PEREIRA DE LYRA & PTB AL & REC FEDERAL 2010 & Fazenda Santo Inacio, R\$56.487,66 & $\mathrm{NI}$ & NĂO INFORMOU (NI) \\
\hline 270940 & VIÇOSA & AL & $\begin{array}{l}\text { TEOTONIO BRANDÃO VILELA } \\
\text { FILHO }\end{array}$ & PSDB AL & REC FEDERAL 2010 & 1/7 DO IMOVEL RURAL SÃO FRANCISCO & $\mathrm{NI}$ & NÃO INFORMOU (NI) \\
\hline $\mathrm{x}$ & $x$ & AL & $\begin{array}{l}\text { CARLOS ALBERTO MOREIRA } \\
\text { DE MENDONÇA CANUTO } \\
\end{array}$ & PMDB AL & REC FEDERAL 2010 & Fazendas N. S. Do Carmo E São São Sebastiäo. & $\mathrm{NI}$ & NÄO INFORMOU (NI) \\
\hline $\mathrm{x}$ & $x$ & AL & $\begin{array}{l}\text { CARLOS ALBERTO MOREIRA } \\
\text { DE MENDONÇA CANUTO } \\
\end{array}$ & PMDB AL & REC FEDERAL 2010 & Área De Terra Encravada, $418,84 \mathrm{Ha}$ & 418,84 & GRANDE PROPRIEDADE \\
\hline $\mathrm{x}$ & $\begin{array}{l}\text { MARGEM DIREITA DO } \\
\text { PARANA SUMAUMA SUPIA } \\
\end{array}$ & $\mathrm{AM}$ & $\begin{array}{l}\text { JOÄO THOMÉ VERÇOSA } \\
\text { MESTRINHO DE MEDEIROS } \\
\text { RAPOSO }\end{array}$ & PMDB AM & REC FEDERAL 2010 & $\begin{array}{l}4 \text { Terrenos A Margem Direita Do Parana Do } \\
\text { Sumauma Supia }\end{array}$ & $1.200,00$ & GRANDE PROPRIEDADE \\
\hline $\mathrm{x}$ & $\begin{array}{l}\text { MARGEM ESQUERDA DO } \\
\text { RIO MANACAPURU }\end{array}$ & $\mathrm{AM}$ & $\begin{array}{l}\text { JOÄO THOMÉ VERÇOSA } \\
\text { MESTRINHO DE MEDEIROS } \\
\text { RAPOSO }\end{array}$ & PMDB AM & REC FEDERAL 2010 & $\begin{array}{l}\text { Terreno A Margem Esquerda Do Rio Manacapuru } \\
\text { Adquirido Em } 1998\end{array}$ & 322,00 & MÉDIA PROPRIEDADE \\
\hline $\mathrm{x}$ & $x$ & $\mathrm{AM}$ & JOSE ARISTODEMO PINOTTI & PMDB SP & REC FEDERAL 2006 & $\begin{array}{l}\text { Gleba De } 3.000 \text { Alqueires Em Comunata - } \\
\text { Amazonas }\end{array}$ & $8.160,00$ & GRANDE PROPRIEDADE \\
\hline 160030 & MACAPÁ & AP & ANTONIO DA JUSTA FEIJÄO & PTC AP & REC FEDERAL 2010 & Imóvel Rural Retiro llha Grande $(\mathrm{R} \$ 5.630 .000,00)$ & $\mathrm{NI}$ & NÃO INFORMOU (NI) \\
\hline 290250 & BAIANÓPOLIS & $\mathrm{BA}$ & JOSÉ WELLINGTON ROBERTO & PR PB & REC FEDERAL 2010 & Imovel Rural C/ $934 \mathrm{Ha}$, Adq. 14.03.91 & 934,00 & MÉDIA PROPRIEDADE \\
\hline 290320 & BARREIRAS & $\mathrm{BA}$ & $\begin{array}{l}\text { JUSMARI TEREZINHA DE } \\
\text { SOUZA OLIVEIRA }\end{array}$ & PFL BA & REC FEDERAL 2008 & Uma Chacara Denominada Tia Lidia & $\mathrm{NI}$ & NÄO INFORMOU (NI) \\
\hline 290320 & BARREIRAS & BA & EDMAR DE SOUZA ARRUDA & PSC PR & REC FEDERAL 2010 & $24,691 \%$ De 4.983,23 Ha, Fazenda Maringa & $1.230,35$ & GRANDE PROPRIEDADE \\
\hline 290340 & BELMONTE & BA & FÁBIO LOUREIRO SOUTO & DEM BA & REC FEDERAL 2010 & Fazenda - Belmonte & $\mathrm{NI}$ & NÄO INFORMOU (NI) \\
\hline 290340 & BELMONTE & $\mathrm{BA}$ & FÁBIO LoUREIRO SOUTO & DEM BA & REC FEDERAL 2010 & \begin{tabular}{|l} 
Fazenda Santa Helena Belmonte \\
\end{tabular} & $\mathrm{NI}$ & NÃO INFORMOU (NI) \\
\hline 290340 & BELMONTE & $\mathrm{BA}$ & FÁBIO LOUREIRO SOUTO & DEM BA & REC FEDERAL 2010 & Faz Nova Floresta - Belmonte & $\mathrm{NI}$ & NÃO INFORMOU (NI) \\
\hline 290390 & BOM JESUS DA LAPA & BA & JOSE ALVES ROCHA & PRBA & REC FEDERAL 2010 & $\begin{array}{l}\text { Loteamento Agricola Sito No Perimetro Irrigado De } \\
\text { Formoso A }\end{array}$ & $\mathrm{NI}$ & NÄO INFORMOU (NI) \\
\hline 290390 & BOM JESUS DA LAPA & BA & JOSE ALVES ROCHA & PRBA & REC FEDERAL 2010 & $\begin{array}{l}\text { Lote Agricola Ha Situado No Perimetro Irrigado De } \\
\text { Formoso }\end{array}$ & $\mathrm{NI}$ & NÃ̃o INFORMOU (NI) \\
\hline 290390 & BOM JESUS DA LAPA & BA & JOSE ALVES ROCHA & PRBA & REC FEDERAL 2010 & $\begin{array}{l}\text { Lote Agricola Sito No Perimetro Irrigado De } \\
\text { Formoso A Area Empresarial } \\
\end{array}$ & $\mathrm{NI}$ & NÄ̃O INFORMOU (NI) \\
\hline 290390 & BOM JESUS DA LAPA & BA & JOSE ALVES ROCHA & PRBA & REC FEDERAL 2010 & Uma Gleba De Terra & 4,00 & MINIFUNDIO \\
\hline 290430 & BREJÖES & BA & JOÄO ALMEIDA DOS SANTOS & PSDB BA & REC FEDERAL 2002 & $\begin{array}{l}\text { Fazenda Santo Antonio adquirida em } 2000 \text { de } \\
\text { Marina de Sant'ana Lopes CPF-06596150534 }\end{array}$ & $\mathrm{NI}$ & NÃO INFORMOU (NI) \\
\hline 290430 & BREJÖES & $\mathrm{BA}$ & JOĀO ALMEIDA DOS SANTOS & PSDB BA & REC FEDERAL 2002 & $\begin{array}{l}\text { Fazenda São Bernardo, com rebanho adquirida em } \\
1973\end{array}$ & 398,00 & MÉDIA PROPRIEDADE \\
\hline 290440 & BREJOLÂNDIA & $\mathrm{BA}$ & $\begin{array}{l}\text { JOSE LOURENÇO MORAIS DA } \\
\text { SILVA }\end{array}$ & PMDB BA & REC FEDERAL 1998 & FAZ SĀO MARTINHO E SÃO JOSÉ & $5.500,00$ & GRANDE PROPRIEDADE \\
\hline 290440 & BREJOLÂNDIA & $\mathrm{BA}$ & $\begin{array}{l}\text { JOSE LOURENÇO MORAIS DA } \\
\text { SILVA }\end{array}$ & PMDB BA & REC FEDERAL 1998 & FAZ SÃO MARTINHO 2 & $2.224,00$ & GRANDE PROPRIEDADE \\
\hline 290440 & BREJOLÂNDIA & $\mathrm{BA}$ & $\begin{array}{l}\text { JOSE LOURENÇO MORAIS DA } \\
\text { SILVA }\end{array}$ & PMDB BA & REC FEDERAL 1998 & FAZ SÃO PAULO & $1.980,00$ & GRANDE PROPRIEDADE \\
\hline 290440 & BREJOLÂNDIA & $\mathrm{BA}$ & $\begin{array}{l}\text { JOSE LOURENÇO MORAIS DA } \\
\text { SILVA }\end{array}$ & PMDB BA & REC FEDERAL 1998 & FAZ SÃo PAULO & $1.980,00$ & GRANDE PROPRIEDADE \\
\hline 290570 & CAMAÇARI & BA & $\begin{array}{l}\text { ARTHUR DE OLIVEIRA MAIA DA } \\
\text { SILVA }\end{array}$ & PMDB BA & REC FEDERAL 2010 & Área De Terra No Distrito De Abrantes & $\mathrm{NI}$ & NÃ̃ INFORMOU (NI) \\
\hline 290700 & CARDEAL DA SILVA & BA & $\begin{array}{l}\text { JOÄO LUIZ CORREIA ARGÔLO } \\
\text { DOS SANTOS }\end{array}$ & PPBA & REC FEDERAL 2010 & Fazenda Maringa & $\mathrm{NI}$ & NÃO INFORMOU (NI) \\
\hline 290700 & CARDEAL DA SILVA & BA & $\begin{array}{l}\text { JOĀO LUIZ CORREIA ARGÔLO } \\
\text { DOS SANTOS }\end{array}$ & PPBA & REC FEDERAL 2010 & Fazenda Parecida & $\mathrm{NI}$ & NĂO INFORMOU (NI) \\
\hline 290700 & CARDEAL DA SILVA & BA & $\begin{array}{l}\text { JOÃO LUIZ CORREIA ARGÔLO } \\
\text { DOS SANTOS }\end{array}$ & PPBA & REC FEDERAL 2010 & Fazenda Moreira & 13,00 & MINIFUNDIO \\
\hline 290810 & $\operatorname{cocos}$ & $\mathrm{BA}$ & $\begin{array}{l}\text { JOSÉ DOS SANTOS FREIRE } \\
\text { JUNIOR } \\
\end{array}$ & PMDB TO & REC FEDERAL 2010 & $\begin{array}{l}1 \text { Gleba De Terra Cadastrada No Incra N } \\
302023717757 \text { Cocos Ba }\end{array}$ & $\mathrm{NI}$ & NÄ̃ INFORMOU (NI) \\
\hline 290910 & CORIBE & $\mathrm{BA}$ & JOSE ALVES ROCHA & PRBA & REC FEDERAL 2010 & Sitio A Rua Cleriston Andrade & $\mathrm{NI}$ & NÃO INFORMOU (NI) \\
\hline 290910 & CORIBE & $\mathrm{BA}$ & JOSE ALVES ROCHA & PRBA & REC FEDERAL 2010 & Fazenda Volta Do Rego & $\mathrm{NI}$ & NÃO INFORMOU (NI) \\
\hline 290910 & CORIBE & $\mathrm{BA}$ & JOSE ALVES ROCHA & PR BA & REC FEDERAL 2010 & Fazenda Jacu & $\mathrm{NI}$ & NÄO INFORMOU (NI) \\
\hline 290910 & CORIBE & BA & JOSE ALVES ROCHA & PRBA & REC FEDERAL 2010 & Loteamento Agricola Sito Na Fazenda Formoso & $\mathrm{NI}$ & NÃO INFORMOU (NI) \\
\hline 290910 & CORIBE & BA & JOSE ALVES ROCHA & PRBA & REC FEDERAL 2010 & Fazenda Denominada Sao Lourenço & $\mathrm{NI}$ & NÃO INFORMOU (NI) \\
\hline 290910 & CORIBE & BA & JOSE ALVES ROCHA & PRBA & REC FEDERAL 2010 & Loteamento Agricola & $\mathrm{NI}$ & NÃO INFORMOU (NI) \\
\hline 290910 & CORIBE & $\mathrm{BA}$ & JOSE ALVES ROCHA & PRBA & REC FEDERAL 2010 & Fazenda Denominada Volta O Rego & $\mathrm{NI}$ & NÃ̃O INFORMOU (NI) \\
\hline 290910 & CORIBE & $\mathrm{BA}$ & JOSE ALVES ROCHA & PRBA & REC FEDERAL 2010 & Fazenda Denominada Sao Lourenço & $\mathrm{NI}$ & NÄO INFORMOU (NI) \\
\hline 290910 & CORIBE & BA & JOSE ALVES ROCHA & PRBA & REC FEDERAL 2010 & Fazenda Denominada S Lourenç̧o & $\mathrm{NI}$ & NÃO INFORMOU (NI) \\
\hline 290910 & CORIBE & BA & JOSE ALVES ROCHA & PRBA & REC FEDERAL 2010 & Fazenda Denominada Bonito & $\mathrm{NI}$ & NÄO INFORMOU (NI) \\
\hline
\end{tabular}




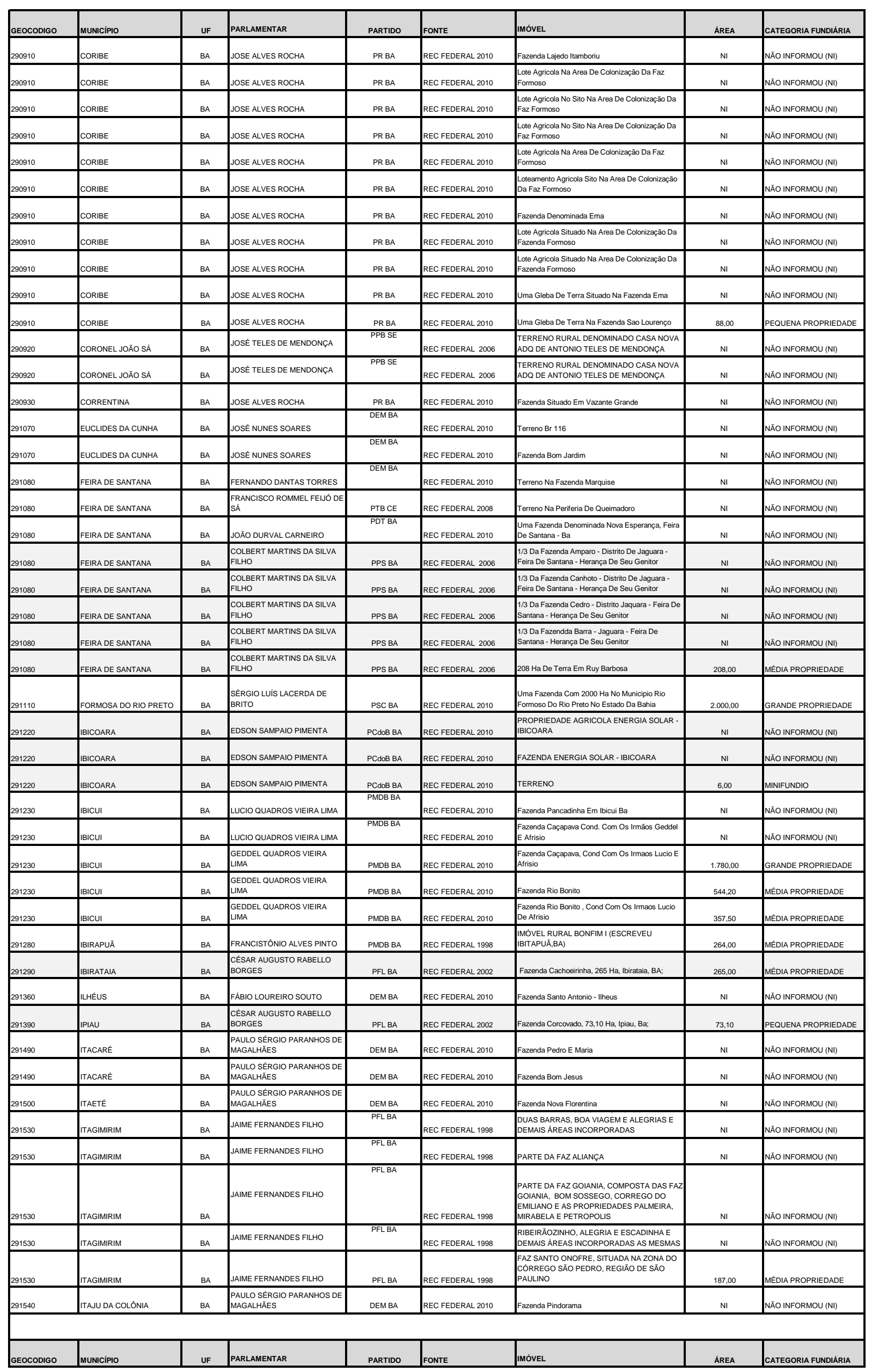




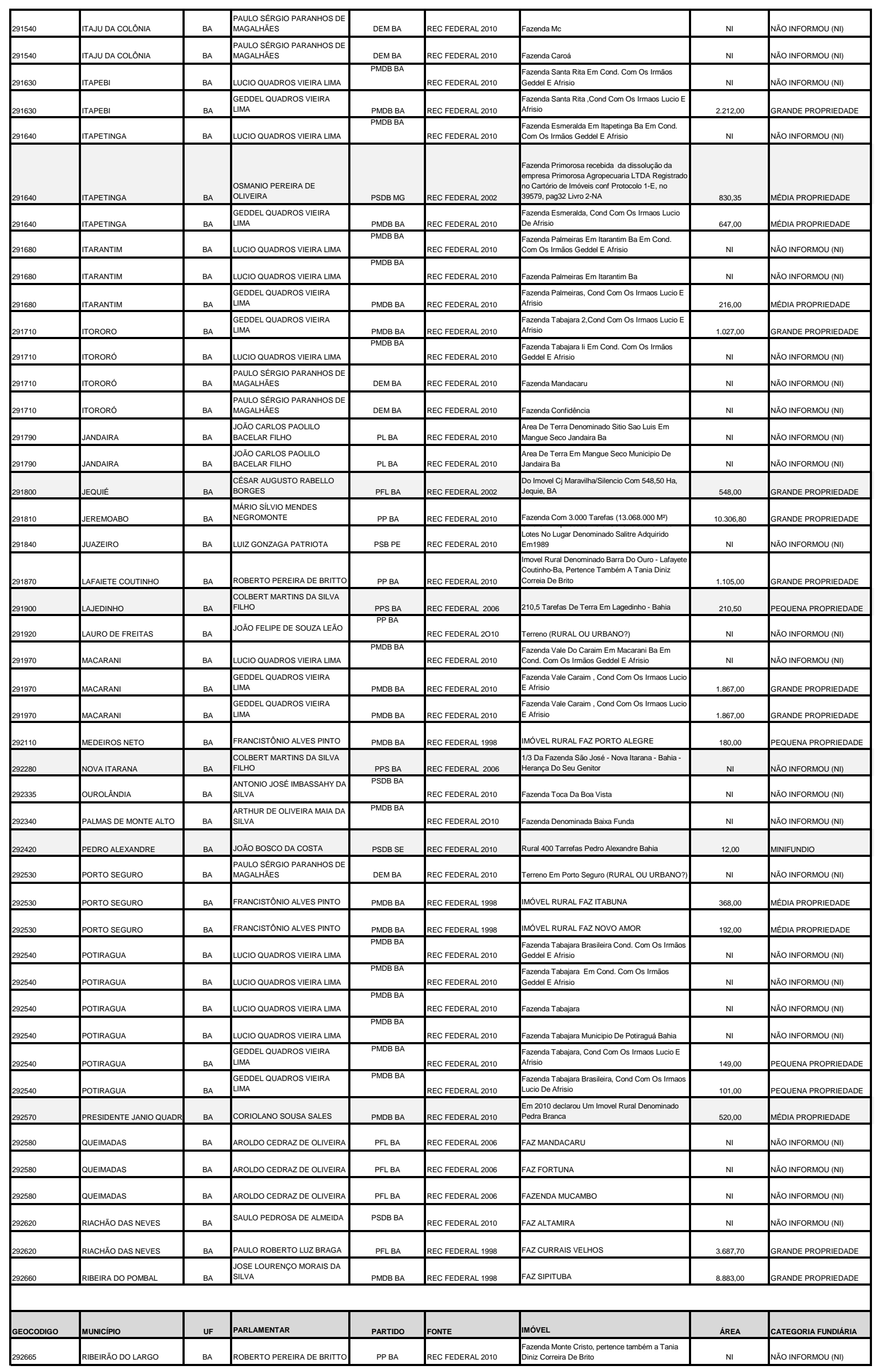




\begin{tabular}{|c|c|c|c|c|c|c|c|c|}
\hline 292720 & RUY BARBOSA & BA & $\begin{array}{l}\text { COLBERT MARTINS DA SILVA } \\
\text { FILHO }\end{array}$ & PPS BA & REC FEDERAL 2006 & 37 Ha De Terra Em Ruy Barbosa & 37,00 & MINIFUNDIO \\
\hline 292740 & SALVADOR & BA & MARCOS ANTONIO MEDRADO & $\overline{\text { PDT BA }}$ & REC FEDERAL 2010 & $\begin{array}{l}\text { Uma Chacara Rua Bejamin De Souza Sao Tome } \\
\text { De Paripe }\end{array}$ & $\mathrm{NI}$ & NÄO INFORMOU (NI) \\
\hline 292740 & SALVADOR & BA & MARCOS ANTONIO MEDRADO & PDT BA & REC FEDERAL 2010 & $\begin{array}{l}\text { Um Terreno Na Fazenda Noelia Burgos Sao Tome } \\
\text { De Paripe }\end{array}$ & $\mathrm{NI}$ & NĀO INFORMOU (NI) \\
\hline 292805 & SANTA LUZIA & $\mathrm{BA}$ & FELIX DE ALMEIDA MENDONÇA & DEM BA & REC FEDERAL 1998 & $\begin{array}{l}\text { IMÓVEL AGRíCOLA DENOMINADO CONJUNTO } \\
\text { CONCEIÇÃO, ZONA RIO SÄO PEDRO, NO } \\
\text { IISTRIT JACARANDÁ APREST. REGISTRO N } \\
\text { INCRA (ESCREVEU SANTA LUIZA,BA) }\end{array}$ & $\mathrm{NI}$ & NÃO INFORMOU (NI) \\
\hline 292840 & $\begin{array}{l}\text { SANTA RITA DE CASSIA } \\
\text { (antiga Rio Preto e lbipetuba) }\end{array}$ & BA & $\begin{array}{l}\text { SÉRGIO LUIS LACERDA DE } \\
\text { BRITO }\end{array}$ & PSC BA & REC FEDERAL 2010 & $\begin{array}{l}\text { Uma Fazenda } 2000 \text { Ha No Livro N } 2 \text { Matricula } 4195 \\
\text { No Monicipio De Santa Rita De Cassia No Estado } \\
\text { Da Bahia }\end{array}$ & $2.000,00$ & GRANDE PROPRIEDADE \\
\hline 292840 & $\begin{array}{l}\text { SANTA RITA DE CASSIA } \\
\text { (antiga Rio Preto e lbipetuba) }\end{array}$ & $\mathrm{BA}$ & $\begin{array}{l}\text { SÉRGIO LUÍS LACERDA DE } \\
\text { BRITO }\end{array}$ & PSC BA & REC FEDERAL 2010 & $\begin{array}{l}\text { Uma Fazenda Com } 2000 \text { Ha No Livro } 02 \text { Matricula } \\
4197\end{array}$ & $2.000,00$ & GRANDE PROPRIEDADE \\
\hline 292840 & $\begin{array}{l}\text { SANTA RITA DE CASSIA } \\
\text { (antiga Rio Preto e lbipetuba) }\end{array}$ & $\mathrm{BA}$ & $\begin{array}{l}\text { SÉRGIO LUÍS LACERDA DE } \\
\text { BRITO }\end{array}$ & PSC BA & REC FEDERAL 2010 & $\begin{array}{l}\text { Uma Fazenda Com } 2000 \text { Ha No Livro N } 2 \text { Matricula } \\
4196\end{array}$ & $2.000,00$ & GRANDE PROPRIEDADE \\
\hline 292840 & $\begin{array}{l}\text { SANTA RITA DE CASSIA } \\
\text { (antiga Rio Preto e lbipetuba) }\end{array}$ & BA & PAULO ROBERTO LUZ BRAGA & PFLBA & REC FEDERAL 1998 & FAZ PRIMAVERA & $1.375,00$ & GRANDE PROPRIEDADE \\
\hline 292860 & SANTO AMARO & $\mathrm{BA}$ & $\begin{array}{l}\text { JOSÉ CARLOS LEĀO DE } \\
\text { ARAUJO }\end{array}$ & PDTBA & REC FEDERAL 2010 & 1 Terreno Na Praia De Cabussu & $\mathrm{NI}$ & NÃO INFORMOU (NI) \\
\hline 292860 & SANTO AMARO & $\mathrm{BA}$ & MARCOS ANTONIO MEDRADO & PDT BA & REC FEDERAL 2010 & $\begin{array}{l}\text { Uma Fazenda De Nome Araripe Calafati Santo } \\
\text { Amaro No Incra }\end{array}$ & $\mathrm{NI}$ & NÃO INFORMOU (NI) \\
\hline 292890 & SÃO DESIDÉRIO & BA & JOĀO ALMEIDA DOS SANTOS & PSDB BA & REC FEDERAL 2002 & Fazenda Rancho Cacheado, adq 1998 & 900,00 & MÉDIA PROPRIEDADE \\
\hline 292905 & SÃO FELIX DO CORIBE & $\mathrm{BA}$ & JOSE ALVES ROCHA & PRBA & REC FEDERAL 2010 & Sitio Na Rodovia Ba & $\mathrm{NI}$ & NÄO INFORMOU (NI) \\
\hline 292980 & SAÚdE & $\mathrm{BA}$ & SEVERIANO ALVES DE SOUZA & PDT BA & REC FEDERAL 2002 & $\begin{array}{l}\text { Uma área de terra rural, Fazenda Cardoso de Fora } \\
\text { com } 200 \text { tarefas matrícula n²-02-M37 }\end{array}$ & 284,00 & MÉDIA PROPRIEDADE \\
\hline 293270 & URUÇUCA & $\mathrm{BA}$ & $\begin{array}{l}\text { PAULO SÉRGIO PARANHOS DE } \\
\text { MAGALHÄES }\end{array}$ & DEM BA & REC FEDERAL 2010 & Fazenda Bom Sossego & $\mathrm{NI}$ & NÃO INFORMOU (NI) \\
\hline 293270 & |URUÇUCA & $\mathrm{BA}$ & $\begin{array}{l}\text { PAULO SÉRGIO PARANHOS DE } \\
\text { MAGALHÄES }\end{array}$ & DEM BA & REC FEDERAL 2010 & Fazenda Dois Irmăos & $\mathrm{NI}$ & NÃO INFORMOU (NI) \\
\hline 293300 & VALENTE & $\mathrm{BA}$ & AROLDO CEDRAZ DE OLIVEIRA & PFLBA & REC FEDERAL 2006 & FAZ ALTO BONITO & $\mathrm{NI}$ & NÄO INFORMOU (NI) \\
\hline 293350 & VENCESLAU GUIMARÃES & $\mathrm{BA}$ & $\begin{array}{l}\text { LEUR ANTONIO DE BRITO } \\
\text { LOMANTO }\end{array}$ & PMDB BA & REC FEDERAL 1998 & ÁREA DE TERRAS DEN FAZ ÁGUA SANTA & 53,00 & PEQUENA PROPRIEDADE \\
\hline$x$ & $\mathrm{x}$ & BA & SEVERIANO ALVES DE SOUZA & PDT BA & REC FEDERAL 2002 & $\begin{array}{l}\text { Uma área de terra rural, Fazenda da Serraria com } \\
164 \text { ha matricula n R-04-M347 }\end{array}$ & 164,00 & PEQUENA PROPRIEDADE \\
\hline 230100 & ABAIARA & CE & $\begin{array}{l}\text { FRANCISCO ROMMEL FEIJÓ DE } \\
\text { SÁ }\end{array}$ & PTB CE & REC FEDERAL 2008 & Parte De Terra No Sitio & $\mathrm{NI}$ & NÃO INFORMOU (NI) \\
\hline 230020 & ACARAu & $\mathrm{CE}$ & ANIBAL FERREIRA GOMES & PMDB CE & REC FEDERAL 2010 & Uma Glepa De Terra No Entrame Do Rio & 24,00 & PEQUENA PROPRIEDADE \\
\hline 230100 & AQUIRAZ & $\mathrm{CE}$ & LUIS BARBOSA ALVES & PSDB RR & REC FEDERAL 2010 & UM TERRENO NO LUGAR MARIUBAS & $\mathrm{NI}$ & NÄO INFORMOU (NI) \\
\hline 230100 & AQUIRAZ & $\mathrm{CE}$ & ROBERTO SOARES PESSOA & PLCE & REC FEDERAL 2008 & Um Lote De Terra & $\mathrm{NI}$ & NÃO INFORMOU (NI) \\
\hline 230170 & AURORA & $\mathrm{CE}$ & $\begin{array}{l}\text { RAIMUNDO ANTONIO DE } \\
\text { MACEDO (RAIMUNDÃO) }\end{array}$ & PMDB CE & REC FEDERAL 2010 & Um Terra No Sitio Denominado Cacimba Velha & $\mathrm{NI}$ & NÃO INFORMOU (NI) \\
\hline 230170 & AURORA & $\mathrm{CE}$ & $\begin{array}{l}\text { RAIMUNDO ANTONIO DE } \\
\text { MACEDO (RAIMUNDÄO) }\end{array}$ & PMDBCE & REC FEDERAL 2010 & Uma Parte De Terra & NI & NÄO INFORMOU (NI) \\
\hline 230170 & AURORA & $\mathrm{CE}$ & $\begin{array}{l}\text { RAIMUNDO ANTONIO DE } \\
\text { MACEDO (RAIMUNDÃO) }\end{array}$ & PMDB CE & REC FEDERAL 2010 & Uma Parte De Terra No Sitio Pacote & $\mathrm{NI}$ & NÃO INFORMOU (NI) \\
\hline 230170 & AURORA & $\mathrm{CE}$ & $\begin{array}{l}\text { RAIMUNDO ANTONIO DE } \\
\text { MACEDO (RAIMUNDÃO) }\end{array}$ & PMDB CE & REC FEDERAL 2010 & Uma Parte De Terra & $\mathrm{NI}$ & NÄO INFORMOU (NI) \\
\hline 230170 & AURORA & $\mathrm{CE}$ & $\begin{array}{l}\text { RAIMUNDO ANTONIO DE } \\
\text { MACEDO (RAIMUNDÄO) }\end{array}$ & PMDB CE & REC FEDERAL 2010 & Uma Parte De Terra & $\mathrm{NI}$ & NÃO INFORMOU (NI) \\
\hline 230170 & AURORA & $\mathrm{CE}$ & $\begin{array}{l}\text { RAIMUNDO ANTONIO DE } \\
\text { MACEDO (RAIMUNDÃO) }\end{array}$ & PMDB CE & REC FEDERAL 2010 & Parte De Terra & $\mathrm{NI}$ & NÃO INFORMOU (NI) \\
\hline 230170 & AURORA & $\mathrm{CE}$ & $\begin{array}{l}\text { RAIMUNDO ANTONIO DE } \\
\text { MACEDO (RAIMUNDÄO) }\end{array}$ & PMDB CE & REC FEDERAL 2010 & Uma Parte De Terra & $\mathrm{NI}$ & NÃO INFORMOU (NI) \\
\hline 230190 & BARBALHA & CE & $\begin{array}{l}\begin{array}{l}\text { FRANCISCO ROMMEL FEIJÓ DE } \\
\text { SÁ }\end{array} \\
\end{array}$ & PTB CE & REC FEDERAL 2008 & Terreno Localizado No Sitio Santa Cruz & $\mathrm{NI}$ & NÃO INFORMOU (NI) \\
\hline 230200 & BARRo & $\mathrm{CE}$ & $\begin{array}{l}\text { RAIMUNDO ANTONIO DE } \\
\text { MACEDO (RAIMUNDÃO) }\end{array}$ & PMDB CE & REC FEDERAL 2010 & Uma Parte De Terra No Sitio Serrote Do Cachimbo & $\mathrm{NI}$ & NÄO INFORMOU (NI) \\
\hline 230200 & BARRO & CE & $\begin{array}{l}\text { RAIMUNDO ANTONIO DE } \\
\text { MACEDO (RAIMUNDÃO) }\end{array}$ & PMDB CE & REC FEDERAL 2010 & $\begin{array}{l}\text { Uma Propriedade De Terra No Sitio Cachimbo E } \\
\text { Cajeirinha }\end{array}$ & $\mathrm{NI}$ & NÃO INFORMOU (NI) \\
\hline 230280 & CANINDÉ & $\mathrm{CE}$ & FRANCISCO PINHEIRO LANDIM & PMDB CE & REC FEDERAL 2002 & PROPRIEDADE SELA E TAQUARI & $3.600,00$ & GRANDE PROPRIEDADE \\
\hline 230370 & CAUCAIA & CE & $\begin{array}{l}\text { VICENTE FERREIRA DE } \\
\text { ARRUDA COELHO }\end{array}$ & PRCE & REC FEDERAL 2010 & $\begin{array}{l}\text { Um Lote C } 06 \text { Na Fazenda Soledade Havido Por } \\
\text { Heranca De Seu Pai Falecido Em } 1968\end{array}$ & $\mathrm{NI}$ & NÄO INFORMOU (NI) \\
\hline 230370 & CAUCAIA & CE & $\begin{array}{l}\text { VICENTE FERREIRA DE } \\
\text { ARRUDA COELHO }\end{array}$ & PRCE & REC FEDERAL 2010 & $\begin{array}{l}\text { Um Lote A } 03 \text { Na Fazenda Soledade Havido Por } \\
\text { Heranca De Seu Pai Falecido Em } 1968\end{array}$ & $\mathrm{NI}$ & NÃO INFORMOU (NI) \\
\hline 230370 & CAUCAIA & CE & $\begin{array}{l}\text { VICENTE FERREIRA DE } \\
\text { ARRUDA COELHO }\end{array}$ & PRCE & REC FEDERAL 2010 & $\begin{array}{l}\text { De Soledade Havido Por Heranca De Seu Pai } \\
\text { Falecido Em } 1968\end{array}$ & $\mathrm{NI}$ & NÃO INFORMOU (NI) \\
\hline 230370 & CAUcala & $\mathrm{CE}$ & $\begin{array}{l}\text { VICENTE FERREIRA DE } \\
\text { ARRUDA COELHO }\end{array}$ & PRCE & REC FEDERAL 2010 & $\begin{array}{l}\text { Caucaia Havido Por Heranca De Seu Pai Falecido } \\
\text { Em } 1968\end{array}$ & $\mathrm{NI}$ & NÃO INFORMOU (NI) \\
\hline 230370 & CAUCAIA & $\mathrm{CE}$ & $\begin{array}{l}\text { VICENTE FERREIRA DE } \\
\text { ARRUDA COELHO }\end{array}$ & PR CE & REC FEDERAL 2010 & $\begin{array}{l}\text { Lote F08 Na Fazenda Soledade Havido Por } \\
\text { Heranca De Seu Pai Falecido Em } 1968\end{array}$ & $\mathrm{NI}$ & NÄO INFORMOU (NI) \\
\hline 230370 & CAUCAIA & CE & $\begin{array}{l}\text { VICENTE FERREIRA DE } \\
\text { ARRUDA COELHO }\end{array}$ & PRCE & REC FEDERAL 2010 & $\begin{array}{l}\text { Caucaia Havido Por Heranca De Seu Pai Falecido } \\
\text { Em } 1968\end{array}$ & $\mathrm{NI}$ & NÄO INFORMOU (NI) \\
\hline 230440 & FORTALEZA & $\mathrm{CE}$ & ROBERTO SOARES PESSOA & PLCE & REC FEDERAL 2008 & Um Lote De Terra & $\mathrm{NI}$ & NÃO INFORMOU (NI) \\
\hline 230730 & JUAZEIRO DO NORTE & CE & $\begin{array}{l}\text { FRANCISCO ROMMEL FEIJÓ DE } \\
\text { SAA }\end{array}$ & PTB CE & REC FEDERAL 2008 & $\begin{array}{l}\text { 1/4 De Sobra De Terrenos De Espolio De Jose } \\
\text { Feijó De Sá }\end{array}$ & $\mathrm{NI}$ & NÄO INFORMOU (NI) \\
\hline 230730 & JUAZEIRO DO NORTE & CE & $\begin{array}{l}\begin{array}{l}\text { FRANCISCO ROMMEL FEIJÓ DE } \\
\text { SAA }\end{array} \\
\end{array}$ & PTB CE & REC FEDERAL 2008 & Terreno Na Lagoa Seca & $\mathrm{NI}$ & NÃO INFORMOU (NI) \\
\hline 230730 & JUAZEIRO DO NORTE & $\mathrm{CE}$ & $\begin{array}{l}\text { RAIMUNDO ANTONIO DE } \\
\text { MACEDO (RAIMUNDÄO) }\end{array}$ & PMDB CE & REC FEDERAL 2010 & Um Terreno Agricola Situado No Sitio Sta Clara & $\mathrm{NI}$ & NÃO INFORMOU (NI) \\
\hline 230750 & LAVRAS DA MANGABEIRA & CE & EUNICIO LOPES OLIVEIRA & PMDB CE & REC FEDERAL 2010 & $\begin{array}{l}\text { Parte De Um Imovel Rural Sito No Sitio Varzea } \\
\text { Grande }\end{array}$ & $\mathrm{NI}$ & NÃO INFORMOU (NI) \\
\hline
\end{tabular}




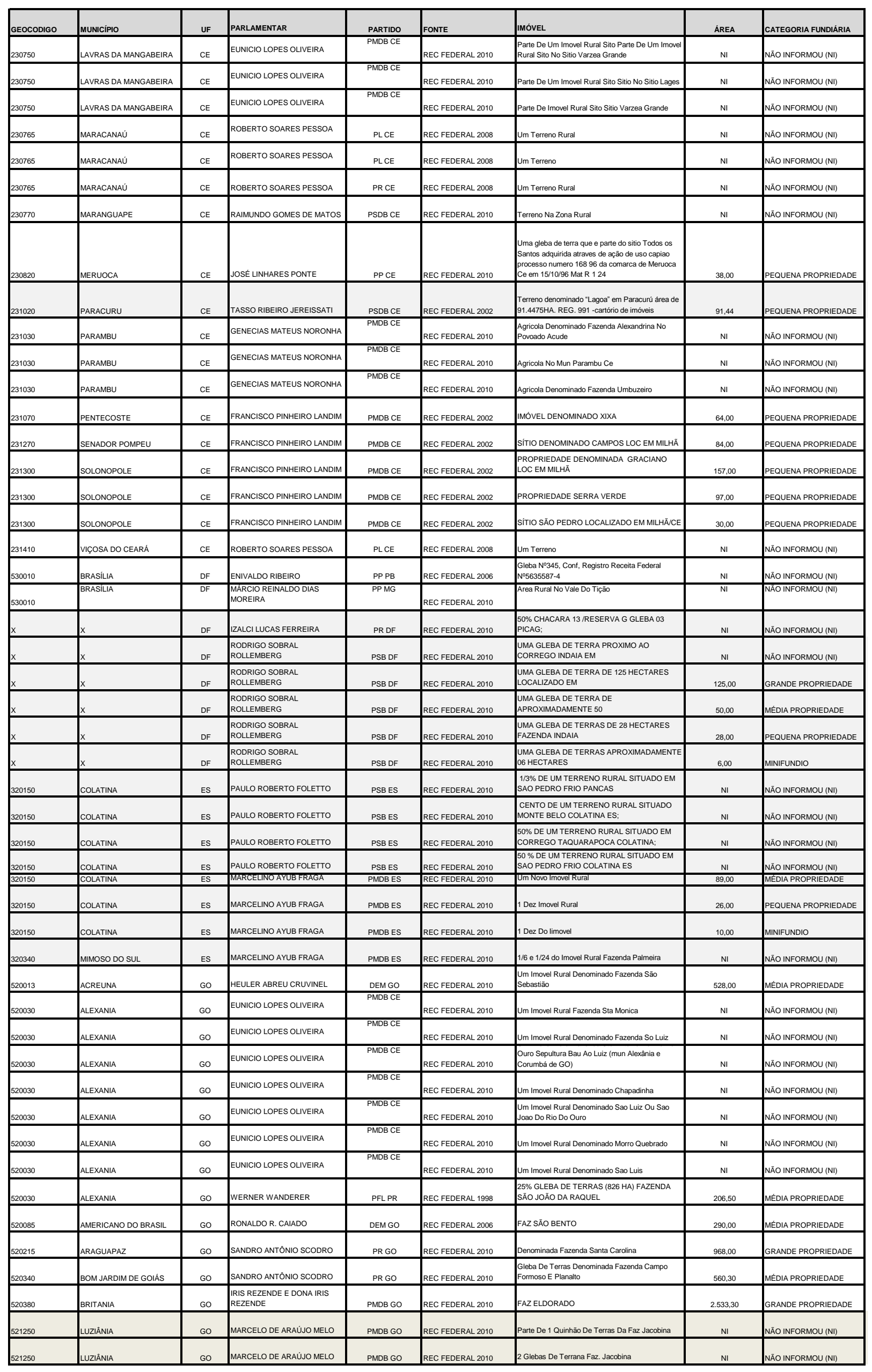




\begin{tabular}{|c|c|c|c|c|c|c|c|c|}
\hline GEOCODIGO & MUNICiPIO & uF & PARLAMENTAR & PARTIDO & FONTE & IMÓvel & ÁREA & CATEGORIA FUNDIÁRIA \\
\hline 520549 & CIDADE OCIDENTAL & GO & $\begin{array}{l}\text { HUMBERTO GUIMARAES } \\
\text { SOUTO }\end{array}$ & PPS GO & REC FEDERAL 2010 & $\begin{array}{l}\text { Um Imovel Rural Com Todas As Benfeitorias } \\
\text { Denominado Fazenda Bebedouro }\end{array}$ & $\mathrm{NI}$ & NÄO INFORMOU (NI) \\
\hline 520380 & BRITANIA & Go & $\begin{array}{l}\text { IRIS REZENDE E DONA IRIS } \\
\text { REZENDE }\end{array}$ & PMDB GO & REC FEDERAL 2010 & FAZ MUTIRAMA & $2.332,60$ & GRANDE PROPRIEDADE \\
\hline 520380 & BRITANIA & Go & $\begin{array}{l}\text { IRIS REZENDE E DONA IRIS } \\
\text { REZENDE }\end{array}$ & PMDB GO & REC FEDERAL 2010 & TERRAS & 459,80 & MÉDIA PROPRIEDADE \\
\hline 520380 & BRITANIA & Go & $\begin{array}{l}\text { IRIS REZENDE E DONA IRIS } \\
\text { REZENDE }\end{array}$ & PMDB GO & REC FEDERAL 2010 & TERRAS & 58,08 & PEQUENA PROPRIEDADE \\
\hline 520380 & BRITANIA & GO & $\begin{array}{l}\text { IRIS REZENDE E DONA IRIS } \\
\text { REZENDE }\end{array}$ & PMDB GO & REC FEDERAL 2010 & TERRAS & 53,30 & PEQUENA PROPRIEDADE \\
\hline 520380 & BRITANIA & Go & $\begin{array}{l}\text { IRIS REZENDE E DONA IRIS } \\
\text { REZENDE }\end{array}$ & PMDB GO & REC FEDERAL 2002 & FAZ MUTIRAMA & 49,26 & MINIFUNDIO \\
\hline 520425 & CACHOEIRA DOURADA & Go & JOSE G. ROCHA & PSD GO & REC FEDERAL 2010 & GLEBA DE TERRA FAZ ILHA DO CASCALHO* & 9,68 & MINIFUNDIO \\
\hline 520530 & CAVALCANTE & Go & AELTON JOSE DE FREITAS & PPMG & REC FEDERAL 2010 & $50 \%$ DA FAZENDA GAVIÄO & $\mathrm{NI}$ & NÃO INFORMOU (NI) \\
\hline 520580 & CORUMBA DE GOIÁS & GO & EUNICIO LOPES OLIVEIRA & PMDBCE & REC FEDERAL 2010 & $\begin{array}{l}\text { Um Imovel Rural Fazenda Mannuela (Corumbá, } \\
\text { GO) }\end{array}$ & $\mathrm{NI}$ & NÃO INFORMOU (NI) \\
\hline 520580 & CORUMBÁ DE GOIÁS & GO & EUNICIO LOPES OLIVEIRA & PMDBCE & REC FEDERAL 2010 & Um Imovel Rural Denominado Sao Joao Sao Luiz & $\mathrm{NI}$ & NÄO INFORMOU (NI) \\
\hline 520580 & CORUMBÁ DE GOIÁS & GO & EUNICIO LOPES OLIVEIRA & PMDBCE & REC FEDERAL 2010 & Um Imovel Rural Denominado Fazenda Bau & $\mathrm{NI}$ & NÃO INFORMOU (NI) \\
\hline 520580 & CORUMBÁ DE GOIÁS & GO & EUNICIO LOPES OLIVEIRA & PMDBCE & REC FEDERAL 2010 & $\begin{array}{l}\text { Um Imovel Rural Denominado Fazenda Gongonhas } \\
\text { (declarou mun Corumbá,GO) }\end{array}$ & $\mathrm{NI}$ & NÃO INFORMOU (NI) \\
\hline 520580 & CORUMBÁ DE GOIÁS & GO & EUNICIO LOPES OLIVEIRA & PMDBCE & REC FEDERAL 2010 & $\begin{array}{l}\text { Um Imovel Rural Denominado Fazenda Gonconhas } \\
\text { Dos Alves }\end{array}$ & $\mathrm{NI}$ & NÃO INFORMOU (NI) \\
\hline 520580 & CORUMBÁ DE GOIÁS & Go & EUNICIO LOPES OLIVEIRA & PMDBCE & REC FEDERAL 2010 & $\begin{array}{l}\text { Um Imovel Rural Denominado Fazenda Baua Lages } \\
\text { Buenos E Lages Dos Bicudos }\end{array}$ & $\mathrm{NI}$ & NÃO INFORMOU (NI) \\
\hline 520580 & CORUMBÁ DE GOIÁS & GO & EUNICIO LOPES OLIVEIRA & PMDBCE & REC FEDERAL 2010 & Um Imovel Rural Denominado Cercado E Retiro & $\mathrm{NI}$ & NÃO INFORMOU (NI) \\
\hline 520580 & CORUMBÁ DE GOIÁS & Go & EUNICIO LOPES OLIVEIRA & PMDBCE & REC FEDERAL 2010 & $\begin{array}{l}\text { Um Imovel Rural Denominado Fazenda } \\
\text { Dgongonhas Dos Alves }\end{array}$ & $\mathrm{NI}$ & NÄO INFORMOU (NI) \\
\hline 520580 & CORUMBÁ DE GOIÁS & GO & EUNICIO LOPES OLIVEIRA & PMDB CE & REC FEDERAL 2010 & Um Imovel Rural Denominado Lage E Gongonhas & $\mathrm{NI}$ & NÃO INFORMOU (NI) \\
\hline 520580 & CORUMBÁ DE GOIÁS & GO & EUNICIO LOPES OLIVEIRA & PMDBCE & REC FEDERAL 2010 & $\begin{array}{l}\text { Um Imovel Rural Denominado Rural Denominado } \\
\text { Gongonhas Dos Alves }\end{array}$ & $\mathrm{NI}$ & NÄO INFORMOU (NI) \\
\hline 520580 & CORUMBÁ DE GOIÁS & GO & EUNICIO LOPES OLIVEIRA & PMDBCE & REC FEDERAL 2010 & $\begin{array}{l}\text { Um Imovel Rural Denominado Gongonhas Dos } \\
\text { Alves Municipio Corumba De Goias Go }\end{array}$ & $\mathrm{NI}$ & NÃO INFORMOU (NI) \\
\hline 520580 & CORUMBÁ DE GOIÁS & GO & EUNICIO LOPES OLIVEIRA & PMDBCE & REC FEDERAL 2010 & $\begin{array}{l}\text { Um Imovel Rural Denominado Pasto Grande } \\
\text { Corumba De Goias Go }\end{array}$ & $\mathrm{NI}$ & NÃO INFORMOU (NI) \\
\hline 520580 & CORUMBÁ DE GOIÁS & GO & EUNICIO LOPES OLIVEIRA & PMDBCE & REC FEDERAL 2010 & $\begin{array}{l}\text { Um Imovel Rural Denominado Lages Municipio De } \\
\text { Corrumba De Goias Go }\end{array}$ & $\mathrm{NI}$ & NÄO INFORMOU (NI) \\
\hline 520580 & CORUMBÁ DE GOIÁS & Go & EUNICIO LOPES OLIVEIRA & PMDBCE & REC FEDERAL 2010 & $\begin{array}{l}\text { Um Imovel Rural Denominado Gongonhas Dos } \\
\text { Alves Municipio Corumba De Goias Go Area } 259\end{array}$ & $\mathrm{NI}$ & NÃO INFORMOU (NI) \\
\hline 520580 & CORUMBÁ DE GOIÁS & GO & EUNICIO LOPES OLIVEIRA & PMDB CE & REC FEDERAL 2010 & $\begin{array}{l}\text { Um Imovel Rural Denominado Fazenda Sao } \\
\text { Geraldo Municipio Corumba De Goias }\end{array}$ & $\mathrm{NI}$ & NÃO INFORMOU (NI) \\
\hline 520580 & CORUMBÁ DE GOIÁS & GO & EUNICIO LOPES OLIVEIRA & PMDBCE & REC FEDERAL 2010 & $\begin{array}{l}\text { Buenos Ou Buenos E Olaria Municipio Corumba De } \\
\text { Goias }\end{array}$ & $\mathrm{NI}$ & NÃO INFORMOU (NI) \\
\hline 520580 & CORUMBÁ DE GOIÁS & GO & EUNICIO LOPES OLIVEIRA & PMDBCE & REC FEDERAL 2010 & $\begin{array}{l}\text { Um Imovel Rural Denominado Mato Da Cruz } \\
\text { Municipio Corumba De Goias Go }\end{array}$ & $\mathrm{NI}$ & NÃO INFORMOU (NI) \\
\hline 520580 & CORUMBÁ DE GOIÁS & Go & EUNICIO LOPES OLIVEIRA & PMDB CE & REC FEDERAL 2010 & $\begin{array}{l}\text { Um Imovel Rural Denominado Olaria E Mato Do } \\
\text { Meio Municipio Corumba De Goias Go }\end{array}$ & $\mathrm{NI}$ & NÄO INFORMOU (NI) \\
\hline 520580 & CORUMBÁ DE GOIÁS & GO & EUNICIO LOPES OLIVEIRA & PMDBCE & REC FEDERAL 2010 & Um Imovel Rural Denominado Sao Luiz & $\mathrm{NI}$ & NÃO INFORMOU (NI) \\
\hline 520580 & CORUMBÁ DE GOIÁS & Go & EUNICIO LOPES OLIVEIRA & PMDBCE & REC FEDERAL 2010 & $\begin{array}{l}\text { Um Imovel Rural Denominado Cocal Gongonhas } \\
\text { Lages Dos Bicudos }\end{array}$ & $\mathrm{NI}$ & NÃO INFORMOU (NI) \\
\hline 520580 & CORUMBÁ DE GOIÁS & Go & EUNICIO LOPES OLIVEIRA & PMDBCE & REC FEDERAL 2010 & Um Imovel Rural Denominado Bau Funil & $\mathrm{NI}$ & NÃO INFORMOU (NI) \\
\hline 520580 & CORUMBÁ DE GOIÁS & Go & EUNICIO LOPES OLIVEIRA & PMDBCE & REC FEDERAL 2010 & Um Imovel Rural Denominado Beija Mao & $\mathrm{NI}$ & NÃO INFORMOU (NI) \\
\hline 520580 & CORUMBÁ DE GOIÁS & Go & EUNICIO LOPES OLIVEIRA & PMDBCE & REC FEDERAL 2010 & Um Imovel Rural Denominado Faz Cercado & $\mathrm{NI}$ & NĀO INFORMOU (NI) \\
\hline 520580 & CORUMBÁ DE GOIÁS & Go & EUNICIO LOPES OLIVEIRA & PMDBCE & REC FEDERAL 2010 & Um Imovel Rural Denominado Bau & $\mathrm{NI}$ & NÃO INFORMOU (NI) \\
\hline 520580 & CORUMBÁ DE GOIÁS & Go & EUNICIO LOPES OLIVEIRA & PMDBCE & REC FEDERAL 2010 & Um Imovel Rural Denominado Funil & $\mathrm{NI}$ & NÃO INFORMOU (NI) \\
\hline 520580 & CORUMBÁ DE GOIÁS & Go & EUNICIO LOPES OLIVEIRA & PMDBCE & REC FEDERAL 2010 & Um Imovel Rural Denominado Bau & $\mathrm{NI}$ & NÃO INFORMOU (NI) \\
\hline 520580 & CORUMBÁ DE GOIÁS & Go & EUNICIO LOPES OLIVEIRA & PMDBCE & REC FEDERAL 2010 & Um Imovel Rural Denominado Bau & $\mathrm{NI}$ & NÃO INFORMOU (NI) \\
\hline 520580 & CORUMBÁ DE GOIÁS & GO & EUNICIO LOPES OLIVEIRA & PMDB CE & REC FEDERAL 2010 & Um Imovel Rural Denominado Sao Luis & $\mathrm{NI}$ & NĀO INFORMOU (NI) \\
\hline 520580 & CORUMBÁ DE GOIÁS & Go & EUNICIO LOPES OLIVEIRA & PMDBCE & REC FEDERAL 2010 & Um Imovel Rural Denominado Sao Joao Parte & $\mathrm{NI}$ & NÃO INFORMOU (NI) \\
\hline 520580 & CORUMBÁ DE GOIÁS & Go & EUNICIO LOPES OLIVEIRA & PMDBCE & REC FEDERAL 2010 & Um Imovel Rural Denominado Retiro & $\mathrm{NI}$ & NÃO INFORMOU (NI) \\
\hline 520580 & CORUMBÁ DE GOIÁS & GO & EUNICIO LOPES OLIVEIRA & PMDBCE & REC FEDERAL 2010 & Um Imovel Rural Denominado Funil & $\mathrm{NI}$ & NÃO INFORMOU (NI) \\
\hline 520580 & CORUMBÁ DE GOIÁS & Go & EUNICIO LOPES OLIVEIRA & PMDBCE & REC FEDERAL 2010 & Um Imovel Rural Denominado Funil & $\mathrm{NI}$ & NÃO INFORMOU (NI) \\
\hline 520580 & CORUMBÁ DE GOIÁS & Go & EUNICIO LOPES OLIVEIRA & PMDB CE & REC FEDERAL 2010 & Um Imovel Rural Denominado Bau & $\mathrm{NI}$ & NÄO INFORMOU (NI) \\
\hline 520580 & CORUMBÁ DE GOIÁS & Go & EUNICIO LOPES OLIVEIRA & PMDBCE & REC FEDERAL 2010 & $\begin{array}{l}\text { Um Imovel Rural Denominado Congonhas Dos } \\
\text { Alves }\end{array}$ & $\mathrm{NI}$ & NÃO INFORMOU (NI) \\
\hline 520580 & CORUMBÁ DE GOIÁS & Go & EUNICIO LOPES OLIVEIRA & PMDBCE & REC FEDERAL 2010 & Um Imovel Rural Denominado Sepultura & $\mathrm{NI}$ & NÃO INFORMOU (NI) \\
\hline 520580 & CORUMBÁ DE GOIÁS & GO & EUNICIO LOPES OLIVEIRA & PMDBCE & REC FEDERAL 2010 & Um Imovel Rural Denominado So Luiz & $\mathrm{NI}$ & NÃO INFORMOU (NI) \\
\hline 520580 & CORUMBÁ DE GOIÁS & Go & EUNICIO LOPES OLIVEIRA & PMDBCE & REC FEDERAL 2010 & $\begin{array}{l}\text { Um Imovel Rural Denominado Lages Do Rio Do } \\
\text { Ouro }\end{array}$ & $\mathrm{NI}$ & NÃO INFORMOU (NI) \\
\hline 520580 & CORUMBÁ DE GOIÁS & GO & EUNICIO LOPES OLIVEIRA & PMDB CE & REC FEDERAL 2010 & Um Imovel Rural Denominado Congonha Dos Alves & $\mathrm{NI}$ & NÄO INFORMOU (NI) \\
\hline 520580 & CORUMBÁ DE GOIÁS & Go & EUNICIO LOPES OLIVEIRA & PMDBCE & REC FEDERAL 2010 & Um Imovel Rural Denominado Congonha Dos Alves & $\mathrm{NI}$ & NÃO INFORMOU (NI) \\
\hline 520580 & CORUMBÁ DE GOIÁS & Go & EUNICIO LOPES OLIVEIRA & PMDBCE & REC FEDERAL 2010 & $\begin{array}{l}\text { Um Imovel Rural Denominado Sao Luis Mun } \\
\text { Corumba De Goias }\end{array}$ & $\mathrm{NI}$ & NÃO INFORMOU (NI) \\
\hline
\end{tabular}




\begin{tabular}{|c|c|c|c|c|c|c|c|c|}
\hline GEOCODIGO & MUNICiPIO & UF & PARLAMENTAR & PARTIDO & FONTE & IIMÓvel & ÁREA & CATEGORIA FUNDIÁRIA \\
\hline 520580 & CORUMBÁ DE GOIÁS & Go & EUNICIO LOPES OLIVEIRA & PMDBCE & REC FEDERAL 2010 & $\begin{array}{l}\text { Um Imovel Rural Denominado Beja A Mao Mun } \\
\text { Corumba De Goias Go }\end{array}$ & $\mathrm{NI}$ & NÃO INFORMOU (NI) \\
\hline 520580 & CORUMBÁ DE GOIÁS & Go & EUNICIO LOPES OLIVEIRA & PMDB CE & REC FEDERAL 2010 & $\begin{array}{l}\text { Um Imovel Rural Denominado So Luiz Mun } \\
\text { Corumba De Goias }\end{array}$ & $\mathrm{NI}$ & NÃO INFORMOU (NI) \\
\hline 520580 & CORUMBÁ DE GOIÁS & Go & EUNICIO LOPES OLIVEIRA & PMDBCE & REC FEDERAL 2010 & $\begin{array}{l}\text { Um Imovel Rural Denominado Sao Luis Mun De } \\
\text { Corumba De Go }\end{array}$ & $\mathrm{NI}$ & NÄO INFORMOU (NI) \\
\hline 520580 & CORUMBÁ DE GOIÁS & GO & EUNICIO LOPES OLIVEIRA & PMDBCE & REC FEDERAL 2010 & $\begin{array}{l}\text { Um Imovel Rural Denominado Lages Do Rio Do } \\
\text { Ouro Mun De Corumba De Goias Go }\end{array}$ & $\mathrm{NI}$ & NÃO INFORMOU (NI) \\
\hline 520580 & CORUMBÁ DE GOIÁS & Go & EUNICIO LOPES OLIVEIRA & PMDBCE & REC FEDERAL 2010 & $\begin{array}{l}\text { Um Imovel Rural Denominado Capuava Mun } \\
\text { Corumba De Goias }\end{array}$ & $\mathrm{NI}$ & NÃO INFORMOU (NI) \\
\hline 520580 & CORUMBÁ DE GOIÁS & Go & EUNICIO LOPES OLIVEIRA & PMDBCE & REC FEDERAL 2010 & $\begin{array}{l}\text { Um Imovel Rural Denominado Funil Mun Corumba } \\
\text { De Goias }\end{array}$ & $\mathrm{NI}$ & NÄO INFORMOU (NI) \\
\hline 520580 & CORUMBÁ DE GOIÁS & GO & EUNICIO LOPES OLIVEIRA & PMDBCE & REC FEDERAL 2010 & $\begin{array}{l}\text { Um Imovel Rural Denominado Mata Do Rio } \\
\text { Corumba }\end{array}$ & $\mathrm{NI}$ & NÃO INFORMOU (NI) \\
\hline 520580 & CORUMBÁ DE GOIÁS & Go & EUNICIO LOPES OLIVEIRA & PMDBCE & REC FEDERAL 2010 & Um Imovel Rural Denominado Pasto Grande & $\mathrm{NI}$ & NÄO INFORMOU (NI) \\
\hline 520580 & CORUMBÁ DE GOIÁS & GO & EUNICIO LOPES OLIVEIRA & PMDBCE & REC FEDERAL 2010 & Um Imovel Rural Denominado Mata Do Corumba & $\mathrm{NI}$ & NÃO INFORMOU (NI) \\
\hline 520580 & CORUMBÁ DE GOIÁS & Go & EUNICIO LOPES OLIVEIRA & PMDB CE & REC FEDERAL 2010 & $\begin{array}{l}\text { Um Imovel Rural Denominado Congonha Dos } \\
\text { Buenos }\end{array}$ & $\mathrm{NI}$ & NÃO INFORMOU (NI) \\
\hline 520580 & CORUMBÁ DE GOIÁS & Go & EUNICIO LOPES OLIVEIRA & PMDBCE & REC FEDERAL 2010 & Um Imovel Rural Denominado Congonha Dos Alves & $\mathrm{NI}$ & NÄO INFORMOU (NI) \\
\hline 520580 & CORUMBÁ DE GOIÁS & GO & EUNICIO LOPES OLIVEIRA & PMDBCE & REC FEDERAL 2010 & $\begin{array}{l}\text { Um Imovel Rural Denominado Retiro E Congonha } \\
\text { Dos Alves }\end{array}$ & $\mathrm{NI}$ & NÃO INFORMOU (NI) \\
\hline 520580 & CORUMBÁ DE GOIÁS & GO & EUNICIO LOPES OLIVEIRA & PMDBCE & REC FEDERAL 2010 & $\begin{array}{l}\text { Um Imovel Rural Denominado Congonha Do } \\
\text { Buenos E Buenos }\end{array}$ & $\mathrm{NI}$ & NÃO INFORMOU (NI) \\
\hline 520580 & CORUMBÁ DE GOIÁS & Go & EUNICIO LOPES OLIVEIRA & PMDBCE & REC FEDERAL 2010 & Um Imovel Rural Denominado Congonha Dos Alves & $\mathrm{NI}$ & NÃO INFORMOU (NI) \\
\hline 520580 & CORUMBÁ DE GOIÁS & Go & EUNICIO LOPES OLIVEIRA & PMDB CE & REC FEDERAL 2010 & Um Imovel Rural Denominado Mata Do Curumba & $\mathrm{NI}$ & NÄO INFORMOU (NI) \\
\hline 520580 & CORUMBÁ DE GOIÁS & Go & EUNICIO LOPES OLIVEIRA & PMDBCE & REC FEDERAL 2010 & $\begin{array}{l}\text { Um Imovel Rural Denominado Denominado Cocal } \\
\text { Parte }\end{array}$ & $\mathrm{NI}$ & NÃO INFORMOU (NI) \\
\hline 520580 & CORUMBÁ DE GOIÁS & Go & EUNICIO LOPES OLIVEIRA & PMDB CE & REC FEDERAL 2010 & Um Imovel Rural Denominado Cocal Parte & $\mathrm{NI}$ & NÃO INFORMOU (NI) \\
\hline 520580 & CORUMBÁ DE GOIÁS & Go & EUNICIO LOPES OLIVEIRA & PMDBCE & REC FEDERAL 2010 & $\begin{array}{l}\text { Um Imovel Rural Denominado Fazenda Catingueiro } \\
\text { Grande }\end{array}$ & $\mathrm{NI}$ & NÄO INFORMOU (NI) \\
\hline 520580 & CORUMBÁ DE GOIÁS & Go & EUNICIO LOPES OLIVEIRA & PMDB CE & REC FEDERAL 2010 & $\begin{array}{l}\text { Um Imovel Rural Denominado Fazenda Mata Do } \\
\text { Corumba }\end{array}$ & $\mathrm{NI}$ & NÃO INFORMOU (NI) \\
\hline 520580 & CORUMBÁ DE GOIÁS & Go & EUNICIO LOPES OLIVEIRA & PMDBCE & REC FEDERAL 2010 & Um Imovel Rural Denominado Olaria & $\mathrm{NI}$ & NÃO INFORMOU (NI) \\
\hline 520580 & CORUMBÁ DE GOIÁS & Go & EUNICIO LOPES OLIVEIRA & PMDBCE & REC FEDERAL 2010 & Um Imovel Rural Denominado Funil & $\mathrm{NI}$ & NÃO INFORMOU (NI) \\
\hline 520580 & CORUMBÁ DE GOIÁS & Go & EUNICIO LOPES OLIVEIRA & PMDBCE & REC FEDERAL 2010 & Um Imovel Rural Denominado Capuava & $\mathrm{NI}$ & NÃO INFORMOU (NI) \\
\hline 520830 & DIVINÓPOLIS DE GOIÁS & Go & $\begin{array}{l}\text { JOSÉ DOS SANTOS FREIRE } \\
\text { JUNIOR }\end{array}$ & PMDB TO & REC FEDERAL 2010 & Fazenda & 895,40 & MÉDIA PROPRIEDADE \\
\hline 520830 & DIVINÓPOLIS DE GOIÁS & Go & $\begin{array}{l}\text { JOSÉ DOS SANTOS FREIRE } \\
\text { JUNIOR }\end{array}$ & РMDB TO & REC FEDERAL 2010 & 1 Gleba Terra & 250,00 & MÉDIA PROPRIEDADE \\
\hline 520725 & DOVERLÂNDIA & GO & LEANDRO VILELA VELLOSO & PMDB GO & REC FEDERAL 2010 & $\begin{array}{l}\text { Parte Das Terras Da Fazenda Lebre Em } \\
\text { Doverlandia Goias }\end{array}$ & $\mathrm{NI}$ & NÄO INFORMOU (NI) \\
\hline 520800 & FORMOSA & Go & AROLDO CEDRAZ DE OLIVEIRA & PFLBA & REC FEDERAL 2006 & FAZENDA VELHA & 820,00 & GRANDE PROPRIEDADE \\
\hline 520800 & FORMOSA & Go & FRANCISCO GRAZIANO NETO & PSDB SP & REC FEDERAL 1998 & $\begin{array}{l}\text { FAZ DA GROTA (INCRA 931063021423-0) } \\
\text { CONFERIR }\end{array}$ & 369,00 & MÉDIA PROPRIEDADE \\
\hline 520870 & GOIANIA & Go & LUCIA VANIA A. COSTA & PSD GO & REC FEDERAL 2010 & FAZ CAVEIRAS & $\mathrm{NI}$ & NÃO INFORMOU (NI) \\
\hline 520870 & GOIANIA & GO & LUCIA VANIA A. COSTA & PSD GO & REC FEDERAL 2010 & FAZ CAVEIRAS & $\mathrm{NI}$ & NÃO INFORMOU (NI) \\
\hline 520870 & GOIANIA & Go & LUCIA VANIA A. COSTA & PSD GO & REC FEDERAL 2010 & FAZ CAVEIRAS & $\mathrm{NI}$ & NÄO INFORMOU (NI) \\
\hline 520870 & GOIANIA & Go & LUCIA VANIA A. COSTA & PSD GO & REC FEDERAL 2010 & FAZ SANTA RITA & $\mathrm{NI}$ & NÃO INFORMOU (NI) \\
\hline 520870 & GOIANIA & Go & LUCIA VANIA A. COSTA & PSD GO & REC FEDERAL 2010 & FAZ SALINO & $\mathrm{NI}$ & NÃO INFORMOU (NI) \\
\hline 520870 & GOIANIA & Go & LUCIA VANIA A. COSTA & PSD GO & REC FEDERAL 2010 & FAZ QUEBRA ANZOL & $\mathrm{NI}$ & NÃO INFORMOU (NI) \\
\hline 520870 & GOIANIA & Go & LUCIA VANIA A. COSTA & PSD GO & REC FEDERAL 2010 & TERRENO MARGENS CORREGO CASCAVEL & $\mathrm{NI}$ & NÃO INFORMOU (NI) \\
\hline 520870 & GOIANIA & Go & LUCIA VANIA A. COSTA & PSD GO & REC FEDERAL 2010 & FAZ ARRANCA TOCO & $\mathrm{NI}$ & NÄO INFORMOU (NI) \\
\hline 520870 & GOIANIA & Go & LUCIA VANIA A. COSTA & PSD GO & REC FEDERAL 2010 & FAZ BOA VISTA & 19,36 & PEQUENA PROPRIEDADE \\
\hline 520870 & GOIANIA & GO & LUCIA VANIA A. COSTA & PSDGO & REC FEDERAL 2010 & CHACARA SANTA RITA & 15,33 & PEQUENA PROPRIEDADE \\
\hline 520870 & GOIÂNIA & Go & GIOVANNI CORREA QUEIROZ & PDT PA & REC FEDERAL 1998 & CHÁCARA SANTO ANTONIO & 4,84 & MINIFUNDIO \\
\hline 520920 & GUAPO & Go & $\begin{array}{l}\text { IRIS REZENDE E DONA IRIS } \\
\text { REZENDE }\end{array}$ & PMDB GO & REC FEDERAL 2010 & TERRAS FAZ BOA VISTA & 35,02 & PEQUENA PROPRIEDADE \\
\hline 520920 & GUAPO & Go & $\begin{array}{l}\text { |IRIS REZENDE E DONA IRIS } \\
\text { REZENDE }\end{array}$ & PMDB GO & REC FEDERAL 2010 & FAZ BOA VISTA DO RIBEIRAO & 26,91 & PEQUENA PROPRIEDADE \\
\hline 520920 & GUAPO & Go & $\begin{array}{l}\text { IRIS REZENDE E DONA IRIS } \\
\text { REZENDE }\end{array}$ & PMDB GO & REC FEDERAL 2010 & FAZ BOA VISTA DO RIBEIRAO & 24,20 & PEQUENA PROPRIEDADE \\
\hline 520920 & GUAPO & Go & $\begin{array}{l}\text { |RIS REZENDE E DONA IRIS } \\
\text { REZENDE }\end{array}$ & PMDB GO & REC FEDERAL 2010 & TERRAS FAZ BOA VISTA & 19,33 & PEQUENA PROPRIEDADE \\
\hline 521000 & INHUMAS & GO & ROBERTOBALESTRA & PPB GO & REC FEDERAL 2006 & GLEBADE TERRAFAZ LIMEIRA & 471,90 & GRANDE PROPRIEDADE \\
\hline 521000 & INHUMAS & GO & ROBERTOBALESTRA & PPB GO & REC FEDERAL 2006 & GLEBADE TERRASFAZ SAO PEDRO & 323,00 & MÉDIA PROPRIEDADE \\
\hline 521000 & IINHUMAS & GO & ROBERTOBALESTRA & PPB GO & REC FEDERAL 2006 & GLEBA DE TERRAS FAZ CAPOEIRAO & 242,24 & MÉDIA PROPRIEDADE \\
\hline 521000 & IINHUMAS & GO & ROBERTO BALESTRA & PPB GO & REC FEDERAL 2006 & GLEBA DE TERRA, FAZ MANACA & 180,82 & MÉDIA PROPRIEDADE \\
\hline 521000 & INHUMAS & GO & ROBERTO BALESTRA & PPB GO & REC FEDERAL 2006 & GLEBA DE TERRA FAZ QUILOMBO & 58,08 & PEQUENA PROPRIEDADE \\
\hline 521000 & INHUMAS & Go & ROBERTO BALESTRA & PPB GO & REC FEDERAL 2006 & GLEBA DE TERRAS FAZ BOA ESPERANÇA & 44,80 & PEQUENA PROPRIEDADE \\
\hline 521040 & |TABERAI & GO & RONALDOR.CAIADO & DEMGO & REC FEDERAL 2006 & FAZCONQUISTA & 795,60 & GRANDE PROPRIEDADE \\
\hline 521080 & ITAJA & Go & $\begin{array}{l}\text { JULLO FRANCISCO SEMEGHINI } \\
\text { NETO }\end{array}$ & PSDB SP & REC FEDERAL 210 & Terra Nua & $\mathrm{NI}$ & NÃO INFORMOU (NI) \\
\hline 521080 & ITAJA & Go & $\begin{array}{l}\text { JULLO FRANCISCO SEMEGHINI } \\
\text { NETO }\end{array}$ & PSDB SP & REC FEDERAL 210 & Terreno & $\mathrm{NI}$ & NÄO INFORMOU (NI) \\
\hline 521080 & ITAJA & GO & $\begin{array}{l}\text { JULIO FRANCISCO SEMEGHINI } \\
\text { NETO }\end{array}$ & PSDB SP & REC FEDERAL 210 & Terreno & $\mathrm{NI}$ & NÄO INFORMOU (NI) \\
\hline 521150 & ITUMBIARA & GO & JOSE G. ROCHA & PSD GO & REC FEDERAL 2010 & GLEBA DE TERRASFAZ PANAMA & 678,78 & GRANDE PROPRIEDADE \\
\hline
\end{tabular}

\begin{tabular}{|c|c|c|c|c|c|c|c|c|}
\hline GEOCODIGO & MUNICIPIO & UF & PARLAMENTAR & PARTIDO & FONTE & IMÓVEeL & ÁREA & CATEGORIA FUNDIÁRIA \\
\hline
\end{tabular}




\begin{tabular}{|c|c|c|c|c|c|c|c|c|}
\hline 521150 & ITUMBIARA & $\mathrm{GO}$ & JOVAIR ARANTES & PTB GO & REC FEDERAL 2010 & Gleba naFazenda Panama & 213,70 & TMÉDIA PROPRIEDADE \\
\hline 521150 & ITUMBIARA & Go & JOSE G. ROCHA & PSD GO & REC FEDERAL 2009 & GLEBA DE TERRAS FAZ LAGEADO DE BAIXO & 138,99 & MÉDIA PROPRIEDADE \\
\hline 521150 & ITUMBIARA & Go & JOSE G. ROCHA & PSD GO & REC FEDERAL 2010 & GLEBA DE TERRAS FAZ LAGEADO DE BAIXO & 80,91 & PEQUENA PROPRIEDADE \\
\hline 521150 & ITUMBIARA & Go & JOSE G. ROCHA & PSD GO & REC FEDERAL 2010 & GLEBA DE TERRAS FAZ LAGEADO DE BAIXO & 59,10 & PEQUENA PROPRIEDADE \\
\hline 521150 & ITUMBIARA & Go & JOSE G. ROCHA & PSD GO & REC FEDERAL 1998 & GLEBA DE TERRAS FAZ N.O., SANT.L e GUA & 9,68 & MINIFUNDIO \\
\hline 521250 & LUZIÂNIA & GO & JULIO JOSE DE CAMPOS & DEMMT & REC FEDERAL 2010 & Chacara Luziana Go Ad 1988 Reg 274137 & $\mathrm{NI}$ & NAO INFORMOU (NI) \\
\hline 521250 & |LUZIÂNIA & Go & $\begin{array}{l}\text { JOSÉ RIBAMAR FERREIRA DE } \\
\text { ARAÚJO COSTA (SARNEY) }\end{array}$ & PMDB AP & REC FEDERAL 1998 & $\begin{array}{l}\text { FAZENDA SÃO JOSÉ DO PERICUMÄ } \\
\text { PERICUMĀ (COM BENFEITORIAS) REG INCRA } \\
\text { 931071015210-3 E } 931071017540\end{array}$ & 268,00 & MÉDIA PROPRIEDADE \\
\hline 521250 & LUZIÂNIA & GO & \begin{tabular}{|l|l|} 
MARCONDES IRAN BENEVIDES \\
GADELHA
\end{tabular} & PSC PB & REC FEDERAL 2010 & Chácara Localizada Jo Jd Estrela Dalva & 2,00 & MINIFUNDIO \\
\hline 521260 & MAIRIPOTABA & GO & SANDRO ANTÔNIO SCODRO & PR GO & REC FEDERAL 2010 & $\begin{array}{l}\text { Gleba De Terras, Denominada De Estancia Santa } \\
\text { Claudia }\end{array}$ & 387,20 & MÉDIA PROPRIEDADE \\
\hline 521280 & MARA ROSA & $\mathrm{GO}$ & RONALDO R. CAIADO & DEMGO & REC FEDERAL 2006 & GLEBA DE TERRAS ALDEIA MARIA & $1.243,88$ & GRANDE PROPRIEDADE \\
\hline 521280 & MARA ROSA & GO & RONALDO R. CAIADO & DEMGO & REC FEDERAL 2006 & FAZ ESCALADA & 968,00 & MEDIA PROPRIEDADE \\
\hline 521305 & MIMOSO DE GOIAS & GO & JOSE FUSCALDI CESILIO & PSD DF & REC FEDERAL 2002 & Uma Fazenda denominanda Bonifo & 648,00 & MEDIA PROPRIEDADE \\
\hline 521308 & MINAÇU & Go & $\begin{array}{l}\text { CARLOS ALBERTO LERÉIA DA } \\
\text { SILVA }\end{array}$ & PSDB GO & REC FEDERAL 2010 & $\begin{array}{l}\text { Fazenda Santa Rita I - Incra 92611 140008273-2 E } \\
\text { Receita Federal 3.447.258-4 Na Zona Rural De } \\
\text { Miną̧u, Logradouro Via Palmeirópolis }\end{array}$ & $\mathrm{NI}$ & NÃO INFORMOU (NI) \\
\hline 521308 & MINAÇU & Go & $\begin{array}{l}\text { CARLOS ALBERTO LERÉIA DA } \\
\text { SILVA }\end{array}$ & PSDB GO & REC FEDERAL 2010 & $\begin{array}{l}\text { Uma Gleba De Terra Rural, Do Quinhăo Da } \\
\text { Fazenda Queixada Do Curriola }\end{array}$ & $\mathrm{NI}$ & NÃO INFORMOU (NI) \\
\hline 521308 & MINAÇU & GO & $\begin{array}{l}\text { CARLOS ALBERTO LERÉIA DA } \\
\text { SILVA }\end{array}$ & PSDB GO & REC FEDERAL 2010 & $\begin{array}{l}\text { Tres Glebas De Terra Rural, Do Logradouro São } \\
\text { Lourenço Do Paraiso }\end{array}$ & $\mathrm{NI}$ & NÄO INFORMOU (NI) \\
\hline 521308 & MINAÇU & GO & $\begin{array}{l}\text { CARLOS ALBERTO LERÉIA DA } \\
\text { SILVA }\end{array}$ & PSDB GO & REC FEDERAL 2010 & $\begin{array}{l}\text { Fazenda Esplanada - Incra 926140004227-7 E } \\
\text { Rec.Federal 3.460.146-5, no Logradouro Săo } \\
\text { Lourenço Do Parásiso-Regiäo Fazenda Maranhäo, } \\
\text { Com Área De 383.61,92 Há }\end{array}$ & 383,61 & MÉDIA PROPRIEDADE \\
\hline 521308 & MINAÇU & GO & $\begin{array}{l}\text { CARLOS ALBERTO LERÉIA DA } \\
\text { SILVA }\end{array}$ & PSDB GO & REC FEDERAL 2010 & Área Às Margens Do Lago Serra Da Mesa & 4,84 & MINIFUNDIO \\
\hline 521308 & MINAÇU & Go & $\begin{array}{l}\text { CARLOS ALBERTO LERÉIA DA } \\
\text { SILVA }\end{array}$ & PSDB GO & REC FEDERAL 2010 & $\begin{array}{l}\text { Lote Rural, N. 28, Localizado No Loteamento São } \\
\text { Lourenço Do Paraiso }\end{array}$ & 4,76 & MINIFUNDIO \\
\hline 521483 & NOVA CRIXAS & GO & RONALDO R.CAIADO & DEMGO & $\begin{array}{l}\text { REC FEDERAL } 2006 \\
\end{array}$ & SANTAMARIA & $1.120,45$ & GRANDE PROPRIEDADE \\
\hline 521483 & NOVA CRIXÁS & GO & RONALDOR.CAIADO & DEMGO & REC FEDERAL 2006 & FAZSEGREDO & 754,31 & MÉDIA PROPRIEDADE \\
\hline 521730 & PIRENÓPOLIS & $\mathrm{GO}$ & JOSE FUSCALDICESILIO & PSD DF & REC FEDERAL 2002 & Uma Fazenda denominada Brinco de Ouro & 600,00 & GRANDE PROPRIEDADE \\
\hline 522020 & SÄO MIGUEL DO ARAGUAIA & GO & SANDRO ANTONIOSCODRO & PRGO & REC FEDERAL 2010 & Denominada Fazenda Santa Camila & $1.860,00$ & GRANDE PROPRIEDADE \\
\hline 522020 & SÄO MIGUEL DO ARAGUAIA & GO & SANDRO ANTONIOSCODRO & PRGO & REC FEDERAL 2010 & Denominada Fazenda Santa Camila & $1.860,00$ & GRANDE PROPRIEDADE \\
\hline 522140 & TRINDADE & Go & $\begin{array}{l}\text { IRIS REZENDE E DONA IRIS } \\
\text { REZENDE }\end{array}$ & PMDB GO & REC FEDERAL 2010 & FAZ BOA VISTA DO RIBEIRAO & 711,27 & MÉDIA PROPRIEDADE \\
\hline 210005 & AÇALÂANDIA & MA & HÉLIO BATISTA DOS SANTOS & PSDB MA & REC FEDERAL 2010 & Terreno Rural No Loteamento Colonia Gurupi & $\mathrm{NI}$ & NÃO INFORMOU (NI) \\
\hline 210005 & AÇALÂANDIA & MA & HÉLIO BATISTA DOS SANTOS & PSDB MA & REC FEDERAL 2010 & Gleba De Terras & $\mathrm{NI}$ & NÃO INFORMOU (NI) \\
\hline 210015 & $\begin{array}{l}\text { ÁGUA DOCE DO } \\
\text { MARANHĀO }\end{array}$ & MA & $\begin{array}{l}\text { IRACEMA MARIA PORTELLA } \\
\text { NUNES NOGUEIRA LIMA }\end{array}$ & PPPI & REC FEDERAL 2010 & Uma Gleba De Terra Localizada & $\mathrm{NI}$ & NÃO INFORMOU (NI) \\
\hline 210095 & ARAme & MA & PEDRO FERNANDES RIBEIRO & PTB MA & REC FEDERAL 2010 & Propriedade Rural No Pov Centro Cabôclo & $\mathrm{NI}$ & NÃO INFORMOU (NI) \\
\hline 210120 & BACABAL & MA & JOSÉ VIEIRA LINS & PRMA & $\begin{array}{l}\text { REC FEDERAL } 2010 \\
\end{array}$ & $\begin{array}{l}\text { Propriedade Rural C/300 Ha Na Estrada De } \\
\text { Bacabal E Bela Vista Km 10-Fazenda Dois Irmäos }\end{array}$ & 300,00 & MÉDIA PROPRIEDADE \\
\hline 210120 & BACABAL & MA & JOSÉ VIEIRA LINS & PR MA & REC FEDERAL 2010 & $\begin{array}{l}\text { Propriedade Rural C/24 Ha Situada Na Av João } \\
\text { Alberto Na Cidade De Bacabal Adquirida Em } \\
\text { 28.12.2000 }\end{array}$ & 24,00 & MINIFUNDIO \\
\hline 210120 & BACABAL & MA & JOSÉ VIEIRA LINS & PR MA & $\begin{array}{l}\text { REC FEDERAL } 2010 \\
\end{array}$ & $\begin{array}{l}\text { Terreno C/13.570 M2 Na Br } 316 \text { Km } 361 \\
\text { Bacabal/Ma }\end{array}$ & 1,35 & MINIFUNDIO \\
\hline 210120 & BACABAL & $\mathrm{MA}$ & JOSÉ VIEIRA LINS & PRMA & REC FEDERAL 2010 & Terreno C/10179,62M2 Em Bacabal/Ma & 1,01 & MINIFUNDIO \\
\hline 210140 & BALSAS & MA & JOSE G. ROCHA & PSD GO & REC FEDERAL 2010 & $\begin{array}{l}\text { GLEBA DE TERRAS FAZ ST LUZIA D RIO D } \\
\text { BALSAS }\end{array}$ & $4.595,04$ & GRANDE PROPRIEDADE \\
\hline 210140 & BALSAS & MA & JOSE G. ROCHA & PSDGO & REC FEDERAL 2010 & GLEBADE TERRASFAZ SANTALUZIA & $2.127,72$ & GRANDE PROPRIEDADE \\
\hline 210140 & BALSAS & $\mathrm{MA}$ & JOSEG. ROCHA & PSDGO & REC FEDERAL 2010 & GLEBADE TERRAS FAZ MANGABA & $1.460,88$ & GRANDE PROPRIEDADE \\
\hline 210140 & BALSAS & $\mathrm{MA}$ & JOSE G. ROCHA & PSD GO & REC FEDERAL 2010 & GLEBADE TERRASFAZ BEIJAFLORTE II & 968,45 & MÉDIA PROPRIEDADE \\
\hline 210140 & BALSAS & $\mathrm{MA}$ & JOSEG. ROCHA & PSD GO & REC FEDERAL 2010 & GLEBADE TERRASFAZ SANRODRIGO & 634,68 & MÉDIA PROPRIEDADE \\
\hline 210140 & BALSAS & MA & $\begin{array}{l}\text { FRANCISCO DE ASSIS } \\
\text { MLLHOMEM COELHO }\end{array}$ & PFLMA & REC FEDERAL 1998 & BREJÃ̃o & 367,00 & GRANDE PROPRIEDADE \\
\hline 210140 & BALSAS & $\mathrm{MA}$ & JOSEG. ROCHA & PSDGO & REC FEDERAL 2010 & GLEBADE TERRASFAZ IIUMBIARA & 356,30 & MEDIA PROPRIEDADE \\
\hline 210270 & CANTANHEDE & MA & $\begin{array}{l}\text { ELISEU BARROSO DE } \\
\text { CARVALHO MOURA }\end{array}$ & PPMA & REC FEDERAL 2008 & Área & $1.270,00$ & GRANDE PROPRIEDADE \\
\hline 210270 & CANTANHEDE & MA & $\begin{array}{l}\text { ELISEU BARROSO DE } \\
\text { CARVALHO MOURA }\end{array}$ & & REC FEDERAL 2010 & PROPRIEDADE RURAL & $1.270,00$ & GRANDE PROPRIEDADE \\
\hline 210300 & CAXIAS & MA & $\begin{array}{l}\text { PAULO CELSO FONSECA } \\
\text { MARINHO }\end{array}$ & PL MA & REC FEDERAL 2010 & PROPRIEDADES I & $\mathrm{NI}$ & NÃO INFORMOU (NI) \\
\hline 210300 & CAXIAS & MA & $\begin{array}{l}\text { PAULO CELSO FONSECA } \\
\text { MARINHO }\end{array}$ & PLMA & REC FEDERAL 2010 & PROPRIEDADE & $\mathrm{NI}$ & NĀO INFORMOU (NI) \\
\hline 210300 & CAXIAS & MA & $\begin{array}{l}\text { PAULO CELSO FONSECA } \\
\text { MARINHO }\end{array}$ & PLMA & REC FEDERAL 2010 & UMA PROPRIEDADE RETIRO & $\mathrm{NI}$ & NÄO INFORMOU (NI) \\
\hline 210300 & CAXIAS & MA & $\begin{array}{l}\text { PAULO CELSO FONSECA } \\
\text { MARINHO JUNIOR }\end{array}$ & PL MA & REC FEDERAL 2002 & No Lugar Denominado Melancia, Data Prata, № 30 & 343,20 & MÉDIA PROPRIEDADE \\
\hline 210300 & CAXIAS & MA & $\begin{array}{l}\text { PAULO CELSO FONSECA } \\
\text { MARINHO JUNIOR }\end{array}$ & PL MA & REC FEDERAL 2010 & No Lugar Baixa Funda, Bairro Panpulha & 75,80 & PEQUENA PROPRIEDADE \\
\hline 210300 & CAXIAS & MA & $\begin{array}{l}\text { PAULO CELSO FONSECA } \\
\text { MARINHO JUNIOR }\end{array}$ & PL MA & REC FEDERAL 2010 & ? Localizado Na Usina Velha, S/N, Bairro Salobo & 4,46 & MINIFUNDIO \\
\hline 210330 & CODÓ & MA & $\begin{array}{l}\text { ANTONIO JOAQUIM ARAUJO } \\
\text { FILHO }\end{array}$ & PPB MA & REC FEDERAL 2010 & $1039 \mathrm{Ha}$ De Terras Da Fazenda Vidal, Codo-Ma & $1.039,00$ & GRANDE PROPRIEDADE \\
\hline 210330 & CODÓ & MA & $\begin{array}{l}\text { ANTONIO JOAQUIM ARAUJO } \\
\text { FILHO }\end{array}$ & PPB MA & REC FEDERAL 2010 & $\begin{array}{l}23,63 \text { Ha De Terras Na Palmeira Do Norte, Codo- } \\
\text { Ma }\end{array}$ & 423,63 & MÉDIA PROPRIEDADE \\
\hline 210330 & CODÓ & MA & $\begin{array}{l}\text { ANTONIO JOAQUIM ARAUJO } \\
\text { FILHO }\end{array}$ & PPB MA & REC FEDERAL 2010 & $287 \mathrm{Ha}$ De Terras Junto A Fazenda Vidal, Codo-Ma & 287,00 & MÉDIA PROPRIEDADE \\
\hline 210330 & CODÓ & MA & $\begin{array}{l}\text { ANTONIO JOAQUIM ARAUJO } \\
\text { FILHO }\end{array}$ & PPB MA & REC FEDERAL 2010 & 199,57 Ha De Terras Em Codó-Ma & 199,57 & PEQUENA PROPRIEDADE \\
\hline 210390 & DUQUE BACELAR & MA & $\begin{array}{l}\text { PEDRO NOVAIS LIMA } \\
\text { (LICENCIADO) }\end{array}$ & PMDB MA & REC FEDERAL 2010 & $50 \%$ De 365 Ha De Terra No Lugar Jabuti & 182,50 & PEQUENA PROPRIEDADE \\
\hline 210530 & IMPERATRIZ & MA & $\begin{array}{l}\text { PAULO CELLO FONSECA } \\
\text { MARINHO JUNIOR }\end{array}$ & PLMA & REC FEDERAL 2010 & UMA GLEBA DE TERRA & 40,87 & MINIFUNDIO \\
\hline 210610 & LORETO & MA & NELSON PADOVANI & PSC PR & REC FEDERAL 2010 & $\begin{array}{l}50 \% \text { De Um Imovel Rural Com Area De } \\
12,457,00 \mathrm{Has} \text { Reg. Na Mat. 01-605, No Registro } \\
\text { De Imoveis Da Comarca De Loreto, Ma }\end{array}$ & 12,45 & PEQUENA PROPRIEDADE \\
\hline 210750 & PAÇO DO LUMIAR & MA & $\begin{array}{l}\text { JOSÉ RIBAMAR FERREIRA DE } \\
\text { ARAÚJO COSTA (SARNEY) }\end{array}$ & PMDB AP & REC FEDERAL 2006 & $\begin{array}{l}\text { UM TERRENO DENOM JAGUAREMA, LOC À } \\
\text { MARGEM ESQUERDA DA RODOVIA MA-53, NO } \\
\text { TRECHO OLHO D'ÁGUA-ARAÇAGI }\end{array}$ & $\mathrm{NI}$ & NÃO INFORMOU (NI) \\
\hline
\end{tabular}




\begin{tabular}{|c|c|c|c|c|c|c|c|c|}
\hline GEOCODIGO & MUNICIPIO & $\mathrm{UF}$ & PARLAMENTAR & PARTIDO & TFONTE & IIMOVEL & AREA & CATEGORIA FUNDIÁRIA \\
\hline 210750 & PAÇO DO LUMIAR & MA & $\begin{array}{l}\text { LOURIVAL MENDES DA } \\
\text { FONSECA FILHO }\end{array}$ & PTdoB MA & REC FEDERAL 2010 & $\begin{array}{l}\text { 04 Lotes De Terra Própria Em Área Rural, Iguaiba, } \\
\text { Loteamento Denominado Jardim Das Mansōes }\end{array}$ & $\mathrm{NI}$ & NẴo INFORMOU (NI) \\
\hline 210750 & PAÇO DO LUMIAR & MA & $\begin{array}{l}\text { LOURIVAL MENDES DA } \\
\text { FONSECA FILHO }\end{array}$ & PTdoB MA & REC FEDERAL 2010 & $\begin{array}{l}\text { Uma Área Rural, Constituida De } 24 \text { Lotes De } \\
\text { Terreno Com } 1800 \text { Pés De Coco Irrigados }\end{array}$ & $\mathrm{NI}$ & NÃO INFORMOU (NI) \\
\hline 210780 & PARNARAMA & MA & JOSÉ VIEIRA LINS & PRMA & REC FEDERAL 2010 & $\begin{array}{l}\text { Propriedade Rural C/9821, } 16, \text { Sendo } 7415,5 \mathrm{Ha} \text { Da } \\
\text { Fazenda Ollo Dóagua E } 2405,66 \mathrm{Ha} \text { Da Fazenda } \\
\text { Tiuba, No Municipio De Parnarama }\end{array}$ & $9.821,16$ & GRANDE PROPRIEDADE \\
\hline 210810 & PAULO RAMOS & MA & ADAUTO PEREIRA DE LIMA & PFLPB & REC FEDERAL 2002 & $\begin{array}{l}\text { FAZENDA SĀO FRANCISCO, LOC NO LUGAR } \\
\text { DENOM CENTRO DOS ANGICOS }\end{array}$ & $\mathrm{NI}$ & NÃO INFORMOU (NI) \\
\hline 210810 & Paulo ramos & MA & ADAUTO PEREIRA DE LIMA & PFLPB & REC FEDERAL 2002 & $\begin{array}{l}\text { IMOVEL RURAL INDEPENDENCIA, HOJE } \\
\text { DENOM TYANA (DECLAROU PAULO } \\
\text { RAMOS/PB) }\end{array}$ & $\mathrm{NI}$ & NĀO INFORMOU (NI) \\
\hline 210810 & PAULO RAMOS & MA & PEDRO FERNANDES RIBEIRO & РТВ MA & REC FEDERAL 2010 & Propriedade Rural & $\mathrm{NI}$ & NÃO INFORMOU (NI) \\
\hline 210810 & PAULO RAMOS & MA & PEDRO FERNANDES RIBEIRO & PFLMA & REC FEDERAL 2010 & $\begin{array}{l}\text { PROPRIEDADE RURAL NO MUNICIPIO DE } \\
\text { PAULO RAMOS MA }\end{array}$ & $\mathrm{NI}$ & NĀO INFORMOU (NI) \\
\hline 210945 & RAPOSA & MA & ROSEANA SARNEYMURAD & PFLMA & REC FEDERAL 2010 & 1/4 DaFazenda Curupu, Em Raposa-Ma & $\overline{N I}$ & NAO INFORMOU (NI) \\
\hline 210945 & RAPOSA & MA & ROSEANA SARNEYMURAD & PFLMA & REC FEDERAL 2010 & Area Rural No Distrito Federal, Com Area & 4,20 & MINIFUNDIO \\
\hline 210950 & RIACHÄO & MA & \begin{tabular}{|l} 
FRANCISCO DE ASSIS \\
MLLHOMEM COELHO \\
\end{tabular} & PFLMA & REC FEDERAL 1998 & FAZENDA FERRADO & $4.148,00$ & GRANDE PROPRIEDADE \\
\hline 210950 & RIACHÄO & MA & \begin{tabular}{|l} 
FRANCISCO DE ASSIS \\
MLLHOMEM COELHO \\
\end{tabular} & PFLMA & REC FEDERAL 1998 & FAZENDA AGUA BRANCA & $2.000,00$ & GRANDE PROPRIEDADE \\
\hline 210950 & RIACHÄO & MA & $\begin{array}{l}\text { FRANCISCO DE ASSIS } \\
\text { MILHOMEM COELHO }\end{array}$ & PFL MA & REC FEDERAL 1998 & FAZENDA AGUA BRANCA & $2.000,00$ & GRANDE PROPRIEDADE \\
\hline 210950 & RIACHÄO & MA & $\begin{array}{l}\text { FRANCISCO DE ASSIS } \\
\text { MLLHOMEM COELHO } \\
\end{array}$ & PFL MA & REC FEDERAL 1998 & FAZENDA RIO SERENO & 900,00 & MÉDIA PROPRIEDADE \\
\hline 210990 & SANTA INÊS & MA & JOSÉ RIBAMAR COSTA ALVES & PSB MA & REC FEDERAL 2002 & Terra nua no povoado de São José dos Aterrados & 34,00 & MINIFUNDIO \\
\hline 211027 & $\begin{array}{l}\text { SANTO AMARO DO } \\
\text { MARANHÄO }\end{array}$ & MA & $\begin{array}{l}\text { JOSÉ RIBAMAR FERREIRA DE } \\
\text { ARAúJO COSTA (SARNEY) }\end{array}$ & PMDB AP & REC FEDERAL 2006 & $\begin{array}{l}\text { TERRENO ADQ ZEFERINO DE SOUZA } \\
\text { MARREIROS (mun Santo Amaro,MA) }\end{array}$ & $\mathrm{NI}$ & NÄO INFORMOU (NI) \\
\hline 211027 & SANTO AMARO DO MARANH & MA & $\begin{array}{l}\text { JOSÉ RIBAMAR FERREIRA DE } \\
\text { ARAÚJO COSTA (SARNEY) } \\
\end{array}$ & PMDB AP & REC FEDERAL 2006 & $\begin{array}{l}\text { TERRENO TERRA NUA, ADQUIRIDO DE } \\
\text { CLODOALDO GARCIA LIRA CONFLITO DE } \\
\text { TERRAS NESTE TERRENO } \\
\end{array}$ & 200,00 & PEQUENA PROPRIEDADE \\
\hline 211027 & SANTO AMARO DO MARANH & MA & $\begin{array}{l}\text { JOSÉ RIBAMAR FERREIRA DE } \\
\text { ARAÚJO COSTA (SARNEY) }\end{array}$ & PMDB AP & REC FEDERAL 2006 & $\begin{array}{l}\text { TERRENO ADQ DE SALUSTIANO DA SILVA } \\
\text { SOUZA (declarou mun Santo Amaro,MA }\end{array}$ & 0,28 & MINIFUNDIO \\
\hline 211070 & $\begin{array}{l}\text { SÄO DOMINGOS DO } \\
\text { MARANHĀOO }\end{array}$ & MA & PEDRO FERNANDES RIBEIRO & PTB MA & REC FEDERAL 2010 & Propriedade Rural No Pov Centro Dos Macacos & $\mathrm{NI}$ & NÃO INFORMOU (NI) \\
\hline 211070 & $\begin{array}{l}\text { SÄO DOMINGOS DO } \\
\text { MARANHĀO }\end{array}$ & MA & PEDRO FERNANDES RIBEIRO & PTB MA & REC FEDERAL 2010 & Propriedade Rural No Pov Centro Velho & $\mathrm{NI}$ & NÃO INFORMOU (NI) \\
\hline 211120 & SÃO JOSÉ DE RIBAMAR & MA & PEDRO FERNANDES RIBEIRO & PFLMA & REC FEDERAL 2010 & $\begin{array}{l}\text { LOTE RURAL NO POV TAJAÇOABA EM SÃo } \\
\text { JOSÉ DE }\end{array}$ & $\mathrm{NI}$ & NÃO INFORMOU (NI) \\
\hline 211120 & SÃO JOSÉ DO RIBAMAR & MA & PEDRO FERNANDES RIBEIRO & PTB MA & REC FEDERAL 2010 & Lote Rural No Pov Tajaçoaba & $\mathrm{NI}$ & NÄO INFORMOU (NI) \\
\hline 211200 & TASSO FRAGOSO & MA & JOSE G. ROCHA & PSD GO & REC FEDERAL 1998 & GLEBA DE TERRAS FAZ N.O., SANT.L e GUA & $1.287,32$ & GRANDE PROPRIEDADE \\
\hline 211230 & TUNTUM & MA & $\begin{array}{l}\text { ANTONIO JOAQUIM ARAUJO } \\
\text { FILHO }\end{array}$ & PPB MA & REC FEDERAL 2010 & $4300 \mathrm{Ha}$ De Terras Em Tuntum-Ma & $4.300,00$ & GRANDE PROPRIEDADE \\
\hline 211230 & TUNTUM & MA & $\begin{array}{l}\text { ANTONIO JOAQUIM ARAUJO } \\
\text { FLLHO }\end{array}$ & PPB MA & REC FEDERAL 2010 & $2500 \mathrm{Ha}$ De Terras Em Tuntum-Ma & $2.500,00$ & GRANDE PROPRIEDADE \\
\hline$x$ & $x$ & MA & $\begin{array}{l}\text { ELISEU BARROSO DE } \\
\text { CARVALHO MOURA }\end{array}$ & PPMA & REC FEDERAL 2008 & Faz. Matinha & $\mathrm{NI}$ & NÃO INFORMOU (NI) \\
\hline $\mathrm{x}$ & $\mathrm{x}$ & MA & $\begin{array}{l}\text { ELISEU BARROSO DE } \\
\text { CARVALHO MOURA }\end{array}$ & PPMA & REC FEDERAL 2008 & Faz. Tabuado & $\mathrm{NI}$ & NÃO INFORMOU (NI) \\
\hline $\mathrm{x}$ & $\mathrm{x}$ & MA & \begin{tabular}{|l} 
ELISEU BARROSO DE \\
CARVALHO MOURA
\end{tabular} & PPMA & REC FEDERAL 2008 & Faz. Pirapemas Sede & $\mathrm{NI}$ & NÃO INFORMOU (NI) \\
\hline$x$ & $x$ & MA & PEDRO FERNANDES RIBEIRO & PFL MA & REC FEDERAL 2010 & $\begin{array}{l}\text { PROPRIEDADE RURAL NO POV CENTRO DOS } \\
\text { MACACOS EM }\end{array}$ & $\mathrm{NI}$ & NĀO INFORMOU (NI) \\
\hline $\mathrm{x}$ & $x$ & MA & PEDRO FERNANDES RIBEIRO & PFLMA & REC FEDERAL 2010 & $\begin{array}{l}\text { PROPRIEDADE RURAL NO POV CENTRO } \\
\text { VELHO EM SÄO } \\
\end{array}$ & $\mathrm{NI}$ & NÃO INFORMOU (NI) \\
\hline $\mathrm{x}$ & $x$ & MA & PEDRO FERNANDES RIBEIRO & PFL MA & REC FEDERAL 2010 & $\begin{array}{l}\text { PROPRIEDADE RURAL NO POV CENTRO } \\
\text { CABÔCLO NO } \\
\end{array}$ & $\mathrm{NI}$ & NÃO INFORMOU (NI) \\
\hline$x$ & $\mathrm{x}$ & MA & JosÉ RIBAMAR COSTA ALVES & PSB MA & REC FEDERAL 2002 & Terra nua área Fazenda Maranhão & $2.999,72$ & GRANDE PROPRIEDADE \\
\hline 310160 & ALFENAS & MG & $\begin{array}{l}\text { GERALDO THADEU PEREIRA } \\
\text { DOS SANTOS }\end{array}$ & PPS MG & REC FEDERAL 2010 & $\begin{array}{l}\text { Uma Chacara De № } 26 \text { Escritura 11406, Valor } \\
\text { Gastos Em Reforma No Ano De 2009 R\$ } \\
\text { 25.800,00 }\end{array}$ & $\mathrm{NI}$ & NÄO INFORMOU (NI) \\
\hline 310290 & ANTONIO CARLOS & MG & HERCULANO ANGHINETTI & PMDBMG & RECCFEDERAL 2010 & Faz. Paraiso 2 & NI & NAO INFORMOU (NI) \\
\hline 310290 & ANTOONIO CARLOS & MG & $\begin{array}{l}\text { JOSE BONIFACIO TAMM DE } \\
\text { ANDRADA }\end{array}$ & PSDBMG & REC FEDERAL 2010 & $\begin{array}{l}\text { CASA NA LOC DE BATALHA PERTENCE A FAZ } \\
\text { BELEM }\end{array}$ & NI & NAAO INFORMOU (NI) \\
\hline 310290 & ANTÓNIO CARLOS & MG & $\begin{array}{l}\text { JOSE BONIFACIO TAMM DE } \\
\text { ANDRADA }\end{array}$ & PSDBMG & REC FEDERAL 2010 & $\begin{array}{l}\text { PARTE DAS TERRAS E BENFEITORIAS QUE } \\
\text { FORMAM O CONDOMINIO DA SEDE DA FAZ } \\
\text { BORDA DO CAMPO }\end{array}$ & $\mathrm{NI}$ & NĀO INFORMOU (NI) \\
\hline 310290 & ANTONIO CARLOS & MG & $\begin{array}{l}\text { JOSE BONIFACIO TAMM DE } \\
\text { ANDRADA }\end{array}$ & PSDBMG & REC FEDERAL 2010 & $\begin{array}{l}\text { 3PROPRIEDADES DE TERRAS (adquiridas De } \\
\text { Carlos Augusto Viana) }\end{array}$ & $\overline{\mathrm{NI}}$ & NAO INFORMOU (NII) \\
\hline 310290 & ANTONIO CARLOS & MG & $\begin{array}{l}\text { JOSE BONIFACIO TAMM DE } \\
\text { ANDRADA }\end{array}$ & PSDBMG & REC FEDERAL 2010 & FAZ BELEM - Doação De Henrique Diniz Andrada & $\overline{\mathrm{NI}}$ & NAO INFORMOU (NI) \\
\hline 310290 & ANTÔNIO CARLOS & MG & $\begin{array}{l}\text { JOSE BONIFACIO TAMM DE } \\
\text { ANDRADA }\end{array}$ & PSDBMG & REC FEDERAL 2010 & TERRAS NA FAZ BORDA DO CAMPO & 924,44 & GRANDE PROPRIEDADE \\
\hline 310340 & ARAÇUAI & MG & $\begin{array}{l}\text { ADEMIR CAMILO PRATES } \\
\text { RODRIGUES }\end{array}$ & PDT MG & REC FEDERAL 2010 & Fazenda Taioba Córrego Taioba & 289,77 & MÉDIA PROPRIEDADE \\
\hline 310370 & ARAPONGA & MG & DANILO DE CASTRO & PFLMG & REC FEDERAL 2002 & $\begin{array}{l}10 \% \text { DO IMÓVEL RURAL DENOMINADO SÁO } \\
\text { DOMINGOS E OUTROS }\end{array}$ & $\mathrm{NI}$ & NÂO INFORMOU (NI) \\
\hline 310370 & ARAPONGA & MG & RODRIGO DE CASTRO & PSDB MG & REC FEDERAL 2010 & \begin{tabular}{|l}
$5 \%$ Da Compra Terreno Rural Em Terras De \\
Cultura E Pasto Situado No Lugar Denominanado \\
Fazenda Da Conceiçăo Sitio Sagrado Coraçăão De \\
Jesus Ou Săo Domingos Zona Rural
\end{tabular} & $\mathrm{NI}$ & NÄO INFORMOU (NI) \\
\hline 310370 & ARAPONGA & MG & DANILO DE CASTRO & PFL MG & REC FEDERAL 2002 & $\begin{array}{l}\text { PROPRIEDADE RURAL DENOMINADA SÄO } \\
\text { DOMINGOS }\end{array}$ & 184,14 & MÉDIA PROPRIEDADE \\
\hline 310370 & ARAPONGA & MG & RODRIGO DE CASTRO & PSDB MG & REC FEDERAL 2010 & $\begin{array}{l}\text { Aquisição De Um Terreno Situado Na Serra Da } \\
\text { Grama }\end{array}$ & 33,00 & PEQUENA PROPRIEDADE \\
\hline 310560 & BARBACENA & MG & $\begin{array}{l}\text { JOSE BONIFACIO TAMM DE } \\
\text { ANDRADA }\end{array}$ & PSDBMG & REC FEDERAL 2010 & $\begin{array}{l}\text { Siftio Estiva Mäe Maria Distrito De Padre Brito } \\
\text { (Adquirido De Helenice Maria Laguardia De } \\
\text { Oliveira) }\end{array}$ & $\mathrm{NI}$ & NAO INFORMOU (NI) \\
\hline 310560 & BARBACENA & MG & $\begin{array}{l}\text { JOSE BONIFACIO TAMM DE } \\
\text { ANDRADA }\end{array}$ & PSDBMG & REC FEDERAL 2010 & SITIO NA COLONIA RODRIGO SILVA & $\mathrm{NI}$ & NÂO INFORMOU (NI) \\
\hline 310665 & BERIZAL & MG & NEWTONCARDOSO & PMDBMG & RECFEDERAL 2010 & Fazenda Muribeca & $\mathrm{NIT}$ & |NAOINFORMOU(NI) \\
\hline 310670 & BETIM & MG & CARLAILE JESUS PEDROSA & PSDBMG & REC FEDERAL 2010 & Fazenda Saraiva E Betim & $\mathrm{NI}$ & NAO INFORMOU (NI) \\
\hline 310670 & BETIM & 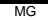 & CARLAILE JESUS PEDROSA & PSDBMG & REC FEDERAL 2010 & Sitio Na Fazenda Saraiva Em Betim & NI & NAO INFORMOU (NI) \\
\hline 310670 & BETIM & MG & JOSE SARAIVA FELIPE & PMDB MG & REC FEDERAL 2010 & Lotes Na litha Arquipelogo Verde ( $(\mathrm{R} \$ 367.000,00$ ) & $\mathrm{NI}$ & NAO INFORMOU (NI) \\
\hline 310720 & BOCAINA DE MINAS & MG & $\begin{array}{l}\text { DIMAS FABIANO TOLEDO } \\
\text { JÚNIOR }\end{array}$ & PPMG & REC FEDERAL 2010 & $\begin{array}{l}\text { Um Terço Da Fraçăo Ideal De Alguns Hectares De } \\
\text { Terra Em Pastos Carrascais Cerrados E Pedreiras } \\
\text { Situado No Imovel Grao Mongol De Baixo Em } \\
\text { Bocaina De Minas Mg }\end{array}$ & $\overline{\mathrm{NI}}$ & NAO INFORMOU (NI) \\
\hline
\end{tabular}




\begin{tabular}{|c|c|c|c|c|c|c|c|c|}
\hline GEOCODIGO & MUNICIPIO & UF & PARLAMENTAR & PARTIDO & FONTE & JIMOVEL & AREA & CATEGORIA FUNDIÁRIA \\
\hline 310720 & BOCAINA DE MINAS & MG & $\begin{array}{l}\text { DIMAS FABIANO TOLEDO } \\
\text { JÚNIOR }\end{array}$ & PPMG & REC FEDERAL 2010 & $\begin{array}{l}\text { Um Terço De Sessenta Hectares De Terras Na } \\
\text { Regiäo Sertão Bocaina De Minas Doada Pelo } \\
\text { Genitor }\end{array}$ & 20,00 & MINIFUNDIO \\
\hline 310770 & BOM JESUS DO AMPARO & MG & $\begin{array}{l}\text { JOSE SANTANA DE } \\
\text { VASCONCELLOS MOREIRA }\end{array}$ & PRMG & REC FEDERAL 2010 & $\begin{array}{l}\text { Imovel Rural - 114ha - 6km Do Munic. De Bom } \\
\text { Jesus Do Amparo/Mg }\end{array}$ & 114,00 & MÉDIA PROPRIEDADE \\
\hline 311110 & CAMPINA VERDE & MG & $\begin{array}{l}\text { JOSÉ THOMAZ DA SILVA NONÔ } \\
\text { NETTO }\end{array}$ & \begin{tabular}{|l|l} 
DEM AL \\
\end{tabular} & REC FEDERAL 2010 & Faz Brejao - (Reserva Ambiental) & $\mathrm{NI}$ & NÄO INFORMOU (NI) \\
\hline 3111200 & CAMPO BELO & MG & MAURO RIBEIROLOPES & DEMMG & $\begin{array}{l}\text { REC FEDERAL } 2010 \\
\end{array}$ & FAZ TECELAO & 204,14 & MÉDIA PROPRIEDADE \\
\hline 311180 & CANAPÓLIS & MG & JOÃO JOSÉ PEREIRA DE LYRA & PTB AL & REC FEDERAL 2010 & $\begin{array}{l}\text { Propriedade Rural, Fazenda Trialcool, R\$ } \\
950.966,00\end{array}$ & $\mathrm{NI}$ & NĀO INFORMOU (NI) \\
\hline 311340 & CARATINGA & MG & ANIVALDO JUVENIL VALE & PSDB PA & REC FEDERAL 2002 & $\begin{array}{l}\text { IMÓVEL RURAL LOCALIZADO EM CÓRREGO } \\
\text { SUIÇO (NÂOO DECLAROU EM 2010) }\end{array}$ & 159,00 & MÉDIA PROPRIEDADE \\
\hline 311455 & CARNEIRINHO & MG & JOSÉ SILVA SOARES & PDT MG & REC FEDERAL 2010 & $\begin{array}{l}\text { Imóvel Rural, Com AŻrea De 48,4 Ha-Carneirinhos- } \\
\text { Mg, Conforme Registro E Matricula R1/15.239 }\end{array}$ & 48,40 & PEQUENA PROPRIEDADE \\
\hline 311930 & COROMANDEL & MG & SILAS BRASILEIRO & PMDBMG & RECFEDERAL 2010 & Fazenda Chapada Dos Borges & $\mathrm{NI}$ & NAO INFORMOU (NI) \\
\hline 311930 & COROMANDEL & MG & $\begin{array}{l}\text { ROMEU FERREIRA DE } \\
\text { QUEIROZ }\end{array}$ & PTBMG & REC FEDERAL 2006 & FAZ SERRA VERDE & 903,70 & GRANDE PROPRIEDADE \\
\hline 311930 & COROMANDEL & MG & $\begin{array}{l}\text { ROMEU FERREIRA DE } \\
\text { QUEIROZ }\end{array}$ & PTBMG & REC FEDERAL 2006 & FAZ SUCURI E ARAUJOS & 440,00 & MÉDIA PROPRIEDADE \\
\hline 311930 & COROMANDEL & MG & $\begin{array}{l}\text { ROMEU FERREIRA DE } \\
\text { QUEIROZ }\end{array}$ & PTBMG & REC FEDERAL 2006 & FAZ COROMANDEL & 233,40 & MÉDIA PROPRIEDADE \\
\hline 311930 & COROMANDEL & $\overline{M G}$ & $\begin{array}{l}\text { ROMEU FERREIRA DE } \\
\text { QUEIROZ }\end{array}$ & $\begin{array}{l}\text { PTB MG } \\
\end{array}$ & REC FEDERAL 2006 & FAZ SUCURI E ARAUJOS & 100,00 & PEQUENA PROPRIEDADE \\
\hline 312230 & DIVINÓPOLIS & $\overline{M G}$ & $\begin{array}{l}\text { DOMINGOS SÁVIO CAMPOS } \\
\text { RESENDE }\end{array}$ & PSDB MG & REC FEDERAL 2010 & Fazenda Em Divinópolis & $\mathrm{NI}$ & NĀO INFORMOU (NI) \\
\hline 312230 & DIVINOPOLIS & MG & $\begin{array}{l}\text { DOMINGOS SAVIO CAMPOS } \\
\text { RESENDE }\end{array}$ & PSDBMG & REC FEDERAL 2010 & Benfeitorias Na Fazenda Em Divinóóolis & $\mathrm{NI}$ & NAAO INFORMOU (NI) \\
\hline 312400 & ERVALIA & MG & DANILO DE CASTRO & $\begin{array}{l}\text { PFLMG } \\
\end{array}$ & REC FEDERAL 2002 & $\begin{array}{l}\text { PROPRIEDADE RURAL DENOMINADA } \\
\text { TURVĀO }\end{array}$ & 30,05 & PEQUENA PROPRIEDADE \\
\hline 312400 & ERVÁLIA & MG & DANILO DE CASTRO & PFLMG & REC FEDERAL 2002 & ÁREA & 10,48 & MINIFUNDIO \\
\hline 312400 & ERVÁLIA & MG & DANILO DE CASTRO & PFLMG & REC FEDERAL 2002 & $\begin{array}{l}\text { PROPRIEDADE RURAL DENOMINADA } \\
\text { TURVÄO - ANEXO }\end{array}$ & 7,54 & PEQUENA PROPRIEDADE \\
\hline 312400 & ERVÁLIA & MG & DANILO DE CASTRO & PFLMG & $\begin{array}{l}\text { REC FEDERAL } 2002 \\
\end{array}$ & TERRA NO SITIO CAIXA DÁGUA & 3,00 & MINIFUNDIO \\
\hline 312680 & FREI GASPAR & MG & $\begin{array}{l}\text { ADEMIR CAMILO PRATES } \\
\text { REDRIGUES }\end{array}$ & PDT MG & REC FEDERAL 2010 & $\begin{array}{l}\text { Fazenda Calíórnia, 223,9Ha-Córrego Conceiçăo- } \\
\text { Frei Gaspar-Mg }\end{array}$ & 223,90 & MÉDIA PROPRIEDADE \\
\hline 312710 & FRUTAL & $\overline{M G}$ & $\begin{array}{l}\text { NARCIO RODRIGUES DA } \\
\text { SILVEIRA }\end{array}$ & PSDBMG & REC FEDERAL 2010 & Uma Chacara Denominada Manga Sul & $\mathrm{NI}$ & NAO INFORMOU (NI) \\
\hline 312780 & GRÃO MOGOL & MG & $\begin{array}{l}\text { ROMEU FERREIRA DE } \\
\text { QUEIROZ }\end{array}$ & PTBMG & REC FEDERAL 2006 & 1/2 FAZ TAMANDUÁ & $1.682,57$ & GRANDE PROPRIEDADE \\
\hline 312940 & BEERTIOGA & MG & HERCULANO ANGHINETTI & PMDBMG & REC FEDERAL 2010 & Faz. Paraiso & $\mathrm{NI}$ & NAO INFORMOU (NI) \\
\hline 312940 & IBERTIOGA & $\overline{M G}$ & HERCULANO ANGHINETTI & PMDBMG & REC FEDERAL 2010 & Faz. Estrela Da Manhã & NI & NAO INFORMOU (NI) \\
\hline 312950 & BBA & MG & ARACELY DE PAULA & PRMG & REC FEDERAL 2010 & $\begin{array}{l}\text { AQUIS DE GLEBA DE TERRAS DENOMINADA } \\
\text { CHACOEIRA GRANDE }\end{array}$ & $\mathrm{NI}$ & NAO INFORMOU (NI) \\
\hline 312950 & |BIA & $\overline{M G}$ & ARACELY DE PAULA & PRMG & REC FEDERAL 2010 & $\begin{array}{l}2 \text { GLEBAS DE TERRA NA FAZ CHAPADA } \\
\text { (Direito Hereditario Deixado Por Maria Jacinra } \\
\text { Borges) }\end{array}$ & $\overline{\mathrm{NI}}$ & NAO INFORMOU (NII) \\
\hline 312950 & |BIIÁ & MG & ARACELY DE PAULA & PRMG & REC FEDERAL 2010 & $\begin{array}{l}\text { FAZ CACHOEIRA GRANDE Diretio Hereditario } \\
\text { Adquirido De Rodrigues Charles }\end{array}$ & 242,00 & MÉDIA PROPRIEDADE \\
\hline 312950 & |BIAA & MG & ARACELY DE PAULA & PRMG & REC FEDERAL 2010 & $\begin{array}{l}\text { GLEBA DE TERRAS FAZ SAO BENTO LOC } \\
\text { DISTRITO DE ARGENITA }\end{array}$ & 119,00 & PEQUENA PROPRIEDADE \\
\hline 312950 & $|B| A \mid A$ & MG & ARACELY DE PAULA & PRMG & REC FEDERAL 2010 & $\begin{array}{l}\text { FAZ SOBRADO LOC DISTRITO DE } \\
\text { ARGENTIADQ POR Direito Hereditário POR } \\
\text { Ananias Candido Borges }\end{array}$ & 7,26 & PEQUENA PROPRIEDADE \\
\hline 313170 & ITABIRA & MG & $\begin{array}{l}\text { JOSE SANTANA DE } \\
\text { VASCONCELLOS MOREIRA }\end{array}$ & PRMG & REC FEDERAL 2010 & $\begin{array}{l}\text { Propriedade Rural - Fazenda Da Fortuna/ Dist. De } \\
\text { lpoema }\end{array}$ & 958,67 & GRANDE PROPRIEDADE \\
\hline 313210 & |TACARAMBI & $\overline{M G}$ & $\begin{array}{l}\text { CLEUBER BRANDAO } \\
\text { CARNEIRO }\end{array}$ & PTBMG & REC FEDERAL 2006 & $\begin{array}{l}\text { Uma Parte De Terras Na Faz. Junco Em Pastada E } \\
\text { Cercada }\end{array}$ & $\mathrm{NI}$ & NAO INFORMOU (NI) \\
\hline 313210 & |TACARAMBI & MG & $\begin{array}{l}\text { CLEUBER BRANDĀO } \\
\text { CARNEIRO }\end{array}$ & PTBMG & REC FEDERAL 2006 & $\begin{array}{l}\text { Uma Parte De Terras Na Faz. Geral Junco Denom. } \\
\text { Faz. Loreninha }\end{array}$ & $\overline{\mathrm{NI}}$ & NĀO INFORMOU (NI) \\
\hline 313210 & |TACARAMBI & MG & $\begin{array}{l}\text { CLEUBER BRANDAO } \\
\text { CARNEIRO }\end{array}$ & PTBMG & REC FEDERAL 2006 & Uma Area De Terras Na Faz. Juco & 117,78 & PEQUENA PROPRIEDADE \\
\hline 313370 & ITATIAIUÇU & MG & WALTER DA ROCHA TOSTA & PMN MG & REC FEDERAL 2010 & $\begin{array}{l}\text { Um Tererno Na Chapada No Municíio De } \\
\text { Itatiaiuçu }\end{array}$ & $\overline{\mathrm{NI}}$ & $\overline{N A \bar{O} \text { INFORMOU (NI) }}$ \\
\hline 313370 & ITATIAIUÇU & MG & WALTER DA ROCHA TOSTA & PMNMG & REC FEDERAL 2010 & $\begin{array}{l}\text { Um Terreno Em Local Denominado Araujos No } \\
\text { Municipio De ltatiaiuçu }\end{array}$ & NI & NAO INFORMOU (NI) \\
\hline 313370 & ITATIAIUÇU & $\overline{M G}$ & WALTER DA ROCHA TOSTA & PMNMG & REC FEDERAL 2010 & Um Imovel Rural Em Itatiaiuçu & $\overline{\mathrm{NI}}$ & NAO INFORMOU (NI) \\
\hline 313440 & ITURAMA & $\overline{M G}$ & AELTON JOSE DE FREITAS & PPBMG & REC FEDERAL 2010 & $\begin{array}{l}\text { Imovel Rural FAZ Monte Alto Adquirida De Neldon } \\
\text { Berbamini }\end{array}$ & $\overline{\mathrm{NI}}$ & NAO INFORMOU (NI) \\
\hline 313440 & ITURAMA & MG & AELTON JOSE DE FREITAS & PPBMG & REC FEDERAL 2010 & $\begin{array}{l}\text { Imovel Rural FAZ Monte Alto Adquirida De Oscar } \\
\text { Sebastiäo De Oliveira }\end{array}$ & $\mathrm{NI}$ & NÄO INFORMOU (NI) \\
\hline 313440 & TITURAMA & MG & AELTON JOSE DE FREITAS & PPBMG & REC FEDERAL 2010 & Propriedade RuRAL FAZ Santa Rosa & NI & NAO INFORMOU (NI) \\
\hline 313440 & |TURAMA & MG & AELTON JOSE DE FREITAS & PPBMG & REC FEDERAL 2010 & $\begin{array}{l}\text { Imovel Rural FAZ Monte Alto Adquirda De José } \\
\text { Gonçalves }\end{array}$ & $\overline{N I}$ & NAO INFORMOU (NI) \\
\hline 313440 & ITURAMA & MG & $\begin{array}{l}\text { ANTONIO DUARTE NOGUEIRA } \\
\text { JUNIOR }\end{array}$ & PSDB SP & REC FEDERAL 210 & Hectares & NI & NÃO INFORMOU (NI) \\
\hline 313440 & ITURAMa & MG & $\begin{array}{l}\text { JOSÉ THOMAZ DA SILVA NONÔ } \\
\text { NETTO }\end{array}$ & DEM AL & REC FEDERAL 2010 & Faz. Monte Alto & $\mathrm{NI}$ & NÃO INFORMOU (NI) \\
\hline 313440 & ITURAMA & MG & $\begin{array}{l}\text { JOSÉ THOMAZ DA SILVA NONÔ } \\
\text { NETTO }\end{array}$ & DEM AL & REC FEDERAL 2010 & Faz. Barreiro & NI & NÃO INFORMOU (NI) \\
\hline 313490 & JACUTINGA & MG & JOSE CARLOS ELIAS & PTBES & REC FEDERAL 2010 & TERRA NUA & 28,36 & PEQUENA PROPRIEDADE \\
\hline 313630 & JOÄO PINHEIRO & MG & $\begin{array}{l}\text { ARNALDO CALIL PEREIRA } \\
\text { JARDIM }\end{array}$ & PP SP & REC FEDERAL 210 & Um Imovél Rural, Situado No Distrito De Veredas & $\mathrm{NI}$ & NÃO INFORMOU (NI) \\
\hline 313730 & LAGOA DOS PATOS & MG & $\begin{array}{l}\text { ODELMO LEÄO CARNEIRO } \\
\text { SOBRINHO }\end{array}$ & PP MG & REC FEDERAL 2008 & $\begin{array}{l}\text { Uma Gleba De Terras Const. Pelo Lote Ne } 09 \text { Do } \\
\text { Loteamento Poço Azul, Denominada Fazenda } \\
\text { Lagoa Dos Patos - Area De 205,00,82 Ha }\end{array}$ & 205,00 & MÉDIA PROPRIEDADE \\
\hline 313850 & LIBERDADE & MG & $\begin{array}{l}\text { DIMAS FABIANO TOLEDO } \\
\text { JÚNIOR }\end{array}$ & PPMG & REC FEDERAL 2010 & $\begin{array}{l}\text { Noventa Por Cento De Um Imovel Rural No } \\
\text { Municipio De Liberdade Mg Adquirido De Paulo } \\
\text { Cesar Andrade Jr }\end{array}$ & $\mathrm{NI}$ & NAOO INFORMOU (NI) \\
\hline 313850 & LIBERDADE & MG & JJOSE MILLTAOCOSTA & PTBMG & REC FEDERAL 2006 & \begin{tabular}{|l} 
Area De Terra \\
\end{tabular} & $\overline{386,00}$ & MEDIA PROPRIEDADE \\
\hline 313850 & LIBERDADE & MG & JOSE MILITAO COSTA & PTBMG & REC FEDERAL 2006 & Area De Terra & 62,75 & PEQUENA PROPRIEDADE \\
\hline 313850 & LIBERDADE & MG & JOSE MILITAO COSTA & PTBMG & REC FEDERAL 2006 & Area De Terra & 42,60 & PEQUENA PROPRIEDADE \\
\hline 313850 & LIBERDADE & MG & JOSE MILITAO COSTA & PTBMG & REC FEDERAL 2006 & Area De Terra & 21.56 & PEQUENA PROPRIEDADE \\
\hline 314090 & MATIPO & MG & JOAO MAGALHAES BIFANO & PMDBMG & REC FEDERAL 2010 & Terreno em Matipó (rural ou urbano?) & $\mathrm{NI}$ & NAO INFORMOU (NII) \\
\hline 314390 & MURIAE & MG & RENZO DO AMARAL BRAZ & PPMG & REC FEDERAL 2010 & $\begin{array}{l}\text { 33,33\% DE } 2 \text { HA Setenta E Oito Ares E Cinquenta } \\
\text { E Oito Centiares E Fraçāo De Terras Com As } \\
\text { Benfeitorias Existentes Situadas Na Faz Santa } \\
\text { Laura }\end{array}$ & NI & NAO INFORMOU (NI) \\
\hline 313660 & NOVA UNIÃ̃o & MG & $\begin{array}{l}\text { JOSE SANTANA DE } \\
\text { VASCONCELLOS MOREIRA }\end{array}$ & PRMG & REC FEDERAL 2010 & $\begin{array}{l}\text { Imovel Rural - Fazenda Vale Verde - 170.15.62ha- } \\
\text { Nova Uniao E Bom Jesus Do }\end{array}$ & 170,15 & MÉDIA PROPRIEDADE \\
\hline 314530 & NOVO CRUZEIRO & MG & $\begin{array}{l}\text { ADEMIR CAMILO PRATES } \\
\text { REDRIGUES }\end{array}$ & PDT MG & REC FEDERAL 2010 & $\begin{array}{l}\text { Gleba De Terras,Denominada Fazenda Piauí- } \\
\text { Cidade De Carai-Mg, Adquirida Em 31/10/2007, } \\
\text { Registrado No Livro 2Ab, Fls. } 117\end{array}$ & 766,00 & GRANDE PROPRIEDADE \\
\hline 314530 & NOVO CRUZEIRO & MG & $\begin{array}{l}\text { ADEMIR CAMILO PRATES } \\
\text { REDRIGUES }\end{array}$ & PDT MG & REC FEDERAL 2010 & $\begin{array}{l}\text { Denominada Fazenda Santana-Cidade De Caraí- } \\
\text { Mg, Adquirida Em 31/02/2007-Registrada No Livro } \\
\text { 2Ab, Fls.181 }\end{array}$ & 574,00 & GRANDE PROPRIEDADE \\
\hline
\end{tabular}




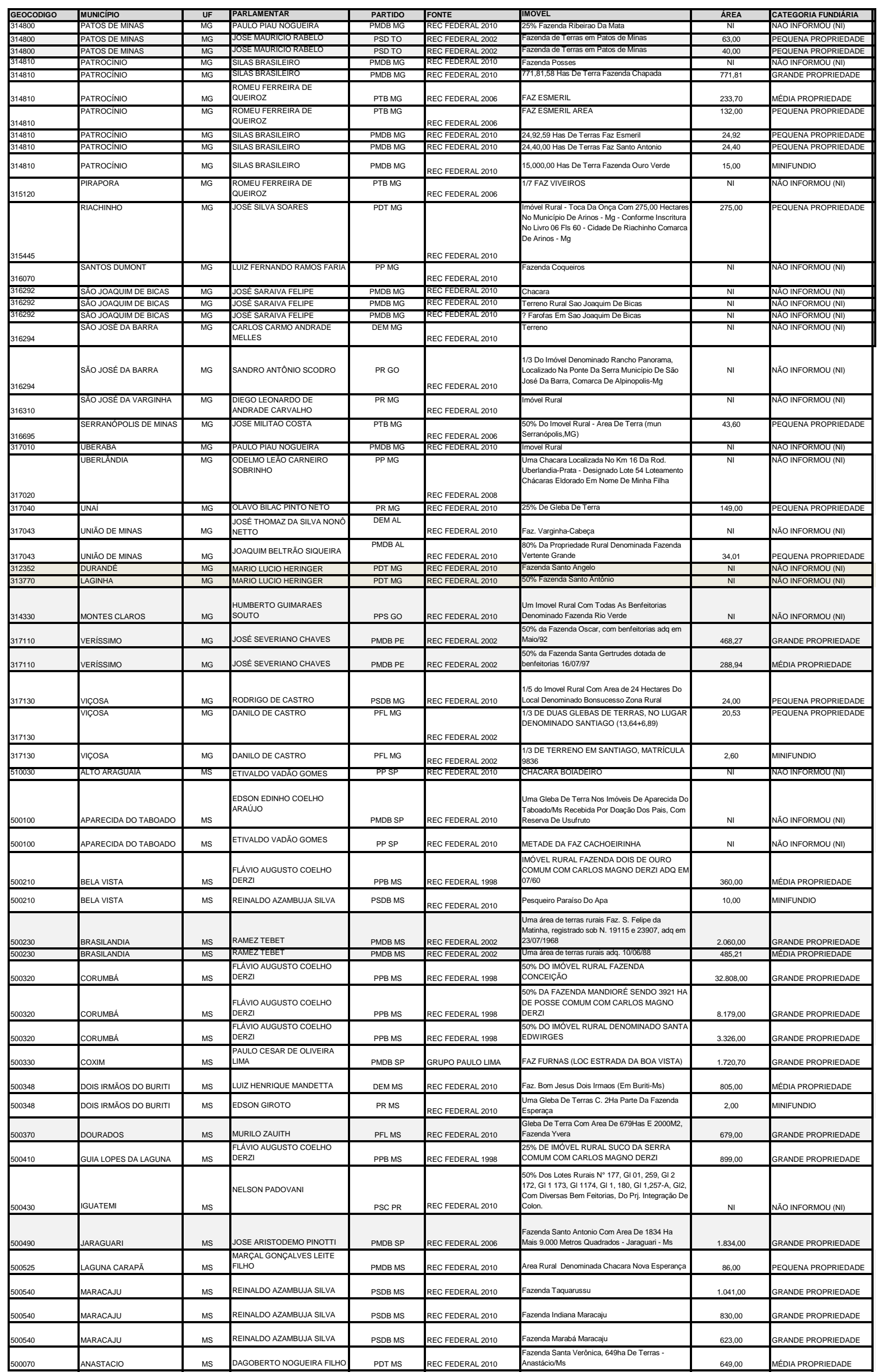




\begin{tabular}{|c|c|c|c|c|c|c|c|c|}
\hline 500110 & AQUIDAUANA & Ms & DAGOBERTO NOGUEIRA FILHO & PDT MS & REC FEDERAL 2010 & Fazenda Mariana, $1066 \mathrm{ha}$ - Aquidauana/Ms & $1.066,00$ & MÉDIA PROPRIEDADE \\
\hline 5006 & NOVA ALVORADA DO SUL & Ms & RAMEZ TEBET & РМDB MS & REC FEDERAL 2002 & $\begin{array}{l}\text { Uma área de terras Fazenda Angelina 12/09/97 } \\
\text { adq. De Agropastoril Laucidio Coelho S/C LTDA, }\end{array}$ & $1.394,00$ & GaAnd \\
\hline
\end{tabular}




\begin{tabular}{|c|c|c|c|c|c|c|c|c|}
\hline GEOCODIGO & MUUNICIPIO & UF & PARLAMENTAR & PARTIDO & FONTE & JIMOVEL & AREA & |CATEGORIA FUNDIÁRIA \\
\hline 500600 & NOVA ALVORADA DO SUL & MS & RAMEZ TEBET & PMDB MS & REC FEDERAL 2002 & $\begin{array}{l}\text { Uma área de terras rurais adq. 15/12/2000 } \\
\text { denominada Fazenda Vo Tuffi no RGI sob N. } 9470 \\
\text { com pagamento parcelado }\end{array}$ & $1.273,57$ & GRANDE PROPRIEDADE \\
\hline 500600 & NOVA ALVORADA DO SUL & MS & RAMEZ TEBET & PMDB MS & REC FEDERAL 2002 & $\begin{array}{l}\text { Uma área de Terras rurais Fazenda Angelina, adq. } \\
\text { De Agropastoril Laucidio Coelho S/C LTDA, em } \\
\text { 08/10/1989 }\end{array}$ & 700,00 & GRANDE PROPRIEDADE \\
\hline 500600 & NOVA ALVORADA DO SUL & MS & RAMEZ TEBET & PMDB MS & REC FEDERAL 2002 & $\begin{array}{l}\text { Uma área de terras que passa a integrar a Fazenda } \\
\text { vo Tuffi adq. } 26 / 1 / 1 / 2001\end{array}$ & 227,00 & GRANDE PROPRIEDADE \\
\hline 510626 & NOVO MUNDO & MS & NELSON PADOVANI & PSC PR & REC FEDERAL 2010 & $\begin{array}{l}\text { Um Imovel Rural Constituidos Pelos Lotes 170, } 179 \\
179 \mathrm{~A} \text { E } 178\end{array}$ & $\mathrm{NI}$ & NÃO INFORMOU (NI) \\
\hline 500640 & PEDRO GOMES & MS & $\begin{array}{l}\text { PAULO CESAR DE OLIVEIRA } \\
\text { LIMA }\end{array}$ & PMDB SP & REC FEDERAL 2010 & $50 \%$ FAZ SANTA ROSA & $\mathrm{NI}$ & NÃO INFORMOU (NI) \\
\hline 500640 & PEDRO GOMES & MS & $\begin{array}{l}\text { PAULO CESAR DE OLIVEIRA } \\
\text { LIMA }\end{array}$ & PMDB SP & REC FEDERAL 2010 & 50\% FAZ SANTA REGINA & $\mathrm{NI}$ & NÃO INFORMOU (NI) \\
\hline 500640 & PEDRO GOMES & MS & $\begin{array}{l}\text { PAULO CESAR DE OLIVEIRA } \\
\text { LIMA }\end{array}$ & PMDB SP & REC FEDERAL 2010 & $50 \%$ FAZ SÃO PEDRO & $\mathrm{NI}$ & NÃ̃ INFORMOU (NI) \\
\hline 500755 & SANTA RITA DO PARDO & MS & RAMEZ TEBET & PMDB MS & REC FEDERAL 2002 & $\begin{array}{l}\text { Uma área de terras rurais adq de Antonio Lopes } \\
\text { Viana em 04/08/98 }\end{array}$ & 470,00 & MÉDIA PROPRIEDADE \\
\hline 500755 & SANTA RITA DO PARDO & Ms & RAMEZ TEBET & PMDB MS & REC FEDERAL 2002 & \begin{tabular}{|l} 
Uma área de terras rurais adq em 22/03/89, \\
registrado FLS. 39/40, Livro 25
\end{tabular} & 279,55 & MÉDIA PROPRIEDADE \\
\hline 500830 & TRES LAGOAS & MS & RAMEZ TEBET & PMDB MS & REC FEDERAL 2002 & $\begin{array}{l}\text { Uma área de terras rurais Rancho Marrecas } \\
\text { registrado sob N. 208622, Livro 3-AQ }\end{array}$ & 56,31 & PEQUENA PROPRIEDADE \\
\hline$x$ & $\mathrm{x}$ & MS & RAMEZ TEBET & PMDB MS & REC FEDERAL 2002 & $\begin{array}{l}\text { Uma área de terras rurais adq. 29/1/2/92 do formal } \\
\text { de partilha de Farid Nassar }\end{array}$ & $1.124,00$ & GRANDE PROPRIEDADE \\
\hline 510025 & ALTA FLORESTA & MT & $\begin{array}{l}\text { WELLINGTON ANTONIO } \\
\text { FAGUNDES }\end{array}$ & PRMT & REC FEDERAL 2010 & AREA DE TERRENO (FAZ. BAHIA) & 508,80 & MÉDIA PROPRIEDADE \\
\hline 510160 & BARÃO DE MELGAÇO & MT & JAYME VERISSIMO CAMPOS & DEM MT & REC FEDERAL 2010 & $\begin{array}{l}\text { Uma Area De Terra } 203 \text { Has Na Baia Dos Quatos } \\
\text { Barāo De Melgaço-Mt }\end{array}$ & $\mathrm{NI}$ & NÃO INFORMOU (NI) \\
\hline 510160 & BARÃO DE MELGAÇO & MT & JÚLIO JOSÉ DE CAMPOS & DEM MT & REC FEDERAL 2010 & $\begin{array}{l}\text { Fazenda Sao Jose Do Piquiri Barao De Melgaco } \\
\text { 8443Ha Com Infra Estrutura Completa }\end{array}$ & $8.443,00$ & GRANDE PROPRIEDADE \\
\hline 510170 & BARRA DOS BUGRES & MT & MARCOS MONTES CORDEIRO & DEM MG & REC FEDERAL 2010 & \begin{tabular}{|l|} 
Imovel Rural Denominado Fazenda Mineira I Barra \\
Dos Bugres
\end{tabular} & $\mathrm{NI}$ & NÃO INFORMOU (NI) \\
\hline 510250 & CACERES & MT & JAYME VERISSIMO CAMPOS & DEM MT & REC FEDERAL 2010 & Fazenda Boa Esperança Municipio De Cáceres & $\mathrm{NI}$ & NÃO INFORMOU (NI) \\
\hline 510250 & CACERES & MT & JAYME VERÍSSIMO CAMPOS & DEM MT & REC FEDERAL 2010 & $\begin{array}{l}\text { Lote De Terra Municipio De Caceres-Mt Parte Ideal } \\
\text { Da Fazenda Boa Esperançaa }\end{array}$ & $\mathrm{NI}$ & NÄO INFORMOU (NI) \\
\hline 510250 & CACERES & MT & JAYME VERISSIMO CAMPOS & DEM MT & REC FEDERAL 2010 & $\begin{array}{l}\text { Uma Area De Terra Com } 537,50 \text { Has No Lugar } \\
\text { Denominado Sesmaria Palmares De Cidade }\end{array}$ & 537,50 & MÉDIA PROPRIEDADE \\
\hline 510263 & CAMPO NOVO DO PARECIS & MT & BLAIRO BORGES MAGGI & PRMT & REC FEDERAL 2010 & $\begin{array}{l}\text { Area Faz Santo Anonio Na Gleba Juruena li } \\
\text { Matrícula N. 11.434, Foi Averbada No Cri De } \\
\text { Sapezal Sob O N. } 510\end{array}$ & 468,20 & MÉDIA PROPRIEDADE \\
\hline 510268 & CAMPOS DE JULHO & MT & NELSON PADOVANI & PSC PR & REC FEDERAL 2010 & $\begin{array}{l}\text { 1/3 De Uma Area Rural C 32,888, 1890Ha, Denom } \\
\text { Faz. Carolina E Tiaraju, }\end{array}$ & 32,88 & PEQUENA PROPRIEDADE \\
\hline 510270 & CANARAMA & MT & $\begin{array}{l}\text { IRIS REZENDE E DONA IRIS } \\
\text { REZENDE }\end{array}$ & PMDB GO & REC FEDERAL 2010 & FAZ VALE DO XINGU & $1.394,00$ & GRANDE PROPRIEDADE \\
\hline 510270 & CANARAMA & MT & $\begin{array}{l}\text { IRIS REZENDE E DONA IRIS } \\
\text { REZENDE }\end{array}$ & PMDB GO & REC FEDERAL 2002 & GLEBA DE TERRAS & 386,00 & MÉDIA PROPRIEDADE \\
\hline 510270 & CANARANA & MT & \begin{tabular}{|l} 
IRIS REZENDE E DONA IRIS \\
REZENDE
\end{tabular} & PMDB GO & REC FEDERAL 2010 & FAZ ESTRELA & $9.558,90$ & GRANDE PROPRIEDADE \\
\hline 510270 & CANARANA & MT & $\begin{array}{l}\text { IRIS REZENDE E DONA IRIS } \\
\text { REZENDE }\end{array}$ & PMDB GO & REC FEDERAL 2010 & FAZ N SENHORA APARECIDA & $5.284,00$ & GRANDE PROPRIEDADE \\
\hline 510270 & CANARANA & MT & $\begin{array}{l}\text { IRIS REZENDE E DONA IRIS } \\
\text { REZENDE }\end{array}$ & PMDB GO & REC FEDERAL 2010 & FAZ ALVORADA & $1.394,00$ & GRANDE PROPRIEDADE \\
\hline 510270 & CANARANa & MT & $\begin{array}{l}\text { IRIS REZENDE E DONA IRIS } \\
\text { REZENDE }\end{array}$ & PMDB GO & REC FEDERAL 2002 & GLEBA DE TERRAS & 866,00 & MÉDIA PROPRIEDADE \\
\hline 510270 & CANARANA & MT & $\begin{array}{l}\text { IRIS REZENDE E DONA IRIS } \\
\text { REZENDE }\end{array}$ & PMDB GO & REC FEDERAL 2010 & UMA GLEBA DE TERRAS & 386,80 & MÉDIA PROPRIEDADE \\
\hline 510285 & CASTANHEIRA & MT & JAYME VERÍSSIMO CAMPOS & DEM MT & REC FEDERAL 2010 & $\begin{array}{l}\text { Fazenda Santa Clara No Municipio De } \\
\text { Castanheiras - Mt }\end{array}$ & $\mathrm{NI}$ & NÃO INFORMOU (NI) \\
\hline 510310 & COCALINHO & MT & SANDRO ANTÔNIO SCODRO & PR GO & REC FEDERAL 2010 & $\begin{array}{l}\text { Denominada Fazenda Santa Luiza, Município De } \\
\text { Cocalzinho/Mt }\end{array}$ & $9.000,00$ & GRANDE PROPRIEDADE \\
\hline 510310 & COCALINHO & MT & BLAIRO BORGES MAGGI & PRMT & REC FEDERAL 2010 & $\begin{array}{l}\text { Faz Fauna, Matrícula } 5518 \text { Do Cri De Agua Boa - } \\
\text { Mt }\end{array}$ & 155,00 & PEQUENA PROPRIEDADE \\
\hline 510310 & COCALINHO & MT & BLAIRO BORGES MAGGI & PRMT & REC FEDERAL 2010 & $\begin{array}{l}\text { Uma Area De Terra De 150,0 Ha, FAZ Flora } \\
\text { Matricula N. 5.517 Do Cri De Agua Boa - Mt }\end{array}$ & 150,00 & PEQUENA PROPRIEDADE \\
\hline 510340 & CUIABÁ & MT & $\begin{array}{l}\text { JOÄO AUGUSTO RIBEIRO } \\
\text { NARDES }\end{array}$ & PPB RS & REC FEDERAL 2002 & TERRAS ADQUIRIDAS EM 28/01/1982 & 220,00 & MÉDIA PROPRIEDADE \\
\hline 510340 & CUIABÁ & MT & RICARTE DE FREITAS JUNIOR & РТВ мт & REC FEDERAL 2006 & AREA DE TERRAS NA SESMARIA MUTUCA & 9,15 & MINIFUNDIO \\
\hline 510340 & CUIABÁ & MT & JONAS PINHEIRO DA SILVA & DEM MT & REC FEDERAL 2010 & UMA CHÁCARA À MARGEM DO RIO COCHOPÓ & 0,77 & MINIFUNDIO \\
\hline 510360 & DOM AQUINO & MT & CARLOS GOMES BEZERRA & PMDBMT & REC FEDERAL 2010 & FAZ SAOCARLOS & 65,00 & $\begin{array}{l}\text { PEQUENA PROPRIEDADE } \\
\end{array}$ \\
\hline 510410 & GUARANTÄO DO NORTE & MT & $\begin{array}{l}\text { ROGÉRIO LUCIO SOARES DA } \\
\text { SILVA }\end{array}$ & PMDB MT & REC FEDERAL 2008 & $\begin{array}{l}\text { Area De Terras Denominada Gleba Triângulo, Com } \\
\text { 2.371,6202ha, No Munic De Guarantä Do Norte/Mt }\end{array}$ & $2.371,62$ & GRANDE PROPRIEDADE \\
\hline 510520 & JUSCIMEIRA & MT & $\begin{array}{l}\text { WELLINGTON ANTONIO } \\
\text { FAGUNDES }\end{array}$ & PR MT & REC FEDERAL 2010 & $\begin{array}{l}\text { 50\% De Uma Area } 30 \text { Hec. De Terras Pastais E } \\
\text { Lavadias C/Balneario De Aguas Quentes E DEM } \\
\text { MTais Benfeiturias C/58.632 Has }\end{array}$ & 30,00 & MINIFUNDIO \\
\hline 510300 & $\begin{array}{l}\text { Na Rod que Liga Chapara dos } \\
\text { Guimarães a Localidade De } \\
\text { Cachoeira Rica }\end{array}$ & MT & $\begin{array}{l}\text { ELIENE JOSÉ DE LIMA (ELIENE } \\
\text { LIMA) }\end{array}$ & PP MT & REC FEDERAL 2010 & $\begin{array}{l}\text { Fazenda Santa Rita Na Rod Que Liga Chapara Dos } \\
\text { Guimarães A Localidade De Cachoeira Rica }\end{array}$ & $\mathrm{NI}$ & NÃO INFORMOU (NI) \\
\hline 510340 & Na Rodovia Emanuel Pinheiro & MT & $\begin{array}{l}\text { ELIENE JOSÉ DE LIMA (ELIENE } \\
\text { LIMA) }\end{array}$ & PP MT & REC FEDERAL 2010 & $\begin{array}{l}\text { Chacara Com } 7 \text { Has As Margens Do Rio } \\
\text { Coxipozinho Na Rodovia Emanuel Pinheiro }\end{array}$ & 7,00 & MINIFUNDIO \\
\hline 510610 & $\begin{array}{l}\text { NOSSA SENHORA DO } \\
\text { LIVRAMENTO }\end{array}$ & MT & JAYME VERISSIMO CAMPOS & DEM MT & REC FEDERAL 2010 & $\begin{array}{l}\text { Uma Area De Terra Com } 251 \text { Has E } 5451 \text { M2 } \\
\text { Taruma Em Livramento - Mt }\end{array}$ & 251,00 & PEQUENA PROPRIEDADE \\
\hline 510610 & $\begin{array}{l}\text { NOSSA SENHORA DO } \\
\text { LIVRAMENTO }\end{array}$ & мт & JAYME VERISSIMO CAMPOS & DEM MT & REC FEDERAL 2010 & $\begin{array}{l}\text { 20\% De Uma Area De Terra Com } 251 \text { Has E } 5451 \\
\text { M2 Em Taruma Livramento-Mt }\end{array}$ & 50,20 & MINIFUNDIO \\
\hline 510610 & $\begin{array}{l}\text { NOSSA SENHORA DO } \\
\text { LIVRAMENTO }\end{array}$ & MT & JAYME VERISSIMO CAMPOS & DEM MT & REC FEDERAL 2010 & 40 Has De Terra No & 40,00 & MINIFUNDIO \\
\hline 510610 & $\begin{array}{l}\text { NOSSA SENHORA SO } \\
\text { LIVRAMENTO }\end{array}$ & MT & HOMERO ALVES PEREIRA & PRMT & REC FEDERAL 2010 & $\begin{array}{l}\text { USUFRUTO DE ÁREA DE TERRAS - NOSSA } \\
\text { SENHORA DAS GRAÇAS }\end{array}$ & $6.810,25$ & GRANDE PROPRIEDADE \\
\hline 510610 & $\begin{array}{l}\text { NOSSA SENHORA SO } \\
\text { LIVRAMENTO }\end{array}$ & MT & HOMERO ALVES PEREIRA & PRMT & REC FEDERAL 2010 & FAZ PARAíso & 679,64 & MÉDIA PROPRIEDADE \\
\hline 510628 & NOVO SAO JOAQUIM & MT & HOMERO ALVES PEREIRA & PRMT & REC FEDERAL 2010 & AREADE TERRAS & 840,00 & MEDIA PROPRIEDADE \\
\hline 510630 & PARANATINGA & MT & CARLOS GOMES BEZERRA & PMDB MT & REC FEDERAL 2010 & Direfto De Posse De Uma Area De Terra & $4.600,00$ & GRANDE PROPRIEDADE \\
\hline 510718 & $\begin{array}{l}\text { RIBEIIAOADE } \\
\text { CASCALHEIRAS }\end{array}$ & MT & ROMEL ANIZIO JORGE & PPMG & REC FEDERAL 2010 & 1/3 DE UM IMOVEL RURAL & $\frac{\mathrm{NI}}{\mathrm{NI}}$ & NAO INFORMOU (NII) \\
\hline 510340 & Rodovia Cuiaba-Santo Antonio & MT & $\begin{array}{l}\text { ELIENE JOSÉ DE LIMA (ELIENE } \\
\text { LIMA) }\end{array}$ & PPMT & REC FEDERAL 2010 & Chacara Na Rodovia Cuiaba-Santo Antonio & $\mathrm{NI}$ & NÃO INFORMOU (NI) \\
\hline 510340 & Rodovia Cuiaba-Santonio & MT & $\begin{array}{l}\text { ELIENE JOSÉ DE LIMA (ELIENE } \\
\text { LIMA) }\end{array}$ & PP MT & REC FEDERAL 2010 & Chacara Rodovia Cuiaba-Santonio Com 2 Has & 2,00 & MINIFUNDIO \\
\hline 510760 & RONDONOPOLIS & MT & BLAIROBORGESMAGGI & PRMT & REC FEDERAL 2010 & Uma Area De Terra Matricula N. 45.991 & 897,10 & MEDIA PROPRIEDADE \\
\hline 510760 & RONDONÓPOLIS & MT & BLAIRO BORGES MAGGI & PRMT & REC FEDERAL 2010 & 50\% de uma Area Matricula 237 & 180,00 & PEQUENA PROPRIEDADE \\
\hline
\end{tabular}




\begin{tabular}{|c|c|c|c|c|c|c|c|c|}
\hline GEOCODIGO & MUNICIPIO & $\mathrm{UF}$ & PARLAMENTAR & PARTIDO & FONTE & TIMOVEL & ÁREA & CATEGORIA FUNDIÁRIA \\
\hline 510760 & RONDONÓPOLIS & $\overline{M T}$ & BLAIRO BORGES MAGGI & PRMT & REC FEDERAL 2010 & $\begin{array}{l}\text { 1/3 De Uma Chacara LOC Nas M,Argens Do Rio } \\
\text { Vermelho, Matricula } 28669\end{array}$ & 9,00 & MINIFUNDIO \\
\hline 510760 & RONDONÓPOLIS & MT & $\begin{array}{l}\text { WELLINGTON ANTONIO } \\
\text { FAGUNDES }\end{array}$ & PRMT & REC FEDERAL 2010 & $\begin{array}{l}\text { Uma Area De Terras P/Const. C/19.991,50 M2 } \\
\text { Parte Fazenda Santo Antônio, Localizado A Av. } \\
\text { Presidente Kennedy }\end{array}$ & 1,90 & MINIFUNDIO \\
\hline 510770 & ROSARIO OESTE & MT & JAYME VERÍSSIMO CAMPOS & DEM MT & REC FEDERAL 2010 & Fazenda Bonanza Municipio De Rosario Oeste & $\mathrm{NI}$ & NÃO INFORMOU (NI) \\
\hline 510770 & ROSARIO OESTE & MT & JAYME VERISSIMO CAMPOS & DEM MT & REC FEDERAL 2010 & $\begin{array}{l}\text { Complexa Denominada Fazenda Ines Constituida } \\
\text { Imovel Investido Cujo Complex Possui 25\% C/ } 4217 \\
\text { Has Em Rosário Oeste-MT }\end{array}$ & $1.054,00$ & MÉDIA PROPRIEDADE \\
\hline 510780 & SANTO ANTONIO DO LEVEF & MT & JONAS PINHEIRO DA SILVA & DEM MT & REC FEDERAL 2010 & LOTE DE TERRAS & 9,00 & MINIFUNDIO \\
\hline 510785 & SÃO FELIX DO ARAGUAIA & MT & JOSÉ MUCIO MONTEIRO FILHO & PFLPE & REC FEDERAL 2006 & $\begin{array}{l}\text { Tdas Oriundas De Desapropriação Da Fazenda } \\
\text { Chica Boa Em Sao Felix Do Araguaia - Em } \\
\text { Custodia Na Cef }\end{array}$ & $\mathrm{NI}$ & NÃO INFORMOU (NI) \\
\hline 510787 & SAPEZAL & MT & BLAIROBORGESMAGGI & PRMT & REC FEDERAL 2010 & Faz Maggi, Matricula 509 & 495,04 & MEDIA PROPRIEDADE \\
\hline 510790 & SINOP & MT & RICARTE DE FREITAS JUNIOR & РТВ MT & REC FEDERAL 2006 & LOTE RURAL -GLEBA CELESTE & 60,50 & MINIFUNDIO \\
\hline 510794 & TABAPORÃ & MT & $\begin{array}{l}\text { ROGÉRIO LUCIO SOARES DA } \\
\text { SILVA }\end{array}$ & PMDBMT & REC FEDERAL 2008 & $\begin{array}{l}\text { Área De Terras Dest. Do Lote Denominado } \\
\text { Cruzeiro Do Sul No Munic De Tabaporaz, Comarca } \\
\text { De Porto Dos GauchosiMt, Adq Em 30/08/1999 }\end{array}$ & $\mathrm{NI}$ & NÃ̃ INFORMOU (NI) \\
\hline 510794 & TABAPORÃ & MT & $\begin{array}{l}\text { ROGÉRIIO LUCIO SOARES DA } \\
\text { SILVA }\end{array}$ & PMDB MT & REC FEDERAL 2008 & $\begin{array}{l}\text { Área De Terras Rurais, Destac Do Lot Denominado } \\
\text { Cruzeiro, Com 2.288na No Munic De Tabaporä, } \\
\text { Comarca De Porto Dos Gauchos, Adq Em }\end{array}$ & $2.288,00$ & GRANDE PROPRIEDADE \\
\hline 510794 & TABAPORÃ & MT & $\begin{array}{l}\text { ROGÉRIO LUCIO SOARES DA } \\
\text { SILVA }\end{array}$ & PMDB MT & REC FEDERAL 2008 & $\begin{array}{l}\text { Area De Terras Dest. Do Lt Denominado Cruzeiro } \\
\text { Do Sul, Com 1.650,6ha, No Munic De Tabaporă, } \\
\text { Comarca De Porto Dos Gauchos:Mt, Adq }\end{array}$ & $1.650,60$ & GRANDE PROPRIEDADE \\
\hline 510794 & TABAPORÃ & MT & $\begin{array}{l}\text { ROGÉRIO LUCIO SOARES DA } \\
\text { SILVA }\end{array}$ & PMDB MT & REC FEDERAL 2008 & $\begin{array}{l}\text { Área De Terras Rurais, Dest. Do Lt Denomin. São } \\
\text { Gabriel, Com } 314 \text {.6ha No Munic De Tabaporãă, } \\
\text { Comarca De Porto Dos Gauchos, Mt, Adq }\end{array}$ & 314,60 & PEQUENA PROPRIEDADE \\
\hline 510794 & TABAPORÃ & MT & $\begin{array}{l}\text { ROGÉRIO LUCIO SOARES DA } \\
\text { SILVA }\end{array}$ & PMDB MT & REC FEDERAL 2008 & $\begin{array}{l}\text { Área De Terras Destac. Do Lt Prudente, Munic. De } \\
\text { Tabaporă, Comarca De Porto Dos Gauchos Mt Adq } \\
\text { Em 30/08/1999, De Silvio De Pauli }\end{array}$ & $\mathrm{NI}$ & NÃO INFORMOU (NI) \\
\hline 510794 & TABAPORÃ & MT & $\begin{array}{l}\text { ROGÉRIO LUCIO SOARES DA } \\
\text { SILVA }\end{array}$ & PMDBMT & REC FEDERAL 2008 & $\begin{array}{l}\text { Área De Terra Rurais, Dest. Do Lt. Denominado } \\
\text { Prudente Com 474ha No Munic De Tabaporă, } \\
\text { Comarca De Porto Dos Gaúchos, Adq Em 23/0 }\end{array}$ & 474,00 & MÉDIA PROPRIEDADE \\
\hline $\mathrm{x}$ & $\mathrm{x}$ & MT & $\begin{array}{l}\text { WELLINGTON ANTONIO } \\
\text { FAGUNDES }\end{array}$ & PRMT & REC FEDERAL 2010 & TERRAS PASTAIS E LAVRADIS GLEBA PAURO & 746,00 & MÉDIA PROPRIEDADE \\
\hline $\mathrm{x}$ & $\mathrm{x}$ & MT & $\begin{array}{l}\text { WELLINGTON ANTONIO } \\
\text { FAGUNDES }\end{array}$ & PRMT & REC FEDERAL 2010 & $\begin{array}{l}\text { TERRAS PASTAIS LAVRADIS LAGO AZUL NA } \\
\text { FAZ. JURIGUE }\end{array}$ & 101,00 & PEQUENA PROPRIEDADE \\
\hline$x$ & $\mathrm{NI}$ & $\mathrm{NI}$ & JAYME VERISSIMO CAMPOS & DEM MT & REC FEDERAL 2010 & 311 Has Adq Plinio Antonio Marais E Esposa & 311,00 & MÉDIA PROPRIEDADE \\
\hline$x$ & $\mathrm{NI}$ & $\mathrm{NI}$ & $\begin{array}{l}\text { JOÄO HERRMANN NETO (1946- } \\
\text { 2009) }\end{array}$ & PDT SP & REC FEDERAL 2006 & AREA & 29,52 & MINIFUNDIO \\
\hline$x$ & $x$ & $\mathrm{NI}$ & $\begin{array}{l}\text { PAULO CESAR DE OLIVEIRA } \\
\text { LIMA }\end{array}$ & PMDB SP & REC FEDERAL 2010 & IMOVEL RURAL CHACARA SÃO CRISTIVÃO & $\mathrm{NI}$ & NÃO INFORMOU (NI) \\
\hline $\bar{x}$ & $x$ & $\mathrm{NI}$ & HOMERO BARBOSATNETO & PMDB PR PR & REC FEDERAL 2006 & NAODECLAROU PROPRIEDADE RURAL- & $\mathrm{NI}$ & NĀO INFORMOU (NI) \\
\hline $\mathrm{X}$ & $x$ & $\mathrm{NI}$ & DILCEUJOAO SPERAFICO & PR PR & REC FEDERAL 2010 & Lote Rural-Fazenda Britania & $\mathrm{NI}$ & NĀO INFORMOU (NI) \\
\hline $\mathrm{x}$ & $x$ & $\mathrm{NI}$ & DILCEUJOAO SPERAFICO & PRPR & REC FEDERAL 2010 & Lotes Ruralis C 2 Armazens & $\mathrm{NI}$ & NĀO INFORMOU (NI) \\
\hline $\mathrm{x}$ & $x$ & $\mathrm{NI}$ & DILCEUJOAO SPERAFICO & PRPR & REC FEDERAL 2010 & Chacara & $\mathrm{NI}$ & NÄO INFORMOU (NI) \\
\hline $\mathrm{X}$ & $x$ & $\mathrm{NI}$ & DILCEUJOAO SPERAFICO & PRPR & REC FEDERAL 2010 & Area De Terras Rural & $\mathrm{NI}$ & NĀO INFORMOU (NI) \\
\hline $\mathrm{X}$ & $x$ & $\mathrm{NI}$ & DILCEU JOAO SPERAFICO & PRPR & REC FEDERAL 2010 & Lote Rural - Permietro Da Faz. Britania & $\mathrm{NI}$ & NĀO INFORMOU (NI) \\
\hline $\mathrm{x}$ & $x$ & $\mathrm{NI}$ & DILCEU JOÄO SPERAFICO & PRPR & REC FEDERAL 2010 & $\begin{array}{l}\text { Área De Terras Correspondente A Parte De Lote } \\
\text { (R\$ } 1.077 .292,86)\end{array}$ & $\mathrm{NI}$ & NÃO INFORMOU (NI) \\
\hline $\mathrm{x}$ & $x$ & $\mathrm{NI}$ & LUCIANO PIZZATTO & DEM PR & REC FEDERAL 2010 & ferreno Rural com Reflorestamento & $\mathrm{NI}$ & NĀO INFORMOU (NI) \\
\hline $\mathrm{x}$ & $\mathrm{x}$ & $\mathrm{NI}$ & LEOPOLDO COSTA MEYER & PSB PR & RE FEDERAL 2010 & 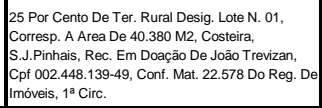 & $\mathrm{NI}$ & NÃO INFORMOU (NI) \\
\hline $\mathrm{x}$ & $\mathrm{x}$ & $\mathrm{NI}$ & $\begin{array}{l}\text { CÉSAR AUGUSTO RABELLO } \\
\text { BORGES }\end{array}$ & PFL BA & REC FEDERAL 2002 & $\begin{array}{l}\text { Condominio Fazenda Corcovado/Cj } \\
\text { Maravilha/Silencio E Condominio De Imoveis } \\
\text { Waldomiro Borges }\end{array}$ & $\mathrm{NI}$ & NÃO INFORMOU (NI) \\
\hline$x$ & $x$ & $\mathrm{NI}$ & IZALCI LUCAS FERREIRA & PRDF & REC FEDERAL 2010 & AREA/RURAL SOMA VALOR R $\$ 508.000,00$ & $\mathrm{NI}$ & NÃO INFORMOU (NI) \\
\hline$x$ & $x$ & $\mathrm{NI}$ & JAYME VERISSIMO CAMPOS & DEM MT & REC FEDERAL 2010 & $\begin{array}{l}11.060,50 \text { Has De Uma Area Denominada De } \\
\text { Fazenda Santa Amalia }\end{array}$ & $11.060,50$ & GRANDE PROPRIEDADE \\
\hline $\mathrm{x}$ & $x$ & $\mathrm{NI}$ & $\begin{array}{l}\text { SERGIO SEBASTIAO DE } \\
\text { BARROS }\end{array}$ & PMN AC & REC FEDERAL 2006 & $95 \%$ De Uma Area Rural - Fazenda Jaraguar & $10.000,00$ & GRANDE PROPRIEDADE \\
\hline$\hat{x}$ & $\frac{1}{x}$ & $\frac{\mathrm{NI}}{\mathrm{NI}}$ & TOAO HERRMANNNETO & PDT SP & REC FEDERAL 2006 & GLEBA & $3.812,30$ & GRANDE PROPRIEDADE \\
\hline $\mathrm{x}$ & $\mathrm{x}$ & $\mathrm{NI}$ & $\begin{array}{l}\text { SALOMAO AFONSO DE SOUSA } \\
\text { CRUZ }\end{array}$ & PTB RR & REC FEDERAL 2010 & FAZ AREIA BRANCA & $2.600,00$ & GRANDE PROPRIEDADE \\
\hline$x$ & $\mathrm{x}$ & $\mathrm{NI}$ & JAYME VERÍSSIMO CAMPOS & DEM MT & REC FEDERAL 2010 & Um Area De Terra Com 2.500,037 Has & $2.500,00$ & GRANDE PROPRIEDADE \\
\hline $\mathrm{x}$ & $x$ & $\mathrm{NI}$ & JAYME VERISSIMO CAMPOS & DEM MT & REC FEDERAL 2010 & Uma Area De Terra Com 2.499,9648 Has & $2.499,90$ & GRANDE PROPRIEDADE \\
\hline$x$ & $x$ & $\mathrm{NI}$ & JAYME VERISSIMO CAMPOS & DEM MT & REC FEDERAL 2010 & $\begin{array}{l}\text { 2.155,96 Has Adq. De Vital Vilela Assunçao Cri } \\
39869\end{array}$ & $2.155,96$ & GRANDE PROPRIEDADE \\
\hline$x$ & $x$ & $\mathrm{NI}$ & JAYME VERISSIMO CAMPOS & DEM MT & REC FEDERAL 2010 & $\begin{array}{l}\text { 2.112,50 Has Parte Ideal Da Fazenda Boa } \\
\text { Esperança }\end{array}$ & $2.112,50$ & GRANDE PROPRIEDADE \\
\hline $\bar{x}$ & $x$ & $\mathrm{NI}$ & JOAO HERRMANNNETO & PDT SP & REC FEDERAL 2006 & GLEBA & $1.871,43$ & GRANDE PROPRIEDADE \\
\hline$x$ & $x$ & $\mathrm{NI}$ & $\begin{array}{l}\text { JOÄO HERRMANN NETO (1946- } \\
\text { 2009) }\end{array}$ & PDT SP & REC FEDERAL 2006 & GLEBA & $1.871,43$ & GRANDE PROPRIEDADE \\
\hline $\mathrm{x}$ & $\mathrm{x}$ & $\mathrm{NI}$ & OSVALDO DE SOUZA COELHO & PFL PE & REC FEDERAL 2010 & Propriedade Fazenda Caminho Novo & $1.796,00$ & GRANDE PROPRIEDADE \\
\hline $\mathrm{x}$ & $x$ & $\mathrm{NI}$ & $\begin{array}{l}\mid \text { IRIS REZENDE E DONA IRIS } \\
\text { REZENDE }\end{array}$ & PMDB GO & REC FEDERAL 2010 & FAZ SÃO LUIZ & $1.037,40$ & GRANDE PROPRIEDADE \\
\hline $\mathrm{x}$ & $\mathrm{x}$ & $\mathrm{NI}$ & JOHNATHAN DE JESUS & PRB RR & REC FEDERAL 2010 & $\begin{array}{l}\text { Tres Imovel, Sitio Säo Francisco Medindo } \\
120 \mathrm{Ha} \text {,Capim Verde Medindo } 461,9 \mathrm{Ha} \text {, Sitio Sonho } \\
\text { Azzul Medindo 403,3 }\end{array}$ & 985,20 & MÉDIA PROPRIEDADE \\
\hline $\mathrm{x}$ & $x$ & $\mathrm{NI}$ & $\begin{array}{l}\text { ODELMO LEĀO CARNEIRO } \\
\text { SOBRINHO }\end{array}$ & PPMG & REC FEDERAL 2008 & $\begin{array}{l}\text { Uma Gleba De Terras Constituida Pelo Lote № } 10 \\
\text { Do Loteamento Poço Azul, Denominada Faz. Lagoa } \\
\text { Dos Patos }\end{array}$ & 959,90 & GRANDE PROPRIEDADE \\
\hline $\mathrm{x}$ & $x$ & $\mathrm{NI}$ & $\begin{array}{l}\text { ODELMO LEÃO CARNEIRO } \\
\text { SOBRINHO }\end{array}$ & PPMG & REC FEDERAL 2008 & $\begin{array}{l}\text { Uma Gleba De Terras Denominada Fazenda Sant } \\
\text { Ana, Constituida a Pelo Lote } 24 \text { Loteamento Poço A } \\
\text { Zul Com Area De } 824,57,36 \text { Ha }\end{array}$ & 824.57 & MÉDIA PROPRIEDADE \\
\hline $\mathrm{x}$ & $x$ & $\mathrm{NI}$ & $\begin{array}{l}\text { FRANCISCO DE ASSIS } \\
\text { MLLOMEM COELHO }\end{array}$ & PFL MA & REC FEDERAL 1998 & FAZENDA KARAIBAS & 750,00 & MÉDIA PROPRIEDADE \\
\hline$x$ & $x$ & $\mathrm{NI}$ & $\begin{array}{l}\text { WELLINGTON ANTONIO } \\
\text { FAGUNDES }\end{array}$ & PRMT & REC FEDERAL 2010 & $\begin{array}{l}\text { 50\%-De Uma Area Terras Pastais E Lavradias } \\
\text { Denom Gleba Em 14/12/85 Da Empresa } \\
\text { Colonizadora Agro-Pastoril }\end{array}$ & 746,00 & MÉDIA PROPRIEDADE \\
\hline
\end{tabular}




\begin{tabular}{|c|c|c|c|c|c|c|c|c|}
\hline GEOCODIGO & MUNICIPIO & $\mathrm{UF}$ & PARLAMENTAR & PARTIDO & FONTE & IIMOVEL & ÁREA & CATEGORIA FUNDIÁRIA \\
\hline$x$ & $\mathrm{x}$ & $\mathrm{NI}$ & $\begin{array}{l}\text { ODELMO LEÄO CARNEIRO } \\
\text { SOBRINHO }\end{array}$ & PP MG & REC FEDERAL 2008 & $\begin{array}{l}\text { 01 Imovel Rural Denominado Fazenda Gaucha lii, } \\
\text { Com Area De } 645,88,68 \text { Ha, Designado P/Gleba } \\
\text { 01-Lote } 14 \text { E Parte Do Lote } 20 \text { Do Lot }\end{array}$ & 645,88 & MÉDIA PROPRIEDADE \\
\hline $\mathrm{x}$ & $\mathrm{x}$ & $\mathrm{NI}$ & $\begin{array}{l}\text { CÉSAR AUGUSTO RABELLO } \\
\text { BORGES }\end{array}$ & PFL BA & REC FEDERAL 2002 & $\begin{array}{l}\text { Fazenda Provisao Com } 645,36 \text { Ha Incorporada Ao } \\
\text { Conj Maraviha/Siliencio; }\end{array}$ & 645,36 & MÉDIA PROPRIEDADE \\
\hline$x$ & $x$ & $\mathrm{NI}$ & $\begin{array}{l}\text { JoÄO HERRMANN NETO (1946- } \\
\text { 2009) }\end{array}$ & PDT SP & REC FEDERAL 2006 & GLEBA & 634,04 & MÉDIA PROPRIEDADE \\
\hline $\mathrm{x}$ & $\mathrm{x}$ & $\mathrm{NI}$ & JAYME VERISSIMO CAMPOS & DEM MT & REC FEDERAL 2010 & $\begin{array}{l}\text { Aquisicaão De } 100 \text { 0/0 Pqarte De Benedito S. C. } \\
\text { Braga Do Total De } 10 \text { 0/0 Sobre } 5554 \text { Has }\end{array}$ & 555,40 & MÉDIA PROPRIEDADE \\
\hline $\mathrm{x}$ & $\mathrm{x}$ & $\mathrm{NI}$ & $\begin{array}{l}\text { CLEUBER BRANDĀO } \\
\text { CARNEIRO }\end{array}$ & PTBMG & REC FEDERAL 2006 & Uma Area De Terras Faz. Junco & 346,50 & MÉDIA PROPRIEDADE \\
\hline $\mathrm{x}$ & $\mathrm{x}$ & $\mathrm{NI}$ & JAYME VERISSIMO CAMPOS & DEM MT & REC FEDERAL 2010 & $\begin{array}{l}\text { Aquisição } 100 \text { 0/0 Parte De Jorge A. P. Miranda, } \\
\text { Jose C. Costa E } 15 \text { o/o Benedito S. C. Braga Do } \\
\text { Total De } 4217 \text { Has }\end{array}$ & 323,00 & MÉDIA PROPRIEDADE \\
\hline $\mathrm{x}$ & $\mathrm{x}$ & $\mathrm{NI}$ & JAYME VERISSIMO CAMPOS & DEM MT & REC FEDERAL 2010 & $\begin{array}{l}244,81 \text { Has Adquirido De Jose Carlos Lameira } \\
\text { Otero }\end{array}$ & 244,81 & MÉDIA PROPRIEDADE \\
\hline$x$ & $x$ & $\mathrm{NI}$ & $\begin{array}{l}\text { THEMISTOCLES DE SAMPAIO } \\
\text { PEREIRA }\end{array}$ & PMDB PI & REC FEDERAL 2008 & $\begin{array}{l}\text { 01 Propriedade Denominada Curralinhos I, Registro } \\
\text { De Imóvel No Livro 02-B № } 07 \text { Fls. } 69 \text { Sob № Reg. } \\
\text { 02, Matricu }\end{array}$ & 203,28 & MÉDIA PROPRIEDADE \\
\hline $\mathrm{x}$ & $\mathrm{x}$ & $\mathrm{NI}$ & JAYME VERISSIMO CAMPOS & DEM MT & REC FEDERAL 2010 & $\begin{array}{l}166 \text { Has E } 2.086 \text { M2 Parte Ideal Fazenda Boa } \\
\text { Esperança }\end{array}$ & 166,00 & PEQUENA PROPRIEDADE \\
\hline $\mathrm{x}$ & $\mathrm{x}$ & $\mathrm{NI}$ & $\begin{array}{l}\text { THEMISTOCLES DE SAMPAIO } \\
\text { PEREIRA }\end{array}$ & PMDB PI & REC FEDERAL 2008 & $\begin{array}{l}\text { 01 Propriedade Rural Denominada Capote, em } \\
\text { 21.01.69 Registro De Imóvel Ás Fls. } 215 \text { A 217, } \\
\text { Sob N. 4.196 Lv. 03-04, Em E }\end{array}$ & 150,00 & PEQUENA PROPRIEDADE \\
\hline$x$ & $\mathrm{x}$ & $\mathrm{NI}$ & $\begin{array}{l}\text { THEMISTOCLES DE SAMPAIO } \\
\text { PEREIRA }\end{array}$ & PMDB PI & REC FEDERAL 2008 & $\begin{array}{l}01 \text { Propriedade Rural Denominada Canto Das } \\
\text { Serragens E Araçâs - Vinagreira, Registrada No } \\
\text { Livro N. 081, Fls. }\end{array}$ & 150,00 & PEQUENA PROPRIEDADE \\
\hline $\mathrm{x}$ & $x$ & $\mathrm{NI}$ & $\begin{array}{l}\text { ODELMO LEĀO CARNEIRO } \\
\text { SOBRINHO }\end{array}$ & PPMG & REC FEDERAL 2008 & $\begin{array}{l}\text { Uma Gleba De Terras Constituida Pelo Lote № } 13 \\
\text { Do Loteamento Poço Azul, Denominada Faz. Agua } \\
\text { Azul II }\end{array}$ & 149,24 & PEQUENA PROPRIEDADE \\
\hline$x$ & $\mathrm{x}$ & $\mathrm{NI}$ & $\begin{array}{l}\text { JÚLLO CESAR DE CARVALHO } \\
\text { LIMA }\end{array}$ & DEM PI & REC FEDERAL 2010 & Terra Nua & 135,50 & PEQUENA PROPRIEDADE \\
\hline$x$ & $x$ & $\mathrm{NI}$ & $\begin{array}{l}\text { ODELMO LEAO CARNEIRO } \\
\text { SOBRINHO }\end{array}$ & PPMG & REC FEDERAL 2008 & $\begin{array}{l}\text { Um Imovel Rural Denominado Fazenda Gauchal, } \\
\text { Com A Area De } 122,17,73 \text { Ha Designado P/Gleba } 04 \\
\text { Parte Do Lote } 20 \text { D Loteamento Poço A }\end{array}$ & 129,17 & PEQUENA PROPRIEDADE \\
\hline $\mathrm{x}$ & $\mathrm{x}$ & $\mathrm{NI}$ & $\begin{array}{l}\text { JORGE ALBERTO TELES } \\
\text { PRADO }\end{array}$ & PMDB SE & REC FEDERAL 2006 & $\begin{array}{l}\text { PROPRIEDADE RURAL ADQ DE LUIZ } \\
\text { ALBERTO TELES PRADO }\end{array}$ & 100,00 & PEQUENA PROPRIEDADE \\
\hline$x$ & $x$ & $\mathrm{NI}$ & $\begin{array}{l}\text { JORGE ALBERTO TELES } \\
\text { PRADO }\end{array}$ & PMDB SE & REC FEDERAL 2006 & $\begin{array}{l}\text { PROPRIEDADE RURAL ADQ DE LUIZ } \\
\text { ALBERTO TELES PRADO }\end{array}$ & 100,00 & PEQUENA PROPRIEDADE \\
\hline $\mathrm{x}$ & $x$ & $\mathrm{NI}$ & $\begin{array}{l}\text { CLEUBER BRANDĀOO } \\
\text { CARNEIRO }\end{array}$ & PTBMG & REC FEDERAL 2006 & $\begin{array}{l}\text { Uma Area De Terras Na Faz. Junco Denom. Faz. } \\
\text { Luciana }\end{array}$ & 78,00 & |PEQUENA PROPRIEDADE \\
\hline $\mathrm{x}$ & $x$ & $\mathrm{NI}$ & J JOSE CARLOS ELIAS & PTBES & REC FEDERAL 2010 & TERRA NUA & 74,85 & |PEQUENA PROPRIEDADE \\
\hline$x$ & $x$ & $\mathrm{NI}$ & $\begin{array}{l}\text { SERGIO SEBASTIAO DE } \\
\text { BARROS } \\
\end{array}$ & PMNAC & REC FEDERAL 2006 & 50\% ÁREA DO SITIO JARI & 67,00 & PEQUENA PROPRIEDADE \\
\hline$x$ & $x$ & $\mathrm{NI}$ & $\begin{array}{l}\text { FRANCISCO OTAVIO BECKERT } \\
\text { (CHICO DA PRINCESA) }\end{array}$ & PR PR & REC FEDERAL 2010 & Área & 61,00 & MÉDIA PROPRIEDADE \\
\hline$x$ & $\mathrm{x}$ & $\mathrm{NI}$ & AGNALDO MUNIZ & PSC RO & REC FEDERAL 2010 & $\begin{array}{l}\text { Terra Rural, Setor 5, Gleba Cunia, Margem Direita } \\
\text { Linha C-20, Terras Centrais, Altura Do Im 3,5 }\end{array}$ & 50,00 & PEQUENA PROPRIEDADE \\
\hline$x$ & $x$ & $\mathrm{NI}$ & $\begin{array}{l}\text { JOÄO HERRMANN NETO (1946- } \\
\text { 2009) }\end{array}$ & PDT SP & REC FEDERAL 2006 & PROPRIEDADE RURAL & 35,09 & MINIFUNDIO \\
\hline$x$ & $x$ & $\mathrm{NI}$ & $\begin{array}{l}\text { THEMISTOCLES DE SAMPAIO } \\
\text { PEREIRA }\end{array}$ & PMDB PI & REC FEDERAL 2008 & $\begin{array}{l}\text { Um Lote De Terra, i Terras De Lavarar E Criar } \\
\text { Demarcadas Em Uma Gleba De Lugar Denominado } \\
\text { Curralinho }\end{array}$ & 34,78 & MINIFUNDIO \\
\hline $\mathrm{x}$ & $\mathrm{x}$ & $\mathrm{NI}$ & $\begin{array}{l}\text { FRANCISCO OTAVIO BECKERT } \\
\text { (CHICO DA PRINCESA) }\end{array}$ & PR PR & REC FEDERAL 2010 & Área & 27,00 & PEQUENA PROPRIEDADE \\
\hline $\mathrm{x}$ & $\mathrm{x}$ & $\mathrm{NI}$ & $\begin{array}{l}\text { THEMISTOCLES DE SAMPAIO } \\
\text { PEREIRA }\end{array}$ & PMDB PI & REC FEDERAL 2008 & $\begin{array}{l}\text { 01 Gleba De Terra Denominada Alto Bonito (02 } \\
\text { Lotes De 12,00 Ha), - Registro De Imóveis As Fls. } \\
215 \text { A } 217 \text { Sob. N. 4. }\end{array}$ & 24,00 & PEQUENA PROPRIEDADE \\
\hline$x$ & $x$ & $\mathrm{NI}$ & GIOVANNI CORREA QUEIROZ & PDT PA & REC FEDERAL 2010 & Area Rural Lote 25 Da Gleba Cacula & 16,00 & MINIFUNDIO \\
\hline$x$ & $x$ & $\mathrm{NI}$ & JOÄO BATISTA MATOS & PMDB SC & REC FEDERAL 2002 & $\begin{array}{l}\text { LOTE RURAL EM LINHA CAMINHO SUBIDA } \\
\text { (USUCAPIÄO POR POSSA A MAIS DE } 20 \text { ANOS }\end{array}$ & 14,64 & MINIFUNDIO \\
\hline$x$ & $x$ & $\mathrm{NI}$ & OSVALDO DE SOUZA COELHO & PFL PE & REC FEDERAL 2010 & Área De Terra No Sítio & 10,00 & MINIFUNDIO \\
\hline $\mathrm{x}$ & $\mathrm{x}$ & $\mathrm{NI}$ & ADAUTO PEREIRA DE LIMA & PFL PB & REC FEDERAL 2002 & $\begin{array}{l}\text { TERRENO DEN RIACHO DO ? (ONDE SE } \\
\text { INSTALA A ANTENA DA RÁDIO MARINGÁ DE } \\
\text { POMBAL LTDA.) }\end{array}$ & 7,50 & MINIFUNDIO \\
\hline$x$ & $x$ & $\mathrm{NI}$ & $\begin{array}{l}\text { JOÅO HERRMANN NETO (1946- } \\
\text { 2009) }\end{array}$ & PDT SP & REC FEDERAL 2006 & GLEBA & 4,70 & MINIFUNDIO \\
\hline $\bar{x}$ & $x$ & $\mathrm{NI}$ & ENIVALDO RIBEIRO & PPPB & REC FEDERAL 2006 & Granja Lagoa Seca & 4,20 & MINIFUNDIO \\
\hline$x$ & $x$ & $\mathrm{NI}$ & $\begin{array}{l}\text { JOÄO HERRMANN NETO (1946- } \\
\text { 2009) }\end{array}$ & PDT SP & REC FEDERAL 2006 & GLEBA & 2,09 & MINIFUNDIO \\
\hline$x$ & $x$ & $\mathrm{NI}$ & $\begin{array}{l}\text { JOÄO HERRMANN NETO (1946- } \\
\text { 2009) }\end{array}$ & PDT SP & REC FEDERAL 2006 & GLEBA & 0,60 & MINIFUNDIO \\
\hline$x$ & $x$ & $\mathrm{NI}$ & $\begin{array}{l}\text { AUGUSTO RODRIGUES } \\
\text { COUTINHO DE MELO }\end{array}$ & DEM PE & REC FEDERAL 2010 & $\begin{array}{l}\text { Lote } 09 \text { Da Fazenda Asa Branca Com Área Aprox. } \\
\text { De } 1.643 \mathrm{M}^{2}\end{array}$ & 0,16 & MINIFUNDIO \\
\hline 150034 & AGUA AZUL DO NORTE & $\mathrm{PA}$ & JOÄO CAMPOS DE ARAÚJO & PSDB GO & REC FEDERAL 2010 & $\begin{array}{l}\text { Terreno Rural Situado No Lote 67/95-Gleba Divisa } \\
\text { Denom Fazenda Progresso, Locazido No Municipio } \\
\text { Agua Azul Do Norte-Pa, Equivalente A } 18.6734 \text { HA }\end{array}$ & 18,67 & MINIFUNDIO \\
\hline 150080 & ANANINDEUA & $P A$ & JOSUE BENGTSON & PTB PA & REC FEDERAL 2010 & Br $316 \mathrm{Km} 7$ Con Rondon & $\mathrm{NI}$ & NAO INFORMOU (NI) \\
\hline 150095 & AURORADOPARA & $P A$ & J JADER BARBALHO & PMDB PA & RECFEDERAL 2010 & Imovel Rural Retiro cháo Preto & $\mathrm{NT}$ & NAOINFORMOU(NI) \\
\hline 150145 & BELTERRA & PA & JOAQUIM DE LIRA MAIA & DEM PA & REC FEDERAL 2010 & $\begin{array}{l}\text { Terreno Rural, Na Gleba 27, Lote } 26 \text { Do Projeto } \\
\text { Integrado De Colonização De Itaituba, Km } 108 \mathrm{Da} \\
\mathrm{Br} 163\end{array}$ & $\mathrm{NI}$ & NÃO INFORMOU (NI) \\
\hline 150250 & CHAVES & $\mathrm{PA}$ & BENEDITO DIAS DE CARVALHO & PPB AP & REC FEDERAL 2002 & $\begin{array}{l}\text { Participacão em 50\% nas Fazendas Såo Luiz e } \\
\text { Liberdade }\end{array}$ & $\mathrm{NI}$ & NÃO INFORMOU (NI) \\
\hline 150270 & CONCEIÇÃO DO ARAGUAIA & $\mathrm{PA}$ & GIOVANNI CORREA QUEIROZ & PDT PA & REC FEDERAL 2010 & LOTES & $\mathrm{NI}$ & NÄO INFORMOU (NI) \\
\hline 150270 & CONCEIÇÃO DO ARAGUAIA & $\mathrm{PA}$ & GIOVANNI CORREA QUEIROZ & PDT PA & REC FEDERAL 2010 & LOTES & $\mathrm{NI}$ & NÃO INFORMOU (NI) \\
\hline 150270 & CONCEIÇÃO DO ARAGUAIA & $\mathrm{PA}$ & $\begin{array}{l}\text { NILTON GOMES DE OLIVEIRA } \\
\text { (NLITON BAIANO) }\end{array}$ & PPB ES & REC FEDERAL 2002 & $\begin{array}{l}\text { Sitio com } 1759 \text { há, com casa residencial - } \\
\text { Conceiçăo do AraguaialPA }\end{array}$ & $1.759,00$ & GRANDE PROPRIEDADE \\
\hline 150360 & ITAITUBA & $\mathrm{PA}$ & OLÁVIO SILVA ROCHA & PMDB & REC FEDERAL 2008 & $\begin{array}{l}\text { Faz. Três Poderes Rodovia Transamazônica, Km } \\
\text { 215, Vicinal Belmonte Km 80, Novo Repartimento }\end{array}$ & $\mathrm{NI}$ & NÃO INFORMOU (NI) \\
\hline 150550 & PARAGOMINAS & $P A$ & JADER BARBALHO & PMDB PA & REC FEDERAL 2010 & Imovel Rural Denominado, Cinderela & $\mathrm{NI}$ & NAO INFORMOU (NI) \\
\hline 150616 & RIO MARIA & PA & GIOVANNI CORREA QUEIROZ & PDT PA & REC FEDERAL 2010 & MOGNOPORA & $1.840,10$ & GRANDE PROPRIEDADE \\
\hline 150655 & SANTALUZIADOPARA & $P A$ & JOSUEBENGTSON & PTB PA & REC FEDERAL 2010 & Lote Agricolas & $\mathrm{NI}$ & NAOINFORMOU(NII) \\
\hline 750655 & SANTALUZIADOPARA & $P A$ & JOSUE BENGTSON & PTB PA & REC FEDERAL 2010 & Agricolas Piracema Sta Luzia Do Para & $\mathrm{NT}$ & NAOINFORMOU(NII) \\
\hline
\end{tabular}




\begin{tabular}{|c|c|c|c|c|c|c|c|c|}
\hline GEOCODIGO & MUNICIPIO & $\mathrm{UF}$ & PARLAMENTAR & PARTIDO & FONTE & TIMOVEL & AREA & CATEGORIA FUNDIÁRIA \\
\hline 150655 & SANTA LUZIA DO PARA & PA & OLÁVIO SILVA ROCHA & PMDB & REC FEDERAL 2008 & $\begin{array}{l}\text { Faz. Maria Bonita, Rod. Br 222, Km 86, Vicinal } \\
\text { Santa Luzia Km } 12\end{array}$ & $\mathrm{NI}$ & NÄO INFORMOU (NI) \\
\hline 150655 & SANTA LUZIA DO PARA & $\mathrm{PA}$ & JOSUEBENGISON & PTBPA & REC FEDERAL 2010 & Agricola & $1.500,00$ & GRANDE PROPRIEDADE \\
\hline 150655 & SANTA LUZIA DO PARA & $\mathrm{PA}$ & JOSUEBEENGISON & PTBPA & REC FEDERAL 2010 & Agricola Com & $1.400,00$ & GRANDE PROPRIEDADE \\
\hline 150655 & SANTA LUZIA DO PARA & $\mathrm{PA}$ & JOSUE BENGTSON & $\begin{array}{c}\text { PTB PA } \\
\end{array}$ & REC FEDERAL 2010 & Agricola & 650,00 & MÉDIA PROPRIEDADE \\
\hline 150655 & SANTA LUZIA DO PARA & $\mathrm{PA}$ & JOSUE BENGTSON & PTB PA & REC FEDERAL 2010 & Terreno Agricola & 300,00 & MÉDIA PROPRIEDADE \\
\hline 150680 & SANTARÉM & PA & JOAQUIM DE LIRA MAIA & DEM PA & REC FEDERAL 2010 & $\begin{array}{l}\text { Imovel Rural Na Colonia Limoal, No Municipio De } \\
\text { Santarem-Pa, Adquirido Por Compra Do Sr. } \\
\text { Norberto Pinto }\end{array}$ & $\mathrm{NI}$ & NÄO INFORMOU (NI) \\
\hline 150680 & SANTARÉM & $\mathrm{PA}$ & JOAQUIM DE LIRA MAIA & DEM PA & REC FEDERAL 2010 & $\begin{array}{l}\text { Imovel Urbano Adquirido Por Compra Do Sr. Pedro } \\
\text { M. Da Silva, Na Vila De Alter-Do-Chão VER SE É } \\
\text { MESMO URBANO }\end{array}$ & $\mathrm{NI}$ & NÃO INFORMOU (NI) \\
\hline 150680 & SANTARÉM & PA & JOAQUIM DE LIRA MAIA & DEM PA & REC FEDERAL 2010 & $\begin{array}{l}\text { Terreno Rural loc na Colonia Cipoal adquirido Por } \\
\text { Compra, No Ano } 1997\end{array}$ & $\mathrm{NI}$ & NÃO INFORMOU (NI) \\
\hline 150680 & SANTARÉm & $\mathrm{PA}$ & JOAQUIM DE LIRA MAIA & DEM PA & REC FEDERAL 2010 & $\begin{array}{l}\text { Terreno Rural loc na Colonia Cipoal Adquirido Por } \\
\text { Compra Do Sr. Pedro Laurentino Félix }\end{array}$ & $\mathrm{NI}$ & NÃO INFORMOU (NI) \\
\hline 150680 & SANTARÉM & $\mathrm{PA}$ & JOAQUIM DE LIRA MAIA & DEM PA & REC FEDERAL 2010 & $\begin{array}{l}\text { Terreno Rural loc Na Colonia Terra Preta Lote 753, } \\
\text { Gleba Mojui Dos Campos. }\end{array}$ & $\mathrm{NI}$ & NÃO INFORMOU (NI) \\
\hline$\frac{150680}{156680}$ & $\begin{array}{l}\text { SANTARÉM } \\
\text { SANTAREM }\end{array}$ & $\frac{P A}{P A}$ & $\begin{array}{l}\text { JOAQUIM DE LIRA MAIA } \\
\text { JOAQUIMDELIRAMAIA }\end{array}$ & $\frac{\text { DEM PA }}{\text { DEMPA }}$ & $\begin{array}{l}\text { REC FEDERAL } 2010 \\
\text { RECFEDERAL } 2010\end{array}$ & $\begin{array}{l}\text { Terreno Rural, Localizado Na Gleba Mojui Dos } \\
\text { Campos, No Municipio De Santarem-ja, Com } \\
158,31 \text { Ha, Adquirido Em } 2005 . \\
\text { Imovel Ruralloc na Colonia Cipoal }\end{array}$ & $\frac{158,31}{3,50}$ & PEQUENA PROPRIEDADE \\
\hline 150680 & SANTARÉm & $\mathrm{PA}$ & JOAQUIM DE LIRA MAIA & DEM PA & REC FEDERAL 2010 & $\begin{array}{l}\text { Terreno Rural Na Colonia Cipoal, Adquirido Por } \\
\text { Compra Do Sr. Jose Antonio Reis Pereira. }\end{array}$ & 2,10 & MINIFUNDIO \\
\hline 750730 & SAOFELIXDOXINGU & $P A$ & CASILDOJOAOMALDANER & PMDBSC & REC FEDERAL 2010 & Lote De Terras, Projeto Trairáa & $\mathrm{N} I$ & NAO INFORMOU(NI) \\
\hline 150730 & SÃO FELIX DO XINGU & $\mathrm{PA}$ & RAIMUNDO COIMBRA JÚNIOR & PMDB TO & REC FEDERAL 2010 & Uma Fazenda Com 500 Alqueires & $1.360,00$ & GRANDE PROPRIEDADE \\
\hline 150740 & SÃO FRANCISCO DO PARÁ & PA & MÁRIO COUTO FILHO & PSDB PA & REC FEDERAL 2006 & Parte De Fazenda 528ha - São Francisco Do Pará & 528,00 & MÉDIA PROPRIEDADE \\
\hline 150830 & VISEU & $P A$ & A ANIVALDO JUVENIL VALE & PSDB PA & REC FEDERAL 2010 & Imovel Rural Faz Tiptinga & $\mathrm{NI}$ & NAOINFORMOU(NI) \\
\hline 150830 & VISEU & $\mathrm{PA}$ & ANIVALDO JUVENIL VALE & PSDB PA & REC FEDERAL 2010 & $\begin{array}{l}\text { Imovel Rural Fazenda Nelore Situada Na Rod } 316 \\
\text { Kms } 83 \text { A } 89 \text { Na Colonia De Piria }\end{array}$ & $\mathrm{NI}$ & NÄO INFORMOU (NI) \\
\hline 150830 & VISEU & $\overline{P A}$ & ANIVALDO JUVENIILVALE & $\overline{\text { PSDBPA }}$ & REC FEDERAL 2002 & |IMOVEL RURALFAZENDA TIPITINGA & $1.198,00$ & GRANDE PROPRIEDADE \\
\hline 150830 & VISEU & $\mathrm{PA}$ & ANIIVALDO JUVENILVALE & $\overline{P S D B P A}$ & REC FEDERAL 2010 & IMOVEL RURAL, NOKm 81 Da Br 316 & 551,00 & MÉDIA PROPRIEDADE \\
\hline 150830 & VISEU & $\mathrm{PA}$ & ANIVALDO JUVENIL VALE & PSDB PA & REC FEDERAL 2010 & $\begin{array}{l}\text { IIMÓVEL RURAL FAZENDA NELORE, LOC KM } \\
316\end{array}$ & 551,00 & MÉDIA PROPRIEDADE \\
\hline 150830 & VISEU & $\mathrm{PA}$ & ANIVALDO JUVENIL VALE & PSDBPA & REC FEDERAL 2010 & IMOVEL RURAL, No Km 81 Da Br 316 & 529,00 & MÉDIA PROPRIEDADE \\
\hline 150830 & VISEU & $\mathrm{PA}$ & $\begin{array}{l}\text { ANIVALDO JUVENIL VALE } \\
\end{array}$ & $\overline{P S D B P A}$ & REC FEDERAL 2010 & Imovel Rural Loc No Km 80 Da Br 316 & 125,00 & PEQUENA PROPRIEDADE \\
\hline 150830 & VISEU & $\mathrm{PA}$ & ANIVALDO JUVENILVALE & PSDBPA & REC FEDERAL 2010 & IIMOVEL RURAL, NOKm 80 DaBr 316 & 125,00 & PEQUENA PROPRIEDADE \\
\hline 150840 & XINGUARA & $\mathrm{PA}$ & $\begin{array}{l}\text { JOÁO BATISTA DE JESUS } \\
\text { RIBEIRO }\end{array}$ & PRTO & REC FEDERAL 2010 & TERRA RURAL & 713,19 & GRANDE PROPRIEDADE \\
\hline 150555 & PAU D'ARCO & $\mathrm{PA}$ & GIOVANNI CORREA QUEIROZ & PDT PA & REC FEDERAL 2010 & AREA RURAL & $4.356,00$ & GRANDE PROPRIEDADE \\
\hline 150680 & SANTARÉm & $\mathrm{PA}$ & JOAQUIM DE LIRA MAIA & DEM PA & REC FEDERAL 2010 & $\begin{array}{l}\text { Imóvel Rural loc na Colonia Limoal Adquirido Por } \\
\text { Compra, Medindo Aproximadamente } 150 \mathrm{Ha}\end{array}$ & 150,00 & MÉDIA PROPRIEDADE \\
\hline 150635 & STA BARBARA DO PARA & $\mathrm{PA}$ & ANIVALDO JUVENILVALE & 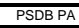 & REC FEDERAL 2010 & Imovel Rural & 84,36 & GRANDE PROPRIEDADE \\
\hline 150830 & VISEU & $\mathrm{PA}$ & ANIVALDO JUVENIL VALE & PSDB PA & REC FEDERAL 2010 & Imovel Rural Localizado No Km 81 Da Br 316 & 551,00 & MÉDIA PROPRIEDADE \\
\hline 150830 & VISEU & $\mathrm{PA}$ & $\begin{array}{l}\text { ANIVALDO JUVENIL VALE } \\
\end{array}$ & PSDBPA & REC FEDERAL 2010 & Imovel Rural Faz Nelore LOC No Km 316 & 551,00 & MÉDIA PROPRIEDADE \\
\hline 150830 & VISEU & $\mathrm{PA}$ & ANIVALDO JUVENIL VALE & PSDB PA & REC FEDERAL 2010 & Imovel Rural Localizado No Km $81 \mathrm{Da} \mathrm{Br} 316$ & 529,00 & MÉDIA PROPRIEDADE \\
\hline 250100 & ARARUNA & PB & J JOSE TARGINO MARANHAO & PMDBPB & REC FEDERAL 2010 & Propriedade Rural Denominada Carnauba & $\mathrm{NI}$ & NAO INFORMOU (NII) \\
\hline 250100 & ARARUNA & PB & JOSÉ TARGINO MARANHÃO & PMDB PB & REC FEDERAL 2010 & $\begin{array}{l}\text { Propriedade Denominada Lagoa Da Mata Ou } \\
\text { Primavera }\end{array}$ & $\mathrm{NI}$ & NÃO INFORMOU (NI) \\
\hline 250100 & ARARUNA & $\mathrm{PB}$ & $\begin{array}{l}\text { BENJAMIM GOMES MARANHAO } \\
\text { NETO }\end{array}$ & PMDB PB & REC FEDERAL 2002 & $\begin{array}{l}\text { IMÓVELL RURAL EXPLORADO SÁO MATEUS E } \\
\text { FAZENDA JARDIM }\end{array}$ & 48,00 & PEQUENA PROPRIEDADE \\
\hline 250110 & AREIA & PB & ADAUTO PEREIRA DE LIMA & PFLPB & REC FEDERAL 2002 & $\begin{array}{l}\text { IMÓVEL RURAL DENOMINADO MACACOS } \\
\text { (INCRA 210003000893) }\end{array}$ & $\mathrm{NI}$ & NÄO INFORMOU (NI) \\
\hline 250250 & BOQUEIRÄO & $\mathrm{PB}$ & CARLOS MARQUES DUNGA & PTB PB & REC FEDERAL 2010 & Propriedade Rural Denominada Faz.São José & 103,60 & PEQUENA PROPRIEDADE \\
\hline 250400 & CAMPINA GRANDE & $\mathrm{PB}$ & ENIVALDO RIBEIRO & PPPB & REC FEDERAL 2006 & $\begin{array}{l}\text { Propriedade em Bodocongo } \\
\end{array}$ & 20,00 & PEQUENA PROPRIEDADE \\
\hline 251640 & CAMPO DE SANTANA & $\mathrm{PB}$ & JOSE TARGINO MARANHAO & PMDB PB & REC FEDERAL 2010 & Propriedade Rural Denominada Acaua & $\frac{\mathrm{N} I \mathrm{NI}}{\mathrm{N}}$ & NAO INFORMOU (NI) \\
\hline 251640 & CAMPO DE SANTANA & PB & JOSÉ TARGINO MARANHÃO & PMDB PB & REC FEDERAL 2010 & Propriedade Rural Denominada Olho Dagua & $\mathrm{NI}$ & NÄO INFORMOU (NI) \\
\hline 251640 & CAMPO DE SANTANA & PB & JOSÉ TARGINO MARANHÃO & PMDB PB & REC FEDERAL 2010 & Propriedade Rural Denominada Lagoa Seca & $\mathrm{NI}$ & NÃO INFORMOU (NI) \\
\hline 251640 & CAMPODE SANTANA & PB & JOSE TARGINOMARANHAO & PMDBPB & REC FEDERAL 2010 & Propriedade Rural Denominada Mocoto & NIT & NAOINFORMOU(NII) \\
\hline 251640 & CAMPODE SANTANA & PB & JOSE TARGINOMARANHAO & PMDBPB & RECTFEDERAL 2010 & Propriedade Rural Denominada Volta & NIT & NAOINFORMOU(NII) \\
\hline 251640 & CAMPO DE SANTANA & PB & JOSÉ TARGINO MARANHÃO & PMDB PB & REC FEDERAL 2010 & Propriedade Rural Denominada Joao Pereira & $\mathrm{NI}$ & NĀO INFORMOU (NI) \\
\hline 251640 & CAMPO DE SANTANA & $\mathrm{PB}$ & J JOSE TARGINO MARANHAO & PMDB PB & REC FEDERAL 2010 & Propriedade Rural Denominada Baixio & $\frac{\mathrm{NI}}{\mathrm{NI}}$ & NAO INFORMOU (NI) \\
\hline 250435 & CATURITÉ & $\mathrm{PB}$ & CARLOS MARQUES DUNGA & РTB РB & REC FEDERAL 2010 & $\begin{array}{l}\text { Propriedade Rural Denominada Malhada Da Cruz E } \\
\text { Caturité }\end{array}$ & 140,60 & MÉDIA PROPRIEDADE \\
\hline 250560 & DIAMANTE & $P B$ & ARMANDO ABILIOVIEIRA & PTBPB & REC FEDERAL 2010 & Propriedade Denominada Sabonete & $\mathrm{N} T$ & NAOINFORMOU(NII) \\
\hline 250560 & DIAMANTE & PB & ARMANDO ABILIOVIEIRA & PTBPB & REC FEDERAL 2010 & Propriedade Denominada Lagoa Seca & $\mathrm{NT}$ & NAOINFORMOU(NI) \\
\hline 250560 & DIAMANTE & $\mathrm{PB}$ & ARMANDO ABILIO VIEIRA & PTB PB & REC FEDERAL 2010 & St Sabonete & $\mathrm{NI}$ & NAO INFORMOU (NI) \\
\hline 250570 & DONAINES & $\mathrm{PB}$ & JOSE TARGINO MARANHAO & PMDB PB & REC FEDERAL 2010 & Propriedade Rural Denominada Cafundo & NI & NAO INFORMOU (NI) \\
\hline 250600 & ESPERANÇA & $\mathrm{PB}$ & ARMANDO ABILIO VIEIRA & PTB PB & REC FEDERAL 2010 & St Lagoa De Pedra & $\mathrm{NI}$ & NAO INFORMOU (NI) \\
\hline 250600 & ESPERANÇA & $\mathrm{PB}$ & ARMANDO ABILIO VIEIRA & PTB PB & REC FEDERAL 2010 & St Lagoa De Pedra & $\mathrm{NI}$ & NAO INFORMOU(NI) \\
\hline 250770 & JUAZEIRINHO & PB & ADAUTO PEREIRA DE LIMA & $\begin{array}{l}\text { PFLPB } \\
\end{array}$ & REC FEDERAL 2002 & $\begin{array}{l}\text { IMOVEL RURAL MENDONÇA (INCRA } \\
\text { 202087002445) }\end{array}$ & $\mathrm{NI}$ & NẴO INFORMOU (NI) \\
\hline 250830 & LAGOASECA & PB & ARMANDO ABILIO VIEIRA & PIBPB & REC FEDERAL 2010 & Granja Las Vegas & $\mathrm{NT}_{\mathrm{T}}$ & NAOO INFORMOU(NII) \\
\hline 250860 & LUCENA & PB & ENIVALDO RIBEIRO & PPPB & REC FEDERAL 2006 & \begin{tabular}{|l} 
Gleba No03 E 05, Conf. Registro Na Receita \\
Federal No4123770-6
\end{tabular} & $\mathrm{NI}$ & NÃO INFORMOU (NI) \\
\hline 250870 & MÃE D'ÁGUA & PB & $\begin{array}{l}\text { JOAO TOTA SOARRES DE } \\
\text { FIGUEIRREDO }\end{array}$ & PP AC & REC FEDERAL 2006 & TERRENO RURAL & $\mathrm{NI}$ & NÃO INFORMOU (NI) \\
\hline 251070 & PAssagem & $\mathrm{PB}$ & ADAUTO PEREIRA DE LIMA & PFLPB & REC FEDERAL 2002 & $\begin{array}{l}\text { IIMÓVEL RURAL TRIGUEIRO (INCRA } \\
\text { 207179000787) }\end{array}$ & $\mathrm{NI}$ & NÃO INFORMOU (NI) \\
\hline 251080 & PATOS & PB & ADAUTO PEREIRA DE LIMA & PFLPB & REC FEDERAL 2002 & $\begin{array}{l}\text { IMÓVEL RURAL MOCAMBO (INCRA } \\
\text { 207182004553) }\end{array}$ & $\mathrm{NI}$ & NÃO INFORMOU (NI) \\
\hline 251210 & POMBAL & PB & ADAUTO PEREIRA DE LIMA & PFLPB & REC FEDERAL 2002 & $\begin{array}{l}\text { TRES PARTES DA TERRA NO IMÓVEL RURAL } \\
\text { PADRE ANTONIO (INCRA 207217010950-0) }\end{array}$ & $\mathrm{NI}$ & NÃO INFORMOU (NI) \\
\hline 251210 & POMBAL & $\mathrm{PB}$ & ADAUTO PEREIRA DE LIMA & $\begin{array}{l}\text { PFLPB } \\
\end{array}$ & REC FEDERAL 2002 & PARTE NO IMÓVEL RURAL SANTA MONICA & $\mathrm{NI}$ & NÃO INFORMOU (NI) \\
\hline 292580 & QUEIMADAS & PB & $\begin{array}{l}\text { VITAL DO RÉGO FILHO } \\
\text { (VITALZINHO) }\end{array}$ & PMDB PB & REC FEDERAL 2010 & Fazenda Denominada Campo De Boi & $\mathrm{NI}$ & NÃO INFORMOU (NI) \\
\hline 251270 & REEMIGIO & PB & ARIMANDO ABILTOVIEIRA & PIBPB & RECTFEDERAL 2010 & StCapim De Cheiro & TाT & NAOINFORMOU (NI) \\
\hline 251270 & REMIGIO & PB & ARMANDO ABILO VIEIRA & PIBPB & REC FEDERAL 2010 & Sitio Genipapo & $\mathrm{NI}$ & |NAOINFORMOU (NII) \\
\hline 251270 & REEMIGIO & $P B$ & ARMANDO ABILIOVIEIRA & PIBPB & REC FEDERAL 2010 & St Urubu & ता & NAOINFORMOU(NI) \\
\hline 251270 & REEMIGIO & PB & ARMANDOABILIOVIEIRA & PTBPB & REC FEDERALL 2010 & St Sao Bento De Cima & $\mathrm{NI}$ & NAO INFORMOU(NII) \\
\hline 251270 & REMIGIO & PB & ARMANDOABILIOVIEIRA & PIBPB & REC FEDERAL 2010 & St Capim De Cheiro & TाT & NAOINFORMOU(NI) \\
\hline 251270 & REMIGIO & PB & ARMANDO ABILIO VIEIRA & PTBPB & REC FEDERAL 2010 & St Palma De Baixo & NII & NAOINFORMOU (NII) \\
\hline 251340 & SANTA LUZIA & $\mathrm{PB}$ & $\begin{array}{l}\text { EFRAIM DE ARAUJO DE } \\
\text { MORAIS }\end{array}$ & PFL PB & REC FEDERAL 2010 & Propriedade Rural (mun Santa Luiza) & 70,00 & PEQUENA PROPRIEDADE \\
\hline 251370 & SANTARITA & PB & ADAUTO PEREIRA DE LIMA & PFLPB & RECTFEDERAL 2002 & 7/5DAPROPRIEDADEDENOMVIGARIO & NIT & NAOTINFORIMOU(NI) \\
\hline
\end{tabular}




\begin{tabular}{|c|c|c|c|c|c|c|c|c|}
\hline GEOCODIGO & MUNICIPIO & $\mathrm{UF}$ & PARLAMENTAR & PARTIDO & FONTE & JIMOVEL & ÁREA & CATEGORIA FUNDIÁRIAA \\
\hline 251610 & SOLEDADE & $\mathrm{PB}$ & CARLOS MARQUES DUNGA & РТВ РВ & REC FEDERAL 2010 & Imóvel Rural Espirito Santo, com Benfeitorias & $1.800,00$ & GRANDE PROPRIEDADE \\
\hline 251710 & VARZEA & $\mathrm{PB}$ & $\begin{array}{l}\text { EFRAIM DE ARAUJO DE } \\
\text { MORAIS }\end{array}$ & PFLPB & REC FEDERAL 2010 & Imovél Rural Denom. Cacimba E Jua & 358,00 & MÉDIA PROPRIEDADE \\
\hline 251710 & VARZEA & $\mathrm{PB}$ & $\begin{array}{l}\text { EFRAIM DE ARAUJO DE } \\
\text { MORAIS }\end{array}$ & PFL PB & REC FEDERAL 2010 & Fazenda Nova Floresta & 348,00 & MÉDIA PROPRIEDADE \\
\hline 251710 & VARZEA & PB & $\begin{array}{l}\text { EFRAIM DE ARAUJO DE } \\
\text { MORAIS }\end{array}$ & PFL PB & REC FEDERAL 2010 & Propriedade Rural Fazenda Uiassaba & 183,00 & PEQUENA PROPRIEDADE \\
\hline 251710 & VARZEA & PB & $\begin{array}{l}\text { EFRAIM DE ARAUJO DE } \\
\text { MORAIS }\end{array}$ & PFLPB & REC FEDERAL 2010 & $\begin{array}{l}\text { Imové́ Rural Denominado Barra Do Navio E I pueira } \\
\text { Do Couro }\end{array}$ & 100,00 & PEQUENA PROPRIEDADE \\
\hline$x$ & $\mathrm{x}$ & PB & $\begin{array}{l}\text { BENJAMIM GOMES MARANHAO } \\
\text { NETO }\end{array}$ & PMDB PB & REC FEDERAL 2002 & IMÓVEL RURAL EXPLORADO SÃO MATEUS & 21,00 & PEQUENA PROPRIEDADE \\
\hline 260640 & GRAVATA & PE & $\begin{array}{l}\text { LUIZ PIAUHYLINO DE MELLOO } \\
\text { MONTEIRO }\end{array}$ & PSDB PE & REC FEDERAL 2002 & $\begin{array}{l}\text { FAZ SAMPAIO (ADQ DE ANTONIO MANOEL DO } \\
\text { NASCIMENTO }\end{array}$ & $\mathrm{NI}$ & NÃO INFORMOU (NI) \\
\hline 260640 & GRAVATA & PE & $\begin{array}{l}\text { LUIZ PIAUHYLINO DE MELLO } \\
\text { MONTEIRO }\end{array}$ & PSDB PE & REC FEDERAL 2002 & $\begin{array}{l}\text { LOTE } 21 \text { E } 22 \text { DA QUADRA C DA FAZ } \\
\text { GRAMADO (ADQ 08/1986) }\end{array}$ & $\mathrm{NI}$ & NÄO INFORMOU (NI) \\
\hline 260680 & IGARAsSU & PE & $\begin{array}{l}\text { SEVERINO SÉRGIO ESTELLITA } \\
\text { GUERRA }\end{array}$ & PSDB PE & REC FEDERAL 2010 & Imovel Localizada No Municipio De Igarassu & $\mathrm{NI}$ & NÃO INFORMOU (NI) \\
\hline 260890 & LIMOEIRO & $\mathrm{PE}$ & $\begin{array}{l}\text { SEVERINO SÉRGIO ESTELITA } \\
\text { GUERRA (SÉRGIO GUERRA) }\end{array}$ & PSDB PE & REC FEDERAL 2010 & Terra Nua Na Pe $5 \mathrm{Km}$ Com 123 Hectares & 123,00 & MÉDIA PROPRIEDADE \\
\hline 260890 & LIMOEIRO & $\mathrm{PE}$ & $\begin{array}{l}\text { SEVERINO SÉRGIO ESTELITA } \\
\text { GUERRA (SÉRGIO GUERRA) }\end{array}$ & PSDB PE & REC FEDERAL 2010 & Terra Nua Na Pe 5 Km 69 Municipio De Limoeiro & 69,00 & PEQUENA PROPRIEDADE \\
\hline 260890 & LIMOEIRO & $\mathrm{PE}$ & $\begin{array}{l}\text { SEVERINO SÉRGIO ESTELITA } \\
\text { GUERRA (SÉRGIO GUERRA) }\end{array}$ & PSDB PE & REC FEDERAL 2010 & 14 Hectares & 14,00 & MINIFUNDIO \\
\hline 261110 & PETROLINA & $\mathrm{PE}$ & LUIZ GONZAGA PATRIOTA & PSB PE & REC FEDERAL 2010 & $\begin{array}{l}\text { Uma } 01 \text { Chacara Denominada Giglyolla Patricia } \\
\text { Adquirida Em } 1981\end{array}$ & $\mathrm{NI}$ & NÃO INFORMOU (NI) \\
\hline 261110 & PETROLINA & PE & LUIZ GONZAGA PATRIOTA & PSB PE & REC FEDERAL 2010 & $\begin{array}{l}\text { Uma } 01 \text { Propriedade Rural Denominada Serra Da } \\
\text { Santa Minadouro Adquirida Em } 1989\end{array}$ & $\mathrm{NI}$ & NĂO INFORMOU (NI) \\
\hline 261110 & \begin{tabular}{|l} 
PETROLINA \\
\end{tabular} & PE & LUIZ GONZAGA PATRIOTA & PSB PE & REC FEDERAL 2010 & $\begin{array}{l}\text { Um } 01 \text { Lote Para Chacaras Adquiridos Em } 1979 \\
\text { Loteamento Giglyolla Patricia }\end{array}$ & $\mathrm{NI}$ & NÃO INFORMOU (NI) \\
\hline 261110 & PETROLINA & $\mathrm{PE}$ & LUIZ GONZAGA PATRIOTA & PSB PE & REC FEDERAL 2010 & $\begin{array}{l}1 \text { Gleba De Terra No Topo Da Serra Da Santa } \\
\text { Adq Em } 2008\end{array}$ & $\mathrm{NI}$ & NÃO INFORMOU (NI) \\
\hline 261110 & PETROLINA & $\mathrm{PE}$ & LUIZ GONZAGA PATRIOTA & PSB PE & REC FEDERAL 2010 & $\begin{array}{l}\text { Uma } 01 \text { Propriedade Rural Com Casa Residencial } \\
\text { Denominada Repouso Do Guerreiro Na llida De } \\
\text { Coqueiro Adq Em } 1993\end{array}$ & $\mathrm{NI}$ & NÃO INFORMOU (NI) \\
\hline 261110 & PETROLINA & PE & OSVALDO DE SOUZA COELHO & PFLPE & REC FEDERAL 2010 & Lote Deterreno № 5 Na Caixa D'água & $\mathrm{NI}$ & NÃO INFORMOU (NI) \\
\hline 261110 & PETROLINA & $\mathrm{PE}$ & OSVALDO DE SOUZA COELHO & PFL PE & REC FEDERAL 2010 & $\begin{array}{l}\text { Uma Propriedade Denominada Sitio Carneiro } \\
\text { Situada Na Estrada De Pedrinha }\end{array}$ & 42,00 & MINIFUNDIO \\
\hline 261110 & PETROLINA & $\mathrm{PE}$ & OSVALDO DE SOUZA COELHO & PFL PE & REC FEDERAL 2010 & $\begin{array}{l}\text { Propriedade Sítio São Pedro Situado Na Fazenda } \\
\text { Trapera }\end{array}$ & 41,00 & MINIFUNDIO \\
\hline 2611101 & PETROLINA & PE & OSVALDO DE SOUZA COELHO & PFL PE & REC FEDERAL 2010 & $\begin{array}{l}\begin{array}{l}\text { Propriedade Denominda Sitio Andorinha Fazenda } \\
\text { Roncado }\end{array} \\
\end{array}$ & 11,00 & MINIFUNDIO \\
\hline 261130 & ромвоS & $\mathrm{PE}$ & JOÄO BASTOS COLAÇO DIAS & PSB PE & REC FEDERAL 1998 & $\begin{array}{l}\text { PROPRIEDADE AGRICOLA DENOMINADA } \\
\text { CAJOCA ADQ EM 11/01/74 }\end{array}$ & $\mathrm{NI}$ & NÄO INFORMOU (NI) \\
\hline 261150 & QUIPAPÁ & PE & $\begin{array}{l}\text { ARTHUR CÉSAR PEREIRA DE } \\
\text { LIRA }\end{array}$ & PMDB AL & REC FEDERAL 2010 & Faz Taguari & $\mathrm{NI}$ & NÄO INFORMOU (NI) \\
\hline 261220 & SALGUEIRO & $\mathrm{PE}$ & LUIZ GONZAGA PATRIOTA & PSB PE & REC FEDERAL 2010 & $\begin{array}{l}\text { Uma } 01 \text { Propriedade Rural Denominada De Barra } \\
\text { Da Favela Adquirida Em } 1988\end{array}$ & $\mathrm{NI}$ & NÄO INFORMOU (NI) \\
\hline 261220 & Salgueiro & PE & LUIZ GONZAGA PATRIOTA & PSB PE & REC FEDERAL 2010 & $\begin{array}{l}\text { Uma } 01 \text { Propriedade Rural Denominada De Barra } \\
\text { Da Serra Da Fazenda Angico Adquirido Em } 1991\end{array}$ & $\mathrm{NI}$ & NÄO INFORMOU (NI) \\
\hline 261220 & Salgueiro & $\mathrm{PE}$ & LUIZ GONZAGA PATRIOTA & PSB PE & REC FEDERAL 2010 & $\begin{array}{l}1 \text { Propriedade Rural Denominada Urubu Adq Em } \\
2007\end{array}$ & $\mathrm{NI}$ & NÄO INFORMOU (NI) \\
\hline 261220 & SALGueiro & PE & LUIZ GONZAGA PATRIOTA & PSB PE & REC FEDERAL 2010 & $\begin{array}{l}\text { lo1 Gleba De Terra No Topo Da Serra Da Torre De } \\
\text { Tv }\end{array}$ & $\mathrm{NI}$ & NÄO INFORMOU (NI) \\
\hline 261320 & SÃO JOÃO & $\mathrm{PE}$ & $\begin{array}{l}\text { JOSÉ CARLOS LEÃO DE } \\
\text { ARAUJO }\end{array}$ & $\begin{array}{l}\text { PDT BA } \\
\end{array}$ & REC FEDERAL 2010 & $\begin{array}{l}\text { Fazenda Denominada Sao Jose (não informou a } \\
\text { UF, pode ser PR ou PE) }\end{array}$ & $\mathrm{NI}$ & NÃO INFORMOU (NI) \\
\hline 261390 & SERRA TALHADA & $\mathrm{PE}$ & $\begin{array}{l}\text { INOCÉNCIO GOMES DE } \\
\text { OLIVEIRA }\end{array}$ & PRPE & REC FEDERAL 2010 & $\begin{array}{l}\text { Terreno C } 2500 \text { Metros Na Fazenda Cachichola } 1 \\
\text { Distrito Serra Talhada Pe }\end{array}$ & 0,25 & MINIFUNDIO \\
\hline 261410 & SERTANIA & PE & LUIZ GONZAGA PATRIOTA & PSB PE & REC FEDERAL 2010 & $\begin{array}{l}\text { Uma } 01 \text { Gleba De Terra No Lugar Denominada } \\
\text { Gogo Da Gata Destinada A Instalacaoo De } \\
\text { Equipamentos De Uma Radio Fm Adquirida Em } \\
1999\end{array}$ & $\mathrm{NI}$ & NÃO INFORMOU (NI) \\
\hline 261485 & TAMandaré & PE & $\begin{array}{l}\text { AUGUSTO RODRIGUES } \\
\text { COUTINHO DE MELO }\end{array}$ & DEM PE & REC FEDERAL 2010 & \begin{tabular}{|l|}
$1 / 4$ Do Imóvel Constitivido Pela Gleba 36 \\
Desmembrado Da Área Remanescente Da Antiga \\
lileba 4 Da Fazenda Estivas Ou Mamucabinhas No \\
Município De Tamadare
\end{tabular} & $\mathrm{NI}$ & NÄO INFORMOU (NI) \\
\hline 220120 & BARRAS & $\mathrm{PI}$ & $\begin{array}{l}\text { THEMISTOCLES DE SAMPAIO } \\
\text { PEREIRA }\end{array}$ & PMDB PI & REC FEDERAL 2008 & $\begin{array}{l}1 \text { Pequena Propriedade Rural Ainda Por Ser } \\
\text { Registrada Em Cartório, Lagoa Dos Lages Com } 06 \\
\text { He }\end{array}$ & 6,00 & MINIFUNDIO \\
\hline 220150 & BATALHA & $\mathrm{PI}$ & $\begin{array}{l}\text { THEMISTOCLES DE SAMPAIO } \\
\text { PEREIRA }\end{array}$ & PMDB PI & REC FEDERAL 2008 & $\begin{array}{l}01 \text { Gleba De Terra Rural Denominada Luiz Da Silva } \\
\text { (Monte Verde), Encravada Na Data Caraibas }\end{array}$ & 497,34 & MÉDIA PROPRIEDADE \\
\hline 220150 & BATALHA & $\mathrm{PI}$ & $\begin{array}{l}\text { THEMISTOCLES DE SAMPAIO } \\
\text { PEREIRA }\end{array}$ & PMDB PI & REC FEDERAL 2008 & $\begin{array}{l}\text { 02 Propriedades Denominadas Santo Antonio } \\
(221,00) \text { E Lagoa Seca }(90,60) \text {, Registradas Sob } \\
\text { № 34, Fls. } 171 \text { vilit4 }\end{array}$ & 311,60 & MÉDIA PROPRIEDADE \\
\hline 220150 & BATALHA & $\mathrm{PI}$ & $\begin{array}{l}\text { THEMISTOCLES DE SAMPAIO } \\
\text { PEREIRA }\end{array}$ & PMDB PI & REC FEDERAL 2008 & FAZ NA LOCALIDADE POÇAO & 300,00 & MÉDIA PROPRIEDADE \\
\hline 220150 & BATALHA & $\mathrm{PI}$ & $\begin{array}{l}\text { THEMISTOCLES DE SAMPAIO } \\
\text { PEREIRA }\end{array}$ & PMDB PI & REC FEDERAL 2008 & $\begin{array}{l}01 \text { Propriedade denominada Santa Adelaide, } \\
\text { Registrada No Livo } 2 \text { - Fls. } 001 \text { R }\end{array}$ & 204,87 & PEQUENA PROPRIEDADE \\
\hline 220150 & BAtalHa & $\mathrm{PI}$ & $\begin{array}{l}\text { THEMISTOCLES DE SAMPAIO } \\
\text { PEREIRA }\end{array}$ & PMDB PI & REC FEDERAL 2008 & $\begin{array}{l}01 \text { Gleba De Terra Denominada Pau D } D_{i} \text { arco Com } \\
\text { Área De } 108,80 \mathrm{Há,E} \text { Encravada Na Data Caraibas, } \\
\text { Municíioio De Batalha-Pi, Registrado N }\end{array}$ & 108,80 & PEQUENA PROPRIEDADE \\
\hline 220150 & BATALHA & $\mathrm{PI}$ & $\begin{array}{l}\text { THEMISTOCLES DE SAMPAIO } \\
\text { PEREIRA }\end{array}$ & PMDB PI & REC FEDERAL 2008 & O1 Gleba De Terra Rural Denominada Caraibas & 95,00 & PEQUENA PROPRIEDADE \\
\hline 220150 & BATALHA & $\mathrm{PI}$ & $\begin{array}{l}\text { THEMISTOCLES DE SAMPAIO } \\
\text { PEREIRA }\end{array}$ & PMDB PI & REC FEDERAL 2008 & $\begin{array}{l}01 \text { Gleba De Terra Rural Denominada Catingueiras } \\
\text { (Porçao li, Encravada Na Data Caraibas }\end{array}$ & 61,31 & PEQUENA PROPRIEDADE \\
\hline 220150 & BATALHA & $\mathrm{PI}$ & $\begin{array}{l}\text { THEMISTOCLES DE SAMPAIO } \\
\text { PEREIRA }\end{array}$ & PMDB PI & REC FEDERAL 2008 & $\begin{array}{l}\text { Gleba De Terra Denominada Betel (Porção } \\
\text { 1),Encravada Na Data Caraibas }\end{array}$ & 61,31 & PEQUENA PROPRIEDADE \\
\hline 220150 & BATALHA & $\mathrm{PI}$ & $\begin{array}{l}\text { THEMISTOCLES DE SAMPAIO } \\
\text { PEREIRA }\end{array}$ & PMDB PI & REC FEDERAL 2008 & \begin{tabular}{|l|} 
Gleba De Terra Denominada Saco (Monte Verde), \\
Data Cararabas, Registrada No Livo De Not
\end{tabular} & 10,39 & MINIFUNDIO \\
\hline 311170 & CANAã & $\mathrm{PI}$ & $\begin{array}{l}\text { JÚLLIO CESAR DE CARVALHO } \\
\text { LIMA }\end{array}$ & DEMPI & REC FEDERAL 2010 & Canaả Com 50 Há & 50,00 & PEQUENA PROPRIEDADE \\
\hline 220370 & ESPERANTINA & $\mathrm{PI}$ & $\begin{array}{l}\text { THEMISTOCLES DE SAMPAIO } \\
\text { PEREIRA }\end{array}$ & PMDB PI & REC FEDERAL 2008 & $\begin{array}{l}01 \text { Terreno Rural Medindo } 80 \times 80 \text { M, Registro De } \\
\text { Imóvel Ás Fls. } 243 / 244 \text { Do Livo 04 N. 4.292 }\end{array}$ & $\mathrm{NI}$ & NÃO INFORMOU (NI) \\
\hline 220370 & ESPERANTINA & $\mathrm{PI}$ & $\begin{array}{l}\text { THEMISTOCLES DE SAMPAIO } \\
\text { PEREIRA }\end{array}$ & PMDB PI & REC FEDERAL 2008 & $\begin{array}{l}\text { 01 Propriedade Rural Denominada Barrocão, } \\
\text { Registro De Imóvel Âs Fls. } 281 / 282 \text { Do Livro } 03 \text { ¿ } \\
N^{\star} 4.468\end{array}$ & $\mathrm{NI}$ & NÃO INFORMOU (NI) \\
\hline
\end{tabular}




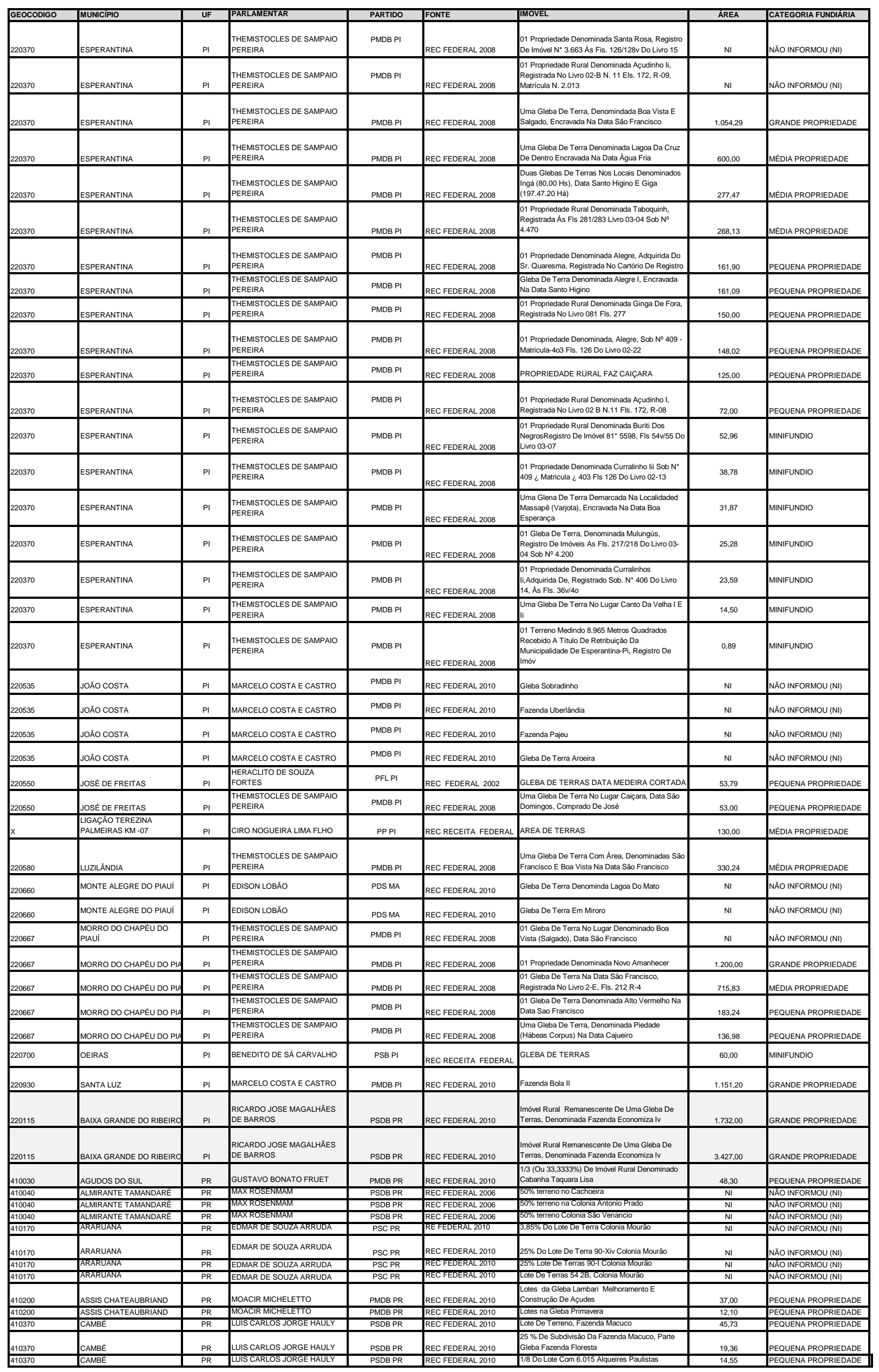




\begin{tabular}{|c|c|c|c|c|c|c|c|c|}
\hline 410425 & CAMPO MAGRO & PR & GUSTAVO BONATO FRUET & PMDB PR & REC FEDERAL 2010 & $\begin{array}{l}\text { 116 (OOU 16,6666\%) De Imóvel Rural Com Area De } \\
125,6 \mathrm{Ha} \text { Em Juriqul }\end{array}$ & 125,60 & MÉDIA PROPRIEDADE \\
\hline 410450 & CAPANEMA & PR & BLAIRO BORGES MAGGI & PRMT & REC FEDERAL 2010 & $\begin{array}{l}\text { 10\% De Um Lote Rural N.57 Da Gleba N. 135.Cp } \\
\text { Do Núcelo AreaMatrícula } 24.257\end{array}$ & 11,60 & MINIFUNDIO \\
\hline
\end{tabular}




\begin{tabular}{|c|c|c|c|c|c|c|c|c|}
\hline GEOCODIGO & MUNICIPIO & UF & PARLAMIENTAR & PARTIDO & FONTE & IIMOVEL & ÁREA & CATEGORIA FUNDIÁRIA \\
\hline 410470 & CARLOPÓLIS & $\mathrm{PR}$ & $\begin{array}{l}\text { FRANCISCO OTAVIO BECKERT } \\
\text { (CHICO DA PRINCESA) }\end{array}$ & PR PR & RE FEDERAL 2010 & $\begin{array}{l}\text { 1/110 Terreno - Illa } 29 \text { Do Reservatório De } \\
\text { Xavantes/Ribeiräo Claro }\end{array}$ & $\mathrm{NI}$ & NÃO INFORMOU (NI) \\
\hline 410480 & Cascavel & $\mathrm{PR}$ & NELSON PADOVANI & PSC PR & REC FEDERAL 2010 & $\begin{array}{l}\text { Lote Rural No } 26 \text { Da Gleba Cascavel, Sem Bem } \\
\text { Feitorias }\end{array}$ & $\mathrm{NI}$ & NÃO INFORMOU (NI) \\
\hline 410480 & CASCAVEL & $\mathrm{PR}$ & NELSON PADOVANI & PSC PR & REC FEDERAL 2010 & $\begin{array}{l}\text { 1/3 Do Lote De Terras, Cascavel /Pr Matricula } \\
64,5951 \text { of }\end{array}$ & $\mathrm{NI}$ & NÃO INFORMOU (NI) \\
\hline 410480 & Cascavel & $\mathrm{PR}$ & NELSON PADOVANI & PSC PR & REC FEDERAL 2010 & $\begin{array}{l}\text { Chacara } 109 \text { A2, Subdivisão Da Chacara } 1094 \text { Da } \\
\text { Gl }\end{array}$ & $\mathrm{NI}$ & NÃO INFORMOU (NI) \\
\hline 410590 & COLORADO & $\mathrm{PR}$ & ALEX CANZIANI SILVEIRA & PTB PR & REC FEDERAL 2010 & $\begin{array}{l}\text { Parte Ideal De 1/4 Da Fazenda Estrela, Na } \\
\text { Comarca De Colorado Havida Por Herança }\end{array}$ & 471,80 & GRANDE PROPRIEDADE \\
\hline 410850 & GENERAL CARNEIRO & $\mathrm{PR}$ & LUCIANO PIZZATTO & DEM PR & REC FEDERAL 2010 & Terreno Rural Com Benfeitorias E Reflorestamento & $\mathrm{NI}$ & NÃO INFORMOU (NI) \\
\hline 410860 & GOIOERE & $\mathrm{PR}$ & OSMAR FERNANDES DIAS & PDT PR & REC FEDERAL 2010 & $\begin{array}{l}\text { Lote Rural Com 222, } 64 \text { Ha, Da Fazenda Santa } \\
\text { Lucia, Gleba N. } 13 \text { Da Colonia Goioere }\end{array}$ & 222,64 & MÉDIA PROPRIEDADE \\
\hline 410890 & GUAIRACA & $\mathrm{PR}$ & LUIZ CARLOS SETIM & DEMPR & REC FEDERAL 2010 & Terras De Pastagem & 340,00 & GRANDE PROPRIEDADE \\
\hline 410890 & GUAIRACA & $\mathrm{PR}$ & LUIZ CARLOS SETIM & DEMPR & $\begin{array}{l}\text { REC FEDERAL } 2010 \\
\end{array}$ & 3.248.496 Ha De Terra Nua & 324,85 & GRANDE PROPRIEDADE \\
\hline 410890 & GUAIRACA & $\mathrm{PR}$ & LUIZCARLOSSETIM & DEMPR & REC FEDERAL 2010 & Fazenda Ouro Branco & 292,00 & MEDIA PROPRIEDADE \\
\hline 410890 & GUAIRACA & $\mathrm{PR}$ & LUIZCARLOSSETIM & DEMPR & REC FEDERAL 2010 & Terras De Pastagens & 196,00 & MEDIA PROPRIEDADE \\
\hline 410890 & GUAIRACA & $\mathrm{PR}$ & LUIZCARLOS SETIM & DEMPR & REC FEDERAL 2010 & Colonia Paranaval & 96,00 & PEQUENA PROPRIEDADE \\
\hline 410890 & GUAIRACA & $\mathrm{PR}$ & LUIZ CARLOSSETIM & DEMPR & REC FEDERAL 2010 & Trerras De Pastagens & 83,00 & PEQUENA PROPRIEDADE \\
\hline 410890 & GUAIRACA & $\mathrm{PR}$ & LUIZCARLOSSETTM & DEM PR & $\begin{array}{ll}\text { REC FEDERAL } 2010 \\
\end{array}$ & ferra & 48,00 & $\begin{array}{l}\text { PEQUENA PROPRIEDADE } \\
\end{array}$ \\
\hline 410940 & GUARAPUAVA & $\mathrm{PR}$ & $\begin{array}{l}\text { CEZAR AUGUSTO CAROLLO } \\
\text { SILVESTRI }\end{array}$ & PPS PR & REC FEDERAL 2010 & Terreno Rural C/ 258,14 Ha. Faz. Santa Carlota & 258,14 & MÉDIA PROPRIEDADE \\
\hline 410940 & GUARAPUAVA & $\mathrm{PR}$ & $\begin{array}{l}\text { CEZAR AUGUSTO CAROLLO } \\
\text { SILVESTRI }\end{array}$ & $\begin{array}{l}\text { PPS PR } \\
\end{array}$ & REC FEDERAL 2010 & Terreno Rural Faz. Santo Antonio - Goioxim & 147,33 & PEQUENA PROPRIEDADE \\
\hline 410940 & GUARAPUAVA & $\mathrm{PR}$ & $\begin{array}{l}\text { CEZAR AUGUSTO CAROLLO } \\
\text { SILVESTRI }\end{array}$ & PPS PR & REC FEDERAL 2010 & Terreno Rural Faz. Santo Antonio - Goioxim & 131,95 & PEQUENA PROPRIEDADE \\
\hline 410940 & GUARAPUAVA & $\mathrm{PR}$ & $\begin{array}{l}\text { CEZAR AUGUSTO CAROLLO } \\
\text { SILVESTRI }\end{array}$ & PPS PR & REC FEDERAL 2010 & Terreno Rural & 111,78 & MÉDIA PROPRIEDADE \\
\hline 410940 & GUARAPUAVA & $\mathrm{PR}$ & $\begin{array}{l}\text { CEZAR AUGUSTO CAROLLO } \\
\text { SILVESTRI }\end{array}$ & PPS PR & REC FEDERAL 2010 & Terreno Rural Faz. Santa Carlota & 100,02 & PEQUENA PROPRIEDADE \\
\hline 410940 & GUARAPUAVA & $\mathrm{PR}$ & $\begin{array}{l}\text { CEZAR AUGUSTO CAROLLO } \\
\text { SILVESTRI } \\
\end{array}$ & PPS PR & REC FEDERAL 2010 & $\begin{array}{l}\text { Terreno Rural Faz. Dos Porcos Cf. Matric. } 16.388 \\
\text { Do } 1^{\circ} \text { Of. De Guarapuava/Pr,Adq. De Isabel } \\
\text { Virmond De Virmond (Usufruto) }\end{array}$ & 94,38 & PEQUENA PROPRIEDADE \\
\hline 410940 & GUARAPUAVA & $\mathrm{PR}$ & $\begin{array}{l}\text { CEZAR AUGUSTO CAROLLO } \\
\text { SILVESTRI }\end{array}$ & PPS PR & REC FEDERAL 2010 & $\begin{array}{l}\text { Vr. De 50\% De Um Terreno Rural Com 92,13 Ha. } \\
\text { Imóvel Monte Alvão Matrícula № } 19395 \text { Do } 3^{\circ} \\
\text { Ofício de Guarapuava/PR }\end{array}$ & 92,13 & MÉDIA PROPRIEDADE \\
\hline 410940 & GUARAPUAVA & $P R$ & $\begin{array}{l}\begin{array}{l}\text { CEZAR AUGUSTO CAROLLOO } \\
\text { SILVESTRI }\end{array} \\
\end{array}$ & PPS PR & REC FEDERAL 2010 & Terreno Rural, Quinhăo 7 Santa Carlota & 33,14 & PEQUENA PROPRIEDADE \\
\hline 410960 & GUARATUBA & $\mathrm{PR}$ & LUCIANO PIZZATTO & DEM PR & REC FEDERAL 2010 & Terreno Rural & $\mathrm{NI}$ & NÄO INFORMOU (NI) \\
\hline 410960 & GUARATUBA & $\mathrm{PR}$ & MAX ROSENMAM & PSDB PR & REC FEDERAL 2006 & terreno & $\mathrm{NI}$ & NÂO INFORMOU (NI) \\
\hline 411190 & JAGUAPITÃ & $\mathrm{PR}$ & ALEX CANZIANI SILVEIRA & PTB PR & REC FEDERAL 2010 & $\begin{array}{l}\text { Parte Ideal De } 1 / 4 \text { Do Imovel Rural Sovai - 05, } \\
\text { Havido Por Doacăa Dos Avos, A/8 Da Outra } \\
\text { Metade Havida Por Herança Paterna }\end{array}$ & 345,40 & GRANDE PROPRIEDADE \\
\hline 411190 & JAGUAPITÃ & $\mathrm{PR}$ & ALEX CANZIANI SILVEIRA & PTB PR & REC FEDERAL 2010 & 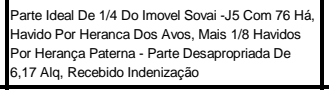 & 90,90 & MÉDIA PROPRIEDADE \\
\hline 411370 & LONDRINA & $\mathrm{PR}$ & ALEX CANZIANI SILVEIRA & PTBPR & REC FEDERAL 2010 & \begin{tabular}{|l|} 
Parte Ideal De $1 / 4$ De 50 De 1.10 Em 18 Chacaras \\
Em Comum Situadas Na Gleba Primavera Havidas \\
Por Herança
\end{tabular} & $\mathrm{NI}$ & NÃO INFORMOU (NI) \\
\hline 411370 & LONDRINA & $\mathrm{PR}$ & ALEX CANZIANI SILVEIRA & PTB PR & REC FEDERAL 2010 & \begin{tabular}{|l|} 
Parte Ideal De $1 / 4$ Do Sitio Santa Rosa Do Viterbio \\
Com 22,5 Alq No Distrito Warta, Havido Por \\
Doaaçao Dos Avos
\end{tabular} & 54,45 & PEQUENA PROPRIEDADE \\
\hline 412820 & UNIÄO DA VITORIA & $\mathrm{PR}$ & AIRTON BERBARDO ROVEDA & PR PR & REC FEDERAL 2010 & $\begin{array}{l}\text { Imovel Rural Sob Mat Nr } 6416 \text { N A Fazenda } \\
\text { Invernadinha }\end{array}$ & $\mathrm{NI}$ & NĀO INFORMOU (NI) \\
\hline 411760 & PALMAS & $\mathrm{PR}$ & AIRTON BERBARDO ROVEDA & PR PR & REC FEDERAL 2010 & $\begin{array}{l}\text { Imovel Rural Com Mat Nrs } 10245 \text { Com } 631 \text { Alq } \\
\text { Fazenda Horizonte }\end{array}$ & 231,98 & MÉDIA PROPRIEDADE \\
\hline 412560 & SÃo MATEUS DO SUL & $\mathrm{PR}$ & AIRTON BERBARDO ROVEDA & PR PR & REC FEDERAL 2010 & $\begin{array}{l}\text { Imovel Rural Loc Na Col Passo Do Iguaçu Dist De } \\
\text { Fluviopolis }\end{array}$ & 18,00 & PEQUENA PROPRIEDADE \\
\hline 410290 & BITURUNA & PR & AIRTON BERBARDO ROVEDA & PR PR & REC FEDERAL 2010 & $\begin{array}{l}\text { Imovel Rural Com Mat Nr } 38673 \text { Fazenda Santa } \\
\text { Barbara }\end{array}$ & $\mathrm{NI}$ & NÃO INFORMOU (NI) \\
\hline 410290 & BITURUNA & $\mathrm{PR}$ & AIRTON BERBARDO ROVEDA & PR PR & REC FEDERAL 2010 & $\begin{array}{l}\text { Imovel Rural Com Mat Nr } 6947 \text { E } 6969 \text { Fazenda } \\
\text { Santa Barbara }\end{array}$ & $\mathrm{NI}$ & NÄO INFORMOU (NI) \\
\hline 411390 & MALLET & $\mathrm{PR}$ & GUSTAVO BONATO FRUET & PMDB PR & REC FEDERAL 2010 & $\begin{array}{l}1 / 12 \text { (Ou 8,333\%) De Imóvel Rural Denominado } \\
\text { Fazenda Palmital }\end{array}$ & $1.136,30$ & GRANDE PROPRIEDADE \\
\hline 411430 & MANDIRITUBA & $\mathrm{PR}$ & IRIS XAVIER SIMŌES & PTB PR & REC FEDERAL 2010 & $\begin{array}{l}\text { Terreno Rural } 1 \text { LITRO E MEIO em Lagoa Dos } \\
\text { Ferreiras }\end{array}$ & $\mathrm{NI}$ & NÃO INFORMOU (NI) \\
\hline 411430 & MANDIRITUBA & $\mathrm{PR}$ & IRIS XAVIER SIMÖES & PTB PR & REC FEDERAL 2010 & Terreno Ref Lotes $02 \mathrm{E} 13 \mathrm{Em}$ Rio Das Antas & $\mathrm{NI}$ & NÃO INFORMOU (NI) \\
\hline 411430 & MANDIRITUBA & $\mathrm{PR}$ & IIRIS XAVIER SIMOES & PTBPR & REC FEDERAL 2010 & Area De Terra em Lagoa Dos Ferreiras & 70,18 & MÉDIA PROPRIEDADE \\
\hline 411430 & MANDIRITUBA & $\mathrm{PR}$ & |RIS XAVIER SIMOES & PTB PR & REC FEDERAL 2010 & \begin{tabular}{|l} 
Terreno Rural Loc Bracatingal \\
\end{tabular} & 21,78 & PEQUENA PROPRIEDADE \\
\hline 411430 & MANDIRITUBA & $\mathrm{PR}$ & IRIS XAVIER SIMOES & PTB PR & REC FEDERAL 2010 & Terreno Rio Da Varzea & 16,94 & PEQUENA PROPRIEDADE \\
\hline 411430 & MANDIRITUBA & $\mathrm{PR}$ & IRIS XAVIER SIMOES & PTBPR & REC FEDERAL 2010 & Terreno Rural Em Lagoa Dos Ferreiras & 7,20 & $\begin{array}{l}\text { PEQUENA PROPRIEDADE } \\
\end{array}$ \\
\hline 411430 & MANDIRITUBA & $\mathrm{PR}$ & IRIS XAVIER SIMÖES & PTB PR & REC FEDERAL 2010 & Terreno Rural em Santo Amaro Areia Branca & 2,42 & MINIFUNDIO \\
\hline 411430 & MANDIRITUBA & $\mathrm{PR}$ & IRIS XAVIER SIITOES & PTBPR & REC FEDERAL 2010 & Area De Terra em Lagoa & 2,42 & MINIFUNDIO \\
\hline 411780 & PALMITAL & $\mathrm{PR}$ & $\begin{array}{l}\text { CEZAR AUGUSTO CAROLLO } \\
\text { SILVESTRI }\end{array}$ & PPS PR & REC FEDERAL 2010 & Terreno Rural Fazenda São Francisco & 398,50 & GRANDE PROPRIEDADE \\
\hline 411780 & PALMITAL & PR & $\begin{array}{l}\text { CEZAR AUGUSTO CAROLLO } \\
\text { SILVESTRI }\end{array}$ & PPS PR & REC FEDERAL 2010 & Terreno Rural Ha. Faz. São Francisco & 162,56 & MÉDIA PROPRIEDADE \\
\hline 411780 & PALMITAL & PR & $\begin{array}{l}\text { CEZAR AUGUSTO CAROLLO } \\
\text { SILVESTRI }\end{array}$ & PPS PR & REC FEDERAL 2010 & Terreno Rural Faz. São Francisco & 40,64 & PEQUENA PROPRIEDADE \\
\hline 411840 & PARANAVAí & $\mathrm{PR}$ & $\begin{array}{l}\text { SERGIO SIQUEIRA DE } \\
\text { CARVALHO } \\
\end{array}$ & PSDB RO & REC FEDERAL 2002 & $\begin{array}{l}\text { 25\% IMÓVEL RURAL FLOR DE MAIO (HAVIDO } \\
\text { POR PARTILHA) }\end{array}$ & 624,40 & GRANDE PROPRIEDADE \\
\hline 411780 & PLANALTINA DO PA & $\mathrm{PR}$ & LUIZCARLOS SETIM & DEM PR & REC FEDERAL 2010 & Terra De Pastagens & 441,00 & GRANDE PROPRIEDADE \\
\hline 411780 & \begin{tabular}{|l} 
PLANALTINA DO PARANA \\
\end{tabular} & $\mathrm{PR}$ & LUIZCARLOS SETIM & DEMPR & $\begin{array}{l}\text { REC FEDERAL } 2010 \\
\end{array}$ & terra & 185,00 & MEDIA PROPRIEDADE \\
\hline 411780 & PLANALTINA DO PARANA & $\mathrm{PR}$ & LUIZCARLOS SETIM & DEMPR & REC FEDERAL 2010 & Terras De Pastagem & 96,00 & PEQUENA PROPRIIEDADE \\
\hline 411990 & PONTA GROSSA & $\mathrm{PR}$ & LUIZCARLOS SETIM & DEMPR & $\begin{array}{l}\text { REC FEDERAL } 2010 \\
\end{array}$ & T Terreno Na Colonia Dona Luiza & $\mathrm{NI}$ & NÁOO INFORMOU (NI) \\
\hline 412000 & PORECATU & $\mathrm{PR}$ & ALVAROFERINANDES DIAS & PSDB PR & RECFEDERAL 2006 & Area Rural De Terras & 144,07 & |MÉDIA PROPRIEDADE \\
\hline 412000 & PORECATU & $\mathrm{PR}$ & ALVAROFERINANDESDIAS & PSDB PR & REC FEDERAL 2006 & Fazenda Barra & 144,00 & MÉDIA PROPRIEDADE \\
\hline 412050 & PRIMEIRO DE MAIO & $\mathrm{PR}$ & LUIS CARLOS JORGE HAULY & PSDB PR & $\begin{array}{l}\text { REC FEDERAL } 2010 \\
\end{array}$ & $1 / 3$ De Chacara Com Benfetitorias & $\mathrm{NI}$ & NÄO INFORMOU (NII) \\
\hline 412135 & RANCHO ALEGRE DO OEST & PR & OSMAR FERNANDES DIAS & PDT PR & REC FEDERAL 2010 & $\begin{array}{l}\text { Lote Rural Com } 116 \text { Alqueires, Fazenda Ipe, } \\
\text { Localizado Em Barreirinha }\end{array}$ & 315,52 & GRANDE PROPRIEDADE \\
\hline 412135 & RANCHO ALEGRE DO OEST & $\mathrm{PR}$ & OSMAR FERNANDES DIAS & PDT PR & REC FEDERAL 2010 & $\begin{array}{l}\text { Lote Rural Com } 3 \text { Alqueires, Fazenda lpe, } \\
\text { Localizado Em Barreirinha }\end{array}$ & 8,16 & MINIFUNDIO \\
\hline 412240 & ROLANDIA & $\mathrm{PR}$ & LUIS CARLOS JORGE HAULY & PSDB PR & REC FEDERAL 2010 & \begin{tabular}{|l|}
$25 \%$ Do Lote De Terras, Gleba Ribeirão Vermelho, \\
Fazenda Ouro Verde
\end{tabular} & 79,61 & PEQUENA PROPRIEDADE \\
\hline 412240 & ROLANDIA & $\mathrm{PR}$ & LUIS CARLOS JORGE HAULY & PSDB PR & REC FEDERAL 2010 & Lote De Terreno Com 2,9 Alqueires Paulistas & 7,00 & PEQUENA PROPRIEDADE \\
\hline 412390 & SANTA MARIANA & $\mathrm{PR}$ & OLAVO BILAC PINTO NETO & PRMG & REC FEDERAL 2010 & Com Cento E Vinte Oito Alqueires Em S Mariana Pr & 309,76 & GRANDE PROPRIEDADE \\
\hline 412390 & SANTA MARIANA & $\mathrm{PR}$ & OLAVO BILAC PINTO NETO & PRMG & REC FEDERAL 2010 & Com Nove E Vinte E Nove Ha Em Mariana Pr (??) & 29,00 & PEQUENA PROPRIEDADE \\
\hline 412410 & SANTO ANTONIO DA PLATIN & PR & $\begin{array}{l}\text { FRANCISCO OTAVIO BECKERT } \\
\text { (CHICO DA PRINCESA) }\end{array}$ & PR PR & RE FEDERAL 2010 & Área & 62,00 & PEQUENA PROPRIEDADE \\
\hline
\end{tabular}




\begin{tabular}{|c|c|c|c|c|c|c|c|c|}
\hline$\frac{412410}{412500}$ & \begin{tabular}{|l} 
SANTO ANTONIO DA PLATI \\
SAO JOAO DO IVAI
\end{tabular} & $\frac{P R}{P R}$ & $\begin{array}{l}\text { FRANCISCO OTAVIO BECKERT } \\
\text { (CHICO DA PRINCESA) } \\
\text { ODILIO BALBIINOIII }\end{array}$ & $\begin{array}{c}\text { PR PR } \\
\text { PMDB PR PR }\end{array}$ & $\begin{array}{l}\text { REC FEDERAL } 2010 \\
\text { REC FEDERAL } 2010\end{array}$ & $\begin{array}{l}\text { Imóvel Rural Sitio Simone } \\
\text { Lote De Terras daFazz. Uba }\end{array}$ & $\frac{24,00}{1,56}$ & $\begin{array}{l}\text { PEQUENA PROPRIEDADE } \\
\text { MMIIIFUNDIO }\end{array}$ \\
\hline 412550 & SÃO JOSE DOS PINHAIS & $\mathrm{PR}$ & $\begin{array}{l}\text { ABELARDO LUIZ LUPIO } \\
\text { MELLO }\end{array}$ & DEM PR & REC FEDERAL 2010 & \begin{tabular}{|l}
$\begin{array}{l}\text { Um Terco De Terras Com B } \\
\text { Boa Vista }\end{array}$ \\
\end{tabular} & $\mathrm{NI}$ & NÃO INFORMOU (NI) \\
\hline 412550 & SÃO JOSE DOS PINHAIS & $\mathrm{PR}$ & $\begin{array}{l}\text { ABEEARDO LUIZ LUPION } \\
\text { MELLO }\end{array}$ & DEMPR & REC FEDERAL 2010 & Area De Terras & $\mathrm{NI}$ & NĀo INFORMOU (NI) \\
\hline
\end{tabular}




\begin{tabular}{|c|c|c|c|c|c|c|c|c|}
\hline GEOCODIGO & MUNICIPIO & UF & PARLAMENTAR & PARTIDO & FONTE & JIMOVEL & ÁREA & |CATEGORIA FUNDIÁRIAA \\
\hline 412550 & SÄO JOSE DOS PINHAIS & $\mathrm{PR}$ & LUIZCARLOSSETIM & DEM PR & REC FEDERAL 2010 & Lote De Terreno Rural & 15,26 & PEQUENA PROPRIEDADE \\
\hline 412550 & SĀO JOSE DOS PINHAIS & $\mathrm{PR}$ & LUIZ CARLOS SETIM & DEM PR & REC FEDERAL 2010 & Terreno Rural & 14,65 & PEQUENA PROPRIEDADE \\
\hline 412550 & SĀO JOSE DOS PINHAIS & $\mathrm{PR}$ & LUIZ CARLOS SETIM & DEMPR & REC FEDERAL 2010 & Area De Terra Nua & 0,73 & MINIFUNDIO \\
\hline 412570 & SÃO MIGUEL DO IGUAÇU & PR & BLAIRO BORGES MAGGI & PRMT & REC FEDERAL 2010 & $\begin{array}{l}\text { 10\% De Uma Chacara N. 128, Uma Csa Em } \\
\text { Alvenaria Com 729,10 M2, Matricula N. } 11.774 \\
\text { S.M. Iguacu - Pr }\end{array}$ & 49,50 & PEQUENA PROPRIEDADE \\
\hline 412570 & SÃO MIGUEL DO IGUAÇU & $\mathrm{PR}$ & BLAIRO BORGES MAGGI & PRMT & REC FEDERAL 2010 & $\begin{array}{l}\text { 10\% De Chacara N. 145, Matrícula 7.094 Do Cri De } \\
\text { S.M. Iguacu }\end{array}$ & 6,30 & MINIFUNDIO \\
\hline 412570 & SÃO MIGUEL DO IGUAÇU & $\mathrm{PR}$ & BLAIRO BORGES MAGGI & PRMT & REC FEDERAL 2010 & $\begin{array}{l}10 \% \text { Um Im[Ovel Rural Parte Chacara } 123 \text { E } 153 \text {, } \\
\text { Matricula 9.154 Cri De S.M. Iguacu - Pr }\end{array}$ & 4,41 & MINIFUNDIO \\
\hline 412570 & SÃo Miguel do IGUAÇU & $\mathrm{PR}$ & BLAIRO BORGES MAGGI & PRMT & REC FEDERAL 2010 & $\begin{array}{l}\text { 10\% De Uma Chacara N. } 129 \text {, Com } 4,395 \mathrm{Ha} \text {, } \\
\text { Matricula N. 9.920 No Cri De S.M. Iguacu - Pr }\end{array}$ & 4,39 & MINIFUNDIO \\
\hline 412570 & SÃo MIGUEL do IGUAÇU & $\mathrm{PR}$ & BLAIRO BORGES MAGGI & PRMT & REC FEDERAL 2010 & \begin{tabular}{|l}
$10 \%$ Um Imóvel Rural Lote 123, Matrícula N. \\
10.367 Do Cri De S.M. Iguacu - Pr
\end{tabular} & 2,90 & MINIFUNDIO \\
\hline 432010 & SARANDI & $\mathrm{PR}$ & ODILLIOB BALBINOTIT & PMDB PR & REC FEDERAL 2010 & Lote De Terras & $\mathrm{NI}$ & NAO INFORMOU (NI) \\
\hline 432010 & SARANDI & $\mathrm{PR}$ & OOILILIOBALBINOTTI & PMDB PR & REC FEDERAL 2010 & Lote De Terras Ruralis & $\overline{\mathrm{NI}}$ & NAO INFORMOU (NI) \\
\hline 432010 & SARANDI & $\mathrm{PR}$ & OOILLIOBALBINOTIT & PMDB PR & REC FEDERAL 2010 & Trerras Ruralis Agricultavels & 96,80 & MÉDIA PROPRIEDADE \\
\hline 412788 & TUNAS DO PARANA & $\mathrm{PR}$ & MAXROSENMAM & PSDB PR & REC FEDERAL 2006 & area de terras & $\mathrm{NI}$ & NÁO INFORMOU (NI) \\
\hline 412788 & TUNAS DO PARANÁ & $\mathrm{PR}$ & MAXROSENIMAM & PSDB PR & REC FEDERAL 2006 & area de terras & $\mathrm{NI}$ & NĀO INFORMOU (NI) \\
\hline 412788 & TUNAS DO PARANA & $\mathrm{PR}$ & |MAX ROSENMAM & PSDB PR & REC FEDERAL 2006 & area de terras & $\mathrm{NI}$ & NAAO INFORMOU (NI) \\
\hline 412810 & UMUARAMA & $\mathrm{PR}$ & OSMAR JOSE SERRAGLIO & PMDB PR & REC FEDERAL 2010 & Lote Rural $14 \mathrm{~N}$, Gleba 12 & $\mathrm{NI}$ & NÄO INFORMOU (NI) \\
\hline 330480 & SÁO FIDELIS & RJ & Paulo Fernando Feijo Torres & PRRJ & REC FEDERAL 2010 & GLEBADE TERRA & 54,00 & MÉDIA PROPRIEDADE \\
\hline 240260 & CEARÁ - MIRIM & $\mathrm{RN}$ & $\begin{array}{l}\text { HENRIQUE EDUARDO LYRA } \\
\text { ALVES }\end{array}$ & PMDB RN & REC FEDERAL 2010 & $\begin{array}{l}50 \% \text { da Propriedade Rural Denominada Fazenda } \\
\text { Gertudes }\end{array}$ & 32,00 & PEQUENA PROPRIEDADE \\
\hline 240430 & GOVERNADOR DIX-SEPT Rd & $\mathrm{RN}$ & $\begin{array}{l}\text { CARLOS ALBERTO DE SOUZA } \\
\text { ROSADO }\end{array}$ & DEM RN & REC FEDERAL 2010 & Propriedade Rural & 554,80 & MÉDIA PROPRIEDADE \\
\hline 240430 & GOVERNADOR DIX-SEPT Rd & RN & $\begin{array}{l}\text { CARLOS ALBERTO DE SOUZA } \\
\text { ROSADO }\end{array}$ & DEM RN & REC FEDERAL 2010 & Propriedade Rural & 115,00 & PEQUENA PROPRIEDADE \\
\hline 240430 & GOVERNADOR DIX-SEPT Rd & $\mathrm{RN}$ & $\begin{array}{l}\text { CARLOS ALBERTO DE SOUZA } \\
\text { ROSADO }\end{array}$ & DEM RN & REC FEDERAL 2010 & Propriedade Rural, Faz. Poco Do Meio & 115,00 & PEQUENA PROPRIEDADE \\
\hline 240560 & JARDIM DE PIRANHAS & $\mathrm{RN}$ & JOÁO DA SILVA MAIA & PRRN & REC FEDERAL 2010 & Na Zona Rural & 29,00 & MIIIIFUNDIO \\
\hline 240560 & JARDIM DE PIRANHAS & $\mathrm{RN}$ & JOÁO DA SILVA MAIA & PRRN & REC FEDERAL 2010 & Parte De Terra Nua & 6,54 & MINIFUNDIO \\
\hline 110140 & MONTE NEGRO & Ro & ERNANDES SANTOS AMORIM & РТВ & REC FEDERAL 2006 & 2 Lotes Rurais Na Br 421 Linha C-35 - Montenegro & $\mathrm{NI}$ & NÃO INFORMOU (NI) \\
\hline 110020 & PORTO VELHO & RO & $\begin{array}{l}\text { JOÄO EDUARDO DADO LEITE } \\
\text { DE CARVALHO }\end{array}$ & PDT SP & REC FEDERAL 2010 & Areas De Terras $583,0 \mathrm{Ha}$ - Porto Velho Ro & 583,00 & MÉDIA PROPRIEDADE \\
\hline 110020 & PORTO VELHO & RO & AGNALDO MUNIZ & PSC RO & REC FEDERAL 2010 & $\begin{array}{l}\text { Terra Rural 68, Setor 5, Gleba Cunia, Margem } \\
\text { Direita Linha C - 20, Terras Centrais, Den. Sitio } \\
\text { Parecis }\end{array}$ & 490,00 & MÉDIA PROPRIEDADE \\
\hline 110020 & PORTO VELHO & RO & AGNALDO MUNIZ & PSC RO & REC FEDERAL 2010 & $\begin{array}{l}\text { Terra Rural, No } 72 \text { (Parte A E B) Den. Fazenda } \\
\text { Cunaa, Ambito Setor 05, Gleba Cunia, Margem } \\
\text { Direita Br 319, Terras Centrais, Altura Km } 44\end{array}$ & 460,00 & MÉDIA PROPRIEDADE \\
\hline 110020 & PORTO VELHO & RO & AGNALDO MUNIZ & PSC RO & REC FEDERAL 2010 & $\begin{array}{l}\text { Terra Rural 75, Setor 5, Gleba Cunia, Margem } \\
\text { Esquerda Linha C-20, Terras Centrais, } \\
\text { Denominaçăo Sitio Indaia }\end{array}$ & 450,00 & MÉDIA PROPRIEDADE \\
\hline 110020 & PORTO VELHO & RO & AGNALDO MUNIZ & PSC RO & REC FEDERAL 2010 & $\begin{array}{l}\text { Terra Rural 77, Setor 5, Gleba Cunia, Margem } \\
\text { Estuuerda Linha C-20, Terras Centrais,Denominado } \\
\text { Sitio Tambaqui }\end{array}$ & 450,00 & MÉDIA PROPRIEDADE \\
\hline 110020 & PORTO VELHO & RO & AGNALDO MUNIZ & PSC RO & REC FEDERAL 2010 & $\begin{array}{l}\text { Terra Rual, } 78 \text {, Setor 5, Gleba Cunhia, Margem } \\
\text { Direita Linha C-20, Denominado Sitio Assai }\end{array}$ & 450,00 & MÉDIA PROPRIEDADE \\
\hline 110020 & PORTO VELHO & RO & AGNALDO MUNIZ & PSC RO & REC FEDERAL 2010 & $\begin{array}{l}\text { Terra Rural, 80/Parte, Setor 05, Gleba Cunia, } \\
\text { Margem Esquerda Linha C-20, Altura Km 5,0, } \\
\text { DENOM Sitio Guaranata }\end{array}$ & 448,00 & MÉDIA PROPRIEDADE \\
\hline 110020 & PORTO VELHO & RO & $\begin{array}{l}\text { JOĀO EDUARDO DADO LEITE } \\
\text { DE CARVALHO }\end{array}$ & PDT SP & REC FEDERAL 2010 & Area De Terras $100 \mathrm{Ha}$ - Porto Velho Ro & 100,00 & PEQUENA PROPRIEDADE \\
\hline 110028 & ROLIM DE MOURA & RO & $\begin{array}{l}\text { EXPEDITO GONÇALVES } \\
\text { FERREIRA JUNIOR }\end{array}$ & PPS RO & REC FEDERAL 2010 & $\begin{array}{l}\text { Lotes De Terras Rurais 34B, 33A e 33B Gleba } 2 \\
\text { Projeto Integracao De Col Jiparana Str }\end{array}$ & 121,00 & PEQUENA PROPRIEDADE \\
\hline 110030 & VILHENA & RO & VALDIR COLATTO & PMDB SC & REC FEDERAL 2010 & Areas De Terras & $1.063,00$ & GRANDE PROPRIEDADE \\
\hline 110030 & VILHENA & $\mathrm{RO}$ & VALDIR COLATTO & PMDB SC & REC FEDERAL 2010 & Area De Terra 1.000 & $1.000,00$ & GRANDE PROPRIEDADE \\
\hline 110030 & VILHENA & RO & VALDIRCOLATTO & PMDBSC & REC FEDERAL 2010 & Area De Terras & 99,73 & PEQUENA PROPRIEDADE \\
\hline 110030 & VILHENA & RO & VALDIRCOLATTO & PMDB SC & REC FEDERAL 2010 & $25 \%$ De Uma Area De Terra & 97,21 & PEQUENA PROPRIEDADE \\
\hline $\mathrm{X}$ & $x$ & $\mathrm{RO}$ & MAURO NAZIF RASUL & PSB RO & REC FEDERAL 2010 & Oor Lotes De Terra Rural & $\mathrm{NI}$ & NAAO INFORMOU (NI) \\
\hline $\mathrm{x}$ & $x$ & RO & MAURO NAZIF RASUL & PSB RO & REC FEDERAL 2010 & for Area De Lote Rural & $\mathrm{NI}$ & NĀO INFORMOU (NI) \\
\hline 140010 & BOA VISTA & $\mathrm{RR}$ & LUIS BARBOSAALVES & PSDB RR & REC FEDERAL 2010 & T/3 STIOHORIZONTE & 343,28 & MÉDIA PROPRIEDADE \\
\hline 140010 & BOA VISTA & $\mathrm{RR}$ & LUIS BARBOSA ALVES & PSDB RR & REC FEDERAL 2010 & 1/3 DAS TERRAS DA FAZ NOVO HORIZONTE & 273,04 & PEQUENA PROPRIEDADE \\
\hline 140010 & BOA VISTA & $\mathrm{RR}$ & LUIS BARBOSA ALVES & PSDB RR & REC FEDERAL 2010 & FAZNOVO HORIZONTE & 273,04 & PEQUENA PROPRIEDADE \\
\hline 140010 & BOAVISTA & $\mathrm{RR}$ & LUIS BARBOSA ALVES & PSDBRR & REC FEDERAL 2010 & 1/3STITOCANAA & 69,60 & MINIFUNDIO \\
\hline 430160 & BAGE & RS & JOSÉ AFONSO EBERT HAMM & PPRS & REC FEDERAL 2010 & Uma Area 01A De Campo No Distrito De Joca & 111,00 & PEQUENA PROPRIEDADE \\
\hline 430160 & BAGE & RS & JOSÉ AFONSO EBERT HAMM & PP RS & REC FEDERAL 2010 & $\begin{array}{l}\text { Uma Area } 5 \text { A De Campo No Distrito De Joca } \\
\text { Tavares }\end{array}$ & 8,00 & MINIFUNDIO \\
\hline 430850 & FREDERICO WESTPHALEN & RS & VILSON LUIZ COVATTI & PP RS & REC FEDERAL 2010 & Uma Fracao De Terras, Na Linha Alto Alegre & 0,77 & MINIFUNDIO \\
\hline 430850 & FREDERICO WESTPHALEN & $\mathrm{RS}$ & VILSON LUIZ COVATTI & PPRS & REC FEDERAL 2010 & Fracao De Terras Na Linha Alto Alegre & 0,74 & MINIFUNDIO \\
\hline 430850 & FREDERICO WESTPHALEN & RS & VILSON LUIZ COVATTI & PP RS & REC FEDERAL 2010 & $\begin{array}{l}\text { Fracao Ideal De Terras No Lote Rural N } 89 \mathrm{Em} \\
\text { Alto Alegre }\end{array}$ & 0,66 & MINIFUNDIO \\
\hline 430850 & FREDERICO WESTPHALEN & RS & VILSON LUIZ COVATTI & PPRS & REC FEDERAL 2010 & $\begin{array}{l}\text { Fracao Ideal De Terras Do Lote Rural } 89 \mathrm{Na} \\
\text { Localidade De Alto Alegre }\end{array}$ & 0,22 & MINIFUNDIO \\
\hline 430920 & Gravataí & RS & PAULO JOSE GOUVEA & PLRS & REC FEDERAL 2002 & \begin{tabular}{|l|}
$\begin{array}{l}\text { Sitio, Repassado A Maristela Nunes De Souza } \\
\text { Como Divisāo De Bens Na Partiliha De Separaçāo } \\
\text { Judicial }\end{array}$ \\
\end{tabular} & 1,00 & MINIFUNDIO \\
\hline 431365 & PALMARES DO SUL & RS & ELISEU LEMOS PADILHA & PMDB RS & REC FEDERAL 2010 & $\begin{array}{l}\text { Trinta E Seis Por Cento Dos Direitos Prossessórios } \\
\text { Fazenda Girivá Em Quintão }\end{array}$ & $\mathrm{NI}$ & NÃO INFORMOU (NI) \\
\hline 510675 & PONTES E LACERDA & RS & ELISEU LEMOS PADILHA & PMDB RS & REC FEDERAL 2010 & $\begin{array}{l}\text { Oitenta Por Cento De Quarenta Hequitares } \\
\text { Sesmaria Da Lavrinha Ou Rosario Municipio De } \\
\text { Pontes E Lacenda Mt }\end{array}$ & $1.853,20$ & GRANDE PROPRIEDADE \\
\hline 431570 & RIO PARDO & RS & NELSON MARCHEZAN JÚNIOR & PSDB RS & REC FEDERAL 2010 & 106 Ha E 1342Mq, Em Capivarita & 106,00 & MÉDIA PROPRIEDADE \\
\hline 431680 & SANTA CRUZ DO SUL & RS & TELMO JOSÉ KIRST & PP RS & REC FEDERAL 1998 & $\begin{array}{l}\text { IMÓVEL EM LINHA JOÄO ALVES, LOC NO } 1^{9} \\
\text { DISTRITO DE SANTA CRUZ DO SUL - CRI } \\
20690\end{array}$ & 18,10 & MINIFUNDIO \\
\hline 431680 & SANTA CRUZ DO SUL & RS & TELMO JOSÉ KIRST & PPRS & REC FEDERAL 1998 & \begin{tabular}{|l} 
IMÓVEL EM LINHA JOÄO ALVES, LOC NO ${ }^{2}$ \\
DISTRITO DE SANTA CRUZ DO SUL - CRI \\
21.965
\end{tabular} & 7,00 & MINIFUNDIO \\
\hline 431680 & SANTA CRUZ DO SUL & $\mathrm{RS}$ & SÉRGIO IVAN MORAES & PTBRS & REC FEDERAL 2010 & 01 Terra Em Carro Alegre & 1,00 & MINIFUNDIO \\
\hline 431695 & SANTA MARIA DO HERVAL & $\mathrm{RS}$ & RENATO DELMAR MOLLING & PPRS & REC FEDERAL 2010 & Parte De Are A De Terras Com $12 \mathrm{Ha} 1 \mathrm{~A}$ & 12,00 & MINIFUNDIO \\
\hline 431760 & SANTO ANTONIO DA PATRU & RS & CEZAR AUGUSTO SCHIRMER & PMDB RS & REC FEDERAL 2008 & ÁREA DE TERRAS & 33,18 & PEQUENA PROPRIEDADE \\
\hline 431760 & SANTO ANTONIO DA PATRY & RS & RENATO DELMAR MOLLING & PP RS & REC FEDERAL 2010 & $\begin{array}{l}\text { Area De Terras Com Galpão De } 10 \text { Metros } \\
\text { Quadrados Em Sertao Do Canta Galo }\end{array}$ & 17,00 & MINIFUNDIO \\
\hline 431760 & SANTO ANTONIO DA PATRL & RS & RENATO DELMAR MOLLING & PPRS & REC FEDERAL 2010 & $\begin{array}{l}\text { Area De Terras Loclaizada Em Sertao Do Canta } \\
\text { Gala }\end{array}$ & 17,00 & MINIFUNDIO \\
\hline 431760 & SANTO ANTONIO DA PATRL & RS & RENATO DELMAR MOLLING & PP RS & REC FEDERAL 2010 & $\begin{array}{l}\text { Area De Terras Localizada Em Sertao Do Canta } \\
\text { Galo }\end{array}$ & 3,00 & MINIFUNDIO \\
\hline
\end{tabular}




\begin{tabular}{|c|c|c|c|c|c|c|c|c|}
\hline GEOCODIGO & MUNICIPIO & UF & PARLAMENTAR & PARTIDO & FONTE & IIMOVEL & ÁREA & CATEGORIA FUNDIÁRIA \\
\hline 431800 & SÃ̃O BORJA & RS & LUIZ CARLOS HEINZE & PPRS & REC FEDERAL 2010 & $\begin{array}{l}\text { Uma Fracao De Campos E Matos, Lugar Den. } \\
\text { Rincao Do Inhatium }\end{array}$ & 175,00 & MÉDIA PROPRIEDADE \\
\hline 431800 & SÃO BORJA & RS & LUIZ CARLOS HEINZE & PP RS & REC FEDERAL 2010 & $\begin{array}{l}\text { Uma Fracao De Campos E Matos,Lugar } \\
\text { Denominado Rincao Do Inhatuim }\end{array}$ & 175,00 & MÉDIA PROPRIEDADE \\
\hline 431800 & SÃO BORJA & RS & LUIZ CARLOS HEINZE & PP RS & REC FEDERAL 2010 & $\begin{array}{l}\text { Uma Fracao De Campos E Matos, Lugar } \\
\text { Denominado Santo Inacio E Angico }\end{array}$ & 162,00 & MÉDIA PROPRIEDADE \\
\hline 431800 & SÃO BORJA & RS & LUIZ CARLOS HEINZE & PP RS & REC FEDERAL 2010 & $\begin{array}{l}\text { Uma Fracao De Campos E Matos, Lugar Den. } \\
\text { Rincao Do Inhatium }\end{array}$ & 147,00 & MÉDIA PROPRIEDADE \\
\hline 431800 & SÃO BORJA & RS & LUIZ CARLOS HEINZE & PPRS & REC FEDERAL 2010 & $\begin{array}{l}\text { Uma Fracao De Campos E Matos, Lugar Den. } \\
\text { Santo Inacio E Angico }\end{array}$ & 138,00 & MÉDIA PROPRIEDADE \\
\hline 431800 & SÃO BORJA & RS & LUIZ CARLOS HEINZE & PPRS & REC FEDERAL 2010 & $\begin{array}{l}\text { Uma Fracao De Campos E Matos Lugar Den. } \\
\text { Rincao Do Inhatium }\end{array}$ & 110,00 & MÉDIA PROPRIEDADE \\
\hline 431800 & SÃO BORJA & RS & LUIZ CARLOS HEINZE & PP RS & REC FEDERAL 2010 & $\begin{array}{l}\text { Uma Fracao De Campos E Matos, Lugar Den. } \\
\text { Rincao Inhatium }\end{array}$ & 101,00 & MÉDIA PROPRIEDADE \\
\hline 431800 & SÃO BORJA & RS & LUIZ CARLOS HEINZE & PPRS & REC FEDERAL 2010 & $\begin{array}{l}\text { Uma Fracao De Campos E Matos Situada No } \\
\text { Primeiro Distrito }\end{array}$ & 67,00 & PEQUENA PROPRIEDADE \\
\hline 431800 & SÃO BORJA & RS & LUIZ CARLOS HEINZE & PPRS & REC FEDERAL 2010 & $\begin{array}{l}\text { Uma Fracao De Campos E Matos Lugar Den. } \\
\text { Rincao Do Inhatium }\end{array}$ & 62,00 & PEQUENA PROPRIEDADE \\
\hline 431800 & SÃO BORJA & RS & LUIZ CARLOS HEINZE & PP RS & REC FEDERAL 2010 & $\begin{array}{l}\text { Uma Fracao De Campos E Matos Lugar Den. } \\
\text { Rincao Do Inhatium }\end{array}$ & 60,00 & PEQUENA PROPRIEDADE \\
\hline 431800 & SÃO BORJA & RS & LUIZ CARLOS HEINZE & PPRS & REC FEDERAL 2010 & Uma Fracao De Campos E Matos, Den.Timbauva & 58,00 & PEQUENA PROPRIEDADE \\
\hline 431800 & SÃO BORJA & RS & LUIZ CARLOS HEINZE & PPRS & REC FEDERAL 2010 & $\begin{array}{l}\text { Uma Fracao De Campos E Matos, Lugar Den. } \\
\text { Rincao Das Tunas }\end{array}$ & 49,00 & PEQUENA PROPRIEDADE \\
\hline 431800 & SÃO BORJA & RS & LUIZ CARLOS HEINZE & PP RS & REC FEDERAL 2010 & $\begin{array}{l}\text { 1/3 De Uma Fracao De Campos Lugar Den. } \\
\text { Rincao Do Inhatium }\end{array}$ & 35,00 & PEQUENA PROPRIEDADE \\
\hline 431800 & SÃO BORJA & RS & LUIZ CARLOS HEINZE & PP RS & REC FEDERAL 2010 & $\begin{array}{l}\text { Uma Fracao De Campos E Matos, Lugar } \\
\text { Denominado Santa Clara }\end{array}$ & 31,00 & PEQUENA PROPRIEDADE \\
\hline 431800 & SÃO BORJA & RS & LUIZ CARLOS HEINZE & PP RS & REC FEDERAL 2010 & $\begin{array}{l}\text { Uma Fracao De Campos E Matos Lugar Den. } \\
\text { Rincao Do Inhatium }\end{array}$ & 30,00 & PEQUENA PROPRIEDADE \\
\hline 431800 & SÃO BORJA & RS & LUIZ CARLOS HEINZE & PP RS & REC FEDERAL 2010 & $\begin{array}{l}\text { Uma Fracao De Campos E Matos Com 29Ha 04A } \\
\text { ittuada No } 10 \text { Distrito. Em Nome Da Filha Natalia } \\
\text { Batista Heinze }\end{array}$ & 29,00 & PEQUENA PROPRIEDADE \\
\hline 431800 & SÃ̃O BORJA & RS & LUIZ CARLOS HEINZE & PP RS & REC FEDERAL 2010 & $\begin{array}{l}\text { 1/3 de uma Fracao De Campos Lugar Den. Rincao } \\
\text { Do Inhatium }\end{array}$ & 27,00 & PEQUENA PROPRIEDADE \\
\hline 431800 & SÃO BORJA & RS & LUIZ CARLOS HEINZE & PPRS & REC FEDERAL 2010 & $\begin{array}{l}\text { Uma Fracao De Campos E Matos 20Ha Lugar Den. } \\
\text { Rincao Do Inhatium }\end{array}$ & 20,00 & PEQUENA PROPRIEDADE \\
\hline 431800 & SÃO BORJA & RS & LUIZ CARLOS HEINZE & PPRS & REC FEDERAL 2010 & $\begin{array}{l}\text { Uma Fracao De Campos E Matos Den. Rincao Do } \\
\text { Innatium }\end{array}$ & 20,00 & PEQUENA PROPRIEDADE \\
\hline 431800 & SÃO BORJA & RS & LUIZ CARLOS HEINZE & PPRS & REC FEDERAL 2010 & $\begin{array}{l}\text { Uma Fracao De Campo Com Loc. No Rincao Do } \\
\text { Inhatium }\end{array}$ & 18,00 & PEQUENA PROPRIEDADE \\
\hline 431800 & SÃ̃ BORJA & RS & LUIZ CARLOS HEINZE & PP RS & REC FEDERAL 2010 & $\begin{array}{l}\text { Uma Fracao De Campos E Matos Den. Rincao Do } \\
\text { Inhatium }\end{array}$ & 15,00 & MINIFUNDIO \\
\hline 431800 & SÃO BORJA & RS & LUIZ CARLOS HEINZE & PP RS & REC FEDERAL 2010 & $\begin{array}{l}\text { Uma Fracao De Campos E Matos, Lugar Den. } \\
\text { Rincao Do Inhatium }\end{array}$ & 10,00 & MINIFUNDIO \\
\hline 431800 & SÃO BORJA & RS & LUIZ CARLOS HEINZE & PP RS & REC FEDERAL 2010 & $\begin{array}{l}\text { Uma Fracao De Campos, Lugar Denominado Santa } \\
\text { Clara }\end{array}$ & 4,00 & MINIFUNDIO \\
\hline 431880 & SÁO LOURENÇO DO SUL & RS & GIOVANICHERIIII & PDT RS & REC FEDERAL 2010 & movel Rural & $\mathrm{NI}$ & \begin{tabular}{|l|l|} 
NÁO INFORMOU (NII) \\
\end{tabular} \\
\hline $\begin{array}{ll}431880 \\
\end{array}$ & SÁO LOURENCÇO DO SUL & RS & GIOVANICHERIIII & PDTRS & REC FEDERAL 2010 & Propriedade Rural & $\mathrm{NI}$ & NÄO INFORMOU (NI) \\
\hline 431880 & SÁO LOURENÇO DO SUL & RS & GIOVANICHERINII & PDT RS & REC FEDERAL 2010 & 75,4 Ha Terras, Campos, Matos E Lavoura & 75,40 & MEDIA PROPRIEDADE \\
\hline 432232 & TURUÇu & RS & $\begin{array}{l}\text { ADOLFO ANTONIO FETTER } \\
\text { JUNIOR }\end{array}$ & PPB RS & REC FEDERAL 2010 & Uma Fração De Terras, Den. Granja Do Turuçu & 228,90 & MÉDIA PROPRIEDADE \\
\hline 432232 & TURUÇU & RS & $\begin{array}{l}\text { ADOLFO ANTONIO FETTER } \\
\text { JUNIOR }\end{array}$ & PPB RS & REC FEDERAL 2010 & $\begin{array}{l}\text { Uma Fração De Terras, Den. Estancia São } \\
\text { Francisco }\end{array}$ & 228,36 & MÉDIA PROPRIEDADE \\
\hline 432232 & TURUÇU & RS & $\begin{array}{l}\text { ADOLFO ANTONIO FETTER } \\
\text { JUNIOR }\end{array}$ & PPB RS & REC FEDERAL 2010 & $\begin{array}{l}\text { Uma Fração De Terras, Den. Fazenda Do } \\
\text { Adolfinho }\end{array}$ & 141,34 & PEQUENA PROPRIEDADE \\
\hline $\mathrm{x}$ & $\mathrm{x}$ & RS & ELISEU LEMOS PADILHA & PMDB RS & REC FEDERAL 2010 & $\begin{array}{l}\text { Fazanda Girivá Em Quintáo Quarenta Dos Direitos } \\
\text { Prossessorios }\end{array}$ & $\mathrm{NI}$ & NÃO INFORMOU (NI) \\
\hline 420010 & ABELARDO LUZ & $\mathrm{SC}$ & VALDIR COLATTO & PMDB SC & REC FEDERAL 2010 & Parte De Uma Área De Terra & 4,22 & MINIFUNDIO \\
\hline 420010 & ABELARDO LUZ & $\mathrm{sc}$ & $\begin{array}{l}\text { VALDIR COLATTO } \\
\end{array}$ & PMDB SC & REC FEDERAL 2010 & Área De Terras & 0,97 & MINIFUNDIO \\
\hline 420110 & ANITÁPOLIS & sc & $\begin{array}{l}\text { JOÁOA ALBERTO PIZZOLATTI } \\
\text { (JÚNIOR) }\end{array}$ & PPSC & REC FEDERAL 2010 & $80 \%$ De Um Terreno Rural Sem Benfeitorias & 119,75 & MÉDIA PROPRIEDADE \\
\hline 420110 & ANITÁPOLIS & sc & $\begin{array}{l}\begin{array}{l}\text { JOÄO ALBERTO PIZZOLATTI } \\
\text { (JÚNIOR) }\end{array} \\
\end{array}$ & PP SC & REC FEDERAL 2010 & 80\% DE Um Terreno Rural Sem Benfeitoria & 75,00 & PEQUENA PROPRIEDADE \\
\hline 420250 & BOM JARDIM DA SERRA & $\mathrm{sc}$ & RRONALDO JOSE BENEDET & PMDBSC & REC FEDERAL 2010 & Gleba De Terras & 35,00 & PEQUENA PROPRIEDADE \\
\hline 420250 & BOM JARDIM DA SERRA & $\mathrm{sc}$ & RONALDO JOSE BENEDET & PMDB SC & REC FEDERAL 2010 & terreno Rural & 20,00 & PEQUENA PROPRIEDADE \\
\hline 420515 & DOUTOR PEDRINHO & sc & $\begin{array}{l}\begin{array}{l}\text { JOÄO ALBERTO PIZZOLATTI } \\
\text { (JÚNIOR) }\end{array} \\
\end{array}$ & PP SC & REC FEDERAL 2010 & Rural Com 360.200M2 & 36,02 & PEQUENA PROPRIEDADE \\
\hline 420690 & IBIRAMA & sc & JOÃO BATISTA MATOS & PMDB SC & REC FEDERAL 2002 & $\begin{array}{l}1 \text { TERRENO RURAL SITUADO EM ALTO RIO } \\
\text { RAFAEL CEDRO, Escr. N } 070 \text { A } 078 \text { Do LV 020, } \\
\text { F1 } 070 \text { Do Reg Imov. }\end{array}$ & 342,94 & GRANDE PROPRIEDADE \\
\hline 421320 & POMERODE & sc & \begin{tabular}{|l} 
JOÄO ALBERTO PIZZOLATTI \\
(JÚNIOR)
\end{tabular} & PP SC & REC FEDERAL 2010 & LOTE Rural Em Ribeirao Herdt & 9,95 & MINIFUNDIO \\
\hline 421320 & POMERODE & sc & $\begin{array}{l}\text { JOÄO ALBERTO PIZZOLATTI } \\
\text { (JÚNIOR) }\end{array}$ & PP SC & REC FEDERAL 2010 & LOTE Rural No Ribeirao Herdt & 8,00 & MINIFUNDIO \\
\hline 421430 & RANCHO QUEIMADO & sc & $\begin{array}{l}\text { ESPERIDIÄO AMIN HELOU } \\
\text { FILHO }\end{array}$ & PP SC & REC FEDERAL 2010 & $\begin{array}{l}\text { Rural Com Área De 8,3 Hectares Em Rio Da } \\
\text { Schar,, }\end{array}$ & 8,30 & MINIFUNDIO \\
\hline 421760 & SIDEROPOLIS & SC & RONALDO JOSEBENEDET & PMDB SC & REC FEDERAL 2010 & Terreno Rural, Rio Jordăo, & 23,00 & PEQUENA PROPRIEDADE \\
\hline 421760 & SIDERÓPOLIS & Sc & RONALDO JOSÉ BENEDET & PMDB SC & REC FEDERAL 2010 & $50 \%$ De Terreno Rural, Rio Manin & 14,00 & MINIFUNDIO \\
\hline 421760 & SIDERÓPOLIS & $\mathrm{sc}$ & $\begin{array}{l}\text { RONALDO JOSÉ BENEDET } \\
\end{array}$ & PMDB SC & REC FEDERAL 2010 & Terreno Rural, Rio Jordão & 11,00 & MINIFUNDIO \\
\hline 421760 & SIDERÓPOLIS & $\mathrm{SC}$ & RONALDO JOSÉ BENEDET & PMDB SC & REC FEDERAL 2010 & Terreno Rural Rio Jordäo & 11,00 & MINIFUNDIO \\
\hline 421760 & SIDERÓPOLIS & $\mathrm{sc}$ & $\begin{array}{l}\text { RONALDO JOSÉ BENEDET } \\
\end{array}$ & PMDB SC & REC FEDERAL 2010 & Terreno Rural, Secção Jordão & 11,00 & MINIFUNDIO \\
\hline 280030 & ARACAJU & SE & JOSÉ CARLOS MACHADO & PFLSP & REC FEDERAL 2010 & Sítio São Carlos No Mosqueiro ¿ Aracaju/Se & $\mathrm{NI}$ & NÄO INFORMOU (NI) \\
\hline 280230 & FREI PAULO & SE & JOSE CARLOSTMACHADO & PFLSP & REC FEDERAL 2010 & 600\% DaFazenda Salgado Em Frei Paulolse & $\mathrm{NI}$ & NÄO INFORMOU (NII) \\
\hline 280260 & GRACCHO CARDOSO & $\mathrm{SE}$ & $\begin{array}{l}\text { JORGE ALBERTO TELES } \\
\text { PRADO }\end{array}$ & PMDB SE & REC FEDERAL 2006 & $\begin{array}{l}\text { FAZ SÄO JORGE (IMÓVEL RURAL } \\
\text { EXPLORADO) }\end{array}$ & 400,00 & MÉDIA PROPRIEDADE \\
\hline 280260 & GRACCHO CARDOSO & SE & $\begin{array}{l}\text { JORGE ALBERTO TELES } \\
\text { PRADO }\end{array}$ & PMDB SE & REC FEDERAL 2006 & $\begin{array}{l}\text { FAZ SÁO JORGE (IMÓVEL RURAL } \\
\text { EXPLORADO) }\end{array}$ & 400,00 & MÉDIA PROPRIEDADE \\
\hline 280260 & GRACCHO CARDOSO & SE & $\begin{array}{l}\text { JJORGE ALBERTO TELES } \\
\text { PRADO }\end{array}$ & PMDB SE & REC FEDERAL 2006 & $\begin{array}{l}\text { FAZ SERRA GRANDE (IMÓVEL RURAL } \\
\text { EXPLORADO) }\end{array}$ & 80,00 & PEQUENA PROPRIEDADE \\
\hline 280260 & GRACCHO CARDOSO & $\mathrm{SE}$ & $\begin{array}{l}\text { JORGE ALBERTO TELES } \\
\text { PRADO }\end{array}$ & PMDB SE & REC FEDERAL 2006 & $\begin{array}{l}\text { FAZ SERRA GRANDE (IMÓVEL RURAL } \\
\text { EXPLORADO) }\end{array}$ & 80,00 & PEQUENA PROPRIEDADE \\
\hline 280290 & ITABAIANA & SE & JOSÉ CARLOS MACHADO & PFLSP & REC FEDERAL 2010 & $50 \%$ Da Fazenda Telha, Campo Do Brito/tabaiana & $\mathrm{NI}$ & NÃO INFORMOU (NI) \\
\hline 280290 & ITABAIANA & SE & JOSE CARLOSTMACHADO & PFLSP & REC FEDERAL 2010 & Chacara Săo Sivestre Em ltabalana/Se & $\frac{\mathrm{NI}}{\mathrm{N}}$ & NÄO INFORMOU (NI) \\
\hline 280290 & ITABAiana & SE & JOSÉ CARLOS MACHADO & PFLSP & REC FEDERAL 2010 & $\begin{array}{l}\text { Uma Área De Terra No Povoado Batula Em } \\
\text { Itabaiana/Se }\end{array}$ & $\mathrm{NI}$ & NÃO INFORMOU (NI) \\
\hline 280410 & MOITA BONITA & SE & JOÄO BOSCO DA COSTA & PSDB SE & REC FEDERAL 2010 & $\begin{array}{l}\text { Rural } 22824 \text { Hectares Jacoca Moita Bonita (Área } \\
\text { muito grande, pode haver equívoco na informaçăo }\end{array}$ & $\mathrm{NI}$ & NÃO INFORMOU (NI) \\
\hline 280410 & MOITA BONITA & SE & JOAOBOSCO DA COSTA & PSDB SE & REC FEDERAL 2010 & 100 Tarrefas Moita Bonita & 3,00 & \begin{tabular}{|l|l|l|l|l|l} 
MINUNIO \\
\end{tabular} \\
\hline 280410 & MOITA BONITA & SE & JOÄO BOSCO DA COSTA & PSDB SE & REC FEDERAL 2010 & $\begin{array}{l}\text { Silitio Agricola Campo Grande Moita Bonita Se } 50 \\
\text { Tarrefas }\end{array}$ & 1,50 & MINIFUNDIO \\
\hline 280410 & MOITA BONITA & SE & JOAOBOSCODACOSTA & PSDBSE & REC FEDERAL 2010 & Rural 7 Tarefas Motia Bonita & 0,21 & MINIFUNDIO \\
\hline 280410 & MOITA BONITA & SE & JOAOBOSCODACOSTA & PSDBSE & REC FEDERAL 2010 & Sition 2 Tarrefas Moita Bonita & 0,06 & MINIFUNDIO \\
\hline 280420 & MONTE ALEGRE DE SERGIF & SE & JOAO BOSCO DA COSTA & PSDB SE & REC FEDERAL 2010 & 524 Tarreas Monte Santo Monte Alegre & 15,72 & MINIFUNDIO \\
\hline 280450 & NOSSA SENHORA DA GLOR & SE & JOAO BOSCO DACOSTA & PSDBSE & AREC FEDERAL 2010 & 6 Tarrefas Nossa Senhora Da Gloria Se & 0,18 & MINIFUNDIO \\
\hline
\end{tabular}




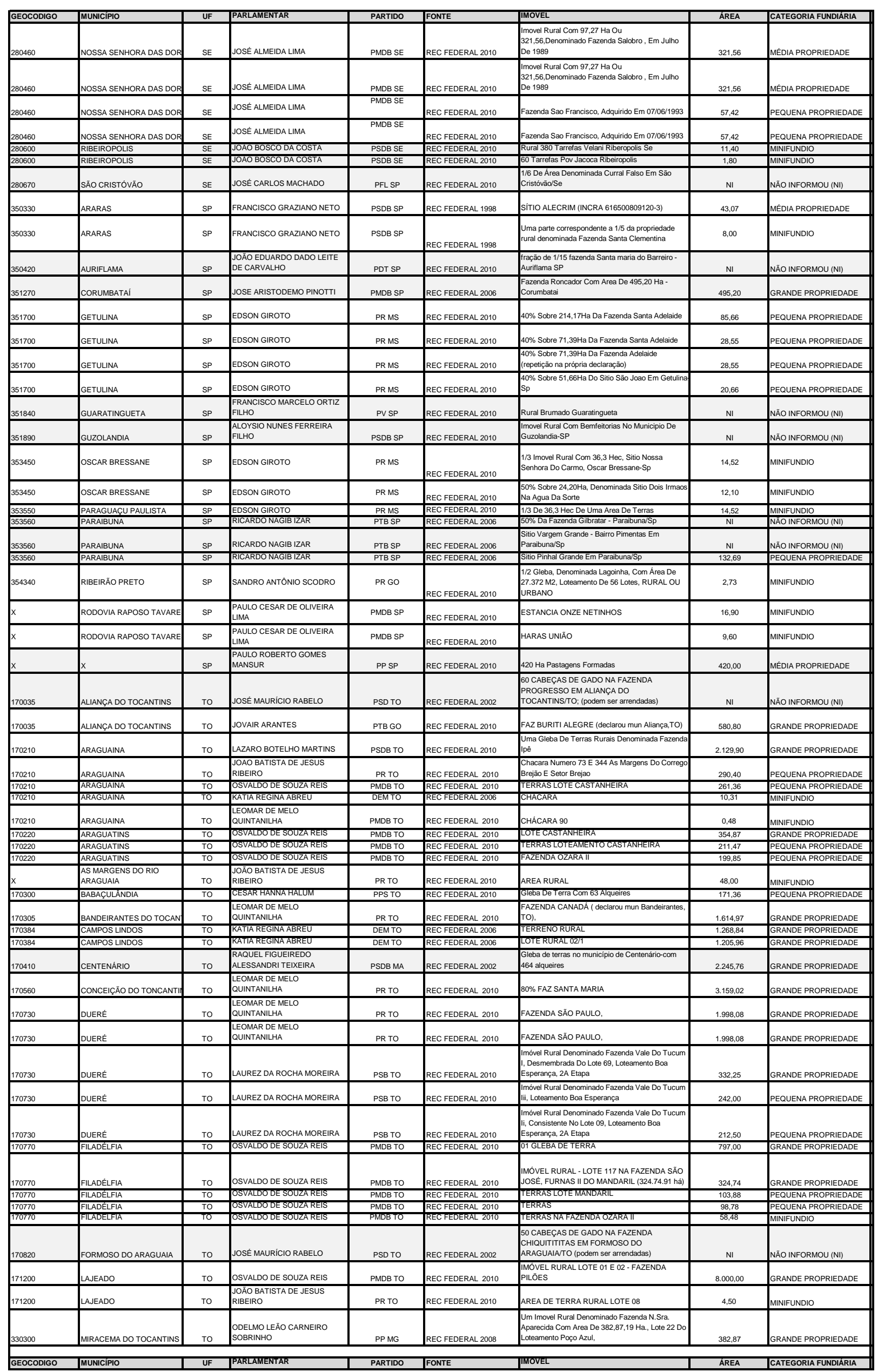




\begin{tabular}{|c|c|c|c|c|c|c|c|c|}
\hline 330300 & MIRACEMA DO TOCANTINS & TO & $\begin{array}{l}\text { ODELMO LEAO CARNEIRO } \\
\text { SOBRINHO }\end{array}$ & PPMG & REC FEDERAL 2008 & $\begin{array}{l}\text { Um Imovel Rural Com Area De 103,34,22 Ha, } \\
\text { Designado Pela Gleba 03, Parte Do Lote } 20 \text { Do } \\
\text { Loteamento Poço Azul }\end{array}$ & 103,34 & PEEQUENA PROPRIEDADE \\
\hline 172100 & PALMAS & то & AMARILDO MARTINS DA SILVA & РРВ ТО & REC FEDERAL 2002 & \begin{tabular}{|l|}
500 Hectares de terras adquirido o direito do \\
loteamento Bom Sossego para ser negociado com o \\
Basa
\end{tabular} & 500,00 & MÉDIA PROPRIEDADE \\
\hline 172100 & PALMAS & то & $\begin{array}{l}\text { LEOMAR DE MELO } \\
\text { QUINTANILHA }\end{array}$ & PRTO & REC FEDERAL 2010 & CHÁCARA № 38 , SETOR LESTE, & 9,67 & MINIFUNDIO \\
\hline 172100 & Palmas & то & $\begin{array}{l}\text { RAQUEL FIGUEIREDO } \\
\text { ALESSANDRI TEIXEIRA }\end{array}$ & PSDB MA & REC FEDERAL 2002 & $\begin{array}{l}\text { Uma chácara de aproximadamente } 1 \text { alqueire no } \\
\text { município de Palmas }\end{array}$ & 4,84 & MINIFUNDIO \\
\hline 171790 & PONTE ALTA DO TOCANTIN & то & VICENTE ALVES DE OLIVEIRA & PR TO & REC FEDERAL 2010 & $\begin{array}{l}\text { FAZENDA SĀO JUDAS DO BALSAS PONTE } \\
\text { ALTA DO }\end{array}$ & $\mathrm{NI}$ & NÃO INFORMOU (NI) \\
\hline 171820 & PORTO NACIONAL & Tо & AMARILDO MARTINS DA SILVA & РРВ ТO & REC FEDERAL 2002 & $\begin{array}{l}\text { Gleba de terras denominada Fazenda chequinha } \\
\text { (Barra Mansa II) }\end{array}$ & 417,60 & MÉDIA PROPRIEDADE \\
\hline 172030 & SÃO SEBASTIÃO DO TOCAN & то & OSVALDO DE SOUZA REIS & PMDB TO & REC FEDERAL 2010 & $\begin{array}{l}\text { TERRA NA FAZENDA OZARA II, LOTE PRAIA } \\
\text { CHATA }\end{array}$ & $1.581,85$ & GRANDE PROPRIEDADE \\
\hline 172030 & SÄOA SEBASTIÄO DO TOCAN & TO & OSVALDO DE SOUZA REIS & PMDB TO & REC FEDERAL 2010 & FAZENDA OZARAIII, LOTE PRAIA CHATA & 503,00 & GRANDE PROPRIEDADE \\
\hline 172030 & SÃO SEBASTIĀO DO TOCAN & то & OSVALDO DE SOUZA REIS & PMDB TO & REC FEDERAL 2010 & LOTE DE TERRAS NA PRAIA CHATA & 423,21 & GRANDE PROPRIEDADE \\
\hline 172030 & SÃO SEBASTIÃO DO TOCAN & Tо & OSVALDO DE SOUZA REIS & PMDB TO & REC FEDERAL 2010 & FAZENDA OZARA IILLOTE PRAIA CHATA & 350,00 & GRANDE PROPRIEDADE \\
\hline 172030 & SÃO SEBASTIÄO DO TOCAN & Tо & OSVALDO DE SOUZA REIS & PMDB TO & REC FEDERAL 2010 & TERRAS LOTE PRAIA CHATA & 334,91 & GRANDE PROPRIEDADE \\
\hline 172030 & SÃO SEBASTIÄO DO TOCAN & то & OSVALDO DE SOUZA REIS & PMDB TO & REC FEDERAL 2010 & TERRAS LOTE PRAIA CHATA & 130,45 & PEQUENA PROPRIEDADE \\
\hline 172030 & $\begin{array}{l}\text { SAO SEBASTIAO DO } \\
\text { TOCANTINS }\end{array}$ & то & OSVALDO DE SOUZA REIS & РMDB TO & REC FEDERAL 2010 & $\begin{array}{l}\text { TERRAS NA FAZENDA OZARA III, LOTE PRAIA } \\
\text { CHATA (mun Säo Sebastiâo,T) }\end{array}$ & 62,00 & MINIFUNDIO \\
\hline 172030 & $\begin{array}{l}\text { SAO SEBASTIAO DO } \\
\text { TOCANTINS }\end{array}$ & то & OSVALDO DE SOUZA REIS & PMDB TO & REC FEDERAL 2010 & TERRAS LOTE PRAIA CHATA & 30,22 & MINIFUNDIO \\
\hline 172030 & $\begin{array}{l}\text { SAO SEBASTIAO DO } \\
\text { TOCANTINS }\end{array}$ & то & OSVALDO DE SOUZA REIS & PMDB TO & REC FEDERAL 2010 & TERRAS & 28,06 & MINIFUNDIO \\
\hline $\mathrm{x}$ & $x$ & то & VICENTE ALVES DE OLIVEIRA & PR TO & REC FEDERAL 2010 & $\begin{array}{l}\text { FAZENDA SAO PEDRO COM } 2864 \text { HECTARES } \\
\text { CONFORME A }\end{array}$ & $2.864,00$ & GRANDE PROPRIEDADE \\
\hline
\end{tabular}

Organizado por: COSTA, S. H. G., 2012 
ANEXO F - TERRAS DAS EMPRESAS VINCULADAS AOS PARLAMENTARES RURALISTAS, INCRA (2003)

\begin{tabular}{|c|c|c|c|c|c|c|c|c|c|c|c|c|}
\hline GEOCÓDIGO & PARLAMENTAR & PARTIDO & FONTE & IMÓVEL & MUNICIPIO & UF & AREA & classe frewewn & $\begin{array}{c}\text { AREA } \\
\text { REGISTRAD } \\
\text { A }\end{array}$ & $\begin{array}{l}\text { DECLARADA } \\
\text { COMO POSSE }\end{array}$ & EMPRESA & VINCULO COM O PARLAMENTAR \\
\hline 120060 & $\begin{array}{l}\text { EDUAADO FRANCISCO } \\
\text { SCIARRA }\end{array}$ & PFL PR & $\begin{array}{l}\text { INCRA } \\
2003\end{array}$ & $\begin{array}{c}\text { GLEBA } \\
\text { PARANACLE }\end{array}$ & TARAUACA & $\mathrm{AC}$ & 195.309 & macomenewe & 224.406 & 0 & $\begin{array}{l}\text { PARANACRE CIA } \\
\text { PARANAENSE DE } \\
\text { COLONIZ AGROP } \\
\text { E IND DO ACRE }\end{array}$ & $\begin{array}{l}\text { EDUARDO FRANCISCO SCIARRA } \\
\text { DECLAROU AÇOES NESTA } \\
\text { EMPRESA }\end{array}$ \\
\hline 270860 & $\begin{array}{l}\text { JOÃO JOSÉ PEREIRA } \\
\text { DE LYRA }\end{array}$ & PTB AL & INCRA 2003 & $\begin{array}{l}\text { FAZENDA } \\
\text { RETIRO }\end{array}$ & $\begin{array}{l}\text { SÁO MIGUEL } \\
\text { DOS CAMPOS }\end{array}$ & AL & 25 & unewaso & 25 & 0 & USINA CAETE SA & $\begin{array}{l}\text { Pertence ao Grupo Carlos Lyra (ex- } \\
\text { senador irmäo de Joäo Lyra) }\end{array}$ \\
\hline 270860 & $\begin{array}{c}\text { JOÃo JOSÉ PEREIRA } \\
\text { DE LYRA }\end{array}$ & PTB AL & $\begin{array}{l}\text { INCRA } \\
2003\end{array}$ & $\begin{array}{l}\text { FAZENDA } \\
\text { RETIRO }\end{array}$ & $\begin{array}{l}\text { SÃO MIGUEL } \\
\text { DOS CAMPOS }\end{array}$ & AL & 39 & & 39 & 0 & USINA CAETE SA & $\begin{array}{l}\text { Pertence ao Grupo Carios Lyra (ex- } \\
\text { senador imäa de Joấ Lyra) }\end{array}$ \\
\hline 270860 & $\begin{array}{c}\text { JOĂo JOSÉ PEREIRA } \\
\text { DE LYRA }\end{array}$ & PTB AL & $\begin{array}{l}\text { INCRA } \\
2003\end{array}$ & $\begin{array}{l}\text { FAZENDA } \\
\text { TABATINGA }\end{array}$ & $\begin{array}{l}\text { SÃO MIGUEL } \\
\text { DOS CAMPOS }\end{array}$ & $\mathrm{AL}$ & 465 & procontense & 0 & 465 & USINA CAETE SA & $\begin{array}{l}\text { Pertence ao Grupo Carlos Lyra (ex- } \\
\text { senador immâo de Joäo Lyra) }\end{array}$ \\
\hline 270860 & $\begin{array}{c}\text { JOÅo JOSÉ PEREIRA } \\
\text { DE LYRA }\end{array}$ & PTB AL & $\begin{array}{l}\text { INCRA } \\
2003\end{array}$ & $\begin{array}{l}\text { FAZENDA CHA } \\
\text { DO OURO }\end{array}$ & \begin{tabular}{|l|} 
SÁO MIGUEL \\
DOS CAMPOS \\
\end{tabular} & AL & 147 & 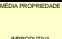 & 0 & 147 & USINA CAETE SA & $\begin{array}{l}\text { Pertence ao Grupo Carlos Lyra (ex- } \\
\text { senador irmāo de Joâo Lyra) }\end{array}$ \\
\hline 270860 & $\begin{array}{c}\text { JOÃo JOSÉ PEREIRA } \\
\text { DE LYRA }\end{array}$ & PTB AL & $\begin{array}{l}\text { INCRA } \\
2003\end{array}$ & $\begin{array}{l}\text { FAZENDA } \\
\text { CAROBAS }\end{array}$ & $\begin{array}{l}\text { SÃO MIGUEL } \\
\text { DOS CAMPOS }\end{array}$ & $\mathrm{AL}$ & 1.742 & proconemase & 0 & 1.742 & USINA CAETE SA & $\begin{array}{l}\text { Pertence ao Grupo Carios Lyra (ex- } \\
\text { senador irmāo de Joäo Lyra) }\end{array}$ \\
\hline 270860 & $\begin{array}{l}\text { JOÃo JOSÉ PEREIRA } \\
\text { DE LYRA. }\end{array}$ & PTB AL & $\begin{array}{l}\text { INCRA } \\
2003\end{array}$ & $\begin{array}{c}\text { FAZENDA PAU } \\
\text { BRASIL }\end{array}$ & $\begin{array}{l}\text { SÁO MIGUEL } \\
\text { DOS CAMPOS }\end{array}$ & AL & 1.800 & procontense & 1.800 & 0 & USINA CAETE SA & $\begin{array}{l}\text { Pertence ao Grupo Carlos Lyra (ex- } \\
\text { senador irmäo de Joäo Lyra) }\end{array}$ \\
\hline 270860 & $\begin{array}{c}\text { JOÃo JOSÉ PEREIRA } \\
\text { DE LYRA }\end{array}$ & PTB AL & $\begin{array}{l}\text { INCRA } \\
2003\end{array}$ & $\begin{array}{l}\text { FAZENDA PAU } \\
\text { AMARELO }\end{array}$ & $\begin{array}{l}\text { SÃO MIGUEL } \\
\text { DOS CAMPOS }\end{array}$ & AL & 381 & Phosirma & 0 & 381 & USINA CAETE SA & $\begin{array}{l}\text { Pertence ao Grupo Carlos Lyra (ex- } \\
\text { senador irmāo de Joâo Lyra) }\end{array}$ \\
\hline 270100 & $\begin{array}{l}\text { JOÅO JOSÉ PEREIRA } \\
\text { DE LYRA }\end{array}$ & PTB AL & $\begin{array}{l}\text { INCRA } \\
2003\end{array}$ & $\begin{array}{l}\text { FAZENDA } \\
\text { RIACHAO }\end{array}$ & BOCA DA MATA & $\mathrm{AL}$ & 46 & & 46 & 0 & USINA CAETE SA & $\begin{array}{l}\text { Pertence ao Grupo Carlos Lyra (ex- } \\
\text { senador irmāo de Joäo Lyra) }\end{array}$ \\
\hline
\end{tabular}




\begin{tabular}{|c|c|c|c|c|c|c|c|c|c|c|c|c|}
\hline GEOCÓDIGO & PARLAMENTAR & PARTIDO & FONTE & IMOVEL & MunicíPIo & uF & AREA & cuassensoniman & \begin{tabular}{|c|} 
AREA \\
REGISTRAD \\
A
\end{tabular} & $\begin{array}{l}\text { DEELAAADA } \\
\text { COMO POSSE }\end{array}$ & EMPRESA & VINCULO COM O PARLAMENTAR \\
\hline 270100 & $\begin{array}{l}\text { JOĂO JOSÉ PERERAR } \\
\text { DE LYRA }\end{array}$ & Pтв AL & $\begin{array}{l}\text { INCRA } \\
2003\end{array}$ & $\begin{array}{l}\text { FAZENDA } \\
\text { RIACHAO }\end{array}$ & BOCA DA MATA & AL & 1.200 & 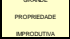 & 1.200 & 0 & USINA CAETE SA & $\begin{array}{l}\text { Pertence ao Ginpo Cartos Lyra (ex- } \\
\text { serador immào de Joôo Lyra) }\end{array}$ \\
\hline 270100 & \begin{tabular}{|c|c|} 
JOÁO JOSÉ PEREERA \\
DE LYRA
\end{tabular} & PtB AL & $\begin{array}{l}\text { INCRA } \\
2003 \\
\end{array}$ & $\begin{array}{l}\text { FAZENDA } \\
\text { RAACHAO }\end{array}$ & BOCA DA MATA & AL & 6 & neswaso & 6 & 0 & USINA CAETE SA & 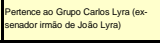 \\
\hline 270230 & $\begin{array}{l}\text { JOÅO JOSÉ PEREIRA } \\
\text { DE LYRA }\end{array}$ & Ртв AL & $\begin{array}{l}\text { INCRA } \\
2003\end{array}$ & \begin{tabular}{|l|} 
FAZENDA SAO \\
JOSE II
\end{tabular} & CoRURIPE & AL & 303 & & 0 & 303 & USINA CAETE SA & $\begin{array}{l}\text { Pertence ao Ginpo Cartos Lyra (ex- } \\
\text { senador imäo de Joầo Lyra) }\end{array}$ \\
\hline 270230 & $\begin{array}{c}\text { JOÃO JOSÉ PEREERA } \\
\text { DE LYRA }\end{array}$ & PtBal & $\begin{array}{l}\text { INCRA } \\
2003\end{array}$ & $\begin{array}{l}\text { SITIO RUA } \\
\text { NOVA }\end{array}$ & CORURIPE & AL & 66 & & 66 & 0 & USINA CAETE SA & $\begin{array}{l}\text { Pertence ao Ginpo Cartos Lyra (ex- } \\
\text { senador immäo de Joäo Lyra) }\end{array}$ \\
\hline 270230 & $\begin{array}{l}\text { JOÅO JOSÉ PEREERA } \\
\text { DE LYRA }\end{array}$ & PTB AL & $\begin{array}{l}\text { INCRA } \\
2003\end{array}$ & $\begin{array}{l}\text { FAZENDA } \\
\text { CABECO }\end{array}$ & CORURIPE & $\mathrm{AL}$ & 143 & & 143 & 0 & USINA CAETE SA & $\begin{array}{l}\text { Pertence ao Ginpo Cartos Lyra (ex- } \\
\text { senador imäo de Joốo Lyra) }\end{array}$ \\
\hline 270230 & \begin{tabular}{|l} 
JOĂO JOSÉ PEREERA \\
DE LYRA
\end{tabular} & PTB AL & $\begin{array}{l}\text { INCRA } \\
2003 \\
\end{array}$ & FAZENDA BOTE & CORURIPE & $\mathrm{AL}$ & 65 & & 65 & 0 & USINA CAETE SA & 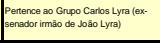 \\
\hline 270430 & $\begin{array}{l}\text { JOÄO JOSÉ PERERA } \\
\text { DE LYRA }\end{array}$ & PtB AL & $\begin{array}{l}\text { INCRA } \\
2003\end{array}$ & $\begin{array}{c}\text { FAZENDA SAO } \\
\text { BENTO }\end{array}$ & MACEIO & AL & 1.254 & 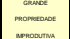 & 1.254 & ${ }^{0}$ & $\begin{array}{l}\text { USINA } \\
\text { CACHOEIRA SA }\end{array}$ & $\begin{array}{l}\text { Pertence ao Ginpo Cartos L Lyra (ex- } \\
\text { senador imáo de Joôo Lyra) }\end{array}$ \\
\hline 270430 & $\begin{array}{l}\text { JOÁO JOSÉ PEREERA } \\
\text { DE LYRA }\end{array}$ & Ptв AL & $\begin{array}{l}\text { INCRA } \\
2003\end{array}$ & $\begin{array}{c}\text { FAZENDA } \\
\text { PEDRA PRETA } \\
\end{array}$ & MACEIO & $\mathrm{AL}$ & 113 & $\begin{array}{l}\text { monomenove } \\
\text { minourra }\end{array}$ & 113 & 0 & $\begin{array}{l}\text { USINA } \\
\text { CACHOEIRA SA }\end{array}$ & $\begin{array}{l}\text { Pertence ao Gupo Caros L Lyra (ex- } \\
\text { serador imäo de Joöo Lyra) }\end{array}$ \\
\hline 2700430 & \begin{tabular}{|l} 
JOĀo JOSÉ PEREERA \\
DE LYRA
\end{tabular} & PTB AL & $\begin{array}{l}\text { INCFA } \\
2003\end{array}$ & $\begin{array}{c}\text { IFMAOS BEUA } \\
\text { FLOR }\end{array}$ & MACÉ́́ & $\mathrm{AL}$ & 120 & phor & 120 & 0 & \begin{tabular}{|c|} 
USINA \\
CACHOEIRA SA \\
\end{tabular} & $\begin{array}{l}\text { Pertenceo ao Grupo Cartos Lyra (ex- } \\
\text { senador imáa de Joâo Lyra) }\end{array}$ \\
\hline 270430 & $\begin{array}{l}\text { JOÅO JOSÉ PERERAR } \\
\text { DE LYRA }\end{array}$ & PTB AL & $\begin{array}{l}\text { INCRA } \\
2003\end{array}$ & \begin{tabular}{|l} 
RIACHAO OU \\
SELA BARRIGA
\end{tabular} & Macelo & AL & 524 & manoreave & 121 & 0 & $\begin{array}{l}\text { USINA } \\
\text { CACHOERA SA }\end{array}$ & $\begin{array}{l}\text { Perterce ao Guppo Cartos Lyra (ex- } \\
\text { senador imāo de Joôo Lyra) }\end{array}$ \\
\hline 270780 & $\begin{array}{c}\text { JOǺ JOSÉ PERERA } \\
\text { DE LYRA }\end{array}$ & PtB AL & $\begin{array}{l}\text { INCRA } \\
2003\end{array}$ & $\begin{array}{l}\text { FAZENDA } \\
\text { MESSIAS }\end{array}$ & ROTEIRO & AL & 275 & & 275 & 0 & USINA CAETE SA & $\begin{array}{l}\text { Pertence ao Ginpo Carlos Lyra (ex- } \\
\text { senador imáa de Joöo Lyra) }\end{array}$ \\
\hline 270060 & $\begin{array}{l}\text { JOÅO JOSÉ PERERA } \\
\text { DE LYRA }\end{array}$ & PtB AL & $\begin{array}{l}\text { INCRA } \\
2003\end{array}$ & $\begin{array}{l}\text { ANDORINHA } \\
\text { ROSA }\end{array}$ & $\begin{array}{l}\begin{array}{l}\text { BARRA DE Sáo } \\
\text { MiGGUL }\end{array} \\
\end{array}$ & AL & 211 & & 211 & 0 & $\begin{array}{c}\text { AGROPECUARIA } \\
\text { LTDA }\end{array}$ & 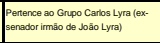 \\
\hline
\end{tabular}




\begin{tabular}{|c|c|c|c|c|c|c|c|c|c|c|c|c|}
\hline GEOCÓDIGO & PARLAMENTAR & PARtioo & FONTE & IMOVEL & Municipio & UF & AREA & 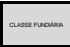 & $\begin{array}{c}\text { AREA } \\
\text { REGISTRAD } \\
\text { A }\end{array}$ & $\begin{array}{l}\text { DECLARADA } \\
\text { COMO POSSE }\end{array}$ & EMPRESA & VINCULO COM O PARLAMENTAR \\
\hline 270060 & $\begin{array}{l}\text { JOĂO JOSÉ PEREERA } \\
\text { DE LYRA }\end{array}$ & PтB AL & $\begin{array}{r}\text { INCRA } \\
2003\end{array}$ & $\begin{array}{l}\text { PERPETUO } \\
\text { SOCORRO }\end{array}$ & \begin{tabular}{|l|} 
BARRA DE SÁO \\
MIGUEL
\end{tabular} & AL & 2 & nerenoso & 2 & 0 & USINA CAETE SA & $\begin{array}{l}\text { Pentence ao Gripo Cartos Lyra (ex- } \\
\text { senador irmào de Joẫo Lyra) }\end{array}$ \\
\hline 270100 & $\begin{array}{c}\text { JOÅO JOSÉ PEREEIRA } \\
\text { DE LYRA }\end{array}$ & Pтв AL & $\begin{array}{l}\text { INCRA } \\
2003 \\
\end{array}$ & SITIO RIACHAO & BOCA DA MATA & AL & 16 & nesenoso & 16 & 0 & USINA CAETE SA & $\begin{array}{l}\text { Pertence ao Ginpo Cartos Lyra (ex- } \\
\text { senador imäo de Joäo Lyra) }\end{array}$ \\
\hline 270230 & $\begin{array}{c}\text { JOÅo JOSÉ PEREERA } \\
\text { DE LYRA }\end{array}$ & PtB AL & $\begin{array}{l}\text { INCRA } \\
2003\end{array}$ & $\begin{array}{l}\text { SITIO RUA } \\
\text { NOVAA }\end{array}$ & CORURIPE & AL & 80 & & 80 & 0 & USINA CAETE SA & 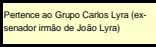 \\
\hline 270780 & $\begin{array}{l}\text { JOÁO JOSÉ PEREE|RA } \\
\text { DE LYRA }\end{array}$ & Pтв AL & $\begin{array}{l}\text { INCRA } \\
2003\end{array}$ & FAZENDA & ROTEIRO & AL & 210 & & 210 & 0 & USINA CAETE SA & $\begin{array}{l}\text { Pertence ao Grupo Caros Lyra (ex- } \\
\text { senador imäo de Joäo Lyya) }\end{array}$ \\
\hline 270780 & $\begin{array}{l}\text { JOÁO JOSÉ PEREERA } \\
\text { DE LYRA }\end{array}$ & PTB AL & $\begin{array}{l}\text { INCRA } \\
2003\end{array}$ & FAZENDA & ROTEIRO & AL & 206 & & 0 & 206 & USINA CAETE SA & 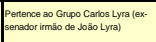 \\
\hline 270780 & $\begin{array}{c}\text { JOĂO JOSÉ PERERARA } \\
\text { DE LYRA }\end{array}$ & PtB AL & $\begin{array}{l}\text { INCRA } \\
2003 \\
\end{array}$ & $\begin{array}{c}\begin{array}{c}\text { ROTEIROE } \\
\text { PECO }\end{array} \\
\end{array}$ & ROTEIRO & AL & 1.006 & 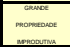 & 1.006 & 0 & USINA CAETE SA & $\begin{array}{l}\text { Pertence ao Grupo Cartos Lyra (ex- } \\
\text { serador imáo de Joöo Lyra) }\end{array}$ \\
\hline 270780 & \begin{tabular}{|l|} 
JOÁo JOSÉ PERERARA \\
DE LYRA
\end{tabular} & PTB AL & $\begin{array}{l}\text { INCRA } \\
2003\end{array}$ & \begin{tabular}{|l|} 
FAZENDA RIO \\
DOMEIO
\end{tabular} & ROTERO & AL & 187 & & 0 & 187 & USINA CAETE SA & 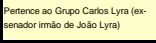 \\
\hline 270860 & $\begin{array}{l}\text { JOÁo JOSÉ PEREERA } \\
\text { DE LYRA }\end{array}$ & Pтв AL & $\begin{array}{c}\text { INCRA } \\
2003 \\
\end{array}$ & \begin{tabular}{|l} 
FAZENDA \\
LAGOA BONITA
\end{tabular} & $\begin{array}{l}\text { SÁ MIGUEL } \\
\text { DOS CAMPOS }\end{array}$ & AL & 1.224 & moromenowe & 1.224 & 0 & USINA CAETE SA & 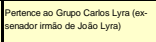 \\
\hline 270860 & $\begin{array}{c}\text { JOÅO JOSÉ PERERARA } \\
\text { DE LYRA }\end{array}$ & PtB AL & $\begin{array}{l}\text { INCRA } \\
2003\end{array}$ & $\begin{array}{c}\text { FAZENDA SAO } \\
\text { JOAO }\end{array}$ & \begin{tabular}{|l} 
SÁO MIGUEL \\
DOS CAMPOS
\end{tabular} & AL & 929 & 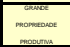 & 929 & 0 & USINA CAETE SA & $\begin{array}{l}\text { Pertence ao Grupo Cartos Lyra (ex- } \\
\text { senador imaáo de Joäo Lyra) }\end{array}$ \\
\hline 270860 & $\begin{array}{l}\text { JOÁo JOSÉ PEREERA } \\
\text { DE LYRA }\end{array}$ & PtB AL & $\begin{array}{c}\text { INCRA } \\
2003 \\
\end{array}$ & $\begin{array}{l}\text { FAZENDA } \\
\text { MORAO }\end{array}$ & $\begin{array}{l}\text { SÁO MIGUEL } \\
\text { DOS CAMPOS }\end{array}$ & AL & 235 & & 217 & 0 & USINA CAETE SA & 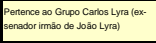 \\
\hline 270660 & $\begin{array}{l}\text { JOÅo JOSÉ PEREERA } \\
\text { DE LYRA }\end{array}$ & PTB AL & $\begin{array}{l}\text { INCRA } \\
2003\end{array}$ & $\begin{array}{l}\text { JOSE RIACHOE } \\
\text { U SUSPEN }\end{array}$ & $\begin{array}{l}\text { SÁO MIGUEL } \\
\text { DOS CAMPOS }\end{array}$ & AL & 4.147 & monomence & 4.147 & 0 & USINA CAETE SA & $\begin{array}{l}\text { Pertence ao Grupo Carbos Lyra (ex- } \\
\text { senador imăo de Joäo Lyra) }\end{array}$ \\
\hline 270860 & $\begin{array}{c}\text { JOĂO JOSE PEREIRA } \\
\text { DE LYRA }\end{array}$ & Pтв AL & $\begin{array}{l}\text { INCRA } \\
2003\end{array}$ & \begin{tabular}{|c|} 
FAZENDA \\
MONTE ALEGRE
\end{tabular} & $\begin{array}{l}\text { SAO MIGUEL } \\
\text { DOS CAMPOS }\end{array}$ & AL & 486 & 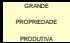 & 0 & 486 & USINA CAETE SA & 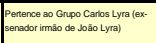 \\
\hline
\end{tabular}




\begin{tabular}{|c|c|c|c|c|c|c|c|c|c|c|c|c|}
\hline GEOCOODIOSO & PARLAMENTAR & PARTIDO & FONTE & IMOVEL & MunicíPIo & uF & AREA & cuassensoniman & \begin{tabular}{|c|} 
AREA \\
REGISTRAD \\
A
\end{tabular} & $\begin{array}{l}\text { DEELAAADA } \\
\text { COMO POSSE }\end{array}$ & EMPRESA & VINCULO COM O PARLAMENTAR \\
\hline 270230 & $\begin{array}{l}\text { JOĂO JOSÉ PEREREA } \\
\text { DE LYRA }\end{array}$ & PTB AL & $\begin{array}{c}\text { INCRA } \\
2003 \\
\end{array}$ & $\begin{array}{l}\text { FAZENDA FLOR } \\
\text { DO SAPUCAY }\end{array}$ & MACEIO & AL & 25 & & 25 & 0 & \begin{tabular}{|l} 
USINA \\
CACHOEIRA SA \\
\end{tabular} & 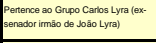 \\
\hline 270230 & \begin{tabular}{|c|c|} 
JOÁO JOSÉ PEREERA \\
DE LYRA
\end{tabular} & PtB AL & $\begin{array}{l}\text { INCRA } \\
2003\end{array}$ & $\begin{array}{l}\text { FAZENDA } \\
\text { SAUDINHA II } \\
\end{array}$ & MACÉ́́ & AL & 1.667 & 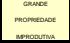 & 836 & 0 & \begin{tabular}{|c|} 
USINA \\
CACHOERA SA \\
\end{tabular} & 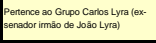 \\
\hline 270230 & $\begin{array}{l}\text { JOÅO JOSÉ PEREIRA } \\
\text { DE LYRA }\end{array}$ & Ртв AL & $\begin{array}{l}\text { INCRA } \\
2003\end{array}$ & $\begin{array}{l}\text { FAZENDA } \\
\text { SAUDINHA II }\end{array}$ & Macelo & AL & 2.824 & Pancreatence & 2.824 & 0 & $\begin{array}{c}\text { USINA } \\
\text { CACHOEIRA SA }\end{array}$ & $\begin{array}{l}\text { Pertence ao Ginpo Carlos Lyra (ex- } \\
\text { senador imäo de Joầo Lyra) }\end{array}$ \\
\hline 270230 & $\begin{array}{c}\text { JOÃO JOSÉ PEREERA } \\
\text { DE LYRA }\end{array}$ & PTBAL & $\begin{array}{l}\text { INCRA } \\
2003\end{array}$ & $\underset{\substack{\text { CACHOEIRA DO } \\
\text { MEIRIM }}}{\text {. }}$ & Maceló & AL & 1.092 & $\begin{array}{l}\text { pnomenture } \\
\text { prosouma }\end{array}$ & 1.092 & 0 & $\begin{array}{l}\text { USINA } \\
\text { CACHOEIRA SA }\end{array}$ & $\begin{array}{l}\text { Pertence ao Ginpo Cartos Lyra (ex- } \\
\text { senador imäo de Joöo Lyra) }\end{array}$ \\
\hline 270230 & $\begin{array}{l}\text { JOÅO JOSÉ PEREERA } \\
\text { DE LYRA }\end{array}$ & PTB AL & $\begin{array}{l}\text { INCRA } \\
2003\end{array}$ & $\begin{array}{l}\text { FAZENDA } \\
\text { GENIPAPO }\end{array}$ & Maceí & $\mathrm{AL}$ & 313 & 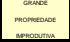 & 313 & 0 & \begin{tabular}{|c|} 
USINA \\
CACHOEIRA SA
\end{tabular} & $\begin{array}{l}\text { Pertence ao Grupo Caros Lyra (ex- } \\
\text { senador imăo de Joỗo Lyra) }\end{array}$ \\
\hline & \begin{tabular}{|l} 
JOĂO JOSÉ PEREERA \\
DE LYRA
\end{tabular} & PTB AL & $\begin{array}{c}\text { INCRA } \\
2003\end{array}$ & $\begin{array}{c}\text { FAZENDA } \\
\text { APOLINARIO }\end{array}$ & PLLAR & $\mathrm{AL}$ & 249 & inocentense & 249 & 0 & USINA CAETE SA & 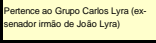 \\
\hline & $\begin{array}{l}\text { JOÄO JOSÉ PERERA } \\
\text { DE LYRA }\end{array}$ & PtB AL & $\begin{array}{l}\text { INCRA } \\
2003\end{array}$ & $\begin{array}{c}\text { FAZENDA BENA } \\
\text { FLOR }\end{array}$ & RIO LARGO & AL & 69 & & 0 & 69 & $\begin{array}{l}\text { USINA } \\
\text { CACHOEIRA SA }\end{array}$ & $\begin{array}{l}\text { Pertence ao Ginpo Cartos L Lyra (ex- } \\
\text { senador imáo de Joôo Lyra) }\end{array}$ \\
\hline 270040 & $\begin{array}{l}\text { JOÁO JOSÉ PEREERA } \\
\text { DE LYRA }\end{array}$ & PTB AL & INCRA 2005 & $\underset{\substack{\text { FAZENDA JOAO } \\
\text { DIAS }}}{-}$ & Atalala & $\mathrm{AL}$ & 342 & 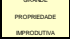 & 342 & 0 & 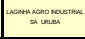 & \\
\hline 270040 & $\begin{array}{l}\text { JOÃO JOSÉ PEREERA } \\
\text { DE LYRA }\end{array}$ & PtB AL & INCRA 2003 & $\begin{array}{l}\text { FAZENDA } \\
\text { PALMEIRA }\end{array}$ & ATALAla & AL & 342 & 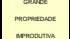 & 342 & 0 & 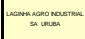 & \\
\hline 270040 & $\begin{array}{l}\text { JOÁO JOSÉ PEREERA } \\
\text { DE LYRA }\end{array}$ & PTB AL & INCRA 2005 & $\begin{array}{c}\text { FAZENDA } \\
\text { URUBA }\end{array}$ & ATALAla & AL & 332 & 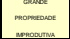 & 332 & 0 & 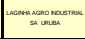 & \\
\hline 270040 & $\begin{array}{c}\text { JOǺ JOSÉ PERERA } \\
\text { DE LYRA }\end{array}$ & PTB AL & INCRA 2005 & $\underset{\substack{\text { FAZENDA AGUA } \\
\text { ENTRA }}}{ }$ & Atalala & $\mathrm{AL}$ & 358 & $\begin{array}{l}\text { monchenewe } \\
\text { rnosuma }\end{array}$ & 358 & 0 & 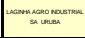 & \\
\hline 270040 & $\begin{array}{l}\text { JOÄO JOSÉ PERERAA } \\
\text { DE LYRA }\end{array}$ & Ptв AL & INCRA 2003 & $\begin{array}{l}\text { FAZENDA } \\
\text { ERMITAO }\end{array}$ & ATALALA & AL & 580 & 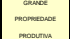 & 580 & 0 & 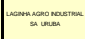 & \\
\hline
\end{tabular}




\begin{tabular}{|c|c|c|c|c|c|c|c|c|c|c|c|c|}
\hline GEOCÓDIGO & PARLAMENTAR & PARTIDO & FONTE & IMOVEL & MunicíPIo & uF & AREA & cuassensoniman & \begin{tabular}{|c|} 
AREA \\
REGISTRAD \\
A
\end{tabular} & $\begin{array}{l}\text { DEELAAADA } \\
\text { COMO POSSE }\end{array}$ & EMPRESA & VINCULO COM O PARLAMENTAR \\
\hline 270040 & $\begin{array}{c}\text { JOÄO JOSÉ PERERA } \\
\text { DE LYRA }\end{array}$ & PTB AL & INCRA 2003 & $\begin{array}{l}\text { FAZENDA } \\
\text { ESPELHO }\end{array}$ & ATALALA & AL & 282 & 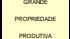 & 282 & 0 & 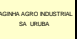 & \\
\hline 270040 & \begin{tabular}{|c|c|c|} 
JOÁO JOSÉ PEREERA \\
DE LYRA
\end{tabular} & PtB AL & INCRA 2003 & $\begin{array}{l}\text { FAZENDA } \\
\text { GRAVATA } \\
\end{array}$ & Atalala & AL & 267 & 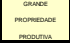 & 267 & 0 & 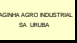 & \\
\hline 270040 & $\begin{array}{c}\text { JOÅO JOSÉ PERERAA } \\
\text { DE LYRA }\end{array}$ & Ртв AL & INCRA 2005 & $\begin{array}{l}\text { FAZENDAA } \\
\text { PARANA }\end{array}$ & AtAlala & AL & 921 & Proverenowe & 913 & 0 & 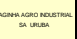 & \\
\hline 270040 & $\begin{array}{c}\text { JOÃO JOSÉ PEREERA } \\
\text { DE LYRA }\end{array}$ & PTBAL & INCRA 2003 & $\begin{array}{l}\text { SANTO } \\
\text { ANTONIO }\end{array}$ & Atalala & AL & 369 & 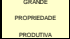 & 369 & 0 & 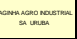 & \\
\hline 270040 & $\begin{array}{c}\text { JOÅO JOSÉ PERERARA } \\
\text { DE LYRA }\end{array}$ & PTB AL & INCRA 2008 & $\begin{array}{l}\text { FAZENDA SAO } \\
\text { FRANCISCO }\end{array}$ & ATALAlA & AL & 457 & Pnonentave & 457 & 0 & 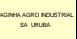 & \\
\hline 270040 & $\begin{array}{c}\text { JOĂO JOSÉ PERERA } \\
\text { DE LYRA }\end{array}$ & PtB AL & INCRA 2003 & $\begin{array}{l}\text { FAZENDA } \\
\text { PUREZA }\end{array}$ & Atalala & $\mathrm{AL}$ & 72 & & 72 & 0 & 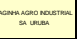 & \\
\hline 270040 & $\begin{array}{c}\text { JOÄO JOSÉ PERERA } \\
\text { DE LYRA }\end{array}$ & PTB AL & INCRA 2003 & $\begin{array}{l}\text { FAZENDA } \\
\text { QUANDU }\end{array}$ & ATALAla & AL & ${ }^{1.078}$ & & 1.078 & ${ }^{0}$ & 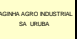 & \\
\hline 270040 & $\begin{array}{l}\text { JOÁO JOSÉ PEREERA } \\
\text { DE LYRA }\end{array}$ & PtBal & INCRA 2005 & $\begin{array}{c}\text { FAZENDA } \\
\text { SANTA RITA } \\
\end{array}$ & Atalala & $\mathrm{AL}$ & 475 & & 475 & 0 & 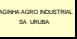 & \\
\hline 270040 & $\begin{array}{c}\text { JOÃO JOSÉ PERERARA } \\
\text { DE LYRA }\end{array}$ & PtB AL & INCRA 2003 & $\begin{array}{c}\text { FAZENDA } \\
\text { CALDEROES }\end{array}$ & ATALAla & AL & 230 & & 230 & 0 & 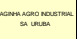 & \\
\hline 270040 & $\begin{array}{c}\text { JOÁO JOSÉ PEREERA } \\
\text { DE LYRA }\end{array}$ & PTB AL & INCRA 2005 & $\begin{array}{c}\text { FAZENDA } \\
\text { PASSAGEM }\end{array}$ & ATALAla & AL & 221 & & 221 & 0 & 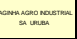 & \\
\hline 270040 & $\begin{array}{c}\text { JOǺ JOSÉ PERERA } \\
\text { DE LYRA }\end{array}$ & PTB AL & INCRA 2005 & $\begin{array}{l}\text { FAZENDA } \\
\text { URUPEMA }\end{array}$ & Atalala & $\mathrm{AL}$ & 236 & & 236 & 0 & 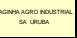 & \\
\hline 270170 & $\begin{array}{l}\text { JOÅO JOSÉ PEREERA } \\
\text { DE LYRA }\end{array}$ & PtB AL & INCRA 2003 & $\begin{array}{l}\text { FAZENDA } \\
\text { TAMOATAI }\end{array}$ & CAPELA & $\mathrm{AL}$ & 195 & & 195 & 0 & 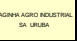 & \\
\hline
\end{tabular}




\begin{tabular}{|c|c|c|c|c|c|c|c|c|c|c|c|c|}
\hline GEOCÓDIGO & PARLAMENTAR & PARTIDO & FONTE & IMOVEL & MunicíPIo & uF & AREA & cuassensoniman & \begin{tabular}{|c|} 
AREA \\
REGISTRAD \\
A
\end{tabular} & $\begin{array}{l}\text { DEELAAADA } \\
\text { COMO POSSE }\end{array}$ & EMPRESA & VINCULO COM O PARLAMENTAR \\
\hline 270230 & $\begin{array}{c}\text { JOÄO JOSÉ PERERA } \\
\text { DE LYRA }\end{array}$ & PTB AL & INCRA 2003 & $\begin{array}{l}\text { FAZENDA SAO } \\
\text { JOAO }\end{array}$ & CORURIPE & AL & 560 & 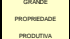 & 560 & 0 & 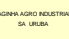 & \\
\hline 270690 & \begin{tabular}{|c|c|c|} 
JOÁO JOSÉ PEREERA \\
DE LYRA
\end{tabular} & PtB AL & INCRA 2003 & $\begin{array}{l}\begin{array}{l}\text { FAZENDA } \\
\text { IMBURY }\end{array} \\
\end{array}$ & PLLAR & AL & 122 & & 122 & 0 & 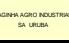 & \\
\hline 270230 & $\begin{array}{c}\text { JOÅO JOSÉ PERERAA } \\
\text { DE LYRA }\end{array}$ & Ртв AL & INCRA 2005 & $\begin{array}{l}\text { FAZENDA } \\
\text { SEGUROI }\end{array}$ & CoRURIPE & AL & 1.145 & Procovenowe & 1.145 & 0 & 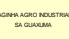 & \\
\hline 270230 & $\begin{array}{c}\text { JOÃO JOSÉ PEREERA } \\
\text { DE LYRA }\end{array}$ & PTBAL & INCRA 2003 & $\begin{array}{l}\text { FAZENDA } \\
\text { TURIACU }\end{array}$ & CORURIPE & AL & 600 & 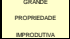 & 600 & 0 & 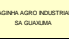 & \\
\hline 270230 & $\begin{array}{l}\text { JOÅO JOSÉ PEREERA } \\
\text { DE LYRA }\end{array}$ & PTB AL & INCRA 2005 & $\begin{array}{l}\text { FAZENDA } \\
\text { CRUZEIRO }\end{array}$ & CORURIPE & AL & 666 & 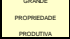 & 600 & 0 & 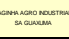 & \\
\hline 270230 & $\begin{array}{c}\text { JOĂO JOSÉ PERERA } \\
\text { DE LYRA }\end{array}$ & PtB AL & INCRA 2003 & $\begin{array}{l}\text { FAZENDA } \\
\text { ESPINHO }\end{array}$ & CORURIPE & $\mathrm{AL}$ & 229 & & 229 & 0 & 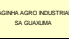 & \\
\hline 270230 & $\begin{array}{c}\text { JOÄO JOSÉ PERERA } \\
\text { DE LYRA }\end{array}$ & PtB AL & INCRA 2003 & $\begin{array}{l}\text { FAZENDA } \\
\text { PEDREIIRAS }\end{array}$ & CORURIPE & AL & 300 & & 303 & ${ }^{0}$ & 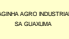 & \\
\hline 270230 & $\begin{array}{l}\text { JOÁO JOSÉ PEREERA } \\
\text { DE LYRA }\end{array}$ & PTB AL & INCRA 2005 & $\begin{array}{c}\text { FAZENDA RUA } \\
\text { NOVAI }\end{array}$ & CORURIPE & $\mathrm{AL}$ & 128 & & 128 & 0 & 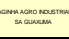 & \\
\hline 270230 & $\begin{array}{c}\text { JOÃO JOSÉ PERERARA } \\
\text { DE LYRA }\end{array}$ & PtB AL & INCRA 2003 & $\begin{array}{c}\text { FAZENDA RUA } \\
\text { NOVA II }\end{array}$ & CORURIPE & AL & 193 & & 193 & 0 & 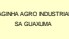 & \\
\hline 270230 & $\begin{array}{c}\text { JOÁO JOSÉ PEREERA } \\
\text { DE LYRA }\end{array}$ & PTB AL & INCRA 2005 & $\begin{array}{c}\text { VARZEA DAS } \\
\text { CANOAS }\end{array}$ & CORURIPE & $\mathrm{AL}$ & 150 & & 150 & 0 & 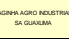 & \\
\hline 270230 & $\begin{array}{c}\text { JOǺ JOSÉ PERERA } \\
\text { DE LYRA }\end{array}$ & PTB AL & INCRA 2005 & $\begin{array}{l}\text { FAZENDA } \\
\text { FORJOS V }\end{array}$ & CORURIPE & $\mathrm{AL}$ & 156 & & 156 & 0 & 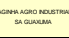 & \\
\hline 270230 & $\begin{array}{l}\text { JOÅO JOSÉ PEREERA } \\
\text { DE LYRA }\end{array}$ & PtB AL & INCRA 2008 & $\begin{array}{l}\text { FAZENDA } \\
\text { FORJOSI }\end{array}$ & CORURIPE & $\mathrm{AL}$ & 161 & & 161 & 0 & 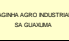 & \\
\hline
\end{tabular}




\begin{tabular}{|c|c|c|c|c|c|c|c|c|c|c|c|c|}
\hline GEOCÓDIGO & PARLAMENTAR & PARTIDO & FONTE & IMOVEL & MunicíPIo & uF & AREA & cuassensoniman & \begin{tabular}{|c|} 
AREA \\
REGISTRAD \\
A
\end{tabular} & $\begin{array}{l}\text { DEELAAADA } \\
\text { COMO POSSE }\end{array}$ & EMPRESA & VINCULO COM O PARLAMENTAR \\
\hline 270230 & $\begin{array}{c}\text { JOÄO JOSÉ PERERA } \\
\text { DE LYRA }\end{array}$ & PTB AL & INCRA 2003 & $\begin{array}{l}\text { FAZENDA } \\
\text { FORNOS II }\end{array}$ & CORURIPE & AL & 167 & & 168 & 0 & 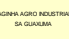 & \\
\hline 270230 & $\begin{array}{c}\text { JOǺ JOSÉ PEREERA } \\
\text { DE LYRA }\end{array}$ & PtB AL & INCRA 2003 & $\begin{array}{c}\text { LAGOA DO PAU } \\
11\end{array}$ & CORURIPE & AL & 378 & & 579 & 0 & 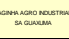 & \\
\hline 270230 & $\begin{array}{c}\text { JOÅO JOSÉ PERERAA } \\
\text { DE LYRA }\end{array}$ & Ртв AL & INCRA 2005 & $\begin{array}{c}\text { LAGOA DO PAU } \\
\vee\end{array}$ & CoRURIPE & AL & 165 & & 152 & 0 & 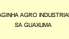 & \\
\hline 270230 & $\begin{array}{c}\text { JOÃO JOSÉ PEREERA } \\
\text { DE LYRA }\end{array}$ & PTBAL & INCRA 2003 & $\begin{array}{c}\text { FAZENDA } \\
\text { SANTA MARIA } \\
\end{array}$ & CORURIPE & AL & 290 & & 290 & 0 & 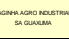 & \\
\hline 270230 & $\begin{array}{l}\text { JOÅO JOSÉ PEREERA } \\
\text { DE LYRA }\end{array}$ & PTB AL & INCRA 2005 & FAZENDA BICAS & CORURIPE & AL & 10 & nesenoso & 10 & 0 & 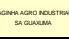 & \\
\hline 270230 & $\begin{array}{c}\text { JOĂO JOSÉ PERERA } \\
\text { DE LYRA }\end{array}$ & PtB AL & INCRA 2003 & $\begin{array}{c}\text { FAZENDA RUA } \\
\text { NOVA III }\end{array}$ & CORURIPE & AL & 18 & nesenoo & 18 & 0 & 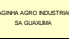 & \\
\hline 270230 & $\begin{array}{c}\text { JOÄO JOSÉ PERERA } \\
\text { DE LYRA }\end{array}$ & PtB AL & INCRA 2003 & $\begin{array}{c}\text { FAZENDA BETA } \\
\text { HARA }\end{array}$ & CORURIPE & AL & 75 & & 75 & ${ }^{0}$ & 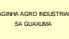 & \\
\hline 270230 & $\begin{array}{l}\text { JOÁO JOSÉ PEREERA } \\
\text { DE LYRA }\end{array}$ & PTB AL & INCRA 2005 & $\begin{array}{l}\text { FAZENDA } \\
\text { FOFNOS III }\end{array}$ & CORURIPE & $\mathrm{AL}$ & 90 & & 91 & 0 & 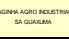 & \\
\hline 270230 & $\begin{array}{c}\text { JOÃO JOSÉ PERERARA } \\
\text { DE LYRA }\end{array}$ & PtB AL & INCRA 2003 & $\begin{array}{l}\text { FAZENDA } \\
\text { FORJOS VII }\end{array}$ & CORURIPE & AL & 63 & & ${ }^{63}$ & 0 & 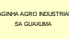 & \\
\hline 270230 & $\begin{array}{c}\text { JOÁO JOSÉ PEREERA } \\
\text { DE LYRA }\end{array}$ & PTB AL & INCRA 2005 & $\begin{array}{l}\text { FAZENDA } \\
\text { FOTJOS IV }\end{array}$ & CORURIPE & AL & 95 & & 95 & 0 & 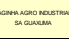 & \\
\hline 270230 & $\begin{array}{c}\text { JOǺ JOSÉ PERERA } \\
\text { DE LYRA }\end{array}$ & PTB AL & INCRA 2005 & $\begin{array}{l}\text { FAZENDA } \\
\text { GRAVATAI }\end{array}$ & CORURIPE & $\mathrm{AL}$ & 62 & & 105 & 0 & 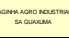 & \\
\hline 270230 & $\begin{array}{l}\text { JOÅO JOSÉ PERERA } \\
\text { DE LYRA }\end{array}$ & Pтв AL & INCRA 200: & $\begin{array}{l}\text { FAZENDA } \\
\text { GRAVATAII }\end{array}$ & CORURIPE & AL & 42 & & 42 & 0 & 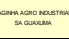 & \\
\hline
\end{tabular}




\begin{tabular}{|c|c|c|c|c|c|c|c|c|c|c|c|c|}
\hline GEOCÓDIGO & PARLAMENTAR & PARTIDO & FONTE & IMOVEL & MunicíPIo & uF & AREA & cuassensoniman & \begin{tabular}{|c|} 
AREA \\
REGISTRAD \\
A
\end{tabular} & $\begin{array}{l}\text { DEELAAADA } \\
\text { COMO POSSE }\end{array}$ & EMPRESA & VINCULO COM O PARLAMENTAR \\
\hline 270230 & $\begin{array}{c}\text { JOÄO JOSÉ PERERA } \\
\text { DE LYRA }\end{array}$ & PTB AL & INCRA 2003 & $\begin{array}{c}\text { FAZENDA } \\
\text { GRAVATA III }\end{array}$ & CORURIPE & AL & 70 & & 70 & 0 & 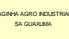 & \\
\hline 270230 & \begin{tabular}{|c|c|c|} 
JOÁO JOSÉ PEREERA \\
DE LYRA
\end{tabular} & PtB AL & INCRA 2003 & $\begin{array}{c}\text { LAGOA DA PAU } \\
\mathrm{V}\end{array}$ & CORURIPE & AL & 99 & & 99 & 0 & 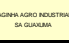 & \\
\hline 270230 & $\begin{array}{c}\text { JOÅO JOSÉ PERERAA } \\
\text { DE LYRA }\end{array}$ & Ртв AL & INCRA 2005 & \begin{tabular}{|c|} 
FAZENDA \\
LAGOA DO PAU I
\end{tabular} & CoRURIPE & AL & 85 & & 85 & 0 & 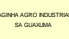 & \\
\hline 270230 & $\begin{array}{c}\text { JOÃO JOSÉ PEREERA } \\
\text { DE LYRA }\end{array}$ & PTBAL & INCRA 2003 & $\begin{array}{c}\text { LAGOA DO PAU } \\
\text { III }\end{array}$ & CORURIPE & AL & 68 & & 80 & 0 & 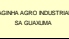 & \\
\hline 270230 & $\begin{array}{l}\text { JOÅO JOSÉ PEREERA } \\
\text { DE LYRA }\end{array}$ & PTB AL & INCRA 2003 & $\begin{array}{c}\text { FAZENDA } \\
\text { PISSARREIRAS } \\
\end{array}$ & CORURIPE & AL & 30 & & 30 & 0 & 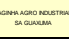 & \\
\hline 270230 & $\begin{array}{c}\text { JOĂO JOSÉ PERERA } \\
\text { DE LYRA }\end{array}$ & PtB AL & INCRA 2003 & $\begin{array}{c}\text { FAZENDA } \\
\text { POXIM }\end{array}$ & CORURIPE & $\mathrm{AL}$ & 53 & & 53 & 0 & 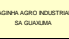 & \\
\hline 270230 & $\begin{array}{c}\text { JOÄO JOSÉ PERERA } \\
\text { DE LYRA }\end{array}$ & PtB AL & INCRA 2003 & $\begin{array}{c}\text { SANTA } \\
\text { CRISTINAI }\end{array}$ & CORURIPE & AL & ${ }^{120}$ & & 120 & ${ }^{0}$ & 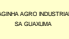 & \\
\hline 270230 & $\begin{array}{l}\text { JOÁO JOSÉ PEREERA } \\
\text { DE LYRA }\end{array}$ & PTB AL & INCRA 2005 & $\begin{array}{c}\text { SANTA } \\
\text { MARLENE }\end{array}$ & CORURIPE & $\mathrm{AL}$ & 45 & & 45 & 0 & 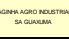 & \\
\hline 270400 & $\begin{array}{c}\text { JOÃO JOSÉ PERERARA } \\
\text { DE LYRA }\end{array}$ & PtB AL & INCRA 2003 & $\underset{\text { MARIA }}{\text { CORACAO DE }}$ & JunOuEiro & AL & 150 & & 150 & 0 & 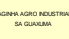 & \\
\hline 270400 & $\begin{array}{c}\text { JOÁO JOSÉ PEREERA } \\
\text { DE LYRA }\end{array}$ & PTB AL & INCRA 2005 & $\begin{array}{c}\text { FAZENDA SSAO } \\
\text { JOSE }\end{array}$ & Junaueiro & $\mathrm{AL}$ & 164 & & 164 & 0 & 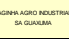 & \\
\hline 270400 & $\begin{array}{c}\text { JOǺ JOSÉ PERERA } \\
\text { DE LYRA }\end{array}$ & PTB AL & INCRA 2005 & $\begin{array}{c}\text { FAZENDA } \\
\text { GRUTA FUNDA } \\
\end{array}$ & Junaueiro & $\mathrm{AL}$ & 30 & nexenaso & 0 & 30 & 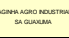 & \\
\hline 270400 & $\begin{array}{c}\text { JOÄO JOSÉ PERERA } \\
\text { DE LYRA }\end{array}$ & PTB AL & INCRA 2003 & $\begin{array}{c}\text { FAZENDA } \\
\text { FOLHA LARGA }\end{array}$ & Junaueiro & AL & 17 & nerenoso & 58 & 0 & 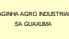 & \\
\hline
\end{tabular}




\begin{tabular}{|c|c|c|c|c|c|c|c|c|c|c|c|c|}
\hline GEOCÓDIGO & PARLAMENTAR & PARTIDO & FONTE & IMOVEL & MunicíPIo & uF & AREA & CATEGORIA & \begin{tabular}{|c|} 
AREA \\
REGISTRAD \\
A
\end{tabular} & $\begin{array}{l}\text { DECLAAADA } \\
\text { COMO POSSE }\end{array}$ & EMPRESA & VINCULO COM O PARLAMENTAR \\
\hline 270400 & $\begin{array}{c}\text { JOÄO JOSÉ PERERA } \\
\text { DE LYRA }\end{array}$ & PTB AL & INCRA 2003 & $\begin{array}{l}\text { JOSE DA } \\
\text { RiBEIRA }\end{array}$ & JunQueiro & AL & 100 & & 100 & 0 & 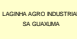 & \\
\hline 270400 & \begin{tabular}{|c|c|c|} 
JOÁO JOSÉ PEREERA \\
DE LYRA
\end{tabular} & PtB AL & INCRA 2003 & $\begin{array}{c}\text { VARZEA DE } \\
\text { CIMA } \\
\end{array}$ & JunOUEIRO & AL & 68 & & 68 & 0 & 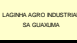 & \\
\hline 270880 & $\begin{array}{c}\text { JOÅO JOSÉ PERERAA } \\
\text { DE LYRA }\end{array}$ & Ртв AL & INCRA 2005 & $\begin{array}{l}\text { FAZENDA } \\
\text { PEDRAS }\end{array}$ & $\begin{array}{l}\text { SÁo } \\
\text { SEBASTIÁo }\end{array}$ & AL & 300 & & 300 & 0 & 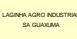 & \\
\hline 270110 & $\begin{array}{c}\text { JOÃO JOSÉ PEREERA } \\
\text { DE LYRA }\end{array}$ & PtBal & INCRA 2003 & $\begin{array}{c}\text { FAZENDA } \\
\text { BEBEDOURO } \\
\end{array}$ & BRANQUINHA & AL & 400 & 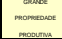 & 400 & 0 & 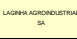 & \\
\hline 270110 & $\begin{array}{l}\text { JOÅO JOSÉ PEREERA } \\
\text { DE LYRA }\end{array}$ & Ptв AL & INCRA 2003 & $\begin{array}{c}\text { FAZENDA } \\
\text { MONTE VERDE }\end{array}$ & BRANQUINHA & AL & 500 & 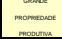 & 500 & 0 & sa & \\
\hline 270110 & $\begin{array}{c}\text { JOĂO JOSÉ PERERA } \\
\text { DE LYRA }\end{array}$ & PtB AL & INCRA 2003 & $\begin{array}{c}\text { FAZENDA } \\
\text { PALMEIRAL }\end{array}$ & BRANOUINHA & AL & 545 & 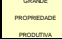 & 475 & 0 & 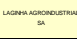 & \\
\hline 270110 & $\begin{array}{c}\text { JOÄO JOSÉ PERERA } \\
\text { DE LYRA }\end{array}$ & PtB AL & INCRA 2003 & $\begin{array}{c}\text { FAZENDA } \\
\text { SANTA TEREZA }\end{array}$ & BRANaUINHA & AL & 330 & 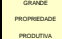 & 330 & ${ }^{0}$ & 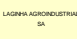 & \\
\hline 270110 & $\begin{array}{c}\text { JOÁO JOSÉ PERERARA } \\
\text { DE LYRA }\end{array}$ & PTB AL & INCRA 2005 & $\begin{array}{c}\text { ANTONIO DA } \\
\text { BOA VISTA } \\
\end{array}$ & BRANQUINHA & $\mathrm{AL}$ & 753 & 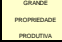 & 753 & 0 & 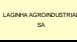 & \\
\hline 270110 & $\begin{array}{c}\text { JOÃO JOSÉ PERERARA } \\
\text { DE LYRA }\end{array}$ & PtB AL & INCRA 2003 & $\begin{array}{c}\text { FAZENDA } \\
\text { BATATEIRA }\end{array}$ & BRANQUINHA & AL & 233 & & ${ }^{233}$ & 0 & 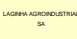 & \\
\hline 270110 & $\begin{array}{c}\text { JOÁO JOSÉ PEREERA } \\
\text { DE LYRA }\end{array}$ & PTB AL & INCRA 2005 & $\begin{array}{l}\text { FAZENDA } \\
\text { SOARES }\end{array}$ & BRANQUINHA & AL & 120 & & 120 & 0 & 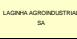 & \\
\hline 270110 & $\begin{array}{c}\text { JOǺ JOSÉ PERERA } \\
\text { DE LYRA }\end{array}$ & PTB AL & INCRA 2005 & $\begin{array}{l}\text { FAZENDA } \\
\text { BRANCA }\end{array}$ & BRANQUINHA & $\mathrm{AL}$ & 100 & & 100 & 0 & 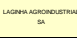 & \\
\hline 270110 & $\begin{array}{l}\text { JOÄO JOSÉ PEREERA } \\
\text { DE LYRA }\end{array}$ & PtB AL & INCRA 2008 & $\begin{array}{c}\text { FAZENDA } \\
\text { CAMPO VERDE }\end{array}$ & BRANQUINHA & AL & 120 & & 120 & 0 & 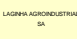 & \\
\hline
\end{tabular}




\begin{tabular}{|c|c|c|c|c|c|c|c|c|c|c|c|c|}
\hline GEOCÓDIGO & PARLAMENTAR & PARTIDO & FONTE & IMOVEL & MunicíPIo & uF & AREA & cuassensoniman & \begin{tabular}{|c|} 
AREA \\
REGISTRAD \\
A
\end{tabular} & $\begin{array}{l}\text { DEELAAADA } \\
\text { COMO POSSE }\end{array}$ & EMPRESA & VINCULO COM O PARLAMENTAR \\
\hline 270110 & $\begin{array}{c}\text { JOÄO JOSÉ PERERA } \\
\text { DE LYRA }\end{array}$ & PTB AL & INCRA 2008 & \begin{tabular}{|l} 
FAZENDA ILHA \\
DAS FLORES
\end{tabular} & BRANaUINHA & AL & 140 & & 140 & 0 & 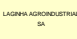 & \\
\hline 270110 & \begin{tabular}{|c|c|c|} 
JOÁO JOSÉ PEREERA \\
DE LYRA
\end{tabular} & PtB AL & INCRA 2003 & $\begin{array}{c}\text { FAZENDA DUAS } \\
\text { BARRAS }\end{array}$ & BRANQUINHA & AL & 28 & & 28 & 0 & 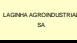 & \\
\hline 270170 & $\begin{array}{c}\text { JOÅO JOSÉ PERERAA } \\
\text { DE LYRA }\end{array}$ & Ртв AL & INCRA 2005 & \begin{tabular}{|c|} 
FAZENDA \\
BONSUCESSO
\end{tabular} & CAPELA & AL & 350 & 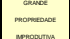 & 450 & 0 & 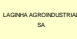 & \\
\hline 270170 & $\begin{array}{c}\text { JOÃO JOSÉ PEREERA } \\
\text { DE LYRA }\end{array}$ & PTB AL & INCRA 2003 & $\begin{array}{c}\text { GRAVATA SAO } \\
\text { SEBASTIAO }\end{array}$ & CAPELA & AL & 1.000 & 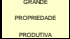 & 998 & 0 & 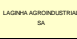 & \\
\hline 2700170 & $\begin{array}{c}\text { JOÅO JOSÉ PERERARA } \\
\text { DE LYRA }\end{array}$ & Ptв AL & INCRA 2003 & $\begin{array}{l}\text { FLORDE } \\
\text { SATUBA }\end{array}$ & CAPELA & AL & 300 & Pnonentave & 300 & 0 & a & \\
\hline 270230 & $\begin{array}{c}\text { JOĂO JOSÉ PERERA } \\
\text { DE LYRA }\end{array}$ & PtB AL & INCRA 2003 & SITIO NOVO & CORURIPE & AL & 290 & & 60 & 230 & 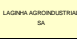 & \\
\hline 270230 & $\begin{array}{c}\text { JOÄO JOSÉ PERERA } \\
\text { DE LYRA }\end{array}$ & PtB AL & INCRA 2003 & $\begin{array}{l}\text { FAZENDA } \\
\text { AROIIIIAA }\end{array}$ & CORURIPE & AL & 226 & & 226 & ${ }^{0}$ & 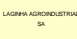 & \\
\hline 270230 & $\begin{array}{l}\text { JOÁO JOSÉ PEREERA } \\
\text { DE LYRA }\end{array}$ & PTB AL & INCRA 2005 & $\begin{array}{l}\text { FAZENDA } \\
\text { ESMANAME }\end{array}$ & CORURIPE & $\mathrm{AL}$ & ${ }^{228}$ & & 228 & 0 & 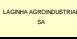 & \\
\hline 270915 & $\begin{array}{c}\text { JOÃO JOSÉ PERERARA } \\
\text { DE LYRA }\end{array}$ & PtB AL & INCRA 2003 & $\begin{array}{c}\text { FAZ CAPOEIRA } \\
\text { DE DENTRO }\end{array}$ & $\begin{array}{l}\text { TEOTOONNIO } \\
\text { VILELAA }\end{array}$ & AL & 28 & nerenaso & 28 & 0 & 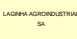 & \\
\hline 270930 & $\begin{array}{c}\text { JOÁO JOSÉ PEREERA } \\
\text { DE LYRA }\end{array}$ & PTB AL & INCRA 2005 & $\begin{array}{l}\text { FAZENDA } \\
\text { MUNDAU }\end{array}$ & $\begin{array}{l}\text { UNIÁ̄o DOS } \\
\text { PALMARES }\end{array}$ & AL & 102 & & 102 & 0 & 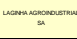 & \\
\hline 270930 & $\begin{array}{c}\text { JOǺ JOSÉ PERERA } \\
\text { DE LYRA }\end{array}$ & PTB AL & INCRA 2005 & $\begin{array}{l}\text { VARZEA } \\
\text { GRANDE }\end{array}$ & $\begin{array}{l}\text { UNMÁ̄o DOS } \\
\text { PALMARES }\end{array}$ & $\mathrm{AL}$ & 100 & & 100 & 0 & 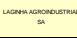 & \\
\hline 270930 & $\begin{array}{c}\text { JOÄO JOSÉ PERERA } \\
\text { DE LYRA }\end{array}$ & Pтв AL & INCRA 2003 & $\begin{array}{l}\text { FAZENDA } \\
\text { ACUCENA }\end{array}$ & $\begin{array}{l}\text { UNIÁo dos } \\
\text { PALMARES }\end{array}$ & AL & 1.020 & Procentense & 1.020 & 0 & 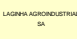 & \\
\hline
\end{tabular}




\begin{tabular}{|c|c|c|c|c|c|c|c|c|c|c|c|c|}
\hline GEOCÓDIGO & PARLAMENTAR & PARTIDO & FONTE & IMOVEL & MunicíPIo & uF & AREA & cuassensoniman & \begin{tabular}{|c|} 
AREA \\
REGISTRAD \\
A
\end{tabular} & $\begin{array}{l}\text { DEELAAADA } \\
\text { COMO POSSE }\end{array}$ & EMPRESA & VINCULO COM O PARLAMENTAR \\
\hline 270930 & $\begin{array}{c}\text { JOÄO JOSÉ PERERA } \\
\text { DE LYRA }\end{array}$ & PTB AL & INCRA 2008 & $\begin{array}{l}\text { FAZENDA } \\
\text { AMOLAR }\end{array}$ & $\begin{array}{l}\text { UNIÁo DOS } \\
\text { PALMARES }\end{array}$ & AL & 462 & 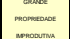 & 462 & 0 & Warst & \\
\hline 270930 & \begin{tabular}{|c|c|c|} 
JOÁO JOSÉ PEREERA \\
DE LYRA
\end{tabular} & PtB AL & INCRA 2003 & $\begin{array}{l}\text { FAZENDA } \\
\text { LAVAGEM }\end{array}$ & $\begin{array}{l}\text { UNAİo DOS } \\
\text { PALMARES }\end{array}$ & AL & 1.843 & 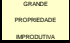 & 1.843 & 0 & 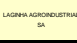 & \\
\hline 270930 & $\begin{array}{c}\text { JOÅO JOSÉ PERERAA } \\
\text { DE LYRA }\end{array}$ & Ртв AL & INCRA 2005 & $\begin{array}{l}\text { ARRAAAL } \\
\text { CABOREGE }\end{array}$ & $\begin{array}{l}\text { UNIÍá DOS } \\
\text { PALMARES }\end{array}$ & AL & 550 & 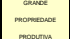 & 89 & 460 & 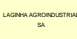 & \\
\hline 270930 & $\begin{array}{c}\text { JOÃO JOSÉ PEREERA } \\
\text { DE LYRA }\end{array}$ & PtBal & INCRA 2003 & $\begin{array}{l}\text { FAZENDA } \\
\text { JACINTO }\end{array}$ & $\begin{array}{l}\text { UNIÄO DOS } \\
\text { PALMARES }\end{array}$ & AL & 533 & 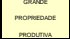 & 300 & 233 & 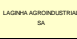 & \\
\hline 270930 & $\begin{array}{c}\text { JOÅO JOSÉ PERERARA } \\
\text { DE LYRA }\end{array}$ & Ptв AL & INCRA 2008 & \begin{tabular}{|c|} 
FAZENDA \\
CAJAZEIRAS
\end{tabular} & $\begin{array}{l}\text { UNIÁO DOS } \\
\text { PALMARES }\end{array}$ & AL & 690 & 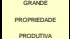 & 630 & 60 & a & \\
\hline 270930 & $\begin{array}{c}\text { JOĂO JOSÉ PERERAR } \\
\text { DE LYRA }\end{array}$ & PtB AL & INCRA 2003 & $\begin{array}{l}\text { GOMES E } \\
\text { SAPUCAIA }\end{array}$ & $\begin{array}{l}\text { UNAIÁO DOS } \\
\text { PALMARES }\end{array}$ & $\mathrm{AL}$ & 463 & 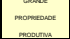 & 463 & 0 & 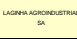 & \\
\hline 270930 & $\begin{array}{c}\text { JOÄO JOSÉ PERERA } \\
\text { DE LYRA }\end{array}$ & PtB AL & INCRA 2003 & \begin{tabular}{|c|} 
FAZENDA \\
LAGINHAIE
\end{tabular} & $\begin{array}{l}\text { UNIÁO DOS } \\
\text { PALMARES }\end{array}$ & AL & 300 & 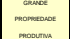 & 300 & ${ }^{0}$ & 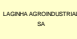 & \\
\hline 270930 & $\begin{array}{c}\text { JOÁO JOSÉ PERERARA } \\
\text { DE LYRA }\end{array}$ & PTB AL & INCRA 2005 & $\begin{array}{c}\text { FAZENDA } \\
\text { MANGABEIRA II }\end{array}$ & $\begin{array}{l}\text { UUNİ̊a DOS } \\
\text { PALMARES }\end{array}$ & $\mathrm{AL}$ & 581 & 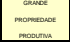 & 581 & 0 & 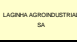 & \\
\hline 270930 & $\begin{array}{c}\text { JOÃO JOSÉ PERERARA } \\
\text { DE LYRA }\end{array}$ & PtB AL & INCRA 2003 & $\begin{array}{l}\text { FAZENDA } \\
\text { PINDOBAL }\end{array}$ & $\begin{array}{l}\text { UNIÄO DOS } \\
\text { PALMARES }\end{array}$ & AL & 250 & 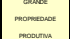 & 250 & 0 & 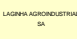 & \\
\hline 270930 & $\begin{array}{c}\text { JOÁO JOSÉ PEREERA } \\
\text { DE LYRA }\end{array}$ & PTB AL & INCRA 2005 & $\begin{array}{c}\text { FAZENDA } \\
\text { TMMBO }\end{array}$ & $\begin{array}{l}\text { UNIÁO DOS } \\
\text { PALMARES }\end{array}$ & $\mathrm{AL}$ & 1.257 & 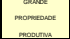 & 1.257 & 0 & 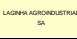 & \\
\hline 270930 & $\begin{array}{c}\text { JOǺ JOSÉ PERERA } \\
\text { DE LYRA }\end{array}$ & PtB AL & INCRA 2005 & $\begin{array}{c}\text { TERRA CAVADA } \\
1\end{array}$ & $\begin{array}{l}\text { UNMÁá DoS } \\
\text { PALMARES }\end{array}$ & $\mathrm{AL}$ & 100 & & 35 & 65 & 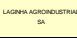 & \\
\hline 270930 & $\begin{array}{c}\text { JOÄO JOSÉ PERERA } \\
\text { DE LYRA }\end{array}$ & Pтв AL & INCRA 2003 & FAZENOA NOVA & $\begin{array}{l}\text { UNIÁo DOS } \\
\text { PaLMARES }\end{array}$ & AL & 70 & & 70 & 0 & 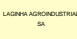 & \\
\hline
\end{tabular}




\begin{tabular}{|c|c|c|c|c|c|c|c|c|c|c|c|c|}
\hline GEOCOODIOSO & PARLAMENTAR & PARTIDO & FONTE & IMOVEL & Municipio & uF & AREA & cuassensacivan & \begin{tabular}{|c|} 
AREA \\
REGISTRAD \\
A
\end{tabular} & $\begin{array}{l}\text { DECLAARADA } \\
\text { COMO POSSE }\end{array}$ & EMPRESA & VINCULO COM O PARLAMENTAR \\
\hline 270930 & $\begin{array}{l}\text { JOÄO JOSÉ PERERARA } \\
\text { DE LYRA }\end{array}$ & PTB AL & INCRA 2003 & $\begin{array}{c}\text { TERRA CAVADA } \\
\text { II }\end{array}$ & $\begin{array}{l}\text { UNIÁO DOS } \\
\text { PALMARES }\end{array}$ & AL & 68 & & 68 & 0 & 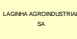 & \\
\hline 270930 & $\begin{array}{l}\text { JOÅO JOSÉ PERERARA } \\
\text { DE LYRA }\end{array}$ & PTB AL & INCRA 2003 & $\begin{array}{c}\text { FAZENDA } \\
\text { VIEIRA }\end{array}$ & \begin{tabular}{|l} 
UnNláo DOS \\
PALMARES
\end{tabular} & AL & 100 & & 0 & 100 & s & \\
\hline 270930 & \begin{tabular}{|l} 
JOÅO JOSÉ PEREERA \\
DE LYRA
\end{tabular} & Pтв AL & INCRA 200: & FAZENDA INGA & $\begin{array}{l}\text { UNIÁO DOS } \\
\text { PALMARES }\end{array}$ & AL & 113 & & 113 & 0 & 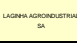 & \\
\hline 270930 & $\begin{array}{l}\text { JOÅO JOSÉ PERERA } \\
\text { DE LYRA }\end{array}$ & PtB AL & INCRA 2003 & $\begin{array}{l}\text { FAZENDA } \\
\text { JACARE }\end{array}$ & $\begin{array}{l}\text { UnNáo DOS } \\
\text { PALMARES }\end{array}$ & AL & 130 & & 130 & 0 & sa & \\
\hline 270930 & $\begin{array}{l}\text { JOÅO JOSÉ PERERARA } \\
\text { DE LYRA }\end{array}$ & PTB AL & INCRA 2005 & SITIO CAIPE & $\begin{array}{l}\text { Uuläo DOS } \\
\text { PALMARES }\end{array}$ & AL & 2 & nerenowo & 14 & 0 & 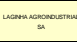 & \\
\hline 270930 & $\begin{array}{l}\text { JOǺ JOSÉ PERERAR } \\
\text { DE LYRA }\end{array}$ & PtB AL & INCRA 2003 & \begin{tabular}{|c|} 
FAZENDA \\
MANGABEIRAS \\
\end{tabular} & \begin{tabular}{|l} 
Unláo dos \\
PaLMARESS
\end{tabular} & AL & 37 & & ${ }^{37}$ & 0 & 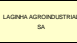 & \\
\hline 270930 & $\begin{array}{l}\text { JOÅO JOSÉ PEREIRA } \\
\text { DE LYRA }\end{array}$ & Pтв AL & INCRA 2003 & $\begin{array}{c}\text { MANGABEIRAS } \\
\text { III }\end{array}$ & $\begin{array}{l}\text { UNMÁO DOS } \\
\text { PALMARES }\end{array}$ & AL & 40 & & ${ }^{40}$ & 0 & 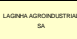 & \\
\hline 270930 & $\begin{array}{l}\text { JOÁO JOSÉ PEREREA } \\
\text { DE LYRA }\end{array}$ & PTB AL & INCRA 2003 & SITIO DO MEIO & $\begin{array}{l}\text { UnNĀo DOS } \\
\text { PALMARES }\end{array}$ & AL & 41 & & 41 & 0 & 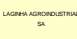 & \\
\hline 270400 & $\begin{array}{l}\text { TEOTÓNIO BRANOÁ } \\
\text { VILELA JUNIOR }\end{array}$ & PSDB AL & $\begin{array}{l}\text { INCRA } \\
2003\end{array}$ & $\begin{array}{l}\text { FAZENDA } \\
\text { CAICARA }\end{array}$ & JunOUEiro & AL & 1.352 & 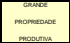 & 1.352 & 0 & $\begin{array}{l}\text { REUNIDAS } \\
\text { SERESTA SA }\end{array}$ & $\begin{array}{l}\text { Teototonio Brandäo Viliel Fiho declarou } \\
\text { aabeses Usinas Reuridas Seresta S.A. }\end{array}$ \\
\hline 270915 & $\begin{array}{l}\text { TEOTÓNIO BRANDß́O } \\
\text { VILLLA JUNIOR }\end{array}$ & PSDB AL & $\begin{array}{l}\text { INCRA } \\
20033\end{array}$ & \begin{tabular}{|l} 
FAZENDA RISCO \\
E PLANALTO
\end{tabular} & $\begin{array}{l}\text { TEOTÓNIO } \\
\text { VILELA }\end{array}$ & AL & 555 & 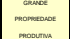 & 555 & 0 & $\begin{array}{l}\text { REUNIDAS } \\
\text { SERESTASA }\end{array}$ & 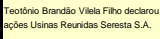 \\
\hline 270915 & $\begin{array}{l}\text { TEOTÓNIO BRAND́̊́O } \\
\text { VILLA JUNIOR }\end{array}$ & PSDB AL & \begin{tabular}{|l}
$\mid$ INCRA \\
2003
\end{tabular} & $\begin{array}{l}\text { FAZENDA } \\
\text { GULANDIM }\end{array}$ & $\begin{array}{l}\text { TEOTOONIO } \\
\text { VILELA }\end{array}$ & AL & 929 & monchencere & 929 & 0 & $\begin{array}{l}\text { REUNIDAS } \\
\text { SERESTA SA }\end{array}$ & $\begin{array}{l}\text { Teotônio Brandáo Vilela Fino declarou } \\
\text { acobes Usinas Reuridas Seresta S.A. }\end{array}$ \\
\hline 270400 & $\begin{array}{l}\text { TEOTÓNIO BRANDḰO } \\
\text { VILELA JUNIOR }\end{array}$ & PSDB AL & $\begin{array}{l}\text { INCRA } \\
2003\end{array}$ & $\begin{array}{l}\text { FAZENDA } \\
\text { MADEIRA }\end{array}$ & JuNQUEIRO & AL & 1.391 & monamenove & 1.391 & 0 & $\begin{array}{l}\text { REUNDAA } \\
\text { SERESTA SA }\end{array}$ & $\begin{array}{l}\text { Teototorio Brandáo Vilela Fino declarou } \\
\text { ag̣oes Usinas Reuridas Seresta S.A. }\end{array}$ \\
\hline
\end{tabular}




\begin{tabular}{|c|c|c|c|c|c|c|c|c|c|c|c|c|}
\hline GEOCODIGO & PARLAMENTAR & PARTIDO & FONTE & IMOVEL & MUNICIPIO & UF & AREA & CLasse funcuiner & $\begin{array}{c}\text { AREA } \\
\text { REGISTRAD } \\
\text { A } \\
\end{array}$ & $\begin{array}{l}\text { DECLARADA } \\
\text { COMO POSSE }\end{array}$ & EMPRESA & VINCULO COM O PARLAMENTAR \\
\hline 270230 & $\begin{array}{l}\text { TEOTOONIO BRANDÄO } \\
\text { VILELA JUNIOR }\end{array}$ & PSDB AL & $\begin{array}{l}\text { INCRA } \\
2003\end{array}$ & $\begin{array}{l}\text { IMBURI DO } \\
\text { MAIAO }\end{array}$ & CORURIPE & AL & 438 & & 438 & 0 & $\begin{array}{l}\text { REUNIDAS } \\
\text { SERESTA SA }\end{array}$ & $\begin{array}{l}\text { Teotônio Brandāo Vilela Fiho declarou } \\
\text { apọes Usinas Reuridas Seresta S.A. }\end{array}$ \\
\hline 270880 & $\begin{array}{l}\text { TEOTOONIO BRANDÁO } \\
\text { VILELA JUNIOR }\end{array}$ & PSDB AL & $\begin{array}{l}\text { INCRA } \\
2003\end{array}$ & $\begin{array}{l}\text { PORTEIRAE T } \\
\text { DE DENTRO }\end{array}$ & $\begin{array}{l}\text { SÁO } \\
\text { SEBASTLĀO }\end{array}$ & AL & 263 & & 263 & 0 & $\begin{array}{l}\text { REUNIDAS } \\
\text { SERESTA SA }\end{array}$ & $\begin{array}{l}\text { Teotónio Brandảo Vilela Fiho declarou } \\
\text { apộes Usinas Reuridas Seresta S.A. }\end{array}$ \\
\hline 270915 & $\begin{array}{l}\text { TEOTÓNIO BRANDÁO } \\
\text { VILELA JUNIOR }\end{array}$ & PSDB AL & $\begin{array}{l}\text { INCRA } \\
2003\end{array}$ & $\begin{array}{l}\text { BRAVAE } \\
\text { BREJAO }\end{array}$ & $\begin{array}{l}\text { TEOTÓNIO } \\
\text { VILELA }\end{array}$ & AL & 390 & 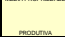 & 390 & 0 & $\begin{array}{l}\text { REUNIDAS } \\
\text { SERESTA SA }\end{array}$ & $\begin{array}{l}\text { Teotônio Brandāo Vilela Fîho declarou } \\
\text { açôes Usinas Reuridas Seresta S.A. }\end{array}$ \\
\hline 270915 & $\begin{array}{c}\text { TEOTOONIO BRANDǺO } \\
\text { VILELA JUNIOR }\end{array}$ & PSDB AL & $\begin{array}{l}\text { INCRA } \\
2003\end{array}$ & $\begin{array}{l}\text { FAZENDA } \\
\text { MUTUNS }\end{array}$ & $\begin{array}{l}\text { TEOTOONIO } \\
\text { VILELA }\end{array}$ & AL & 218 & Prous & 218 & 0 & $\begin{array}{l}\text { REUNIDAS } \\
\text { SERESTA SA }\end{array}$ & $\begin{array}{l}\text { Teotônio Brandão Vilela Fiho declarou } \\
\text { açōes Usinas Reuridas Seresta S.A. }\end{array}$ \\
\hline 270915 & $\begin{array}{c}\text { TEOTOONIO BRANDÁO } \\
\text { VILELA JUNIOR }\end{array}$ & PSDB AL & $\begin{array}{l}\text { INCRA } \\
2003\end{array}$ & FAZENDA PIAUI & $\begin{array}{l}\text { TEOTÓNIO } \\
\text { VILELA }\end{array}$ & AL & 428 & & 429 & 0 & $\begin{array}{l}\text { REUNIDAS } \\
\text { SERESTA SA }\end{array}$ & $\begin{array}{l}\text { Teotônio Brandão Vilela Fiho declarou } \\
\text { açōes Usinas Reuridas Seresta S.A. }\end{array}$ \\
\hline 270915 & $\begin{array}{l}\text { TEOTOONIO BRANDÁOO } \\
\text { VILELA JUNIOR }\end{array}$ & PSDB AL & $\begin{array}{l}\text { INCRA } \\
2003\end{array}$ & $\begin{array}{l}\text { FAZENDA } \\
\text { POUSADA III }\end{array}$ & $\begin{array}{l}\text { TEOTÓNIO } \\
\text { VILELA }\end{array}$ & AL & 242 & proournia & 242 & 0 & $\begin{array}{l}\text { REUNIDAS } \\
\text { SERESTA SA }\end{array}$ & $\begin{array}{l}\text { Teotônio Brandäo Vilela Fitho declarou } \\
\text { aọōes Usinas Reuridas Seresta S.A. }\end{array}$ \\
\hline 270880 & $\begin{array}{l}\text { TEOTÓNIO BRANDÁO } \\
\text { VILELA JUNIOR }\end{array}$ & PSDB AL & $\begin{array}{l}\text { INCRA } \\
2003\end{array}$ & $\begin{array}{l}\text { FAZENDA } \\
\text { BREJINHO }\end{array}$ & $\begin{array}{l}\text { SAOO } \\
\text { SEBASTIÁO }\end{array}$ & AL & 150 & menocontra & 150 & 0 & $\begin{array}{l}\text { REUNIDAS } \\
\text { SERESTA SA }\end{array}$ & $\begin{array}{l}\text { Teotônio Brandão Vilela Fiho declarou } \\
\text { açóes Usinas Reuridas Seresta S.A. }\end{array}$ \\
\hline 270915 & $\begin{array}{l}\text { TEOTOONIO BRANDÄO } \\
\text { VILELA JUNIOR }\end{array}$ & PSDB AL & $\begin{array}{l}\text { INCRA } \\
2003\end{array}$ & $\begin{array}{l}\text { FAZENDA } \\
\text { MARTE JEANE }\end{array}$ & $\begin{array}{l}\text { TEOTÓNIO } \\
\text { VILELA }\end{array}$ & AL & 232 & & 232 & 0 & $\begin{array}{l}\text { REUNIDAS } \\
\text { SERESTA SA }\end{array}$ & $\begin{array}{l}\text { Teotónio Brandão Vileala Fiho declarou } \\
\text { açóes Usinas Reuridas Seresta S.A. }\end{array}$ \\
\hline 270230 & $\begin{array}{l}\text { TEOTOONIO BRANDÁO } \\
\text { VILELA JUNIOR }\end{array}$ & PSDB AL & $\begin{array}{l}\text { INCRA } \\
2003\end{array}$ & $\begin{array}{l}\text { GROTADA } \\
\text { PRENSA }\end{array}$ & CORURIPE & AL & 18 & mwronao & 18 & 0 & $\begin{array}{l}\text { REUNIDAS } \\
\text { SERESTA SA }\end{array}$ & $\begin{array}{l}\text { Teotônio Brandão Vilela Fiho declarou } \\
\text { ap̣ōes Usinas Reuridas Seresta S.A. }\end{array}$ \\
\hline 270230 & $\begin{array}{l}\text { TEOTOONIO BRANDĀO } \\
\text { VILELA JUNIOR }\end{array}$ & PSDB AL & $\begin{array}{l}\text { INCRA } \\
2003\end{array}$ & $\begin{array}{l}\text { RIACHO DO } \\
\text { BARRO }\end{array}$ & CORURIPE & AL & 11 & merenso & 11 & 0 & $\begin{array}{l}\text { REUNIDAS } \\
\text { SERESTA SA }\end{array}$ & $\begin{array}{l}\text { Teotônio Brandāo Vilela Faho declarou } \\
\text { ap̣ōes Usinas Reuridas Seresta S.A. }\end{array}$ \\
\hline 270400 & $\begin{array}{l}\text { TEOTOONNIO BRANDÁO } \\
\text { VILELA JUNIOR }\end{array}$ & PSDB AL & $\begin{array}{l}\text { INCRA } \\
2003\end{array}$ & $\begin{array}{l}\text { FAZENDA } \\
\text { TUCUM }\end{array}$ & JUNQUEIRO & AL & 20 & nesurado & 20 & 0 & $\begin{array}{l}\text { REUNIDAS } \\
\text { SERESTA SA }\end{array}$ & $\begin{array}{l}\text { Teotônio Brandão Vilela Fiho declarou } \\
\text { acọoes Usinas Reuridas Seresta S.A. }\end{array}$ \\
\hline 270915 & $\begin{array}{l}\text { TEOTOONNO BRANDĂO } \\
\text { VILELA JUNIOR }\end{array}$ & PSDB AL & $\begin{array}{l}\text { INCRA } \\
2003\end{array}$ & $\begin{array}{l}\text { SANTOS } \\
\text { TEIXEIRA }\end{array}$ & $\begin{array}{l}\text { TEOTONIO } \\
\text { VILELA }\end{array}$ & AL & 9 & nerwaso & 9 & 0 & $\begin{array}{l}\text { REUNIDAS } \\
\text { SERESTA SA }\end{array}$ & $\begin{array}{l}\text { Teotônio Brandão Vilela Fiho declarou } \\
\text { apọoses Usinas Reuridas Seresta S.A. }\end{array}$ \\
\hline
\end{tabular}




\begin{tabular}{|c|c|c|c|c|c|c|c|c|c|c|c|c|}
\hline GEOCODIGO & PARLAMENTAR & PARTIDO & FONTE & IMOVEL & Municipio & uF & AREA & ciassenscaivan & \begin{tabular}{|c|} 
AREA \\
REGISTRAD \\
A
\end{tabular} & $\begin{array}{l}\text { DECLARADA } \\
\text { COMO POSSE } \\
\end{array}$ & EMPRESA & VINCULO COM O PARLAMENTAR \\
\hline 270915 & $\begin{array}{l}\text { TEOTÓNIO BRANOḰOO } \\
\text { VILLA JUNIOR }\end{array}$ & PSDB AL & $\begin{array}{l}\mid \text { INCRA } \\
2003\end{array}$ & $\begin{array}{l}\text { FAZENDA } \\
\text { FLEXEIRAS }\end{array}$ & $\begin{array}{l}\text { TEOTÓNIO } \\
\text { VILELA }\end{array}$ & AL & 21 & nesenoo & 21 & 0 & $\begin{array}{l}\text { REUNIDAS } \\
\text { SERESTA SA }\end{array}$ & $\begin{array}{l}\text { Teototorio Brandáa Viela Fiho declarou } \\
\text { acobes Usinas Reuridas Seresta S.A. }\end{array}$ \\
\hline 270230 & $\begin{array}{l}\text { TEOTOONIO BRANDÁO } \\
\text { VILLA JUNIOR }\end{array}$ & PSDB AL & \begin{tabular}{|l}
$\mid$ NCRA \\
2003
\end{tabular} & \begin{tabular}{|c|} 
FAZENDA \\
CXACHOEIRA \\
\end{tabular} & CORURIPE & AL & 58 & & 58 & 0 & $\begin{array}{l}\text { REUNIDAS } \\
\text { SERESTA SA } \\
\end{array}$ & $\begin{array}{l}\text { Teototrio Brandäo Vilela Fino declarou } \\
\text { apbes Usinas Reuridas Seresta S.A. }\end{array}$ \\
\hline 270880 & \begin{tabular}{|c|} 
TEOTÓNIO BRANDÁO \\
VILLA JUNIOR
\end{tabular} & PSDB AL & \begin{tabular}{|l} 
NCRA \\
2003
\end{tabular} & $\begin{array}{c}\text { FAZENDA SAO } \\
\text { GERALDO }\end{array}$ & $\begin{array}{l}\text { SÁO } \\
\text { SEBASTIÁO }\end{array}$ & $\mathrm{AL}$ & 98 & & 98 & 0 & $\begin{array}{c}\text { REUNIDAS } \\
\text { SERESTA SA } \\
\end{array}$ & $\begin{array}{l}\text { Teotônio Brandäo Vilela Fino declarou } \\
\text { acoes Usinas Reuridas Seresta S.A. }\end{array}$ \\
\hline 270915 & $\begin{array}{l}\text { TEOTÓNIO BRANDÁO } \\
\text { VILLA JUNIOR }\end{array}$ & PSDB AL & $\begin{array}{l}\mid \text { NCRA } \\
2003\end{array}$ & $\begin{array}{c}\text { FAZENDA SAO } \\
\text { MATHEUS }\end{array}$ & $\begin{array}{l}\text { TEOTOONNIO } \\
\text { VILELA }\end{array}$ & AL & 124 & & ${ }^{124}$ & 0 & $\begin{array}{l}\text { REUNIDAS } \\
\text { SERESTA SA }\end{array}$ & $\begin{array}{l}\text { Teototônio Brandáa Vilila Fino declarou } \\
\text { acóes Usinas Reuridas Seresta S.A. }\end{array}$ \\
\hline 270915 & 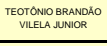 & PSDB AL & $\begin{array}{l}\text { INCRA } \\
2003\end{array}$ & $\begin{array}{l}\text { RIACHO DO } \\
\text { BARRO }\end{array}$ & $\begin{array}{l}\text { TEOTÓNIO } \\
\text { VILELAA }\end{array}$ & AL & 39 & & 39 & 0 & $\begin{array}{l}\text { REUNIDAS } \\
\text { SERESTA SA }\end{array}$ & $\begin{array}{l}\text { Teototrio Brandăo Viliela Fiho declarou } \\
\text { apōes Usinas Reuridas Seresta S.A. }\end{array}$ \\
\hline 270690 & $\begin{array}{c}\text { CARLOS ALBERTO } \\
\text { MORERAAD } \\
\text { MENDONCA CANUTO }\end{array}$ & PMDB AL & INCRA 2000 & $\begin{array}{c}\text { FAZENDA SAO } \\
\text { JOAO }\end{array}$ & PLLAR & AL & 156 & & 156 & 0 & $\begin{array}{l}\text { USINA TERRA } \\
\text { NOVA SA }\end{array}$ & \\
\hline 270690 & $\begin{array}{c}\text { CARLOS ARERTTO } \\
\text { MORELRADE } \\
\text { MENDONCA CANUTO }\end{array}$ & PMDB AL & INCRA 2003 & $\begin{array}{c}\text { FAZENDA } \\
\text { IMBURI IRETTA } \\
\end{array}$ & PlLAR & AL & 571 & & 571 & 0 & $\begin{array}{l}\text { USINA TERRA } \\
\text { NOVA SA }\end{array}$ & \\
\hline 270690 & $\begin{array}{c}\text { CARLOS ALBERTO } \\
\text { MORERARE } \\
\text { MENDONCA CANUTO }\end{array}$ & PMDB AL & INCRA 2003 & $\begin{array}{c}\text { FAZENDA } \\
\text { TERRA NOVA }\end{array}$ & PILAR & AL & 508 & monemente & 508 & 0 & $\begin{array}{l}\text { USINA TERRA } \\
\text { NOVA SA }\end{array}$ & \\
\hline
\end{tabular}




\begin{tabular}{|c|c|c|c|c|c|c|c|c|c|c|c|c|}
\hline GEOCODICO & PARLAMENTAR & PARTIDO & FONTE & IMOVEL & MUNicipio & uF & AREA & cusesentucivon & $\begin{array}{c}\text { AREA } \\
\text { REGISTRAD } \\
\text { A }\end{array}$ & $\begin{array}{l}\text { DECLARADA } \\
\text { COMO POSSE }\end{array}$ & EMPRESA & VINCULO COM O PARLAMENTAR \\
\hline 270690 & $\begin{array}{l}\text { CARLOS ALBERTO } \\
\text { MOREIRAA DE } \\
\text { MENDONCGA CANUTO }\end{array}$ & PMDB AL & INCRA 2003 & $\begin{array}{c}\text { FAZENDA } \\
\text { BUENOS AYRES } \\
\text { NUVENS }\end{array}$ & PLAR & AL & 480 & we & 480 & 0 & $\begin{array}{l}\text { USINA TERRA } \\
\text { NOVA SA }\end{array}$ & \\
\hline 270690 & $\begin{array}{l}\text { CARLOS ALBERTO } \\
\text { MORERARA DE } \\
\text { MENDONGCA CANUTO }\end{array}$ & PMDB AL & INCRA 2003 & $\begin{array}{c}\text { FAZENDA } \\
\text { MANGABEIRA } \\
\text { ESQUERDA }\end{array}$ & PLLAR & AL & 436 & & 436 & 0 & $\begin{array}{l}\text { USINA TERRA } \\
\text { NOVA SA }\end{array}$ & \\
\hline 270690 & $\begin{array}{l}\text { CARLOS ALBERTO } \\
\text { MOREIRADE } \\
\text { MENDONGA CANUTO }\end{array}$ & PMDB AL & INCRA 2003 & $\begin{array}{c}\text { FAZENDA } \\
\text { FIMBURI } \\
\text { ESOUERDA }\end{array}$ & PILAR & AL & 116 & & 116 & 0 & $\begin{array}{l}\text { USINA TERRA } \\
\text { NOVA SA }\end{array}$ & \\
\hline 270690 & $\begin{array}{l}\text { CARLOS ALBERTO } \\
\text { MOOEERAA DE } \\
\text { MENDONGA CANUTO }\end{array}$ & PMDB AL & INCRA 2003 & $\begin{array}{l}\text { FAZENDA } \\
\text { SUMAUMA }\end{array}$ & PILAR & AL & 136 & & 136 & 0 & $\begin{array}{l}\text { USINA TERRA } \\
\text { NOVA SA }\end{array}$ & \\
\hline 270690 & $\begin{array}{l}\text { CARLOS ALBERTO } \\
\text { MOOEERAA DE } \\
\text { MENDONÇA CANUTO }\end{array}$ & PMDB AL & INCRA 2003 & $\begin{array}{l}\text { FAZENDA SAO } \\
\text { JOSE }\end{array}$ & PILAR & AL & 452 & manosestove & 452 & 0 & $\begin{array}{l}\text { USINA TERRA } \\
\text { NOVA SA }\end{array}$ & \\
\hline 270690 & $\begin{array}{l}\text { CARLOS ALBERTO } \\
\text { MORERARA DE } \\
\text { MENDONGQA CANUTO }\end{array}$ & PMDB AL AL & INCRA 2003 & $\begin{array}{c}\text { FAZENDA } \\
\text { MANGABEIIRA } \\
\text { DIREITA }\end{array}$ & PlLAR & AL & 86 & & 86 & 0 & $\begin{array}{l}\text { USINA TERRA } \\
\text { NOVA SA }\end{array}$ & \\
\hline
\end{tabular}




\begin{tabular}{|c|c|c|c|c|c|c|c|c|c|c|c|c|}
\hline GEOCODIGO & PARLAMENTAR & PARTIDO & FONTE & IMÓVEL & MUNICIPIO & UF & AREA & cLasse Hurcivar & $\begin{array}{c}\text { AREA } \\
\text { REGISTRAD } \\
\text { A }\end{array}$ & $\begin{array}{l}\text { DECLARADA } \\
\text { COMO POSSE }\end{array}$ & EMPRESA & VINCULO COM O PARLAMENTAR \\
\hline 292250 & $\begin{array}{l}\text { PAULO SERGIO } \\
\text { PARANHOS DE } \\
\text { MAGALHAES }\end{array}$ & DEM BA & INCRA 2003 & $\begin{array}{l}\text { FAZENDA } \\
\text { BARAUNA }\end{array}$ & $\begin{array}{l}\text { MUQUEM DE } \\
\text { SÁO } \\
\text { FRANCISCO }\end{array}$ & BA & 4.000 & 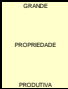 & 4.000 & 0 & $\begin{array}{c}\text { PARANHOS SA } \\
\text { AGRICULTURA E } \\
\text { PECUARIA? }\end{array}$ & \\
\hline 520549 & $\begin{array}{l}\text { JOSÉ RIBAMAR } \\
\text { FERREIRA DE ARAÚJO } \\
\text { COSTA }\end{array}$ & PMDB AP & $\begin{array}{l}\text { INCRA } \\
2003\end{array}$ & $\begin{array}{l}\text { SITIO MARIA } \\
\text { PEREIRA }\end{array}$ & $\begin{array}{l}\text { CIDADE } \\
\text { OCIDENTAL }\end{array}$ & Go & 654 & Procontence & 659 & 0 & $\begin{array}{c}\text { MANIL SA } \\
\text { EMPREENDIMEN } \\
\text { TOS E } \\
\text { PARTICIPACOES) } \\
;\end{array}$ & $\begin{array}{l}\text { MATRICULA DECLRADA POR } \\
\text { SARNEY EM LUIZIANIA (CIDADE } \\
\text { OCIDENTAL VIZINHA DE LUIZIANIA }\end{array}$ \\
\hline 520870 & $\begin{array}{l}\text { LUCIA VANIA ABRÁO } \\
\text { COSTA }\end{array}$ & PSD GO & INCRA 2003 & FAZ CAVEIRAS & GOIÂNIA & Go & 3 & neruseo & 3 & 0 & $\begin{array}{l}\text { CONSTRUCAO } \\
\text { LTDA); }\end{array}$ & \\
\hline 520320 & $\begin{array}{l}\text { LUCIA VANIA ABRAO } \\
\text { COSTA }\end{array}$ & PSD GO & INCRA 2003 & FAZ PORTEIRAS & BARRO ALTO & Go & 6.490 & 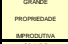 & 6.490 & 0 & $\begin{array}{l}\text { AGROPECUARIA } \\
\text { LTDA): }\end{array}$ & $\begin{array}{l}\text { LUCIA VANIA DECLAROU Firma } \\
\text { Vera CrIz S A }\end{array}$ \\
\hline 520320 & $\begin{array}{l}\text { LUCIA VANIA ABRAO } \\
\text { COSTA }\end{array}$ & PSD GO & INCRA 2003 & $\begin{array}{l}\text { ANTONIO DA } \\
\text { LAGUANA }\end{array}$ & BARRO ALTO & Go & 1.384 & 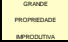 & 1.384 & 0 & $\begin{array}{l}\text { AGROPECUARIA } \\
\text { LTDA); }\end{array}$ & $\begin{array}{l}\text { LUCIA VANIA DECLAROU Firma } \\
\text { Vera Criz S A }\end{array}$ \\
\hline 520860 & $\begin{array}{l}\text { LUCIA VANIA ABRR̃o } \\
\text { COSTA }\end{array}$ & PSD GO & INCRA 2003 & $\begin{array}{l}\text { FAZSAO } \\
\text { CARLOS }\end{array}$ & GOIANÉSIA & Go & 975 & 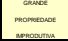 & 975 & 0 & $\begin{array}{l}\text { AGROPECUARIA } \\
\text { LTDA); }\end{array}$ & $\begin{array}{l}\text { LUCIA VANIA DECLAROU Firma, } \\
\text { Vera Criz S A }\end{array}$ \\
\hline 520860 & $\begin{array}{l}\text { LUCIA VANIA ABRÃO } \\
\text { COSTA }\end{array}$ & PSD GO & INCRA 2008 & $\begin{array}{l}\text { FAZENDA } \\
\text { COVOA }\end{array}$ & GOIANÉSIA & Go & 1.044 & proconemence & 1.044 & 0 & $\begin{array}{c}\text { AGROPECUARIA } \\
\text { LTDA); }\end{array}$ & $\begin{array}{l}\text { LUCIA VANIA DECLAROU Firma } \\
\text { Vera Criz S A }\end{array}$ \\
\hline 520860 & $\begin{array}{l}\text { LUCIA VANIA ABRÃO } \\
\text { COSTA }\end{array}$ & PSD GO & INCRA 2003 & $\begin{array}{l}\text { FAZENDA VERA } \\
\text { CRUZ }\end{array}$ & GOIANESLAA & Go & 6.190 & 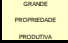 & 6.190 & 0 & $\begin{array}{l}\text { AGROPECUARIA } \\
\text { LTDA); }\end{array}$ & $\begin{array}{l}\text { LUCIA VANIA DECLAROU Firma } \\
\text { Vera Criz S A }\end{array}$ \\
\hline 521935 & $\begin{array}{l}\text { LUCIA VANIA ABRÁO } \\
\text { COSTA }\end{array}$ & PSD GO & INCRA 2003 & FAZ CODORA & SANTA ISABEL & Go & 4.356 & 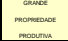 & 4.356 & 0 & $\begin{array}{l}\text { AGROPECUARIA } \\
\text { LTDA): }\end{array}$ & $\begin{array}{l}\text { LUCIA VANIA DECLAROU Firma } \\
\text { Vera Craz S A }\end{array}$ \\
\hline 522020 & $\begin{array}{l}\text { LUCIA VANIA ABRÁO } \\
\text { COSTA }\end{array}$ & PSD GO & INCRA 2003 & $\begin{array}{l}\text { DE SAO } \\
\text { SEBASTIAO }\end{array}$ & GOIANÉSIA & Go & 176 & provourma & 176 & 0 & $\begin{array}{l}\text { AGROPECUARIA } \\
\text { LTDA); }\end{array}$ & $\begin{array}{l}\text { LUCIA VANIA DECLAROU Firma } \\
\text { Vera Cruz SA }\end{array}$ \\
\hline
\end{tabular}




\begin{tabular}{|c|c|c|c|c|c|c|c|c|c|c|c|c|}
\hline GEOCODIGO & PARLAMENTAR & PARTIDO & FONTE & IMOVEL & Municipio & uF & AREA & cuassensoniman & \begin{tabular}{|c|} 
AREA \\
REGISTRAD \\
A
\end{tabular} & $\begin{array}{l}\text { DECLARADA } \\
\text { COMO POSSE }\end{array}$ & EMPRESA & VINCULO COM O PARLAMENTAR \\
\hline 520380 & $\begin{array}{l}\text { LUCIA VANIAABRÃo } \\
\text { COSTA }\end{array}$ & PSDGO & INCRA 2003 & $\begin{array}{l}\text { CHACARA SOL } \\
\text { NASCENTE }\end{array}$ & BRITÁNIA & во & 9 & newnoo & 6 & 3 & $\begin{array}{l}\text { PARTICIPACOES } \\
\text { LTDA): }\end{array}$ & $\begin{array}{l}\text { LUCIA VANIA DECLAROU Firma } \\
\text { Vera CrIz S A }\end{array}$ \\
\hline 522020 & $\begin{array}{l}\text { SANDROA ANTÓNIO } \\
\text { SCODRO (SANDRO } \\
\text { MABEL) }\end{array}$ & PRGO & INCRA 2005 & \begin{tabular}{|c|} 
FAZENDA \\
MINAS GOASS
\end{tabular} & $\begin{array}{l}\text { SAO MIGUEL } \\
\text { DO ARAGUAAA }\end{array}$ & go & 1.938 & 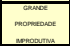 & 1.936 & 0 & $\begin{array}{c}\text { CONSTRUCOES } \\
\text { LTDA); }\end{array}$ & $\begin{array}{l}\text { Quotas Da Empresa Sk Agropecuária } \\
\text { Ldoa }\end{array}$ \\
\hline 522020 & $\begin{array}{c}\text { SANDRO ANTÓNIO } \\
\text { SCODRO SAANDRO } \\
\text { MABELL) }\end{array}$ & PR GO & INCRA 2008 & $\begin{array}{c}\text { RECANTO DA } \\
\text { PRAIA }\end{array}$ & $\begin{array}{l}\text { SẮ MIGUEL } \\
\text { DO ARAGUAAA }\end{array}$ & go & 1.403 & monoreave & 1.403 & 0 & $\begin{array}{c}\text { CONSTRUCOES } \\
\text { LTDA); }\end{array}$ & \\
\hline 210300 & $\begin{array}{l}\text { PAULO CELSO } \\
\text { FONSECA MARINHO }\end{array}$ & PLMA & $\begin{array}{l}\text { INCFA } \\
2003\end{array}$ & $\begin{array}{l}\text { FAZENDA } \\
\text { ESTRELA }\end{array}$ & CAXIAS & MA & 1.322 & 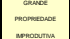 & 1.322 & 0 & $\begin{array}{l}\text { EINDUSTRIA } \\
\text { LIMITADA }\end{array}$ & $\begin{array}{l}\text { MARIINHO, DECLAROU CARGO } \\
\text { NESTA EMPAEA }\end{array}$ \\
\hline 310020 & NEWTON CARDOSO & PMDB MG & $\begin{array}{l}\text { INCRA } \\
2003\end{array}$ & $\begin{array}{l}\text { ALEGRIA E } \\
\text { VARJOES }\end{array}$ & AвAETE & MG & 108 & 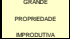 & 1.843 & 0 & $\begin{array}{c}\text { SIOERURGICA } \\
\text { PITANGUI }\end{array}$ & $\begin{array}{l}\text { nevton cardoso declarou companhia } \\
\text { siderngica pitangui }\end{array}$ \\
\hline 310020 & NEWTON CARDOSO & Рмов мG & $\begin{array}{c}\text { INCRA } \\
2003\end{array}$ & \begin{tabular}{|l} 
FAZENDA BOM \\
JARDIM
\end{tabular} & ABAETÉ & MG & 52 & 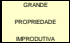 & 793 & 0 & \begin{tabular}{|l}
$\begin{array}{c}\text { SIDERURGICA } \\
\text { PITANGUI }\end{array}$ \\
\end{tabular} & $\begin{array}{l}\text { Renton cardoso declarou compantia } \\
\text { siderengica pitangui }\end{array}$ \\
\hline 310070 & $\begin{array}{l}\text { JOÅO JOSÉ PERERA } \\
\text { DE LYRA }\end{array}$ & Ptв AL & $\begin{array}{c}\text { INCRA } \\
2003\end{array}$ & $\begin{array}{l}\text { PONTALE } \\
\text { ESTIVAS }\end{array}$ & $\begin{array}{l}\text { AGUA } \\
\text { COMPRIDA }\end{array}$ & Ma & 18 & & 243 & 0 & USINA CAETE SA & 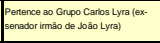 \\
\hline 310070 & $\begin{array}{l}\text { JOÁO JOSÉ PEREREA } \\
\text { DE LYRA }\end{array}$ & PTB AL & $\begin{array}{l}\text { INCRA } \\
2003\end{array}$ & $\begin{array}{c}\text { SEBASTIAO DO } \\
\text { BURITI }\end{array}$ & $\begin{array}{l}\text { AGUA } \\
\text { COMPRIDA }\end{array}$ & MG & 9 & & 96 & 0 & USINA CAETE SA & $\begin{array}{l}\text { Pertence ao Ginpo Cartos L Lyra (ex- } \\
\text { serador imáo de Joâo Lyra) }\end{array}$ \\
\hline 311730 & $\begin{array}{l}\text { JOÅO JOSÉ PEREERA } \\
\text { DE LYRA }\end{array}$ & PtBal & $\begin{array}{l}\text { INCRA } \\
2003\end{array}$ & $\begin{array}{l}\text { PONTE DE } \\
\text { TERRA }\end{array}$ & $\begin{array}{l}\text { CONCEICAÁA } \\
\text { DAS ALAGOAS }\end{array}$ & MG & 31 & 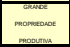 & 397 & 0 & USINA CAETE SA & $\begin{array}{l}\text { Pertence ao Grupo Cartos Lyra (ex- } \\
\text { senador immao de Joôo Lyra) }\end{array}$ \\
\hline 311730 & $\begin{array}{l}\text { JOÁO JOSÉ PEREERA } \\
\text { DE LYRA }\end{array}$ & PtB AL & $\begin{array}{l}\text { INCRA } \\
2003\end{array}$ & $\begin{array}{l}\text { FAZENDA } \\
\text { LAMBARI }\end{array}$ & $\begin{array}{l}\text { CONCEICÁAO } \\
\text { DAS ALAGOAS }\end{array}$ & MG & 22 & & 284 & 0 & USINA CAETE SA & $\begin{array}{l}\text { Perterce ao Ginpo Carlos Lyra (ex- } \\
\text { senador immào de Joöo Lyra) }\end{array}$ \\
\hline 311730 & $\begin{array}{l}\text { JOÅO JOSÉ PERERA } \\
\text { DE LYRA }\end{array}$ & PtB AL & $\begin{array}{l}\text { INCRA } \\
2003\end{array}$ & $\begin{array}{l}\text { FAZENDA } \\
\text { CACHOEIRA }\end{array}$ & $\begin{array}{l}\text { CONCEIĆÁ } \\
\text { DAS ALAGOAS }\end{array}$ & MG & 19 & & 238 & 0 & USINA CAETE SA & 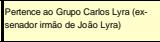 \\
\hline 311730 & $\begin{array}{l}\text { JOÅO JOSÉ PEREIRA } \\
\text { DE LYRA }\end{array}$ & Pтв AL & $\begin{array}{l}\text { INCRA } \\
2003\end{array}$ & $\begin{array}{l}\text { SANTO } \\
\text { ANTONIO }\end{array}$ & $\begin{array}{l}\text { CONCEICÁAO } \\
\text { DAS ALAGOAS }\end{array}$ & MG & 16 & & 214 & 0 & USINA CAETE SA & $\begin{array}{l}\text { Pertence ao Grupo Cartos Lyra (ex- } \\
\text { senador imăo de Joăo Lyra) }\end{array}$ \\
\hline
\end{tabular}




\begin{tabular}{|c|c|c|c|c|c|c|c|c|c|c|c|c|}
\hline GEOCODIGO & PARLAMENTAR & PARTIDO & FONTE & IMOVEL & MUNICIPIO & uF & AREA & cuassensacivan & \begin{tabular}{|c|} 
AREA \\
REGISTRAD \\
A
\end{tabular} & $\begin{array}{l}\text { DECLAARADA } \\
\text { COMO POSSE }\end{array}$ & EMPRESA & VINCULO COM O PARLAMENTAR \\
\hline 311730 & $\begin{array}{l}\text { JOÄO JOSÉ PERERARA } \\
\text { DE LYRA }\end{array}$ & PTB AL & $\begin{array}{l}\text { INCRA } \\
2003\end{array}$ & $\begin{array}{c}\text { SiTIO CAIAPO } \\
\text { ESPORA }\end{array}$ & $\begin{array}{l}\text { CONCEICÁAO } \\
\text { DAS ALAGOAS }\end{array}$ & MG & 10 & & 133 & 0 & USINA CAETE SA & $\begin{array}{l}\text { Pertence ao Ginpo Cartos L Lyra (ex- } \\
\text { serador imâo de Joâo Lyra) }\end{array}$ \\
\hline 311730 & $\begin{array}{l}\text { JOǺ JOSÉ PEREERA } \\
\text { DE LYRA }\end{array}$ & PtBal & $\begin{array}{l}\text { INCRA } \\
2003\end{array}$ & $\begin{array}{c}\text { PFATADE } \\
\text { BAIXO }\end{array}$ & $\begin{array}{l}\text { CONCEICÁA } \\
\text { DAS ALAGOAS }\end{array}$ & MG & 10 & & 128 & 0 & USINA CAETE SA & $\begin{array}{l}\text { Pertence ao Ginpo Cartos Lyra (ex- } \\
\text { serador imáo de Joöo Lyra) }\end{array}$ \\
\hline 311730 & \begin{tabular}{|l} 
JOÅO JOSÉ PEREERA \\
DE LYRA
\end{tabular} & Pтв AL & $\begin{array}{l}\text { INCFA } \\
2003\end{array}$ & \begin{tabular}{|l|} 
FAZENDA \\
CACHOEIRA \\
\end{tabular} & $\begin{array}{l}\text { CONCEICÁA } \\
\text { DAS ALAGOAS }\end{array}$ & MG & 10 & & 101 & 0 & USINA CAETE SA & 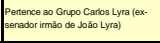 \\
\hline 311730 & $\begin{array}{l}\text { JOÅO JOSÉ PERERA } \\
\text { DE LYRA }\end{array}$ & PtB AL & $\begin{array}{l}\text { INCRA } \\
2003\end{array}$ & $\begin{array}{l}\text { PRATA DE } \\
\text { BAIXO }\end{array}$ & $\begin{array}{l}\text { CONCEICÁAO } \\
\text { DAS ALAGOAS }\end{array}$ & MG & 0 & & 88 & 0 & USINA CAETE SA & $\begin{array}{l}\text { Pertence ao Ginpo Cartos L Lyra (ex- } \\
\text { senador imäo de Joẫo Lyra) }\end{array}$ \\
\hline 311730 & $\begin{array}{l}\text { JOÅO JOSÉ PERERARA } \\
\text { DE LYRA }\end{array}$ & PTB AL & $\begin{array}{l}\text { INCRA } \\
2003\end{array}$ & $\begin{array}{c}\text { PRATADE } \\
\text { BAIXO }\end{array}$ & $\begin{array}{l}\text { CONCEICÁAO } \\
\text { DAS ALAGOAS }\end{array}$ & MG & 0 & & 84 & 0 & USINA CAETE SA & 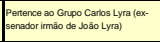 \\
\hline 311730 & $\begin{array}{l}\text { JOǺ JOSÉ PERERARA } \\
\text { DE LYRA }\end{array}$ & PTBAL & $\begin{array}{l}\text { INCRA } \\
2003\end{array}$ & \begin{tabular}{|l|} 
FAZENDA \\
CACHOEIRA \\
\end{tabular} & $\begin{array}{l}\text { CONCEICÁAO } \\
\text { DAS ALAGOAS }\end{array}$ & MG & 0 & & 73 & 0 & USINA CAETE SA & 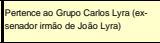 \\
\hline 311730 & $\begin{array}{l}\text { JOÅO JOSÉ PEREIRA } \\
\text { DE LYRA }\end{array}$ & Pтв AL & $\begin{array}{l}\text { INCRA } \\
2003\end{array}$ & $\begin{array}{l}\text { PRATADE } \\
\text { BAIXO }\end{array}$ & $\begin{array}{l}\text { CONCEICÁAO } \\
\text { DAS ALAGOAS }\end{array}$ & MG & 0 & & 60 & 0 & USINA CAETE SA & $\begin{array}{l}\text { Pertence ao Grupo Cartos Lyra (ex- } \\
\text { senador imâo de Joầo Lyra) }\end{array}$ \\
\hline 311730 & $\begin{array}{l}\text { JOÁO JOSÉ PEREREA } \\
\text { DE LYRA }\end{array}$ & PTB AL & $\begin{array}{l}\text { INCRA } \\
2003\end{array}$ & \begin{tabular}{|c|} 
NUNES \\
CREOULOS
\end{tabular} & $\begin{array}{l}\text { CONCEICÁAO } \\
\text { DAS ALAGOAS }\end{array}$ & MG & 0 & & 59 & 0 & USINA CAETE SA & $\begin{array}{l}\text { Pertence ao Ginpo Cartos L Lyra (ex- } \\
\text { serador imáo de Joâo Lyra) }\end{array}$ \\
\hline 311730 & $\begin{array}{l}\text { JOÅO JOSÉ PEREERA } \\
\text { DE LYRA }\end{array}$ & PtB AL & $\begin{array}{l}\text { INCRA } \\
2003\end{array}$ & $\begin{array}{c}\text { BAIXO } \\
\text { CACHOEIRA }\end{array}$ & $\begin{array}{l}\text { CONCEICAÁA } \\
\text { DAS ALAGOAS }\end{array}$ & MG & 0 & & 54 & 0 & USINA CAETE SA & $\begin{array}{l}\text { Pertence ao Grupo Cartos Lyra (ex- } \\
\text { senador immao de Joôo Lyra) }\end{array}$ \\
\hline 311730 & $\begin{array}{l}\text { JOÁO JOSÉ PEREERA } \\
\text { DE LYRA }\end{array}$ & PtB AL & $\begin{array}{l}\text { INCRA } \\
2003\end{array}$ & $\begin{array}{c}\text { PRATADE } \\
\text { BAIXO }\end{array}$ & $\begin{array}{l}\text { CONCEICÁAO } \\
\text { DAS ALAGOAS }\end{array}$ & MG & 0 & & 49 & 0 & USINA CAETE SA & $\begin{array}{l}\text { Perterce ao Ginpo Carlos Lyra (ex- } \\
\text { senador immào de Joöo Lyra) }\end{array}$ \\
\hline 311730 & $\begin{array}{l}\text { JOÅO JOSÉ PERERA } \\
\text { DE LYRA }\end{array}$ & PtB AL & $\begin{array}{l}\text { INCRA } \\
2003 \\
\end{array}$ & $\begin{array}{c}\text { PRATADE } \\
\text { BAIXO }\end{array}$ & $\begin{array}{l}\text { CONCEIĆÁ } \\
\text { DAS ALAGOAS }\end{array}$ & MG & 0 & & 36 & 0 & USINA CAETE SA & 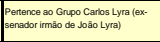 \\
\hline 311730 & $\begin{array}{l}\text { JOÅO JOSÉ PEREIRA } \\
\text { DE LYRA }\end{array}$ & Pтв AL & $\begin{array}{l}\text { INCRA } \\
2003\end{array}$ & $\begin{array}{l}\text { PAATADE } \\
\text { BAIXO }\end{array}$ & $\begin{array}{l}\text { CONCEICÁAO } \\
\text { DAS ALAGOAS }\end{array}$ & MG & 0 & & 29 & 0 & USINA CAETE SA & $\begin{array}{l}\text { Pertence ao Grupo Cartos Lyra (ex- } \\
\text { senador imăo de Joăo Lyra) }\end{array}$ \\
\hline
\end{tabular}




\begin{tabular}{|c|c|c|c|c|c|c|c|c|c|c|c|c|}
\hline GEOCODIGO & PARLAMENTAR & PARTIDO & FONTE & IMOVEL & MunicíPIo & uF & AREA & cuassensoniman & \begin{tabular}{|c|} 
AREA \\
REGISTRAD \\
A
\end{tabular} & $\begin{array}{l}\text { DEELAAADA } \\
\text { COMO POSSE }\end{array}$ & EMPRESA & VINCULO COM O PARLAMENTAR \\
\hline 311730 & $\begin{array}{l}\text { JOĂO JOSÉ PEREREA } \\
\text { DE LYRA }\end{array}$ & PtB AL & $\begin{array}{l}\text { INCRA } \\
2003\end{array}$ & \begin{tabular}{|l|} 
FAZENDA \\
PRATA DE CIMA \\
\end{tabular} & \begin{tabular}{|l} 
CONCEICÁAO \\
DAS ALAGOAS
\end{tabular} & MG & 0 & & 28 & 0 & USINA CAETE SA & $\begin{array}{l}\text { Pertence aa Ginpo Cartos Lyra (ex- } \\
\text { serandor imäa de Joâo Lyra) }\end{array}$ \\
\hline 311730 & \begin{tabular}{|c|c|} 
JOÁO JOSÉ PEREERA \\
DE LYRA
\end{tabular} & PtB AL & $\begin{array}{l}\text { INCRA } \\
2003 \\
\end{array}$ & $\begin{array}{l}\text { FAZENDA } \\
\text { SUCURI }\end{array}$ & $\begin{array}{l}\text { CONCEICÁO } \\
\text { DAS ALAGOAS }\end{array}$ & MG & 0 & & 27 & 0 & USINA CAETE SA & 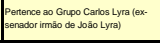 \\
\hline 311730 & $\begin{array}{l}\text { JOÅO JOSÉ PEREIRA } \\
\text { DE LYRA }\end{array}$ & Ртв AL & $\begin{array}{l}\text { INCRA } \\
2003\end{array}$ & $\begin{array}{l}\text { PRATA DE } \\
\text { BAIXO }\end{array}$ & $\begin{array}{l}\text { CONCEIC̆ÁO } \\
\text { DAS ALAGOAS }\end{array}$ & MG & 0 & & 24 & 0 & USINA CAETE SA & $\begin{array}{l}\text { Pertence ao Ginpo Cartos Lyra (ex- } \\
\text { senador imäo de Joầo Lyra) }\end{array}$ \\
\hline 311730 & $\begin{array}{c}\text { JOÃO JOSÉ PEREERA } \\
\text { DE LYRA }\end{array}$ & PtBal & $\begin{array}{l}\text { INCRA } \\
2003\end{array}$ & $\begin{array}{c}\text { FAZENDA } \\
\text { PRATA DE CIMA }\end{array}$ & $\begin{array}{l}\text { CONCEICÁA } \\
\text { DAS ALAGOAS }\end{array}$ & MG & 0 & nerenoso & 23 & 0 & USINA CAETE SA & $\begin{array}{l}\text { Pertence ao Ginpo Cartos Lyra (ex- } \\
\text { senador immäo de Joäo Lyra) }\end{array}$ \\
\hline 311730 & $\begin{array}{l}\text { JOÅO JOSÉ PEREERA } \\
\text { DE LYRA }\end{array}$ & PTB AL & $\begin{array}{l}\text { INCRA } \\
2003\end{array}$ & $\begin{array}{l}\text { PRATA DE } \\
\text { BAIXO }\end{array}$ & $\begin{array}{l}\text { CONCEIČ́AO } \\
\text { DAS ALAGOAS }\end{array}$ & MG & 0 & nerensio & 19 & 0 & USINA CAETE SA & $\begin{array}{l}\text { Pertence ao Ginpo Cartos Lyra (ex- } \\
\text { senador imäo de Joốo Lyra) }\end{array}$ \\
\hline 311730 & \begin{tabular}{|l} 
JOĂO JOSÉ PEREERA \\
DE LYRA
\end{tabular} & PTB AL & $\begin{array}{l}\text { INCRA } \\
2003 \\
\end{array}$ & \begin{tabular}{|l} 
FAZENDA \\
PRATA DE CIMA \\
\end{tabular} & $\begin{array}{l}\text { CONCEIČ́AO } \\
\text { DAS ALAGOAS }\end{array}$ & MG & 0 & nerenoso & 14 & 0 & USINA CAETE SA & 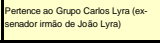 \\
\hline 311730 & $\begin{array}{l}\text { JOÄO JOSÉ PERERA } \\
\text { DE LYRA }\end{array}$ & PtB AL & $\begin{array}{l}\text { INCRA } \\
2003\end{array}$ & \begin{tabular}{|c|} 
FAZENDA \\
PAATA DE CIMA
\end{tabular} & $\begin{array}{l}\text { CONCEICÁAO } \\
\text { DAS ALAGOAS }\end{array}$ & Ma & 0 & neruaco & 14 & ${ }^{0}$ & USINA CAETE SA & $\begin{array}{l}\text { Pertence ao Ginpo Cartos L Lyra (ex- } \\
\text { senador imáo de Joôo Lyra) }\end{array}$ \\
\hline 311730 & $\begin{array}{l}\text { JOÁO JOSÉ PEREERA } \\
\text { DE LYRA }\end{array}$ & PTB AL & $\begin{array}{l}\text { INCRA } \\
2003\end{array}$ & $\begin{array}{c}\text { EMATADA } \\
\text { GUNGA }\end{array}$ & $\begin{array}{l}\text { CONCEICAOA } \\
\text { DAS ALAGOAS }\end{array}$ & MG & 0 & newaso & 12 & 0 & USINA CAETE SA & $\begin{array}{l}\text { Pertence ao Gupo Caros L Lyra (ex- } \\
\text { serador imäo de Joöo Lyra) }\end{array}$ \\
\hline 312125 & $\begin{array}{l}\text { JOÃO JOSÉ PEREERA } \\
\text { DE LYRA }\end{array}$ & PtB AL & $\begin{array}{l}\text { INCRA } \\
2003\end{array}$ & $\begin{array}{c}\text { FAZENDA } \\
\text { SANTO INACIO }\end{array}$ & CONQUISTA & MG & 0 & nerenaso & 14 & 0 & $\begin{array}{l}\text { ACUCARE } \\
\text { ALCOOL }\end{array}$ & $\begin{array}{l}\text { Pertence ao Ginpo Cartos L Lyra (ex- } \\
\text { senador imäo de Joäo Lyra) }\end{array}$ \\
\hline 312090 & NEWTON CARDOSO & PMDB MG & $\begin{array}{l}\text { INCRA } \\
2003\end{array}$ & $\begin{array}{c}\text { CERRADAO DO } \\
\text { AMARAL }\end{array}$ & CURVELO & MG & 60 & monowente & 900 & 0 & $\begin{array}{c}\text { SIDERURGICA } \\
\text { PITANGUI }\end{array}$ & $\begin{array}{l}\text { nevton cardoso declarou companhia } \\
\text { siderurgica pitangui }\end{array}$ \\
\hline 312125 & $\begin{array}{c}\text { JOǺ JOSÉ PERERA } \\
\text { DE LYRA }\end{array}$ & PtB AL & $\begin{array}{l}\text { INCRA } \\
2003\end{array}$ & $\begin{array}{l}\text { FAZENDA } \\
\text { FARTURA }\end{array}$ & DeLta & MG & 30 & $\begin{array}{l}\text { monchenewe } \\
\text { rnosuma }\end{array}$ & 369 & 0 & $\begin{array}{l}\text { ACUCARE } \\
\text { ALCOOL }\end{array}$ & $\begin{array}{l}\text { Pertence ao Ginpo Carlos L Lyra (ex- } \\
\text { senador imáa de Joöo Lyra) }\end{array}$ \\
\hline 312125 & $\begin{array}{l}\text { JOÄO JOSÉ PERERAA } \\
\text { DE LYRA }\end{array}$ & Ptв AL & $\begin{array}{l}\text { INCRA } \\
2003\end{array}$ & $\begin{array}{c}\text { FAZENDA NOVA } \\
\text { ALLANCA }\end{array}$ & DELTA & Ma & 27 & & 343 & 0 & $\begin{array}{l}\text { ACUCARE } \\
\text { ALCOOL }\end{array}$ & $\begin{array}{l}\text { Pertence ao Ginpo Cartos L Lyra (ex- } \\
\text { senador imâo de Joẫo Lyra) }\end{array}$ \\
\hline
\end{tabular}




\begin{tabular}{|c|c|c|c|c|c|c|c|c|c|c|c|c|}
\hline GEOCODIGO & PARLAMENTAR & PARTIDO & FONTE & IMOVEL & MunicíPIo & uF & AREA & cuassensoniman & \begin{tabular}{|c|} 
AREA \\
REGISTRAD \\
A
\end{tabular} & $\begin{array}{l}\text { DEELAAADA } \\
\text { COMO POSSE }\end{array}$ & EMPRESA & VINCULO COM O PARLAMENTAR \\
\hline 312125 & $\begin{array}{l}\text { JOĂO JOSÉ PEREREA } \\
\text { DE LYRA }\end{array}$ & PtB AL & $\begin{array}{l}\text { INCRA } \\
2003\end{array}$ & \begin{tabular}{|l|} 
FAZENDA \\
CALIFORNIA \\
\end{tabular} & DELTA & MG & 22 & & 280 & 0 & $\begin{array}{l}\text { ACUCARE } \\
\text { ALCOOL }\end{array}$ & $\begin{array}{l}\text { Pertence aa Ginpo Cartos Lyra (ex- } \\
\text { serandor imäa de Joâo Lyra) }\end{array}$ \\
\hline 312125 & \begin{tabular}{|c|c|} 
JOÁO JOSÉ PEREERA \\
DE LYRA
\end{tabular} & PtB AL & $\begin{array}{l}\text { INCRA } \\
2003 \\
\end{array}$ & \begin{tabular}{|c|} 
FAZENDA \\
BEBEDOURO \\
\end{tabular} & DELTA & MG & 18 & & 242 & 0 & $\begin{array}{l}\text { ACUCARE } \\
\text { ALCOOL }\end{array}$ & 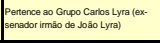 \\
\hline 312125 & $\begin{array}{l}\text { JOÅO JOSÉ PEREIRA } \\
\text { DE LYRA }\end{array}$ & Ртв AL & $\begin{array}{l}\text { INCRA } \\
2003\end{array}$ & $\begin{array}{l}\text { FAZENDA } \\
\text { CANADA }\end{array}$ & DELTA & MG & 18 & & 234 & 0 & $\begin{array}{l}\text { ACUCARE } \\
\text { ALCOOL }\end{array}$ & $\begin{array}{l}\text { Pertence ao Ginpo Cartos Lyra (ex- } \\
\text { serandor imäo de Joầo Lyra) }\end{array}$ \\
\hline 312125 & $\begin{array}{c}\text { JOÃO JOSÉ PEREERA } \\
\text { DE LYRA }\end{array}$ & PtBal & $\begin{array}{l}\text { INCRA } \\
2003\end{array}$ & $\begin{array}{c}\text { FAZENDA } \\
\text { PONTE ALTA } \\
\end{array}$ & DELTA & MG & 16 & & 198 & 0 & $\begin{array}{l}\text { ACUCARE } \\
\text { ALCOOL }\end{array}$ & $\begin{array}{l}\text { Pertence ao Ginpo Cartos Lyra (ex- } \\
\text { senador immäo de Joäo Lyra) }\end{array}$ \\
\hline 312125 & $\begin{array}{l}\text { JOÅO JOSÉ PEREERA } \\
\text { DE LYRA }\end{array}$ & PTB AL & $\begin{array}{l}\text { INCRA } \\
2003\end{array}$ & $\begin{array}{l}\text { FAZENDA } \\
\text { NASCENTE }\end{array}$ & DELTA & MG & 14 & & 181 & 0 & $\begin{array}{l}\text { ACUCARE } \\
\text { ALCOOL }\end{array}$ & $\begin{array}{l}\text { Pertence ao Ginpo Cartos Lyra (ex- } \\
\text { senador imäo de Joốo Lyra) }\end{array}$ \\
\hline 312125 & \begin{tabular}{|l} 
JOĂO JOSÉ PEREERA \\
DE LYRA
\end{tabular} & PTB AL & $\begin{array}{l}\text { INCRA } \\
2003 \\
\end{array}$ & $\begin{array}{c}\text { FAZENDA } \\
\text { GAMELEIRA }\end{array}$ & DELTA & MG & 11 & & 146 & 0 & $\begin{array}{l}\text { ACUCARE } \\
\text { ALCOOL }\end{array}$ & 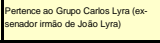 \\
\hline 312125 & $\begin{array}{l}\text { JOÄO JOSÉ PERERA } \\
\text { DE LYRA }\end{array}$ & PtB AL & $\begin{array}{l}\text { INCRA } \\
2003\end{array}$ & $\begin{array}{l}\text { FAZENDA } \\
\text { CANOEIRO }\end{array}$ & DELTA & Ma & 11 & & 145 & ${ }^{0}$ & $\begin{array}{l}\text { ACUCARE } \\
\text { ALCOOL }\end{array}$ & $\begin{array}{l}\text { Pertence ao Ginpo Cartos L Lyra (ex- } \\
\text { senador imáo de Joôo Lyra) }\end{array}$ \\
\hline 312125 & $\begin{array}{l}\text { JOÁO JOSÉ PEREERA } \\
\text { DE LYRA }\end{array}$ & Ptв AL & $\begin{array}{l}\text { INCRA } \\
2003\end{array}$ & $\begin{array}{c}\text { FAZENDA } \\
\text { CACHEERA }\end{array}$ & DELTA & MG & 11 & & 112 & 0 & $\begin{array}{l}\text { ACUCARE } \\
\text { ALCOOL }\end{array}$ & $\begin{array}{l}\text { Pertence ao Gupo Caros L Lyra (ex- } \\
\text { serador imäo de Joöo Lyra) }\end{array}$ \\
\hline 312125 & \begin{tabular}{|l} 
JOĀo JOSÉ PEREERA \\
DE LYRA
\end{tabular} & PTB AL & $\begin{array}{l}\text { INCFA } \\
2003\end{array}$ & \begin{tabular}{|l|} 
FAZENDA \\
COLORADO \\
\end{tabular} & DELTA & MG & 11 & & 111 & 0 & $\begin{array}{l}\text { ACUCARE } \\
\text { ALCOOL }\end{array}$ & $\begin{array}{l}\text { Pertenceo ao Grupo Cartos Lyra (ex- } \\
\text { senador imáa de Joâo Lyra) }\end{array}$ \\
\hline 312125 & $\begin{array}{l}\text { JOÅO JOSÉ PEREERA } \\
\text { DE LYRA }\end{array}$ & PtB AL & $\begin{array}{l}\text { INCRA } \\
2003\end{array}$ & $\begin{array}{l}\text { FAZENDA } \\
\text { ESPINHA }\end{array}$ & DELTA & MG & 0 & & ${ }^{81}$ & 0 & $\begin{array}{l}\text { ACUCARE } \\
\text { ALCOOL }\end{array}$ & $\begin{array}{l}\text { Pertence ao Grupo Cartos Lyra (ex- } \\
\text { Senador immäo de Joôo Lyra) }\end{array}$ \\
\hline 312125 & $\begin{array}{l}\text { JOÅO JOSÉ PERERA } \\
\text { DE LYRA }\end{array}$ & PTB AL & $\begin{array}{l}\text { INCRA } \\
2003\end{array}$ & \begin{tabular}{|l} 
JARDIM DAS \\
OLIVEIRAS \\
\end{tabular} & DELTA & MG & 0 & & 62 & 0 & $\begin{array}{l}\text { ACUCARE } \\
\text { ALCOOL }\end{array}$ & 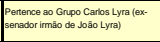 \\
\hline 312125 & $\begin{array}{l}\text { JOÄO JOSÉ PERERAA } \\
\text { DE LYRA }\end{array}$ & Ptв AL & $\begin{array}{l}\text { INCRA } \\
2003\end{array}$ & $\begin{array}{l}\text { FAZENDA } \\
\text { SANTO INACIO }\end{array}$ & DELTA & Ma & 0 & & 27 & 0 & $\begin{array}{l}\text { ACUCARE } \\
\text { ALCOOL }\end{array}$ & $\begin{array}{l}\text { Pertence ao Ginpo Cartos L Lyra (ex- } \\
\text { senador imâo de Joẫo Lyra) }\end{array}$ \\
\hline
\end{tabular}




\begin{tabular}{|c|c|c|c|c|c|c|c|c|c|c|c|c|}
\hline GEOCÓDIGO & PARLAMENTAR & PARTIDO & FONTE & IMOVEL & MunicíPIo & uF & AREA & cuassensoniman & \begin{tabular}{|c|} 
AREA \\
REGISTRAD \\
A
\end{tabular} & $\begin{array}{l}\text { DEELAAADA } \\
\text { COMO POSSE }\end{array}$ & EMPRESA & VINCULO COM O PARLAMENTAR \\
\hline 312125 & $\begin{array}{l}\text { JOÁO JoSÉ PEREIRA } \\
\text { DE LYRA }\end{array}$ & PTB AL & $\begin{array}{l}\text { INCFA } \\
2003\end{array}$ & $\begin{array}{l}\text { FAZENDA } \\
\text { ALEGRIA }\end{array}$ & DELTA & MG & 0 & & 26 & 0 & $\begin{array}{l}\text { ACUCAR E } \\
\text { ALCOOL }\end{array}$ & 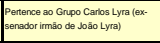 \\
\hline 312770 & LAEL VEEIRA VARELLA & PMDB MG & $\begin{array}{l}\text { INCRA } \\
20033\end{array}$ & $\begin{array}{l}\text { FAZENDA } \\
\text { AROEIRA }\end{array}$ & $\begin{array}{l}\text { GOVERNADOR } \\
\text { VALADARES }\end{array}$ & Ma & 44 & Prncosestave & 1.431 & 0 & $\begin{array}{c}\text { LAEL VARELLA } \\
\text { LTDA }\end{array}$ & LAEL VARELLA \\
\hline 313440 & $\begin{array}{l}\text { ODELMO LEAO } \\
\text { CARNERORO SEBRINHO }\end{array}$ & PPMG & $\begin{array}{l}\text { INCRAA } \\
2003\end{array}$ & \begin{tabular}{|c|} 
FAZENDA \\
SANTA ROSA
\end{tabular} & |TURAMA & ma & 0 & & so & 0 & $\begin{array}{l}\text { BRASIL CENTRAL } \\
\text { DE CARNES S A }\end{array}$ & $\begin{array}{l}\text { ODELMOLEAO ARNNERO } \\
\text { SOBRINHO declarou } 40 \text { cotas }\end{array}$ \\
\hline 313830 & NEWTON CARDOSO & PMDB MG & $\begin{array}{l}\text { INCRA } \\
2003\end{array}$ & $\begin{array}{c}\text { FAZENDA } \\
\text { LAGOA RICA } \\
\end{array}$ & $\begin{array}{l}\text { LEANDRO } \\
\text { FERREIRA }\end{array}$ & MG & 16 & & 240 & 0 & $\begin{array}{c}\text { SIOERURGICA } \\
\text { PITANGUI }\end{array}$ & $\begin{array}{l}\text { nenton cardoso declarou companhia } \\
\text { siderurgica pitangui }\end{array}$ \\
\hline 313830 & NEWTON CARDOSO & Рмов MG & $\begin{array}{l}\text { INCRA } \\
2003\end{array}$ & $\begin{array}{l}\angle A G O A \\
\text { CORACAO }\end{array}$ & $\begin{array}{l}\text { LEANDRO } \\
\text { FERREIRA }\end{array}$ & MG & 11 & & 172 & 0 & $\begin{array}{l}\text { SIDERURGICA } \\
\text { PITANGUI }\end{array}$ & $\begin{array}{l}\text { nenton cardoso declarou compantia } \\
\text { sidenrugica pitangui }\end{array}$ \\
\hline 314050 & NEWTON CARDOSO & PMDB MG & $\begin{array}{l}\text { INCRA } \\
2003\end{array}$ & $\begin{array}{l}\text { BOCAINAE } \\
\text { FECHO }\end{array}$ & $\begin{array}{l}\text { MARTINHO } \\
\text { CAMPOS }\end{array}$ & ma & 58 & monomensure & 884 & 0 & \begin{tabular}{|l} 
SIDERURGICA \\
PITANGUI
\end{tabular} & $\begin{array}{l}\text { nevton cardoso declarou compantia } \\
\text { sidenrugica pitangui }\end{array}$ \\
\hline 314050 & NEWTON CARDOSO & PMDB MG & $\begin{array}{l}\text { INCRA } \\
2003\end{array}$ & $\begin{array}{l}\text { CAPAO RIACHO } \\
\text { DAS PEDRAS }\end{array}$ & $\begin{array}{l}\text { MARTINHO } \\
\text { CAMPOS }\end{array}$ & Ma & ${ }^{33}$ & & 504 & ${ }^{0}$ & \begin{tabular}{|l|} 
SIOERURGICA \\
PITANGUI
\end{tabular} & $\begin{array}{l}\text { nenton cardoso declarou compantia } \\
\text { siderngica pitargui }\end{array}$ \\
\hline 314050 & NEWTON CARDOSO & PMDB MG & $\begin{array}{l}\text { INCRA } \\
2003 \\
\end{array}$ & $\begin{array}{l}\text { FAZENDA } \\
\text { CRISCIUMAA }\end{array}$ & $\begin{array}{l}\text { MARTINHO } \\
\text { CAMPOS }\end{array}$ & MG & 33 & & 496 & 0 & $\begin{array}{c}\text { SIOERURGICA } \\
\text { PITANGUI }\end{array}$ & $\begin{array}{l}\text { nevton cardoso declarou companhia } \\
\text { siderurgica pitangui }\end{array}$ \\
\hline 314050 & NEWTON CARDOSO & PMDB MG & $\begin{array}{l}\text { INCFA } \\
2003\end{array}$ & \begin{tabular}{|l} 
RiACHO BURTIT \\
DOS BORGES
\end{tabular} & $\begin{array}{l}\text { MARTINHO } \\
\text { CAMPOS }\end{array}$ & MG & 32 & & 480 & 0 & \begin{tabular}{|c|}
$\begin{array}{c}\text { SIDERURGICA } \\
\text { PITANGUI }\end{array}$ \\
\end{tabular} & $\begin{array}{l}\text { nemton cardoso declarou companhia } \\
\text { sidenurgica pitarngui }\end{array}$ \\
\hline 314050 & NEWTON CARDOSO & PMDB MG & $\begin{array}{l}\text { INCRA } \\
2003\end{array}$ & $\begin{array}{l}\text { FAZENDA } \\
\text { RASGAO }\end{array}$ & $\begin{array}{l}\text { MARTINHO } \\
\text { CAMPOS }\end{array}$ & Ma & 26 & & 400 & 0 & \begin{tabular}{|l|} 
SIDERURGICA \\
PITANGUI
\end{tabular} & $\begin{array}{l}\text { neenton cardoso declarou compantia } \\
\text { siderergica pitangui }\end{array}$ \\
\hline 314070 & NEWTON CARDOSO & PMDB MG & $\begin{array}{l}\text { INCRA } \\
2003\end{array}$ & \begin{tabular}{|c|} 
FAZENDA \\
SERRA AZUL \\
\end{tabular} & MATEUS LEME & MG & 0 & & 56 & 0 & $\begin{array}{c}\text { SIDERURGICA } \\
\text { PITANGUI }\end{array}$ & $\begin{array}{l}\text { nemton cardoso declarou companhis } \\
\text { siderengrica pitangui }\end{array}$ \\
\hline 314390 & $\begin{array}{l}\text { LAEL VIEIRA VARELLA } \\
\end{array}$ & PMDB MG & $\begin{array}{l}\text { INCRA } \\
2003\end{array}$ & $\begin{array}{l}\text { FAZENDA } \\
\text { ELOORADO }\end{array}$ & MURIAE & MG & 16 & & 382 & 0 & $\begin{array}{c}\text { LAEL VARELAA } \\
\text { LTDA }\end{array}$ & LAEL VARELLA \\
\hline
\end{tabular}




\begin{tabular}{|c|c|c|c|c|c|c|c|c|c|c|c|c|}
\hline GEOCOODISO & PARLAMENTAR & PARTIDO & FONTE & IMOVEL & Municipio & UF & AREA & cuasse frowivan & \begin{tabular}{|c|} 
AREA \\
REGISTRAD \\
A
\end{tabular} & $\begin{array}{l}\text { DECLARADA } \\
\text { COMO POSSE }\end{array}$ & EMPRESA & VINCULO COM O PARLAMENTAR \\
\hline 314390 & LAELL VEIRA VARELLA & PMDBMG & $\begin{array}{l}\text { INCRA } \\
2003\end{array}$ & $\begin{array}{c}\text { FAZENDA } \\
\text { CACHOEIRA } \\
\end{array}$ & MURIAAE & MG & 30 & & 320 & 0 & $\begin{array}{c}\text { LAEL VARELLA } \\
\text { LTDA }\end{array}$ & LAel Varella \\
\hline 314390 & $\begin{array}{l}\text { LAEL VEEIRA VARELLA } \\
\end{array}$ & PMDB MG & $\begin{array}{l}\text { INCRA } \\
2003\end{array}$ & $\begin{array}{c}\text { FAZENDA } \\
\text { CACHOEIRA }\end{array}$ & MURIAE & MG & 15 & & 320 & 0 & $\begin{array}{c}\text { LAEL VARELLA } \\
\text { LTDA }\end{array}$ & LAEL VARELLA \\
\hline 314390 & 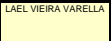 & PMDB MG & $\begin{array}{l}\text { INCRA } \\
2003\end{array}$ & $\begin{array}{c}\text { FAZENDA } \\
\text { ELDORADO }\end{array}$ & MURIAE & Ma & 0 & & 28 & 0 & $\begin{array}{c}\text { LAEL VARELLA } \\
\text { LTDA }\end{array}$ & LAEL VARELLA \\
\hline 315120 & $\begin{array}{l}\text { ROMEU FERREIRA DE } \\
\text { OUEROZ }\end{array}$ & PTBMG & $\begin{array}{l}\text { INCRA } \\
2003\end{array}$ & $\begin{array}{l}\text { FAZ VIVEIROS } \\
\text { FLOBESTAP } \\
\text { BRIGIIDA }\end{array}$ & PIRAPORA & MG & 276 & & 7.214 & 0 & $\begin{array}{l}\text { UNIAGRO UNIAO } \\
\text { AGROPECUARIA } \\
\text { PIRAPORA SA }\end{array}$ & $\begin{array}{l}\text { ROMEU OUEIROZ DECLAROU FAZ } \\
\text { VIVEIRO, ESTEE O OUNICO FA } \\
\text { REGISTOFAZENDA VIVEIRO EM } \\
\text { PIRAPORA }\end{array}$ \\
\hline 315140 & NEWTON CARDOSO & PMDB мG & $\begin{array}{l}\text { INCRA } \\
2003\end{array}$ & $\begin{array}{l}\text { CACHOEIRAE } \\
\text { MRAANDA }\end{array}$ & Pitangul & MG & 78 & nomentint & 751 & 0 & $\begin{array}{c}\text { SIDERURGICA } \\
\text { PITANGUI }\end{array}$ & $\begin{array}{l}\begin{array}{l}\text { nemton cardoso declarou companhia } \\
\text { sidenrugica pitarguii }\end{array} \\
\end{array}$ \\
\hline 315140 & NEWTON CARDOSO & PMDв мG & $\begin{array}{l}\text { INCRA } \\
2003\end{array}$ & $\begin{array}{l}\text { FAZENDA } \\
\text { BARNABE }\end{array}$ & Pitangul & MG & 20 & & 250 & 0 & $\begin{array}{c}\text { SIDERURGICA } \\
\text { PITANGUI }\end{array}$ & $\begin{array}{l}\begin{array}{l}\text { nemton cardoso declarou compantia } \\
\text { siderugica pitangui }\end{array} \\
\end{array}$ \\
\hline 315140 & NEWTON CARDOSO & PMDB ma & $\begin{array}{l}\text { INCRA } \\
2003\end{array}$ & $\begin{array}{l}\text { FAZENDA } \\
\text { ENGENHO }\end{array}$ & Pittangul & Ma & 0 & & 84 & 0 & \begin{tabular}{|c|} 
COMPANHIA \\
SIOERURGICA \\
PITANGUI
\end{tabular} & $\begin{array}{l}\begin{array}{l}\text { nenton cardoso declarou compantia } \\
\text { sidenugica pitarngui }\end{array} \\
\text { a }\end{array}$ \\
\hline 315200 & NEWTON CARDOSO & PMDв мG & $\begin{array}{l}\text { INCRA } \\
2003\end{array}$ & $\begin{array}{l}\text { FAZENDA } \\
\text { DIAMANTE }\end{array}$ & POMPÉU & MG & 303 & maneresed & 4.639 & 0 & \begin{tabular}{|c|} 
COMPANHIA \\
SIOERURGICA \\
PITANGUI
\end{tabular} & $\begin{array}{l}\text { nemton cardoso declarou companhia } \\
\text { siderurgica pitanguil }\end{array}$ \\
\hline 315200 & NEWTON CARDOSO & PMDB MG & $\begin{array}{l}\text { INCRA } \\
2003\end{array}$ & $\begin{array}{l}\text { FAZENDA } \\
\text { RETIRO }\end{array}$ & POMPÉU & MG & 31 & & 469 & 0 & \begin{tabular}{|c|} 
COMPANHIA \\
SIOERRRGGIIAA \\
PITANGUI
\end{tabular} & $\begin{array}{l}\text { nenton cardoso declarou compantia } \\
\text { sidenurgica pitanguil }\end{array}$ \\
\hline
\end{tabular}




\begin{tabular}{|c|c|c|c|c|c|c|c|c|c|c|c|c|}
\hline GEOCÓDIGO & PARLAMENTAR & PARTIDO & FONTE & IMÓVEL & MUNICIPIO & UF & AREA & chases hucivon & $\begin{array}{c}\text { AREA } \\
\text { REGISTRAD } \\
\text { A }\end{array}$ & $\begin{array}{l}\text { DECLARADA } \\
\text { COMO POSSE }\end{array}$ & EMPRESA & VINCULO COM O PARLAMENTAR \\
\hline 316470 & SILAS BRASILEIRO & PMDBMG & $\begin{array}{l}\text { INCFA } \\
2003\end{array}$ & $\begin{array}{l}\text { FAZENDA SAO } \\
\text { JOSE }\end{array}$ & $\begin{array}{l}\text { SEBASTIÅO DO } \\
\text { PARAISO }\end{array}$ & MG & 26 & & 395 & 0 & $\begin{array}{l}\text { ENGENHARIA } \\
\text { LTDA }\end{array}$ & $25 \%$ declarado por silas bras ieiro \\
\hline 317010 & $\begin{array}{l}\text { JOĀO JOSÉ PEREIRA } \\
\text { DE LYRA }\end{array}$ & PTB AL & $\begin{array}{l}\text { INCRA } \\
2003\end{array}$ & FAZENDA JAO & UBERABA & MG & 0 & & 87 & 0 & $\begin{array}{c}\text { USINA DELTA SA } \\
\text { ACUCAR E } \\
\text { ALCOOL }\end{array}$ & $\begin{array}{l}\text { Pertence ao Grupo Carlos Lyra (ex- } \\
\text { senador irmäo de Joấo Lyra) }\end{array}$ \\
\hline 317010 & $\begin{array}{l}\text { JOÅO JOSÉ PEREIRA } \\
\text { DE LYRA }\end{array}$ & PTB AL & $\begin{array}{c}\text { INCRA } \\
2003\end{array}$ & $\begin{array}{l}\text { FAZENDA } \\
\text { ESPINHA }\end{array}$ & UBERABA & MG & 0 & & 87 & 0 & $\begin{array}{l}\text { ACUCARE } \\
\text { ALCOOL }\end{array}$ & $\begin{array}{l}\text { Pertence ao Grupo Carlos Lyra (ex- } \\
\text { senador irmäo de Joâo Lyra) }\end{array}$ \\
\hline 317010 & $\begin{array}{l}\text { JOÅO JOSÉ PEREIRA } \\
\text { DE LYRA. }\end{array}$ & PTB AL & $\begin{array}{c}\text { INCRA } \\
2003\end{array}$ & $\begin{array}{l}\text { FAZENDA } \\
\text { LARANJEIRAS }\end{array}$ & UBERABA & MG & 0 & & 75 & 0 & $\begin{array}{l}\text { ACUCARE } \\
\text { ALCOOL }\end{array}$ & $\begin{array}{l}\text { Pertence ao Grupo Carlos Lyra (ex- } \\
\text { senador irmăo de Joấ Lyra) }\end{array}$ \\
\hline 317010 & $\begin{array}{l}\text { JOÅO JOSÉ PEREIRA } \\
\text { DE LYRA }\end{array}$ & PTB AL & $\begin{array}{l}\text { INCRA } \\
2003\end{array}$ & $\begin{array}{l}\text { ESTANCIA } \\
\text { PONTA PORA }\end{array}$ & UBERABA & MG & 0 & & 58 & 0 & $\begin{array}{l}\text { ACUCARE } \\
\text { ALCOOL }\end{array}$ & $\begin{array}{l}\text { Pertence ao Grupo Carlos Lyra (ex- } \\
\text { senador irmäo de Joäo Lyra) }\end{array}$ \\
\hline 317010 & $\begin{array}{l}\text { JOĂO JOSÉ PEREIRA } \\
\text { DE LYRA }\end{array}$ & PTB AL & $\begin{array}{l}\text { INCRA } \\
2003\end{array}$ & $\begin{array}{l}\text { FAZENDA } \\
\text { PEROBAS }\end{array}$ & UBERABA & MG & 0 & & 37 & 0 & $\begin{array}{l}\text { ACUCARE } \\
\text { ALCOOL }\end{array}$ & $\begin{array}{l}\text { Pertence ao Grupo Carlos Lyra (ex- } \\
\text { senador irmáo de Joäo Lyra) }\end{array}$ \\
\hline 510706 & $\begin{array}{l}\text { BLAIRO BORGES } \\
\text { MAGGI }\end{array}$ & PRMT & $\begin{array}{l}\text { INCRA } \\
2003\end{array}$ & $\begin{array}{l}\text { AGRICOLAE } \\
\text { PECUARIA } \\
\text { MORRO AZUL } \\
\text { LTD }\end{array}$ & QUERĖNCIA & MT & 29.200 & pnoreseave & 29.200 & 0 & $\begin{array}{l}\text { AGRICOLAE } \\
\text { PECUARIA } \\
\text { MORRO AZUL } \\
\text { LTDA }\end{array}$ & 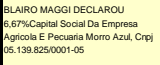 \\
\hline 510706 & $\begin{array}{l}\text { BLAIRO BORGES } \\
\text { MAGGI }\end{array}$ & PRMT & $\begin{array}{l}\text { INCRA } \\
2003\end{array}$ & $\begin{array}{l}\text { AGRICOLAE } \\
\text { PECUARIA } \\
\text { MORRO AZUL } \\
\text { LTD }\end{array}$ & QUERËNCIA & MT & 19.200 & 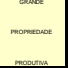 & 19.200 & 0 & $\begin{array}{l}\text { AGRICOLAE } \\
\text { PECUARIA } \\
\text { MORRO AZUL } \\
\text { LTDA }\end{array}$ & $\begin{array}{l}\text { BLAIRO MAGGI DECLAROU } \\
\text { 6,67\%Capital Social Da Empresa } \\
\text { Agricola E Pecuara Morro Azu, Croj } \\
\text { 05.139.825/0001-06 }\end{array}$ \\
\hline 510706 & $\begin{array}{l}\text { BLAIIO BORGES } \\
\text { MAGGI }\end{array}$ & PR MT & $\begin{array}{l}\text { INCRA } \\
2003\end{array}$ & $\begin{array}{l}\text { AGRICOLAE } \\
\text { PECUARIA } \\
\text { MORRO AZUL } \\
\text { LTD }\end{array}$ & QUERĖNCIA & MT & 24.400 & Hochessfraba & 24.200 & 0 & $\begin{array}{l}\text { AGRICOLAE } \\
\text { PECUARIA } \\
\text { MORRO AZUL } \\
\text { LTDA }\end{array}$ & $\begin{array}{l}\text { BLAIRO MAGGI DECLAROU } \\
\text { 6,67\%Capital Social Da Empresa } \\
\text { Agricola E Pecuara Morro Azu, Crej } \\
\text { 05.139.825/0001-07 }\end{array}$ \\
\hline
\end{tabular}




\begin{tabular}{|c|c|c|c|c|c|c|c|c|c|c|c|c|}
\hline GEOCÓDICO & PARLAMENTAR & PARTIDO & FONTE & IMÓVEL & Municíio & uF & AREA & chases necouvan & $\begin{array}{c}\text { AREA } \\
\text { REGISTRAD } \\
\text { A }\end{array}$ & $\begin{array}{l}\text { DECLARADA } \\
\text { COMO POSSE }\end{array}$ & EMPRESA & VINCULO COM O PARLAMENTAR \\
\hline 510268 & $\begin{array}{l}\text { BLAIRO BORGES } \\
\text { MAGGI }\end{array}$ & PR MT & INCRA 2003 & $\begin{array}{c}\text { CAMPOS DE } \\
\text { JULIO }\end{array}$ & $\begin{array}{l}\text { CAMPOS DE } \\
\text { JüLOO }\end{array}$ & MT & 15 & nerenoo & 15 & 0 & $\begin{array}{c}\text { AGROPECUARIA } \\
\text { MAGGI LTDA }\end{array}$ & \\
\hline 510760 & $\begin{array}{l}\text { BLAIRO BORGES } \\
\text { MAGGI }\end{array}$ & PRMT & INCRA 2003 & FAZENDA SM 5 & $\begin{array}{l}\text { RONDONOPOLI } \\
\text { s }\end{array}$ & мT & 998 & pnocentease & 998 & 0 & $\begin{array}{c}\text { AGROPECUARIA } \\
\text { MAGGI LTDA }\end{array}$ & \\
\hline 510760 & $\begin{array}{c}\text { BLAARO BORGES } \\
\text { MAGGI }\end{array}$ & PRMT & INCRA 2008 & FAZENDA SM 4 & \begin{tabular}{|l|} 
RONDONÓPOLL \\
s
\end{tabular} & мт & 809 & & 809 & 0 & \begin{tabular}{|c|} 
AGROPECUAARIA \\
MAGGI LTDA
\end{tabular} & \\
\hline 510787 & $\begin{array}{l}\text { BLAARO BORGES } \\
\text { MAGGI }\end{array}$ & PRMT & INCRA 2003 & $\begin{array}{l}\text { FAZENDA } \\
\text { TUCUNARE }\end{array}$ & SAPEZAL & MT & 13.245 & 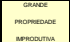 & 13.245 & 0 & $\begin{array}{c}\text { AGROPECUARIA } \\
\text { MAGGILTDA }\end{array}$ & \\
\hline 510787 & $\begin{array}{c}\text { BLAIRO BORGGS } \\
\text { MAGGI }\end{array}$ & PRMT & INCRA 2000 & $\begin{array}{l}\text { PCH SANTA } \\
\text { LUCIA }\end{array}$ & SAPEZAL & MT & 25 & nerewoo & 25 & 0 & $\begin{array}{c}\begin{array}{c}\text { AGROPECUAAIA } \\
\text { MAGGI LTDA }\end{array} \\
\end{array}$ & \\
\hline 510787 & $\begin{array}{l}\text { BLAIRO BORGES } \\
\text { MAGGI }\end{array}$ & PR MT & $\begin{array}{l}\text { INCRA } \\
2003\end{array}$ & $\begin{array}{l}\text { FAZENDA } \\
\text { CIDEZAL }\end{array}$ & SAPEZAL & MT & 609 & & 609 & 0 & \begin{tabular}{c|} 
CIDREZAL \\
AGRICELA LTDA
\end{tabular} & BLAIRO MAGGI DECLAROU \\
\hline 510267 & $\begin{array}{c}\text { BLAIRO BORGES } \\
\text { MAGGI }\end{array}$ & PRMT & INCRA 2003 & $\begin{array}{l}\text { FAZENDAA } \\
\text { TABAJARA }\end{array}$ & CAMPO VERDE & MT & 116 & & 116 & 0 & $\begin{array}{c}\text { SEMENTES } \\
\text { MAGGI LTDA }\end{array}$ & \\
\hline 510460 & $\begin{array}{l}\text { BLAIRO BORGES } \\
\text { MAGGI }\end{array}$ & PRMT & $\begin{array}{l}\text { INCRA } \\
2003\end{array}$ & $\underset{\text { ARMAZENADOR }}{\text { A }}$ & |TIQUiRA & MT & 25 & nevenoo & 25 & 0 & $\begin{array}{l}\text { SEMENTES } \\
\text { MAGGI LTDA }\end{array}$ & \\
\hline 510760 & $\begin{array}{l}\text { BLAIRO BORGES } \\
\text { MAGGI }\end{array}$ & PRMT & INCRA 2003 & $\begin{array}{c}\text { FAZENDA } \\
\text { MINEIRINHO }\end{array}$ & $\begin{array}{l}\text { RONDONOPOLLI } \\
s\end{array}$ & MT & 5 & nexuso & 5 & 0 & $\begin{array}{l}\text { SEMENTES } \\
\text { MAGGILTDA }\end{array}$ & \\
\hline 510760 & $\begin{array}{l}\text { BLAIRO BORGES } \\
\text { MAGGI }\end{array}$ & PRMT & INCRA 2000 & $\begin{array}{l}\text { PONTE DE } \\
\text { PEDRA }\end{array}$ & \begin{tabular}{ll|} 
RONDONÓPOLI \\
s
\end{tabular} & MT & 62 & & 62 & 0 & $\begin{array}{l}\text { SEMENTES } \\
\text { MAGGI LTDA }\end{array}$ & \\
\hline 510330 & $\begin{array}{l}\text { DILSO SPERAFICO E } \\
\text { DILCEUJOÁO } \\
\text { SPERAFICO }\end{array}$ & PSDB MS e & INCPA 2000 & $\begin{array}{c}\text { ESTANCIA } \\
\text { BELEM VIEIRA }\end{array}$ & СоMODORO & мT & 2.738 & manomenowe & 2.738 & 2.738 & $\begin{array}{l}\text { AGRICOLA } \\
\text { SPERAAIICO } \\
\text { LTDA }\end{array}$ & \\
\hline
\end{tabular}




\begin{tabular}{|c|c|c|c|c|c|c|c|c|c|c|c|c|}
\hline GEOCODI@O & PARLAMENTAR & PARTIDO & FONTE & IMOUEL & Municipio & uF & AREA & cheses nucivar & $\begin{array}{c}\text { AREA } \\
\text { REGISTRAD } \\
\text { A }\end{array}$ & $\begin{array}{l}\text { DECLARADA } \\
\text { COMO POSSE }\end{array}$ & EMPRESA & VINCULO COM O PARLAMENTAR \\
\hline 510268 & $\begin{array}{c}\text { DLLSO SPERAFICO E } \\
\text { DILCEN UOAOAO } \\
\text { SPERAFICO } \\
\end{array}$ & PSDBMS E & INCRA 200 & $\begin{array}{c}\text { CHACARA } \\
\text { GLEBA JUINA III } \\
\end{array}$ & $\begin{array}{l}\text { CAMPOS DE } \\
\text { JúLLO }\end{array}$ & мT & 11 & nerewoo & 11 & 0 & $\begin{array}{c}\text { AGFICOLA } \\
\text { SPERAFICO } \\
\text { LTDA } \\
\end{array}$ & \\
\hline 510704 & $\begin{array}{c}\text { DLLSO SPERAFICO E } \\
\text { DILCEU OÁO } \\
\text { SPERAFICO }\end{array}$ & PSDB MS E & INCRA 2003 & \begin{tabular}{|c|} 
FAZENDA \\
LUCIANA \\
PRIIAAVEA DO \\
LESTE
\end{tabular} & $\begin{array}{l}\text { PRIMAVERA DO } \\
\text { LESTE }\end{array}$ & MT & 9 & nerwaso & ${ }^{9}$ & 0 & $\begin{array}{c}\text { AGRICOLA } \\
\text { SPERAFIOO } \\
\text { LTDA }\end{array}$ & \\
\hline 510340 & $\begin{array}{l}\text { DLLSO SPERAFICO E } \\
\text { DILCEU JOÁO } \\
\text { SPERAFICO }\end{array}$ & PSDBMS & INCRA 2003 & $\begin{array}{l}\text { BOA VISTA OU } \\
\text { PAULINA EM } \\
\text { COXIPO }\end{array}$ & CuIABÁ & MT & 16 & nensoo & 16 & 0 & $\begin{array}{l}\text { SPERAFICO DA } \\
\text { AMAZONIA SA }\end{array}$ & \\
\hline 510340 & $\begin{array}{c}\text { DILSO SPERAFICO E } \\
\text { DILCEU OAOO } \\
\text { SPERAFICO }\end{array}$ & PSOB MS E & INCRA 200 & $\begin{array}{c}\text { EXTRACAO } \\
\text { OLEOS CUIABA } \\
\end{array}$ & CUIABÁ & MT & 16 & mentao & 16 & 0 & $\begin{array}{l}\text { SPERAFICO DA } \\
\text { AMAZONIA SA }\end{array}$ & \\
\hline 510268 & $\begin{array}{c}\text { DILSO SPERAFFCO E } \\
\text { DILCEU OÁO } \\
\text { SPERAFFO }\end{array}$ & PSDBMS & INCRA 2003 & $\begin{array}{l}\text { FAZENDA } \\
\text { MASSAFAA }\end{array}$ & $\begin{array}{l}\text { CAMPOS DE } \\
\text { JúLIO }\end{array}$ & MT & 30 & nerenoso & 30 & 0 & $\begin{array}{l}\text { SPERAFICO DA } \\
\text { AMAZONIA SA }\end{array}$ & \\
\hline 510350 & $\begin{array}{l}\text { DILCEU JOÁO } \\
\text { SPERAFICO }\end{array}$ & PSDB MS e & INCRA 200 & TAPURAH & Diamantino & мT & 3 & merwaso & 3 & 0 & $\begin{array}{l}\text { SPERAFICO DA } \\
\text { AMAZONIA SA }\end{array}$ & \\
\hline 251080 & 8 PEREIRA DE & $\mathrm{PFLPB}^{\text {PLPB }}$ & $\begin{array}{l}\text { INCRA } \\
2003\end{array}$ & $\begin{array}{c}\text { SITIO PEDRA } \\
\text { BRANCA }\end{array}$ & PATOS & $\mathrm{PB}$ & 163 & & $15 B$ & 0 & $\begin{array}{c}\text { ALGODOEIRA } \\
\text { HORACIO } \\
\text { NOBREGA SA } \\
\end{array}$ & $\begin{array}{l}\text { ADAUTO PEREIRA DE LIMA TEM } \\
\text { ACOES }\end{array}$ \\
\hline 250030 & ENIVALDO RIBEIRO & PP PB & $\begin{array}{l}\text { INCFA } \\
2003 \\
\end{array}$ & $\begin{array}{c}\text { FAZENDA SAO } \\
\text { JOSE }\end{array}$ & $\begin{array}{l}\text { ALAGOA } \\
\text { GRANDE }\end{array}$ & $\mathrm{PB}$ & 720 & 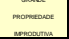 & 720 & 0 & $\begin{array}{c}\text { USINA TANQUES } \\
\text { SIA }\end{array}$ & \\
\hline 250030 & ENIVALDO RIBEIRO & PP PB & $\begin{array}{l}\text { INCFA } \\
2003\end{array}$ & $\begin{array}{c}\text { SERROTINHO } \\
\text { GRUTAO }\end{array}$ & $\begin{array}{l}\text { ALAGOA } \\
\text { GRANDE }\end{array}$ & $\mathrm{PB}$ & 1.750 & 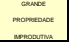 & 1.750 & 0 & $\begin{array}{c}\text { USINA TANQUES } \\
\text { S/A }\end{array}$ & \\
\hline 250030 & ENIVALDO RIBEIRO & PP PB & $\begin{array}{l}\text { INCFA } \\
2003\end{array}$ & $\begin{array}{c}\text { FAZENDA } \\
\text { GURIINHEZINHO }\end{array}$ & $\begin{array}{l}\text { ALAGOA } \\
\text { GRANDE }\end{array}$ & PB & 400 & & 400 & 0 & $\begin{array}{c}\text { USINA TANQUES } \\
\text { SiA }\end{array}$ & \\
\hline
\end{tabular}




\begin{tabular}{|c|c|c|c|c|c|c|c|c|c|c|c|c|}
\hline GEOCÓDICO & PARLAMENTAR & PARTIDO & FONTE & IMÓVEL & MunicíPIO & uF & AREA & cuases nucown & $\begin{array}{c}\text { AREA } \\
\text { REGISTRAD } \\
\text { A }\end{array}$ & $\begin{array}{l}\text { DECLARADA } \\
\text { COMO POSSE }\end{array}$ & EMPRESA & VINCULO COM O PARLAMENTAF \\
\hline 250030 & ENIYALO RIBEIRO & PP PB & $\begin{array}{l}\text { INCRA } \\
2003\end{array}$ & $\begin{array}{c}\text { DO RIO } \\
\text { CRISTALINO }\end{array}$ & $\begin{array}{l}\text { ALAGOA } \\
\text { GRANDE }\end{array}$ & PB & 124 & & 124 & 0 & $\begin{array}{c}\text { USINA TANQUES } \\
\text { SIA }\end{array}$ & \\
\hline 250030 & ENIYALDO RIBEIRO & PP PB & $\begin{array}{l}\text { INCRA } \\
2003\end{array}$ & $\begin{array}{l}\text { FAZENDA } \\
\text { PATOS }\end{array}$ & $\begin{array}{l}\text { ALAGOA } \\
\text { GRANDE }\end{array}$ & $\mathrm{PB}$ & ${ }^{128}$ & & ${ }^{128}$ & 0 & $\begin{array}{c}\text { USINA TANQUES } \\
\text { SiA }\end{array}$ & \\
\hline 250030 & ENIVALDO RIBEIRO & PP PB & $\begin{array}{l}\text { INCRA } \\
2003\end{array}$ & $\begin{array}{l}\text { FAZENDA } \\
\text { MANDAU }\end{array}$ & $\begin{array}{l}\text { ALAGOA } \\
\text { GRANDE }\end{array}$ & PB & 150 & & 150 & 0 & $\underset{\substack{\text { USINA TANOUES } \\
\text { SAA }}}{ }$ & \\
\hline 250030 & ENIYALO RIBEEIRO & PP PB & $\begin{array}{l}\text { INCRA } \\
2003\end{array}$ & ENGENHOCA & $\begin{array}{l}\text { ALAGOA } \\
\text { GRANDE }\end{array}$ & PB & 200 & & 200 & 0 & $\begin{array}{c}\text { USINA TANQUES } \\
\text { S/A }\end{array}$ & \\
\hline 250030 & ENIVALDO RIBEIRO & PP PB & $\begin{array}{l}\text { INCRA } \\
2003 \\
\end{array}$ & JOAO PEREIRA & $\begin{array}{l}\text { ALAGOA } \\
\text { GRANDE }\end{array}$ & $\mathrm{PB}$ & 25 & nesuad & 25 & 0 & \begin{tabular}{|c|} 
USINA TANQUES \\
SIA
\end{tabular} & \\
\hline 250030 & ENIYALO RIBEIRO & PP PB & $\begin{array}{l}\text { INCRA } \\
2003\end{array}$ & LAGOA VERDE & $\begin{array}{l}\text { ALAGOA } \\
\text { GRANDE }\end{array}$ & $\mathrm{PB}$ & 6 & newnoso & 6 & 0 & 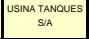 & \\
\hline 250030 & ENIVALDO RIBEEIRO & PP PB & $\begin{array}{l}\text { INCRA } \\
2003\end{array}$ & $\begin{array}{l}\text { FAZENDA } \\
\text { AVENCA }\end{array}$ & $\begin{array}{l}\text { ALAGOA } \\
\text { GRANDE }\end{array}$ & PB & 113 & & 113 & 0 & $\begin{array}{c}\text { USINA TANQUES } \\
\text { S/A }\end{array}$ & \\
\hline 250030 & ENIVALDO RIBEIRO & PP PB & $\begin{array}{l}\text { INCRA } \\
2003\end{array}$ & $\begin{array}{c}\text { FAZENDA JOSE } \\
\text { GARCIA }\end{array}$ & $\begin{array}{l}\text { ALAGOA } \\
\text { GRANOE }\end{array}$ & $\mathrm{PB}$ & 50 & & 50 & 0 & $\mid \begin{array}{c}\text { USINA TANQUES } \\
\text { S/A }\end{array}$ & \\
\hline 250030 & ENIYALD RIBEIRO & PP PB & $\begin{array}{l}\text { INCRA } \\
2003\end{array}$ & $\begin{array}{l}\text { FAZENDA } \\
\text { CAPIVARA }\end{array}$ & $\begin{array}{l}\text { ALAGOA } \\
\text { GRANDE }\end{array}$ & PB & 120 & & 120 & 0 & $\begin{array}{c}\text { USINA TANQUES } \\
\text { S/A }\end{array}$ & \\
\hline 250030 & ENIVALDO RIBEIRO & PP PB & $\begin{array}{l}\text { INCRA }_{2003} \\
\end{array}$ & $\begin{array}{l}\text { SANTO } \\
\text { ANTONIO }\end{array}$ & $\begin{array}{l}\text { ALAGOA } \\
\text { GRANDE }\end{array}$ & PB & 40 & & 40 & 0 & $\begin{array}{c}\text { USINA TANQUES } \\
\text { S/A }\end{array}$ & \\
\hline
\end{tabular}




\begin{tabular}{|c|c|c|c|c|c|c|c|c|c|c|c|c|}
\hline GEOCOODISO & PARLLMENTAR & PARTIDO & FONTE & IMOVEL & Municipio & uF & AREA & cuassersuciven & \begin{tabular}{|c|} 
AREA \\
REGISTRAD \\
A
\end{tabular} & $\begin{array}{l}\text { DECLARADA } \\
\text { COMO POSSE } \\
\end{array}$ & EMPRESA & VINCULO COM O PARLAMENTAR \\
\hline 250030 & ENIYALO RIBEIRO & PP PB & $\begin{array}{l}\text { INCRA } \\
2003\end{array}$ & $\begin{array}{c}\text { FAZENDA } \\
\text { SANTA CLARA }\end{array}$ & $\begin{array}{l}\text { ALAGOA } \\
\text { GRANDE }\end{array}$ & $\mathrm{PB}$ & 72 & & 0 & 72 & $\mid \begin{array}{c}\text { USINA TANQUES } \\
\text { S/A }\end{array}$ & \\
\hline 250030 & ENIYALD RIBEIRO & PPPB & $\begin{array}{l}\text { INCRA } \\
2003\end{array}$ & $\begin{array}{c}\text { FAZENDA } \\
\text { BARRIGUDA }\end{array}$ & $\begin{array}{l}\text { ALAGOA } \\
\text { GRANDE }\end{array}$ & $\mathrm{PB}$ & 110 & & 110 & 0 & $\begin{array}{c}\text { USINA TANQUES } \\
\text { S/A }\end{array}$ & \\
\hline 250030 & ENIVALDO RIBEERO & PP PB & $\begin{array}{l}\text { INCRA } \\
2003\end{array}$ & $\begin{array}{l}\text { FAZENDA } \\
\text { VERTENTE }\end{array}$ & $\begin{array}{l}\text { ALAGOA } \\
\text { GRANDE }\end{array}$ & $\mathrm{PB}$ & 40 & & 40 & 0 & $\begin{array}{c}\text { USINA TANQUES } \\
\text { SAA }\end{array}$ & \\
\hline 250110 & ENIYALO RIBEIRO & PP PB & $\begin{array}{l}\text { INCRA } \\
2003\end{array}$ & $\begin{array}{l}\text { FAZENDA } \\
\text { LAGINHA }\end{array}$ & AREIA & $\mathrm{PB}$ & 186 & & 186 & 0 & $\mid \begin{array}{c}\text { USINA TANQUES } \\
\text { S/A }\end{array}$ & \\
\hline 251150 & ENIYALD RIBEIRO & PP PB & $\begin{array}{l}\text { INCRA } \\
2003\end{array}$ & $\begin{array}{c}\text { FAZENDA } \\
\text { INDEPENDENCI } \\
\text { A }\end{array}$ & PILAR & $\mathrm{PB}$ & 385 & & 385 & 0 & $\begin{array}{c}\text { USINA TANQUES } \\
\text { SIA }\end{array}$ & \\
\hline 260190 & $\begin{array}{l}\text { RICARDO FERREIRA } \\
\text { FUUZA }\end{array}$ & & $\begin{array}{l}\text { INCRA } \\
2003\end{array}$ & $\begin{array}{l}\text { FAZENDA } \\
\text { SANTA FE }\end{array}$ & BEZERROS & PE & 353 & & 353 & 0 & $\begin{array}{c}\text { AGROPECUARIA } \\
\text { JACANA LTDA }\end{array}$ & $\begin{array}{l}\text { FICARDO FIUZA DECLAROU } \\
\text { 331.034 COTAS DA } \\
\text { AGROPECUARIA JAÇANÁ LTDA }\end{array}$ \\
\hline 260190 & $\begin{array}{c}\text { RICARDO FERREIRA } \\
\text { FUUZA }\end{array}$ & & $\begin{array}{l}\text { INCRA } \\
2003 \\
\end{array}$ & $\begin{array}{c}\text { FAZENDA } \\
\text { SANTA FE II }\end{array}$ & BEZERROS & $\mathrm{PE}$ & 255 & & 98 & 156 & $\begin{array}{c}\text { AGROPECUARIA } \\
\text { JACANA LTDA }\end{array}$ & $\begin{array}{l}\text { FICARDO FIUZA DECLAROU } \\
\text { 331.0.04 COATAS DA } \\
\text { AGROPECUARIA JACSANÃ LTDA }\end{array}$ \\
\hline 260190 & $\begin{array}{l}\text { RICARDO FERREIRA } \\
\text { FUUZA }\end{array}$ & & $\begin{array}{l}\text { INCRA } \\
2003\end{array}$ & $\begin{array}{l}\text { RETTROE } \\
\text { JABOTICABA }\end{array}$ & BEZERROS & PE & 59 & & 59 & 0 & $\begin{array}{c}\text { AGROPECUARIA } \\
\text { JACANA LTDA }\end{array}$ & $\begin{array}{l}\text { FiCARDO FIUZA DECLAROU } \\
\text { 331.034 COTAS DA } \\
\text { AGROPECUARIA JACGANÃ LTDA }\end{array}$ \\
\hline
\end{tabular}




\begin{tabular}{|c|c|c|c|c|c|c|c|c|c|c|c|c|}
\hline GEOCÓDIGO & PARLAMENTAR & PARTIDO & FONTE & IMÓVELL & Municírio & UF & AREA & chases hrowive & \begin{tabular}{|c|} 
AREA \\
REGISTRAD \\
A
\end{tabular} & $\begin{array}{l}\text { DECLARADA } \\
\text { COMO POSSE }\end{array}$ & EMPRESA & VINCULO COM O PARLAMENTAR \\
\hline 260190 & $\begin{array}{l}\text { RICARDO FERREIRA } \\
\text { FUUZA }\end{array}$ & $D^{P F L P E}$ & $\begin{array}{l}\text { INCRA } \\
2003\end{array}$ & AREIAL & BEZERROS & $\mathrm{PE}$ & 51 & & 51 & 0 & $\begin{array}{c}\text { AGROPECUAFIA } \\
\text { JACANA LTDA }\end{array}$ & $\begin{array}{l}\text { RICARDO FIUZA DECLAROU } \\
\text { 331.034 COTAS DA } \\
\text { AGROPECUARIA JAÇANÁ LTDA }\end{array}$ \\
\hline 261510 & $\begin{array}{l}\text { CIRO NOGUEIRA LMA } \\
\text { FLHO }\end{array}$ & PPPI & $\begin{array}{l}\text { INCRA } \\
2003\end{array}$ & $\begin{array}{l}\text { FAZENDA } \\
\text { JUNCO }\end{array}$ & TERESINA & $\mathrm{Pl}$ & 1.741 & promentewe & 1.741 & 0 & $\begin{array}{l}\text { CIIRO NOGUEIRA } \\
\text { AROPECUARIAE } \\
\text { IMOVEIS LTDA }\end{array}$ & \\
\hline 261510 & $\begin{array}{l}\text { CIRO NOGUEIRA LIMA } \\
\text { FLHO }\end{array}$ & PPPI & $\begin{array}{l}\text { INCFA } \\
2003\end{array}$ & $\begin{array}{l}\text { JUNCO } \\
\text { SERAFIM }\end{array}$ & TERESINA & $\mathrm{PI}$ & 822 & monomenove & 822 & 0 & $\begin{array}{l}\text { CIRO NOGUEIRA } \\
\text { AROPECUARIA E } \\
\text { IMOVEIS LTDA }\end{array}$ & \\
\hline 220080 & $\begin{array}{l}\text { JÚLLO CESAR DE } \\
\text { CARVALHO LIMA }\end{array}$ & DEM PI & INCRA 2003 & SACO & $\begin{array}{l}\text { ANTÓNIO } \\
\text { ALMEIDA }\end{array}$ & $\mathrm{Pl}$ & 2.026 & ce & 2.026 & 0 & $\begin{array}{l}\text { AGROLUPE } \\
\text { AGROPECUARIA } \\
\text { GUADALUPE }\end{array}$ & \\
\hline 220450 & $\begin{array}{l}\text { JÚLO CESAR DE } \\
\text { CARVALHO LIMA }\end{array}$ & DEM PI & INCRA 2003 & BREJO & GUADalupe & PI & 2.200 & Pnomentenve & 2.200 & 0 & $\begin{array}{l}\text { AGROPECUARIA } \\
\text { GUADALUPE }\end{array}$ & \\
\hline 261510 & $\begin{array}{l}\text { JÚLO CESAR DE } \\
\text { CARVALHO LIMA }\end{array}$ & DEM PI & INCRA 2003 & TALISMA & TERESINA & $\mathrm{Pl}$ & 72 & & 72 & 0 & $\begin{array}{c}\text { FRUTOS CANAA } \\
\text { LTDA }\end{array}$ & \\
\hline 261510 & $\begin{array}{l}\text { JÚLLO CESAR DE } \\
\text { CARVALHO LIMA }\end{array}$ & DEM PI & INCRA 2003 & CANAA & TERESINA & $\mathrm{PI}$ & 50 & & 50 & 0 & $\begin{array}{c}\text { FRUTOS CANAA } \\
\text { LTDA }\end{array}$ & $\begin{array}{l}\text { Capital Da Empres Tropical Fntas } \\
\text { Canaä LIda }\end{array}$ \\
\hline 261510 & $\begin{array}{l}\text { JÚLO CESAR DE } \\
\text { CARVALHO LIMA }\end{array}$ & DEM PI & INCRA 2003 & TALSMAI & TERESINA & $\mathrm{PI}$ & 57 & & 57 & 0 & $\begin{array}{c}\text { FRUTOS CANAA } \\
\text { LTDA }\end{array}$ & \\
\hline
\end{tabular}




\begin{tabular}{|c|c|c|c|c|c|c|c|c|c|c|c|c|}
\hline GEOCÓDISO & PARLAMENTAR & PARTIDO & FONTE & IMOVEL & Muncíipo & uF & AREA & chassersecouna & $\begin{array}{c}\text { AREA } \\
\text { REGISTRAD } \\
\text { A }\end{array}$ & $\begin{array}{l}\text { DECLARADA } \\
\text { COMO POSSE }\end{array}$ & EMPRESA & VINCULO COM O PARLAMENTAR \\
\hline 261510 & $\begin{array}{l}\text { JÚLIO CESAR DE } \\
\text { CARVALHO LIMA }\end{array}$ & DEM PI & INCRA 2008 & TALISMA II & TERESINA & $\mathrm{Pl}$ & 56 & & 56 & o & $\begin{array}{c}\text { FRUTOS CANAA } \\
\text { LTDA }\end{array}$ & \\
\hline 261510 & $\begin{array}{l}\text { THEMISTOCLES DE } \\
\text { SAMPAIO PEREIRA }\end{array}$ & PMDB PI & $\begin{array}{l}\text { INCRA } \\
2003\end{array}$ & $\begin{array}{l}\text { BARRA DO } \\
\text { RIACCOODA } \\
\text { CERCA }\end{array}$ & TERESINA & $\mathrm{Pl}$ & 293 & incometowe & 293 & 0 & $\begin{array}{c}\text { MANGAL FRUTAS } \\
\text { TROPICAIS DE } \\
\text { EXPORTACAOO } \\
\text { LTDA }\end{array}$ & \\
\hline 261510 & $\begin{array}{l}\text { THEMISTOCLES DE } \\
\text { SAMPAIO PEREIRA }\end{array}$ & PMDB PI & $\begin{array}{l}\text { INCRA } \\
2003\end{array}$ & BURITI & TERESINA & $\mathrm{PI}$ & 65 & & 65 & 0 & $\begin{array}{c}\text { MANGAL FRUTAS } \\
\text { TROPICAIS DE } \\
\text { EXPORTACAO } \\
\text { LTDA }\end{array}$ & \\
\hline 110029 & REDITÁRIO CASSOL & PPRO & $\begin{array}{l}\text { INCFA } \\
2003\end{array}$ & FAZCASSOL & $\begin{array}{l}\text { SANTA LUZIA } \\
\text { DOOESTE }\end{array}$ & Ro & 3.521 & monomenowe & 3.372 & o & $\begin{array}{c}\text { AGROPECUARIA } \\
\text { LTDA }\end{array}$ & \\
\hline 110029 & REDITÁRIO CASSOL & PPRO & $\begin{array}{l}\text { INCRA } \\
2003\end{array}$ & FAZCASSOL & $\begin{array}{l}\text { SANTA LUZA } \\
\text { DOOESTE }\end{array}$ & Ro & 1.263 & innomestovere & 1.263 & 0 & $\begin{array}{c}\text { AGROPECUAAIA } \\
\text { LTDA }\end{array}$ & \\
\hline 110029 & REDITARIO CASSOL & PPRO & $\begin{array}{l}\text { INCFA } \\
2003\end{array}$ & & $\begin{array}{l}\text { SANTA LUZIA } \\
\text { DiOESTE }\end{array}$ & Ro & 1.204 & Pnomenence & 1.204 & 0 & $\begin{array}{c}\text { AGROPECUAAIA } \\
\text { LTDA }\end{array}$ & \\
\hline 110029 & REDITÁRIO CASSOL & PPRO & $\begin{array}{l}\text { INCFA } \\
2003\end{array}$ & & $\begin{array}{l}\text { SANTA LUZIA } \\
\text { DIOESTE }\end{array}$ & Ro & 381 & & 361 & 0 & $\begin{array}{c}\text { AGROPECUARIA } \\
\text { LTDA }\end{array}$ & \\
\hline 110029 & REDITARIO CASSOL & PPRO & $\begin{array}{l}\text { INCRA } \\
2003\end{array}$ & & $\begin{array}{l}\text { SANTA LUZA } \\
\text { DOOESTE }\end{array}$ & Ro & 629 & & 629 & 0 & $\begin{array}{c}\text { AGROPECUAAIA } \\
\text { LTDA }\end{array}$ & \\
\hline 110029 & REDITÁRIO CASSOL & PPRO & $\begin{array}{l}\text { INCFA } \\
2003\end{array}$ & & $\begin{array}{l}\text { SANTA LUZIA } \\
\text { DiOESTE }\end{array}$ & Ro & 699 & & 699 & 0 & $\begin{array}{c}\text { AGROPECUARIA } \\
\text { LTDA }\end{array}$ & \\
\hline 110029 & REDITÁRIO CASSOL & Ro & $\begin{array}{l}\text { INCRA } \\
2003\end{array}$ & & $\begin{array}{l}\text { SANTA LUZZA } \\
\text { DiOESTE }\end{array}$ & Ro & 182 & & 182 & 0 & $\begin{array}{c}\text { CASSOL } \\
\text { AGROPECUARIA } \\
\text { LTDA }\end{array}$ & \\
\hline
\end{tabular}




\begin{tabular}{|c|c|c|c|c|c|c|c|c|c|c|c|c|}
\hline GEOCOOIGO & PARLAMENTAR & PARTIDO & FONTE & IMÓVEL & MUNICIPIO & UF & AREA & clusese rucownor & $\begin{array}{c}\text { AREA } \\
\text { REGISTRAD } \\
\text { A }\end{array}$ & $\begin{array}{l}\text { DECLARADA } \\
\text { COMO POSSE }\end{array}$ & EMPRESA & VINCULO COM O PARLAMENTAR \\
\hline 110001 & REDITARIO CASSOL & PP RO & $\begin{array}{l}\text { INCRA } \\
2003\end{array}$ & $\begin{array}{c}\text { SITIO } \\
\text { ELETROSOL }\end{array}$ & $\begin{array}{l}\text { ALTA } \\
\text { FLORESTA } \\
\text { DIOESTE }\end{array}$ & Ro & 217 & PEatew & 217 & 0 & \begin{tabular}{c|} 
ELETROSOL \\
CENTRAIS \\
ELETRICAS \\
CASSOLLTDA \\
\end{tabular} & \\
\hline 110030 & REDITARIO CASSOL & PP RO & $\begin{array}{l}\text { INCRA } \\
2003\end{array}$ & $\begin{array}{c}\text { SITIO } \\
\text { ELETROSOL }\end{array}$ & VILHENA & Ro & 175 & Pnomeneave & 175 & 0 & $\begin{array}{c}\text { ELETROSOL } \\
\text { CENTRAIS } \\
\text { ELETRICAS } \\
\text { CASSOLLTDA }\end{array}$ & \\
\hline 140010 & $\begin{array}{l}\text { SALOMAO AFONSO DE } \\
\text { SOUSA CRUZ }\end{array}$ & PTB RR & $\begin{array}{l}\text { INCRA } \\
2003\end{array}$ & $\begin{array}{l}\text { FAZENDA PAU } \\
\text { RAINHA }\end{array}$ & $B O A$ VISTA & $\mathrm{RR}$ & 2.241 & $\begin{array}{l}\text { Paconemence } \\
\text { mernourma }\end{array}$ & 2.256 & 0 & $\begin{array}{l}\text { AGROPECUARIA } \\
\text { PAU RAINHA SA }\end{array}$ & \\
\hline 430350 & $\begin{array}{l}\text { ADOLFO ANTONIO } \\
\text { FETTER JUNIOR }\end{array}$ & PPRSBRS & $\begin{array}{l}\text { INCRA } \\
2003\end{array}$ & $\begin{array}{l}\text { FAZENDA NOVO } \\
\text { TEMPO }\end{array}$ & CAMAQUÃ. & RS & 943 & 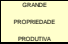 & 932 & 0 & $\begin{array}{l}\text { NOVO TEMPO } \\
\text { LTDA }\end{array}$ & ADOLFO FETTER \\
\hline 421050 & $\begin{array}{l}\text { CASILDO JOÅO } \\
\text { MALDANER }\end{array}$ & PMDB SC & $\begin{array}{l}\text { INCRA } \\
2003\end{array}$ & $\begin{array}{c}2 \text { SUBDIVISAO } \\
165168\end{array}$ & MARAVILHA & $\mathrm{sc}$ & 6 & nerewaio & 6 & 0 & $\begin{array}{l}\text { A MALDANER E } \\
\text { FILHOS LTDA }\end{array}$ & $\begin{array}{l}\text { CASILDO MALDANER Quotas De } \\
\text { Capital - A. Maldaner E Filhos Lidda }\end{array}$ \\
\hline 420125 & $\begin{array}{l}\text { ESPERIDIIÁO AMIN } \\
\text { HELOU FILHO }\end{array}$ & PPSC & $\begin{array}{l}\text { INCRA } \\
2003\end{array}$ & $\begin{array}{l}\text { FAZENDA } \\
\text { JUNDIA }\end{array}$ & APIÚNA & sc & 624 & 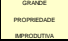 & 624 & 0 & $\begin{array}{l}\text { HEMMER IND E } \\
\text { COM }\end{array}$ & $\begin{array}{l}\text { ESPERIDIÅO AMIN DECLAROU } \\
\text { 2.045 Açoes Da Cia Hemmer }\end{array}$ \\
\hline 420125 & $\begin{array}{l}\text { ESPERIDIÅO AMIN } \\
\text { HELOU FILHO }\end{array}$ & PP SC & $\begin{array}{l}\text { INCRA } \\
2003\end{array}$ & FAZENDINHA & APIÚNA & $\mathrm{sc}$ & 89 & proourrima & 89 & 0 & $\begin{array}{l}\text { HEMMER IND E } \\
\text { COM }\end{array}$ & $\begin{array}{l}\text { ESPERIDIĀO AMIN DECLAROU } \\
\text { 2.045 Açôes Da Cia Hemmer }\end{array}$ \\
\hline 421410 & $\begin{array}{l}\text { ESPERIDIÅO AMIN } \\
\text { HELOU FILHO }\end{array}$ & PP SC & $\begin{array}{l}\text { INCRA } \\
2003\end{array}$ & $\begin{array}{l}\text { FAZENDA } \\
\text { AGRIAO }\end{array}$ & $\begin{array}{l}\text { PRESIDENTE } \\
\text { NEREU }\end{array}$ & sc & 996 & 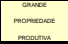 & 996 & 0 & $\begin{array}{l}\text { HEMMER INDE } \\
\text { COM }\end{array}$ & $\begin{array}{l}\text { ESPERIIIIÃO AMIN DECLAROU } \\
2.045 \text { Açóes Da Cia Hemmer }\end{array}$ \\
\hline 421410 & $\begin{array}{l}\text { ESPERIDIÄO AMIN } \\
\text { HELOU FILHO }\end{array}$ & PP sc & $\begin{array}{l}\text { INCRA } \\
2003\end{array}$ & $\begin{array}{l}\text { FAZENDA ITAJAAI } \\
\text { MIRIM }\end{array}$ & $\begin{array}{l}\text { PRESIDENTE } \\
\text { NEREU }\end{array}$ & sc & 337 & 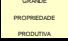 & 337 & 0 & $\begin{array}{l}\text { HEMMER IND E } \\
\text { COM }\end{array}$ & $\begin{array}{l}\text { ESPERIDIÃO AMIN DECLAROU } \\
\text { 2.045 Açôes Da Cia Hemmer }\end{array}$ \\
\hline 420125 & $\begin{array}{l}\text { ESPERIDIÁO AMIN } \\
\text { HELOU FILHO }\end{array}$ & pp sc & $\begin{array}{l}\text { INCRA } \\
2003\end{array}$ & $\begin{array}{l}\text { FAZENDA } \\
\text { APIUNA }\end{array}$ & APIÚNA & sc & 244 & 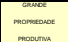 & 244 & 0 & KARSTEN S A & $\begin{array}{l}\text { esperidiláo amin declarou } 142 \text { Açōes } \\
\text { Da Karsten S/A }\end{array}$ \\
\hline 420130 & $\begin{array}{l}\text { ESPERIDIŔO AMIN } \\
\text { HELOU FILHO }\end{array}$ & PP SC & $\begin{array}{l}\text { INCRA } \\
2003\end{array}$ & $\begin{array}{l}\text { FAZENDA } \\
\text { ARAQUARI }\end{array}$ & |ARAQUARI & sc & 837 & 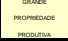 & 837 & 0 & KARSTEN S A & $\begin{array}{l}\text { esperidläo amin declarou } 142 \text { Açōes } \\
\text { Da Karsten S/A }\end{array}$ \\
\hline 420170 & $\begin{array}{l}\text { ESPERIDIÁO AMIN } \\
\text { HELOU FILHO }\end{array}$ & PP SC & $\begin{array}{l}\text { INCRA } \\
2003\end{array}$ & $\begin{array}{c}\text { FAZENDA } \\
\text { GUARICANAS }\end{array}$ & ASCURRA & $\mathrm{sc}$ & 664 & Pnoreseave & 664 & 0 & KARSTEN S A & $\begin{array}{l}\text { esperidiāo amin declarou } 142 \text { Açốes } \\
\text { Da Karsten S/A }\end{array}$ \\
\hline
\end{tabular}




\begin{tabular}{|c|c|c|c|c|c|c|c|c|c|c|c|c|}
\hline GEOCOODISO & PARLAMENTAR & PARTIDO & FONTE & IMOVEL & Municipio & uF & AREA & cuassensacivan & \begin{tabular}{|c|} 
AREA \\
REGISTRAD \\
A
\end{tabular} & $\begin{array}{l}\text { DECLAARADA } \\
\text { COMO POSSE } \\
\end{array}$ & EMPRESA & VINCULO COM O PARLAMENTAR \\
\hline 420170 & $\begin{array}{l}\text { ESPERIDLALOAMIN } \\
\text { HELOU FILHO }\end{array}$ & ppsc & $\begin{array}{l}\text { INCRA } \\
2003\end{array}$ & $\begin{array}{l}\text { FAZENDA } \\
\text { GUARICANASI }\end{array}$ & AscurRA & sc & 125 & & 125 & 0 & KARSTENSA & $\begin{array}{l}\text { esperidalo a amin declarou } 142 \text { Açöes } \\
\text { Da Karsten SiA }\end{array}$ \\
\hline 420750 & $\begin{array}{l}\text { ESPERIDIAOA AMIN } \\
\text { HELOU FIHO }\end{array}$ & ppsc & $\begin{array}{l}\text { INCRA } \\
2003\end{array}$ & $\begin{array}{l}\text { FAZENDA } \\
\text { ENCANO }\end{array}$ & INDALAL & sc & 311 & 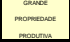 & 311 & 0 & KARSTENS A & $\begin{array}{l}\text { espendidáa amin declarou } 142 \text { Açóes } \\
\text { Da Karsten SiA }\end{array}$ \\
\hline 421470 & $\begin{array}{l}\text { ESPERIILT́ROAMIN } \\
\text { HELOU FILHO }\end{array}$ & Pp Sc & $\begin{array}{l}\text { INCRA } \\
2003\end{array}$ & $\begin{array}{l}\text { FAZENDA AGUA } \\
\text { AMARELA }\end{array}$ & $\begin{array}{l}\text { RIO DOS } \\
\text { CEDROS }\end{array}$ & sc & 409 & & 409 & 0 & KARSTEN SA & $\begin{array}{l}\text { espenidiba amin declarou } 142 \text { Acöes } \\
\text { Da Karsten S/A }\end{array}$ \\
\hline 420800 & ODACIR ZONTA & PPSC & $\begin{array}{l}\text { INCRA } \\
2003\end{array}$ & & $\Pi A$ & sc & 15 & nerenoo & 15 & 0 & $\begin{array}{l}\text { COOPERATIVA } \\
\text { CRED RUAALLA } \\
\text { URACAT SICOOB } \\
\text { SC CREDIAUC }\end{array}$ & $\begin{array}{l}\text { ODACIR ZONTA DECLAROU } \\
\text { Empresa Crediauc Ltda }\end{array}$ \\
\hline 354110 & $\begin{array}{l}\text { JOAO HERRMANN } \\
\text { NETO }\end{array}$ & PDT SP & INCRA 2003 & $\begin{array}{c}\text { FAZENDA SAO } \\
\text { FRANCISCO } \\
\end{array}$ & $\begin{array}{l}\text { PRESIDENTE } \\
\text { ALVES } \\
\end{array}$ & $\mathrm{SP}$ & 360 & pnocenter & 360 & ${ }^{0}$ & $\begin{array}{l}\text { DESTLARIA } \\
\text { GUARICANGA SA }\end{array}$ & $\begin{array}{l}\text { oâa hemman declarouna biografia da } \\
\text { camara que foe diretora, declarou nos } \\
\text { bens capitial guaricanga }\end{array}$ \\
\hline 353870 & $\begin{array}{l}\text { JOAO HERRMANN } \\
\text { NETO }\end{array}$ & PDT SP & INCRA 2003 & $\begin{array}{l}\text { FAZENDA } \\
\text { SANTANA }\end{array}$ & PIRACICABA & $\mathrm{SP}$ & 77 & & 77 & 0 & $\begin{array}{l}\text { SANTA BARBARA } \\
\text { AGRICOLA SA }\end{array}$ & \\
\hline 345990 & $\begin{array}{l}\text { JOÁO HERRMANN } \\
\text { NETO }\end{array}$ & PDT SP & INCRA 2003 & SITIO SALTO & SÁO CARLOS & $\mathrm{sP}$ & 110 & & 110 & 0 & $\begin{array}{l}\text { SANTA BARBARA } \\
\text { AGRICOLA SA }\end{array}$ & \\
\hline 351170 & $\begin{array}{l}\text { JOÁO HERRMANN } \\
\text { NETO }\end{array}$ & PDT SP & INCRA 200: & $\begin{array}{c}\text { FAZENDA SAO } \\
\text { LOURENCO }\end{array}$ & CHARQUEADA & $\mathrm{SP}$ & 627 & 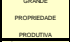 & 627 & 0 & $\begin{array}{l}\text { SANTA BARBARA } \\
\text { AGRICOLA SA } \\
\end{array}$ & \\
\hline 351170 & $\begin{array}{l}\text { JOÁO HERRMANN } \\
\text { NETO }\end{array}$ & PDT SP & INCRA 2003 & \begin{tabular}{|c|} 
FAZENDA \\
SANTA TEREZA
\end{tabular} & CHARQUEADA & $\mathrm{sP}$ & 148 & & 142 & 0 & $\begin{array}{l}\text { SANTA BARBARA } \\
\text { AGRICOLA SA }\end{array}$ & \\
\hline 352690 & $\begin{array}{l}\text { JOAOO HERRMANN } \\
\text { NETO }\end{array}$ & PDT SP & INCRA 200: & \begin{tabular}{|c|} 
FAZENDA \\
PORTO FELIZ \\
\end{tabular} & LIMEIRA & $\mathrm{SP}$ & 493 & 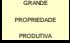 & 493 & 0 & $\begin{array}{l}\text { SANTA BARBARA } \\
\text { AGRICOLA SA }\end{array}$ & \\
\hline 352690 & $\begin{array}{l}\text { JOÁO HERRMANN } \\
\text { NETO }\end{array}$ & PDT SP & INCRA 2003 & \begin{tabular}{|l} 
FAZENDA \\
SOSSEGOIE II
\end{tabular} & LIMEIRA & $\mathrm{SP}$ & 205 & 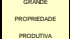 & 205 & 0 & $\begin{array}{l}\text { SANTA BARBARA } \\
\text { AGRICOLA SA }\end{array}$ & \\
\hline
\end{tabular}




\begin{tabular}{|c|c|c|c|c|c|c|c|c|c|c|c|c|}
\hline GEOCÓDIGO & PARLAMENTAR & PARTIDO & FONTE & IMOVEL & MunicíPIo & uF & AREA & cuassensoniman & \begin{tabular}{|c|} 
AREA \\
REGISTRAD \\
A
\end{tabular} & $\begin{array}{l}\text { DEELAAADA } \\
\text { COMO POSSE }\end{array}$ & EMPRESA & VINCULO COM O PARLAMENTAR \\
\hline 352690 & $\begin{array}{l}\text { JOAO HERRMANN } \\
\text { NETO }\end{array}$ & PDT SP & INCRA 2008 & $\begin{array}{c}\text { SITIO MORRO } \\
\text { ALTOO }\end{array}$ & LIMEIRA & $\mathrm{SP}$ & 37 & & 25 & 11 & $\begin{array}{l}\text { SANTA BARBARA } \\
\text { AGRICOLA SA }\end{array}$ & \\
\hline 352690 & $\begin{array}{l}\text { JOÁO HERRMANN } \\
\text { NETO }\end{array}$ & PDT SP & INCRA 2003 & \begin{tabular}{|c|} 
SITO \\
BOSCHERO \\
\end{tabular} & LIMEIRA & $\mathrm{SP}$ & 20 & & 20 & 0 & $\begin{array}{l}\text { SANTA BARBARA } \\
\text { AGRICOLA SA } \\
\end{array}$ & \\
\hline 353180 & $\begin{array}{l}\text { JOAO HERRMANN } \\
\text { NETO }\end{array}$ & PDT SP & INCRA 2005 & CAFUNDE $\|$ & MONTE MOR & SP & 32 & & 32 & 0 & $\begin{array}{l}\text { SANTA BARBARA } \\
\text { AGRICOLA SA }\end{array}$ & \\
\hline 353180 & $\begin{array}{l}\text { JOÁ HERRMANN } \\
\text { NETO }\end{array}$ & PDT SP & INCRA 2003 & $\begin{array}{c}\text { SITIO SANTO } \\
\text { ANTONIO }\end{array}$ & MONTE MOR & $\mathrm{SP}$ & 23 & & 23 & 0 & $\begin{array}{c}\text { SANTA BARBARA } \\
\text { AGRICOLA SA }\end{array}$ & \\
\hline 353970 & $\begin{array}{l}\text { JOÁO HERRMANN } \\
\text { NETO }\end{array}$ & PDT SP & INCRA 2008 & $\begin{array}{c}\text { FAZENDA BELA } \\
\text { VISTA }\end{array}$ & PIIAACICABA & $S P$ & 1.018 & Pnontentave & 1.018 & 0 & $\begin{array}{l}\text { SANTA BARBARA } \\
\text { AGRICOLA SA }\end{array}$ & \\
\hline 353870 & $\begin{array}{l}\text { JOAO HERRMANN } \\
\text { NETO }\end{array}$ & PDT SP & INCRA 2003 & $\begin{array}{l}\text { FAZENDA } \\
\text { CAIAPIA }\end{array}$ & PIRACICABA & $\mathrm{SP}$ & 668 & 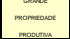 & 668 & 0 & $\begin{array}{c}\text { SANTA BARBARA } \\
\text { AGRICOLA SA }\end{array}$ & $\begin{array}{l}\text { Joäo Herman declarou Administrador. } \\
\text { Fazenda Caiapiá, Priracicaba }\end{array}$ \\
\hline 353870 & $\begin{array}{l}\text { JOÅO HERRMANN } \\
\text { NETO }\end{array}$ & PDT SP & INCRA 2003 & \begin{tabular}{|c|} 
FAZENDA \\
PEDREGULHO
\end{tabular} & PIIAACICABa & $\mathrm{SP}$ & 217 & 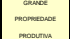 & 217 & ${ }^{0}$ & $\begin{array}{l}\text { SANTA BARBARA } \\
\text { AGRICOLA SA }\end{array}$ & \\
\hline 353870 & $\begin{array}{l}\text { JOAO HERRMANN } \\
\text { NETO }\end{array}$ & PDT SP & INCRA 2005 & $\begin{array}{l}\text { FAZENDA } \\
\text { SANTA FEE }\end{array}$ & PIRACICABA & $\mathrm{SP}$ & 317 & 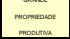 & 317 & 0 & $\begin{array}{c}\text { SANTA BARBARA } \\
\text { AGRICOLA SA }\end{array}$ & \\
\hline 353870 & $\begin{array}{l}\text { JOÁO HERRMANN } \\
\text { NETO }\end{array}$ & PDT SP & INCRA 2003 & \begin{tabular}{|c|} 
FAZENDA \\
SANTA IZABEL
\end{tabular} & PIIRACICABA & $\mathrm{SP}$ & 231 & 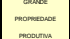 & 231 & 0 & $\begin{array}{c}\text { SANTA BARBARA } \\
\text { AGRICOLA SA }\end{array}$ & \\
\hline 353970 & $\begin{array}{l}\text { JOÁO HERRMANN } \\
\text { NETO }\end{array}$ & PDT SP & INCRA 2008 & \begin{tabular}{|l} 
FAZENDA \\
SANTA JULLA
\end{tabular} & PIIAACICABa & $\mathrm{SP}$ & 721 & 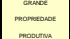 & 721 & 0 & $\begin{array}{l}\text { SANTA BARBARA } \\
\text { AGRICOLA SA }\end{array}$ & \\
\hline 353870 & $\begin{array}{l}\text { JOÁ HERRMANN } \\
\text { NETO }\end{array}$ & PDT SP & INCRA 2005 & $\begin{array}{c}\text { FAZENDA } \\
\text { SANTA LDIA } \\
\end{array}$ & PIRACICABA & $\mathrm{SP}$ & 777 & 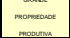 & 777 & 777 & $\begin{array}{c}\text { SANTA BARBARA } \\
\text { AGRICOLA SA }\end{array}$ & \\
\hline 353870 & $\begin{array}{l}\text { JOÁO HERRMANN } \\
\text { NETO }\end{array}$ & PDT SP & INCRA 2003 & $\begin{array}{l}\text { FAZENDA } \\
\text { SANTA ROSA II }\end{array}$ & PIIAACICABA & SP & 1.396 & 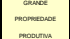 & 1.396 & 0 & $\begin{array}{l}\text { SANTA BARBARA } \\
\text { AGRICOLA SA }\end{array}$ & \\
\hline
\end{tabular}




\begin{tabular}{|c|c|c|c|c|c|c|c|c|c|c|c|c|}
\hline GEOCOODIOSO & PARLAMENTAR & PARTIDO & FONTE & IMOVEL & Municipio & UF & AREA & ciassersuromen & \begin{tabular}{|c|} 
AREA \\
REGISTRAD \\
A
\end{tabular} & $\begin{array}{l}\text { DECLAARADA } \\
\text { COMO POSSE }\end{array}$ & EMPRESA & VINCULO COM O PARLAMENTAR \\
\hline 353970 & $\begin{array}{l}\text { JOAO HERRMANN } \\
\text { NETO }\end{array}$ & PDT SP & INCRA 2003 & $\begin{array}{c}\text { FAZENDA SAO } \\
\text { JOSE }\end{array}$ & PIRACICABA & $\mathrm{SP}$ & 1.131 & 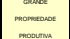 & 1.131 & 1.131 & $\begin{array}{l}\text { SANTA BARBARA } \\
\text { AGRICOLA SA }\end{array}$ & \\
\hline 353870 & $\begin{array}{l}\text { JOAO HERRMANN } \\
\text { NETO }\end{array}$ & PDT SP & INCRA 2003 & $\begin{array}{c}\text { FAZENDA SAO } \\
\text { LUIZ }\end{array}$ & PIRACICABA & $\mathrm{SP}$ & 489 & 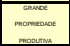 & 489 & 0 & \begin{tabular}{|l|}
$\begin{array}{c}\text { SANTA BARBARA } \\
\text { AGRICOLA SA }\end{array}$ \\
\end{tabular} & \\
\hline 353870 & $\begin{array}{l}\text { JOÁO HERRMANN } \\
\text { NETO }\end{array}$ & PDT SP & INCRA 200: & $\begin{array}{c}\begin{array}{c}\text { FAZENDA SAO } \\
\text { ROQUE }\end{array} \\
\end{array}$ & PIRACICABA & $\mathrm{SP}$ & 439 & Pnorvetence & 439 & 0 & \begin{tabular}{|c|} 
SANTA BARBARA \\
AGRIICLA SA \\
\end{tabular} & \\
\hline 353870 & $\begin{array}{l}\text { JOÁO HERRMANN } \\
\text { NETOO }\end{array}$ & PDT SP & INCRA 2003 & \begin{tabular}{|c|} 
FAZENDA SAO \\
BENEDITO
\end{tabular} & PIRACICABAA & $\mathrm{sP}$ & 491 & 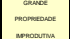 & 491 & 0 & $\begin{array}{l}\text { SANTA BARBARA } \\
\text { AGRICOLA SA }\end{array}$ & \\
\hline 353870 & $\begin{array}{l}\text { JOÁO HERRMANN } \\
\text { NETO }\end{array}$ & PDT SP & INCRA 2005 & $\begin{array}{l}\text { SANTO } \\
\text { ANTONIO }\end{array}$ & PIRACICABA & $\mathrm{SP}$ & 48 & & 48 & 0 & \begin{tabular}{|l} 
SANTA BARBARA \\
AGRICOLA SA
\end{tabular} & \\
\hline 353870 & $\begin{array}{l}\text { JOAO HERRMANN } \\
\text { NETO }\end{array}$ & PDT SP & INCAA 200: & $\begin{array}{l}\text { FAZENDA } \\
\text { BARROCAO }\end{array}$ & PIRACICABA & $\mathrm{SP}$ & 69 & & 69 & 0 & \begin{tabular}{|l} 
SANTA BARBARA \\
AGRILOLA SA
\end{tabular} & \\
\hline 353870 & $\begin{array}{l}\text { JOÅO HERRMANN } \\
\text { NETO }\end{array}$ & PDT SP & INCRA 2003 & \begin{tabular}{|l|} 
FAZENDA \\
COSTA PINTO
\end{tabular} & PIIRACICABA & $\mathrm{SP}$ & 124 & & 124 & 0 & \begin{tabular}{|c|} 
SANTA BARBARA \\
AGRICOLA SA
\end{tabular} & \\
\hline 353870 & $\begin{array}{l}\text { JOAO HERRMANN } \\
\text { NETO }\end{array}$ & PDT SP & INCRA 2003 & $\begin{array}{l}\text { FAZENDA } \\
\text { SANTAFE }\end{array}$ & PIRACICABAA & $\mathrm{SP}$ & 62 & & 62 & 0 & $\begin{array}{l}\text { SANTA BARBARA } \\
\text { AGRICOLA SA }\end{array}$ & \\
\hline 355370 & $\begin{array}{l}\text { JOAO HERRMANN } \\
\text { NETO }\end{array}$ & PDT SP & INCRA 2003 & \begin{tabular}{|c|} 
FAZENDA \\
SANTA ROSA
\end{tabular} & PIIRACICABAA & $\mathrm{SP}$ & 106 & & 110 & 0 & $\begin{array}{l}\text { SANTA BARBARA } \\
\text { AGRICOLA SA }\end{array}$ & \\
\hline 353970 & $\begin{array}{l}\text { JOÁ HERRMANN } \\
\text { NETO }\end{array}$ & PDT SP & INCRA 2003 & TERMINAL & PIRACICABA & $\mathrm{SP}$ & 40 & & 40 & 0 & $\begin{array}{c}\text { SANTA BARBARA } \\
\text { AGRICOLA SA }\end{array}$ & \\
\hline 353870 & $\begin{array}{l}\text { JOÁO HERRMANN } \\
\text { NETOO }\end{array}$ & PDT SP & INCRA 2003 & $\begin{array}{l}\text { TRECHO } \\
\text { FEPASA }\end{array}$ & PIRACICABA & $\mathrm{SP}$ & 23 & & 23 & 0 & \begin{tabular}{|l} 
SANTA BARBARA \\
AGRICOLA SA
\end{tabular} & $\begin{array}{l}\text { Joảo Hemman declarou Administrador } \\
\text { Eazenda Caispias, Priracicaba }\end{array}$ \\
\hline 354580 & $\begin{array}{l}\text { JOAOO HERRMANN } \\
\text { NETO }\end{array}$ & PDT SP & $\begin{array}{l}\text { INCRAA } \\
2003\end{array}$ & \begin{tabular}{|c|} 
AREIA BRANCA \\
BOM RETIRO
\end{tabular} & $\begin{array}{l}\text { BARBARA } \\
\text { DiOESTE }\end{array}$ & sp & 1.209 & minamenowe & 1.210 & 0 & $\begin{array}{l}\text { SANTA BARBARA } \\
\text { AGRICOLA SA }\end{array}$ & \\
\hline
\end{tabular}




\begin{tabular}{|c|c|c|c|c|c|c|c|c|c|c|c|c|}
\hline GEOCODIGO & PARLAMENTAR & PARTIDO & FONTE & IMOVEL & Municipio & UF & AREA & chassersacivan & \begin{tabular}{|l|} 
AREA \\
REGISTRAD \\
\end{tabular} & $\begin{array}{l}\text { DECLAAADA } \\
\text { COMO POSSEE }\end{array}$ & EMPRESA & VINCULO COM O PARLAMENTAR \\
\hline 354580 & $\begin{array}{l}\text { JOAO HERRMANN } \\
\text { NETO }\end{array}$ & PDT SP & INCRA 2003 & $\begin{array}{c}\text { FAZENDA BOM } \\
\text { RETIRO }\end{array}$ & $\begin{array}{l}\text { BÁRBAAA } \\
\text { DiOESTE }\end{array}$ & $\mathrm{SP}$ & 266 & 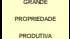 & 266 & 0 & $\begin{array}{l}\text { SANTA BARBARA } \\
\text { AGRICOLA SA }\end{array}$ & \\
\hline 354580 & $\begin{array}{l}\text { JOAO HERRMANN } \\
\text { NETO }\end{array}$ & PDT SP & INCRA 2003 & $\begin{array}{l}\text { FAZENDA } \\
\text { JAMAICA }\end{array}$ & $\begin{array}{l}\text { BARBARA } \\
\text { DOOESTE }\end{array}$ & $\mathrm{SP}$ & 379 & 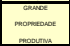 & 379 & 0 & \begin{tabular}{|c|} 
SANTA BARBARA \\
AGRICOLA SA
\end{tabular} & \\
\hline 354580 & $\begin{array}{l}\text { JOÁO HERRMANN } \\
\text { NETO }\end{array}$ & PDT SP & INCRA 200: & $\begin{array}{c}\begin{array}{c}\text { FAZENDA SAO } \\
\text { LUIZ }\end{array} \\
\end{array}$ & $\begin{array}{l}\text { BÁABAAA } \\
\text { DiOESTE }\end{array}$ & $\mathrm{SP}$ & 1.965 & monoreave & 1.965 & 0 & \begin{tabular}{|c|} 
SANTA BARBARA \\
AGRIICLA SA
\end{tabular} & \\
\hline 354580 & $\begin{array}{l}\text { JOÁO HERRMANN } \\
\text { NETO }\end{array}$ & PDT SP & INCRA 2003 & $\begin{array}{c}\text { FAZENDA SAO } \\
\text { PEDRO }\end{array}$ & $\begin{array}{l}\text { BÁRBAAA } \\
\text { DiOESTE }\end{array}$ & $\mathrm{sP}$ & 414 & 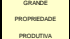 & 414 & 0 & $\begin{array}{l}\text { SANTA BARBARA } \\
\text { AGRICOLA SA }\end{array}$ & \\
\hline 354580 & $\begin{array}{l}\text { JOÁO HERRMANN } \\
\text { NETO }\end{array}$ & PDT SP & INCRA 2005 & $\begin{array}{c}\text { SANTO } \\
\text { ANTONIO }\end{array}$ & $\begin{array}{l}\text { BABBAAA } \\
\text { DiOESTE }\end{array}$ & $\mathrm{SP}$ & 191 & 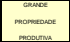 & 191 & 0 & \begin{tabular}{|c|} 
SANTA BARBARA \\
AGRICOLA SA
\end{tabular} & \\
\hline 354580 & $\begin{array}{l}\text { JOAO HERRMANN } \\
\text { NETO }\end{array}$ & PDT SP & INCAA 200: & \begin{tabular}{|c|} 
SITIO \\
FAZENDINHA \\
\end{tabular} & $\begin{array}{l}\text { AABBAAA } \\
\text { DiOESTE }\end{array}$ & $\mathrm{SP}$ & 280 & inocontensere & 280 & 280 & \begin{tabular}{|c|} 
SANTA BARBARA \\
AGRILOLA SA
\end{tabular} & \\
\hline 354580 & $\begin{array}{c}\text { JOÅO HERRMANN } \\
\text { NETO }\end{array}$ & PDT SP & INCRA 2003 & BARRACA & \begin{tabular}{|l} 
BAABAAA \\
DiOESTE
\end{tabular} & $\mathrm{sP}$ & 79 & & 79 & 0 & \begin{tabular}{|c|} 
SANTA BARBARA \\
AGRICOLA SA
\end{tabular} & \\
\hline 354580 & $\begin{array}{l}\text { JOAO HERRMANN } \\
\text { NETO }\end{array}$ & PDT SP & INCRA 2003 & CAFUNDE & $\begin{array}{l}\text { BÁRBARA } \\
\text { DIOESTE }\end{array}$ & $\mathrm{SP}$ & 94 & & 94 & 0 & $\begin{array}{l}\text { SANTA BARBARA } \\
\text { AGRICOLA SA }\end{array}$ & \\
\hline 354580 & $\begin{array}{l}\text { JOAO HERRMANN } \\
\text { NETO }\end{array}$ & PDT SP & INCRA 2003 & CAFUNDE I & $\begin{array}{l}\text { BABBAAA } \\
\text { DOOESTE }\end{array}$ & $\mathrm{SP}$ & 96 & & 96 & 0 & $\begin{array}{l}\text { SANTA BARBARA } \\
\text { AGRICOLA SA }\end{array}$ & \\
\hline 354580 & $\begin{array}{l}\text { JOÁ HERRMANN } \\
\text { NETO }\end{array}$ & PDT SP & INCRA 2003 & CAPUAVA & $\begin{array}{l}\text { BAABAARA } \\
\text { DiOESTE }\end{array}$ & $\mathrm{SP}$ & 72 & & 72 & 0 & $\begin{array}{c}\text { SANTA BARBARA } \\
\text { AGRICOLA SA }\end{array}$ & \\
\hline 354580 & $\begin{array}{l}\text { JOÁO HERRMANN } \\
\text { NETOO }\end{array}$ & PDT SP & INCRA 2003 & \begin{tabular}{|c|} 
FAZENDA SAO \\
PEDRO
\end{tabular} & $\begin{array}{l}\text { BABBAAA } \\
\text { DiOESTE }\end{array}$ & $\mathrm{SP}$ & 128 & & 128 & 0 & \begin{tabular}{|c|} 
SANTA BARBARA \\
AGRICOLA SA
\end{tabular} & \\
\hline 354580 & $\begin{array}{l}\text { JOAOO HERRMANN } \\
\text { NETO }\end{array}$ & PDT SP & INCRA 2005 & JAMAICA PARTE & $\begin{array}{l}\text { BÁRBAAA } \\
\text { DOOESTE }\end{array}$ & sp & 132 & & 125 & 0 & $\begin{array}{c}\text { SANTA BARBARA } \\
\text { AGRICOLA SA }\end{array}$ & \\
\hline
\end{tabular}




\begin{tabular}{|c|c|c|c|c|c|c|c|c|c|c|c|c|}
\hline GEOCOODIGO & PARLLMENTAR & PARTIDO & FONTE & IMOVEL & municipio & uF & AREA & 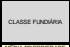 & \begin{tabular}{|c|} 
AREA \\
REGISTRAD \\
A
\end{tabular} & $\begin{array}{l}\text { DECLAARADA } \\
\text { COMO POSSE } \\
\end{array}$ & EMPRESA & VINCULO COM O PARLAMENTAR \\
\hline 354580 & $\begin{array}{l}\text { JOÁ HERRMANN } \\
\text { NETO }\end{array}$ & PDT SP & INCRA 2008 & $\begin{array}{c}\text { JOAO DO MATO } \\
1\end{array}$ & $\begin{array}{l}\text { BÁRBAAA } \\
\text { DiOESTE }\end{array}$ & $\mathrm{SP}$ & 57 & & 57 & 0 & $\begin{array}{c}\text { SANTA BARBARA } \\
\text { AGRICOLA SA }\end{array}$ & \\
\hline 354580 & $\begin{array}{l}\text { JOÁO HERRMANN } \\
\text { NETO }\end{array}$ & PDT SP & INCRA 2005 & $\begin{array}{c}\text { RiBEIRAODOS } \\
\text { BOIS }\end{array}$ & $\begin{array}{l}\text { AARBAAA } \\
\text { DiOESTE }\end{array}$ & $\mathrm{sP}$ & 53 & & 53 & 0 & \begin{tabular}{|c|} 
SANTA BARBARA \\
AGRIICOLA SA
\end{tabular} & \\
\hline 354580 & $\begin{array}{l}\text { JOÁO HERRMANN } \\
\text { NETO }\end{array}$ & PDT SP & INCRA 200: & $\begin{array}{l}\text { SITTO AREIA } \\
\text { BRANCA }\end{array}$ & $\begin{array}{l}\text { BÁABAAA } \\
\text { DiOESTE }\end{array}$ & $\mathrm{SP}$ & 111 & & 111 & 0 & $\begin{array}{c}\text { SANTA BARBARA } \\
\text { AGRIICOLA SA }\end{array}$ & \\
\hline 354580 & $\begin{array}{l}\text { JOÁO HERRMANN } \\
\text { NETO }\end{array}$ & PDT SP & INCRA 2008 & \begin{tabular}{|c|} 
SITIO AREIA \\
BRANCA MINITI
\end{tabular} & $\begin{array}{l}\text { BÁRBAAA } \\
\text { DiOESTE }\end{array}$ & $\mathrm{SP}$ & 60 & & 60 & 0 & $\begin{array}{c}\text { SANTA BARBARA } \\
\text { AGRICOLA SA }\end{array}$ & \\
\hline 354580 & $\begin{array}{l}\text { JOÁO HERRMANN } \\
\text { NETO }\end{array}$ & PDT SP & INCRA 2005 & $\begin{array}{c}\text { SITIO CONCHA } \\
\text { DE OURO }\end{array}$ & $\begin{array}{l}\text { BAABAARA } \\
\text { DiOESTE }\end{array}$ & $\mathrm{SP}$ & 72 & & 72 & 0 & $\begin{array}{c}\text { SANTA BARBARA } \\
\text { AGRIICOLA SA }\end{array}$ & \\
\hline 354580 & $\begin{array}{l}\text { JOAO HERRMANN } \\
\text { NETO }\end{array}$ & PDT SP & INCAA 200: & \begin{tabular}{|c|} 
SITIO SANTO \\
ANTONIO
\end{tabular} & $\begin{array}{l}\text { BÁRBARA } \\
\text { DOOESTE }\end{array}$ & $\mathrm{SP}$ & 60 & & 60 & 0 & \begin{tabular}{|c|} 
SANTA BARBARA \\
AGRICOLA SA
\end{tabular} & \\
\hline 354580 & $\begin{array}{l}\text { JOÁO HERPMANN } \\
\text { NETO }\end{array}$ & PDT SP & INCRA 2003 & TERRA ROXA & $\begin{array}{l}\text { BÁRBARA } \\
\text { DIOESTE }\end{array}$ & $\mathrm{SP}$ & 92 & & 92 & 0 & \begin{tabular}{|c|} 
SANTA BARBARA \\
AGRICOLA SA \\
\end{tabular} & \\
\hline 354580 & $\begin{array}{l}\text { JOÁO HERRMANN } \\
\text { NETO }\end{array}$ & PDT SP & $\begin{array}{l}\text { INCRA } \\
2003\end{array}$ & $\begin{array}{c}\text { AREIA BRANCA } \\
\text { " }\end{array}$ & $\begin{array}{l}\text { AARBAAA } \\
\text { DiOESTE }\end{array}$ & $\mathrm{SP}$ & 9 & nerewoo & 9 & 0 & \begin{tabular}{|c|} 
SANTA BARBARA \\
AGRICOLA SA
\end{tabular} & \\
\hline 354580 & $\begin{array}{l}\text { JOÁO HERRMANN } \\
\text { NETO }\end{array}$ & PDT SP & $\begin{array}{c}\text { INCRA } \\
2003\end{array}$ & $\begin{array}{c}\text { BAIRRO } \\
\text { FAZENDINHA } \\
\end{array}$ & $\begin{array}{l}\text { AARBARA } \\
\text { DiOESTE }\end{array}$ & $\mathrm{SP}$ & 9 & nerenao & 9 & 0 & \begin{tabular}{|c|} 
SANTA BARBARA \\
AGRICOLA SA \\
\end{tabular} & \\
\hline 354580 & $\begin{array}{l}\text { JOÁO HERRMANN } \\
\text { NETO }\end{array}$ & PDT SP & INCRA 2005 & $\begin{array}{c}\text { BAIRRO SANTO } \\
\text { ANTONIO }\end{array}$ & \begin{tabular}{|l} 
BÁRBARA \\
DiOESTE \\
\end{tabular} & $\mathrm{SP}$ & 4 & nesuono & 4 & 0 & \begin{tabular}{|c|} 
SANTA BARBARA \\
AGRICOLA SA \\
\end{tabular} & \\
\hline 354580 & $\begin{array}{l}\text { JOAO HERRMANN } \\
\text { NETO }\end{array}$ & PDT SP & INCRA 2003 & $\begin{array}{l}\text { CHACARA } \\
\text { BARRACA }\end{array}$ & $\begin{array}{l}\text { SANTA } \\
\text { BARBAAAA } \\
\text { DIOESTE }\end{array}$ & $\mathrm{SP}$ & 0 & nerenoo & 0 & 0 & \begin{tabular}{|c|} 
SANTA BARBARA \\
AGRICOLA SA
\end{tabular} & \\
\hline 354580 & $\begin{array}{l}\text { JOÁ HERRMANN } \\
\text { NETO }\end{array}$ & PDT SP & INCRA 2003 & GRIVOL & \begin{tabular}{|l} 
SANTA \\
BARBAARA \\
DiOESTE
\end{tabular} & $\mathrm{SP}$ & 0 & nerenot & 0 & 0 & $\mid \begin{array}{c}\text { SANTA BARBARA } \\
\text { AGRICOLA SA }\end{array}$ & \\
\hline
\end{tabular}




\begin{tabular}{|c|c|c|c|c|c|c|c|c|c|c|c|c|}
\hline GEOCÓDICO & PARLAMENTAR & PARtido & FONTE & IMÓVEL & MUNicipio & uF & AREA & cusesentucivon & \begin{tabular}{|c|} 
AREA \\
REGISTRAD \\
A
\end{tabular} & $\begin{array}{l}\text { DECLARADA } \\
\text { COMO POSSE }\end{array}$ & EMPRESA & VINCULO COM O PARLAMENTAR \\
\hline 354580 & $\begin{array}{l}\text { JOÁO HERRMANN } \\
\text { NETO }\end{array}$ & PDT SP & INCRA 2003 & $\begin{array}{c}\text { JOAO DO MATO } \\
\text { "I }\end{array}$ & $\begin{array}{l}\text { SANTA } \\
\text { BARBARA } \\
\text { DIOESTE }\end{array}$ & $\mathrm{sP}$ & 3 & nerenoso & 3 & 0 & $\begin{array}{c}\text { SANTA BARBARA } \\
\text { AGRICOLA SA }\end{array}$ & \\
\hline 354580 & $\begin{array}{l}\text { JOAO HERRMANN } \\
\text { NETO }\end{array}$ & PDT SP & $\begin{array}{l}\text { INCPA } \\
2003\end{array}$ & AREla brancaI & $\begin{array}{l}\text { BABBARA } \\
\text { DiOESTE }\end{array}$ & SP & 29 & & 29 & 0 & \begin{tabular}{|c|} 
SANTA BARBARA \\
AGRICOLA SA
\end{tabular} & \\
\hline 354580 & $\begin{array}{l}\text { JOÁO HERRMANN } \\
\text { NETO }\end{array}$ & PDT SP & INCRA 2003 & $\begin{array}{c}\text { BELO } \\
\text { HORIZONTE }\end{array}$ & \begin{tabular}{|l} 
BÁABARA \\
DiOESTE
\end{tabular} & $\mathrm{sP}$ & 12 & & 12 & 0 & \begin{tabular}{|c|} 
SANTA BARBARA \\
AGRICOLA SAA
\end{tabular} & \\
\hline 354580 & $\begin{array}{l}\text { JOAO HERRMANN } \\
\text { NETO }\end{array}$ & PDT SP & INCRA 2005 & \begin{tabular}{|c|}
$\begin{array}{c}\text { FAZENDA BOA } \\
\text { VISTA }\end{array}$ \\
\end{tabular} & \begin{tabular}{|l} 
BABBARA \\
DIOESTE
\end{tabular} & $\mathrm{sP}$ & 14 & & 14 & 0 & \begin{tabular}{|c|} 
SANTA BARBARA \\
AGRICOLA SA \\
\end{tabular} & \\
\hline 354580 & $\begin{array}{l}\text { JOAO HERRMANN } \\
\text { NETO }\end{array}$ & PDT SP & INCRA 2003 & MACHADO & \begin{tabular}{|l} 
BABBARA \\
DIOESTE
\end{tabular} & SP & 10 & & 10 & 0 & \begin{tabular}{|c|} 
SANTA BARBARA \\
AGRICOLA SA
\end{tabular} & \\
\hline 354580 & $\begin{array}{l}\text { JOAO HERRMANN } \\
\text { NETO }\end{array}$ & PDT SP & INCRA 2003 & $\begin{array}{l}\text { SITIO BARBOSA } \\
\text { OLHOS D AGUA }\end{array}$ & $\begin{array}{l}\text { BÁABARA } \\
\text { DiOESTE }\end{array}$ & sp & 16 & & 16 & 0 & \begin{tabular}{|c|} 
SANTA BARBARA \\
AGRICOLA SA
\end{tabular} & \\
\hline 354580 & $\begin{array}{l}\text { JOAO HERRMANN } \\
\text { NETO }\end{array}$ & PDT SP & INCRA 2003 & $\begin{array}{c}\text { SITIO } \\
\text { coNSERVA }\end{array}$ & \begin{tabular}{|l|} 
BABBAAA \\
OOOESTE
\end{tabular} & $\mathrm{SP}$ & 14 & & 14 & 0 & \begin{tabular}{|c|} 
SANTA BARBARA \\
AGRICOLA SA
\end{tabular} & \\
\hline 354580 & $\begin{array}{l}\text { JOAOO HERRMANN } \\
\text { NETO }\end{array}$ & PDT SP & INCRA 2003 & $\begin{array}{c}\text { SITIO MONJOLO } \\
\text { VELHO }\end{array}$ & \begin{tabular}{|l} 
BABBARA \\
DIOESTE
\end{tabular} & SP & 31 & & 31 & 0 & \begin{tabular}{|c|} 
SANTA BARBARA \\
AGRICOLA SA
\end{tabular} & \\
\hline 354580 & $\begin{array}{l}\text { JOAO HERRMANN } \\
\text { NETO }\end{array}$ & PDT SP & INCRA 2003 & SITIO PEDROSO & $\begin{array}{l}\text { BAABARA } \\
\text { DiOESTE }\end{array}$ & sp & 12 & & 12 & 0 & \begin{tabular}{|c|} 
SANTA BARBARA \\
AGRICOLA SA
\end{tabular} & \\
\hline 355040 & $\begin{array}{l}\text { JOÁO HERRMANN } \\
\text { NETO }\end{array}$ & PDT SP & INCRA 2003 & \begin{tabular}{|l|} 
FAZENDA BOA \\
ESPERANCA
\end{tabular} & SÁO PEDRO & $\mathrm{sP}$ & 464 & contence & 464 & 464 & $\begin{array}{c}\text { SANTA BARBARA } \\
\text { AGRICOLA SA }\end{array}$ & \\
\hline 353180 & $\begin{array}{l}\text { JOÁO HERRMANN } \\
\text { NETO }\end{array}$ & PDT SP & INCRA 2003 & \begin{tabular}{|c|} 
FAZENDA \\
MONTE BELL
\end{tabular} & MONTE MOR & sp & 782 & teve & 782 & 0 & $\begin{array}{c}\text { SANTA BARBARA } \\
\text { AGRICOLA SA }\end{array}$ & \\
\hline
\end{tabular}




\begin{tabular}{|c|c|c|c|c|c|c|c|c|c|c|c|c|}
\hline GEOCODIGO & PARLAMENTAR & PARtido & FONTE & IMÓVEL & MUNicipio & UF & AREA & 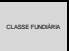 & \begin{tabular}{|c|}
$\begin{array}{c}\text { AREA } \\
\text { REGISTRAD } \\
\text { A }\end{array}$ \\
\end{tabular} & $\begin{array}{l}\text { DECLAARADA } \\
\text { COMO POSSE }\end{array}$ & EMPRESA & VINCULO COM O PARLAMENTAR \\
\hline 354390 & $\begin{array}{l}\text { JOAO HERRMANN } \\
\text { NETO }\end{array}$ & PDT SP & INCRA 2003 & $\begin{array}{l}\text { FAZENDA } \\
\text { PITANGA }\end{array}$ & RIO CLARO & $\mathrm{sP}$ & 1.197 & 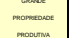 & 1.197 & 0 & $\mid \begin{array}{c}\text { SANTA BARBARA } \\
\text { AGRICOLA SA }\end{array}$ & \\
\hline 355040 & $\begin{array}{l}\text { JOÁ HERRMANN } \\
\text { NETO }\end{array}$ & PDT SP & INCRA 2003 & $\begin{array}{c}\text { FAZENDA SAO } \\
\text { FRANCISCO }\end{array}$ & SAO PEDRO & $\mathrm{sP}$ & 575 & Proconemence & 575 & 575 & $\begin{array}{c}\text { SANTA BARBARA } \\
\text { AGRICOLA SA }\end{array}$ & \\
\hline 350720 & $\begin{array}{l}\text { JOÁO HERRMANN } \\
\text { NETO }\end{array}$ & PDT SP & $\begin{array}{l}\text { INCFA } \\
2003\end{array}$ & AREA MATAO & BORÁ & sp & 125 & & 125 & 0 & $\begin{array}{c}\text { SANTA BARBABAA } \\
\text { AGRICOLA SA }\end{array}$ & $\begin{array}{l}\text { joâo hemman declarou Administrador, } \\
\text { Fazenda Caiapia, Piracicaba }\end{array}$ \\
\hline 351370 & NELSON MARQUEZELLI & Pтв SP & $\begin{array}{l}\text { INCRA } \\
2003\end{array}$ & $\begin{array}{l}\text { FAZENDA } \\
\text { BANDEERA } \\
\text { BRANCA }\end{array}$ & DEscalvado & SP & 204 & ue & 204 & 0 & \begin{tabular}{|c|} 
COOPERETIVA \\
DE CREDITO \\
RURAL DO VALE \\
DO MOGI GUACU
\end{tabular} & nelson marquezeli \\
\hline 351370 & NELSON MARQUEZELL & Pтв SP & $\begin{array}{l}\text { INCRA } \\
2003\end{array}$ & $\begin{array}{c}\text { GRANANA SANTA } \\
\text { TEREZZNHA }\end{array}$ & DEscalvado & $\mathrm{sp}$ & 15 & & 15 & 0 & \begin{tabular}{|c|} 
COOPERETIVA \\
DE CREDITO \\
RURAL DO VALE \\
DO MOGI GUACU
\end{tabular} & nelson marquezeli \\
\hline 351370 & NELSON MARQUEZELI & Pтв SP & $\begin{array}{l}\text { INCPA } \\
2003\end{array}$ & $\begin{array}{c}\text { FAZENDA } \\
\text { JAGUARANDI } \\
\text { SEDE }\end{array}$ & DEScalvado & $\mathrm{sP}$ & 66 & & 66 & 0 & \begin{tabular}{|c|} 
COOPERETTVA \\
DE CREDITO \\
RURAL DO VALE \\
DO MOGI GUACU
\end{tabular} & nelson marquezelli \\
\hline 950010 & $\begin{array}{l}\text { PAULO CESAR DE } \\
\text { OLIVEIRA LIMA }\end{array}$ & PMDB SP & $\begin{array}{l}\text { INCRA } \\
2003\end{array}$ & $\begin{array}{l}\text { CHACARA } \\
\text { CAMDA }\end{array}$ & ADAMANTINA & sp & 5 & nereunao & 5 & 0 & $\begin{array}{l}\text { COOPERATIVA } \\
\text { AGRIC MISTA } \\
\text { ADAMANTINA } \\
\end{array}$ & paubo lima \\
\hline
\end{tabular}




\begin{tabular}{|c|c|c|c|c|c|c|c|c|c|c|c|c|}
\hline GEOCODICO & PARLAMENTAR & PARtido & FONTE & IMÓVEL & MUNicipio & UF & AREA & cusesenencivan & \begin{tabular}{|c|}
$\begin{array}{c}\text { AREA } \\
\text { REGISTRAD } \\
\text { A }\end{array}$ \\
\end{tabular} & $\begin{array}{l}\text { DECLAAADA } \\
\text { COMO POSSE }\end{array}$ & EMPRESA & VINCULO COM O PARLAMENTAR \\
\hline 171610 & $\begin{array}{l}\text { IGOR PUGLESI } \\
\text { AVELINO }\end{array}$ & PMDB TO & $\begin{array}{l}\text { INCFA } \\
2003\end{array}$ & $\begin{array}{c}\text { MONA } \\
\text { AGROPECUARIA } \\
\text { LTDA }\end{array}$ & $\begin{array}{l}\text { PARAISO DO } \\
\text { TOCANTINS }\end{array}$ & то & 3.269 & Pnowereace & 3.224 & 0 & \begin{tabular}{|c|} 
MONA \\
AGRPEECUARIA \\
LTDA
\end{tabular} & $\begin{array}{l}\text { GOR PUGLLESI declarou 15\% DA } \\
\text { EMPRA MONA AGROPECUARIA } \\
\text { LTDA }\end{array}$ \\
\hline 170820 & VALDIR COLATTO & PMDB SC & $\begin{array}{l}\text { INCFA } \\
2003\end{array}$ & $\begin{array}{l}\text { PRONETO RIO } \\
\text { FORMOSO II } \\
\text { ETAPA }\end{array}$ & $\begin{array}{l}\text { ForMOSO DO } \\
\text { ARAGUAIA }\end{array}$ & то & 143 & & 143 & 0 & $\begin{array}{l}\text { AGROPECUARIA } \\
\text { BARRA GRANDE } \\
\text { LTDA }\end{array}$ & $\begin{array}{l}\text { VaLDIR COLATTO dedarouGieba A } \\
\text { Do Projeto Rio Formoso } 4 \text {, }\end{array}$ \\
\hline 170820 & VALDR COLATTO & PMDB SC & $\begin{array}{l}\text { INCFA } \\
2003\end{array}$ & $\begin{array}{l}\text { PROJETO RIO } \\
\text { FORMOSO } 3 \text { ET } \\
\text { MOD L1 }\end{array}$ & $\begin{array}{l}\text { FORMOSO DO } \\
\text { ARAGUAIA }\end{array}$ & то & 43 & menaso & ${ }^{43}$ & 0 & $\begin{array}{c}\text { AGROPECUARIA } \\
\text { IMPUCA LTDA }\end{array}$ & $\begin{array}{l}\text { VALDIR COLATTO declarouGieba A } \\
\text { Do Projeto Rio Formoso } 4\end{array}$ \\
\hline 170820 & VALDR COLATTO & PMDB SC & $\begin{array}{l}\text { INCFA } \\
2003\end{array}$ & $\begin{array}{l}\text { PROUETO RIO } \\
\text { FORMOSOII } \\
\text { ETAPA }\end{array}$ & $\begin{array}{l}\text { FORMOSO DO } \\
\text { ARAGUAIA }\end{array}$ & то & 137 & & 137 & 0 & $\begin{array}{c}\text { AGROPECUAFIA } \\
\text { PORTO RICO } \\
\text { LTDA }\end{array}$ & $\begin{array}{l}\text { VALDIR COLATTO declarouGileba A } \\
\text { Do Projeto Rio Formoso } 4 \text {, }\end{array}$ \\
\hline 170820 & VALDIR COLATTO & PMDB SC & $\begin{array}{l}\text { INCFA } \\
2003\end{array}$ & $\begin{array}{l}\text { PRONETO RIO } \\
\text { FORMOSO } 3 \\
\text { ETAPA }\end{array}$ & $\begin{array}{l}\text { FORMOSO DO } \\
\text { ARAGUAIA }\end{array}$ & то & 849 & & 849 & 0 & \begin{tabular}{|c|} 
AGROVEL \\
AGROINDUSTRIA \\
LVEREDA LTDA
\end{tabular} & $\begin{array}{l}\text { VALDIR COLATTO dedarouGieba A } \\
\text { Do Projeto Rio Formoso } 4\end{array}$ \\
\hline 170820 & VALDIR COLATTO & PMDB SC & $\begin{array}{l}\text { INCRA } \\
2003\end{array}$ & $\begin{array}{l}\text { PRONETO RIO } \\
\text { FORMOSO } 3 \\
\text { ETAPA }\end{array}$ & $\begin{array}{l}\text { ForMOSO DO } \\
\text { ARAGUAIA }\end{array}$ & то & 215 & & 215 & 0 & $\begin{array}{l}\text { AGROVILA VALE } \\
\text { PERDIDO LTDA }\end{array}$ & $\begin{array}{l}\text { VALDIR COLATTO dedarouGileba A } \\
\text { Do Projeto Rio Formoso } 4,\end{array}$ \\
\hline 170820 & VALDIR COLATTO & PMDB SC & $\begin{array}{l}\text { INCRA } \\
2003\end{array}$ & $\begin{array}{l}\text { PROJETO RIO } \\
\text { FORMOSO }\end{array}$ & $\begin{array}{l}\text { FoRMOSO DO } \\
\text { ARAGUAIA }\end{array}$ & то & 685 & & 685 & 0 & \begin{tabular}{|} 
GOIANIA \\
SOCIEDADE \\
AGROPECUAAIA \\
LTDA
\end{tabular} & $\begin{array}{l}\text { VALDIR COLATTO dedarouGieba A } \\
\text { Do Projeto Rio Formoso } 4 \text {, }\end{array}$ \\
\hline
\end{tabular}




\begin{tabular}{|c|c|c|c|c|c|c|c|c|c|c|c|c|}
\hline GEOCÓDICO & PARLAMENTAR & PARTIDO & FONTE & IMÓVEL & Municipio & UF & AREA & 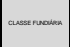 & $\begin{array}{c}\text { AREA } \\
\text { REGISTRAD } \\
\text { A }\end{array}$ & $\begin{array}{l}\text { DECLARADA } \\
\text { COMO POSSE }\end{array}$ & EMPRESA & VINCULO COM O PARLAMENTAR \\
\hline 170820 & VALDIR COLATTO & PMDB SC & $\begin{array}{l}\text { INCRA } \\
2003\end{array}$ & $\begin{array}{c}\text { PROUETO RIO } \\
\text { FORMOSO ॥I } \\
\text { ETAPA }\end{array}$ & \begin{tabular}{|l} 
Formoso DO \\
ARAGUAAIA
\end{tabular} & Tо & 750 & & 750 & 0 & $\begin{array}{c}\text { OASIS } \\
\text { SOCEIEDAEE } \\
\text { AGROPECUAARIA } \\
\text { LTDA }\end{array}$ & 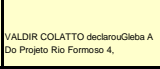 \\
\hline 170020 & VALDIR COLATTO & PMDB SC & $\begin{array}{l}\text { INCRA } \\
2003\end{array}$ & $\begin{array}{c}\begin{array}{c}\text { PROUETO RIO } \\
\text { FORMOSO II } \\
\text { ETAPA }\end{array} \\
\end{array}$ & \begin{tabular}{|l}
$\begin{array}{l}\text { Formoso DO } \\
\text { ARAGUAAIA }\end{array}$ \\
\end{tabular} & то & 750 & & 750 & 0 & $\begin{array}{c}\text { PANTANAL } \\
\text { GOLAANO } \\
\text { SOCIEDADE } \\
\text { AGROPECUARIA } \\
\text { LTDA }\end{array}$ & $\begin{array}{l}\text { VaLDIR COLATTO dedarouGieba A } \\
\text { Do Projeto Rio Formoso } 4 \text {, }\end{array}$ \\
\hline 170820 & VALDIR COLATTO & PMDB SC & $\begin{array}{l}\text { INCRA } \\
2003\end{array}$ & $\begin{array}{c}\text { PROUETO RIO } \\
\text { FORMOSO II } \\
\text { ETAPA }\end{array}$ & $\begin{array}{l}\text { FORMOSO DO } \\
\text { ARAGUAIA }\end{array}$ & то & 750 & & 750 & 0 & $\begin{array}{c}\text { SOCIEDADE } \\
\text { AGRICOLA } \\
\text { LGOA } \\
\text { FORMOSA LTDA }\end{array}$ & $\begin{array}{l}\text { VALDIR COLATTO dedarouGileba A } \\
\text { Do Projeto Rio Formoso } 4 \text {, }\end{array}$ \\
\hline
\end{tabular}

Organizado por: COSTA, S. H. G., 2012 


\section{MATO GROSSO DO SUL}

Ind. Óleo -Fil 37

Faz. Amambai - Fil 02 Sandra Dóris - Fil 03 Lagunita F10 Esc. Amambai - Fil 11 Laguna Carapã Fil 21 Aral Moreira - Fil 22 Dourados Fil27 Cau - Fil 30 Capei Fil 38 Cab. do Apa Fil 39 São Tomáz -Fil40 Jaguarete Fil41 Bataguassu Fil 44

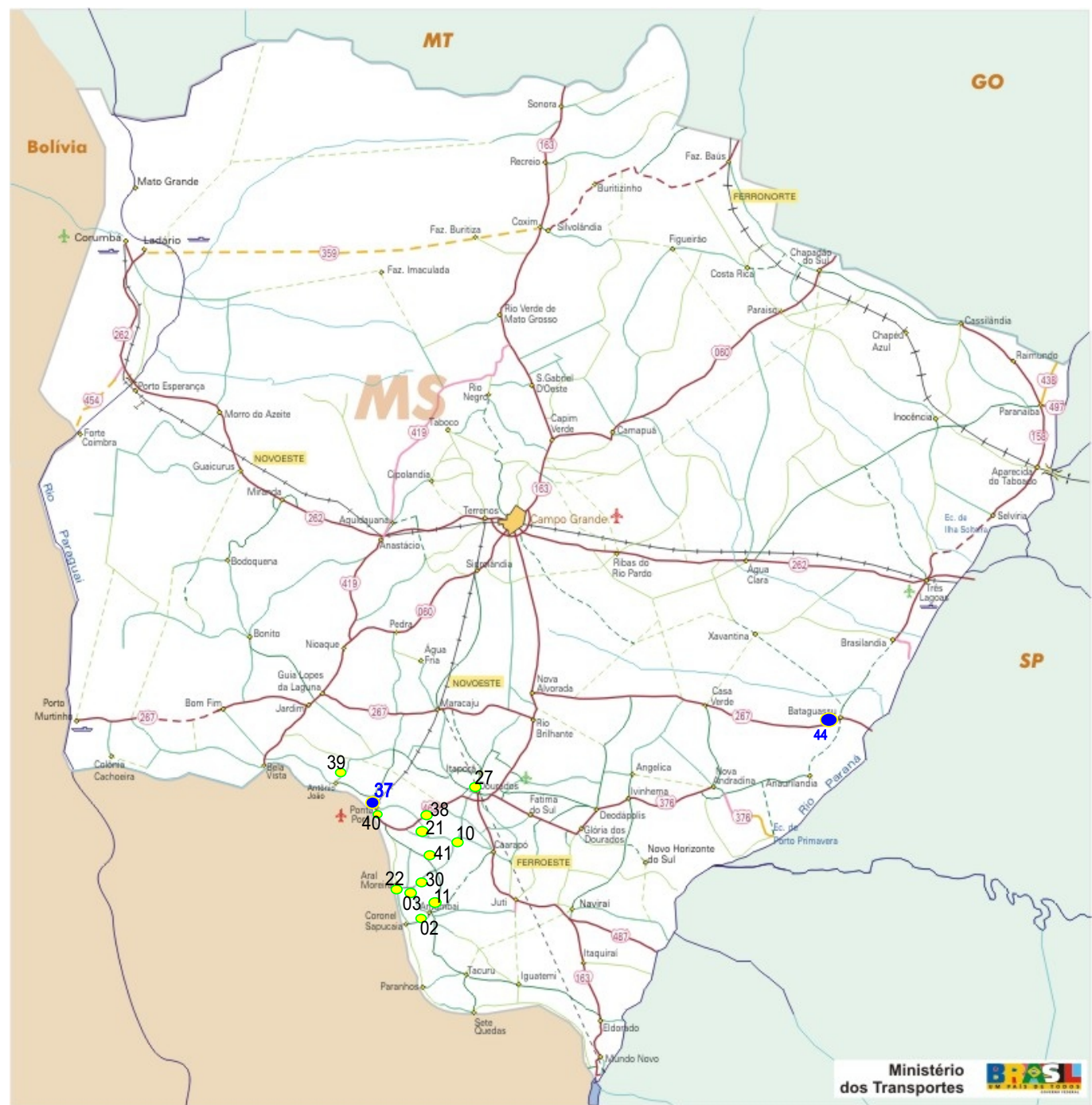




\section{MATO GROSSO}

Faz. Comodoro - Fil32 Ind. Óleo Cuiabá - Fil 33

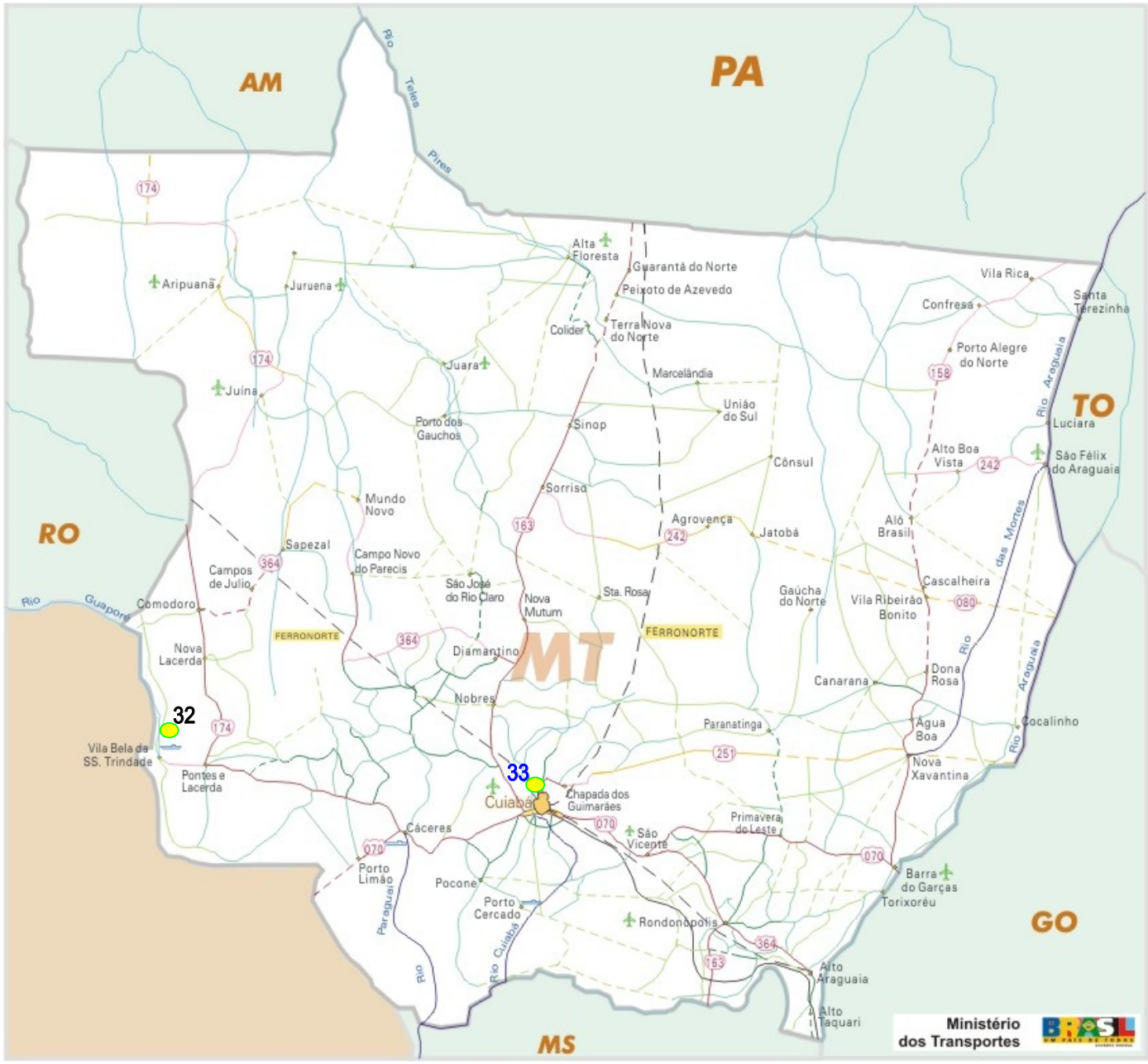




\section{PARANÁ}

MATRIZ - Toledo 1 Ind. Óleo -F26 M.C.Rondon Guaira -F17 V. Nova -F06 Entre Rios Oeste -F49 V. Ipiranga -F14 Sta. Helena -F28 Cascavel -F08 Corbelia -F25 Penha -F29 S.Pedro -F46 Laranjeiras do Sul -F35 Curitiba -F34 Paranaguá F-31 Padrão/Too F-04 Nova Aurora F-07 São Luis/Too - F15 S. Inês/Too-F19 Moinho/Too-F24

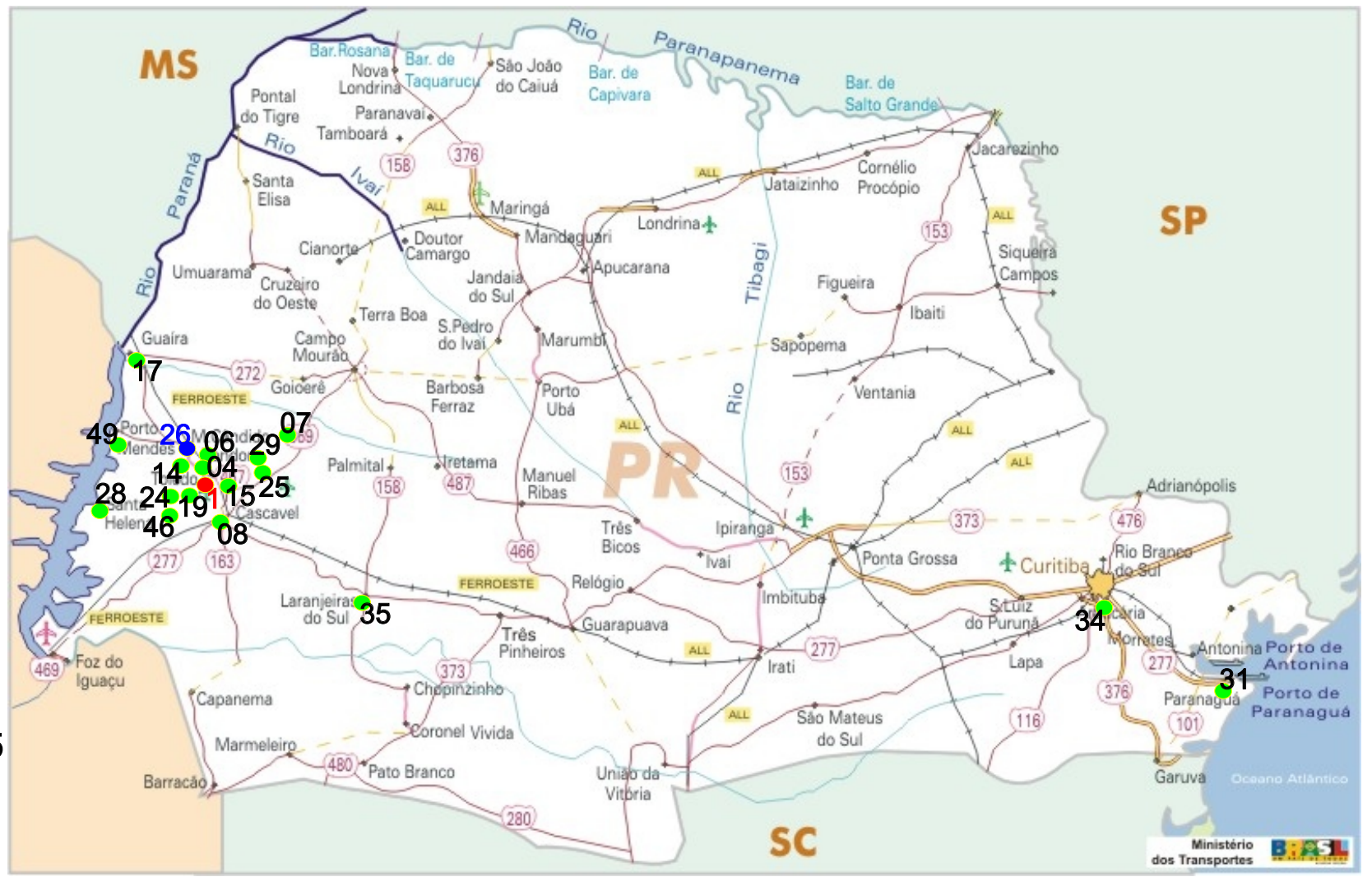


SÃO PAULO

Orlândia -F47

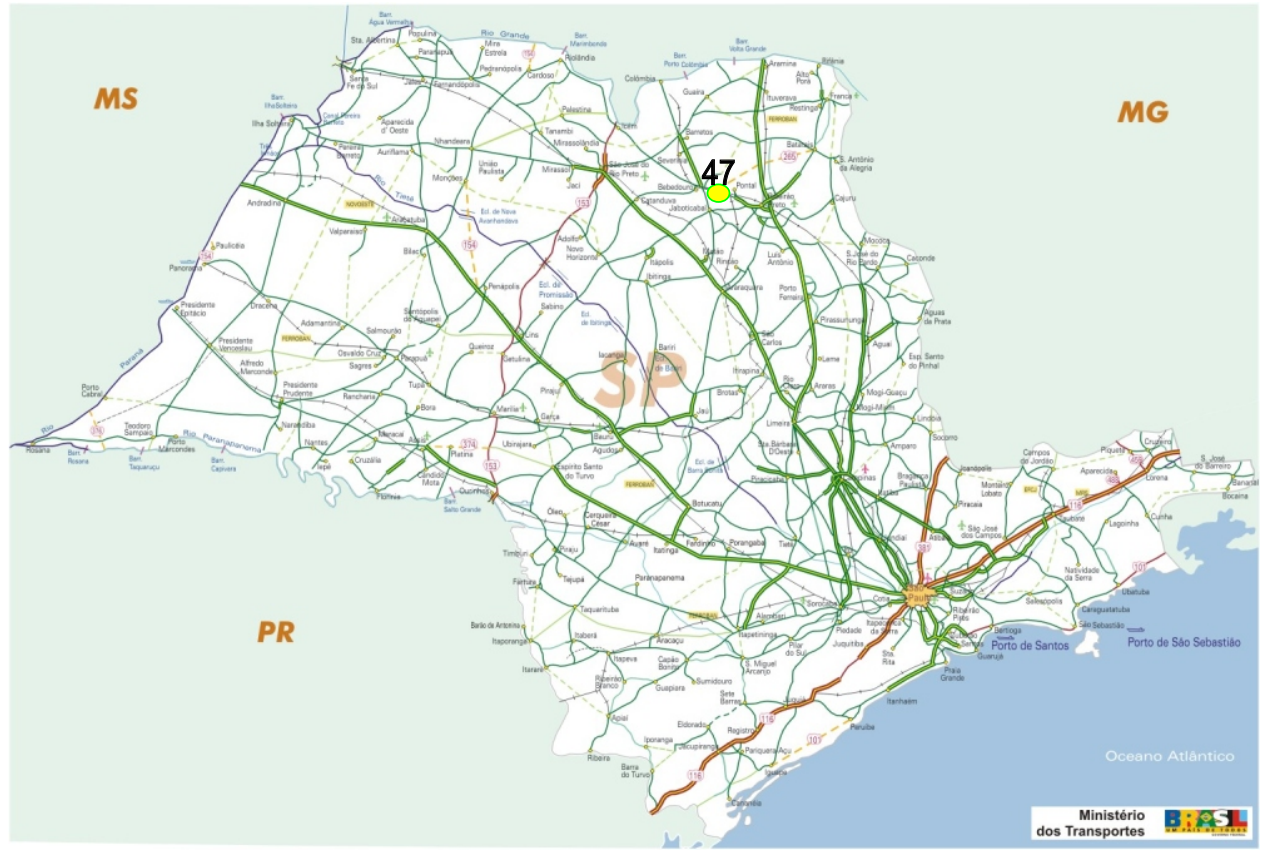

SPERAFICO

$+1$

\section{AGROINDUSTRIAL}




\title{
ANEXO H
}

\author{
CÂMARA DOS DEPUTADOS \\ PROJETO DE LEI N.ำ 5.418, DE 2009 \\ (Do Sr. Lira Maia) \\ Cria Área de Livre Comércio no Município de Santarém, no Estado do Pará, e dá outras providências.
}

DESPACHO:

ÀS COMISSÕES DE:

AMAZÔNIA, INTEGRAÇÃO NACIONAL E DE DESENVOLVIMENTO REGIONAL;

DESENVOLVIMENTO ECONÔMICO, INDÚSTRIA E COMÉRCIO;

FINANÇAS E TRIBUTAÇÃO (MÉRITO E ART. 54, RICD) E

CONSTITUIČÃO E JUSTIÇA E DE CIDADANIA (ART. 54 RICD).

APRECIAÇẨ:

Proposição Sujeita à Apreciação Conclusiva pelas Comissões - Art. 24 ॥

PUBLICAÇÃO INICIAL

Art. 137, caput - RICD

O CONGRESSO NACIONAL DECRETA:

Art. 1o É criada, no território do Município de Santarém, Estado do Pará, área de livre comércio de importação e exportação e de regime fiscal especial, estabelecida com a finalidade de promover o desenvolvimento da região oeste do Pará, a integração econômica do interior da Amazônia com o resto do país e a proteção do meio ambiente.

Parágrafo único. O regime jurídico tributário da área de livre comércio criada por esta lei será àquele aplicável às áreas de que tratam as Leis ns 7.965, de 22 de dezembro de 1989, 8.210, de 19 de junho de 1991, 8.256, de 25 de novembro de 1991, o art. 11 da Lei 8.387, de 30 de dezembro de 1991, e a Lei 8.857, de 8 de março de 1994.

Art. 2o - Esta Lei entra em vigor na data de sua publicação.

Art. $3^{\circ}$ - Revogam-se as disposições em contrário.

\section{JUSTIFICATIVA}

Todos os Estados amazônicos têm, no mínimo, um município reconhecido pela legislação federal como área de livre comércio, a saber, tabatinga, no estado do Amazonas, Guajará-Mirim, no Estado de Rondônia, Macapá e Santana no Estado do Amapá, Brasiléia e Cruzeiro do Sul no Estado do Acre, e Boa Vista e Bonfim no Estado de Roraima. Esta circunstância coloca o Estado do Pará em uma situação de evidente desvantagem fiscal em relação aos seus vizinhos no que tange à atração de investimentos produtivos.

O Estado do Pará tem dimensões territoriais amazônicas. Enquanto as regiões metropolitana, sudeste e nordeste do Estado alcançam altos níveis de crescimento pela atração de investimentos produtivos em grandes projetos privados ligados à geração de energia, mineração, metalurgia e agropecuária, a região Oeste do Pará sofre com o isolamento geográfico e a ameaça de devastação ambiental , último recurso que a população encontra para buscar a sobrevivência econômica.

Dentre do atual cenário da economia e da geopolítica do Pará e do Brasil, Santarém é a cidade paraense que melhor preenche os requisitos para ser contemplada com área de livre comércio, em face de pelo menos quatro razões principais.

1. Santarém está no centro da região da Amazônia brasileira mais ameaçada pela degradação ambiental. A cidade, embora seja o mais importante pólo de trocas da região oeste do Pará, influenciando mais de um milhão de habitantes, tem a expansão da sua área produtiva cerceada por reservas ambientais e florestas nacionais, dificultando o desenvolvimento de sua vocação produtiva na área do agronegócio.

O reconhecimento de Santarém como área de livre comércio permitirá que a cidade encarne, de fato e de direito, a sua vocação de cidade-pólo do Oeste do Pará, responsável pelo abastecimento de milhares de paraenses que vivem nos municípios do entorno, que sofrem com a falta de acesso a produtos de qualidade a preços acessível. A geração de emprego e renda motivada pela implantação da Área de Livre Comércio de Santarém, constituirá uma importante alternativa para o desenvolvimento sustentável da região.

2. Santarém, assim como as demais cidades do oeste do Pará, sofrem com o isolamento territorial e as dificuldades logísticas de transporte. A ausência de uma ligação rodoviária segura e permanente com a capital do Estado e com o resto do país encarece a aquisição de produtos acabados e matérias-primas, praticamente inviabilizando qualquer iniciativa produtiva no setor industrial. A área de livre comércio em Santarém será uma alternativa para compensar, com a desoneração tributária, ônus logístico que a cidade e a região sofrem.

3. Quando comparada com as demais cidades-pólo do Estado, Santarém vem nitidamente perdendo espaço no quadro geral da economia paraense. Marabá, Barcarena e outras cidades do sul e nordeste do Estado são beneficiadas com grandes projetos na área mineral, que aportam investimentos na casa dos bilhões de Reais e emprego na faixa de milhares. Diante disso, o reconhecimento de Santarém como área de livre comércio será uma adequada e necessária contrapartida estatal para equilibrar o desenvolvimento do Estado entre as suas diferentes regiões.

4. A pressão pela divisão territorial do Pará é diretamente proporcional ao abandono e à depreciação econômica de Santarém e cidades vizinhas. O desenvolvimento trazido pela área de livre comércio de Santarém será um importante bálsamo para as feridas causadas por décadas de esquecimento que o povo do oeste do Pará sente em relação ao poder central estabelecido na capital do Estado.

Ressalta-se ainda que Santarém é o principal fornecedor de serviços e produtos da região oeste do Estado do Pará, com localização estratégica para apoio aos Municípios da região, principalmente aos grandes projetos desenvolvimentistas ali implantados, tais como projeto da Alcoa em Juruti; Mineração Rio do Norte em Oriximiná, Hidroelétrica de Belo Monte em Altamira, dentre outros. 
Portanto, já é hora de o governo federal reconhecer que não só os demais Estados da Amazônia, mas também o Estado do Pará, mas especialmente Santarém e o oeste do Estado, também merecem ser contemplados com projetos estratégicos como é o caso da criação da Área de Livre Comércio de Santarém - ALCS, ora proposta.

Pelos motivos expostos e devido a importância econômica para o desenvolvimento sustentável daquela região é que solicito o apoio dos Nobres Pares para que possamos aprovar a presente matéria.

Sala das Sessões, em 15 de junho de 2009.

\section{Joaquim de LIRA MAIA}

Deputado Federal

Vice-Líder - DEM/PA

Fonte: http://www.camara.gov.br/sileg/MostrarIntegra.asp?CodTeor=667771. 
ANEXO I

MPV-458

CÂMARA DOS DEPUTADOS

00205

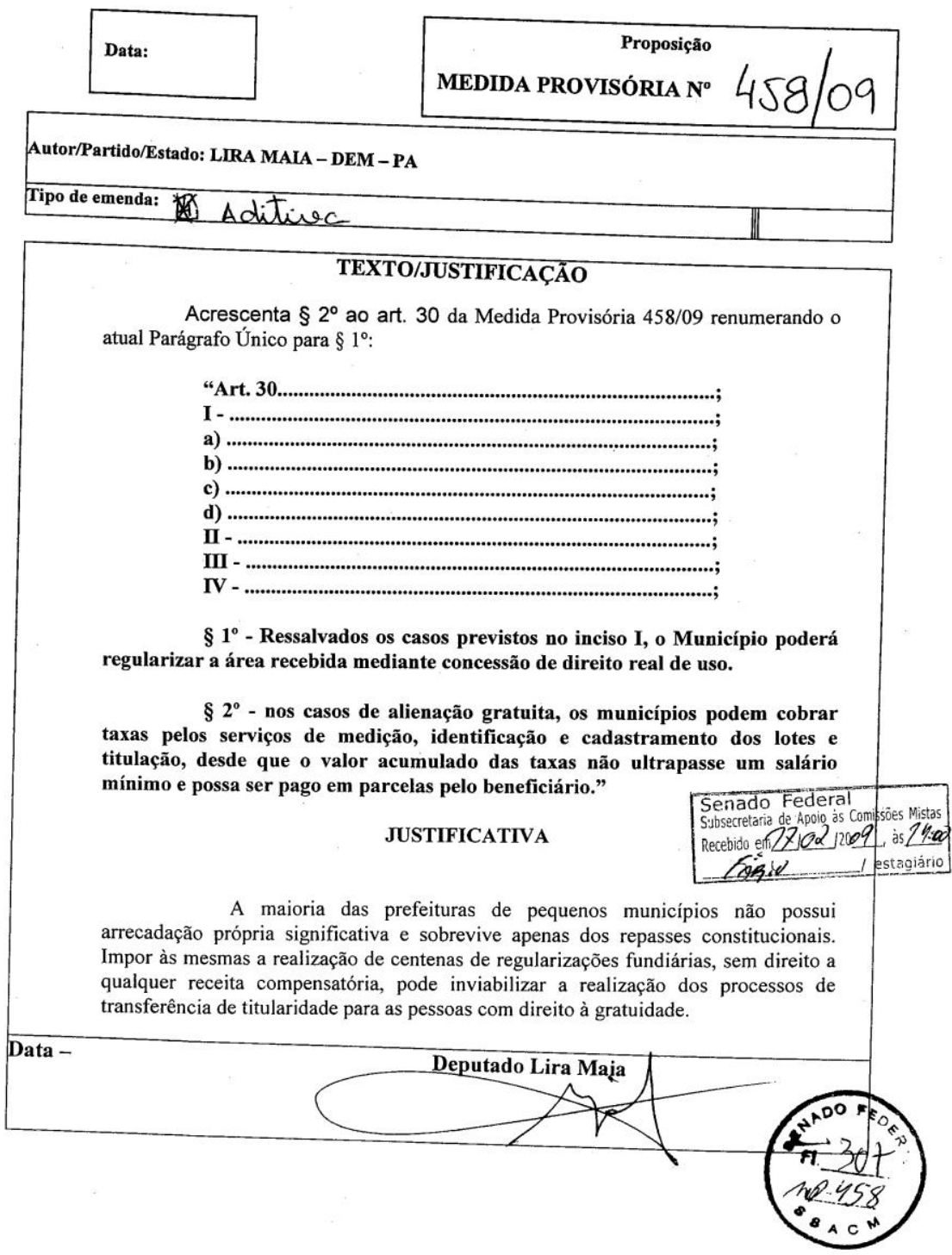


ANEXO J

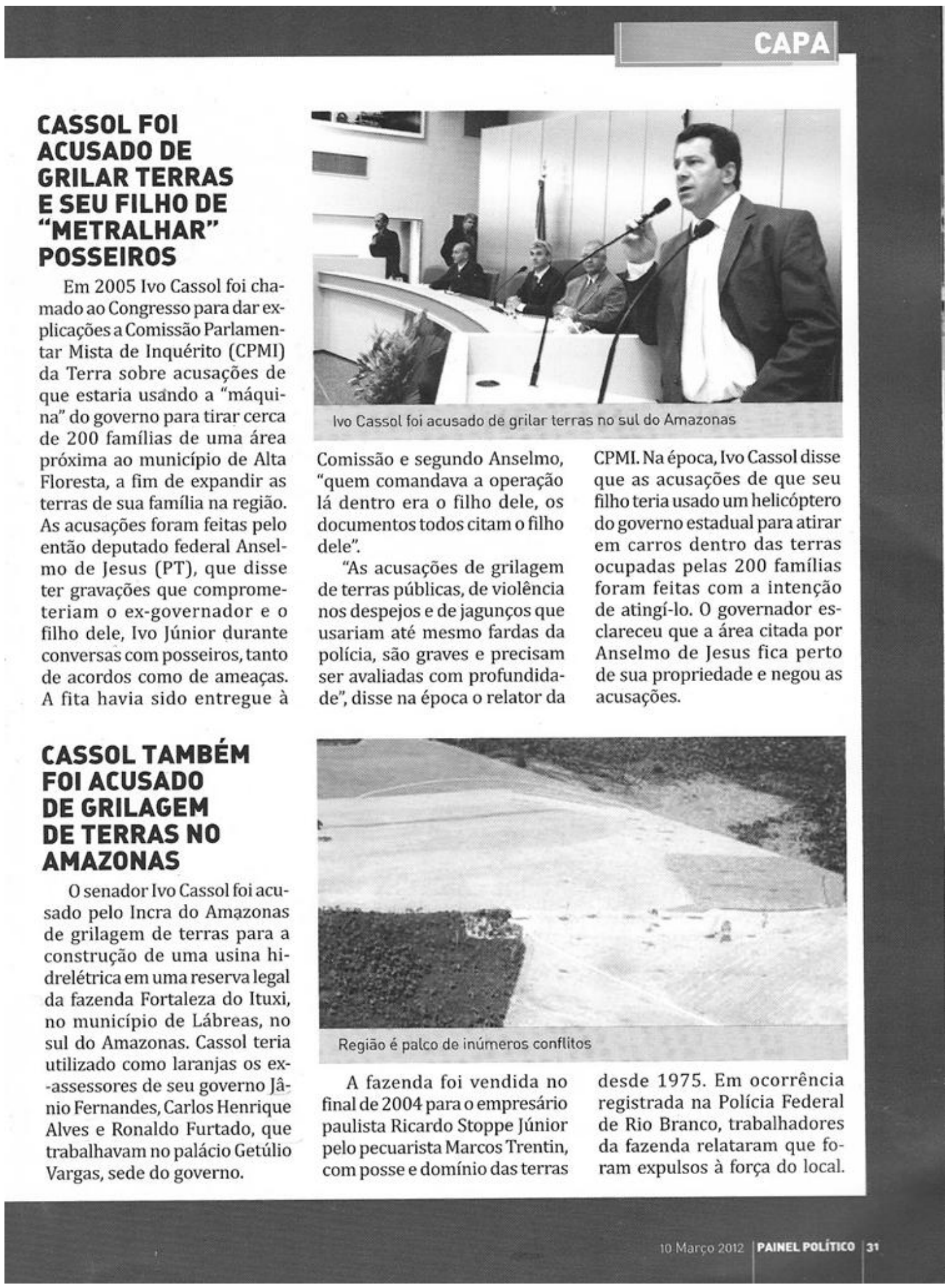




\section{ANEXO L - FAZENDAS DO GRUPO ANDRÉ MAGGI}

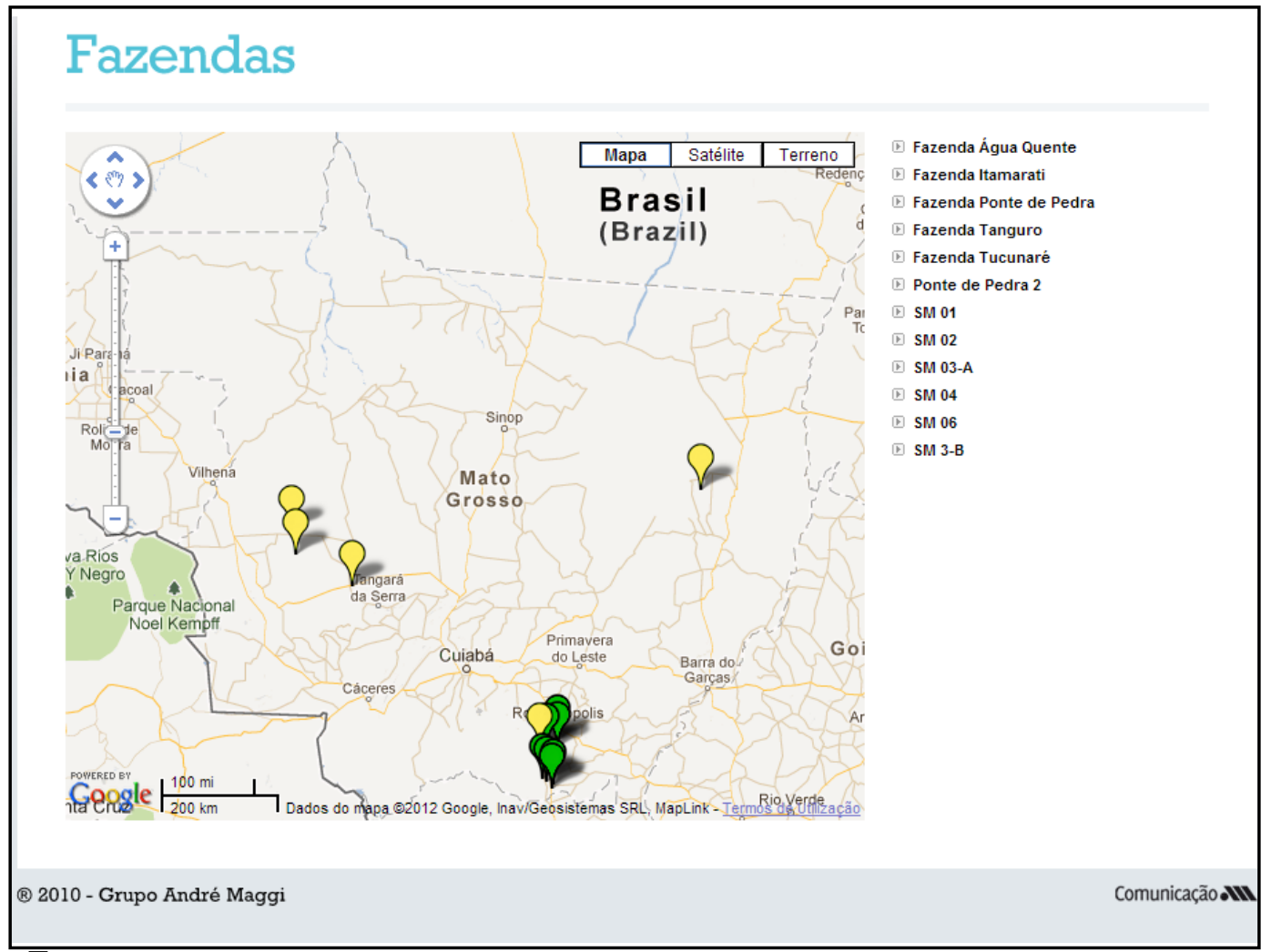

Fonte: http://www.grupoandremaggi.com.br/?page id=78

\section{Fazenda Tanguro}

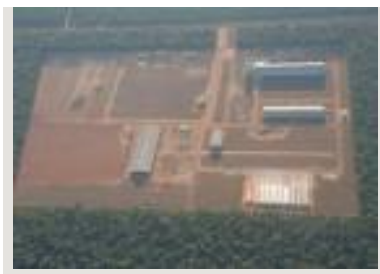

FAZENDA TANGURO, Rod. MT 320 - Km 104, Zona Rural, CEP: 78643-000, Caixa Postal: 104, Querência (MT). Telefone: (66) 3529-3900 Fazenda Itamarati

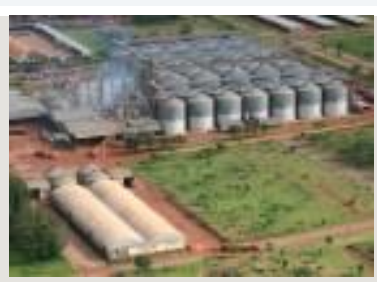

AGR. E PEC. MORRO AZUL LTDA - Faz. Itamarati Norte - Rod. BR 364 entroncamento c/ MT 170 - CEP: 78.360-000 - Campo Novo do Parecis - MT - Telefone: (65) $3349-1130$

\section{Fazenda Ponte de Pedra}

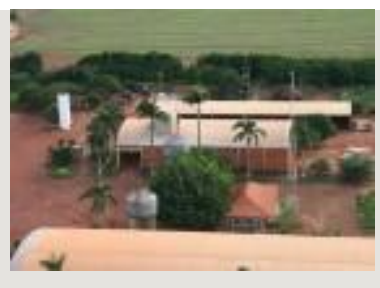

AGROP. MAGGI LTDA. - FAZ. PONTE DE PEDRA - Rod. BR -163 KM - 102, S/NR - Rondonópolis/MT - CEP: 78700-000 


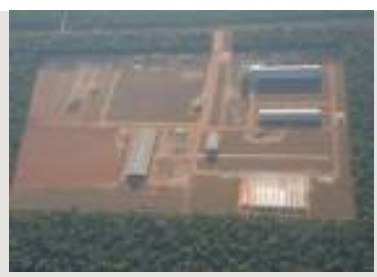

FAZENDA TANGURO, Rod. MT 320 - Km 104, Zona Rural, CEP: 78643-000, Caixa Postal: 104, Querência (MT). Telefone: (66) 3529-3900 Fazenda Tucunaré

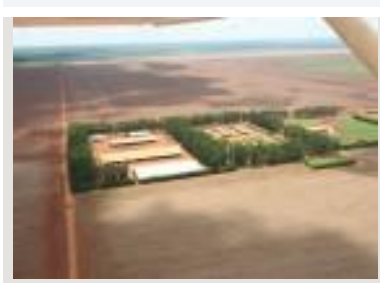

AGROPEC. MAGGI LTDA - TUCUNARÉ 01 - Rod. MT-235, KM-133 - CEP: 78365-000 - Sapezal - MT - Telefone: (65) 3383-1252.

\section{Ponte de Pedra 2}

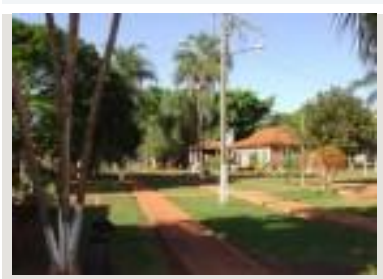

FAZENDA PONTE DE PEDRA 2. Rod. BR 163, KM 102 + 35 a direita, Rondonopolis - MT.

\section{SM 01}

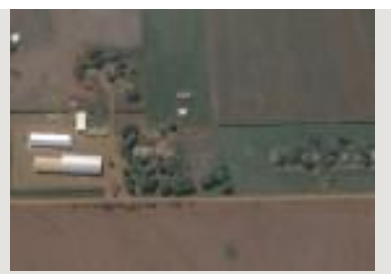

AGROPECUÁRIA MAGGI LTDA - SM 01 - Rod. BR-163 KM-10 - CEP: 78790-000 - Itiquira - MT + 18 Km Direita

\section{SM 02}

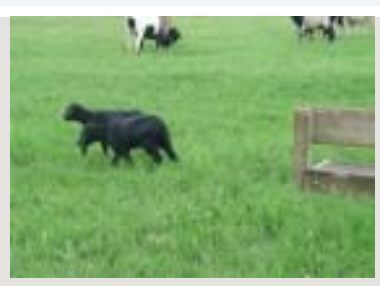

FAZENDA SM 02 - ROD. BR 163, KM 94, RONDONÓPOLIS - MT.

SM 03-A

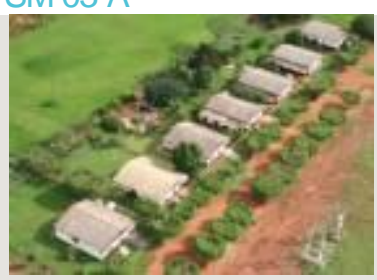

FAZENDA SM-03 - ROD. BR 163, KM 10, ITIQUIRA - MT. 
SM 04

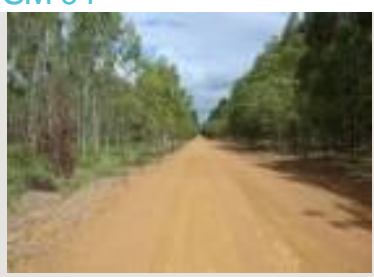

FAZENDA SM 04 - ROD. BR 163, KM 45, RONDONÓPOLIS - MT.

SM 06

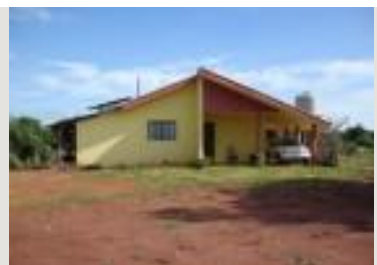

FAZENDA SM 06 - ROD. BR 163 KM 10, ITIQUIRA - MT.

SM 3-B

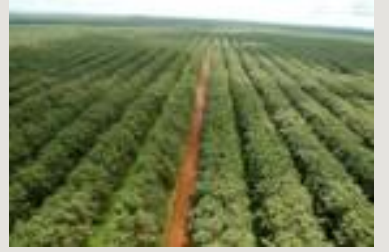

ROD. BR 163 - KM 16,5, ITIQUIRA - MT. 


\begin{tabular}{|c|c|c|c|}
\hline \multicolumn{4}{|c|}{$\begin{array}{l}\text { ANEXO M - NOME, UF, PROFISSÃO DECLARADA E ATUAÇÃO PROFISSIONAL E POLÍTICA OU FONTE } \\
\text { DE RENDA PATRIMONIAL }\end{array}$} \\
\hline NOME & UF & PROFISSÃO DECLARADA & ATUAÇAO PROFISSIONAL \\
\hline $\begin{array}{l}\text { JOÃO TOTA SOARES DE } \\
\text { FIGUEIREDO }\end{array}$ & $A C$ & Membro do Poder Executivo & AGRICULTURA \\
\hline MARCIO MIGUEL BITTAR & $A C$ & Agropecuarista & PECUÁRIA \\
\hline $\begin{array}{l}\text { SERGIO SEBASTIÃO DE } \\
\text { BARROS }\end{array}$ & $A C$ & $\begin{array}{l}\text { Engenheiro Agrimensor, Agropecuarista e } \\
\text { empresário }\end{array}$ & PECUÁRIA \\
\hline $\begin{array}{l}\text { SERGIO DE OLIVEIRA } \\
\text { CUNHA (PETECÃO) }\end{array}$ & $A C$ & Empresário e Técnico em Contabilidade & POLÍTICA \\
\hline $\begin{array}{l}\text { JOAQUIM BELTRÃO } \\
\text { SIQUEIRA }\end{array}$ & $\mathrm{AL}$ & Agricultor e Engenheiro Eletricista & FAZENDAS \\
\hline $\begin{array}{l}\text { JOSÉ THOMAZ NONÔ } \\
\text { NETTO }\end{array}$ & $\mathrm{AL}$ & Agricultor, Advogado e Promotor de Justiça & JUDICIÁRIO \\
\hline $\begin{array}{l}\text { CARLOS ALBERTO } \\
\text { MOREIRA DE MENDONÇA } \\
\text { CANUTO }\end{array}$ & $\widehat{A L}$ & Agropecuarista & PECUÁRIA \\
\hline $\begin{array}{l}\text { ARTHUR CESAR PEREIRA } \\
\text { DE LIRA }\end{array}$ & $A L$ & Agropecuarista, Bacharel em Direito e Empresário & PECUÁRIA \\
\hline RENAN CALHEIROS FILHO & $A L$ & Economista & POLÍTICA \\
\hline JOÃO CALDAS DA SILVA & $\mathrm{AL}$ & Advogado & POLÍTICA \\
\hline LUIZ DANTAS LIMA & $\mathrm{AL}$ & Agrônomo & POLÍTICA \\
\hline OLAVO CALHEIROS FILHO & $\overline{A L}$ & Engenheiro Agrônomo & POLÍTICA \\
\hline $\begin{array}{l}\text { JOSÉ RENAN } \\
\text { VASCONCELOS } \\
\text { CALHEIROS } \\
\end{array}$ & $\mathrm{AL}$ & Advogado & $\begin{array}{l}\text { POLÍTICA E INVESTIMENTOS EM } \\
\text { AGRONEGÓCIO }\end{array}$ \\
\hline BENEDITO DE LIRA & $\mathrm{AL}$ & Advogado, Servidor Público e Pecuarista & SUCROALCOLEIRO \\
\hline $\begin{array}{l}\text { TEOTONIO BRANDAO } \\
\text { VILELA FILHO }\end{array}$ & $A L$ & Economista & SUCROALCOLEIRO \\
\hline $\begin{array}{l}\text { JOAO JOSE PEREIRA DE } \\
\text { LYRA }\end{array}$ & $A L$ & Industrial e Advogado & $\begin{array}{l}\text { SUCROALCOLEIRO (GRUPO CARLOS } \\
\text { LYRA) }\end{array}$ \\
\hline $\begin{array}{l}\text { JOÃO THOMÉ VERÇOSA } \\
\text { MESTRINHO DE MEDEIROS } \\
\text { RAPOSO }\end{array}$ & $\mathrm{AM}$ & Empresário Rural & EMPRESA AGRONEGÓCIO \\
\hline $\begin{array}{l}\text { ATILA SIDNEY LINS } \\
\text { ALBUQUERQUE }\end{array}$ & AM & $\begin{array}{l}\text { Economista, Servidor Público e Bacharel em } \\
\text { Direito }\end{array}$ & POLÍTICA \\
\hline $\begin{array}{l}\text { BENEDITO DIAS DE } \\
\text { CARVALHO }\end{array}$ & AP & Médico e Empresário & MEDICINA \\
\hline $\begin{array}{l}\text { ANTONIO DA JUSTA } \\
\text { FEIJÃO }\end{array}$ & $\mathrm{AP}$ & Geólogo & MINERAÇÃO \\
\hline $\begin{array}{l}\text { JOSÉ RIBAMAR FERREIRA } \\
\text { DE ARAÚJO COSTA (JOSÉ } \\
\text { SARNEY) }\end{array}$ & AP & Bacharel em Ciências Jurídicas e Sociais & POLÍTICA \\
\hline FATIMA LUCIA PELAES & AP & Socióloga & SOCIÓLOGA \\
\hline EDSON SAMPAIO PIMENTA & BA & Agricultor & AGRICULTURA \\
\hline OZIEL ALVES DE OLIVEIRA & BA & Agricultor e Administrador & AGRICULTURA \\
\hline $\begin{array}{l}\text { FELIX DE ALMEIDA } \\
\text { MENDONÇA }\end{array}$ & BA & Engenheiro Civil e Construtor & CONSTRUÇÃO CIVIL \\
\hline $\begin{array}{l}\text { FELIX DE ALMEIDA } \\
\text { MENDONÇA JÚNIOR }\end{array}$ & BA & Não Informou & CONSTRUÇÃO CIVIL \\
\hline $\begin{array}{l}\text { JOÃO FELIPE DE SOUZA } \\
\text { LEÃO }\end{array}$ & BA & Empresário & CONSTRUÇÃO CIVIL \\
\hline $\begin{array}{l}\text { JOAO CARLOS PAOLILO } \\
\text { BACELAR FILHO }\end{array}$ & BA & Engenheiro (Empresário do Agronegócio) & CONSTRUÇÃO CIVIL \\
\hline
\end{tabular}




\begin{tabular}{|c|c|c|c|}
\hline $\begin{array}{l}\text { PAULO SERGGIO } \\
\text { PARANHOS DE } \\
\text { MAGALHÃES }\end{array}$ & BA & Administrador de Empresas & CONSTRUTORA E FAZENDAS \\
\hline $\begin{array}{l}\text { JOÃO CARLOS PAOLILO } \\
\text { BACELAR }\end{array}$ & BA & Industrial (Diretor, firmas de Agropecuária e Constry & $\begin{array}{c}\text { EMPRESA (CONSTRUÇÃO CIVIL, } \\
\text { AGRONEGÓCIO E DISTRIBUIÇÃO DE } \\
\text { COMBUSTÍVEIS E LUBRIFICANTES) }\end{array}$ \\
\hline $\begin{array}{l}\text { LEUR ANTONIO DE BRITO } \\
\text { LOMANTO }\end{array}$ & BA & Advogado & EMPRESA RURAL \\
\hline $\begin{array}{l}\text { COLBERT MARTINS DA } \\
\text { SILVA FILHO }\end{array}$ & BA & Médico & FAZENDAS \\
\hline $\begin{array}{l}\text { SEVERIANO ALVES DE } \\
\text { SOUZA }\end{array}$ & BA & Advogado e Procurador Federal & FAZENDAS \\
\hline $\begin{array}{l}\text { MARIO SILVIO MENDES } \\
\text { NEGROMONTE }\end{array}$ & BA & Advogado & FAZENDAS \\
\hline FÁBIO LOUREIRO SOUTO & BA & Economista & FAZENDAS \\
\hline $\begin{array}{l}\text { CESAR AUGUSTO } \\
\text { RABELLO BORGES }\end{array}$ & BA & Engenheiro Civil & FAZENDAS \\
\hline $\begin{array}{l}\text { JOSE LOURENÇO MORAIS } \\
\text { DA SILVA }\end{array}$ & BA & Economista e Empresário & FAZENDAS E EMPRESA \\
\hline $\begin{array}{l}\text { JUSMARI TEREZINHA DE } \\
\text { SOUZA OLIVEIRA }\end{array}$ & BA & Educadora e agricultora & GRÃOS (SOJA) \\
\hline JOSE ALVES ROCHA & BA & Médico & MEDICINA \\
\hline $\begin{array}{l}\text { JOAO ALMEIDA DOS } \\
\text { SANTOS }\end{array}$ & BA & Geólogo & MINERAÇÃO E AGRICULTURA \\
\hline $\begin{array}{l}\text { FERNANDO DANTAS } \\
\text { TORRES }\end{array}$ & BA & Empresário & MINERAÇÃO E CONSTRUÇÃO CIVIL \\
\hline $\begin{array}{l}\text { ARTHUR DE OLIVEIRA } \\
\text { MAIA DA SILVA }\end{array}$ & BA & Advogado & PECUÁRIA \\
\hline $\begin{array}{l}\text { SAULO PEDROSA DE } \\
\text { ALMEIDA }\end{array}$ & BA & Agropecuarista e Médico & PECUÁRIA \\
\hline $\begin{array}{l}\text { JOAO LUIZ CORREIA } \\
\text { ARGÔLO DOS SANTOS }\end{array}$ & BA & Pecuarista, Empresário e Administrador & PECUÁRIA \\
\hline $\begin{array}{l}\text { PAULO ROBERTO LUZ } \\
\text { BRAGA } \\
\end{array}$ & $\mathrm{BA}$ & Agropecuarista e Administrador de Empresas & PECUÁRIA \\
\hline $\begin{array}{l}\text { FRANCISTÔNIO ALVES } \\
\text { PINTO (07/10/1943 a } \\
16 / 02 / 2010)\end{array}$ & $\overline{B A}$ & Pecuarista & PECUÁRIA \\
\hline $\begin{array}{l}\text { LUCIO QUADROS VIEIRA } \\
\text { LIMA }\end{array}$ & BA & Pecuarista, Engenheiro Agrônomo e Cacauicultor & PECUÁRIA \\
\hline $\begin{array}{l}\text { GEDDEL QUADROS VIEIRA } \\
\text { LIMA } \\
\end{array}$ & BA & Administrador de Empresas e Pecuarista & PECUÁRIA \\
\hline JOÃO DURVAL CARNEIRO & BA & Cirurgião-Dentista e Pecuarista & PECUÁRIA \\
\hline JOSÉ NUNES SOARES & BA & Empresário & PECUÁRIA \\
\hline JAIME FERNANDES FILHO & $\mathrm{BA}$ & $?$ & PECUÁRIA \\
\hline $\begin{array}{l}\text { SERGIO LUIS LACERDA DE } \\
\text { BRITO }\end{array}$ & BA & Servidor Público, Administrador de Empresas e Em| & PECUÁRIA \\
\hline $\begin{array}{l}\text { EUJÁCIO SIMÕES VIANA } \\
\text { FILHO }\end{array}$ & BA & Arquiteto & POLÍTICA \\
\hline \begin{tabular}{|l} 
ANTONIO JOSE \\
IMBASSAHY DA SILVA
\end{tabular} & BA & Engenheiro Eletricista & POLÍTICA \\
\hline $\begin{array}{l}\text { MARCOS ANTONIO } \\
\text { MEDRADO } \\
\end{array}$ & BA & Administrador de Empresas e Radialista & POLÍTICA \\
\hline CORIOLANO SOUSA SALES & BA & Advogado & POLÍTICA \\
\hline $\begin{array}{l}\text { AROLDO CEDRAZ DE } \\
\text { OLIVEIRA }\end{array}$ & BA & Professor Universitário, Empresário e Médico Veteri & POLÍTICA \\
\hline $\begin{array}{l}\text { ROBERTO PEREIRA DE } \\
\text { BRITTO }\end{array}$ & BA & Professor Universitário & POLÍTICA E MEDICINA \\
\hline $\begin{array}{l}\text { JOSÉ CARLOS LEÃO DE } \\
\text { ARAUJO }\end{array}$ & BA & Radialista e Administrador & TECNOLOGIA \\
\hline $\begin{array}{l}\text { FRANCISCO PINHEIRO } \\
\text { LANDIM }\end{array}$ & CE & Empresário & CONSTRUTORA \\
\hline
\end{tabular}




\begin{tabular}{|c|c|c|c|}
\hline $\begin{array}{l}\text { GENECIAS MATEUS } \\
\text { NORONHA }\end{array}$ & CE & Empresário & EMPRESA (MOTOS) \\
\hline $\begin{array}{l}\text { TASSO RIBEIRO } \\
\text { JEREISSATI }\end{array}$ & CE & Empresário & EMPRESA (PECUÁRIA) \\
\hline \begin{tabular}{|l} 
VICENTE FERREIRA DE \\
ARRUDA COELHO \\
\end{tabular} & CE & Advogado, Jornalista e Cientista Político & FAZENDA \\
\hline $\begin{array}{l}\text { RAIMUNDO ANTONIO DE } \\
\text { MACEDO (RAIMUNDÃO) }\end{array}$ & CE & Médico & MEDICINA \\
\hline $\begin{array}{l}\text { RAIMUNDO GOMES DE } \\
\text { MATOS }\end{array}$ & CE & Médico & MEDICINA \\
\hline $\begin{array}{l}\text { FRANCISCO ROMMEL } \\
\text { FEIJÓ DE SÁ }\end{array}$ & CE & Médico e Administrador Hospitalar & MEDICINA \\
\hline ANIBAL FERREIRA GOMES & CE & Agropecuarista e Cirurgião Dentista & PECUÁRIA \\
\hline \begin{tabular}{|l|} 
ROBERTO SOARES \\
PESSOA
\end{tabular} & CE & Agropecuarista, Economista e Contador & PECUÁRIA \\
\hline EUNICIO LOPES OLIVEIRA & CE & Agropecuarista e Empresário & PECUÁRIA \\
\hline $\begin{array}{l}\text { JOSE ARNON CRUZ } \\
\text { BEZERRA DE MENEZES }\end{array}$ & CE & Médico & PECUÁRIA \\
\hline $\begin{array}{l}\text { CARLOS MAURO CABRAL } \\
\text { BENEVIDES }\end{array}$ & CE & Advogado, Jornalista e Professor & POLÍTICA (PAI PECUÁRIA) \\
\hline JOSÉ LINHARES PONTE & CE & Padre & RELIGIOSO (PADRE) \\
\hline IZALCI LUCAS FERREIRA & $\mathrm{DF}$ & Professor e Contador & CONTABILIDADE \\
\hline JAQUELINE MARIA RORIZ & DF & Pedagoga & EMPRESA (AGROPECUÁRIA) \\
\hline JOSÉ FUSCALDI CESÍLIO & $\mathrm{DF}$ & Fazendeiro e Comerciante & FAZENDAS \\
\hline $\begin{array}{l}\text { RODRIGO SOBRAL } \\
\text { ROLLEMBERG }\end{array}$ & DF & Funcionário Público & FUNCIONALISMO PÚBLICO \\
\hline $\begin{array}{l}\text { JOAQUIM DOMINGOS } \\
\text { RORIZ (RENUNCIOU EM } \\
2007 \text { ) }\end{array}$ & DF & Empresário & PECUÁRIA \\
\hline CAMILO COLA & ES & Industrial, Administrador Rural e Empresário de Tral & $\begin{array}{l}\text { AGROPECUARIA E TRANSPORTES } \\
\text { (GRUPO ITAPEMIRIM) }\end{array}$ \\
\hline JOSÉ CARLOS ELIAS & ES & Agricultor e Bancário & CAFEICULTURA \\
\hline JOÃO MIGUEL FEU ROSA & ES & Professor Universitário e Engenheiro & INDUSTRIA E TECNOLOGIA E POLÍTICA \\
\hline $\begin{array}{l}\text { PAULO ROBERTO } \\
\text { FOLETTO }\end{array}$ & ES & Médico & MEDICINA \\
\hline $\begin{array}{l}\text { MARCUS ANTONIO } \\
\text { VICENTE } \\
\end{array}$ & ES & Advogado & NÃO IDENTIFICADO \\
\hline MARCELINO AYUB FRAGA & ES & Agropecuarista & PECUÁRIA \\
\hline $\begin{array}{l}\text { NILTON GOMES OLIVEIRA } \\
\text { (NILTON BAIANO) }\end{array}$ & ES & Médico e Professor Universitário & PECUÁRIA \\
\hline $\begin{array}{l}\text { MARCELO DE ARAUJO } \\
\text { MELO - INESC }\end{array}$ & GO & Não Informou & COMÉRCIO \\
\hline LUIZ JOSE BITTENCOURT & GO & Engenheiro Civil e Professor Universitário & CONSTRUTORA \\
\hline $\begin{array}{l}\text { RAQUEL FIGUEIREDO } \\
\text { ALESSANDRI TEIXEIRA }\end{array}$ & GO & Professora Univrsitária & EDUCAÇÃO \\
\hline $\begin{array}{l}\text { SANDRO ANTONIO } \\
\text { SCODRO }\end{array}$ & GO & Administrador de Empresas & EMPRESA GRUPO MABEL - ALIMENTOS \\
\hline $\begin{array}{l}\text { LUCIA VANIA ABRAO } \\
\text { COSTA }\end{array}$ & GO & Jornalista & $\begin{array}{c}\text { FAMILIA ATUA NA RIZICULTURA E } \\
\text { ALGODOEIRA }\end{array}$ \\
\hline $\begin{array}{l}\text { DEMOSTENES LAZARO } \\
\text { XAVIER TORRES }\end{array}$ & GO & Procurador de Justiça & JUDICIÁRIO \\
\hline \begin{tabular}{|l|} 
JOVAIR DE OLIVEIRA \\
ARANTES \\
\end{tabular} & $\mathrm{GO}$ & Cirurgião Dentista & MEDICINA \\
\hline $\begin{array}{l}\text { LEONARDO MOURA } \\
\text { VILELA }\end{array}$ & GO & Médico, Pecuarista e Professor & PECUÁRIA \\
\hline JOSÉ GOMES DA ROCHA & GO & Agropecuarista e FAZENDAS & PECUÁRIA \\
\hline
\end{tabular}




\begin{tabular}{|c|c|c|c|}
\hline $\begin{array}{l}\text { ROBERTO EGÍDIO } \\
\text { BALLESTRA } \\
\end{array}$ & $\mathrm{GO}$ & $\begin{array}{l}\text { Agropecuarista, Industrial, Advogado, Comerciante } \\
\text { e Técnico em Laticínios }\end{array}$ & PECUÁRIA \\
\hline RONALDO RAMOS CAIADO & $\mathrm{GO}$ & Médico, Professor e Produtor Rural & PECUÁRIA \\
\hline $\begin{array}{l}\text { IRIS DE ARAUJO REZENDE } \\
\text { MACHADO }\end{array}$ & GO & Advogado e Agropecuarista & PECUÁRIA \\
\hline $\begin{array}{l}\text { LEANDRO VILELA } \\
\text { VELLOSO }\end{array}$ & GO & Agricultor & PECUÁRIA \\
\hline HEULER ABREU CRUVINEL & GO & Não Informou (Agrônomo) & PECUÁRIA \\
\hline $\begin{array}{l}\text { CARLOS ALBERTO LERÉIA } \\
\text { DA SILVA }\end{array}$ & $\mathrm{GO}$ & Radialista & PECUÁRIA \\
\hline $\begin{array}{l}\text { IRIS DE ARAUJO REZENDE } \\
\text { MACHADO (DONA IRIS) }\end{array}$ & GO & Administrador Público & PECUÁRIA \\
\hline $\begin{array}{l}\text { JOAO CAMPOS DE } \\
\text { ARAÚJO }\end{array}$ & GO & Delegado de Polícia de Classe Especial & SEGURANÇA PÚBLICA \\
\hline GASTÃO DIAS VIEIRA & MA & Advogado & EDUCAÇÃO \\
\hline $\begin{array}{l}\text { CARLOS ORLEANS } \\
\text { BRANDAO JUNIOR }\end{array}$ & MA & Empresário, Funcionário Público e Médico Veterinál & INDÚSTRIA E COMÉRCIO AGRÍCOLA \\
\hline EDISON LOBÃO & MA & Advogado e Jornalista & INVESTIMENTOS FINANCEIROS \\
\hline $\begin{array}{l}\text { JOSE DE RIBAMAR DA } \\
\text { COSTA ALVES }\end{array}$ & MA & Médico & MEDICINA \\
\hline $\begin{array}{l}\text { ELISEU BARROSO DE } \\
\text { CARVALHO MOURA }\end{array}$ & MA & Engenheiro Civil e Pecuarista & PECUÁRIA \\
\hline JOSÉ VIEIRA LINS & MA & Pecuarista e Empresário & PECUÁRIA \\
\hline $\begin{array}{l}\text { HÉLIO BATISTA DOS } \\
\text { SANTOS }\end{array}$ & MA & Agropecuarista & PECUÁRIA \\
\hline $\begin{array}{l}\text { FRANCISCO DE ASSIS } \\
\text { MILHOMEM }\end{array}$ & MA & Agropecuarista e Engenheiro Agrônomo & PECUÁRIA \\
\hline REMI ABREU TRINTA & MA & Médico e Cirurgião-Dentista & PECUÁRIA \\
\hline $\begin{array}{l}\text { JOAO CASTELO RIBEIRO } \\
\text { GONCALVES }\end{array}$ & MA & Advogado e Empresário & PECUÁRIA \\
\hline $\begin{array}{l}\text { JOSE ALBERTO OLIVEIRA } \\
\text { VELOSO FILHO }\end{array}$ & MA & Bacharel em Direito & PECUÁRIA \\
\hline $\begin{array}{l}\text { PAULO CELSO FONSECA } \\
\text { MARINHO }\end{array}$ & MA & Advogado e Servidor Público & PECUÁRIA \\
\hline $\begin{array}{l}\text { ANTONIO JOAQUIM } \\
\text { ARAUJO FILHO }\end{array}$ & MA & Médico & PECUÁRIA \\
\hline $\begin{array}{l}\text { PEDRO FERNANDES } \\
\text { RIBEIRO }\end{array}$ & MA & Engenheiro Civil e Bancário & PECUÁRIA \\
\hline $\begin{array}{l}\text { PEDRO NOVAIS LIMA } \\
\text { (LICENCIADO) }\end{array}$ & MA & Advogado & POLÍTICA \\
\hline ROSEANA SARNEY MURAD & MA & Socióloga & POLÍTICA \\
\hline NICE LOBÃO & MA & Servidora Pública & POLÍTICA \\
\hline $\begin{array}{l}\text { LOURIVAL MENDES DA } \\
\text { FONSECA FILHO }\end{array}$ & MA & Político e Delegado de Polícia de Classe Especial & SEGURANÇA PÚBLICA \\
\hline JOSÉ MILITÃO COSTA & $M G$ & Servidor Público & ADMINISTRAÇÃO PÚBLICA \\
\hline $\begin{array}{l}\text { CUSTODIO ANTONIO DE } \\
\text { MATTOS }\end{array}$ & MG & Servidor Público & ADMINISTRAÇÃO PÚBLICA \\
\hline $\begin{array}{l}\text { JOSE SILVA SOARES (ZE } \\
\text { SILVA) }\end{array}$ & MG & Agricultor, Agrônomo e Extensionista Rural & AGRICULTURA \\
\hline HERCULANO ANGHINETTI & $M G$ & Corretor de Seguros e Produtor Rural & CAFEICULTURA \\
\hline SILAS BRASILEIRO & $M G$ & Empresário e Produtor Rural & CAFEICULTURA \\
\hline $\begin{array}{l}\text { CARLOS CARMO } \\
\text { ANDRADES MELLES }\end{array}$ & MG & Empresário e Engenheiro Agrônomo & CAFEICULTURA \\
\hline $\begin{array}{l}\text { CARLAILE JESUS } \\
\text { PEDROSA }\end{array}$ & MG & Comerciante & COMÉRCIO \\
\hline $\begin{array}{l}\text { NARCIO RODRIGUES DA } \\
\text { SILVEIRA }\end{array}$ & $M G$ & Jornalista & COMUNICAÇÕES \\
\hline
\end{tabular}




\begin{tabular}{|c|c|c|c|}
\hline $\begin{array}{l}\text { ALEXANDRE SILVEIRA DE } \\
\text { OLIVEIRA }\end{array}$ & MG & Comerciante e Funcionário Público & CONSTRUTORA \\
\hline $\begin{array}{l}\text { ANTONIO EUSTAQUUIO } \\
\text { ANDRADE FERREIRA }\end{array}$ & MG & Engenheiro Civil e Produtor Rural & CONSTRUTORA E AGRICULTURA \\
\hline VITTORIO MEDIOLI & MG & Empresário & EMPRESA \\
\hline $\begin{array}{l}\text { DIEGO LEONARDO DE } \\
\text { ANDRADE CARVALHO }\end{array}$ & MG & Administrador de Empresas & EMPRESA (MERCOGÁS) \\
\hline MAURO RIBEIRO LOPES & MG & Empresário e Bacharel em Direito & $\begin{array}{c}\text { EMPRESA (PETROLEO) E SEGURANÇA } \\
\text { PÚBLICA }\end{array}$ \\
\hline $\begin{array}{l}\text { BONIFACIO JOSE TAMM DE } \\
\text { ANDRADA }\end{array}$ & MG & Advogado e Professor Universitário & $\begin{array}{l}\text { EMPRESA DE ENSINO, FAZENDAS, } \\
\text { INVESTIMENTOS EM VÁRIOS RAMOS }\end{array}$ \\
\hline $\begin{array}{l}\text { JOSÉ PERRELA DE } \\
\text { OLIVEIRA COSTA } \\
\end{array}$ & MG & Empresário & EMPRESA FRIGORÍFICO \\
\hline NEWTON CARDOSO & MG & Advogado, Empresário, Administrador e Sociólogo & $\begin{array}{c}\text { EMPRESA GRUPO NEWTON CARDOSO } \\
\text { (MINERAÇAO E SIDERURGIA, } \\
\text { AGROPECUÁRIA, SILVICULTURA, } \\
\text { CONSTRUÇÃO CIVIL E BEBIDAS) }\end{array}$ \\
\hline $\begin{array}{l}\text { LEONARDO LEMOS } \\
\text { BARROS QUINTÃO }\end{array}$ & MG & Empresário & $\begin{array}{c}\text { EMPRESARIO (FILHO DE POLITICO E } \\
\text { PECUARISTA ) }\end{array}$ \\
\hline RENZO DO AMARAL BRAZ & MG & Administrador de Empresas & INDUSTRIA E COMUNICAÇAO \\
\hline $\begin{array}{l}\text { MARIO LUCIO HERINGER - } \\
\text { INESC }\end{array}$ & MG & Médico e Administrador Hospitalar & MEDICINA \\
\hline $\begin{array}{l}\text { JOSE RAFAEL GUERRA } \\
\text { PINTO COELHO }\end{array}$ & MG & Médico e Professor Universitário & MEDICINA \\
\hline $\begin{array}{l}\text { GERALDO THADEU } \\
\text { PEREIRA DOS SANTOS }\end{array}$ & MG & Dentista & MEDICINA \\
\hline $\begin{array}{l}\text { ADEMIR CAMILO PRATES } \\
\text { RODRIGUES }\end{array}$ & $M G$ & $\begin{array}{l}\text { Advogado, Administrador Hospitalar, Médico } \\
\text { Ginecologista, Obstetra, do Trabalho e Legista }\end{array}$ & MEDICINA \\
\hline $\begin{array}{l}\text { MARCOS MONTES } \\
\text { CORDEIRO }\end{array}$ & MG & Médico, Professor de Medicina e Médico do Trabalh & MEDICINA \\
\hline JOSE SARAIVA FELIPE & MG & Médico e Professor Universitário & MEDICINA \\
\hline WALTER DA ROCHA TOSTA & MG & Autônomo & NÃO IDENTIFICADO \\
\hline $\begin{array}{l}\text { DIMAS FABIANO TOLEDO } \\
\text { JÚNIOR }\end{array}$ & MG & Não Informou & NÃO IDENTIFICADO \\
\hline PAULO PIAU NOGUEIRA & MG & Engenheiro Agrônomo e Produtor Rural & PECUÁRIA \\
\hline LAEL VIEIRA VARELLA & MG & Empresário & PECUÁRIA \\
\hline $\begin{array}{l}\text { ODELMO LEAO CARNEIRO } \\
\text { SOBRINHO }\end{array}$ & MG & Bancário e Produtor Rural & PECUÁRIA \\
\hline AELTON JOSÉ DE FREITAS & MG & Empresário, Engenheiro Agrônomo e Produtor Rura & PECUÁRIA \\
\hline ARACELY DE PAULA & MG & Advogado e Pecuarista & PECUÁRIA \\
\hline ROMEL ANÍZIO JORGE & MG & Agropecuarista e Empresário Rural & PECUÁRIA \\
\hline $\begin{array}{l}\text { LUIZ FERNANDO RAMOS } \\
\text { FARIA }\end{array}$ & MG & $\begin{array}{l}\text { Agropecuarista, Empresário, Administrador e } \\
\text { Engenheiro Mecânico }\end{array}$ & PECUÁRIA \\
\hline $\begin{array}{l}\text { OSMANIO PEREIRA DE } \\
\text { OLIVEIRA }\end{array}$ & MG & Advogado e Empresário & PECUÁRIA \\
\hline $\begin{array}{l}\text { MARCIO REINALDO DIAS } \\
\text { MOREIRA }\end{array}$ & MG & Economista e Analista de Orçamentos & PECUÁRIA \\
\hline JOÃO MAGALHÃES BIFANO & $M G$ & Pecuarista e Cafeicultor & PECUÁRIA E CAFEICULTURA \\
\hline \begin{tabular}{|l|} 
DOMINGOS SAVIO \\
CAMPOS RESENDE \\
\end{tabular} & MG & Médico Veterinário & PECUÁRIA E CAFEICULTURA \\
\hline MARIO DE OLIVEIRA & MG & Ministro Evangélico & PECUÁRIA E RELIGIOSO (PASTOR) \\
\hline $\begin{array}{l}\text { HUMBERTO GUIMARAES } \\
\text { SOUTO - INESC }\end{array}$ & MG & Advogado e Contador & POLÍTICA \\
\hline \begin{tabular}{|l} 
ELISEU RESENDE \\
$(07 / 02 / 1929$ a 01/02/07)
\end{tabular} & MG & Engengeiro Civil & POLÍTICA \\
\hline $\begin{array}{l}\text { RODRIGO BATISTA DE } \\
\text { CASTRO } \\
\end{array}$ & MG & Advogado e Administrador de Empresas & POLÍTICA \\
\hline
\end{tabular}




\begin{tabular}{|c|c|c|c|}
\hline $\begin{array}{l}\text { CLEUBER BRANDÃO } \\
\text { CARNEIRO }\end{array}$ & MG & $\begin{array}{l}\text { Advogado, Professor, Produtor Rural e } \\
\text { Comerciante }\end{array}$ & POLÍTICA \\
\hline DANILO DE CASTRO & MG & Bancário & POLÍTICA \\
\hline OLAVO BILAC PINTO NETO & MG & Advogado & $\begin{array}{c}\text { POLITICA E INVESTIMENTOS NO } \\
\text { AGRONEGÓCIO }\end{array}$ \\
\hline EDMAR BATISTA MOREIRA & MG & Empresário e Produtor Rural & SEGURANÇA PRIVADA \\
\hline JAIME MARTINS FILHO & MG & Advogado, Engenheiro e Empresário & SIDERURGIA E COMUNICAÇÃO \\
\hline $\begin{array}{l}\text { BERNARDO DE } \\
\text { VASCONCELLOS SANTANA }\end{array}$ & MG & Advogado & SILVICULTURA \\
\hline $\begin{array}{l}\text { JOSE SANTANA DE } \\
\text { VASCONCELOS }\end{array}$ & MG & Advogado, Empresário e Produtor Rural & SILVICULTURA \\
\hline $\begin{array}{l}\text { ROMEU FERREIRA DE } \\
\text { QUEIROZ }\end{array}$ & MG & Advogado e Empresário & SILVICULTURA \\
\hline $\begin{array}{l}\text { MARÇAL GONÇALVES } \\
\text { LEITE FILHO }\end{array}$ & MS & Advogado e Radialista & COMUNICAÇÕES \\
\hline MURILO ZAUITH & MS & Engenheiro Civil & EMPRESA ENSINO SUPERIOR \\
\hline DILSO SPERAFICO & MS & Engenheiro Agrônomo e Produtor Rural & EMPRESA GRUPO SPERAFICO - GRÃOS \\
\hline EDSON GIROTO & MS & Engenheiro Civil & FAZENDA \\
\hline $\begin{array}{l}\text { DAGOBERTO NOGUEIRA } \\
\text { FILHO - INESC }\end{array}$ & MS & Não Informou & FUNCIONALISMO PÚBLICO \\
\hline $\begin{array}{l}\text { WALDEMIR MOKA } \\
\text { MIRANDA DE BRITO }\end{array}$ & MS & Médico e Professor & MEDICINA \\
\hline $\begin{array}{l}\text { LUIZ HENRIQUE } \\
\text { MANDETTA }\end{array}$ & MS & Médico & MEDICINA \\
\hline $\begin{array}{l}\text { FLÁVIO AUGUSTO COELHO } \\
\text { DERZI (30/06/1951 a } \\
13 / 08 / 2001)\end{array}$ & MS & Agricultor, Administrador de Empresas e Pecuarista & PECUÁRIA \\
\hline $\begin{array}{l}\text { REINALDO AZAMBUJA } \\
\text { SILVA }\end{array}$ & MS & Agropecuarista & PECUÁRIA \\
\hline \begin{tabular}{|l|} 
RAMEZ TEBET (07/11/1936- \\
$17 / 11 / 2006)$
\end{tabular} & MS & Advogado & PECUÁRIA \\
\hline $\begin{array}{l}\text { ELIENE JOSÉ DE LIMA } \\
\text { (ELIENE LIMA) } \\
\end{array}$ & MT & Engenheiro Civil e Professor & CONSTRUÇAO CIVIL \\
\hline JÚLIO JOSÉ DE CAMPOS & MT & Empresário e Engenheiro Agrônomo & FAZENDAS \\
\hline $\begin{array}{l}\text { JAYME VERISSIMO } \\
\text { CAMPOS }\end{array}$ & MT & Não Informou & FAZENDAS \\
\hline $\begin{array}{l}\text { ROGERIO LUCIO SOARES } \\
\text { DA SILVA } \\
\end{array}$ & MT & Agricultor e Empresário & FAZENDAS \\
\hline BLAIRO BORGES MAGGI & MT & Não Informou & GRÃOS (SOJA) - GRUPO ANDRE MAGGI \\
\hline $\begin{array}{l}\text { JONAS PINHEIRO DA SILVA } \\
(22 / 01 / 1941 \text { a } 19 / 02 / 2008) \\
\end{array}$ & MT & Extensionista Rural e Médico Veterinário & MINERAÇÃO \\
\hline CARLOS GOMES BEZERRA & MT & Industrial, Advogado e Professor & PECUÁRIA \\
\hline HOMERO ALVES PEREIRA & MT & Produtor Rural e Técnico em Agropecuária & PECUÁRIA \\
\hline $\begin{array}{l}\text { WELLINGTON ANTONIO } \\
\text { FAGUNDES }\end{array}$ & MT & Comerciante e Médico Veterinário & PECUÁRIA E CONSTRUÇAO CIVIL \\
\hline $\begin{array}{l}\text { RICARTE DE FREITAS } \\
\text { JUNIOR }\end{array}$ & MT & Advogado & POLÍTICA \\
\hline $\begin{array}{l}\text { JADER FONTENELLE } \\
\text { BARBALHO }\end{array}$ & PA & Advogado e Empresário & COMUNICAÇÕES \\
\hline $\begin{array}{l}\text { ELCIONI THEREZINHA } \\
\text { ZAHLUTH BARBALHO }\end{array}$ & PA & Pedagoga & EDUCAÇÃO \\
\hline MÁRIO COUTO FILHO & PA & Não Informou & FAZENDA \\
\hline $\begin{array}{l}\text { JOSE BENITO PRIANTE } \\
\text { JÚNIOR }\end{array}$ & PA & Advogado & NÃO IDENTIFICADO \\
\hline $\begin{array}{l}\text { ASDRUBAL MENDES } \\
\text { BENTES }\end{array}$ & PA & Advogado e Servidor Público & NÃO IDENTIFICADO \\
\hline
\end{tabular}




\begin{tabular}{|c|c|c|c|}
\hline JOAQUIM DE LIRA MAIA & PA & Engenheiro Agrônomo & PECUÁRIA \\
\hline ANIVALDO JUVENIL VALE & PA & Agropecuarista e Servidor Público & PECUÁRIA \\
\hline OLÁVIO SILVA ROCHA & PA & Pecuarista & PECUÁRIA \\
\hline $\begin{array}{l}\text { GIOVANNI CORREA } \\
\text { QUEIROZ }\end{array}$ & PA & Agropecuarista e Médico & PECUÁRIA \\
\hline $\begin{array}{l}\text { WANDENKOLK PASTEUR } \\
\text { GONÇALVES }\end{array}$ & PA & Engenheiro Agrônomo & PECUÁRIA \\
\hline $\begin{array}{l}\text { ZENALDO RODRIGUES } \\
\text { COUTINHO JÚNIOR }\end{array}$ & PA & Advogado & POLÍTICA \\
\hline JOSUÉ BENGTSON & PA & Pastor Evangélico & RELIGIOSO (PASTOR) \\
\hline $\begin{array}{l}\text { JOSE WELLINGTON } \\
\text { ROBERTO }\end{array}$ & PB & Empresário & CONSTRUÇÃO CIVIL \\
\hline $\begin{array}{l}\text { RONALDO JOSE DA CUNHA } \\
\text { LIMA }\end{array}$ & PB & $\begin{array}{l}\text { Advogado, Promotor de Justiça, Professor de } \\
\text { Direito e Professor de Português }\end{array}$ & JUDICIÁRIO \\
\hline $\begin{array}{l}\text { VITAL DO REGO FILHO } \\
\text { (VITALZINHO) }\end{array}$ & PB & Advogado e Médico & PECUÁRIA \\
\hline ARMANDO ABILIO VIEIRA & PB & Médico & PECUÁRIA \\
\hline CARLOS MARQUES DUNGA & PB & Advogado, Pecuarista e Professor & PECUÁRIA \\
\hline ADAUTO PEREIRA DE LIMA & PB & Agropecuarista e Industrial & PECUÁRIA \\
\hline $\begin{array}{l}\text { JOSÉ TARGINO } \\
\text { MARANHÃO (ZÉ } \\
\text { MARANHÃO) }\end{array}$ & PB & Advogado, Pecuarista e Empresário & PECUÁRIA \\
\hline $\begin{array}{l}\text { BENJAMIM GOMES } \\
\text { MARANHAO NETO }\end{array}$ & PB & Cirurgião-Dentista & PECUÁRIA \\
\hline ENIVALDO RIBEIRO & PB & Advogado e Empresário & PECUÁRIA \\
\hline $\begin{array}{l}\text { AGUINALDO VELLOSO } \\
\text { BORGES RIBEIRO }\end{array}$ & PB & Administrador & POLÍTICA \\
\hline $\begin{array}{l}\text { MARCONDES IRAN } \\
\text { BENEVIDES GADELHA }\end{array}$ & PB & Médico & POLÍTICA \\
\hline $\begin{array}{l}\text { EFRAIM DE ARAUJO DE } \\
\text { MORAIS }\end{array}$ & PB & Engenheiro & POLÍTICA \\
\hline JOSÉ MUCIO MONTEIRO & PE & Engenheiro & AGROINDÚSTRIA \\
\hline $\begin{array}{l}\text { AUGUSTO RODRIGUES } \\
\text { COUTINHO DE MELO }\end{array}$ & PE & Engenheiro Civil & CONSTRUTORA \\
\hline JOSÉ SEVERIANO CHAVES & PE & Engenheiro Civil & CONSTRUTORA \\
\hline $\begin{array}{l}\text { INOCÊNCIO GOMES DE } \\
\text { OLIVEIRA }\end{array}$ & PE & Médico & LATICÍNIO E CONSTRUTORA \\
\hline RICARDO FERREIRA FIUZA & $\mathrm{PE}$ & Advogado & PECUÁRIA \\
\hline $\begin{array}{l}\text { LUIZ PIAUHYLINO DE } \\
\text { MELLO MONTEIRO }\end{array}$ & PE & Advogado & PECUÁRIA \\
\hline $\begin{array}{l}\text { JOSE MENDONÇA } \\
\text { BEZERRA (18/01/1936 a } \\
\text { 24/04/2011) }\end{array}$ & PE & $\begin{array}{l}\text { Advogado, Pecuarista, Bacharel em Direito, } \\
\text { Empresário e Avicultor }\end{array}$ & PECUÁRIA \\
\hline $\begin{array}{l}\text { OSWALDO DE SOUZA } \\
\text { COELHO }\end{array}$ & PE & Advogado, Industrial e Pecuarista & PECUÁRIA \\
\hline $\begin{array}{l}\text { ANTONIO CARLOS VIEIRA } \\
\text { DOS SANTOS (CARLOS } \\
\text { BATATA - INESC) }\end{array}$ & PE & Agropecuarista e Médico Veterinário & PECUÁRIA \\
\hline $\begin{array}{l}\text { SEVERINO SÉRGIO } \\
\text { ESTELITA GUERRA } \\
\text { (SÉRGIO GUERRA) }\end{array}$ & PE & Economista, Pecuarista e Professor & PECUÁRIA \\
\hline LUIZ GONZAGA PATRIOTA & PE & Advogado, Jornalista, Administrador de Empresas e & $\begin{array}{c}\text { PECUARIA COMUNICAÇAO INDUSTRIA } \\
\text { CONTRUTORA }\end{array}$ \\
\hline $\begin{array}{l}\text { FERNANDO BEZERRA DE } \\
\text { SOUZA COELHO FILHO }\end{array}$ & PE & Administrador de Empresas & POLÍTICA \\
\hline $\begin{array}{l}\text { SEVERINO JOSÉ } \\
\text { CAVALCANTI FERREIRA }\end{array}$ & $\mathrm{PE}$ & Membro do Poder Legislativo & POLÍTICA \\
\hline
\end{tabular}




\begin{tabular}{|c|c|c|c|}
\hline $\begin{array}{l}\text { MARCOS ANTONIO DE } \\
\text { BARROS (DE JESUS) }\end{array}$ & PE & Radialista, Pastor Evangélico e Auxiliar de Enfermad & RELIGIOSO (PASTOR) \\
\hline $\begin{array}{l}\text { JOÃO BASTOS COLAÇO } \\
\text { DIAS } \\
\end{array}$ & PE & Proprietário de Estabelecimento Comercial & SUCROALCOLEIRO \\
\hline \begin{tabular}{|l} 
JOAO VICENTE DE \\
MACÊDO CLAUDINO \\
\end{tabular} & $\mathrm{PI}$ & Economista & COMÉRCIO \\
\hline $\begin{array}{l}\text { IRACEMA MARIA } \\
\text { PORTELLA NUNES } \\
\text { NOGUEIRA LIMA }\end{array}$ & PI & Professora e Empresária & EMPRESA \\
\hline $\begin{array}{l}\text { CIRO NOGUEIRA LIMA } \\
\text { FILHO }\end{array}$ & $\mathrm{PI}$ & Advogado & EMPRESA \\
\hline \begin{tabular}{|l|} 
THEMISTOCLES DE \\
SAMPAIO PEREIRA \\
\end{tabular} & $\mathrm{PI}$ & Agricultor, Advogado e Pecuarista & PECUÁRIA \\
\hline $\begin{array}{l}\text { JULIO CESAR DE } \\
\text { CARVALHO LIMA }\end{array}$ & PI & Advogado, Professor e Produtor Rural & PECUÁRIA E CONSTRUTORA \\
\hline \begin{tabular}{|l} 
HERACLITO DE SOUZA \\
FORTES \\
\end{tabular} & $\mathrm{PI}$ & Servidor Público & POLÍTICA \\
\hline ATILA FREITAS LIRA & $\mathrm{PI}$ & Economista e Administrador de Empresas & POLÍTICA \\
\hline $\begin{array}{l}\text { MARCELO COSTA E } \\
\text { CASTRO }\end{array}$ & $\mathrm{PI}$ & Médico & POLÍTICA \\
\hline $\begin{array}{l}\text { BENEDITO DE CARVALHO } \\
\text { SÁ } \\
\end{array}$ & $\mathrm{PI}$ & Médico & POLÍTICA \\
\hline $\begin{array}{l}\text { JOSE FRANCISCO PAES } \\
\text { LANDIM }\end{array}$ & PI & Advogado e Professor & POLÍTICA E FRUTICULTURA \\
\hline \begin{tabular}{|l} 
JACOB ALFREDO \\
STOFFELS KAEFER \\
\end{tabular} & PR & Não Informou & AVICULTURA \\
\hline NELSON PADOVANI & PR & Empresário & $\begin{array}{l}\text { COMÉRCIO DE MÁQUINAS AGRÍCOLAS, } \\
\text { IMOBILIÁRIA E HOTELARIA }\end{array}$ \\
\hline HOMERO BARBOSA NETO & PR & Jornalista e Radialista & COMUNICAÇÕES \\
\hline $\begin{array}{l}\text { EDUARDO FRANCISCO } \\
\text { SCIARRA }\end{array}$ & PR & Engenheiro Civil e Empresário & CONSTRUÇÃO CIVIL \\
\hline OSMAR JOSE SERRAGLIO & PR & Advogado e Professor Universitário & CONSTRUTORA E IMOBILIÁRIA \\
\hline $\begin{array}{l}\text { RICARDO JOSÉ } \\
\text { MAGALHÃES DE BARROS - } \\
\text { INESC }\end{array}$ & PR & Engenheiro Civil e Empresário & EMPRESA \\
\hline DILCEU JOÃO SPERAFICO & PR & $\begin{array}{l}\text { Agropecuarista, Industrial Bacharel em Direito e } \\
\text { Filósofo }\end{array}$ & $\begin{array}{c}\text { EMPRESA GRUPO SPERAFICO - GRAOS } \\
\text { E PECUÁRIA }\end{array}$ \\
\hline $\begin{array}{l}\text { LUIS CARLOS JORGE } \\
\text { HAULY } \\
\end{array}$ & PR & Economista e Professor & FAZENDAS \\
\hline ÁLVARO FERNANDES DIAS & PR & Não Informou & FAZENDAS \\
\hline \begin{tabular}{|l|} 
MOACIR MICHELETTO \\
$(25 / 11 / 1942$ a $30 / 01 / 2012)$ \\
\end{tabular} & PR & $\begin{array}{l}\text { Engenheiro Agrônomo, Administrador Rural e } \\
\text { Extensionista Agrícola }\end{array}$ & GRÃOS (SOJA) \\
\hline GUSTAVO BONATO FRUET & PR & Advogado & JUDICIÁRIO \\
\hline $\begin{array}{l}\text { AIRTON BERBARDO } \\
\text { ROVEDA - INESC } \\
\end{array}$ & PR & Comerciante e Minerador & MINERAÇÃO \\
\hline LEOPOLDO COSTA MEYER & PR & Engenheiro Civil & NÃO IDENTIFICADO \\
\hline $\begin{array}{l}\text { FRANCISCO OTAVIO } \\
\text { BECKERT (CHICO DA } \\
\text { PRINCESA) } \\
\end{array}$ & PR & Contador & NÃO IDENTIFICADO \\
\hline NELSON MEURER & $\mathrm{PR}$ & Agropecuarista & PECUÁRIA \\
\hline JOSÉ RODRIGUES BORBA & PR & Pecuarista e Comerciante & PECUÁRIA \\
\hline \begin{tabular}{|l|} 
ABELARDO LUIZ LUPION \\
MELLO \\
\end{tabular} & PR & Agropecuarista e Empresário & PECUÁRIA \\
\hline \begin{tabular}{|l} 
CEZAR AUGUSTO \\
CAROLLO SILVESTRI
\end{tabular} & PR & Agropecuarista e Engenheiro Civil & PECUÁRIA \\
\hline LUIZ CARLOS SETIM & PR & Agropecuarista, Advogado e Administrador de Empl & PECUÁRIA \\
\hline ODILLIO BALBINOTTI & PR & Agropecuarista e Empresário & PECUÁRIA \\
\hline
\end{tabular}




\begin{tabular}{|c|c|c|c|}
\hline $\begin{array}{l}\text { AFFONSO ALVES DE } \\
\text { CAMARGO NETO } \\
(30 / 04 / 1929 \text { a } 25 / 03 / 2011) \\
\end{array}$ & PR & Engenheiro Civil & PECUÁRIA E FRIGORÍFICOS \\
\hline HERMES PARCIANELLO & PR & Bacharel em Ciências Contábeis & POLÍTICA \\
\hline ALEX CANZIANI SILVEIRA & $\overline{P R}$ & Registrador de Imóveis & POLÍTICA \\
\hline $\begin{array}{l}\text { WERNER WANDERER } \\
\text { (Inativo) }\end{array}$ & PR & Agricultor e Empresário & POLÍTICA E AGRICULTURA \\
\hline $\begin{array}{l}\text { MAX ROSENMANN } \\
(29 / 11 / 1944 \text { a } 25 / 10 / 2008)\end{array}$ & PR & Advogado e Empresário & POLÍTICA E EQUIDICULTURA \\
\hline IRIS XAVIER SIMÕES & PR & Radialista & SILVICULTURA \\
\hline LUCIANO PIZZATTO & PR & Empresário e Engenheiro Florestal & SILVICULTURA \\
\hline $\begin{array}{l}\text { EDMAR DE SOUZA } \\
\text { ARRUDA }\end{array}$ & PR & Economista & SILVICULTURA E CONSTRUÇÃO CIVIL \\
\hline OSMAR FERNANDES DIAS & PR & Engenheiro & SISTEMA BANCÁRIO (AGRONEGÓCIO) \\
\hline $\begin{array}{l}\text { PAULO FERNANDO FEIJO } \\
\text { TORRES }\end{array}$ & RJ & Engenheiro Mecânico & ENGENHARIA \\
\hline $\begin{array}{l}\text { ALEXANDRE JOSÉ DOS } \\
\text { SANTOS }\end{array}$ & RJ & Empresário & MINERAÇÃO \\
\hline $\begin{array}{l}\text { LEONARDO CARNEIRO } \\
\text { PICCIANI }\end{array}$ & RJ & Empresário na Área Pecuarista & PECUÁRIA \\
\hline $\begin{array}{l}\text { LUIZ EDUARDO ALMEIDA } \\
\text { DE OLIVEIRA (LUISINHO) }\end{array}$ & RJ & Político & PECUÁRIA \\
\hline JOSE AGRIPINO MAIA & $\mathrm{RN}$ & Engenheiro Civil & COMUNICAÇÕES \\
\hline $\begin{array}{l}\text { HENRIQUE EDUARDO } \\
\text { LYRA ALVES }\end{array}$ & $\mathrm{RN}$ & Bacharel em Direito & COMUNICAÇÕES \\
\hline JOÃO DA SILVA MAIA & $\mathrm{RN}$ & Economista & EMPRESA \\
\hline $\begin{array}{l}\text { NELIO SILVEIRA DIAS } \\
(22 / 01 / 1945 \text { a } 20 / 07 / 2007)\end{array}$ & $\mathrm{RN}$ & Agropecuarista & PECUÁRIA \\
\hline $\begin{array}{l}\text { CARLOS ALBERTO DE } \\
\text { SOUZA ROSADO }\end{array}$ & $\mathrm{RN}$ & $\begin{array}{l}\text { Economista, Professor Universitário e Engenheiro } \\
\text { Agrônomo }\end{array}$ & POLÍTICA \\
\hline $\begin{array}{l}\text { EXPEDITO GONÇALVES } \\
\text { FERREIRA JUNIOR }\end{array}$ & RO & Professor & EDUCAÇAO E AGRICULTURA \\
\hline $\begin{array}{l}\text { NILTON BALBINO (NILTON } \\
\text { CAPIXABA) }\end{array}$ & RO & Empresário & EMPREENDIMENTOS IMOBILIÁRIOS \\
\hline AGNALDO MUNIZ & RO & Advogado & FAZENDAS \\
\hline MAURO NAZIF RASUL & RO & Médico & MEDICINA \\
\hline $\begin{array}{l}\text { SERGIO SIQUEIRA DE } \\
\text { CARVALHO (23/03/1995 a } \\
\text { ?/?/2003) }\end{array}$ & $\mathrm{RO}$ & Médico & MEDICINA \\
\hline CONFÚCIO AIRES MOURA & $\mathrm{RO}$ & Médico, Pecuarista e Professor & PECUÁRIA \\
\hline $\begin{array}{l}\text { RUBENS MOREIRA } \\
\text { MENDES FILHO }\end{array}$ & RO & Agropecuarista e Advogado & PECUÁRIA \\
\hline $\begin{array}{l}\text { ERNANDES SANTOS } \\
\text { AMORIM }\end{array}$ & $\mathrm{RO}$ & Pecuarista & PECUÁRIA \\
\hline IVO NARCISO CASSOL & RO & Empresário & $\begin{array}{c}\text { PECUARIA, PRODUÇAO DE ENERGIA E } \\
\text { MADEIREIRA }\end{array}$ \\
\hline REDITÁRIO CASSOL & RO & Agropecuarista e Industrial & $\begin{array}{c}\text { PECUARIA, PRODUÇAO DE ENERGIA E } \\
\text { MADEIREIRO }\end{array}$ \\
\hline $\begin{array}{l}\text { MARINHA CELIA ROCHA } \\
\text { RAUPP DE MATOS -INESC }\end{array}$ & RO & Servidora Pública, Professora, Técnica em Assunto & POLÍTICA \\
\hline $\begin{array}{l}\text { RAUL DA SILVA LIMA } \\
\text { SOBRINHO }\end{array}$ & RR & Administrador & COMÉRCIO \\
\hline LUIZ BARBOSA ALVES & $\mathrm{RR}$ & Empresário & CONSTRUTORA \\
\hline $\begin{array}{l}\text { JOHNATHAN PEREIRA DE } \\
\text { JESUS }\end{array}$ & $\mathrm{RR}$ & Empresário & FAZENDA \\
\hline $\begin{array}{l}\text { ALCESTE MADEIRA DE } \\
\text { ALMEIDA }\end{array}$ & $\mathrm{RR}$ & Médico & MEDICINA \\
\hline
\end{tabular}




\begin{tabular}{|c|c|c|c|}
\hline $\begin{array}{l}\text { FRANCISCO MOZARILDO } \\
\text { DE MELO CAVALCANTI }\end{array}$ & RR & Médico e Professor & MEDICINA \\
\hline $\begin{array}{l}\text { SALOMÃO AFONSO DE } \\
\text { SOUZA CRUZ }\end{array}$ & $\mathrm{RR}$ & Engenheiro & MINERAÇÃO \\
\hline $\begin{array}{l}\text { LUCIANO DE SOUZA } \\
\text { CASTRO }\end{array}$ & RR & Economista e Administrador & NÃO IDENTIFICADO \\
\hline $\begin{array}{l}\text { FRANCISCO DE ASSIS } \\
\text { RODRIGUES }\end{array}$ & RR & Engenheiro Agrônomo & PECUÁRIA \\
\hline $\begin{array}{l}\text { PAULO CESAR JUSTO } \\
\text { QUARTIERO }\end{array}$ & RR & Engenheiro Agrônomo & RIZICULTURA \\
\hline GIOVANI CHERINI & RS & Tecnólogo em Cooperativismo & AGROINDÚSTRIA \\
\hline PAULO JOSE GOUVEA & RS & Radialista & COMUNICAÇÕES \\
\hline $\begin{array}{l}\begin{array}{l}\text { JULIO CESAR REDECKER } \\
(12 / 07 / 1956 \text { a 17/07/2007) }\end{array} \\
\end{array}$ & RS & Advogado, Professor Universitário e Empresário & COUREIRO-CALÇADISTA \\
\hline \begin{tabular}{|l|} 
RENATO DELMAR \\
MOLLING
\end{tabular} & RS & $\begin{array}{l}\text { Professor, Administrador, Industriário, Auxiliar de } \\
\text { Escritório e Assessor Político }\end{array}$ & COUREIRO-CALÇADISTA E MOVELEIRO \\
\hline $\begin{array}{l}\text { ONYX DORNELLES } \\
\text { LORENZONI }\end{array}$ & RS & Empresário e Médico Veterinário & EMPRESA (VETERINÁRIA) \\
\hline DARCISIO PAULO PERONDI & RS & Médico & MEDICINA \\
\hline \begin{tabular}{|l|} 
ADOLFO ANTONIO FETTER \\
JUNIOR
\end{tabular} & RS & $\begin{array}{l}\text { Administrador de Empresas e Engenheiro } \\
\text { Agrônomo }\end{array}$ & PECUÁRIA \\
\hline ELISEU LEMOS PADILHA & RS & Advogado e Empresário & POLÍTICA \\
\hline \begin{tabular}{|l} 
DARCI POMPEO DE \\
MATTOS \\
\end{tabular} & RS & Advogado e Bancário & POLÍTICA \\
\hline SÉRGIO IVAN MORAES & RS & Não Informou & POLÍTICA \\
\hline VILSON LUIZ COVATTI & RS & Advogado & POLÍTICA \\
\hline $\begin{array}{l}\text { CEZAR AUGUSTO } \\
\text { SCHIRMER }\end{array}$ & RS & Advogado e Professor Universitário & POLÍTICA \\
\hline TELMO JOSÉ KIRST & RS & Industrial e Advogado & POLÍTICA \\
\hline $\begin{array}{l}\text { NELSON MARCHEZAN } \\
\text { JÚNIOR }\end{array}$ & RS & Advogado & POLÍTICA \\
\hline $\begin{array}{l}\text { JOÃO AUGUSTO RIBEIRO } \\
\text { NARDES }\end{array}$ & RS & Administrador de Empresas & POLÍTICA \\
\hline $\begin{array}{l}\text { JOSE AFONSO EBERT } \\
\text { HAMM }\end{array}$ & RS & Engenheiro Agrônomo & RIZICULTURA \\
\hline LUIS CARLOS HEINZE & RS & Engenheiro Agrônomo e Produtor Rural & TRITICULTURA \\
\hline EDSON BEZ DE OLIVEIRA & SC & Agricultor, Professor, Bancário e Contador & AGRICULTRA \\
\hline VALDIR COLATTO & SC & Engenheiro Agrônomo & AGROINDÚSTRIA \\
\hline ODACIR ZONTA & SC & $\begin{array}{l}\text { Contabilista, Professor, Escriturário, Secretário e } \\
\text { Agricultor Cooperativista }\end{array}$ & ALIMENTOS FRIGORÍFICOS \\
\hline $\begin{array}{l}\text { LEONEL ARCANGELO } \\
\text { PAVAN } \\
\end{array}$ & SC & Comerciante & CONSTRUTORA \\
\hline CELSO MALDANER & $S C$ & Economista e Empresário & EMPRESA E FAZENDAS \\
\hline $\begin{array}{l}\text { JOAO ALBERTO } \\
\text { PIZZOLATTI }\end{array}$ & SC & Engenheiro Civil e Servidor Público & FAZENDA \\
\hline $\begin{array}{l}\text { ESPERIDIAO AMIN HELOU } \\
\text { FILHO }\end{array}$ & SC & Advogado, Professor Universitário e Administrador & INDUSTRIA TEXTIL \\
\hline HUGO MATHIAS BIEHL & $S C$ & Administrador de Empresas e Técnico Agrícola & NÃO IDENTIFICADO \\
\hline CASILDO JOÃO MALDANER & SC & Comerciante & PECUÁRIA \\
\hline RONALDO JOSÉ BENEDET & SC & Advogado & PECUÁRIA \\
\hline GERVASIO JOSE DA SILVA & SC & Empresário, Contador e Corretor de Imóveis & POLÍTICA \\
\hline JOÃO BATISTA MATOS & SC & Administrador de Empresas e Professor & SILVICULTURA \\
\hline $\begin{array}{l}\text { JORGE ALBERTO TELES } \\
\text { PRADO }\end{array}$ & SE & Médico & MEDICINA \\
\hline \begin{tabular}{|l} 
JOSÉ TELES DE \\
MENDONÇA \\
\end{tabular} & SE & Agropecuarista e Empresário & PECUÁRIA \\
\hline
\end{tabular}




\begin{tabular}{|c|c|c|c|}
\hline $\begin{array}{l}\text { JOSÉ CLEONÂNCIO DA } \\
\text { FONSECA } \\
\end{array}$ & SE & Agropecuarista e Citricultor & PECUÁRIA \\
\hline JOSÉ CARLOS MACHADO & SE & Engenheiro Civil e Empresário & PECUÁRIA \\
\hline JOÃO BOSCO DA COSTA & SE & Administrador de Empresas & POLÍTICA \\
\hline JOSÉ ALMEIDA LIMA & SE & Advogado e Professor de Direito & POLÍTICA \\
\hline $\begin{array}{l}\text { RICARDO NAGIB IZAR } \\
(28 / 06 / 1938-02 / 05 / 2008+)\end{array}$ & SP & Advogado & ADMINISTRAÇÃO PÚBLICA \\
\hline $\begin{array}{l}\text { FRANCISCO MARCELO } \\
\text { ORTIZ FILHO }\end{array}$ & SP & Advogado e Professor Universitário & EDUCAÇAO \\
\hline JUNJI ABE & $\mathrm{SP}$ & Empresário Rural e Consultor & HORTIFRUTIGRANGEIROS \\
\hline $\begin{array}{l}\text { JULIO FRANCISCO } \\
\text { SEMEGHINI NETO }\end{array}$ & SP & Engenheiro Eletroeletrônico & INDUSTRIA E TECNOLOGIA \\
\hline RICARDO IZAR JUNIOR & SP & Economista e Empresário & INVESTIMENTOS FINANCEIROS \\
\hline \begin{tabular}{|l} 
ALOYSIO NUNES \\
FERREIRA FILHO
\end{tabular} & $\mathrm{SP}$ & Advogado e Procurador do Estado de São Paulo & JUDICIÁRIO \\
\hline $\begin{array}{l}\text { JOSE ARISTODEMO } \\
\text { PINOTTI (20/12/1934 a + } \\
\text { 01/07/2009) }\end{array}$ & SP & Médico & MEDICINA \\
\hline $\begin{array}{l}\text { LUIZ ANTONIO DE } \\
\text { MEDEIROS NETO }\end{array}$ & SP & Metalúrgico e Torneiro Mecânico & METALURGIA \\
\hline $\begin{array}{l}\text { ARNALDO CALIL PEREIRA } \\
\text { JARDIM }\end{array}$ & SP & Engenheiro Civil e Professor & MINAS E ENERGIA \\
\hline $\begin{array}{l}\text { RUBENEUTON OLIVEIRA } \\
\text { LIMA (NEUTON LIMA) }\end{array}$ & SP & Advogado & NÃO IDENTIFICADO \\
\hline $\begin{array}{l}\text { VALDEMAR CORAUCI } \\
\text { SOBRINHO }\end{array}$ & SP & Advogado, Professor Universitário e Técnico em Co & NÃO IDENTIFICADO \\
\hline $\begin{array}{l}\text { EDSON EDINHO COELHO } \\
\text { ARAÚJO }\end{array}$ & SP & Advogado e Professor & NÃO IDENTIFICADO \\
\hline $\begin{array}{l}\text { JOSE CARLOS VAZ DE } \\
\text { LIMA }\end{array}$ & SP & Agente Fiscal de Rendas & NÃO IDENTIFICADO \\
\hline $\begin{array}{l}\text { ANTONIO DUARTE } \\
\text { NOGUEIRA JUNIOR }\end{array}$ & SP & Agricultor, Pecuarista e Engenheiro Agrônomo & PECUÁRIA \\
\hline $\begin{array}{l}\text { PAULO CESAR DE } \\
\text { OLIVEIRA LIMA } \\
\end{array}$ & $\mathrm{SP}$ & $\begin{array}{l}\text { Engenheiro Civil, Jornalista, Pecuarista, } \\
\text { Empresário e Educador }\end{array}$ & PECUÁRIA (GRUPO PAULO LIMA) \\
\hline ETIVALDO VADÃO GOMES & SP & Pecuarista e Empresário & PECUÁRIA \\
\hline $\begin{array}{l}\text { PAULO ROBERTO GOMES } \\
\text { MANSUR } \\
\end{array}$ & SP & Radialista, Empresário e Engenheiro Eletrônico & PECUÁRIA \\
\hline $\begin{array}{l}\text { ALINE LEMOS CORRÊA DE } \\
\text { OLIVEIRA ANDRADE }\end{array}$ & $\mathrm{SP}$ & Empresária & PECUÁRIA \\
\hline $\begin{array}{l}\text { MILTON ANTONIO } \\
\text { CASQUEL MONTI }\end{array}$ & SP & Economista e Empresário & POLÍTICA \\
\hline $\begin{array}{l}\text { FRANCISCO GRAZIANO } \\
\text { NETO }\end{array}$ & $\mathrm{SP}$ & $\begin{array}{l}\text { Agricultor, Professor Universitário e Engenheiro } \\
\text { Agrônomo }\end{array}$ & POLÍTICA \\
\hline $\begin{array}{l}\text { JOSE ALDO REBELO } \\
\text { FIGUEIREDO } \\
\end{array}$ & SP & Jornalista & POLÍTICA \\
\hline RODRIGO GARCIA & SP & Não Informou & POLÍTICA \\
\hline $\begin{array}{l}\text { LUIZ ANTONIO FLEURY } \\
\text { FILHO }\end{array}$ & SP & Advogado & SEGURANÇA PÚBLICA \\
\hline $\begin{array}{l}\text { JOAO EDUARDO DADO } \\
\text { LEITE DE CARVALHO } \\
\end{array}$ & SP & Agente Fiscal de Rendas & SINDICALISMO \\
\hline NELSON MARQUEZZELLI & SP & Agricultor e Empresário & SUCROALCOLEIRO \\
\hline $\begin{array}{l}\text { JOÃO HERRMANN NETO } \\
(07 / 03 / 1946 \text { a 12/04/2009) } \\
\end{array}$ & SP & Engenheiro Agrônomo & SUCROALCOLEIRO \\
\hline CESAR HANNA HALUM & TO & Médico Veterinário & COMÉRCIO DE ALIMENTÍCIOS \\
\hline $\begin{array}{l}\text { MARCELO DE CARVALHO } \\
\text { MIRANDA (Cassado, não } \\
\text { assumiu) }\end{array}$ & TO & Empresário & EMPRESA \\
\hline
\end{tabular}




\begin{tabular}{|c|c|c|c|}
\hline $\begin{array}{l}\text { LAUREZ DA ROCHA } \\
\text { MOREIRA }\end{array}$ & TO & Advogado & FAZENDAS \\
\hline DARCI MARTINS COELHO & TO & Advogado e Professor Universitário & JUDICIÁRIO \\
\hline $\begin{array}{l}\text { LAZARO BOTELHO } \\
\text { MARTINS } \\
\end{array}$ & TO & Pecuarista e Empresário & PECUÁRIA \\
\hline KATIA REGINA DE ABREU & TO & Empresária Rural e Psicóloga & PECUÁRIA \\
\hline $\begin{array}{l}\text { JOSÉ DOS SANTOS } \\
\text { FREIRE JUNIOR }\end{array}$ & TO & Agropecuarista e Economista & PECUÁRIA \\
\hline $\begin{array}{l}\text { IRAJÁ SILVESTRE FILHO } \\
\text { (IRAJÁ ABREU) }\end{array}$ & TO & Empresário e Produtor Rural & PECUÁRIA \\
\hline $\begin{array}{l}\text { VICENTE ALVES DE } \\
\text { OLIVEIRA }\end{array}$ & TO & $\begin{array}{l}\text { Agropecuarista, Empresário e Piloto Comercial } \\
\text { Multimotores e IFR }\end{array}$ & PECUÁRIA \\
\hline IGOR PUGLIESI AVELINO & TO & Agropecuarista e Empresário & PECUÁRIA \\
\hline JOSÉ MAURÍCIO RABELO & TO & Radialista, Professor, Empresário e Lavrador & PECUÁRIA \\
\hline $\begin{array}{l}\text { JOAO BATISTA DE JESUS } \\
\text { RIBEIRO }\end{array}$ & TO & Empresário & PECUÁRIA \\
\hline $\begin{array}{l}\text { LEOMAR DE MELO } \\
\text { QUINTANILHA }\end{array}$ & TO & Bancário & PECUÁRIA \\
\hline $\begin{array}{l}\text { RAIMUNDO COIMBRA } \\
\text { JÚNIOR }\end{array}$ & TO & Jornalista & PECUÁRIA \\
\hline OSVALDO DE SOUZA REIS & TO & Empresário & $\begin{array}{l}\text { PECUARIA E EMPRESARIO (RAMO NAO } \\
\text { IDENTIFICADO) }\end{array}$ \\
\hline $\begin{array}{l}\text { CARLOS EDUARDO } \\
\text { TORRES GOMES } \\
\text { (EDUARDO GOMES) }\end{array}$ & TO & Empresário e Funcionário Público & POLÍTICA \\
\hline $\begin{array}{l}\text { AMARILDO MARTINS DA } \\
\text { SILVA }\end{array}$ & TO & Empresário e Técnico em Contabilidade & RELIGIOSO (PASTOR) \\
\hline \multicolumn{4}{|l|}{ TOTAL } \\
\hline \multicolumn{4}{|c|}{\begin{tabular}{|l|l|l|} 
LEGENDA DA TABELA & \\
\end{tabular}} \\
\hline \multicolumn{4}{|c|}{ RURALISTAS QUE DECLARARAM PROFISSÕES LIGADAS A ATIVIDADE AGROPECUÁRIA OU AGRONEGÓCIO } \\
\hline \multicolumn{4}{|c|}{ PARLAMENTARES RURALISTAS FALECIDOS } \\
\hline \multicolumn{4}{|c|}{ PARLAMENTARES QUE TEM PARENTES NA POLÍTICA } \\
\hline RURALISTAS IDENTIFICADC & S FOR & DA LISTA DO DIAP & \\
\hline
\end{tabular}

